\title{
MULTICOMPONENT, MULTIPHASE FLOW IN POROUS MEDIA WITH TEMPERATURE VARIATION
}

SUPRI TR 71

$\mathrm{DOE} / \mathrm{BC} / 14126--17$

DE9 I 002201

\section{By}

J. S. Wingard

F. M. Orr, Jr.

October 1990

Work Performed Under Contract No. FG19-87BC14126

Piepared for

U.S. Department of Energy

Assistant Secretary for Fossil Energy

Thomas B. Reid, Project Manager

Bartlesville Project Office

P.O. Box 1398

Bartlesville, OK 74005

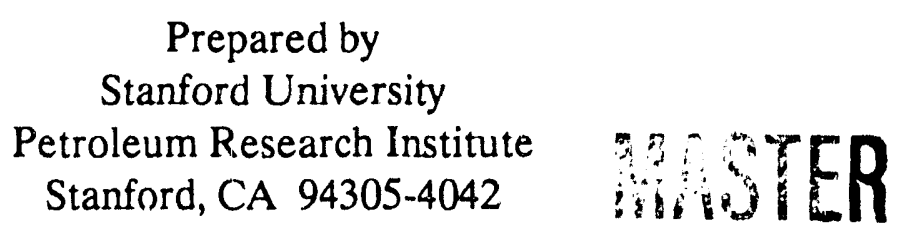

Prepared by

Stanford University

etroleum 


\section{TABLE OF CONTENTS}

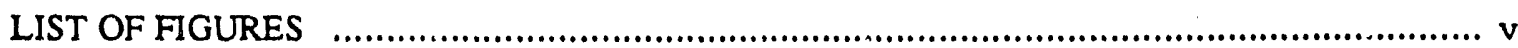

LIST OF TABLES

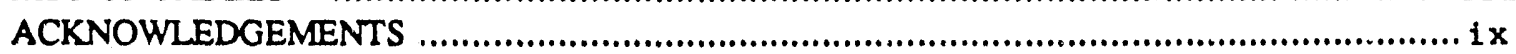

ABSTRACT

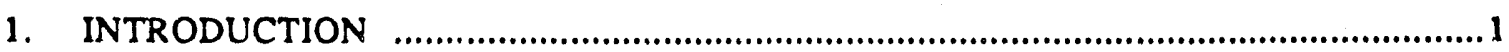

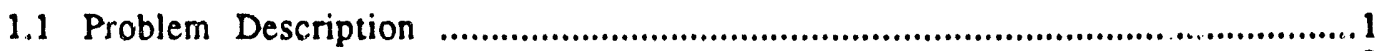

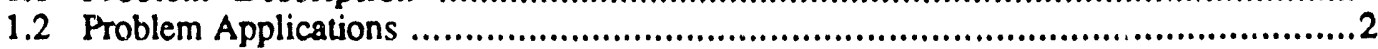

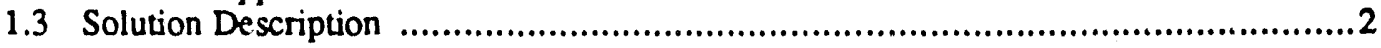

1.3.1 The Reservoir as a Chromatograph ........................................................3

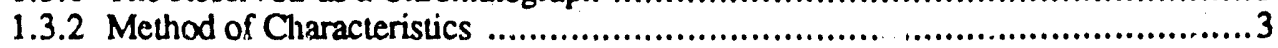

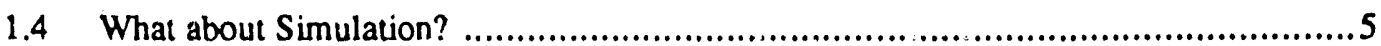

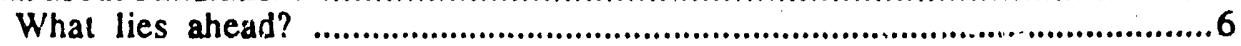

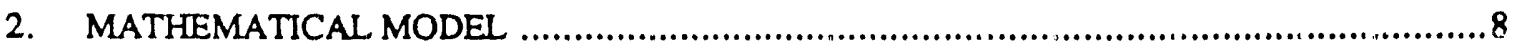

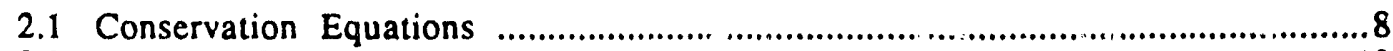

2.2 Method of Characieristics ......................................................................... 10

2.2.1 Calculation of Component and Enthalpy Velaities .................................. 12

Cuherence and the Eigenvalue Problem ................................................ 14

What is Coherence? ............................................................................ 14

Formulation of the Eigenvalue Problem ........................................... 14

2.3 Evaluation of the Derivatives il the Eigenvalue Matrix ................................... 16

2.3.1 Fluid Property Calculation .................................................................... 17

2.3.2 Calculating the Derivativer for the Eigenvalue Problem .............................. 18

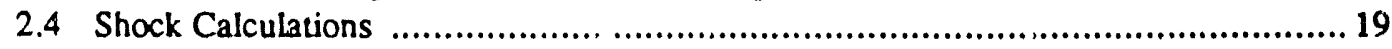

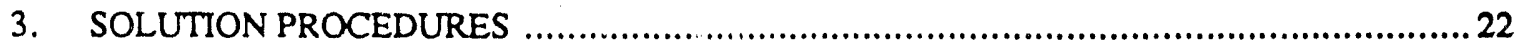

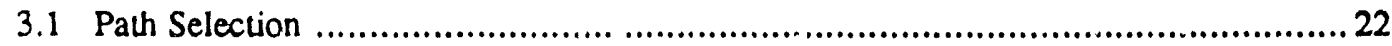

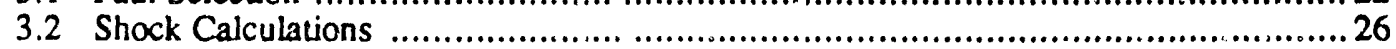

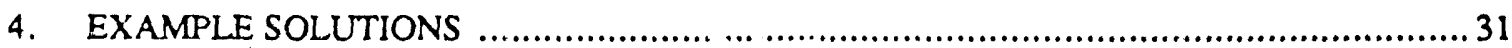

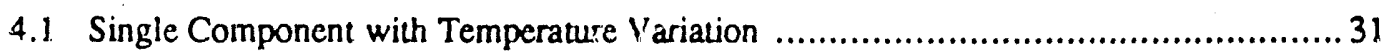

4.1.1 Problem Formulation ......................................................................... 31

Single-Phase Regions .............................................................. 32

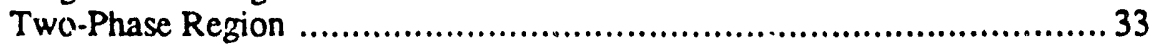

Connecting the Single and Two-Phase Regions .................................... 35

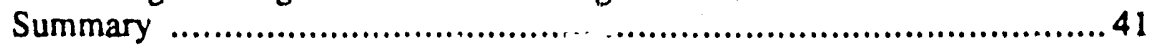

4.2 Two Component - Three-Phase Systems …......................................... 42

4.2.1 Problem Formulation .................................................................42

4.2.2 Example Cases .................................................................................45

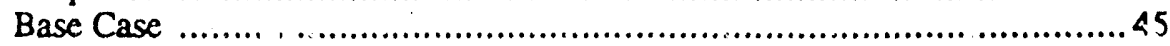

4.2.3 Types of Displacement Patterns ...................................................... 52

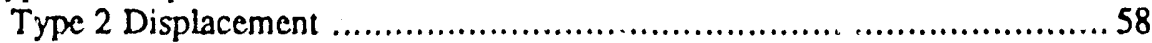

Type 3 Displacement ........................................................... 56

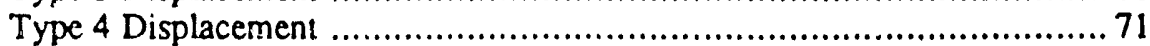

4.2.4 Solution Procedure .......................................................................... 72

4.2.5 Temperature Profile in the Sieam-Oil Region ..................................... 77

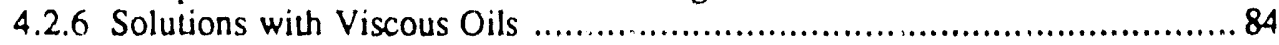

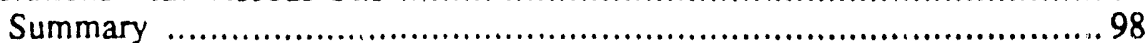


4.3 Three Component - Three-Phase Systems .......................................................100

4.3.1 Problem Formulation ......................................................................

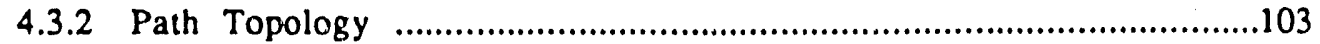

4.3.3 Solution Problems ….................................................................107

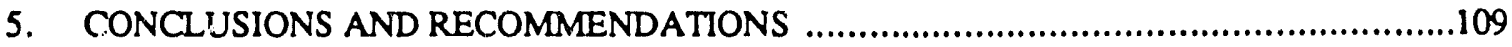

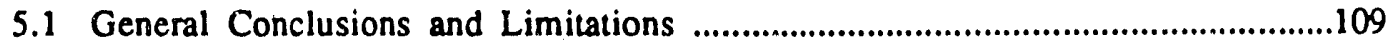

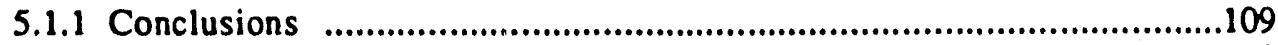

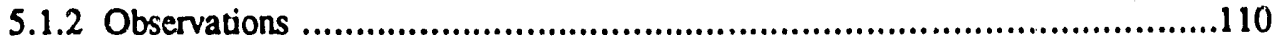

5.1.3 Limitations ...........................................................................110

5.2 Specific Applications ............................................................................111

5.2.1 Single Comonent with Temperature Variation ......................................111

5.2.2 Two-Component, Three-Phase Immiscible System ..................................112

5.2.3 Three-Component, Three-Phase System with Temperature Variation ...............113

5.3 Future Recommendations ..................................................................113

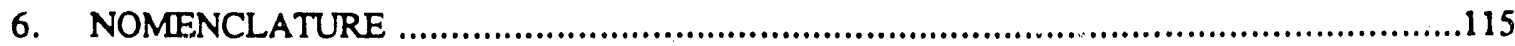

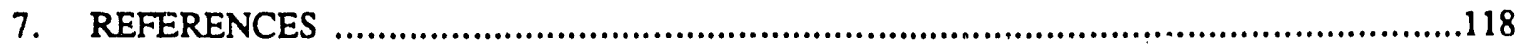

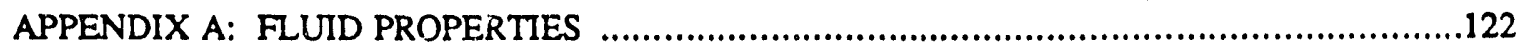

A.1 Steam-Oil-Water Problem ......................................................................122

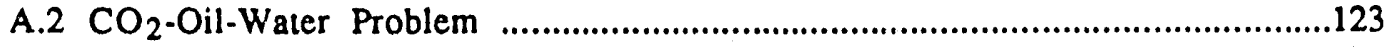

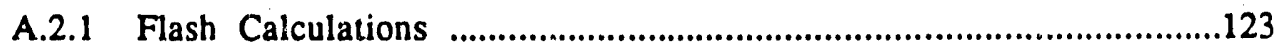

A.2.2 Fluid Properties .......................................................................142

APPENDIX B: ENTHALPY BALAITCE ACROSS A DISCONTINUITY …..............................144

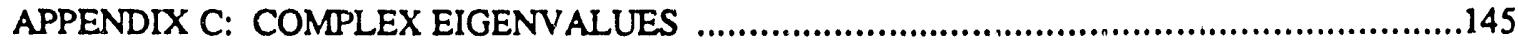

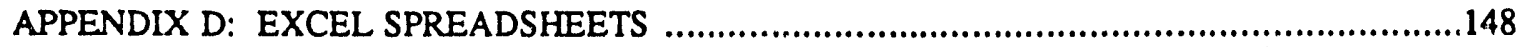

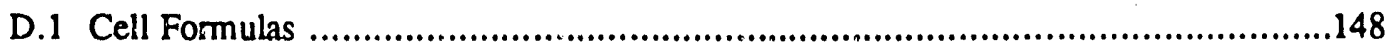

D.2 Changes in Injection Temperature ............................................................148

D.3 Changes in Initial Temperature ...............................................................193

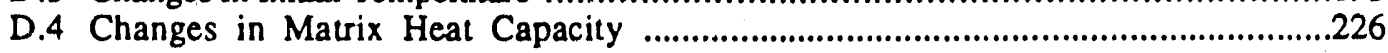

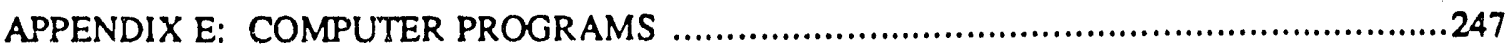




\section{LIST OF FIGURES}

2.1 One-to-one mapping from the space of independent variables, $x$ and $t$ to the space of dependent variables, $C_{1}$ and $C_{2}$.

2.2 Mapping from the space of independent variables, $x$ and t to the space of dependent variables, $C_{1}$ and $C_{2}$. 11

2.3 Map of constant state region from a region in $x-t$ space to a single point in $C_{1}, C_{2}$ space.

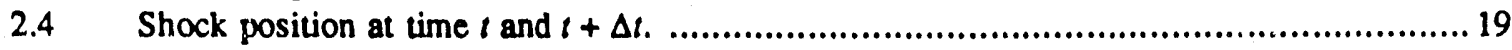

3.1 Illegal composition path for a ternary displacement as the path moves from initial conditions to injection conditions.

3.2 Legal path switch at an equal eigenvalue point for a tenary displacement as the path moves from injection conditions to initial conditions. ..................................... 25

3.3 Legal path switch for a ternary displacement as the path moves from injection conditions to initial conditions.

3.4 Illegal path switch for a temary displacement as the path moves from injection conditions to initial conditions.............................................................................. 27

3.5 Tangent construction for Buckley-Leverett shock front that illustrates how the tangent construction matches shock and wave velocities.

4.1 Solution line in hodograph space for steam injected into liquid water at constant pressure.

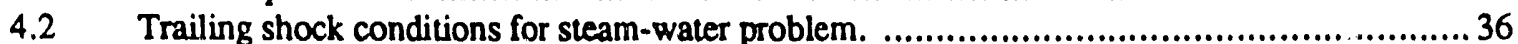

4.3 Wave and shock velocities for trailing shock in steam-water displacement. ........................ 37

4.4 Saturation profile after 1.0 pore volumes of steam injection into water at injection temperatures from $500 \mathrm{~K}-1000 \mathrm{~K}$. ........................................................ 39

4.5 Saturation profile after 1.0 pore volumes steam injection into water at initial temperatures from $300 \mathrm{~K}-450 \mathrm{~K}$.

4.6 Saturation profile after 1.0 pore volumes steam injection into water where the matrix heat capacity varies from $25.0(\mathrm{~kJ} / \mathrm{kg}-\mathrm{K})$ to $0.2(\mathrm{~kJ} / \mathrm{kg}-\mathrm{K})$.

4.7 Hypothetical solution to the two-component, three-phase problem showing the different flow regions.

4.8 Plot of eigenvalues along a constant temperature path ir the water-oil region. ..........................44

4.9 Composition path for the injection of $100 \%$ steam at $650 \mathrm{~K}$ into $94.5 \%$ oil and $6.5 \%$ water at $390.19 \mathrm{~K}$.

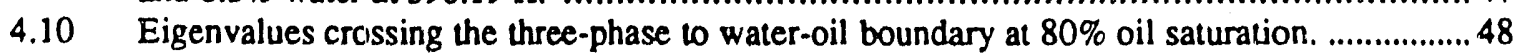

4.11 Saturation profile for the injection of 1.0 pore volumes of $100 \%$ steam at $650 \mathrm{~K}$

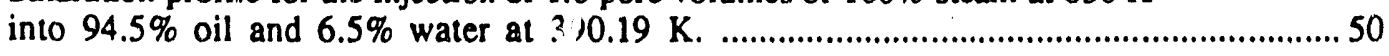

4.12 Temperature profile for the injection $\mathrm{r}$. 1.0 pore volumes of $100 \%$ stcam at $650 \mathrm{~K}$ into $94.5 \%$ oil and $6.5 \%$ water at $390.19 \mathrm{~K}$.

4.13 Flow velocity profile for the injection of 1.0 pore volumes of $100 \%$ steam at

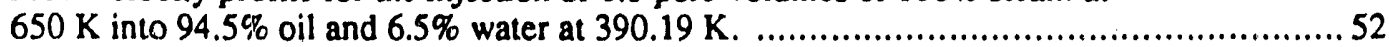

4.14 Composition path that moves between two arbitrary points in the three-phase region.

4.15 Type 2 composition path that moves between two arbitrary points in the three-phase region.

4.16 Type 3 composition path that moves between two arbitrary points in the three-phase region.

4.17 Type 3 composition path that moves between two arbitrary points in the three-phase region.

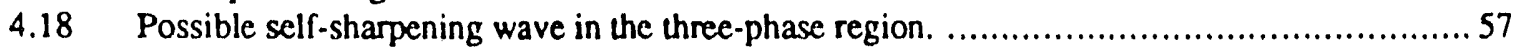


4.19 Limitirg case self-sharpening wave in the three-phase region. ..................................... 58

4.20 Adjusted Type 1 composition path that moves between two arbitrary points

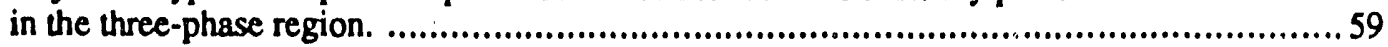

4.21 Adjusted Type 2 composition path that moves between two arbitrary points

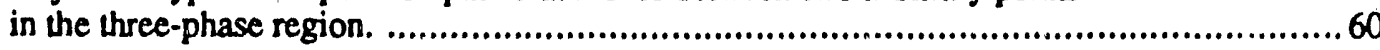

4.22 Adjusted Type 3 composition path that moves between two arbitrary points in the three-phase region. .....................................................................................61

4.23 Composition path for the injection of $100 \%$ steam at $650 \mathrm{~K}$ into $92.4 \%$ oil and $7.6 \%$ water at $412.09 \mathrm{~K}$.

4.24 Temperature profile for the injection of 1.0 pore volumes of $100 \%$ steam at $650 \mathrm{~K}$ into $92.4 \%$ oila nd $7.6 \%$ water at $412.09 \mathrm{~K}$. ..................................................63'

4.25 Flow velocity profile for the injection of 1.0 pore volumes of $100 \%$ steam at $650 \mathrm{~K}$ into $92.4 \%$ oil and $7.6 \%$ water at $412.09 \mathrm{~K}$.

4.26 Saturation profile for the injection of 1.0 pore volumes of $100 \%$ steam at. $650 \mathrm{~K}$ into $92.4 \%$ oil and $7.6 \%$ water at $412.09 \mathrm{~K}$.

4.27 Composition path for the injection of $100 \%$ steam at $650 \mathrm{~K}$ into $91.6 \%$ oil and $8.4 \%$ water at $428.62 \mathrm{~K}$.

4.28 Saturation profile for the injection of $100 \%$ steam at $650 \mathrm{~K}$ into $91.6 \%$ oil and $8.4 \%$ water at $428.62 \mathrm{~K}$.

4.29 Temperature profile for the injection of $100 \%$ steam at $650 \mathrm{~K}$ into $91.6 \%$ oil and $8.4 \%$ water at $428.62 \mathrm{~K}$.

4.30 Flow velocity profile for the injection of $100 \%$ steam at $650 \mathrm{~K}$ into $9.16 \%$ oil and $8.4 \%$ water at $428.62 \mathrm{~K}$.

4.31 Three-phase region of the steam-oil-water displacements showing the regions where the fath path eigenvalues and water saturation permit a jump ints the water-oil region.

4.32 Composition path for the injection of $100 \%$ steam at $650 \mathrm{~K}$ into $88.1 \%$ oil and $11.9 \%$ water at $4.3379 \mathrm{~K}$.

4.33 Saturation profile for the injection of 1.0 pore volumes of $100 \%$ steam at $650 \mathrm{~K}$ into $88.1 \%$ oil and $11.9 \%$ water at $433.79 \mathrm{~K}$.

4.34 Temperature profile for the injection of 1.0 pore volumes of $100 \%$ stearn at $650 \mathrm{~K}$ into $88.1 \%$ oil and $11.9 \%$ water at $433.79 \mathrm{~K}$.

4.35 Flow velocity profile for the injection of 1.0 pore volumes of $100 \%$ steam at $650 \mathrm{~K}$ into $88.1 \%$ oil and $11.9 \%$ water at $433.79 \mathrm{~K}$.

4.36 Composition path for the injection of $100 \%$ steam at $650 \mathrm{~K}$ into $94.8 \%$ oil and $5.24 \%$ water at $402.13 \mathrm{~K}$ with a temperature wave in the steam-oil region.

4.37 Temperature profile for the injection of 1.0 pore volumes of $100 \%$ steam at $650 \mathrm{~K}$ into $94.8 \%$ oil and $5.24 \%$ water at $402.13 \mathrm{~K}$ with a temperature wave in the steam-oil region.

4.38 Flow velocity profile for the injection of 1.0 pore volumes of $100 \%$ steam at $650 \mathrm{~K}$ into $94.8 \%$ oil and $5.24 \%$ water at $402.13 \mathrm{~K}$ with a temperature wave in the steam-oil region.

4.39 Saturation profile for the injection of 1.0 pore volumes of $100 \%$ steam at $650 \mathrm{~K}$ into $94.8 \%$ oil and $5.24 \%$ water at $402.13 \mathrm{~K}$ with a temperature wave in the steam-oil region.

4.40 Saturation profiles in the steam-oil region for the injection of 1.0 pore volumes of $100 \%$ steam at $650 \mathrm{~K}$ into reservoirs with porosities from 0.05 to 0.5 .

4.41 Temperature profiles in the steam-oil region for the injection of 1.0 pore volumes of $100 \%$ steam at $650 \mathrm{~K}$ into reservoirs with porosities from 0.05 to 0.5 .

4.42 Viscosities for water, steam, and three different oils as a function of temperature irom $275 \mathrm{~K}$ to $1000 \mathrm{~K}$.

4.43 Composition path for the injection of $100 \%$ steam at $650 \mathrm{~K}$ into $99.9 \%$ Heavy

Oil $\# 1$ and $0.1 \%$ water at $458.95 \mathrm{~K}$. 
4.44 Saturation profile for the injection of $100 \%$ steam at $650 \mathrm{~K}$ into $99.9 \%$ Heavy

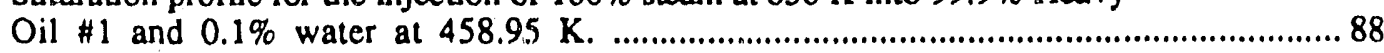

4.45 Temperature profile for the injection of $100 \%$ steam at $650 \mathrm{~K}$ ints $99.9 \%$ Heavy

Oil \#1 and $0.1 \%$ water at $458.95 \mathrm{~K}$. ................................................................. 90

4.46 Flow velocity profile for the injection of $100 \%$ steam at $650 \mathrm{~K}$ intw $99.9 \%$ Heavy

Oil $\# 1$ and $0.1 \%$ water at $458.95 \mathrm{~K}$. ..............................................................91

4.47 Composition path for the injection of $100 \%$ steam at $650 \mathrm{~K}$ into $88.1 \%$ Heavy

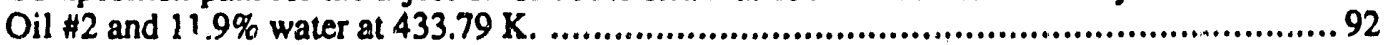

4.48 Saturation profile for the injection of $100 \%$ steam at $650 \mathrm{~K}$ into $88.1 \%$ Heavy

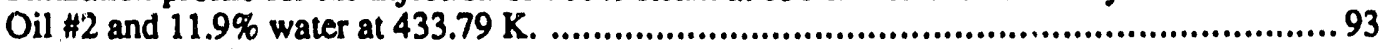

4.49 Temperature profile for the injection of $100 \%$ steam at $650 \mathrm{~K}$ into $88.1 \%$ Heavy

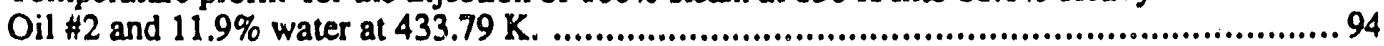

4.50 Flow velocity profile for the injection of $100 \%$ steam at $650 \mathrm{~K}$ into $88.1 \%$ Heavy

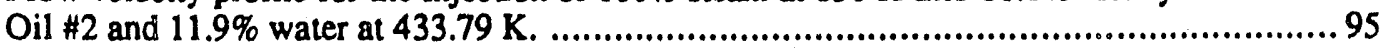

4.51 Phase diagram for a system of $\mathrm{CO}_{2}-$ Oil-Water at temperatures below the saturation temperature of water. .........................................................................101

4.52 Phase diagram for a system of $\mathrm{CO}_{2}$-Oil-Water at temperatures below the saturation temperature of water. ......................................................................102

4.53 Four distinct flow regions in the $\mathrm{CO}_{2}$-Oil-Water system. ..............................................104

4.54 Composition paths for the $\mathrm{CO}_{2}$-Oil-Water system at a constant temperature. .....................105

A.1 Isothermal ternary phase diagram showing the five regions and the 15

composition points.

A.2 Isothermal slice of the complete ternary phase diagram for the $\mathrm{CO}_{2}$-Oil-Water system at $300 \mathrm{~K}$.

A.3 Isothermal slice of the complete ternary phase diagram for the $\mathrm{CO}_{2}$-Oil-Water system at $350 \mathrm{~K}$.

A.4 Isothermal slice of the complete ternary phase diagram for the $\mathrm{CO}_{2}$-Oil-Water system at $375 \mathrm{~K}$.

A.5 Isothermal slice of the complete ternary phase diagram for the $\mathrm{CO}_{2}$-Oil-Water system at $400 \mathrm{~K}$.

A.6 Isothermal slice of the complete temary phase diagram for the $\mathrm{CO}_{2}$-Oil-V/ater system at $425 \mathrm{~K}$.

A.7 Isothermal slice of the complete ternary phase diagram for the $\mathrm{CO}_{2}$-Oil-Water system at $450 \mathrm{~K}$.

A.8 Isothermal slice of the complete ternary phase diagram for the $\mathrm{CO}_{2}$-Oil-Water system at $460 \mathrm{~K}$.

A.9 Isothermal slice of the complete ternary phase diagram for the $\mathrm{CO}_{2}$-Oil-Water system at $470 \mathrm{~K}$.

A.10 Isothermal slice of the complete temary phase diagram for the $\mathrm{CO}_{2}$-Oil-Water system at $475 \mathrm{~K}$.

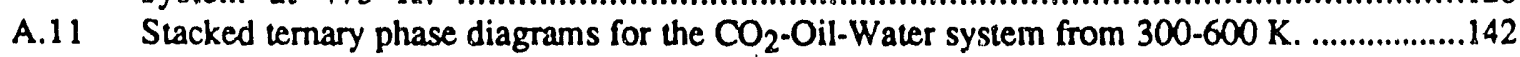

C.1 Region of complex eigenvalues using Stone's model II with $\alpha_{g}=1.0, \alpha_{w}=3.0$,

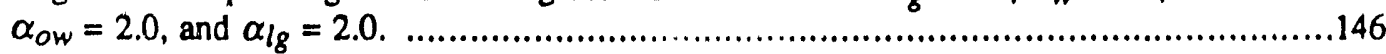

C.2 Region of complex eigenvalues using Stone's model II with $\alpha_{g}=1.0, \alpha_{w}=2.0$, $\alpha_{o w}=2.0$, and $\alpha_{18}=2.0$.

E.1 Basic. Flow Chart for the tracepath Progrim that performs the path integration for the steam-oil-water problem.

E.2. Basic Flow Chart for the jump Program that calculates the shock conditions for the steam-oil-water problem. 


\section{LIST OF TABLES}

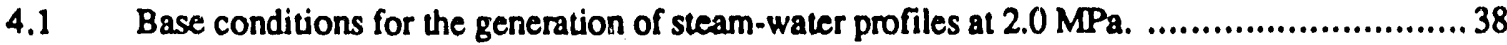

4.2 Three phase relative permeability parameters for Stone's model II. ..................................45

4.3 Composition path for the injection of $100 \%$ steam at $650 \mathrm{~K}$ into $94.5 \%$ oil

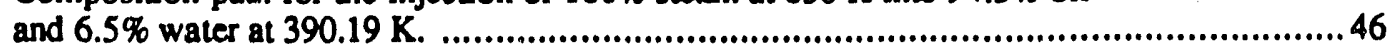

4.4 Abbreviations used to describe the different flow regions. ...........................................46

4.5 The four different three-phease wave profiles for the displacement of oil and water by steam and water.

4.6 Type 2 composition path for injection of $100 \%$ steam at $650 \mathrm{~K}$ into $92.4 \%$ oil and $7.6 \%$ water at $412.09 \mathrm{~K}$.

4.7 Type 3 composition path for injection of $100 \%$ steam at $650 \mathrm{~K}$ into $91.6 \%$ oil and $8.4 \%$ water at $428.62 \mathrm{~K}$.

4.8 Type 4 composition path for injection of $100 \%$ steam at $650 \mathrm{~K}$ into $88.1 \%$ oil and $11.9 \%$ water at $433.79 \mathrm{~K}$.

4.9 Composition path for the injection of $100 \%$ steam at $650 \mathrm{~K}$ into $94.8 \%$ oil and $52.4 \%$ water at $402.13 \mathrm{~K}$ with a temperature wave in the steam-oil region.

4.10 Comparison of composition paths for solutions with and without a temperature variation in the steam-oil region.

4.11 Composition path for the injection of $100 \%$ steam at $650 \mathrm{~K}$ into $99.9 \%$ Heavy

Oil \#1 and $0.01 \%$ w'ser at $458.95 \mathrm{~K}$.

4.12 Composition path Sor the injection of $100 \%$ steam at $650 \mathrm{~K}$ into $88.1 \%$ Heavy

Oil \#2 and $11.9 \%$ water at $433.79 \mathrm{~K}$.

4.13 Non-tie line path in the vapor-liquid region of the $\mathrm{CO}_{2}$-oil-water system. ...........................106

4.14 Typical composition path in the liquid-liquid region of the $\mathrm{CO}_{2}$-oil-water system. ..............107

A.1 Input data for $\mathrm{CO}_{2}$-oil-water phase diagram at $300 \mathrm{~K}$......................................................125

A.2 Input data for $\mathrm{CO}_{2}$-oil-water phase diagram at $350 \mathrm{~K}$...................................................127

A.3 Input data for $\mathrm{CO}_{2}$-oil-water phase diagram at $375 \mathrm{~K}$......................................................129

A.4 Input data for $\mathrm{CO}_{2}$-oil-water phase diagram at $400 \mathrm{~K}$.................................................131

A.5 Input data for $\mathrm{CO}_{2}$-oil-water phase diagram at $425 \mathrm{~K}$. .................................................133

A.6 Input data for $\mathrm{CO}_{2}$-oil-water phase diagram at $450 \mathrm{~K}$. ...................................................135

A.7 Input data for $\mathrm{CO}_{2}$-oil-water phase diagram at $460 \mathrm{~K}$.....................................................137

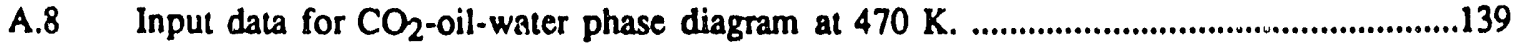

A.9 Input data for $\mathrm{CO}_{2}$-oil-water phase diagram at $475 \mathrm{~K}$..................................................141 


\section{ACKNOWLEDGEMENTS}

Financial support during the course of this study was provided by Stanford University Petroleum Research Institute and Department of Energy Contract \#DE-AC19-87BC14126. 


\begin{abstract}
Recovery of hydrocarbons from porous media is an ongoing concern. Advanced techniques augment conventional recovery methods by injecting fluids that favorably interact with the oil. These fluids interact with the oilby energy transfer, in the case of steam injection, or by mass transfer, as in a miscible gas flood. Often both thermal and compositional considerations are important. An understanding of these injection methods requires knowledge of how temperature variations, phase equilibrium and multiphase flow in porous media interact

The material balance for each component and energy balance are cast as a system of non-strictly hyperbolic partial differential equations. This system of equations is solved using the method of characteristics. The model takes into account the phase behavior by using the Peng-Robinson equation of state to partition the individual c imponents into different phases. Temperature effects are accounted for by the energy balance. Flow effects are modelled by using fractional flow curves and a Stone's three phase relative permeability model.

Three major problems are studied in this dissertation. Each new problem adds an level of interaction to the solution before. The first problem eliminates the phase behavior aspect of the problem by studying the flow of a single component as it undergoes an isothermal phase change. The second problem couples the effects of temperature and flow behavior by including a second component that is immiscible with the original component. Finally, phase behavior is added by using a set of three partially miscible components that partition into two or three separate phases.

Solutions for these equations are formed by spreading waves that propagate in space and time with a constant velocity. The spreading wave regions are connected by jump discontinuities or zones of constant state. The solutions are presented for the three example systems in the form of saturation or composition profiles and solution paths in the composition space. An analysis of the effect of varying some of the important parameters are also presented for each displacement system.
\end{abstract}




\section{INTRODUCTION}

Multiphase, multicomponent flow in porous media occurs in every stage of hydrocarbon recovery, from primary drainage to the most exotic enhanced recovery process. Many times this flow includes temperature variations. The purpose of this study is to present a semi-analytical model that accounts for the interaction between the phase behavior, temperature variation, and fluid flow properies so that a wide variety of cases can be analyzed using this technique.

This chapter begins with a description of the problem and its application to hydrocarbon recovery. The interactions between temperature, phase behavoir and flow properties are discussed. A review of the past efferts using the method of characteristics to solve the differential equations describing conservation laws is contained in the next section. Most of the examples apply to fluid flow in porous media; however, other systems are also mentioned. The reasons for using a semi-analytical model rather than full scale numerical simulations are also covered. Finally, the chapter concludes by describing how the model used in this study expands on the existing models by including temperature gradients.

\subsection{PROBLEM DESCRIPTION}

In any displacement process where there are temperature changes, three major effects interact. These effects are the temperature gradient, the phase behavior, and the flow properties of the fluids and porous medium. These three contributions interact in a complex fashion. The first to be mentioned is the local temperature. The introduction of temperature gradients has a profound effect on the displacement profiles because an increase in temperature directly affects flow properties by reducing the viscosity of the fluids and possibly changing relative permeabilities.

Temperature differences also change the phase compositions by shifting the phase equilibrium. Less volatile components that remain in the liquid phases at iower temperatures move to the vapor phase as the temperature increases. The mass transfer between phases stipulates the degree to which components partition into the mobile phases that are transported downstream.

Phase behavior is the second player in the displacement game. Miscible EOR processes depend on the mass transfer between the injected fluids and the oil to change the flow properties of the hydrocarbons in a favorable way. When $\mathrm{CO}_{2}$ is injected into an oil bearing zone, the $\mathrm{CO}_{2}$ is able to extract significant amounts of intermediate and heavy hydrocarbons into the $\mathrm{CO}_{2}$ rich phase Rathmell et. al. (1971).

Simon and Graue (1965) showed that addition of $\mathrm{CO}_{2}$ to crude oils can sweli the crude to almost twice its original volume for light crude oils. They also reported that the viscosities of the $\mathrm{CO}_{2}$-crude mixtures are lower than those of the dead oil. The changes in saturation and viscosity are the result of mass transfer between the injected $\mathrm{CO}_{2}$ and the hydrocarbon phases.

The solution equilibrium is effected by the temperature and pressure of the system. When the temperature increases, the amount of $\mathrm{CO}_{2}$ that can dissolve into the oil is reduced. When effects of thermai gradients are included in the system, the amount of $\mathrm{CO}_{2}$ that dissolves varies over the length of the displacement, adding to the interplay betwoen the chemical equilibrium and the temperature effects.

Finally, phase behavior changes the heat capacities of the different phases by changing their compositions (Walas 1985). The ability of the various phases to store enthalpy depends on the density of the phase and the heal capacity. These factors determine how the enthalpy partitions between the different flowing phases as well as the matrix. These factors all combine to determine the temperature profile of the displacement.

The third factor in the displacement process is the flow properties of the different phases and the porous medium. The flow properties are usually quantified by fractional nows. These fractional flows 
tell which phases move with higher velocities and which are slower or immobile. In general, fractional flows are nonlinear functions of the phase saturations and viscosities and are the most important factor determining composition and saturation profiles,

Temperature changes have a dramatic effect on the fractional flew relationships. Temperature is a major factor determining the viscosities of the liquid phases which are directly related to the fractional flows. The reduction in viscosity of the hydrocarbon phases is much more dramatic than for water, and is the basis for all thermal recovery processes (Prats 1982). The vapor phase viscosity, on the other hand, shows only a small temperature effect. Ilelow the critical temperature, the viscosity of gases increases proportionally to the square root of the absolute temperature (Reid et al. 1977).

Variations in composition also change the viscosity of the hydrocarton phases. A primary benefit of $\mathrm{CO}_{2}$ miscibility is the reduction of the crude oil viscosity. Correlations relating the composition to the viscosity have been developed for general hydrocarbon systems by Lohrenz et al. (1964) and Lawal (1986). Specific correlations for $\mathrm{CO}_{2}$-heavy oil mixtures are given by a number of authors. Emanual (1985) presents a correlation for predicting the viscosities of crude oil- $\mathrm{CO}_{2}$ mixtures. In his paper Emanual extends a formula by Loeb (1973) to a ternary mixture of $\mathrm{CO}_{2}$, light $\left(\mathrm{C}_{6}^{-}\right)$and heavy $\left(\mathrm{C}_{7}^{+}\right)$ psuedacomponents. A set of equations was presented by Chung et al. (1986) that is suitable for use in numerical simulations. These equations relate crude API gravity to the properties of $\mathrm{CO}_{2}$ crude mixtures.

The interaction of the temperature gradients, chemical equilibrium and fractional now relationships is a web of causes and effects that has been impossible to separate. The purpose of this disseration is to develop a semi-analytical model in which the interactions of these three factors is taken into account.

\subsection{PROBE:EM APPLICATIONS}

The motivation for the study of this problem was to examine the region ahead of an oxygen enriched combustion process. When an inert gas such as nitrogen is removed from the injection air, large amounts of $\mathrm{CO}_{2}$ and steam are produced by the burning reactions. These high temperature products are able to move ahead of the combustion front into the cold reservoir. This process not only transports the heat contained in the product gases downstream but also moves components that can significantly change the properies of the hydrocarbons.

As the combined $\mathrm{CO}_{2}$ and steam move ahead into the reservoir, many things transpire. Fist, the hot gases heat the oil and the surrounding formation. Second, as the steam condenses, a water phase forms that changes the flow characteristics via the saturations. Third, as the $\mathrm{CO}_{2} \mathrm{cools}$ to reservoir temperatures it dissolves in the crude oil changing the density and viscosity of the oil.

The examination of the interactions of the flue gases from the combustion and the original oil is not the only application of multicomponenh, multiphase fir, with temperature variations. A steam drive can be characterized primarily as an energy transport pro ess. The major benefit from steam injection is the increased oil mobility due to viscosity reduction at higher temperatures. Water injected in the form of steam releases some of its enthalpy to the surrounding oil and matrix. This transfer causes the steam to condense and become a hot waterflood ahead of the steam front. Transport properties in the steam-oil region are very different from the water-oil regions.

\subsection{SOLUTION DESCRIPTION}

This section describes the fundamental processes that control how solutions to the model behave. The underlying assumption that is made is that the porous media behaves much like a chromatograph. The difference is that components are partitioned by fractional flow considerations rather than by adsorption onto a stationary column. 


\subsubsection{The Reservoir as \& Chromatograph}

The flow properties of fluids that form during a displacement are determined by the local composition and enthalpy conditions. The loral composition is imposed by the flow conditions upstream. The species present in the most mobile phase move ahead of those components that remain in a less mobile phase. This component separation resembles the adsorptive separation used in multicomponent chromatography. Chromatography separates components by adsorption onto a stationary column. For the case of multicomponent displacements in porous media, the components are separated by partitioning into different phases that travel at different velocities.

The model used in this study is essentially the theory of multicomponent chromatography applied to multiphase flow systems (Helfferich 1981). For different species the fractional flow relationships for multiphase flow are applied in place of adsorption isotherms. The same models used in chromatography can be used, after small adaptations, to describe the flow of multicomponent, multiphase mixtures in porous media.

Simplifying assumptions reduce the material and enthalpy balance equations to a system of quasilinear hyperbolic equations. The solution of these equations is found by the method of characteristics.

\subsubsection{Method of Characteristics}

Use of the method of characteristics to study multiphase flow in porous media dates from the work of Buckley and Leverett (1942) who solved a linear displacement of oil by an immiscible aqueous phase. The authors used a graphical solution where the multivalued saturation velocities were resolved by the use of an "equal area" technique. This matching of areas is essentially the application of the RankineHugoniot conditions at the point of discontinuity. Although the authors did not explicitly use character. istics to arrive at the saturation profiles, Scheidegger (1957) discussed how the method of characteristics could be applied to this problem.

Welge (1961) quantified the effects of phase behavior on a condensing gas drive. By using a technique similar to the method of characteristics, the author developed sets of equations based on material balances that allowed for the calculation of composition paths. It was also shown that along tie lines, where the phase compositions are fixed, the calculations reduce to the Buclsey-Leverett equations.

Temperature effects were introduced by Fayers (1962). The method of characteristics was applied to the problem of hot water injected into an oil filled reservoir. The temperature dependence of the fractional flows appears through the viscosity terms. Densities and heat capacities of the rock and fluids were assumed independent of temperature. One of the important observations made by Fayers was that where there was a discontinuity in temperature, a coincident saturation shock must also be present. This observation was later proved by Hovdan (1986) using Reimann invariants.

Reimann invariants were applied by Rhee et al. (1970) to solve the hyperbolic equations of multicomponent chromatrography. The flow model contained one mobile phase and mass transfer was described using a Langmuir isotherm. Reimann invariants were also used by Hovdan (1986) to solve the hot waterflood problem of Fayers. The entropy condition of Lax (1957) was used to find the physically correct jump conditions.

An isothermal problem similar to the hot waterflood was presented by Temple (1982) for a three cornponent, two phase system of polymer, water and oil. In this paper, viscosity of the aqueous phase was a function of the polymer concentration rather than temperature. Polymer was not allowed to exist in the oil phase and the fractional flows of water and polyme: were general nonconvex' functions of saturations.

\footnotetext{
${ }^{1}$ Nonconvex implies that there is a point of inflection in the fractional flow curve. This inflection point separates the curve into a convex portion and a concave portion of the fractional flow function.
} 
The blending of the theories of multicomponent chromatography with the descriptior of multiphase flow was first advanced by Helfferich (1981). In his paper the coherence criverion, ${ }^{2}$ whic't had been used in chromatography for many years, was applied to general systems of multiphase dlow. Helfferich calculated the velocities of individual components using the method of characteristics in an approach sinilar to the way saturation velocities are calculated in the Buckley-Leverett problem. Coherence is upplied by setuing 2 ii these velocities equal, which turns the system of material balance equations into an eigenvalue problem.

The set of eigenvalues represent the coherent velncities that satisfy the material balance relationships. The associated eigenvectors delineate the paths in composition space that the characteristics associated with a partivalar eigenvalue may follow. A solution profi!e is constructed by marching along these charactsristics from injection to initial conditions.

The integrations of the eigenvectors from the injection to initial condition are the "composition paths." For a multicomponeiit sy: $: m$ there can be many of these paths which emanate from an individual point in composition space. Application of the physical constraint that the faster compositions must reside downstream of slower ones results in a unique and physically correct solution.

The model used by Helfferich as broadened by Dumore (1984) to include volume change on $m$ ixing. As a result the flow velocity was added as a dependerit variable along with the overall composition. This changed the form of the eigenvalue problem from that described by Helfferich to a general eigenvalue problem. Eigenvalues still represented the set of coherent velocities, and the eigenvectors were the directions in the space of dependent variables that characteristics can propagate.

Monroe (1986) extended the model of Dumore to four component systems and used this model to examine the effect of methane on the minimum miscibility pressures of quaternary $\mathrm{CO}_{2}$ hydrocarton systems. Solutions to the four componeni systein required a trial and error procedure to find the unique solution satisfying a given set of initial and injection conditions. The trial and error procedure was necessary because of the existence of an intermediate or "cross-over" tie line. The difficulty stems from the fact that this tie line does not extend through either the initial or injection compositions.

Karankas (1986) used the method of characteristics to solve a combination of the problems given by Fayers (1962) and Temple (i782). The model described the coinjection of hot water and a chemical additive into a viscous oil. The viscosities of the oil and water phases were assumid to be functions of temperature along with the adsorption of the additive onto the matrix. The effect of the additive was to lower the residual oil saturation, changing the relative permeability relationships. Heat capacities for all componeints and the porous medium were assumed cinstant.

This method has been used recently by Pande (1988) to describe the effects of viscous crossflow in a two-layered system. By assuming that crossflow equalizes the pressure differences caused by having a different viscosity profile in each layer, a hyperbolic system of equations results which can be analyzed by the method of characteristics. The displacement with miscible two and three component cases are covered. In this work the effects of volume change on mixing are neglected.

The model developed in this disseration primarily extends ine works of Dumort andMonnoe by relaxing the assumption of constant tempe:ature. The work ad is to the earlier lemperature studies by allowing phase densities, viscosities, heat capacities and phase compositions to de functions of the local temperature. Past effors, with the exception of the general works by Helfferitt: and Dumure and recently Monroe (1985), have also been limited to three total components arid iwo phases. This dissertation expands the system to a four component, three-phase systern.

The method of characteristics maps the independent variables of distance and time in terms of the dependent variables; composition, temperature and flow velocity. The space of dependent variables is

2The coherence criverion is discussed in more denal in \$2.2.2. 
often called hodograph and the transformation from the independant viariables of space and time to composition space is known as the hodograph transformation Courant and Friedrichs (1984). In this model, the te $m$ hodograph space is taken to mean the composition space, which includes the dependent variables temperature and flow velocity as well as the overall composition.

The idea of "coherence" is applied by setting the velocities of all the components as well as the characteristic velocity of the enthalpy equal. Application of the coherence criterion results in a general eigervalue problem, where the eigenvalues represent the permissible coherent velocities and the eigenvectors indicate the direction in monoraph space along which solutions may propagate. The solution in terms of the hodograph variables is obuined by integrating along these coherent paths from an injection condition $t 0$ an initial condition.

When the initial conditions are specified for all space, the problem reduces to the Reimann problem. The Reimann problem is a solution wo the more general Cauchy problem which is given by,

$$
\partial_{1} C(x, t)+\partial_{x} \mathcal{F}(C(x, t))=0
$$

The Reimann problem restricts the system of equations given in Eq. 1.1 to the following initial conditions,

$$
C(x, 0)= \begin{cases}C^{-} & \text {for } x<0 \\ C^{+} & \text {for } x>0\end{cases}
$$

where:

$$
\begin{aligned}
C= & \text { Vector of compositions, temperature and flow velocity } \\
& \left(C_{1}, C_{2} \ldots, C_{n_{C^{-1}}}, T, u\right) \\
\mathcal{F}= & \text { The fractional flow relationships } \\
C^{+}= & \text {Initial conditions to the right of the origin } \\
C^{-}= & \text {Initial conditions to the left of the origin }
\end{aligned}
$$

Solution of this problem tells how an initial discontinuity is resolved into regions of constant state, simple waves and shocks (Dafermos 1983). The constant state regions are bounded by either simple waves or by jump discontinuities, also known as shocks. Since these shocks involve sets of conservation laws, they are known as Rankine-Hugoniot conditions (Jeffrey 1976).

Solution of these balance equations yields a number of possible paths at each point; however, only one of these paths makes physical sense. The route from the injection conditions to the initial state is subject to a velocity constraint: the slower velocities as given by the eigenvalue must lie upstream of the faster velocities. The selection of the correct path using the velocity constraint is the key to obtaining a physically correct solution. Once the proper path is found, the composition, temperature, and saturation profiles are found by relating the composition and temperature at a point to the solution path's eigenvalue.

\subsection{What about Simulation?}

The development of sophisticated compositional and thermal simulators in the recent past raistis an important question: Why not simply use a finite difference simulation to solve these problems? This question has a number of answers.

Numerical simulation of muluphase flow in porous media is rapidly becoming the reservoit engimeer's most used tool. These sophisticated programs solve reservoir engineering problems whose solu- 
tions were unattainable only a few years ago. The use of simulation models has become the standard for present day reservoir engineering.

The use of simulation models does not require an understading of the physical principles that govern multicomponent multiphase flow in porous media. Complete reliance on reservoir simulation programs as "black boxes" to design oil recovery projects carries with it a danger of eliminating the need to understand completely the physical interactions that control oil recovery processes. Study of analytical models permits the reservoir engineer to become more familiar with the physical interactions controlling displacement processes and, in addition, gain insight into the behavior of simulations.

A second use for semi-analytical models is verification of their numerical counterparts. Cieneral models are often too complicated and costly to apply 10 all types of recovery schemes. Spectalized models are developed that apply to a particular crude oil system or reservoir. An indivillual model can incorporate feaures of a formation's lithology or geometry, model the phase behavior of a particular crude oil with an injected solvent, or oxygen requirements of a particular in situ combustion process.

The third motive for studying the analytical solution is identification of the important mechanisms that control the displacements in poruus media. Study of analytical models illustrates what factors a numerical model must consider to retain the ussential features of the governing differential equations. For example, an analytical model can provide insights into how the many clmponents of a crude oil may be combined into fewer pseudocomponents for compositional simulators and till notain the phase behavior features of the fully compositional system.

\section{What lies abead?}

The next chapter will begin by developing the eigenvalue problem from the mass and conservation laws. Relationships for the velocities of the dependent variables are obtained and the eigenvalue problem formulated by setting these velocities equal. The chapter continues by describing how the physically correct solution can be filtered out from among the many possible solution paths using the velocity constraint. Discontinuities in the form of shocks and "self-sharpening" waves are the most difficult parts of the corroct solution, and their properties are covered at the end of the chapter.

Chapter 3 discusses the solution procedure, that is taking the eigenvalue problem and recovering composition, saturation and temperature profiles for specific problems. The chapter opens with a discussion on the basic ideas used to construct these profites. Rules for the integration along the characteristics are covered along with the rules for handling the jump conditions.

Chapter 4 contains three examples of how the model is applied to particular displacement problems. The first of these cases is the injection of steam intw a liquid water filled system. Features of this process include temperature effects and fractional now in a two-phase region. Because this system contains a single component the effects of phase equilibrium are eliminated. The temperature and fractional flow effects are coupled at the discontinuities that carry the solution into and out of the two-phase region.

The steam-water system is extended in the second example by the addition of an oil component. The oil and water phases are assumed immiscible, so phase behavior still has no effect. The new feature is the interaction of temperature and fractional flow properties. This interaction creates temperature profiles in the steam-oil and water-oil regions that are not seen in the single component case.

The third case considered is a three component case with temperature variation. The components are $\mathrm{CO}_{2}$, water, and a heavy oil. The components also form three phases, an aqueous phase that contains liwle $\mathrm{CO}_{2}$ or oil, a $\mathrm{CO}_{2}$ rich phase that can contain a large amount of water vapor at high temperatures, and an oleic phase that can have significant amounts of water and/or $\mathrm{CO}_{2}$ depending on the local temp. eralure. This system contains all the features and interactions mentioned earlier. The interaction of the oleic phase with the other two phases is significant, while the $\mathrm{CO}_{2}$ rich phase and the aqueous phase hardly interact. 
The conclusions and recommendations for future work are discussed in Chapter 5. General statements about the use of the method of characteristics to solve displacement problems are included along with specific comments regarding the interaction of temperature variations, phase behavior and flow ps operties of the sysiems detailed in Chapter 4. 


\section{MATHEMATICAL MODEL}

This chapter covers the development of the mathematical model. The model begirs with the basic conservation equations for mass and total energy. Simplifying assumptions reduce the equations to a system of hyberbolic parial differential equation. The eigenvalue problem is formulated from by a first using the method of characteristics to find the characteristic velocity for each equation and then restricting the solutions to where all the characieristic velocities are equal.

The calculation of the elements in the eigenvalue matrix by finite difference is discussed. Included here are the two and three phase relative permeability models used to calculate fractional flows. Finaliy the chapter concludes with a discussion of the jump conditions. At discontinuities, the conservation equations are transformed from differential form into a system of algebraic equations.

\subsection{CONSERVATION EQUATIONS}

The model proposed in this study is an extension of the multiphase, multicomponient flow model proposed by Dumoré el al. For a system of $n_{c}$ components partitioning into $n_{p}$ phases, if dispersion is neglected, a material balance on the moles of the $i^{\text {th }}$ componient is,

$$
\frac{\partial}{\partial l} \sum_{j=1}^{n_{p}} \phi X_{i j \rho j} s_{j}+\sum_{j=1}^{n_{p}} \nabla \cdot \rho_{j} X_{i j} \vec{u}_{j}=0 \quad i=1 \ldots n_{c}
$$

In addition to the mass of each component, total energy is also conserved. Heat is transported by the flowing phases just as the components are, and the resulting equation, that expresses the conservation of energy is,

$$
\frac{\partial}{\partial t} \sum_{j=1}^{n_{p}} \phi H_{j} \rho_{j} S_{j}+(1-\phi) \rho_{m} H_{m}+\sum_{j=1}^{n_{p}} \nabla \cdot \rho_{j} H_{j} \vec{u}_{j}=0
$$

where

$$
\begin{aligned}
n_{p} & =\text { Number of phases. } \\
n_{c} & =\text { Number of components. } \\
\rho_{j} & =\text { Molar density of phase } j .\left(\mathrm{kg}-\mathrm{mol} / \mathrm{m}^{3}\right) \\
\rho_{m} & =\text { Density of the matrix. }\left(\mathrm{kg} / \mathrm{m}^{3}\right) \\
S_{j} & =\text { Saturation of phase } \mathrm{j} . \\
x_{i j} & =\text { Mole fraction of component } \mathrm{i} \text { in phase } \mathrm{j} . \\
H_{j} & =\text { Specific Molar Enthalpy of phase } \mathrm{j} .\left(\mathrm{kJ} / \mathrm{kg}-\mathrm{mol} \cdot \mathrm{m}^{3}\right) \\
H_{m} & \left.=\text { Specific Enthalpy of the matrix. (kJ/kg } \cdot \mathrm{m}^{3}\right) \\
\vec{u}_{j} & =\text { Phase velocity of phase } j . \text { (Pore Volumes } / \text { Time) } \\
\phi & =\text { Porosity. }
\end{aligned}
$$

Writing the total energy balance equation in this manner makes Eq. 2.2 have the same form as the $n_{c}$ mate: al balance equations. In essence, this model treats heat as another component. 
The balance equations are simplified by using the following definitions,

$$
\begin{aligned}
G_{i} & =\sum_{j=1}^{n_{p}} \rho_{j} X_{i j} S_{j} \\
F_{i} & =\frac{\mu}{\phi} \sum_{j=1}^{n_{p}} \rho_{j} X_{i j} f_{j} \\
\Gamma & =\frac{\mu}{\phi} \sum_{j=1}^{n} \rho_{j} H_{j} S_{j}+\left(\frac{1-\phi}{\phi}\right) \rho_{M} C_{P M} T \\
\Theta & =\frac{\mu}{\phi} \sum_{j=1}^{n_{p}} \rho_{j} H_{j} f_{j}
\end{aligned}
$$

where

$$
\begin{aligned}
& f_{j} \equiv \vec{u}_{j} \cdot \frac{\phi}{u} \quad=\text { Fractional flow of phase } j . \\
& C_{m} \equiv \frac{H_{m}}{T} \quad=\text { Heal capacity of the matrix. }(\mathrm{kJ} / \mathrm{kg} \cdot \mathrm{K})
\end{aligned}
$$

Applying the definitions in Eqs. 2.3-2.6 to Eqs. 2.1 and 2.2 gives the model's basic conservation equations.

$$
\begin{aligned}
& \frac{\partial G_{i}}{\partial t}+\frac{\partial F_{i}}{\partial x}=0 \quad i=1 . . n_{c} \\
& \frac{\partial \Gamma}{\partial t}+\frac{\partial \theta}{\partial x}=0
\end{aligned}
$$

The term $G_{i}$ represents the local overall concentration of component $i$, similar to the local concentration term in the familiar Buckley-Leveretl (1942) equation. The term $F_{i}$ represents the flux of component $i$. The $\Gamma$ term is the energy analog to the local concentration term in the material balance. It differs from the mass terms in that the matrix is also allowed to accumulate heat from the fluids and vice versa. The $\rho_{m} C_{p_{m}} T$ term in the definition of $\Gamma$ accounts for the heat stored in the matrix. Finally, the $Q$ term accounts for the heat flux in the system. Since the matrix is immobile, there is no term for flux due to the matrix.

The model is based on the following assumptions that maintain the hyperbolic nature of the equations. These assumptions are excellent approximations when the flow rates are high compared to the diffusive terms such as thermal conduction, capillary pressure or dispersion.

- Flow is one-dimensional.

- Pressure is constant over the length of the displacement. This assumption is made with respect to the thermodynamic equilibrium. While there must be a pressure gradient in order for flow 10 occur, the solutions are independent of the pressure gradient once the injection velocity is set. 
The assumption of constant pressure also allows the use of the enthalpy of the phase in the arcumulation term. While it is not correct 10 say that enthalpy can be accumulated, because the system is at constant pressure and constant total volume; there is no PV work term to consider. Under these conditions, it is correct to consider the total energy and enthalpy balances as identical.

- Porosity is constant.

- Mass and heat transfer are instantaneous.

- Mass transfer by dispersion and diffusion is neglected.

- Heat transfer by conduction and radiation is neglected.

- Heat capacity of the porous medium is constant over the temperature range of interest.

Because Eqs. 2.7 and 2.8 retain their hyperbolic character, the method of characteristics can be used to solve these coupled equations. If solutions are sought for which a given mixture composition moves at a single velocity, the set of $n_{c}+1$ balance equations is reduced to a general eigenvalue problem where component velocities are given by the eigenvalues and characteristic directions by the associated eigenvectors.

\subsection{METHOD OF CHARACTERISTICS}

In its most basic form, the method of characteristics is a mapping of the solution in the space of the independent variables, $x$ and $t$ to the space of dependent variables, $C_{i}, T$, and $u$. Consider a system of equations with independent variables, $x$ and $t$, and dependent variables, $C_{1}$ and $C_{2}$. When the Jacobian is not singular,

$$
\frac{\partial\left(C, C_{2}\right)}{\partial(x, 8)} \neq 0
$$

there exists a transformation from any point in the space of independent variables to a unique point in the space of dependent variables. The mapping is illustrated in Fig. 2.1.

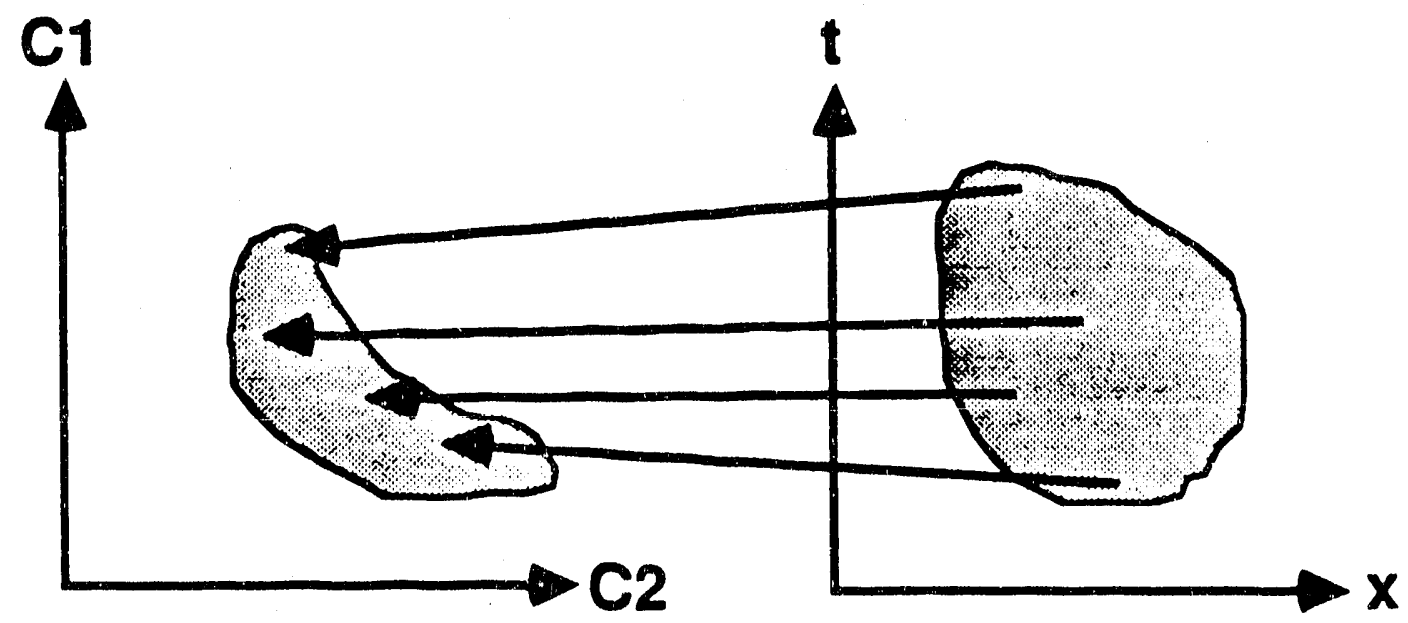

Figure 2.1: One-to-one mapping from the space of independent variables, $x$ and $t$ to the space of dependent variables, $C_{1}$ and $C_{2}$. Jacobian of rank 2 . 
This is type of mapping violates the coherence condition placed on the valid soiutions. When Jacobian given in Eq. 2.9 is not singular then all components travel independenuly of other components. There is no set of composicions that travels together under these conditions. The assumption of coherence and the restriction of the boundary and initial conditions to constant values eliminates this situation from consideration in this dissertation.

Compositions may travel with the same velocity if the Jacobian given by Eq. 2.9 is singular. The Jacubian can be singular if the rank of the Jacobian matrix is either one or zero. When the Jacobian has a rank of one, mapping from a region in $x$ - $t$ space results in a single curve in the space of dependent variables. This mapping is illustrated in Fig. 2.2. A rank one Jacobian implies that the two flow equations are not independent, that is,

$$
C_{1}=\mathscr{r}\left(C_{2}\right)
$$

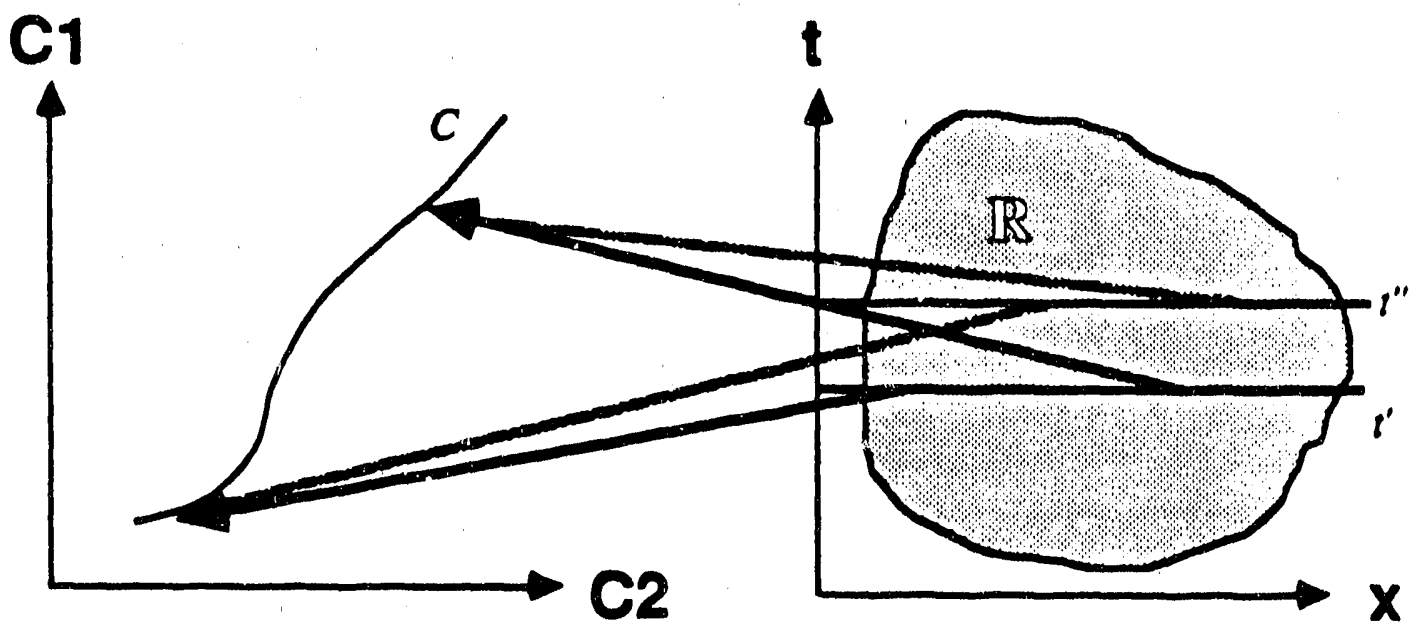

Figure 2.2: Mapping from the space of independent variables, $x$ and $t$ to the space of dependent variables, $C_{1}$ and $C_{2}$. Jacobian is rank 1 .

Only composition points that lie on the curve $\mathbf{C}$ are allowed over the region $\mathbf{R}$. At time $t$ the rompositions along curve $C$ are the local compositions at the positions indicated by the tails of the arrows. At some later time, $t^{\mathrm{n}}$, the new mapping must result in the same set of compositions given by curve $\mathbf{C}$. Although the position of a given composition point will change, the makeup of the composition points does not change. This is the concept of coherence.

Finally, the Jacobian given by Eq. 2.9 can be of rank zero. Fig. 2.3 shows a region of $x-\imath$ space mapped onto a single point in composition space. Because a single composition is spread over a finite region of time and space, this mapping describes a region of constant state. The composition given by point A will exist at a position $x^{\prime}$ from time to to $t$. Similarly, at time $t^{\prime}$, the composition profile will have a value $A$ from $x_{0}$ to $x_{1}$.

It has been shown by Lax (1957) that the solution to the Reimann problem consists of regions of constant state bounded by regions of simple waves. The ordering of these waves follows the physical rule that the faster compositions must lie ahead of the slower compositions. The calculation of the component velocities is key to obtaining a physically correct solution. 


\subsubsection{Calculation of Component and Enthalpy Velocities}

The first step in the development of the eigenvalue problem is to calculate the wave velocities for all the components and the wave velocity for the enthalpy. These velocities are the slopes of the characteristic curves in the $x-1$ plane.

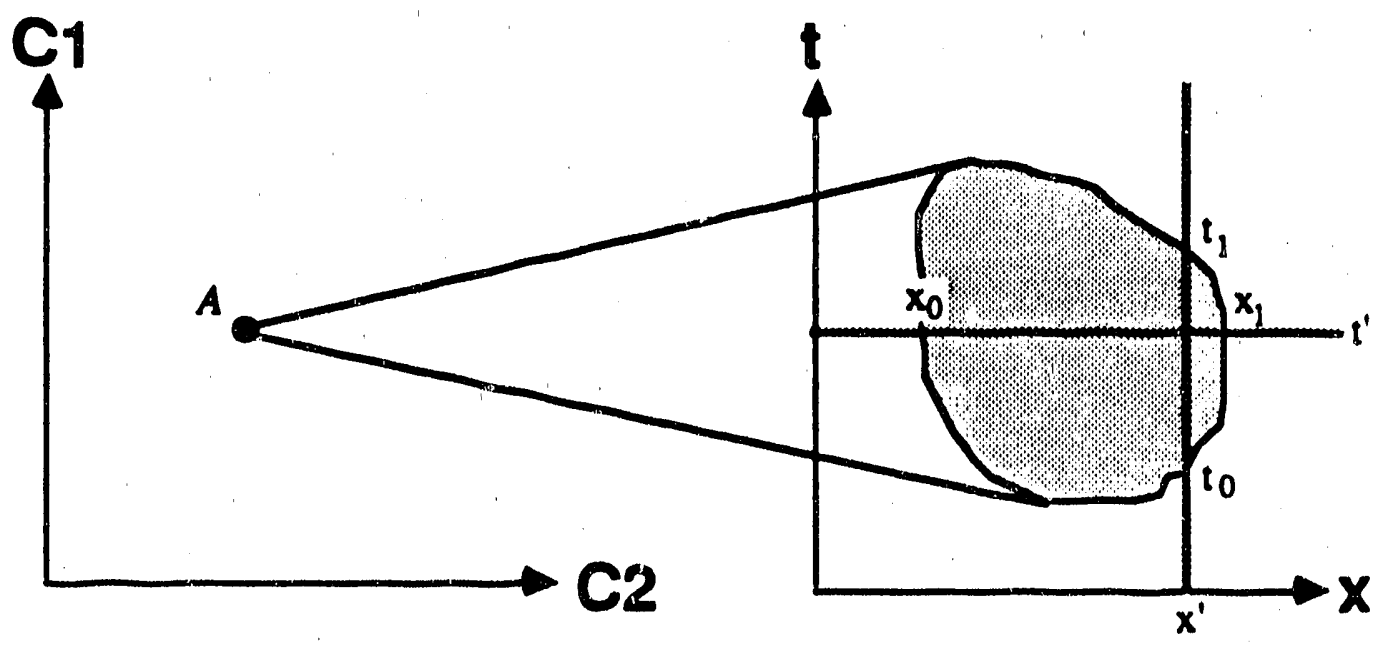

Figure 2.3: Map of constant state region from a region in $x-t$ space to a single peint in $C_{1}, C_{2}$ space. Jacobian is of rank 0 .

Let $\eta$ be a dummy variable that is a function of the independent variables, $x$ and $t$. Writing the basic conservation equations (Eqs. 2.7 and 2.8) in terms of the dummy variable gives,

$$
\frac{d G i}{d \eta} \frac{\partial \eta}{\partial t}+\frac{d F i}{d \eta} \frac{\partial \eta}{\partial x}=0 \quad i=1 \ldots n_{c}
$$

and

$$
\frac{d \Gamma}{d \eta} \frac{\partial \eta}{\partial t}+\frac{d \theta}{d \eta} \frac{\partial \eta}{\partial x}=0
$$

Here the derivatives with respect to $\eta$ are ordinary derivatives since the solution is mapped onto a single variable.

The method of characteristics secks solutions along characteristic lines, that is, where the value of the characteristic variable, $\eta$, is constant.

$$
d \eta=d t \frac{\partial \eta}{\partial t}+d x \frac{\partial \eta}{\partial x}=0
$$

For the $i^{\text {th }}$ component, Eq.2.11 and Eq.2.13 form a set of linear algebraic equations with the characteristic curve parameters, $d \eta / d t$ and $d \eta / d x$ as the unknowns. A non-trivial solution exists if and only if the determinant of the coefficient matrix is zero.

$$
\operatorname{det}\left[\begin{array}{ll}
\frac{d G_{i}}{d \eta} & \frac{d F_{i}}{d \eta} \\
d t & d x
\end{array}\right]=0
$$


A similar relationship holds for all the other $n_{C}-1$ components and the heat, where $\Gamma$ replaces $G_{\mathrm{i}}$ and $\theta$ substitutes for $F_{\mathrm{i}}$.

The characteristic or wave velocity of component $i$ is calculated by expanding tie determinant of the coefficient matrix Eqn. 2.14). Writing the determinant of Eq. 2.14 and solvingfor $d x / d t$ gives,

$$
u_{C_{i}}=\frac{d x}{d t}=\frac{\left(\partial F_{i} / \partial \eta\right)}{\left.\partial G_{i} / \partial \eta\right)}
$$

a similar equation for the velocity of the enthalpy is,

$$
u_{H}=\frac{d x}{d t}=\frac{(\partial \theta / \partial \eta)}{(\partial T / \partial \eta)}
$$

For each of the $n_{c}$ components there is a characteristic velocity described by Eq. 2.15. In addition to these $n_{c}$ component velocities, the total energy balance as shown in Eq.2.:6, gives a characteristic velocity associated with the enthalpy, $u_{H}$, for a total of $n_{c}+1$ different wave velocities. The coherence condition is applied by setting these $n_{c}+1$ velocities equal.

Expanding the terms in the velocity equations (Eqs. 2.15 and 2.16) in terms of the hodograph variables $C_{j}, T$, and $u$, yields;

$$
\begin{aligned}
\frac{d G_{i}}{d \eta} & =\sum_{k=1}^{n_{i}-1} \frac{\partial G_{i}}{\partial C_{k}} \frac{\partial C_{k}}{\partial \eta}+\frac{\partial G_{i}}{\partial T} \frac{\partial T}{\partial \eta} \quad i=1 \ldots n_{c} \\
\frac{d T}{d \eta} & =\sum_{k=1}^{n_{i}-1} \frac{\partial T}{\partial C_{k}} \frac{\partial C_{k}}{\partial \eta}+\frac{\partial T}{\partial T} \frac{\partial T}{\partial \eta} \\
\frac{d T}{d \eta} & =\sum_{k=1}^{n_{1}-1} \frac{\partial F_{i}}{\partial C_{k}} \frac{\partial C_{k}}{\partial \eta}+\frac{\partial F_{i}}{\partial T} \frac{\partial T}{\partial \eta}+\frac{\partial F_{i}}{\partial u} \frac{\partial u}{\partial \eta} \quad i=1 \ldots n_{c} \\
\frac{d \theta}{d \eta} & =\sum_{k=1}^{n_{c}-1} \frac{\partial \theta}{\partial C_{k}} \frac{\partial C_{k}}{\partial \eta}+\frac{\partial \theta}{\partial T} \frac{\partial T}{\partial \eta}+\frac{\partial \theta}{\partial u} \frac{\partial u}{\partial \eta}
\end{aligned}
$$

Substitution of the expressions in Eqs.2.17 and 2.19 into Eq. 2.15 for the $i^{\text {th }}$ component yields,

$$
\sum_{k=1}^{n_{i}-1} \frac{\partial F_{i}}{\partial C_{k}} \frac{\partial C_{k}}{\partial \eta}+\frac{\partial F_{i}}{\partial T} \frac{\partial T}{\partial \eta}+\frac{\partial F_{i}}{\partial u} \frac{\partial u}{\partial \eta}=u_{C_{1}}\left(\frac{\partial G_{i}}{\partial C_{k}} \frac{\partial C_{k}}{\partial \eta}+\frac{\partial G_{i}}{\partial T} \frac{\partial T}{\partial \eta}\right)
$$

a similar substitution using Eqs, 2.18 and 2.20 into Eq. 2.16 gives, for the velocity of the enthalpy wave,

$$
\sum_{k=1}^{n_{c}-1} \frac{\partial \theta}{\partial C_{k}} \frac{\partial C_{k}}{\partial \eta}+\frac{\partial \theta}{\partial T} \frac{\partial T}{\partial \eta}+\frac{\partial \theta}{\partial u} \frac{\partial u}{\partial \eta}=u_{H}\left(\sum_{k=1}^{n_{c}-1} \frac{\partial T}{\partial C_{k}} \frac{\partial C_{k}}{\partial \eta}+\frac{\partial T}{\partial T} \frac{\partial T}{\partial \eta}\right)
$$

There are $\eta$ equations like Eq. 2.21, one for each component,and Eq. 2.22 for the enthalpy velocity giving $n_{c}+1$ separate velocities. The coherence condition states that the only solutions that are stable 
have all $n_{c}+1$ velocities equal. Solutions where these component velocities are equal are found by solving the general eigenvalue problem described in the next section.

\section{Coherence and the Eigenvalue Problem}

The idea of coherence has been used for many years in vie field of multicomponent chromatography and has recently been used by to describe the flow of multicomponent, multiphase mixtures in pomus media. This section discusses the basis for the coherence criteria and how it formulates the system of characteristic equations into an eigenvalue problem.

\section{What is Coherence?}

One of the major assumption of the model is that physically concot rolutions to the hyperbolic conservation equations are formed from regions of constant state surrounded by coherent waves. The term coherent was applied by Helfferich (1981) to mean that the only propagationally stable solutions were those where, at any given point in the displacement, all the waves associated with eawh conserved quantity moved at the same velocity.

The mathematical equivalent of ccherence takes $n_{c}+1$ independent flow equations and couples them by requiring certain sets of compositions to flow together. This eliminates $n_{c}$ of the component velocities and creates the eigenvalue problem.

Physically, coherence is simply a statement of velocity. Given fixed initial and injection conditions, any set of components that exists at a particular location must all have travelled the same distance, $\Delta_{x}$ over the same amount of time, $\Delta_{t}$. The only way these compositions could arrive together is if they all travel with the same velocity.

Sillen (1950) and later Lax (1957) proved that if the solution to the Reimann problem is unique then it is a function of $x / t$ only. The two independent variables can be reduced $t 0$ a single parameter, $\eta=$ $x / t$. This is equivalent to the mapping illustrated earlier in Fig. 2.2. For the Reimann problem, the entire $x-t$ space is mapped into regions of simple waves when the Jacobian is rank one or into regions of constant state if the Jacobian is of rank zero.

Coherence has been used by a number of authors to describe fluid now in porous modia (Fayers 1961 and 1987. Rhee et al. 1970, Monroe 1986, Gorell 1988, and Pande 1988). These authors all solve an eigenvalue problem to determine the composition routes and wave velocities. The velocity constraint is used to isolate the physically correct solution from among the many possible answers.

\section{Formulation of the Eigenvalue Problem}

The $n_{c}+1$ velocities calculated from Eqs. 2.15 and 2.16 represent the rates at which different conserved quantities move through the flow system. Imposing the coherence condition confines the solutions to regions in hodograph space where all these $n_{c}+1$ velocities have the same valuc. This assumption ieads to the formulation of the eigenvalue problem.

The first step is to write the component velocities as functions of the dummy variable, $\eta$.

$$
\mu=\frac{\left(\partial F_{1} / \partial \eta\right)}{\left(\partial G_{1} / \partial \eta\right)}=\frac{\left(\partial F_{2} / \partial \eta\right)}{\left(\partial G_{2} / \partial \eta\right)}=\ldots \frac{\left(\partial F_{m} / \partial \eta\right)}{\left(\partial G_{m} / \partial \eta\right)}=\frac{(\partial \theta / \partial \eta)}{(\partial \theta / \partial \eta)}
$$


Using Eqs. 2.17 and $2.19 \mathrm{in} \mathrm{Eq.} 2.23$ gives for the velocity of component $i$,

$$
\sum_{k=1}^{n} \frac{\partial F_{i}}{\partial C_{k}} \cdot \frac{\partial C_{k}}{\partial \eta}+\frac{\partial F_{i}}{\partial T} \frac{\partial T}{\partial \eta}+\frac{\partial F_{i}}{\partial u} \frac{\partial u}{\partial \eta}=u_{C_{1}}\left(\sum_{k=1}^{n} \frac{-1}{\partial C_{k}} \frac{\partial C_{i}}{\partial \eta}+\frac{\partial C_{i}}{\partial T} \frac{\partial T}{\partial \eta}\right)
$$

and a similar substitution of Eqs. 2.18 and 2.20 into Eq. 2.23 yields the velocity of the enthalpy wave,

$$
\sum_{k=1}^{n_{4}-1} \frac{\partial \theta}{\partial C_{k}} \frac{\partial C_{k}}{\partial \eta}+\frac{\partial \theta}{\partial T} \frac{\partial T}{\partial \eta}+\frac{\partial \theta}{\partial u} \frac{\partial u}{\partial \eta}=u_{H}\left(\sum_{k=1}^{n_{4}-1} \frac{\partial T}{\partial C_{k}} \frac{\partial C_{k}}{\partial \eta}+\frac{\partial T}{\partial T} \frac{\partial T}{\partial \eta}\right)
$$

This gives $n_{c}$ equations in the form of Eq. $? 24$ and one equation from Eq. 2.25, for a total of $n_{c}+1$ velocities. Coherence requires that the velocities for all components and the enthalpy be equal. The systemof $n_{c}+1$ equations, written in matrix form is given in Eq. 2.26, where a single value, $\lambda$, has replaced the $n_{c}+1$ velocities $\left(\mu_{C} 1, u_{C 2}, \ldots, u_{C}{ }_{n c}, u_{H}\right)$.

$$
\left[\begin{array}{cccccc}
\frac{\partial F_{1}}{\partial C_{1}} & \frac{\partial F_{1}}{\partial C_{2}} & \cdots, & \frac{\partial F_{1}}{\partial C_{n c-1}} & \frac{\partial F_{1}}{\partial T} & \frac{\partial F_{1}}{\partial u} \\
\frac{\partial F_{2}}{\partial C_{1}} & \frac{\partial F_{2}}{\partial C_{2}} & \cdots, & \frac{\partial F_{2}}{\partial C_{n c-1}} & \frac{\partial F_{2}}{\partial T} & \frac{\partial F_{2}}{\partial u} \\
\vdots & \vdots & \cdots, & \vdots & \vdots & \vdots \\
\vdots & \vdots & \cdots, & \vdots & \vdots & \vdots \\
\frac{\partial F_{n c}}{\partial C_{1}} & \frac{\partial F_{n c}}{\partial C_{2}} & \cdots, & \frac{\partial F_{n c}}{\partial C_{n c-1}} & \frac{\partial F_{n c}}{\partial T} & \frac{\partial F_{n c}}{\partial u} \\
\frac{\partial C_{1}}{\partial \theta} & \frac{\partial \theta}{\partial C_{2}} & \cdots, & \frac{\partial C^{\prime}}{\partial C_{n c-1}} & \frac{\partial \theta}{\partial T} & \frac{\partial \theta}{\partial u}
\end{array}\right]\left[\begin{array}{c}
\frac{d C_{1}}{d \eta} \\
\frac{d C_{2}}{d \eta} \\
\vdots \\
\vdots \\
\frac{d T}{d \eta} \\
\frac{d u}{d \eta}
\end{array}\right]
$$

$$
\lambda\left[\begin{array}{cccccc}
\frac{\partial G_{1}}{\partial C_{1}} & \frac{\partial G_{1}}{\partial C_{2}} & \cdots, & \frac{\partial G_{1}}{\partial C_{n c-1}} & \frac{\partial G_{1}}{\partial T} & 0 \\
\frac{\partial G_{2}}{\partial C_{2}} & \frac{\partial G_{2}}{\partial C_{2}} & \cdots & \frac{\partial G_{2}}{\partial C_{n c-1}} & \frac{\partial G_{2}}{\partial T} & 0 \\
\vdots & \vdots & \cdots, & \vdots & \vdots & \vdots \\
\vdots & \vdots & \cdots, & \vdots & \vdots & \vdots \\
\frac{\partial G_{n c}}{\partial C_{1}} & \frac{\partial G_{n c}}{\partial C_{2}} & \cdots, & \frac{\partial G_{n c}}{\partial C_{n c-1}} & \frac{\partial G_{n c}}{\partial T} & 0 \\
\frac{\partial T}{\partial C_{1}} & \frac{\partial T}{\partial C_{2}} & \cdots & \frac{\partial T}{\partial C_{n c-1}} & \frac{\partial T}{\partial T} & 0
\end{array}\right]\left[\begin{array}{c}
\frac{d C_{1}}{d \eta} \\
\frac{d C_{2}}{d \eta} \\
\vdots \\
\vdots \\
\frac{d T}{d \eta} \\
\frac{d u}{d \eta}
\end{array}\right]
$$


Eq. 2.26 will, in general contain $n_{c}+1$ eigenvalues. When the conservation equations are hyperbolic, all the eigenvalues are real (Jeffrey 1976), and represent $n_{c}+1$ different coherent velocities.' Recent studies have shuwn that during three phase flow, regions of the composition space are elliptic. Because the firal column in the $\overline{\bar{G}}$ matrix is zero, one of the eigenvalues is infinite. The infinite eigenvalue represents the rate which flow velocity changes propagate through the system (Mchroe 1986), Therefore, there remain $n_{c}$ real, finite velocities for any given coinposition and temperature.

Ai certain singular points, two of the $n_{c}$ eigenvalues can be equal. Systems that exhibit this propenty are called nonstrictly hyperbolic. These equal eigenvaluse points are important in the construxtion of the solution, for at these points, the solution path is free to switch, with no jump in velocity, from one of the characteristics to the other characteristic with the equal eigenvalue. In essence, these points represent a zone of constant state with a length of zero.

Along with the velocities as represented by the eigenvalues, the associated eigenvectors describe the characteristic curve in hodograph space for each of the eigenvalues. These eigenvectors tell what set of compositions and temperature can lie directly ahead of and behind the current composition and still satisfy the material and energy balances.

\subsection{EVALUATION OF THE DERIVATIVES IN THE EIGENVALUE MATRIX}

The derivatives in the eigenvalue matrix (Eq. 2.26) relate how the saturation and flux terms for each component vary when the overall composition, temperature and flow velocity at a particular location change. Evaluation of these derivatives requires that the properties of all the existing phases be known. for a given overall composition and temperature. These properties are:

1. The molar density of each phase.

2. The composition of each phase.

3. The saturation of each phase.

4. The enthalpy of each phase.

5. The viscosity of each phase.

6. The relative permeability of exch phase.

With the exception of the viscosity and the relative permeability, all other properties can be obtained directly from an equation of state. The viscosity is usually given in the form of empirical curves or a correlation relating the phase composition, temperature and pressure to the viscosity of the phase. Relative permeabilities of the existing phases depend not only on the fluid properties, bit on the wetting condition of the matrix as well as the saturation of the coexisting phases. In generai the relative permeabilities of the wetting and gaseous phases are functions of the wetting and gaseous phase saturations, respectively. The remaining phases are given as functions of the two phase relative permeabilities between the intermediate phase and both the wetting and gaseous phases.

\footnotetext{
${ }^{1}$ Recent studies have shown that during three phase flow, regions of the composition space are elliptic. These elliptic regions have complex eigenvalues and can change the nature of the conservation equations. A brief explanation of the elliptic regions is presented in Appendix $D$.
} 


\subsubsection{Fluid Property Calculation}

The method for calculation of the fluid properties depends on the particular system involved. When phase behavior is not included in the problem, correlations relating the viscosity specific volume and enthalpy of the components against temperature are all that are needed to calculate the derivatives in the eigenvalue inatrix. When phase behavior is included as part of the solution, a flash calculation is required. This calculation is usually done by calculating component fugacities using an equation of state. Once the individual phase compositions are found, the physical properties are calculaten fromi the composition, temperature and pressure. Appendix B gives the details on how the fluid properties were obtained for each of the examples presented in Chapter 4.

Relative permeabilities of the wetting and gaseous phases are calculated using a power law

$$
k_{r_{t}}=\sigma_{8}\left(\frac{S_{s}}{1-S_{o r}-S_{w c}}\right)^{\alpha_{t}}
$$

and

$$
k_{r w}=\sigma_{w}\left(\frac{S_{w}-S_{w c}}{1-S_{o r}-S_{w c}}\right)^{\alpha_{w}}
$$

where:

$k_{r g}=$ gaseous phase relative permeability

$k_{r w}=$ wetting phase relative permeability (usually water)

$\sigma_{g}=$ end point (maximum) relative permeability of the gaseous phase

$\sigma_{w}=$ end point (maximum) relative permeability of the wetting phase

$S_{g}=$ gaseous phase saturation

$S_{w}=$ weting phase saturation

$S_{w c}=$ connate wetting phase saturation

$S_{o r}=$ residual intermediate phase saturation

$\alpha_{g}=$ gaseous phase exponent

$\alpha_{w}=$ wetting phase exponent

These equations are used for both the two and three phase relative permeabilities. When three phases are present the relative permeability of the intermediate phase, usually oil, requires further calculations.

This disseration uses two models to calculate the relative permeability of the intermediate phase. Both models combine two phase relative permeability values to calculate the three phase relative permeability. One set of the two phase relationships between the intermediate and wetting phases, oil and water. In this model the oil phase relative permeability in the absence of a free gas phase is given by,

$$
k_{\text {row }}=\sigma_{o w}\left(\frac{S_{0}-S_{m c}}{1-S_{o r}-S_{w c}}\right)^{\alpha_{w}}
$$

where:

$k_{\text {row }}=$ Oil relative permeability in the oil-water system

$\sigma_{w}=$ end point (maximum) relative permeability of the intermediate phase

$S_{0}=$ intermediate phase saturation, $S_{0}=1-S_{w} \cdot S_{8}$

$\alpha_{o w}=$ oil-water exponent 
The second relative permeability relationship is the gas-liquid relationship. This relative permeability relationship is taken at the irreducible water saturation, which is includec in the saturation of the intermediate phase.

$$
k_{r_{g}}=\alpha_{i_{8}}\left(\frac{1-S_{g}-S_{m c}}{1-S_{o r}-S_{m c}}\right)^{\alpha_{4}}
$$

where:

$k_{\text {rig }}=$ Oil relative permeability in the liquid-gas system

$\sigma_{l g}=$ end point (maximum) relative permeability of the intermediate phase

$S_{l}=$ liquid phase saturation, $S_{l}=1-S_{8}$

$\alpha_{l \delta}=$ liquid-gas exponent

Eqs. 2.29 and 2.30 are combined to give the oil phase relative permeability. The model for the intermediate phase permeability is taken from Stone (1970) as given by Aziz and Setarri (1979),

$$
k_{r o}=\frac{\left(k_{r a}+k_{n}\right)\left(k_{n_{t}}+k_{r_{1}}\right)}{k_{\text {row }}}
$$

where:

$k_{\text {rocw }}=$ intermediate phase relative permeability at irreducible water saburation

Once all the fluid properties are calculated for each phase, G, F, $\Gamma$, and are calculated using the definitions given in Eqs. 2.3-2.6.

\subsubsection{Calculating the Derivatives for the Eigenvalue Problem}

The relationships between the overall composition and temperature to the quantities needed for evaluation of the eigenvalue matrix are very complex. Even when calculating fluid properties using an equation of state, evaluation of the derivatives is a costly procedure. Simple forward or backward differences are the easiest to calculate and provide excellent approximations to the true derivatives. Monroe (1986) used this technique to calculate his composition paths.

The primary variables, $G, F, \Gamma$, and $\theta$ are calculated at the current "composition" point ${ }^{2}$ using a flash calculation to obtain the fluid properties. Another flash is performe I at a perturbed composition point by adding a small amount to the mole fraction of one of the components and decreasing the mole fraction of another. A new flash is performed and new values for the four primary variables are calculated. For example, to calculate $\left(\partial F_{1} / \partial C_{2}\right)$ the following difference is used, ${ }^{3}$

$$
\frac{\partial F_{1}}{\partial C_{2}}=\frac{F_{1}\left(C_{1}, C_{2}+\Delta C_{2} \ldots, C_{n c}-\Delta C_{2}, T, u\right)-F_{1}\left(C_{1}, C_{2}, \ldots, C_{n c} T, u\right)}{\Delta C_{2}}
$$

\footnotetext{
2The term composition point means the position in hodograph space. It includes temperature as well as overall composition.

${ }^{3}$ The results of the eigenvalue problem do no: depend on the choice of component from which that $\Delta C_{2}$ is subtracted. As long as the choice is consistent the resultirig eigenvalues and eigenvectors do not change.
} 
There are $n_{c}-1$ compositional derivatives plus a temperature derivative 10 calculate, meaning that at every time the eigenvalue problem is solved, $n_{c}$ flash calculations must be performed." The change in composition at the perturbed points is done on a mole fraction basis so only $n_{c}-1$ of thi: compositions are independent. This requires that the same mole fraction be subtracted from the $C_{n c}$ term in the forward difference. Temperature does not have this restriction so the perturbed point is found by changing the temperature value only. The derivatives of $F$ and $\Theta$ with respect $10 u$ are simple and are calculated analytically.

Near phase boundaries, the changes in saturation and phase properties can be dramatic. In these regions simple forward differences may not accurately represent the true derivative. Equations of state used to represent the phase behavior of the fluid systems contain the information required to construct the derivatives in the eigenvalue matrix (Eq. 2.26) (Nutakki, et al. 1985).

The need for more accurate representation of the derivatives is only necessary near phase boundaries. In regions removed from these boundaries, the simple forward differences give excellent results. When the solution path wavels between regions where the number of phases changes, discontinuities in composition exist which skip over regions near the phase boundaries. This physical feature often causes the solution path to be far enough from the phase boundaries that the simple forward differences are accurate.

The eigenvalnes and the associated eigenvectors are found using an algorithm presented by Moler ano Slewart (1973) as implemented in the IMSL routine EIGZCF. The algorithm uses Householder transformations to reduce the $\bar{F}$ matrix to an upper Hessenberg and the $\bar{G}$ matrix to upper triangular form. These transformed matrices are diagonalized to find the eigenvalues by successive $Q Z$ diagonalizations.

\subsection{SHOCK CALCULATIONS}

When the integration along the characteristics must violate the velocity constraint, it is necessary to introduce discontinuities or shocks into the solution. When conservation laws are applied across the shocks, the relationships between the speed of the shock and the conditions upstream and downstream of the discontinuity are known as Rankine-Hugoniot conditions.

The Rankine-Hugoniot conditions are found by applying a material or heat balance acros; the discontinuity. Consider a shock traveling with a velocity, $\lambda$ as illustrated in Figure 2.4 (from Monroe 1985).

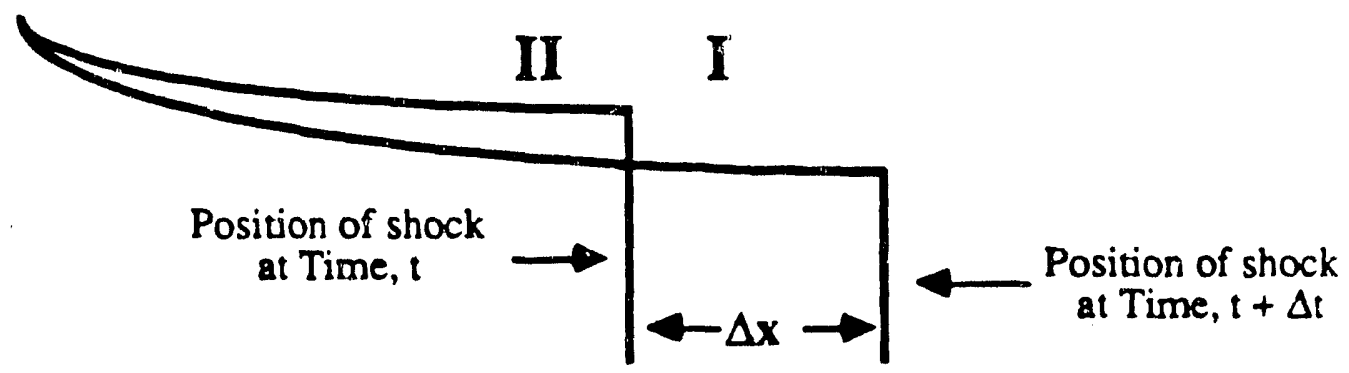

Figure 2.4: Shock position at time $t$ and $\ell+\Delta l$. After Monsoe (1985).

\footnotetext{
'While this may seem like many fash calculations, because the $\Delta C^{\prime}$ is are small, the flash calculation can be provided with an excellent initial guess and the number of iterations is kept low.
} 
Using an approach outlined by Dumort, the shock velocity is given by the material balances,

$$
\Lambda=\frac{1}{\phi}\left(\frac{u^{+} F^{+}-u^{-} F^{-}}{G^{+}-G^{-}}\right)
$$

Where the superscripts $(+)$ and $(-)$ refer to the downstream and upstream sides of the shock, respectully. The same arguments apply to the heat accumulation in a moving shock. The heat balance across the shock is given by,

$$
\Lambda=\frac{1}{\phi}\left(\frac{\mu^{+} \theta^{+}-u^{-} \theta^{-}}{1^{++}-\Gamma^{-}}\right)
$$

These balances are the Rankine-Hugoniol conditions.

Shocks are described completely by three states, the upstream conditions, the downstream conditions, and the shock velocity. Table 2.1 indicates that $2 n_{c}+5$ variables are needed to describe fully a discontunity. The problem is to relate the conditions on one side of the shock to the shock velocity and the conditions on the other side of the discontinuity.

Table 2.1: Unknowns describing a discontinuity in a system with $n_{c}$ components.

\begin{tabular}{rcc|}
\hline & Upstream & Downstream \\
Components & $n_{c}$ & $n_{c}$ \\
Temperature & 1 & 1 \\
Flow Velocity & $\frac{1}{n_{c}+2}$ & $\frac{1}{n_{c}+2}$ \\
TOTALS &
\end{tabular}

These states are related through Rankine-Hugoniot conditions. For a system of $n_{c}$ components the material and energy balances are given by equating $n_{c}+1$ equations like Eq. 2.33 and one given by Eq. 2.34.

$$
\phi \Lambda=\frac{u^{+} F_{1}^{+}-u^{-} F_{1}^{-}}{G_{1}^{+}}=\frac{u^{+} F_{2}^{+}-u_{1}^{-} F_{2}^{-}}{G_{2}^{+}-G_{2}^{-}}=\ldots=\frac{u^{+} F_{u c}^{+}-u^{-} F_{u c}^{-}}{G_{u c}^{+}-G_{n c}^{-}}=\frac{u^{+} \theta^{+}-u^{-} \theta^{-}}{\Gamma^{+}-\Gamma^{-}}
$$

By eliminating $\Lambda$ from this set of equations, we are left with $n_{c}$ independent equations relating the conditions on the upstream side of the shock to the downstream conditions. One of these equalities is used to relate the unknown flow velocity the flow velocity on the opposite side of the shock. Constraints on the mole fraction summations and total saturation provide two additional equations. This leaves $n_{c}+3$ variables that remain to be set.

Seting the conditions on one side of the shock fixes $n_{c}+1$ of the remaining variables. leaving a single degree of freedom. Physical considerations usually require that the shock velocity, $A$, wo be equal to 
one of the wave velocities adjacent to the disc ontinuity. This eliminates the final degree of freedom, fixing the shock conditions.

The model described in this chapter can be applied to a wide variety of multiphase, multicomponent flow problems. It includes the effects of phase behavior, temperature gradients and flow properties in a manner that is only available in complex reservoir simulation programs. Chapter 3 lays the groundwork for application of the model to specific problems. The chapter covers the details of the solution path construction and provides guidelines for how a particular solution is obtained. Details on the formation and behavior of discontunities and how they relate to the solvtion paths is discussed.

Chapter 4 provides specific examples on three displacement systems. These three systems are distinguished by the number of phases and components present. Isothermal solutions and solutions with temperature variation are discussed where appropriate. Saturation or composition profiles illustrate the behavior of the different systems. Each system is studied for the effects of varying one or more relavent parameters such as injection temperature, matrix heal capacity or oil viscosity.

Chapter 5 summarizes the general features and limitations of the model. Difficulties and the methods used to overcome potential pitfalls are stressed. Results for each specific system studied in Chapter 4 are presented. Finally, some ideas for future study and application of the model are mentioned.

\footnotetext{
${ }^{5}$ This matching of the wave velocity and shock velocity results in a shock that is known as an intermediate discontinuity. These intermediate discontinuities are the multidimensional equivalent of the Buckley-Leverell "Langent" shock. These shock classifications are discussed fully in \$3.2.
} 


\section{SOLUTION PROCEDURES}

The solution procedure for the different examples used in this dissertation indicale how the general formulation given in Eq. 2.26 is adapted 10 differen conditions. By using information on how the system is physically constrained to behave, the general formula is modified to conform to the specific problem at hand. This simplification often brings hidden features of the particular system to the surface. In this way the model helps to isolate the reasons behind the behavior of the simplified systems.

A good example of this is provided by the example problem detailed in \$4.1. Briefly, when high temperature steam is injected into a system initially filled with liquid water, a two-phase region forms where the steam and water coexist. Application of the phase rule constrains the flow to be isothermal wherever one component exists in two phases simultaneously. Keeping this restriction in mind and reducing the general formula to a two by two system with saturation and flow velocity as the independent variables reveals that the steam-water system behaves exactly like an immiscible Buckley-Leverett system.

This chapter covers the application of the concept: and equations described in Chapter 2 to specific displacement systems. The chapter begins with the basic rules that are common to all problem formulations. The rules for integrating along the composition paths are explained along with a physical interpretation of the different types of path switches. The chapter concludes with the conditions governing the shocks and discontinuities that are expected in these types of problems.

Many of the concepts and basic solution methods used in Chapter 2 are common to the different problems that this model can describe. First, the dependent variables are chosen. These quantities should be shanging in the regic - $f$ interest. Often the problem will be iroken into different regions where different dependent variai: :s change. For example, in the steam-water problem described later in \$4.1, the two-phase region uses va or saturation as the hodograph variable while in the single-phase region, the temperature is chosen as the dependent variable.

The accumulation and flux derivatives in the general eigenvalue problem (Eq. 2.26) are written with respect to the dependent variables. This means that the problem formulation may be different for the various regions within a single problem. The eigenvalue problem is solved for the velocities (eigenvalues) and the directional derivatives (eigenvectors) in the space of the dependent variables. The solution is integrated along thi: eigenvectors, from the injection to the initial condition, such that the associated eigenvalue increases as the integration moves downstream towards the initial conditions.

\subsection{PATH SEIECTION}

The velocity constraint limits the choice of integration path by requiring the solution to have increasing velocity as the integration proceeds from the injection to the initial conditions in hodograph (dependent variable) space. The following rules control the way the integration from the injection to initial conditions may proceed.

\section{Rule I: The solution must lie along a path}

The eigenvectors indicate which compositions may lie direculy ahead and direculy behind any print in the composition space. To veer off a composition path, as in Figure 3.1, violates the conservation equations (Eqs. 2.1 and 2.2). 


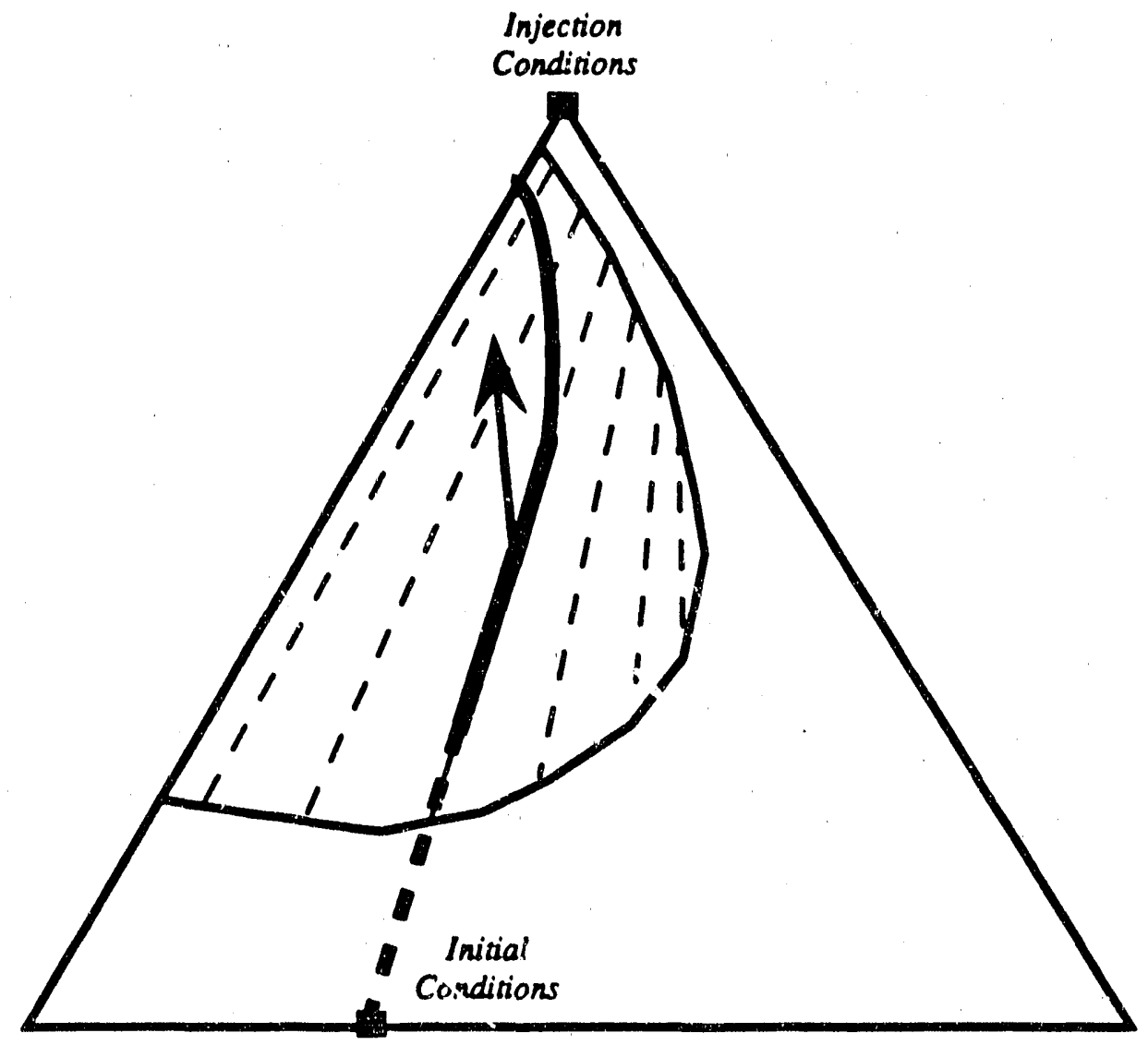

Figure 3.1: Illegal composition path for a temary displacement as the path moves from initial conditions to injection conditions.

Rule II: While travelling exclusively along path, the velocity may increase or decrease regardless of the direction of integration.

As long as the solution remains exciusively along a composition path, the change in velocity along that path determines the type of simple wave that the path represents. Increasing velocity as the solution moves downstream is in accordance with the velocity rule, and these types of simple waves are known as spreading waves. The continuous part of the saturation profile in the Buckley-Leverett solution is an example of a spreading wave. The wave is known as a spreading wave because the body of the wave is associated with steadily increasing velocities. As time passes, the conditions at the leading edge of the wave separate from their neighbors. This spreading occurs over the length of the wave cixising it to grow larger and larger.

The opposite of a spreading wave is a "self-sharpening" wave. These waves are created when the velocity decreases as the integration moves downstream. The portion of the composition path that is self-sharpening resolves into a jump discontinuity, and its velocity is calculated by applying the material balance relationships as if the wave were a genuine shock. An example of this kind of wave is, the Buckley-Leveretl saturation front. 
Between these two cases is an "indifferent" wave that neither spreads $/ 0$ sharpens. When the eigenvalue remains constant along a composition path, the compositions just ahead and just behind travel at the same velocity as the current composition point and remain the same distance from the composition point as time increases. This is equivalent to saying that the two characteristics are parallel.

Mathematically an indifferent wave is equivalent to,

$$
i^{k} \cdot \nabla_{c} \lambda^{k}=0
$$

where $\vec{l}^{k}$ is the directional derivative of the $k^{\text {th }}$ characteristic and $\nabla_{c} \lambda^{k}$ is the gradient of the $t^{\text {th }}$ characteristic velocity in composition space. Jeffrey (1976) showed that these types of waves can only occur in linear or semilinear equations. Where the coefficient matrix, in our case the fractional flow relationships, is strictly non-linear this kind of wave does not appear.

Waves that are only slightly spreading or only slightly sharpening are found ir. some of the problems that have been analyzed previously. Many times the composition paths that travel along non-tieline paths have eigenvalues that show a only a slight change along the solution route. Monroe (1986) found these types of slightly spreading waves in his solutions for $\mathrm{CO}_{2}$-hydrocarbon systems.

Rule III: Path switches may take place at points where two of the eigenvalues are equal.

The hyperbolic system of equations derived in this model has a number of singular points where two of the eigenvalues are equal. The composition path may switch from one path to the ocher at these points.

Figure 3.2 gives an example for a temary system. The eigenvalues are plotted along a tieline in a two-phase region. The dark line indicates the velocities of compositions that are all on the path as it moves from the injection condition to the initial composition. When the path comes to the equal eigenvalue point, labelled $\mathbf{E}$, the path switches from the eigenvalues of the tie-line path to those velocities along non tie-line path.

\section{Rule IV: Switches from a slow path to a faster path are allowed.}

The path switch at the equal eigenvalue point is a smooth transition from one path to the other. Path switches may also be discontinuous. These switches from a slower path to a faster path are permitted as long as the change does not violate the velocity constraint. This means that as the integration moves from the injection to the initial conditions, the only legal switches are from a slower path to a fast path. Figure 3.3 is an example of a legal path switch.

The switch from a slow path to a fast path indicates that a point in composition space has two velocities. This is mathematically equivalent to a region in $x-t$ space that is mapped onto a single point in the composition space. These regions have a single composition point that is constant over a finite intervai in time and distance; for this reason they are known as regions of constant state. This condition is true when the transformation Jacob in given by Eq. 2.9 is of rank zero. 


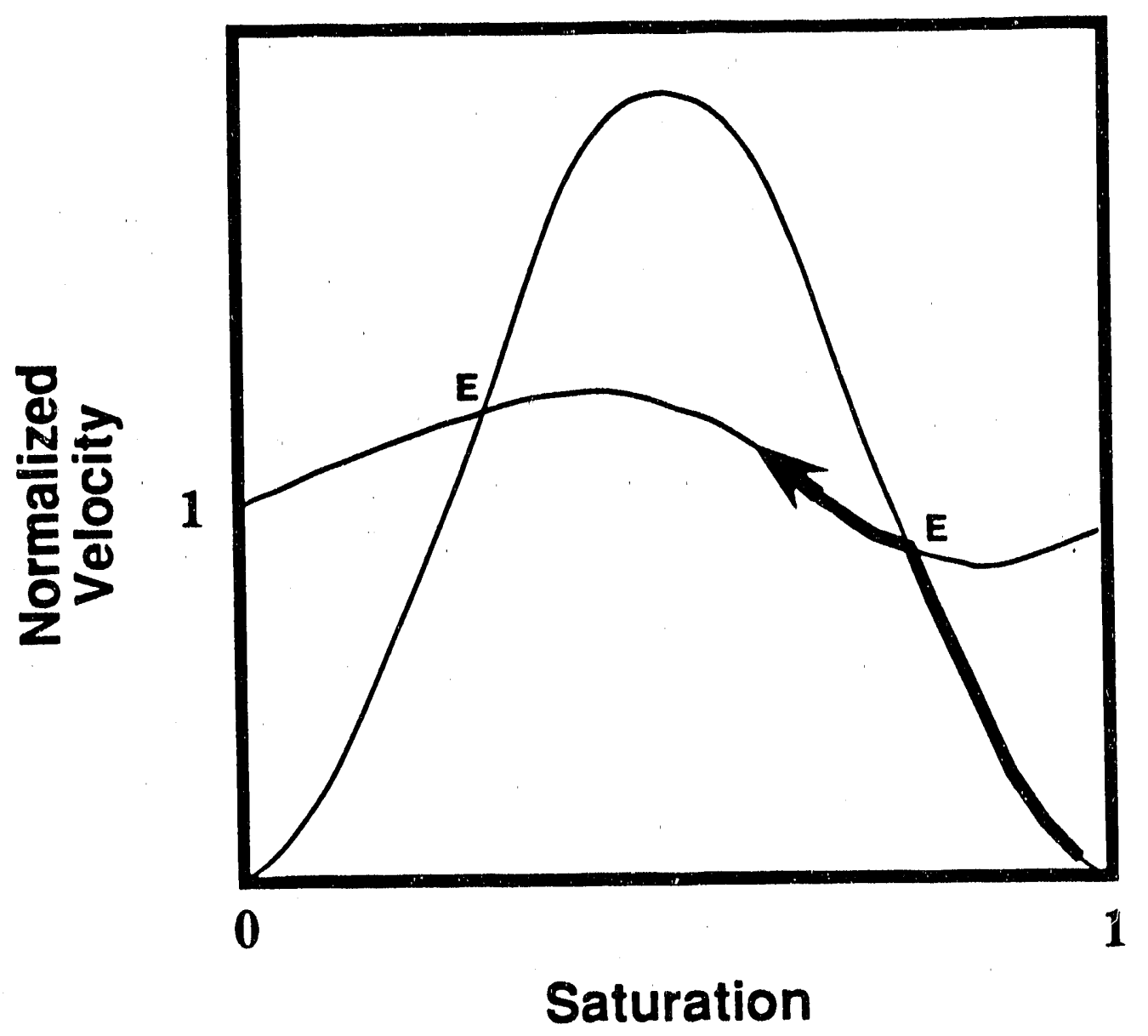

Figure 3.2: Legal path switch at an equal eigenvalue point for a temary displacement as the path moves from injection conditions to initial conditions.

Rule V: Switches from a fast path to a slower path are not allowed.

Path switches that violate the velocity constraint are not allowed. Figure 3.4 shows an example of an illegal path switch that violates the velocity constraint. This kind of switch is similar to the zone of constant state. In both cases the switch indicates that a given composition point has two coherent velocities. However the difference in the illegal switch is that the slower velocities on the non-tie-line path lie downstream of the faster tie-line velocities. This is physically not possible since all the waves began at the same position in $x-t$ space. Solutions that require this type of switch are not physically realizable. 


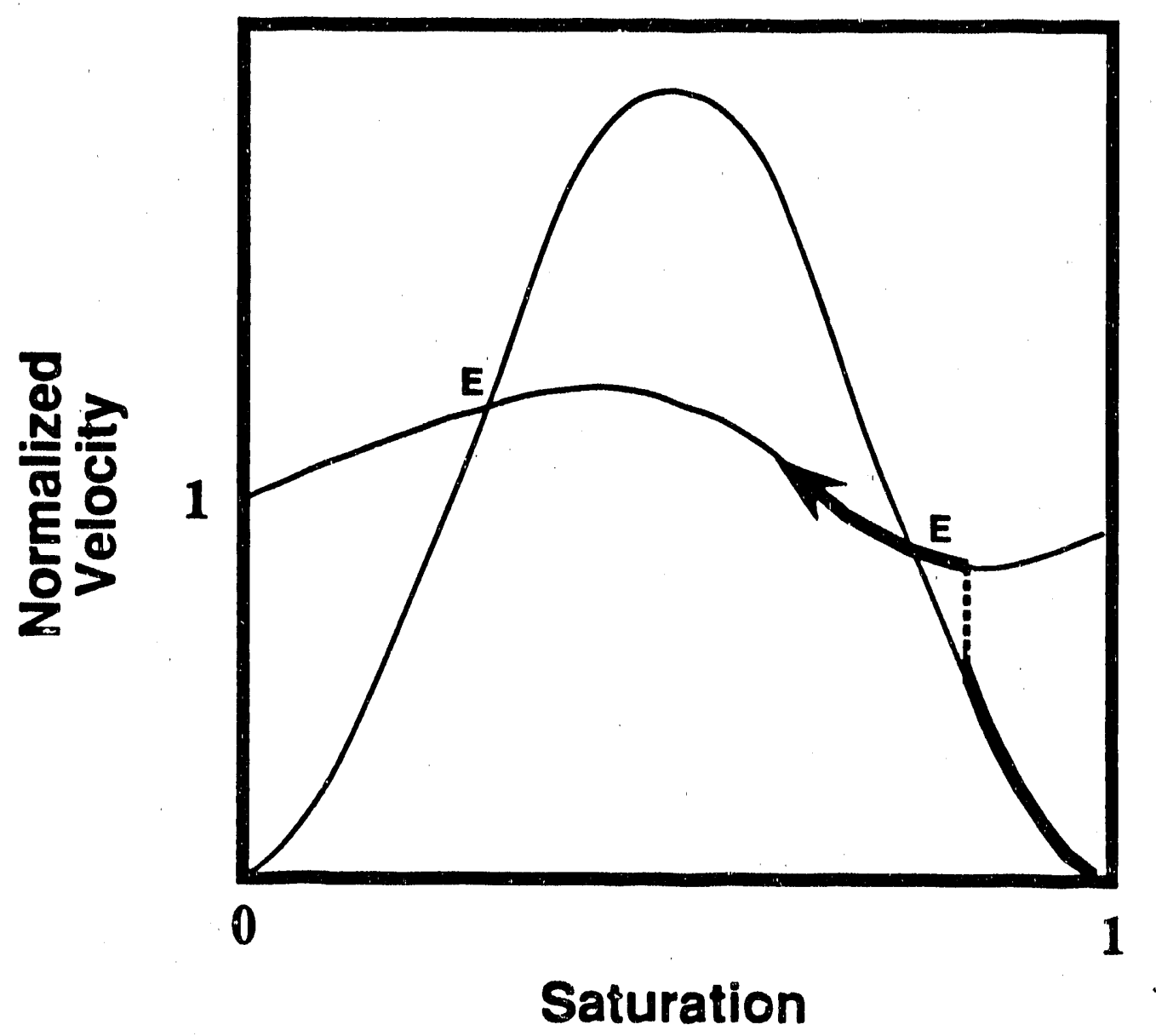

Figure 3.3: Legal path switch for a temary displacement as the path moves from injection conditions to initial conditions.

\subsection{Shock Calculations}

Discontinuities in the form of shocks and/or "self-shappening" waves appear whenever the velocity rule would be violated. Under these conditions, the differential equation along a characteristic becomes an algebraic equation relating conditions on each side of the discontinuity. These algebraic equations are the Rarkine-Hugoniot conditions that relate the hodograph variables on either side of the shock to the shock velocity.

Consider a discontinuity that propagates with a velocity, $L^{k}$. Since the system of equations is hyperbolic, there are $n_{c}+1$ distinct eigenvalues ordered such that $\lambda_{1}<\lambda_{2}<\ldots<\lambda_{n_{c}+1}$ on each side of the shock. The conditions for the shock to be admissible are provided by Lax (1557) and are,

$$
\lambda_{k}^{+} \leq \Lambda_{k} \leq \bar{k}
$$




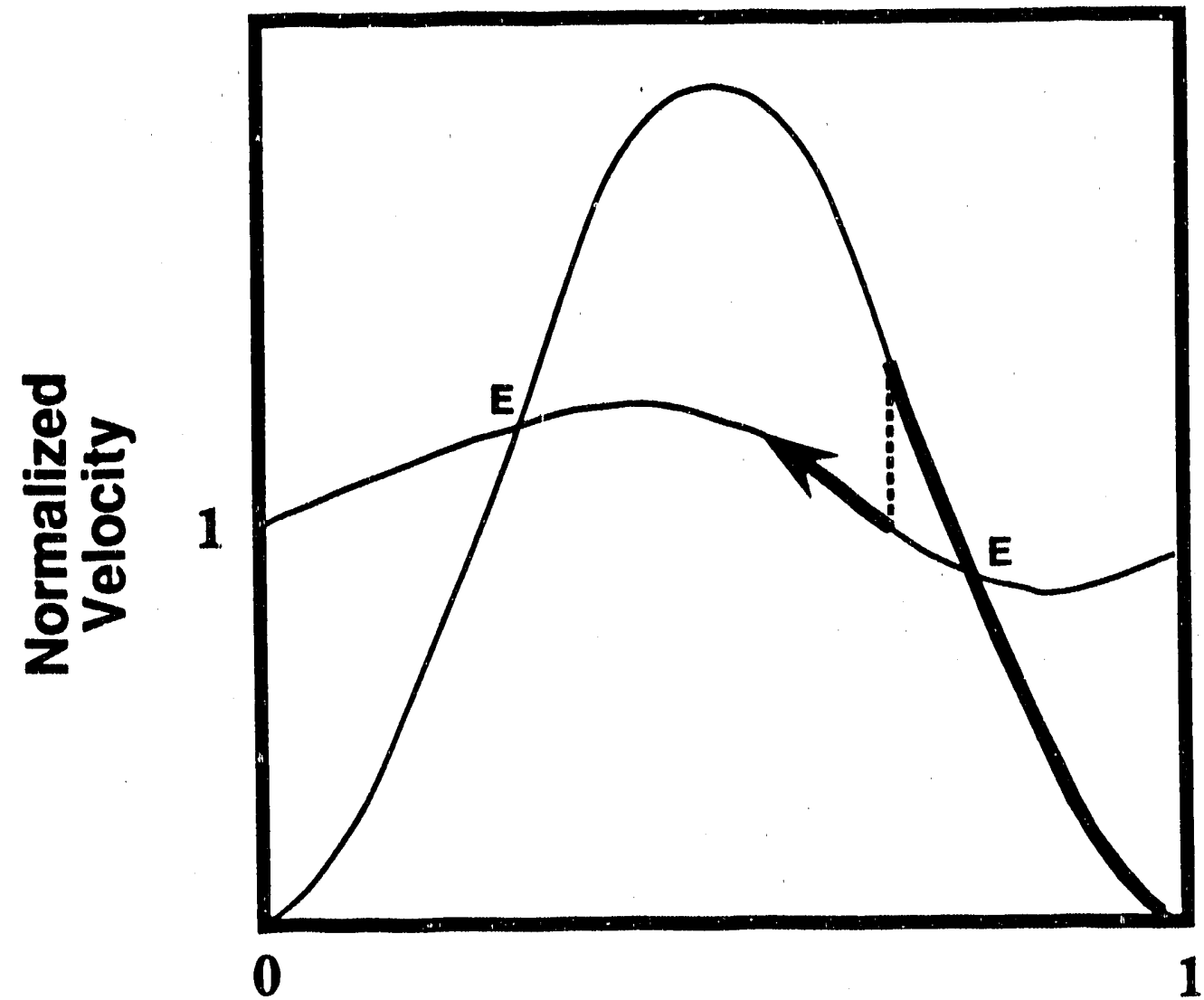

Saturation

Figure 3.4: Illegal path switch for a temary displacement as the path moves from injection conditions to initial conditions.

$$
\begin{array}{lll}
\lambda_{j}^{+}<\Lambda_{k} & \lambda_{j}<\Lambda_{k} & \text { when } j<k, \\
\Lambda_{k}<\lambda_{j}^{+} & \Lambda_{k}<\lambda_{j} & \text { when } j>k
\end{array}
$$

where the superscripts $(+)$ and $(\cdot)$ represent the conditions on the downstream and upstream side of the shock, respectively. Shocks that adhere to these relationships are said to satisfy the Lax Entropy Conditions. Keyfitz and Kranzer (1980) extended the Lax conditions to include systems which are nonstrictly hyperbolic.

Shocks are distinguished by the relationship of the shock velocity to the wave velocities immediately surrounding the discontinuity (Eq. 3.2). The three types of shocks are: contact discontinuity, intermediate discontinuity or semi-shock, and evolutionary or genuine shock (Jeffrey 1976). When the shock velocity matches the wave velocities on both sides of the discontinuity the inequalities in Eq. 3.2 are changed to equalities. These shocks are called contact discontinuities. 
Contact discontinuities are found in problems where the one of the characteristic familles is essentially linear (Keyfitz and Kranzer 1980). The fractional flow relationships for multiphase flow are, in general, nonlinear functions of the dependent variables so this type of shock does not appear in any of the solutions that are presented in this dissertation.

An intermediate discontinuity or semi-shock has a velocity that matches the wave velocity on only one side of the jump. One, but not both of the inequalities in Eq. 3.2 are changed to equalities, so either,

$\alpha$

$$
\lambda_{k}^{-}=\Lambda_{k}>\lambda_{k_{1}}^{+}
$$

$$
\lambda_{k}^{-}>\Lambda_{k}=\lambda_{k}^{+}
$$

These discontinuities are often referred to as contact discontinuities by many authors (Lax, 1957, Keyfitsand Kranzer, 1980, and Temple 1982). Other authors call these discontinuities "tangent" shocks Welge 1961 and Monroe 1986). Where the wave velocity and shock velocity match, the shock is the limit of the spreading wave on that side of the shock. In this dissertation these shocks will be refered to as either upstream intermediate discontinuity (UID) if Eq. 3.5 holds, or downstream inter. mediate discontinuities (DID) when Eq. 3.6 is true.

Equation 3.5 is the condition that controls the front in the Buckley-Leverett problem. Figure 3.5 shows how the tangent construction is represents the matching of the shock and wave, or characteristic velocities. The tangent connecting the downstream side of the shock to the upstream side represents the Rankine-Hugoniot material balance across the shock. The slope of the tangent line is equal to the shock velocity, $\Lambda$.

The wave velocity in the Buckley-Leverett case is given by the velocity of the saturation, $\partial f_{w} / \partial S_{w}$. This represents the eigenvalue on the upstream side of the shock. $\lambda$. When the slope of the fractional flow curve and the material balance (tangent) line are equal, then Eq. 3.5 holds.

A genuine or evolutionary shock has a velocity that does not match the wave velocity on either side of the discontinuity. Both of the inequalities in Eq. 3.2 hold for the genuine shock. These discontinuities are found in solutions where the jump condition is bounded by regions of constant state on both sides of the shock (Monroe 1986 and Pande 1988). Monroe refers to these discontinuities as "nontangent" shocks. Many times these shocks are the result of arriving on an exit tie-line path at a point where an immediate jump to the exit conditions is indicated. The inclusion of genuine discontinuities into the physical solution represents the most difficult aspect of solution path construction. The difficulty arises from the realization that not all the information required is immediately availabie. Many times the shock velocity and/or the conditions on the upstream and downstream sides of the shocks are not known a priori.

The nature of the region adjacent to the shock can provide a starting place for the shock calculation. A region of continuous variation usually means that the shock is an intermediate discontinuity with a region of constant state on the other side of the shock. In this case a multidimensional tangent construction (Welge 1952) can be used to locate the position of the shock if the constant state conditions are known.

On the other hand, when a genuine shock is needed, the conditions surrounding the discontinuity are unknown points on unconnected composition paths. The situation is illustrated in Fig. 3.6.

Points 1 and 2 are on the path that connects to the injection conditions and points $\mathrm{A}-\mathrm{C}$ are on a path that intersects the initial conditions. The narrow dot-dashed lines represent some of the possible jumps from the injection path to the initial path. These lines represent only a small number of the possible jumps. Only one of these lines represents the actual shock that satisfies the velocity constraint. 
Locating the one shock from among all the possibilities is a most difficult task. Often this task becomes the heart of the solution construction problem.

Chapter 4 covers the application of the techniques described in this chapter to different displacement systems. These solutions range from a simple steam-water displacement to a complex system where phase behavior, temperature effects and flow properties interact to control the flow process.

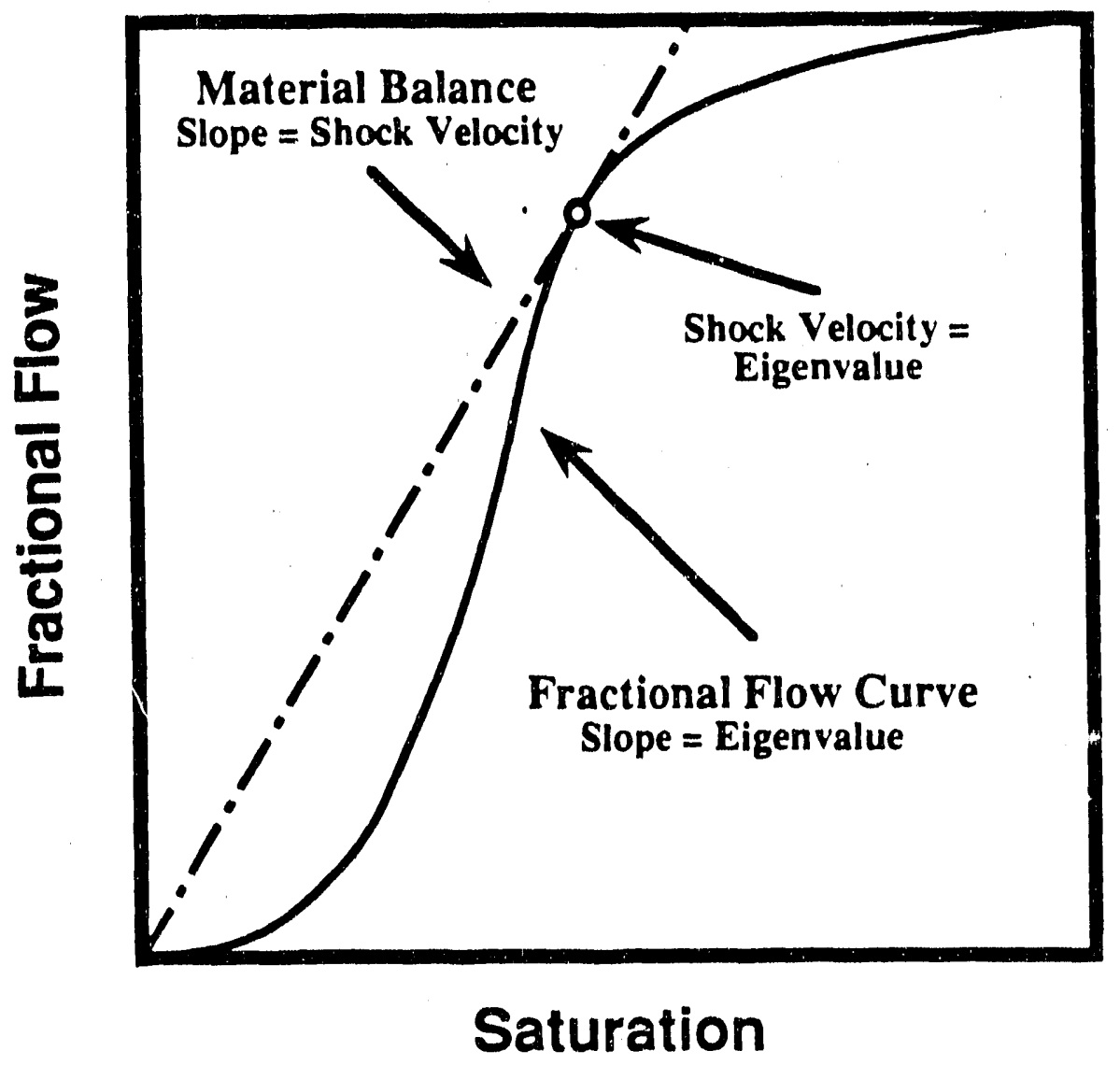

Figure 3.5: Tangent construction for Buckley-Leverett shock front that illustrates how the tangent construction matches shock and wave velocities. 


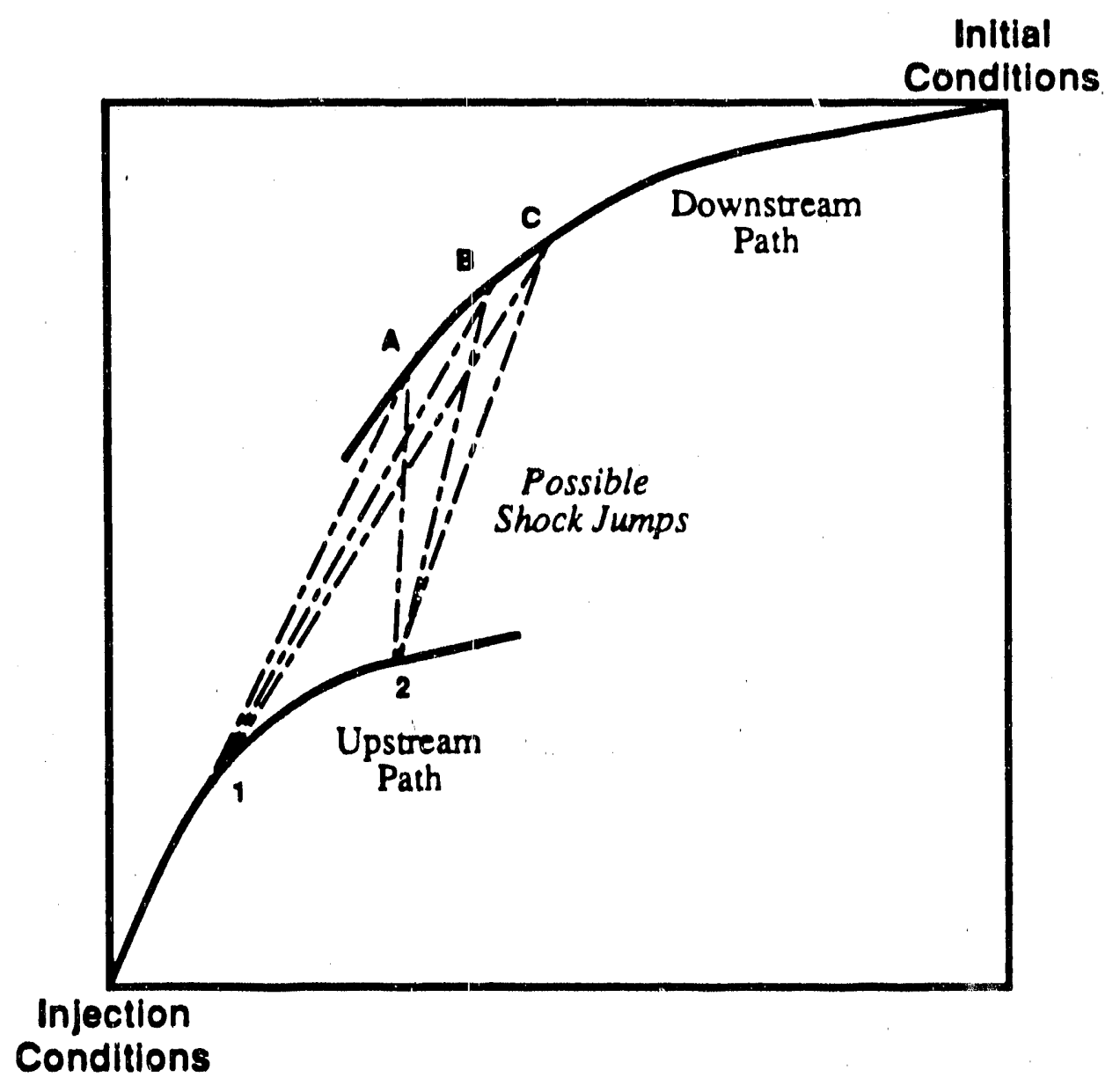

Figure 3.6: Hypothetical shock locations for a three component system. 


\section{EXAMPLE SOLUTIONS}

The solution procedures discussed in Chapler 3 are applied to specific displacement problems. Three different classes of problems are covered in this chapter. In general, the problems are classified by the number of components and the total number of phases. The greater these numbers, the more complex the problem. The progression of problems begins with a single component, two-phase system with temperature variation. This problem is simple in concept, but reveals very interesting results conceming the behavior of these systems.

The second problem adds an immiscible component to the single component problem. This addition adds a new flowing phase to the single component problem. The second phase can lead to the formation of temperature profiles that are not found in the single component case. The new phase also changes the nature of the flow in the isothermal region from that of two-phase to three-phase flow.

The third and final problem extends the two-phase problem by adding the effect of phase behavior. The steam-oil-water system in the second problem is extended by adding a third component that is partially miscible in both the aqueous and oleic phases. The temperature changes the phase behavior by moving the water component from a less mobile liquid phase at low temperatures to the highly mobile vapor phase at high temperatures. The solubility of the third component, represented by $\mathrm{CO}_{2}$, in the oleic phase is also affected by the temperature variations.

\subsection{SINGLE COMPONENT WITH TEMPERATURE VARIATION}

\subsubsection{Problem Formulation}

The injection of steam into liquid water represents a simple type of problem that can be studied using the method of characteristics. By eliminating the compositional variations, the behavior of the temperature waves, especially in the single-phase region can be observed.

The method of characteristics maps from the space of independent variables, $x$ and $t$, into a space that is representative of the quantities that can be measured. In this problem the hodograph variables are vapor saturation, temperature and local flow velocity.

These three variables are not independent. The Gibbs phase rule relates the degrees of freedom to the number of components and the number of coexisting phases. When a single component exists in two phases the phase rule indicates that there is only one degree of freedom. Fixing the pressure also sets the temperature at the saturation temperature corresponding to that pressure. This restriction changes the nature of the problem by forbidding the solution from most of the hodograph space.

Figure 4.1 illustrates where the solution can exist in hodograph space. The allowable solution consists of three straight lines. Above the saturation temperature, the liquid saturation is set at zero and the temperature varies from the injection temperature down to the saturation temperature. The horizontal line represents the two-phase region. When a single component exists in two-phase equilibrium at a constant pressure, the temperature is fixed at the saturation temperature. Finally in the liquid region, the saturation is set at zero and the temperature varies down from the saturation value to the initial value.

Note that flow velocity, which is the third dimension of the hodograph space, is not shown. This is because the flow velocity is not physically related to the temperature and saturation variables. This changes the picture only slighly, changing the three lines into a three dimensional curve in full hodograph space.

The mathematical model for this situation consists of one mass balance and the enthalpy balance. The solution is divided into three distinct regions represented by the three straight lines in Figure 4.1. 


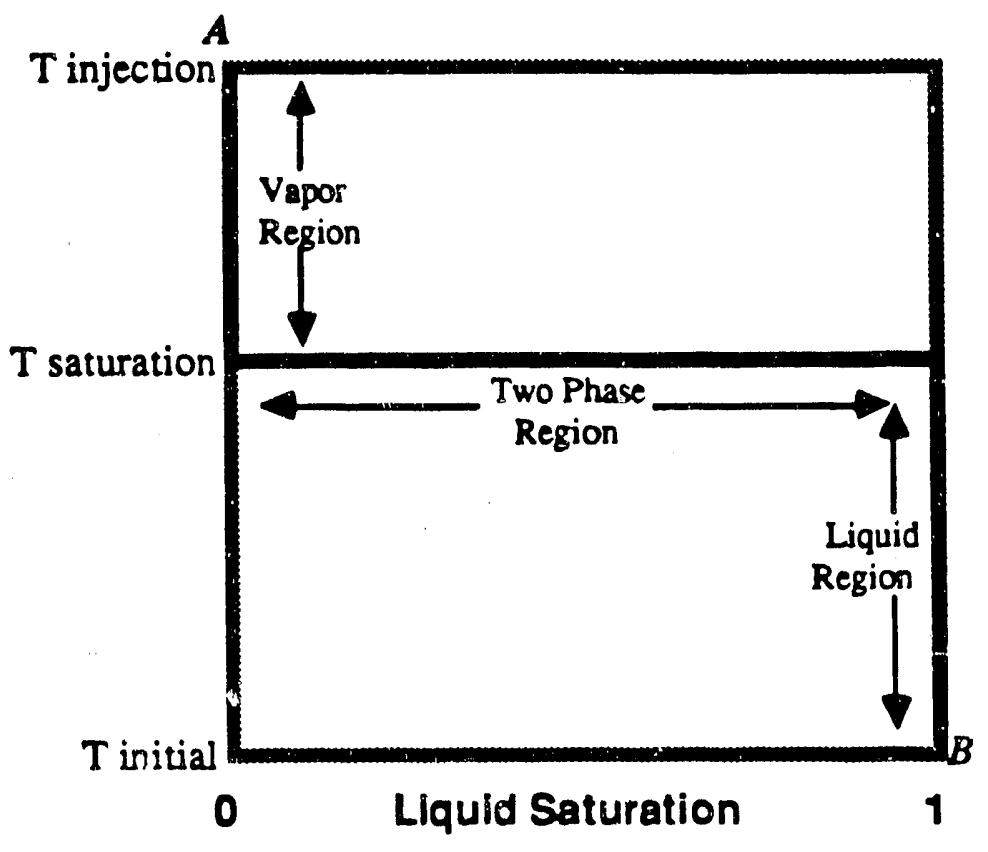

Figure 4.1: Solution line in hodograph space for steam injected into liquid water at constant pressure.

The two-phase region lies between the single-phase regions providing a transition from steam to liquid water. This means that as the solution traverses the hodograph space from point $\mathbf{A}$ to $B$, the eigenvalues must increase.

\section{Single-Phase Regions}

In the single-phase region saturations are fixed, leaving the temperature and the flow velocity as the hodograph variables. The eigenvelue problem for this set of equations is shown below.

$$
\left[\begin{array}{ll}
\frac{\partial F}{\partial T} & \frac{\partial F}{\partial u} \\
\frac{\partial \theta}{\partial T} & \frac{\partial \theta}{\partial u}
\end{array}\right]\left[\begin{array}{l}
\frac{d T}{d \eta} \\
\frac{d u}{d \eta}
\end{array}\right]-\lambda\left[\begin{array}{ll}
\frac{\partial G}{\partial T} & 0 \\
\frac{\partial T}{\partial T} & 0
\end{array}\right]\left[\begin{array}{l}
\frac{d T}{d \eta} \\
\frac{d u}{d \eta}
\end{array}\right]
$$

Since the matrix is two by two, the solution for the two eigenvalues is a quadratic equation. As in the general case (Eq. 2.26) one of the eigenvalues is infinite and the finite value is given by,

$$
\lambda^{\prime}=\frac{\frac{\partial \theta}{\partial T} \frac{\partial F}{\partial u}-\frac{\partial \theta}{\partial u} \frac{\partial F}{\partial T}}{\frac{\partial T}{\partial T} \frac{\partial F}{\partial \nu}-\frac{\partial \theta}{\partial u} \frac{\partial G}{\partial T}}
$$

The associated eigenvector is found by seting one of the components to a predefined value and solving for the other component. Either the mass or enthalpy equation can be used to find the value of this component. Fixing the value of $(\partial T / \partial \eta)$, gives for the velocity change, 


$$
\frac{\partial u}{\partial \eta}=\frac{\frac{\partial T}{\partial \eta}\left(\lambda \frac{\partial G}{\partial T}-\frac{\partial F}{\partial T}\right)}{(\partial F / \partial u)}
$$

In the single-phase region, the evaluation of the derivatives in the eigenvaue matrix is simplified because the saturation and fractional flow terms are either one or zero and drop out of the equations. These derivatives are,

$$
\begin{array}{cc}
\frac{\partial G}{\partial T}=\frac{\partial \rho}{\partial T} & \frac{\partial T}{\partial T}=H \frac{\partial \rho}{\partial T}+\left(\frac{1-\phi}{\phi}\right) \rho_{m} C p_{m} \\
\frac{\partial F}{\partial T}=\frac{1}{\phi}\left[u^{\prime} \frac{\partial \rho}{\partial T}+\rho \frac{\partial u^{\prime}}{\partial T}\right] & \frac{\partial \theta}{\partial T}=\frac{1}{\phi}\left[u^{\prime} \rho \frac{\partial H}{\partial T}+\rho H \frac{\partial u^{\prime}}{\partial T}\right]
\end{array}
$$

and

$$
\frac{\partial F}{\partial u}=\frac{\bar{F}}{u^{T}} \quad \frac{\partial \theta}{\partial u}=\frac{\theta}{u^{T}}
$$

Substituting these values into Eq. 4.2, and remembering that in the single-phase region $Q=F \times H$ gives for the eigenvalue,

$$
\lambda^{\prime}=\frac{u^{\prime}}{\phi}\left[\frac{\rho \frac{\partial H}{\partial T}}{\rho \frac{\partial H}{\partial T}+\left(\frac{1-\phi}{\phi}\right) \rho_{m} C p_{m}}\right]
$$

Eq. 4.5 represents what can be thought of as a "fractional" heat capacity. Every unit of enthalpy that exists at a point in the single-phase region partitions into the fluid phase or the matrix. Eq. 4.5 represents the fraction of the enthalpy that the fluid phase takes over the total enthalpy available.

Enthalpy carried along with the fluids must heat the matrix $t 0$ its temperature before the wave can proceed beyond its location. The ability of the matrix to store enthalpy acts to slow the temperature wave. As the matrix heat capacity decreases, or the porosity increases, a larger fraction of the enthalpy accumulation remains in the mobile phase and the velocity of the temperature wave increases, approaching the flow velocity at a matrix heat capacity of zero.

The temperature profile jumps from the injection temperature in the single-phase region to the saturation temperature in the two-phase region. This initial discontinuity is prevented from spreading in the single-phase region by the nawure of the single phase flow. The enthalpy can only be carried downstream by the flowing fluid. The matrix thermal conductivity is neglected and the matrix is stationary. Therefore, only one velocity, the velocity of the flowing fluid, carries the enthalpy downstream. The lack of a second flowing phase creates the temperature shock in the single-phase region.

\section{Two-Phase Region}

Temperature is constant in the two-phase region, so a hodograph variable other than temperature is needed in this region. The logical choice is one of the phase saturations; in this case the vapor phase saturation is chosen. 
The eigenvalue problem is similar $w$ Eq. 4.1, except that saturation replaces temperature as the hodograph variable. Derivatives with respect to temperature become derivatives taken with respect to vapor saturation.

$$
\left[\begin{array}{ll}
\frac{\partial F}{\partial S_{v}} & \frac{\partial F}{\partial u} \\
\frac{\partial \theta}{\partial S_{v}} & \frac{\partial \theta}{\partial u}
\end{array}\right]\left[\begin{array}{l}
\frac{d S_{v}}{d \eta} \\
\frac{d u}{d \eta}
\end{array}\right]-\lambda\left[\begin{array}{ll}
\frac{\partial G}{\partial S_{v}} & 0 \\
\frac{\partial T}{\partial S_{v}} & 0
\end{array}\right]\left[\begin{array}{l}
\frac{d S_{v}}{d \eta} \\
\frac{d u}{d \eta}
\end{array}\right]
$$

The eigenvalue and tigenvector are calculated in the same manner as in the single-phase regions

$$
\lambda^{\prime \prime}=\frac{\frac{\partial \theta}{\partial S_{v}} \frac{\partial F}{\partial u}-\frac{\partial \theta}{\partial u} \frac{\partial F}{\partial S_{v}}}{\frac{\partial F}{\partial S_{v}} \frac{\partial \theta}{\partial u}-\frac{\partial G}{\partial \nu} \frac{\partial S_{v}}{\partial S^{2}}}
$$

and

$$
\frac{\partial u}{\partial \eta}=\frac{\frac{\partial S_{v}}{\partial \eta}\left(\lambda \frac{\partial G}{\partial T}-\frac{\partial F}{\partial T}\right)}{\frac{\partial F}{\partial u}}
$$

Considerable simplification of the expression for the two-phase velocity is possible because the temperature and fluid properties in the two-phase region are constant. The only changes are the phase saturations and the fractional flows. Using the definitions in Eqs. 2.3-2.6 when the number of phases is two, $n_{c}=2$, gives,

$$
\begin{array}{cc}
\frac{\partial G}{\partial S_{v}}=\rho_{v}-\rho_{l} & \frac{\partial T}{\partial S_{v}}=H_{v} \rho_{v}-H_{l} \rho_{l} \\
\frac{\partial F}{\partial S_{v}}=\frac{u^{\prime \prime}}{\phi}\left(\rho_{v}-\rho_{l}\right) \frac{\partial f_{v}}{\partial S_{v}} & \frac{\partial \theta}{\partial S_{v}}=\frac{u^{\prime \prime}}{\phi}\left(\rho_{v} H_{v}-\rho_{l} H_{l}\right) \frac{\partial S_{v}}{\partial S_{v}}
\end{array}
$$

and

$$
\frac{\partial F}{\partial \nu}=\frac{F}{u^{I I}} \quad \frac{\partial \theta}{\partial \nu}=\frac{\theta}{u^{\prime \prime}}
$$

Substiuting these expressions into Eq. 4.7 gives a very simple expression for $\lambda^{\prime \prime}$

$$
\lambda^{\prime \prime}=\frac{\frac{u^{\prime \prime}}{\varphi}\left[\frac{F}{u^{\prime \prime}}\left(\rho_{v} H_{v}-\rho_{l} H_{1}\right)-\frac{\theta}{u^{\prime \prime}}\left(\rho_{v}-\rho_{l}\right)\right] \frac{\partial \partial_{v}}{\partial S_{v}}}{\left[\frac{F}{u^{I I}}\left(\rho_{v} H_{v}-\rho_{1} H_{l}\right)-\frac{\Theta}{u^{\prime \prime}}\left(\rho_{v}-\rho_{l}\right)\right]}=\frac{u^{\prime \prime}}{\phi}\left(\frac{\partial f_{v}}{\partial S_{v}}\right)
$$


The saturation velocity in this case is the same as the Buckley-Leverett velocity calculated in the two component, immiscible case. The derivative of the fractional flow curve gives the wave velocity in the two-phase region. This analysis makes sense. In the two-phase region, because the system is assumed to be at constant pressure, then it must also be at constant temperature. The steam, water, and matrix coexist with no energy or mass transfer between the phases. The displacement behaves as an isothermal, immiscible displacement.

The velocity component of the eigenvector is calculated using the same equation as in the singlephase crse. Writing the analog to Eq. 4.3 in terms of the saturation derivatives gives

$$
\frac{\partial u}{\partial \eta}=\frac{\partial S_{v}}{\partial \eta} \frac{\lambda \frac{\partial G}{\partial S_{v}}-\frac{\partial F}{\partial S_{v}}}{(\partial F / \partial u))}
$$

Because the densities of the phases remain constant in this region, the change in overall velocity must be zero. This is similar to marching along a tie-line in the immiscible case. This is made apparent by evaluating the derivatives of Eq. 4.13 in the two-phase region. Substitution of the two-phase derivatives into Eq. 4.13 gives

$$
\frac{\partial u}{\partial \eta}=\frac{\partial S_{v} \frac{\partial S_{v}}{\partial S_{v}}\left(\rho_{v}-\rho_{l}\right)-\frac{\partial S_{v}}{\partial S_{v}}\left(\rho_{v}-\rho_{l}\right)}{\frac{\partial F}{\partial u}}=0
$$

The phase rule confines the two-phase region to be at a constant temperature. This also requires the phase densities to be constant. The flow velocity responds to variations in phase density, so in the twophase region the flow velocity is constant.

\section{Connecting the Single and Two-Phase Regions}

The three regions must be physically connocted through a set of shocks. These jumps must occur when the coefficients of the eigenvalue matrix are discontinuous (Jeffrey 1976). Changes in densities and fractional flows at a phase boundaries are the sources of these transition shocks. The problem is to find a pair of shocks that carry the solution into and out of the two-phase region.

The shock solution we seek is described in $\$ 3.2$ as an intermediate discontinuity. The shock velocity matches the wave velocity on only one side of the jump. The correct solution is an intermediate discontinuity where the shock velocity matches the wave velocity in the two-phase region. Monroe calls this :ype of shock a "tangent" shock. Mal'sematically, the equations are,

$$
\Lambda=\frac{1}{\phi} \frac{u^{\prime \prime} F^{\prime \prime}-u^{\prime} F^{\prime}}{G^{\prime \prime}-G^{I}}=\lambda^{\prime \prime}=\frac{u^{\prime \prime}}{\phi}\left(\frac{\partial f_{v}}{\partial S_{v}}\right)
$$

The reason that the velocities must match on the two-phase side of the shock is that in the singlephase region no spreading of the temperature profile can occur. In order to match the shock and wave velosities at the discontinuity, the shock must be the limit of a continuous variation. In the single-phase region, there are no fractional flow effects to partition the enthalpy into a more or less mobile phase. The assumption of local temperature equilibrium requires that the matrix and the fluids be at the same temperature. Since the matrix is immobile, the temperature of the fluid must equal the matrix temperawure before it can move downstream. Therefore, the upstream side of the trailing shock must be at the injection temperature and the downstream side of the leading shock must be at the initial temperature. 
The solution procedure is iterative. The unknowns for this calculation are the conditions on the two-phase side of the shock. The unknowns are the saturation and flow velocity $u^{\prime \prime}$. The trailing shock conditions are calculated first. A diagram of the known conditions in the system is given in Figure 4.2.

The first step is to guess a saturation value on the upstream side of the shock. The primary quantities, G, F, $\Gamma$ and $\theta$ are calculated on both sides of the estimated shock. The two-phase flow velocity, $u^{l l}$, can then be calculated by equating the Rankine-Hugoniot conditions for the mass and the enthalpy and solving for $u^{I l}$. The jump balance is given by,

$$
\frac{u^{\prime \prime} F^{\prime \prime}-u^{l} F^{I}}{G^{\prime \prime}-G^{\prime}}=\frac{u^{\prime \prime} \Theta^{\prime \prime}-u^{\prime} \Theta^{l}}{\Gamma^{\prime \prime}-\Gamma^{\prime}}
$$

\section{Trailing Shock Conditions}

\begin{tabular}{l|l}
\hline \multicolumn{1}{c|}{$\begin{array}{c}\text { Single Phase } \\
\text { Region }\end{array}$} & $\begin{array}{l}\text { Two Phase } \\
\text { Region } \\
\text { Knowns } \\
\text { Flow Velocity } \\
\text { Saturation } \\
\text { Temperature }\end{array}$ \\
Tinjection & $\begin{array}{l}\text { Temperature }= \\
\text { T saturation } \\
\text { Unknowns } \\
\text { Flow Velocity } \\
\text { Vapor Saturation }\end{array}$ \\
\end{tabular}

Figure 4.2: Trailing shock conditions for steam-water problem.

Solving for the velocity ratio yields,

$$
\frac{u^{\prime \prime}}{u^{\prime}}=\frac{F^{\prime}\left(\Gamma^{\prime \prime}-\Gamma^{\prime}\right)-\Theta^{\prime}\left(G^{\prime \prime}-G^{\prime}\right)}{F^{\prime \prime} F^{\prime}\left(\Gamma^{\prime \prime}-\Gamma^{\prime}\right)-\Theta^{\prime \prime}\left(G^{\prime \prime}-G^{\prime}\right)}
$$

Once the two-phase flow velocity is known, the shock velocity can be calculated using the RankineHugoniot conditions for either the mass or enthalpy across the shock. The equation for the mass balance is given by,

$$
\Lambda_{w}=\left(\frac{1}{\phi}\right) \frac{u^{\prime \prime} F^{\prime \prime}-u^{\prime} F^{\prime}}{G^{\prime \prime}-G^{\prime}}
$$

and the enthalpy balance yields, 


$$
\Lambda_{H}=\left(\frac{1}{\phi}\right) \frac{u^{\prime \prime} \Theta^{\prime \prime}-u^{\prime} \Theta^{\prime}}{\Gamma^{\prime \prime}-\Gamma^{I}}
$$

either of these equations can be used to calculate the shork velocity. These two Dalance equations are coupled by the two-phase flow velocity calculation represented by Eq. 4.17.

The last velocity needed is the wave velocity in the two-phase region. Eq. 4.7 is used to calculate the wave velocity. The correct solution is where the shock and wave velocities are equal. A plot of the shock and wave velocities against saturations shows how sensitive the solution is to changes in saturation.

From Figure 4.3, the saturatic $n$ guess can be adjusted depending on the difference between the two velocity values. The saturation is regulated down if the shock velocity exceeds the wave velocity and is increased if the opposite is true.

The calculation for the leading shock is similar to the trailing shock with one exception. The flow velocity on the single-phase side of the shock is the unknown, along with the saturation on the twophase side. The flow velocity on the two-phase side of the shock is equal to the flow velocity calculated at the trailing shock since there is no change in the two-phase region.

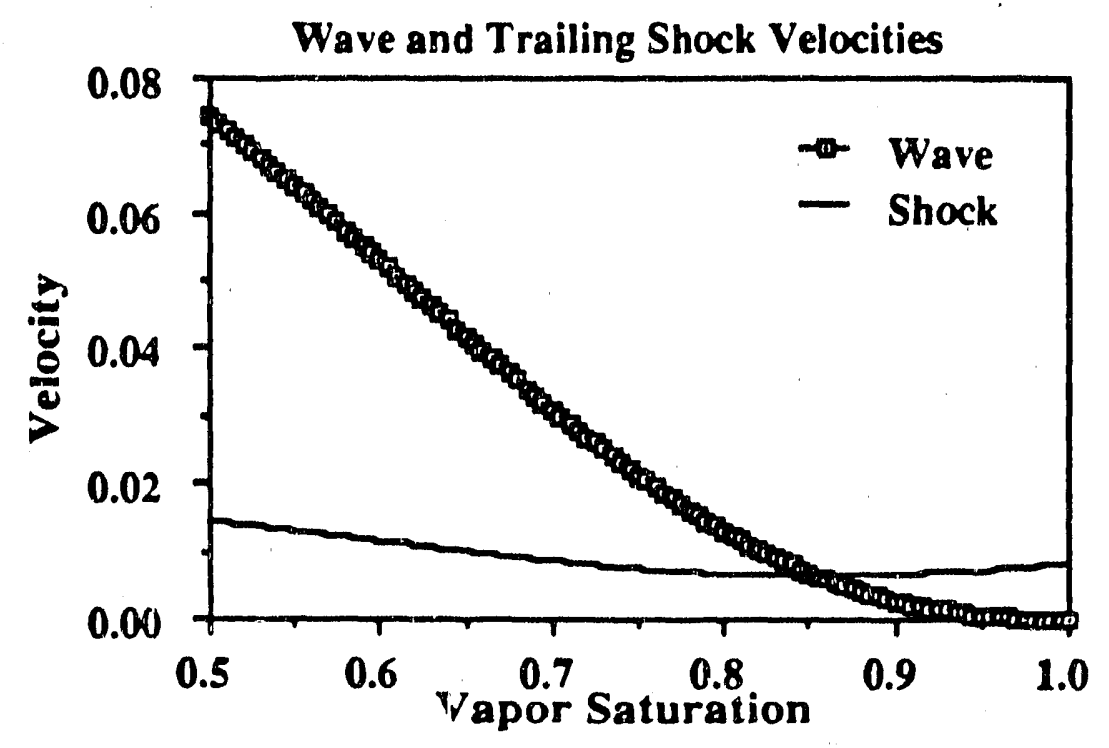

Figure 4.3: Wave and shock velocities for trailing shock in steam-water displacement

After the shock conditions are found, the saturation profile in the two-phase region is constructed by relating the wave velocity calculated by Eq. 4.7 to the saturation value. The wave velocity tells how far a given saturation has moved in after a given number of pore volumes have been injected. The wave velocity is multiplied by the injection amount to give the position of the saturations between the two shock values.

An EXCEL spreadsheet was developed to automate the calculation for a given set of injection and initial conditions. Details of the spreadsheet contents along with the calculations for the examples are found in Appendix D. 
Three parameters were studied for their effect on the saturation profile and location of the trailing and leading shocks. The three variables are: the injection temperature of the steam, the initial temperature of the water, and the heal capacity of the surrounding matrix.

Table 4.1 shows the conditions that are common for all three examples. For all cases the saturation temperature, fluid densities and enthalpies were found by curve fitting a fifth order polynomial in temperature to the values given in Reynolds (1979) for water at $2.0 \mathrm{MPa}$ [290.16 psia]. Viscosities at the saturation pressure were estimated from equations given by Reid (1977). The injection velocity was set at one pore volume of steam per dimensionless time unit.

\begin{tabular}{|rrl|}
\hline Pressure & & $2.0 \mathrm{M} \mathrm{Pa}$ \\
Injection Velocity & & $1.0(\mathrm{~m} / \mathrm{day})$ \\
Saturation Temperature & $T_{\text {sal }}$ & $485.57 \mathrm{~K}$ \\
Irreducible Water Saturation & $S_{w i}$ & 0.0 \\
Steam Phase Exponent & $n_{g}$ & 1.0 \\
Water Phase Exponent & $n_{w}$ & 3.0 \\
\hline Fluid Properties at & Saturation Temperature \\
\hline Steam Viscosity & $\mu_{g}$ & $0.0217(\mathrm{mPa} \cdot \mathrm{sec})$ \\
Water Viscosity & $\mu_{w}$ & $0.1303(\mathrm{mPa} \cdot \mathrm{sec})$ \\
Steam Density & $\rho_{g}$ & $9.970241\left(\mathrm{~kg} / \mathrm{m}^{3}\right)$ \\
Water Density & $P_{w}$ & $850.4723\left(\mathrm{~kg} / \mathrm{m}^{3}\right)$ \\
Steam Enthalpy & $H_{g}$ & $2808.52(\mathrm{~kJ} / \mathrm{kg})$ \\
Water Enthalpy & $H_{w}$ & $901.41(\mathrm{~kJ} / \mathrm{kg})$ \\
\hline Matrix & Properties \\
\hline Density & $\rho_{m}$ & $2650.0\left(\mathrm{~kg} / \mathrm{m}^{3}\right)$ \\
Heat Capacity & $C_{p_{m}}$ & $0.047(\mathrm{~kJ} / \mathrm{kg}-\mathrm{K})$ \\
Porosity & $\phi$ & 0.100 \\
\hline
\end{tabular}

Table 4.1: Base conditions for the generation of steam-water profiles at $2.0 \mathrm{MPa}$

The first example looks at the effects of changing the termperature of the injected steam. Figure 4.4 shows five saturation profiles after the injection of 1.0 pore volume of steam.

The major difference between the five profiles is the position of the leading and trailing shocks as the injection temperature increases. At the leading shock, the shock height remains constant, while the velocity increases with increasing injection temperature. The conditions ahead of the leading shock are constant for all the profiles. This causes the saturation change across the discontinuity to be the same for all the cases. The reason that the position of the shock changes is the reduced flow velocity in the twophase region at higher temperatures. Physically, the lower velocity corresponds to the decreased heat content at higher temperatures. 


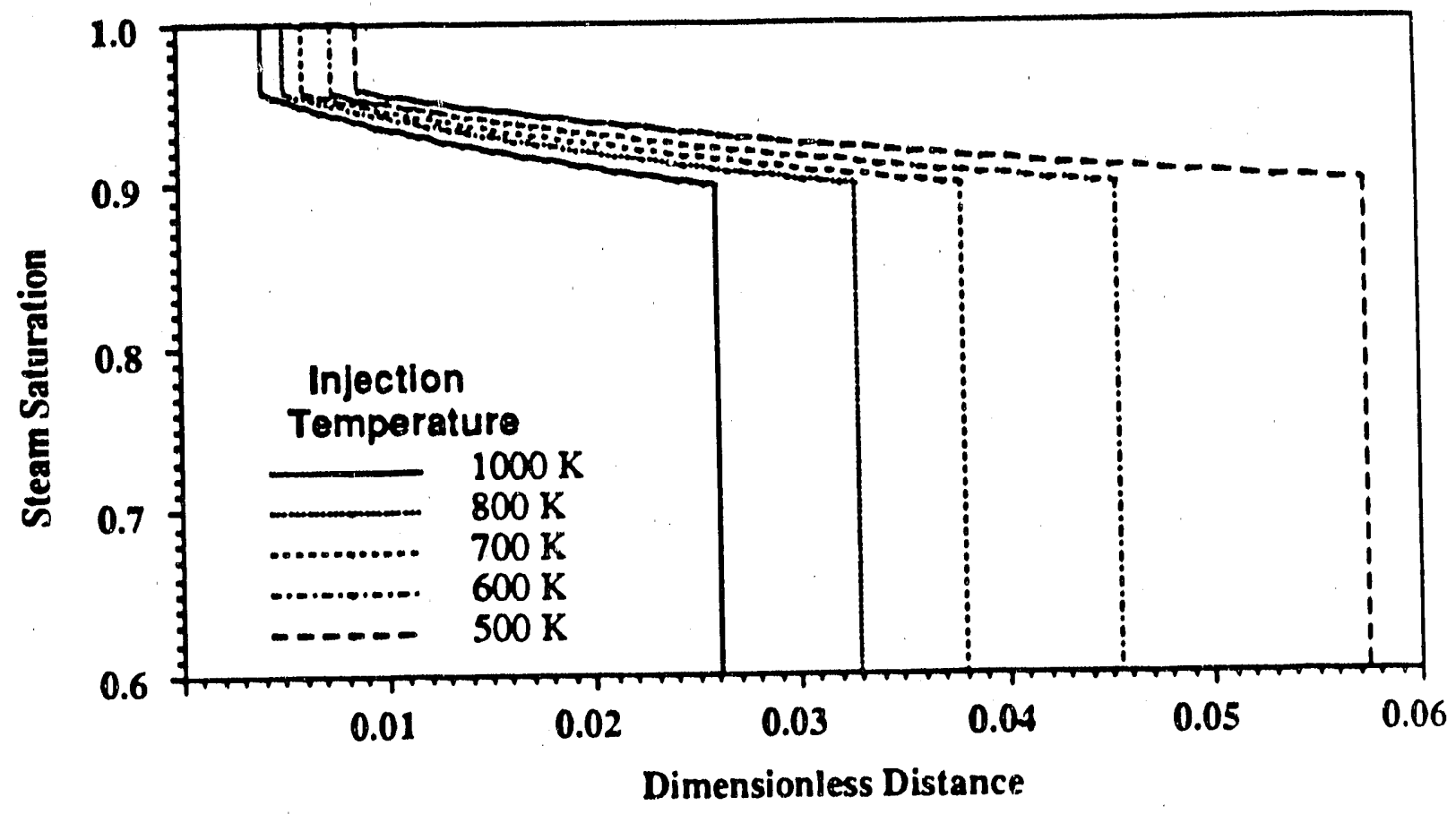

Figure 4.4: Saturation profile after 1.0 pore volumes of steam injection into water at injection temperatures from $500 \mathrm{~K}-1000 \mathrm{~K}$.

The behavior of the trailing shock is explained by looking at the heat balance across the trailing shock. As the injection temperature is increased, the enthalpy difference across the shock increases. To compensate for the increased heat crossing the discontinuity at the higher injection tentperature, the shock velocity decreases. This result is seen in Figure 4.4 .

The second parameter studied was the initial temperature. It causes much smaller and more predictable changes in the saturation profiles than the initial temperature. Figure 4.5 illustrates the change in location of the leading shock as the initial temperature changes from $300 \mathrm{~K}$ to $450 \mathrm{~K}$. The initial temperature only changes the location and height of the leading shock. The hyperbolic nature of the equations means that the conditions downstream do not affect the upstream values. The trailing shock would behave the same regardless of the temperature or saturations downstream of the two-phase region.

The reason for the faster shocks at higher initial temperatures is simply a heating effect. The fluid from the two-phase region must heat the matrix and surroundings from the initial temperature to the saturation temperature before the leading shock can propagate downstream. If the initial temperature is close to the saturation temperature, little enthalpy is needed and the shock has a high velocity and a low height. When the initial temperature is far below the saturation temperature, much more enthalpy is needed and the shock travels slowly. This also requires a larger transfer of enthalpy via condensation, hence a greater shock height.

The third parameter examined was the matrix heat capacity. Figure 4.6 shows how the saturation profile is modified by the matrix heat capacity. As the heal capacity of the matrix is decreased from a value of $25.0(\mathrm{~kJ} / \mathrm{kg} \cdot \mathrm{K})$ to zero, the positions of the leading and trailing shocks are affected while the saturation profile remains unchanged. The explanation for the insensitivity of the two-phase region is clear. Recall that the two-phase region is at constant temperature, therefore no heat transfer can take place 
from the fluids to the matrix or vice versa. This takes the thermal properties of the matrix out of the equations representing the two-phase region.

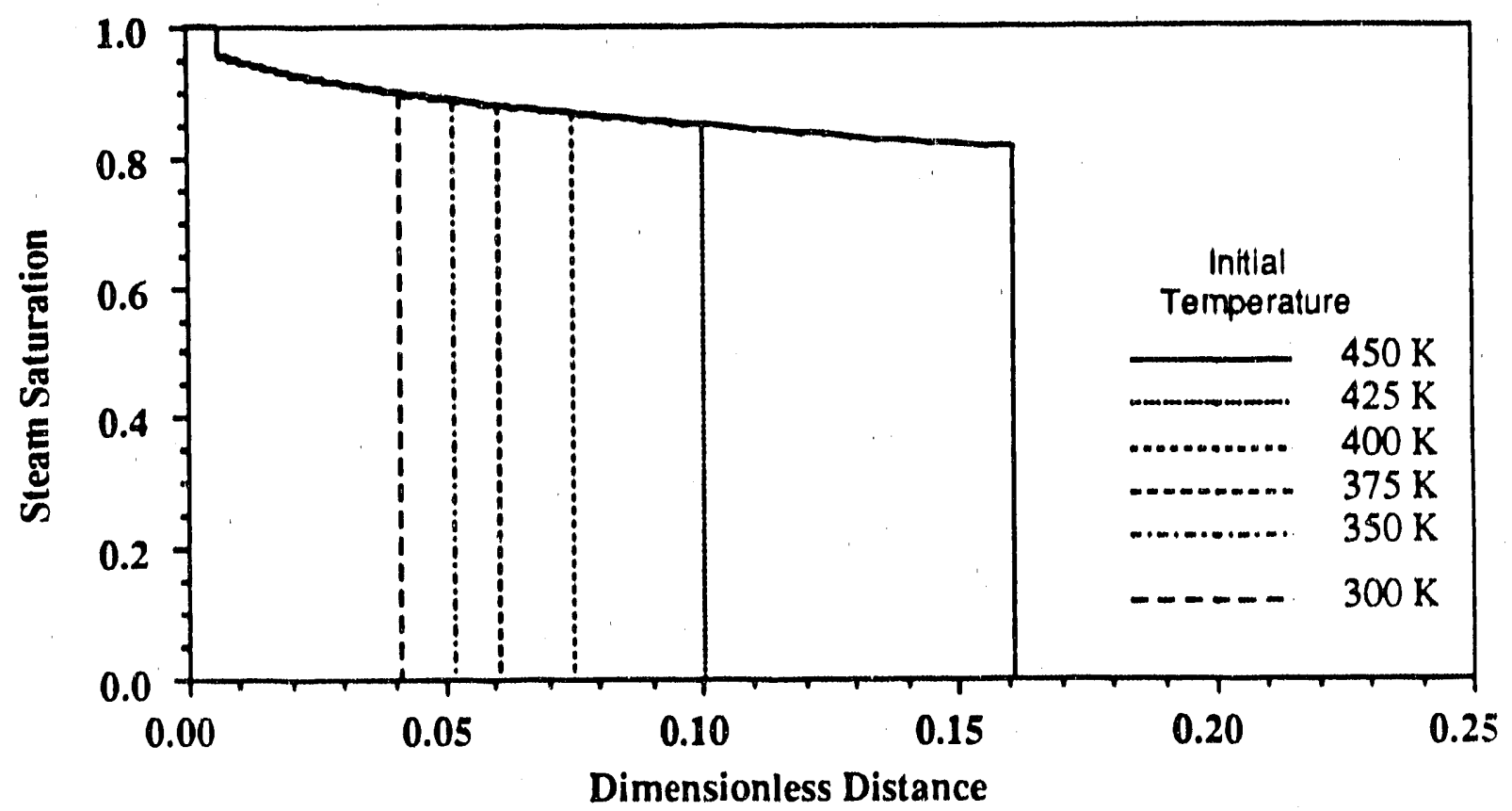

Figure 4.5: Saturation profile after 1.0 pore volumes steam injection into water at initial temperatures from $300 \mathrm{~K}-450 \mathrm{~K}$.

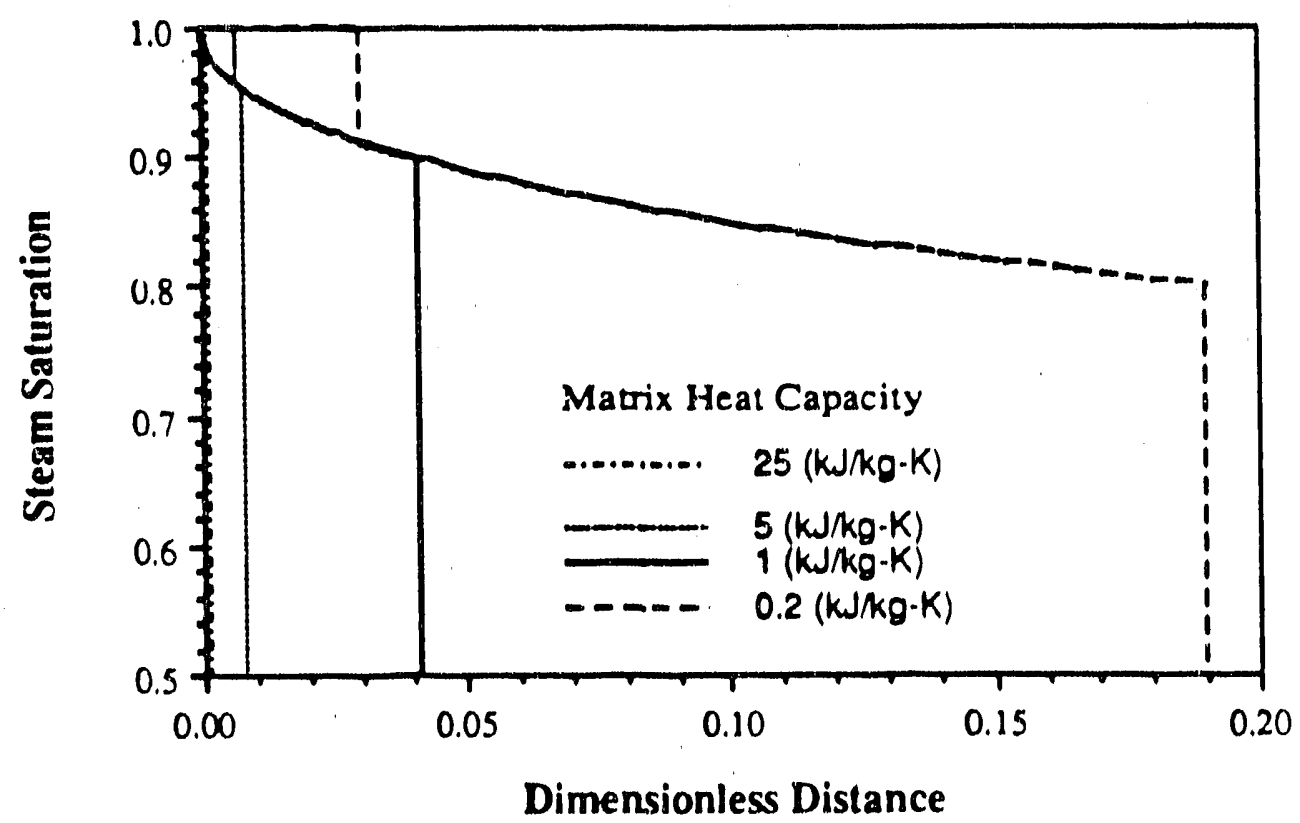

Figure 4.6: Saturation profile after 1.0 pore volumes steam injection into water where the matrix heat capacity varies from $25.0(\mathrm{~kJ} / \mathrm{kg} \cdot \mathrm{K})$ to $0.2(\mathrm{~kJ} / \mathrm{kg}-\mathrm{K})$. 
The velocities of both the trailing and leading shocks increase as the matrix heat capacity decreases. The explanation is essentially identical to that for the initial temperature variation. Lower heat capacity means that less enthalpy is stored per degree of temperature. Less enthalpy is therefore required to raise the matrix from the initial temperature to the saturation temperature and subsequentiy from the saturation temperature to the injection temperature.

\section{Summary}

The displacements in the single component case are characterized by the following features:

1. The displacement has a central, two phase, is thermal region. This region is flanked by singlephase regions containing only vapor towards the injection end and liquid at the leading end.

2. The single-phase regions are regions of constant state. The temperature of the trailing region is at the injection temperature and the leading region is at the initial temperature. This is due to the single-phase flow in these regions.

3. The behavior of the two-phase region is identical to a two component, immiscible displacement Saturation velocity in this region is given by the derivative of the fractional flow curve and the shocks that carry the system into and out of the two-phase region are intermediate discontinuities or tangent shocks.

4. The conditions downstream have no effect on the features of the saturation profile upstream. This means that the initial temperature only effects the leading shock, while the injection temperature can modify the entire saturation profile.

The nature of the single-phase regions can be altered by the addition of a second, immiscible component. These two phases can flow at different velocities creating temperature profiles in the leading and trailing regions. The solution to this problem is described in the next section. 


\subsection{TWO COMPONENT - THREE-PHASE SYSTEMS}

\subsubsection{Problem Formulation}

The second example system examined in this dissertation is an extension of the steam-water problem described in the previous section. A second component, referred to as the oil component, is added to the system. The oil is totally immiscible with both aqueous phases. This has the effect of adding a second phase to the single-phase region and a third phase to the two-phase region of the steam-water problem described in the previous section. Since the oil component forms its own phase, separate from the water component, the presence of the oil has no effect on the phase behavior of the water. The on'v, change the oil makes is on the flow properties of the fluic's.

The addition of the oil component to the steam-water system results in two significant changes. The first of these differences concerns the two-phase oil-water and steam-oil regions. The addition of a second flowing phase can create a temperature profile in these regions. In the single phase case, there was no separation of the enthalpy due to the single phase flow.

Second, a three-phase region replaces the isothermal two-phase region in the previous problem. The region is still at constant temperature because there is no mass transfer in this region, but the effect of the three phase relative permeability problem is added. The solutions in this region are the three phase ana$\log$ to the Buckley-Leverett problem. Figure 4.7 presents a hypothetical saturation profile for this problem. Each region is treated separately and is connected through a series of shocks to form the complete solution.

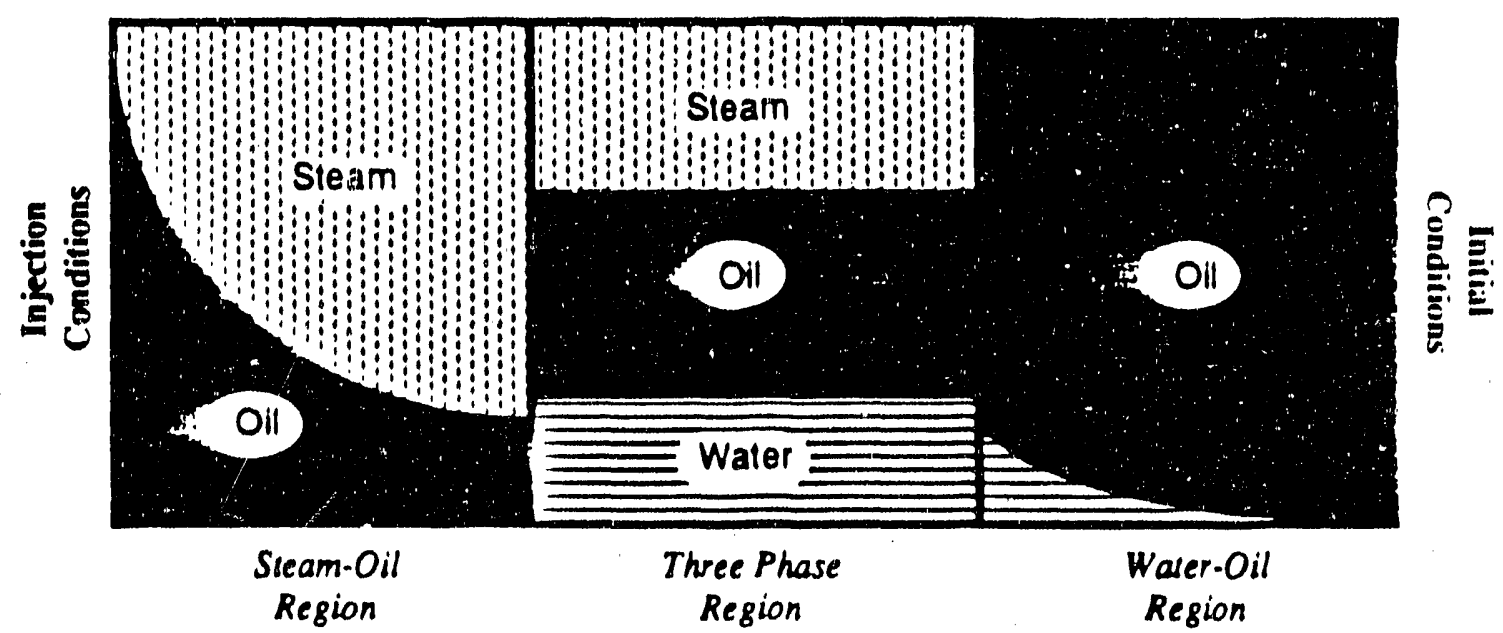

Figure 4.7: Hypothetical solution to the two-component, three-phase problem showing the differen' flow regions.

Adding a second component changes the formulation of the eigenvalue problem from a simple $2 \times 2$ to a $3 \times 3$ system. The formulations for the two-phase and three-phase regions still have different hodograph variables. In the two-phase regions the hodograph variables are the saturation of one of the phases, the temperature and the flow velocity. In the example problems the saturation of the water component was chosen as the hodograph variable. For the steam oil region Eq. 2.26 reduces to where the subscript $w$ refers to the water component, subscript $o$ represents the oleic component, and subscript $v$ refers to the vapor phase. Since the matrix system is $3 \times 3$ the exercise of solving for the eigenvalues and eigenvectors analytically is more complex and will not be done for these systems. 


$$
\left[\begin{array}{lll}
\frac{\partial F_{w}}{\partial S_{v}} & \frac{\partial F_{v}}{\partial T} & \frac{\partial F_{v}}{\partial u} \\
\frac{\partial F_{o}}{\partial S_{v}} & \frac{\partial F_{o}}{\partial T} & \frac{\partial F_{0}}{\partial u} \\
\frac{\partial \theta}{\partial S_{v}} & \frac{\partial \theta}{\partial T} & \frac{\partial \theta}{\partial u}
\end{array}\right]\left[\begin{array}{l}
\frac{d S_{v}}{d \eta} \\
\frac{d T}{d \eta} \\
\frac{d u}{d \eta}
\end{array}\right]-\lambda\left[\begin{array}{lll}
\frac{\partial G_{w}}{\partial S_{v}} & \frac{\partial G_{v}}{\partial T} & 0 \\
\frac{\partial G_{0}}{\partial S_{v}} & \frac{\partial G_{o}}{\partial T} & 0 \\
\frac{\partial T}{\partial S_{v}} & \frac{\partial \Gamma}{\partial T} & 0
\end{array}\right]\left[\begin{array}{l}
\frac{d S_{v}}{d \eta} \\
\frac{d T}{d \eta} \\
\frac{d u}{d \eta}
\end{array}\right]=0
$$

The matrix formulation in the liquid water-oil region is similar to the steam-oil system. The saturation of the vapor phase is replaced by the saturation of the liquid water phase and the following system results, where $S_{w}$ represents the liquid water phase saturation.

$$
\left[\begin{array}{lll}
\frac{\partial F_{w}}{\partial S_{w}} & \frac{\partial F_{w}}{\partial T} & \frac{\partial F_{w}}{\partial u} \\
\frac{\partial F_{0}}{\partial S_{w}} & \frac{\partial F_{0}}{\partial T} & \frac{\partial F_{0}}{\partial u} \\
\frac{\partial \theta}{\partial S_{w}} & \frac{\partial \theta}{\partial T} & \frac{\partial \theta}{\partial \mu}
\end{array}\right]\left[\begin{array}{l}
\frac{d S_{w}}{d \eta} \\
\frac{d T}{d \eta} \\
\frac{d u}{d \eta}
\end{array}\right]-\lambda\left[\begin{array}{lll}
\frac{\partial G_{w}}{\partial S_{w}} & \frac{\partial G_{w}}{\partial T} & 0 \\
\frac{\partial G_{0}}{\partial S_{w}} & \frac{\partial G_{0}}{\partial T} & 0 \\
\frac{\partial \Gamma}{\partial S_{w}} & \frac{\partial T}{\partial T} & 0
\end{array}\right]\left[\begin{array}{l}
\frac{d S_{w}}{d \eta} \\
\frac{d T}{d \eta} \\
\frac{d u}{d \eta}
\end{array}\right]=0
$$

The eigenvalues and eigenvectors in these two regions behave in a similar manner. One of the eigenvectors has a zero component in the temperature direction, $(\partial T / \partial \eta=0)$. This result is expected; in order to move between any two composition points at the same temperature, an isothermal path must be provided. The magnitude of the eigenvalue along this path is the familiar Buckley-Leverett, $(\partial f / \partial S)$ curve. Figure 4.8 demonstrates how the eigenvalues typically vary along a constant temperature path in the water-oil region.

The isothermal path resembles the Buckley-Leveret fractional flow derivative plot. It is large at intermediate saturations but trails off to zero at the saturation end points. The eigenvalue of the nonisothermal path does not show the large variations evident along the isothermal path. However at the points labelled $\mathbf{E}$, the eigenvalues are equal and a continuous path switch may take place at this point according to Rule III as given in $\$ 3.1$.

In addition the isothermal path also has a zero component for the change in the flow velocity. As in the case for the steam-water problem, this is result is directly related to the fact that the phase densities are assumed to be functions of temperature only. Along the non-isothermal path all hodograph variables change.

In the three-phase region, the Gibbs phase rule still demands that the region be at constant temperature. The hodograph variables are chosen to be the steam saturation, the water saturation and the flow velocity. As in the steam-water problem, the flow velocity in this region turns out to be constant. The matrix formulation for this region is given by

$$
\left[\begin{array}{lll}
\frac{\partial F_{w}}{\partial S_{v}} & \frac{\partial F_{w}}{\partial S_{w}} & \frac{\partial F_{w}}{\partial u} \\
\frac{\partial F_{0}}{\partial S_{v}} & \frac{\partial F_{0}}{\partial S_{w}} & \frac{\partial F_{0}}{\partial u} \\
\frac{\partial \theta}{\partial S_{v}} & \frac{\partial \theta}{\partial S_{w}} & \frac{\partial \theta}{\partial u}
\end{array}\right]\left[\begin{array}{l}
\frac{d S_{v}}{d \eta} \\
\frac{d S_{w}}{d \eta} \\
\frac{d u}{d \eta}
\end{array}\right]-\lambda v\left[\begin{array}{lll}
\frac{\partial G_{w}}{\partial S_{v}} & \frac{\partial G_{w}}{\partial S_{w}} & 0 \\
\frac{\partial G_{0}}{\partial S_{v}} & \frac{\partial G_{0}}{\partial S_{w}} & 0 \\
\frac{\partial T}{\partial S_{w}} & \frac{\partial \Gamma}{\partial S_{w}} & 0
\end{array}\right]\left[\begin{array}{c}
\frac{d S_{v}}{d \eta} \\
\frac{d S_{w}}{d \eta} \\
\frac{d u}{d \eta}
\end{array}\right]=0
$$




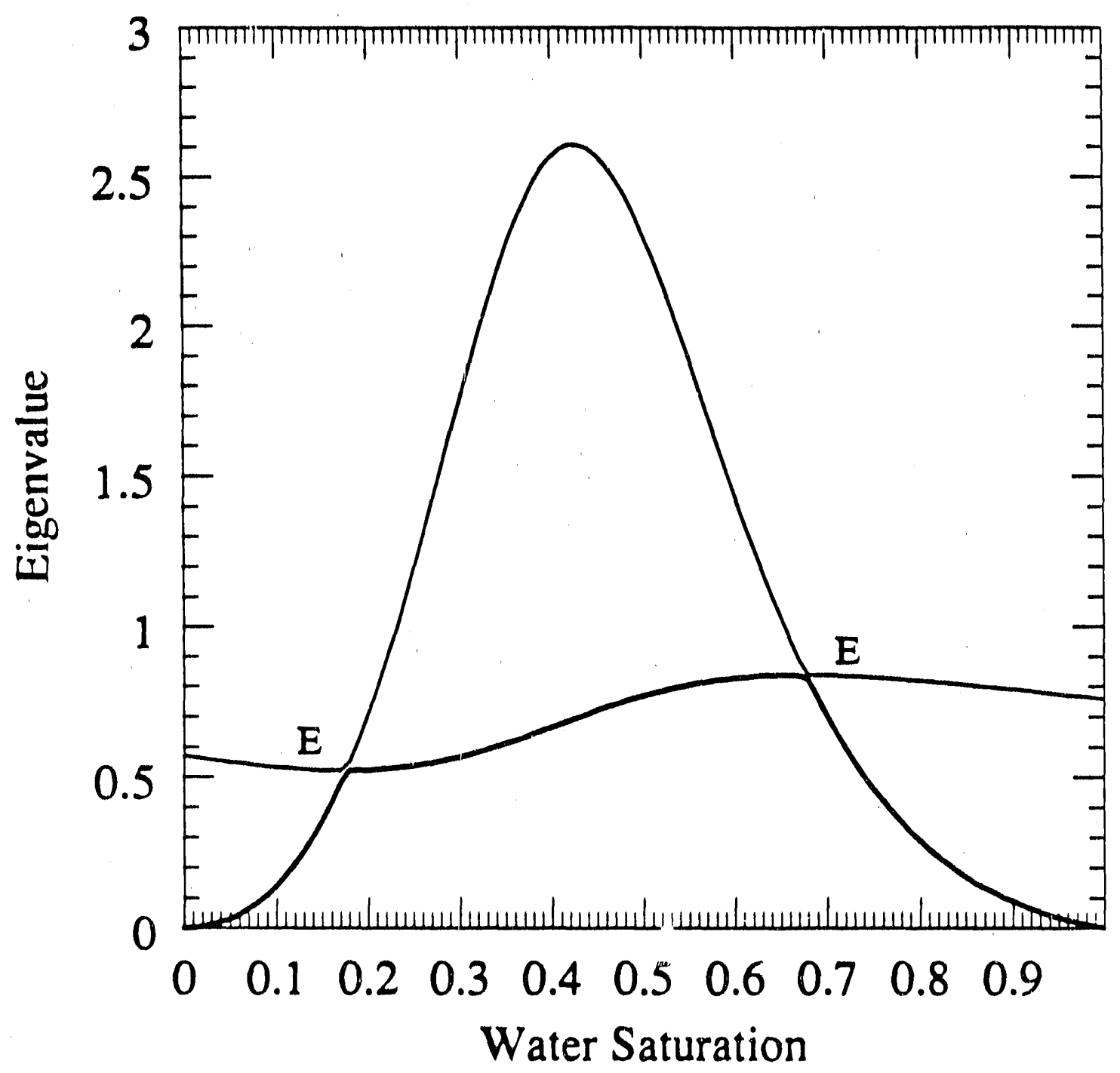

Figure 4.8: Plot of eigenvalues along a constant temperature path in the water-oil region.

This region requires that the three phase relative permeability relationships discussed in $\$ 2.3 .1$ be used to calculate the relative permeability of the oil phase. For most of the examples used in this section, the three phase relative permeability model of Stone (1970), given in Eq. 2.31, is used to calculate the relative permeability of the oil phase.

The three systems presenter are joined to form the solution of the injection of steam into a reservoir filled with oil or oil and water at a lower temperature. The solution is traced from the injection conditions in the steam-oil region, through the three-phase region where steam, oil and water flow simultaneously and into the leading region where oil and water may be nowing. 


\subsubsection{Example Cases}

The solution for the injection of steam into a reservoir initially filled with oil or oil and water at a lower temperature is presented in this section. The procedure for constructing a solution path from injection conditions to initial conditions is detailed. An example solution used as a base case is presented along with the discussion on the solution procedures.

Four basic wave patterns are seen in these displacement systems. The four cases and how they are obtained as variations of the base case are discussed following the base case presentation. The effect of the different displacement types on the saturation and temperature profiles is examined.

The four types of displacement may also be changed by selection of a different path through the injection conditions. Solutions with temperature variations in the steam-oil region, near the injection conditions, are presented and compared to displacements with no temperature profile in the steam-oil region.

Finally, the effect of oil viscosity on the displacement patterns is of concern to the displacement of heavy oils by steam injection. These effects are studied by changing the equation used to represent the oil viscosity. Two "heavy" oils are used, one which has a higher viscosity than the base case oil at all temperatures, and a second that is more viscous at reservoir temperatures, but also has a stronger dependence on temperature and so has a comparable viscosity at injection temperatures.

\section{Base Case}

The base case presented in this section outlines the procedures used to construct a solution from the given fluid properties, relative permeability model and boundary conditions. The equations used to calculate the fluid properties in this model are presented in Appendix A. The densities and viscosities of all the phases are assumed to be functions of temperature only. The oleic phase and the two aqueous phases do not interact by mass transfer. The conditions for the problem are identical to the steam-water problem presented in $\$ 4.1$ in Table 4.1 .

The relative permeability model used is Stone's Model II, given by Eqs. $2.27-2.31$. The parameters used in these example solutions are listed in Table 4.2.

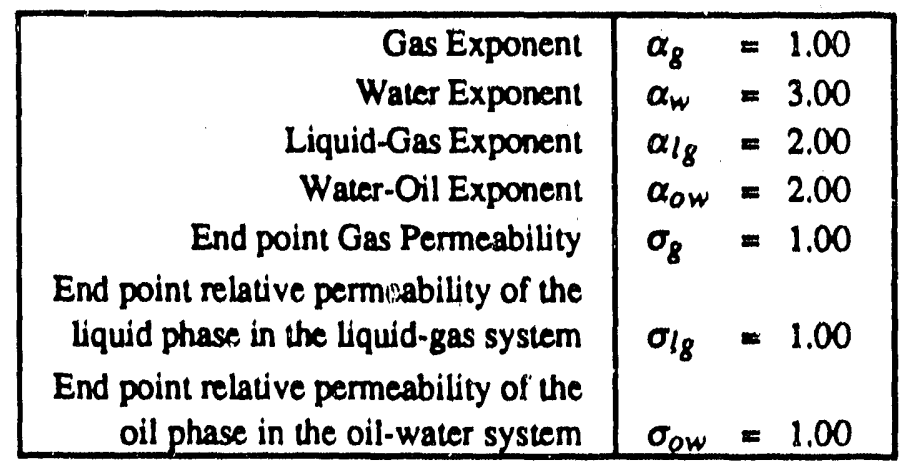

Table 4.2: Three phase relative permeability parameters for Stone's model II.

The construction of the solution begins at the injection conditions. The injection conditions for the base case is given as Point $\mathbf{A}$ in Table $\mathbf{4 . 3}$ and the composition path is shown in Figure 4.9. For the first leg of the solution the slow path is integrated from the injection conditions up to an arbitrary com. position point, Point B. This choice is reasonable because far upstream the compositions must have 
very small wave velocities. At Point B, an upstream intermediate discontinuity (tangent shock) carries the solution into the three-phase region. The choice of this composition is up to the designur of the solution; for a specific displacement, the choice will depend on the initial and injection conditions, and an iterative solution will be required.

\begin{tabular}{|c|c|c|c|c|c|c|c|}
\hline \multirow{3}{*}{ Label } & \multicolumn{5}{|c|}{ Composition Point } & \multirow{3}{*}{$\begin{array}{l}\text { Wave } \\
\text { Velocity }\end{array}$} & \multirow{3}{*}{$\begin{array}{l}\text { Type of } \\
\text { Flow } \\
\text { Region }\end{array}$} \\
\hline & \multicolumn{3}{|c|}{ Saturations } & \multirow[b]{2}{*}{$T(K)$} & \multirow{2}{*}{$\begin{array}{l}\text { Flow } \\
\text { Velocity }\end{array}$} & & \\
\hline & Steam & Oil & Waler & & & & \\
\hline $\begin{array}{l}A \\
B\end{array}$ & $\begin{array}{l}1.00000 \\
0.92000\end{array}$ & $\begin{array}{l}0.00000 \\
0.08000\end{array}$ & $\begin{array}{l}0.00000 \\
0.00000\end{array}$ & $\begin{array}{l}650.00 \\
650.00\end{array}$ & $\begin{array}{l}1.00000 \\
1.00000\end{array}$ & $\begin{array}{l}0.000000 \\
0.010777\end{array}$ & $\begin{array}{l}\text { INJ } \rightarrow \text { SPW } \\
\text { SPW } \rightarrow \text { UID }\end{array}$ \\
\hline $\begin{array}{l}\text { C } \\
\text { D }\end{array}$ & $\begin{array}{c}0.41924 \\
0.4924\end{array}$ & $\begin{array}{l}0.39087 \\
0.39087\end{array}$ & $\begin{array}{l}0.18989 \\
0.18989\end{array}$ & $\begin{array}{l}485.57 \\
485.57\end{array}$ & $\begin{array}{l}0.7058 \\
0.7058\end{array}$ & $\begin{array}{l}0.010777 \\
0.064505\end{array}$ & $\begin{array}{l}\text { UID } \rightarrow \mathrm{ZCS} \\
\mathrm{ZCS} \rightarrow \mathrm{UID}\end{array}$ \\
\hline $\begin{array}{l}E \\
F\end{array}$ & $\begin{array}{l}0.00000 \\
0.00000\end{array}$ & $\begin{array}{l}0.96566 \\
0.96566\end{array}$ & $\begin{array}{l}0.03434 \\
0.03434\end{array}$ & $\begin{array}{l}390.19 \\
390.19\end{array}$ & $\begin{array}{l}0.419 \\
0.0419\end{array}$ & 0.093985 & $\underset{\mathbf{I N I}}{\longrightarrow} \underset{\mathrm{INI}}{\mathrm{UID}}$ \\
\hline
\end{tabular}

Table 4.3 Composition path for the injection of $100 \%$ steam at $650 \mathrm{~K}$ into $94.5 \%$ oil and $6.5 \%$ water at $390.19 \mathrm{~K}$.

\begin{tabular}{|c|l|}
\hline Abbreviation & Flow Region \\
\hline INJ & Injection Conditions \\
INI & Initial Conditions \\
\hline EEP & Equal Eigenvalue Point \\
ZCS & Zone of Constant State \\
\hline SPW & Spreading Wave \\
SSW & Self-Sharpening Wave \\
\hline UID & Upstream Intermediate Discontinuity \\
DD & Downstream Intermediate Discontinuity \\
\hline
\end{tabular}

Table 4.4: Abbreviations used to describe the different flow regions.

There is only one composition point in the three-phase region that satisfies material balances and also the condition that the shuck is an upstream intermediate discontinuity. A discontinuity which satisfies the Rankine-Hugoniot conditions is completely described by fixing two of the three shock parameters, the upstream composition, the downstream composition, and the shock velocity (Jeffrey 1976). The constraint that the shock is an upstream intermadiate discontinuity fixes both the upstream conditions, $B$, and the shock velocity, $\Lambda$. This means that the downstream conditions are uniquely determined.

The point in the three-phase region that is downstream of the intermediate discontinuity is given by Point $C$ in Table 4.3 and Figure 4.9. The dashed line in Figure 4.9 represents the upstream intermediate discontinuity that takes the solution from Point $B$ in the stearn-oil region to $\mathbf{C}$ in the three-phase region. Point C is refered 10 as the "three phase landing" point and Point B is the "jumping point".

In the three-phase region the solution must switch from the slow path to the fast path. With the relative permeability model used in the base case, there are no points in the three-phase region that have equal eigenvalue points. These systems are called strictly hyperbolic systems. The only way 10 switch paths in striculy hyperbolic regions is with a path switch described by Rule IV of $\$ 3.1$. 


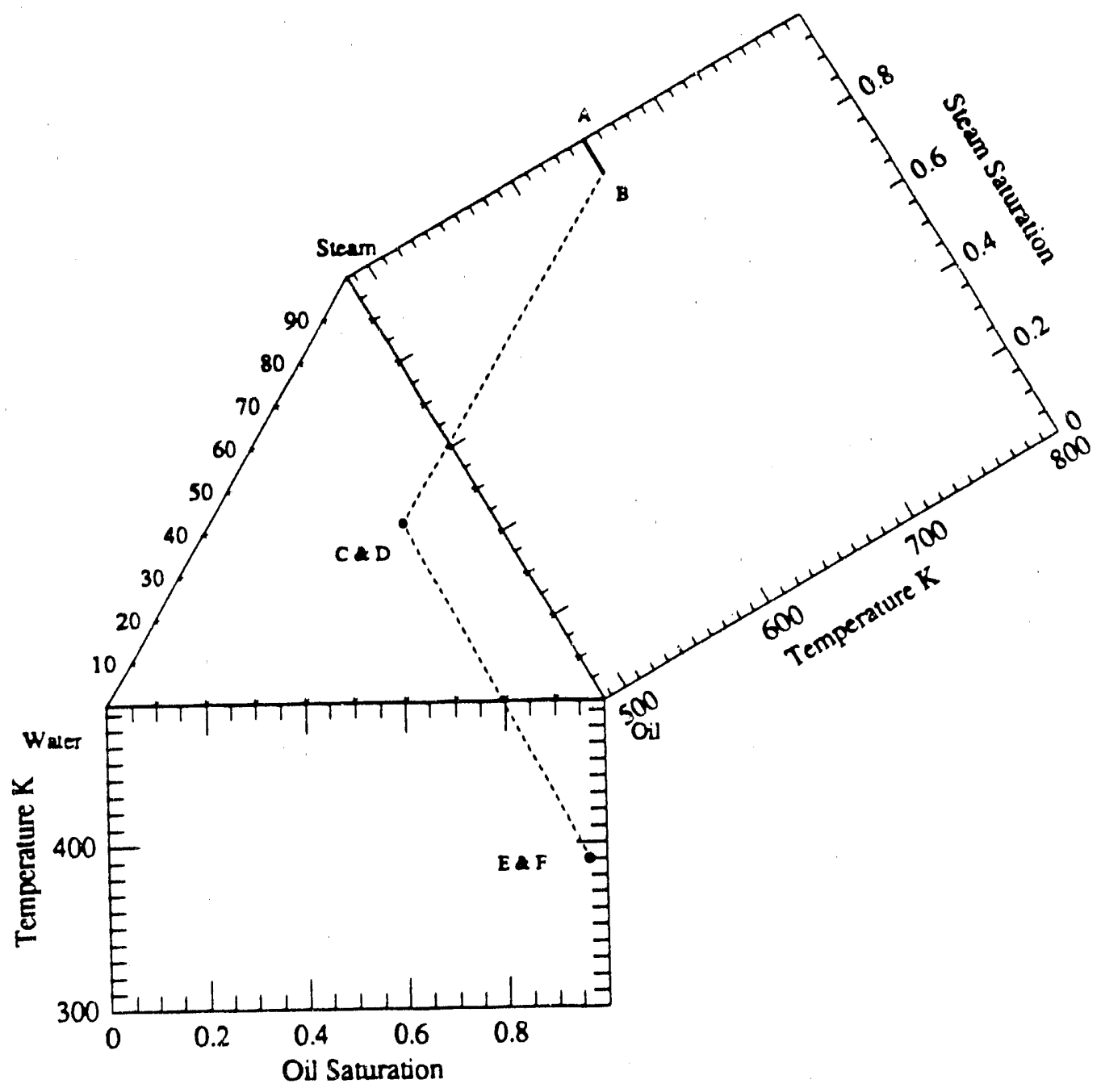

Figure 4.9: Composition path for the injection of $100 \%$ steam at $650 \mathrm{~K}$ into $94.5 \%$ oil and $6.5 \%$ water at $390.19 \mathrm{~K}$.

Point $\mathbf{C}$ has two eigenvalues, both of which are larger than the shock velocity. An immediate jump to the large eigenvalue at $C$ satisfies Rule IV. This jump moves the solution directly onto the fast path at composition $C$. The path switch creates a zone of constant state at Point $\mathbf{C}$ between the positions of the upstream intermediate discontinuity and the composition point on the fast path, Point $D$ in Table 4.3.

The final part of the solution is to jump from Point D directly to the initial conditions. This shock is also an upstream intermediate discontinuity. The arguments used to verify that the three phase landing

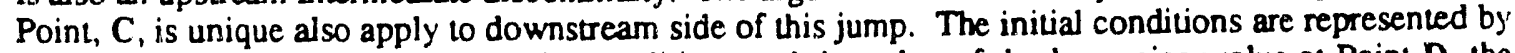
Point $\mathbf{E}$. Using the Rankine-Hugoniot conditions and the value of the large eigenvalue at Point $\mathbf{D}$, the initial conditions are located somewhere in the steam-water region. Table 4.3 and Figure 4.9 show the finished solution paths for the base problem. 
The need to jump directly to the initial conditions is made evident by looking at the eigenvalues in the water-oil region. At the boundary between the three-phase region and the water-oil region one of the eigenvalues is continuous ${ }^{1}$ across the phase boundary while the other eigenvalue has a discontinuity at the phase transition. Figure 4.10 gives an example of this transition at the saturation temperature.

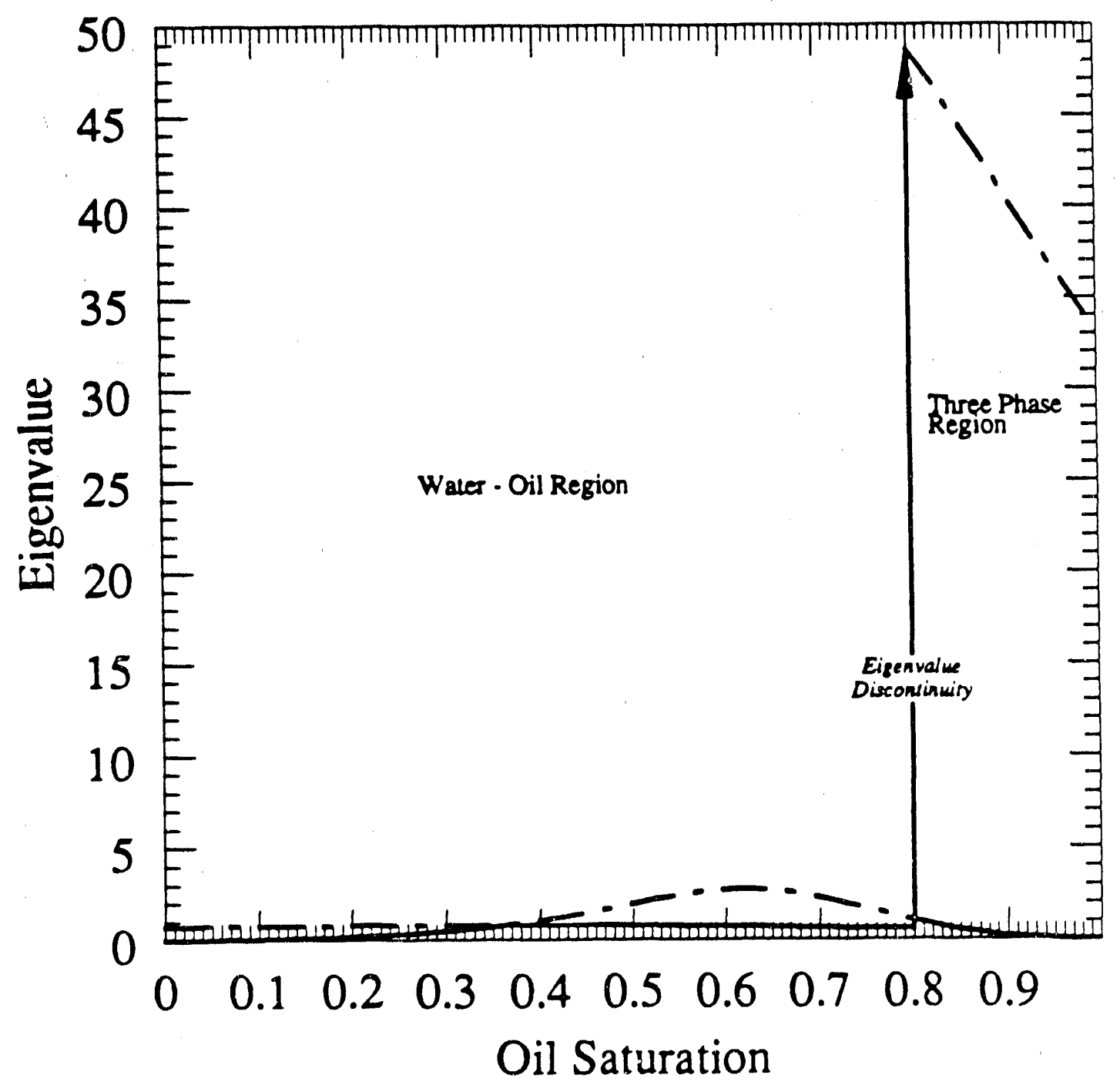

Figure 4.10: Eigenvalues crossing the three phase to water-oil boundary at $80 \%$ oil saturation.

\footnotetext{
1This continuation of the eigenvalue actossi the phase boundary is a consequence of having smooth three. phase relative permeability functions that reduce to the appropriate two phase relationships as the saturation of one of the phases goes to zero. If this is not true, then both the eigenvalues would be discontinuous actoss the phase boundary.
} 
As the solution moves downstream along the fast path in the three-phase region, the velocity is discontinuous at the phase boundary. This jump in the wave velocity requires that the solution have a discontinuity as it moves across this boundary (Jeffrey 1976).

Having shown that a shock is required to go from the three-phase to the water-oil region, it remains to establish the exact landing point in the water-oil region. For the base oil, the landing points in the water-oil region have slower velocities than their corresponding jumping points in the three-phase region. This is primarily due to the low local flow velocity once the steam has condensed into the liquid phase. There can be no solution. path in the water-oil region that is faster than the three phase jumping point This, coupled with the fact that the water-oil region is downstream of the three-phase region leads to the conclusion that there is only one point downstream of the three-phase region. This point must be the initial conditions.

The saturation, temperature and flow velocity profiles after 1.0 pore volumes of steam have been injected are given in Figures 4.11-4.13. Four features about the solution are important to note.

1. When a specific set of initial condition is required, a trial and error procedure similar to that of Monroe is needed. The initial condition may be adjusted by changing the location of the initial jumping off composition, Point B, or by introducing spreading waves into the three-phase region. The introduction of the spreading waves changes the upstream composition point (Point D) of the leading shock, thereby changing the initial conditions that can be reached by the solution path.

2. The velocity profile mimics the temperature profile exaculy. The flow velocity must follow the temperature profile because changes in phase densities, which are functions of temperature only, directly determine the flow velocity.

3. There are no regions where the temperature varies smoothly. All temperature changes are in the forms of shocks that carry the solution from the injection temperature to the saturation temperature and then finally, to the initial temperature.

4. In the three-phase region, the saturations of all three phases remain constant. This zone of constant state over the entire three-phase region is a feature of the specific displacement type used to construct the solution. This represents one limit of the "wave patterns" 2 that can exist in the threephase region. Three other wave patierns are made possible by adding a spreading wave to the region ahead, behind, or on both sides of the zone of constant state. These variations are discussed in the next section.

The example presented has no compositional variation in the three-phase region. This represents only one limiting case of the types of displacement profiles that are seen in the steam-oil-water displacements. The next section describes the different wave patterns and explains how they are constructed. An example solution is presented for the three other wave patterns and a discission of how each type of pattern effects the resulting initial conditions is also included.

\footnotetext{
2The term "wave pataem" is used to describe a progression of wave regimes from the injection end to the initial conditions. For example in the previous example the wave pattern was a spreading wave in the steam-oil region followed by an upstream intermediate discontinuity into the three-phase region. This was followed by a zone of constant state and another upstream intermediate discontinuity to the initial conditions.
} 


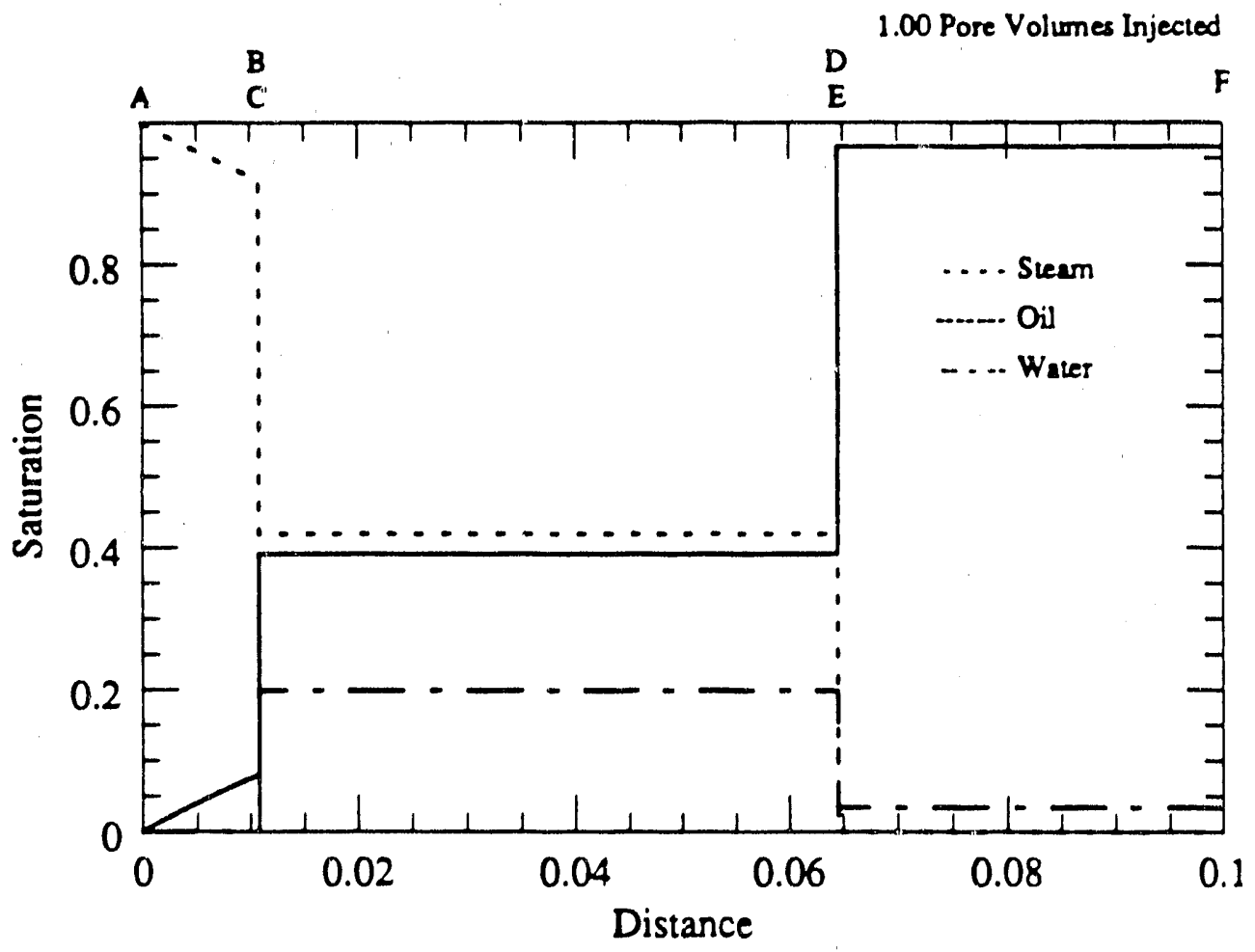

Figure 4.11: Saluration profile for the injection of 1.0 pore volumes of $100 \%$ steam at $650 \mathrm{~K}$ into $94.5 \%$ oil and $6.5 \%$ water at $390.19 \mathrm{~K}$. 


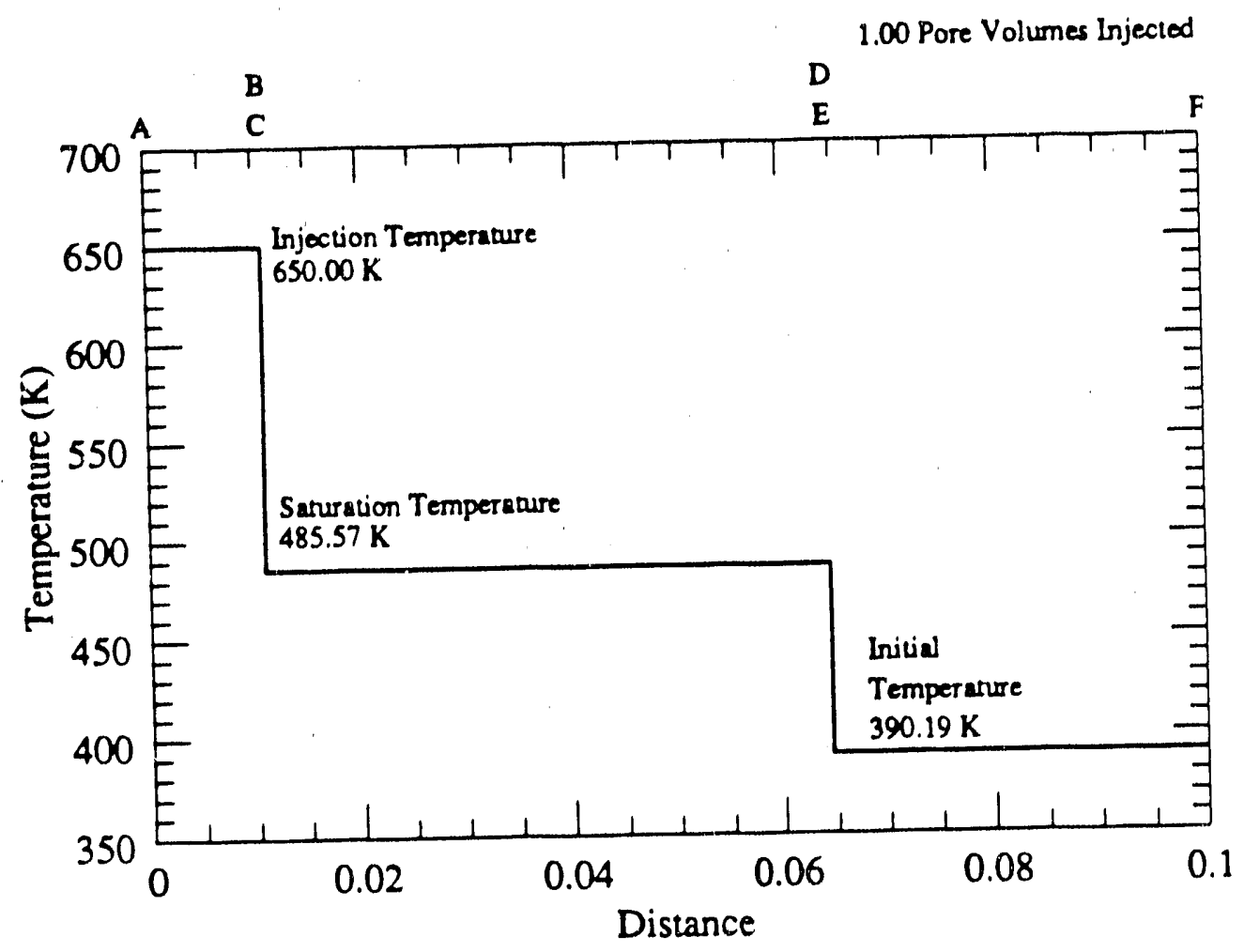

Figure 4.12: Temperature profile for the injection of 1.0 pore volumes of $100 \%$ steam at $650 \mathrm{~K}$ into $94.5 \%$ oil and $6.5 \%$ water at $390.19 \mathrm{~K}$. 


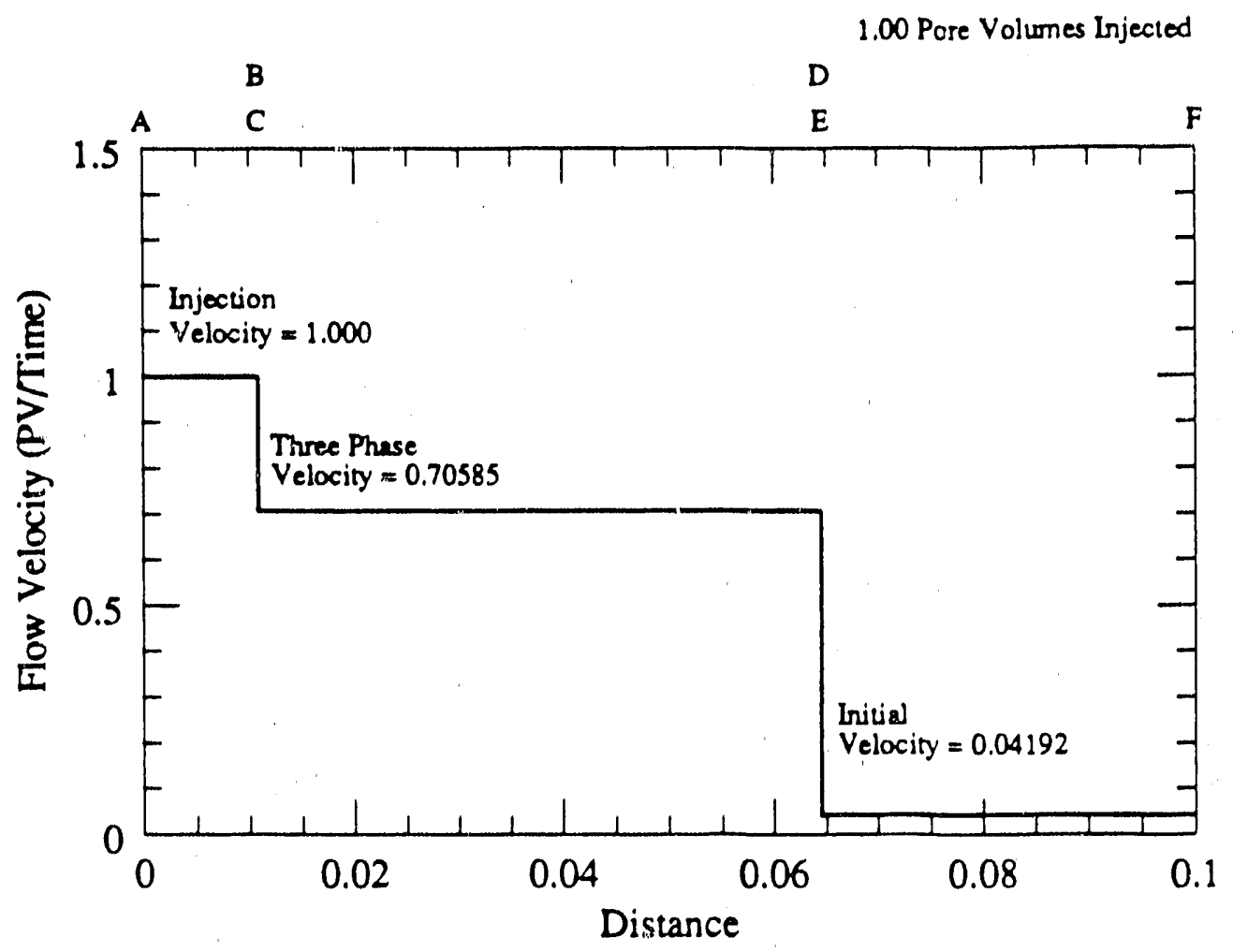

Figure 4.13: Flow velocity profile for the injection of 1.0 pore volumes of $100 \%$ steam at $650 \mathrm{~K}$ into $94.5 \%$ oil and $6.5 \%$ water at $390.19 \mathrm{~K}$.

\subsubsection{Types of Displacement Patterns}

This section discusses the four different wave patterns. Profiles are presented for three new pattems that initially follow the same composition path as the previous case in the steam-oil region. These different cases are distinguished by the type of wave that exists downstream and upstream of the central zone of constant state.

In the three-phase region the object is to move from the slow eigenvalue at the landing point to the fast path at some other point in the three-phase region. This is illustrated by Figure 4.14. The Point $U$ is the landing point from the steam oil region and point $\mathbf{D}$ is the jumpir.g point to the water-oil region downstream. The slower path moving through Point $U$ is illustrated by the dashed curve and the solid line represents the fast path that intersects Point D. The paths intersect at Point $\mathbf{I}$. This divides the three-phase region into three sections:

1. A central zone of constant state as the path switches from the slow path to the fast path at Point $I$.

2. A trailing wave that follows the slow path from Point $\mathbf{U}$ wo Point $\mathbf{I}$.

3. A a leading wave that follows the fast path from Point I to the downstream Point $\mathbf{D}$.

The four displacement types are distinguished by the kind of waves that are upstream and downstream of the central zone of constant state. These four types are listed in Table 4.5. 


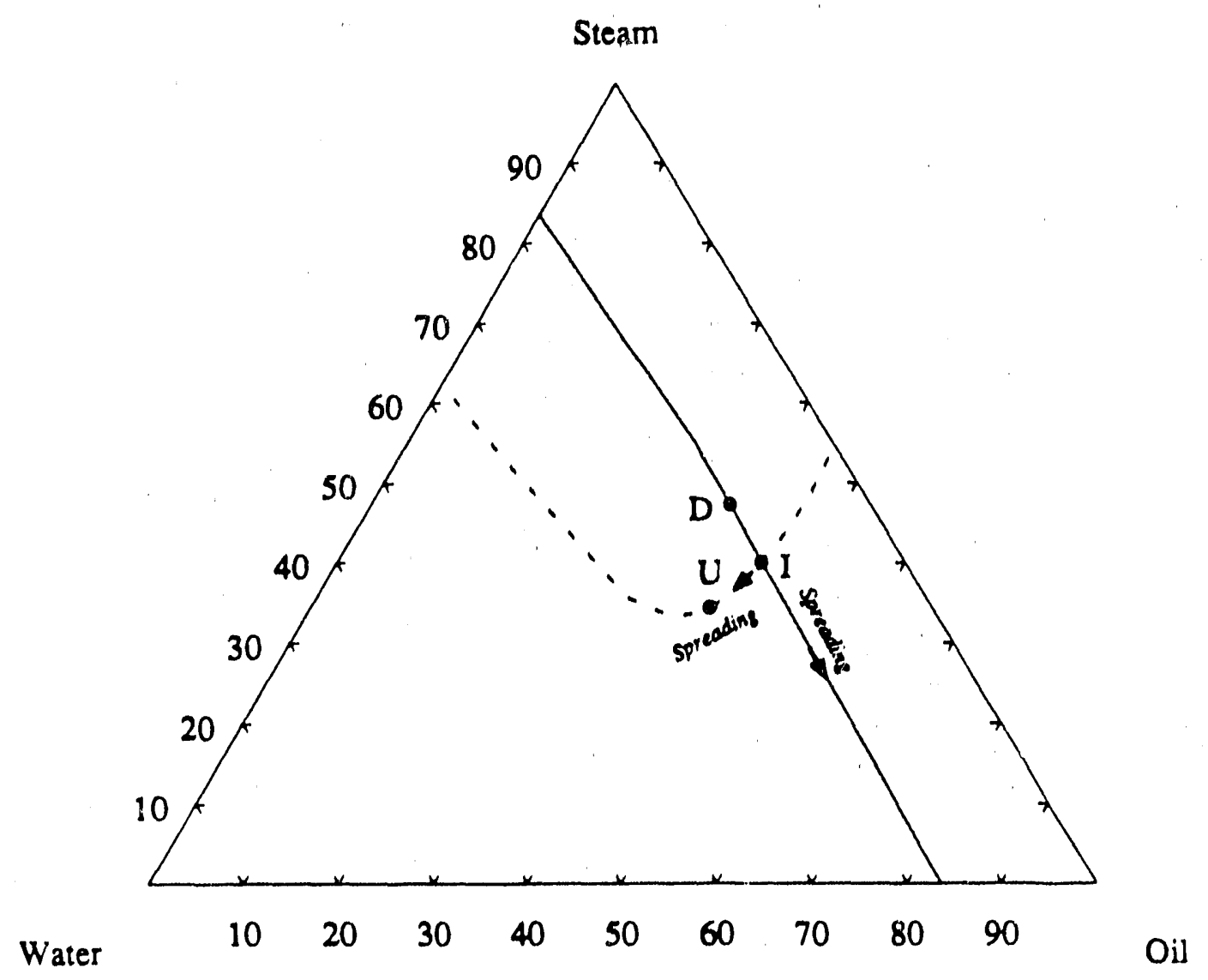

Figure 4.14: Composition path that moves between two arbitrary points in the three-phase region.

\begin{tabular}{|ccc|}
\hline $\begin{array}{c}\text { Displacement } \\
\text { Type }\end{array}$ & $\mathbf{U}-\mathbf{I}^{*}$ & $\mathbf{I}-\mathbf{D}$ \\
\hline 1 & Self Sharpening & Self Sharpening \\
2 & Spreading & Self-Sharpening \\
3 & Self-Sharpening & Spreading \\
4 & Spreading & Spreading \\
\hline
\end{tabular}

$U \longrightarrow I$ represents the composition path from the upstreamlanding point to the point of intersection where the composition path switches from the slow path to the fast path and $I \rightarrow D$ is the portion of the solution that travels along the fast path.

Table 4.5: The four different three phase wave profiles for the displacement of oil and water by steam and water.

The example solution has a self-sharpening wave both upstream and downstream of the central zone of constant state, this corresponds 10 a Type 1 displacement. 
A spreading wave is created when the path eigenvalue increases as the integration proceeds downstream. The spreading wave at the trailing end (from $\mathrm{U}$ to $\mathrm{I}$ ) follows the slow path. This wave is found in the Type 2 wave pattern illustrated in Figur: 4.15 and the Type 4 wave patterns as shown in Figure 4.16. The slow path parallels the contours of constant vapor flow. A integration along the slow path in the spreading direction increases the water saturation and decreases the oll saturation while leaving the saturation of the vapor phase relatively unchanged. The extent of this spreading wave is limited by a maximum velocity on the slow path.

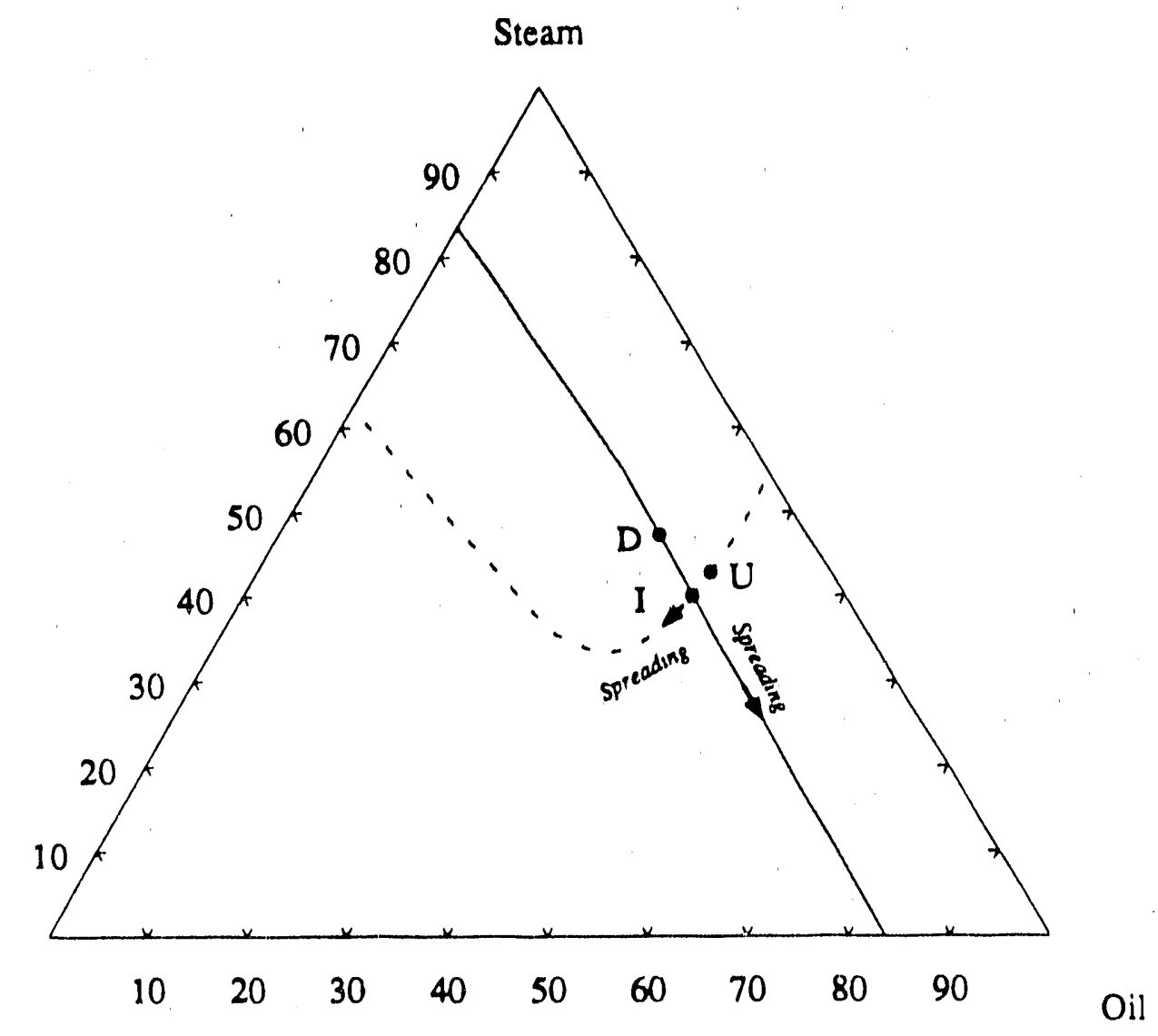

Figure 4.15: Type 2 composition path that moves between two arbitrary points in the three-phase region.

A spreading wave can also exist downstream of the central zone of constant state. This wave is found in the Type 3 and Type 4 wave patterns. The Type 3 composition path is shown in Figure 4.17. The eigervvalues along the fast path change much more rapidly than along the slow path. The fast path nearly parallets lines of constant water saturation. These conditions cause the profiles ahead of the zone of constant state to be spread out to a much greater extent near the initial end of the displacement. 


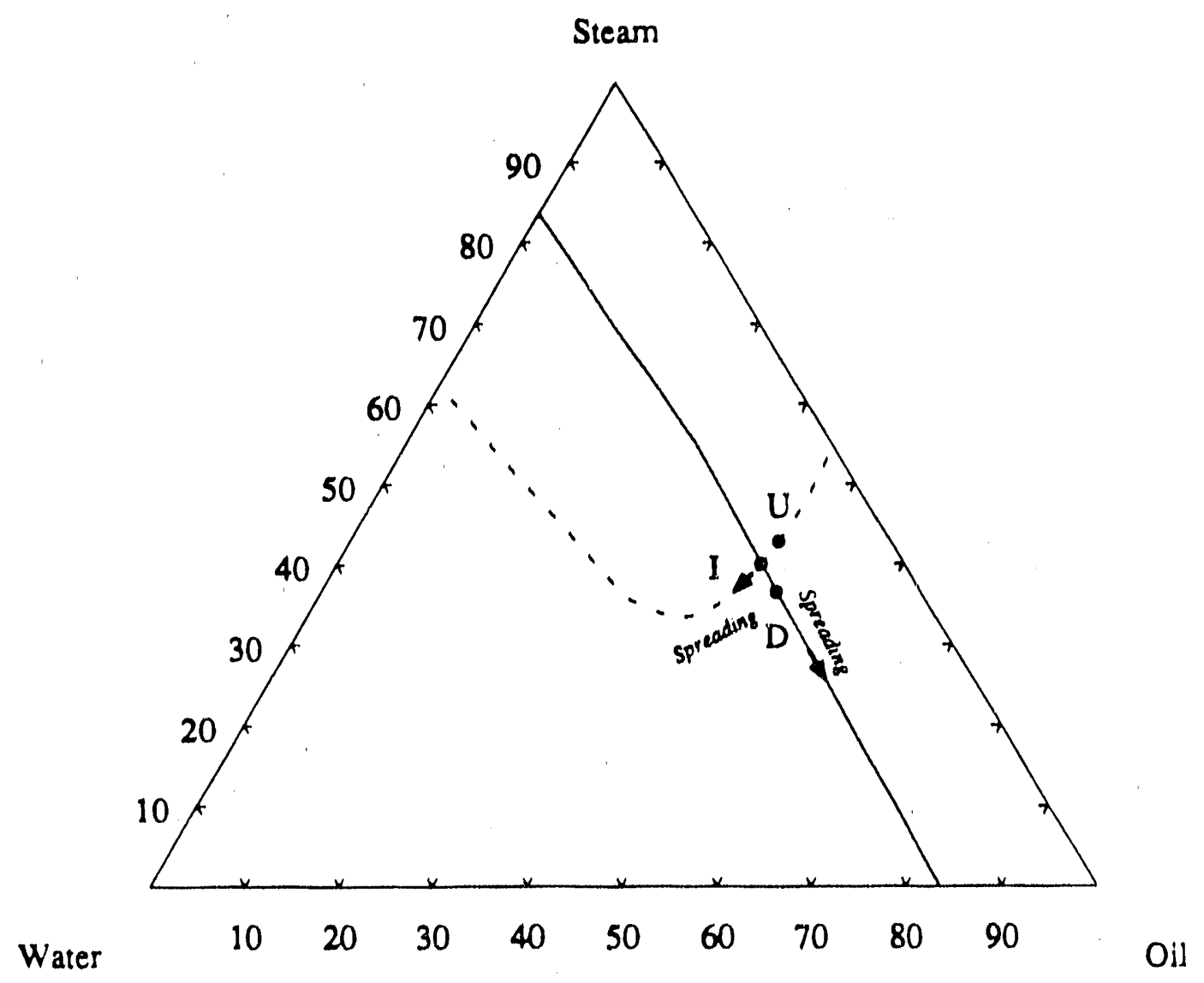

Figure 4.16: Type 4 composition path that moves between two arbitrary points in the three-phase region. 


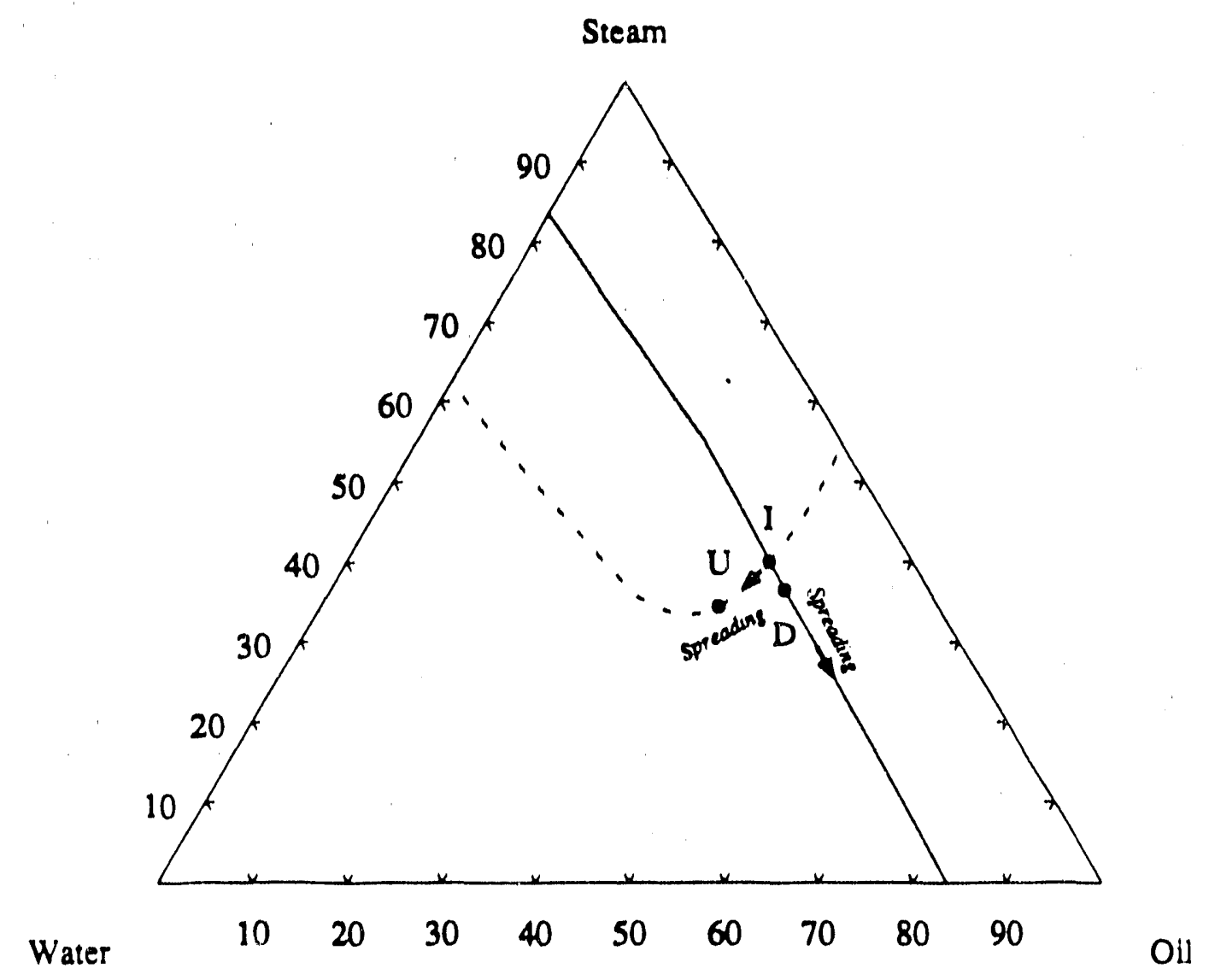

Figure 4.17: Type 3 composition path that moves between two arbitrary points in the three-phase region.

The opposite of the spreading wave is a self-sharpening wave. These waves appear when the eigenvalues decrease as the integration moves downstream. The construction of the self-sharpening waves changes from a continuous integration and becomes a shock calculation. A self-sharpening wave represents the limit of a continuous variation along a composition path. This makes the resolution of the self-shappening wave an insermediate discontinuity.

The trailing self-sharpening wave is restricted to a narrow region just downstream of the phase transition shock. The reasons for this are tied to the velocity constraint. First, and most evident, the self-sharpening wave velocity must be larger than or equal to the velocity of the upstream intermediate discontinuity initially brings the solution into the three-phase region, or,

$$
\lambda_{1}^{+}=\Lambda_{\text {wid }} \leq \Lambda_{\alpha} \leq \lambda_{1}^{-}
$$

where:

$\lambda_{1}^{+}=$Small eigenvalue in the upstream region

$\lambda_{1}^{--}=$Small eigenvalue in the downstream region 
$\Lambda_{\text {uid }}=$ Velocity of the upstream intermediate discontinuity between the upstream and downstream region

$\Lambda_{s s}=$ Velocity of the discontinuity created by the self-sharpening wave

If the self sharpening wave velocity were to be less than the velocity of the upsteam intermediate discontinuity, then a condition downstream, the self-sharpening wave, would have a lower velocity than the upstream condition, the upstream intermediate discontinuity. This is clearly prohibited by the velocity constraint.

When the solution contains a self-sharpening wave, the eigenvalues along the slow path decrease in a smooth fashion between the landing Point $U$ and the intersection Point I. It is known that the shock velocity formed by a self-sharpening wave is intermediate between the endpoints of the path which forms the wave (Jeffrey 1976). That is,

$$
\lambda_{1}^{\prime} \leq \Lambda_{\infty} \leq \lambda_{!}^{U}
$$

Since the downstream point, composition $I$, has a slow path velocity that is less than the velocity of the landing point, I, all self-sharpening wave velocities must be less than the velocity of the small eigenvalue at the landing point, Composition $U$. This eliminates all self sharpening waves with velocities that satisfy the following inequality,

$$
\lambda_{1}^{+}=\Lambda_{\text {aid }}<\lambda_{1}^{--}<\Lambda_{\text {a }}
$$

This only leaves self-sharpening waves that fall in the zone of constant state that usually couples the eigenvalues $\lambda_{1}^{+}$and $\lambda_{1}^{--}$. This type of self sharpening wave is illustrated in Figure 4.18.

There is a zone of constant state that couples the phase shock velocity to the slow eigenvalue in the three-phase region at $U$. The limiting case of the self-sharpening wave is when the zone of constant state vanishes. This condition is equivalent to an immediate jump from the steam-oil region to the large eigenvalue at $I$. The original discontinuity and the self-sharpening wave are added together to give one large shock as illustrated in Figure 4.19. This is the type of shock that was presented in the first example solution in \$4.2.2.

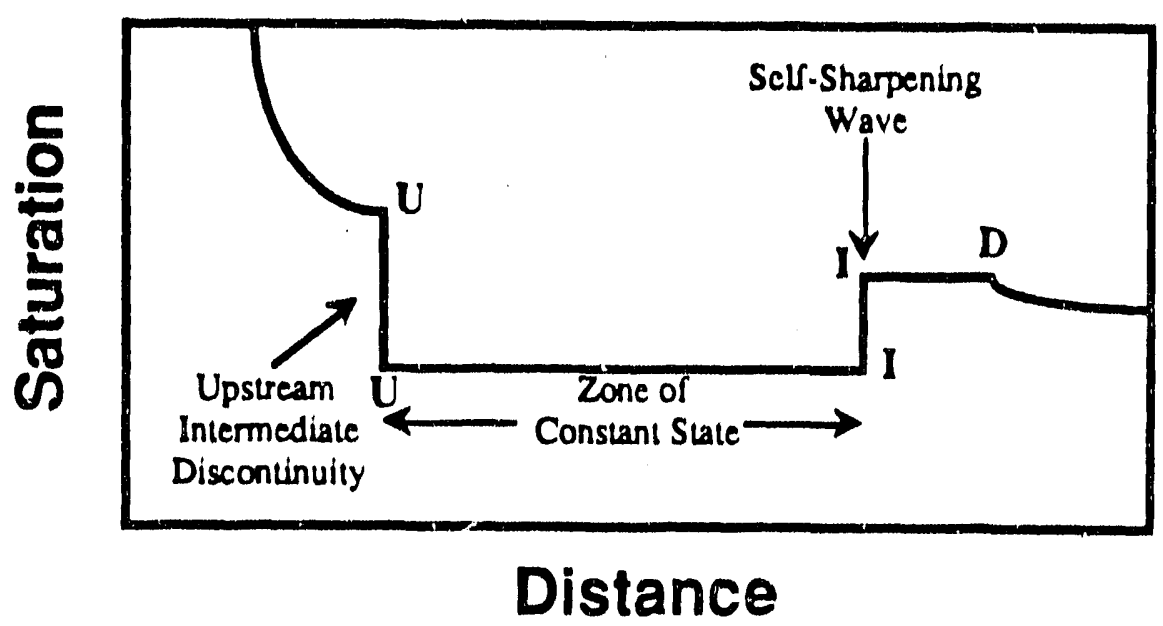

Figure 4.18: Possible self-sharpening wave in the three-phase region. 


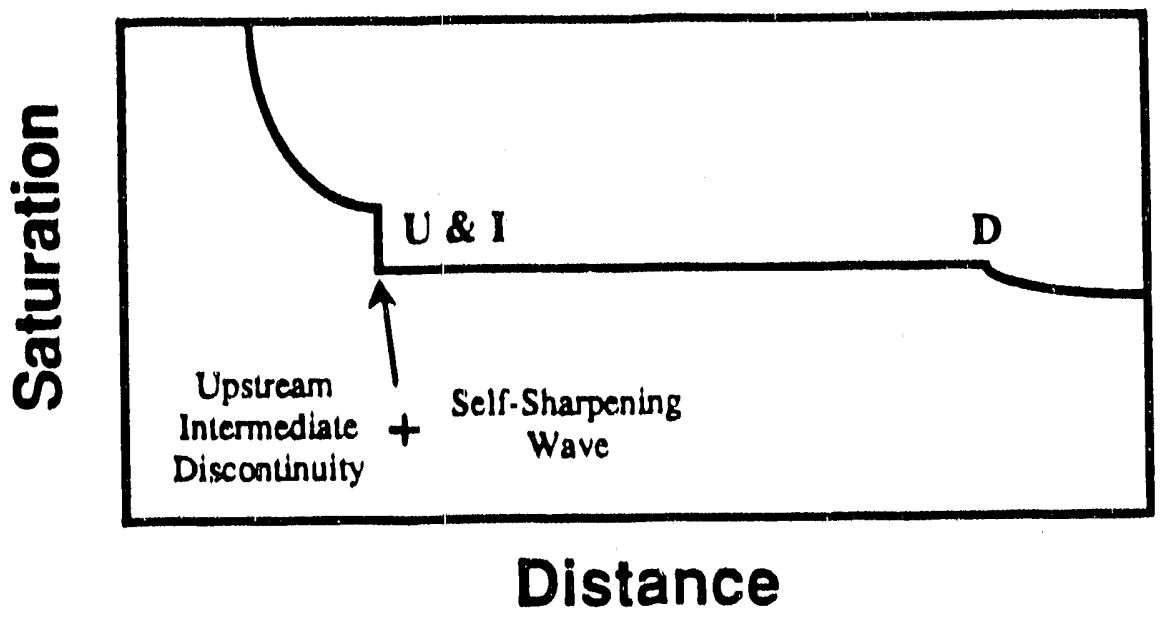

Figure 4.19: Limiting case self-sharpening wave in the three-phase region.

For cases where the two liquid phases have a comparable viscosities, the self-sharpening waves do not move downstream of the limiting case. This has the effect of switching directly to the fast path at the three phase landing point, Point $U$. The three different wave pattems that contain a self-sharpening wave will be presented as the limiting case examples. The "heavy" oil solutions, on the other hand, contain examples of self sharpening waves that do move ahead of the trailing upstream incermediate discontinuity.

The same simation can theoretically occur where the a self sharpening wave can be called for from the intersection Point I to the downstream jumping point, Point D. These kinds of adjustments are not found in these displacements. The zone of constant state that acted as a buffer between the phase change shock and the self-sharpening wave does not exist. Only the limiting case, where the downstream jumping poinh $D$, is equivalent to the intersection point, $I$, is found in these types of displacements.

The self-sharpening waves are resolved by locating an additional upsiream intermediate discontinuity or a downstream intermediate discontinuity that takes the solution from the landing point, $U, 10$ a new point of intersection, $I^{\prime}$. The new landing point, $l^{\prime}$, will not be the saine, as the original intersection point, $I$, on the path defining the self-sharpening wave. The fractional flow relationship across a discontinuity such as a self-sharpening wave is different that the continuous flow variation described by the path from $U$ to $I$. The limiting self-sharpening waves at either of the end points, $U$ or $D$, are adjusted by moving Point $\mathbf{U}$ or $\mathbf{D}$ to the new point of intersection, $\mathbf{1}$. This adjustment for the Type 1 displacement is illustrated in Figure 4.20. The construction of the self-sharpening waves requires that displacement types $1-3$ be changed from composition paths illustrated in Figures 4.14-4.17 to those shown in Figures $4.20-4.22$. The Type 4 wave pattern has spreading waves on both legs of the path in the threephase region and satisfies the velocity constraini without adjustment.

\section{Type 2 Displacement}

The Type 2 displacement has a spreading wave on the upstream side of the three-phase region. This is done by moving along the slow path in the direction of increasing eigenvalues before jumping into the water-oil region. The composition path followed for the Type 2 wave pattern is given by Figure 4.23. The portion of the path in the steam-oil region is the same as the base case. This means that Point $\mathbf{C}$ is also identical to the base case. From Point $\mathbf{C}$ a zone of constant state takes the solution to Point $D$, where the eigenvalue matches the slow path. 
The Type 2 temperature profile as shown in Figure 4.24 is similar to the Type 1 temperature profile given in Figure 4.12. Both profiles have a constant temperatures at the injection temperature until the trailing shock drops the temperature to the thrue phase saturation temperature. The difference between the Type 2 and Type 1 temperature profiles is only seen at the leading shock.

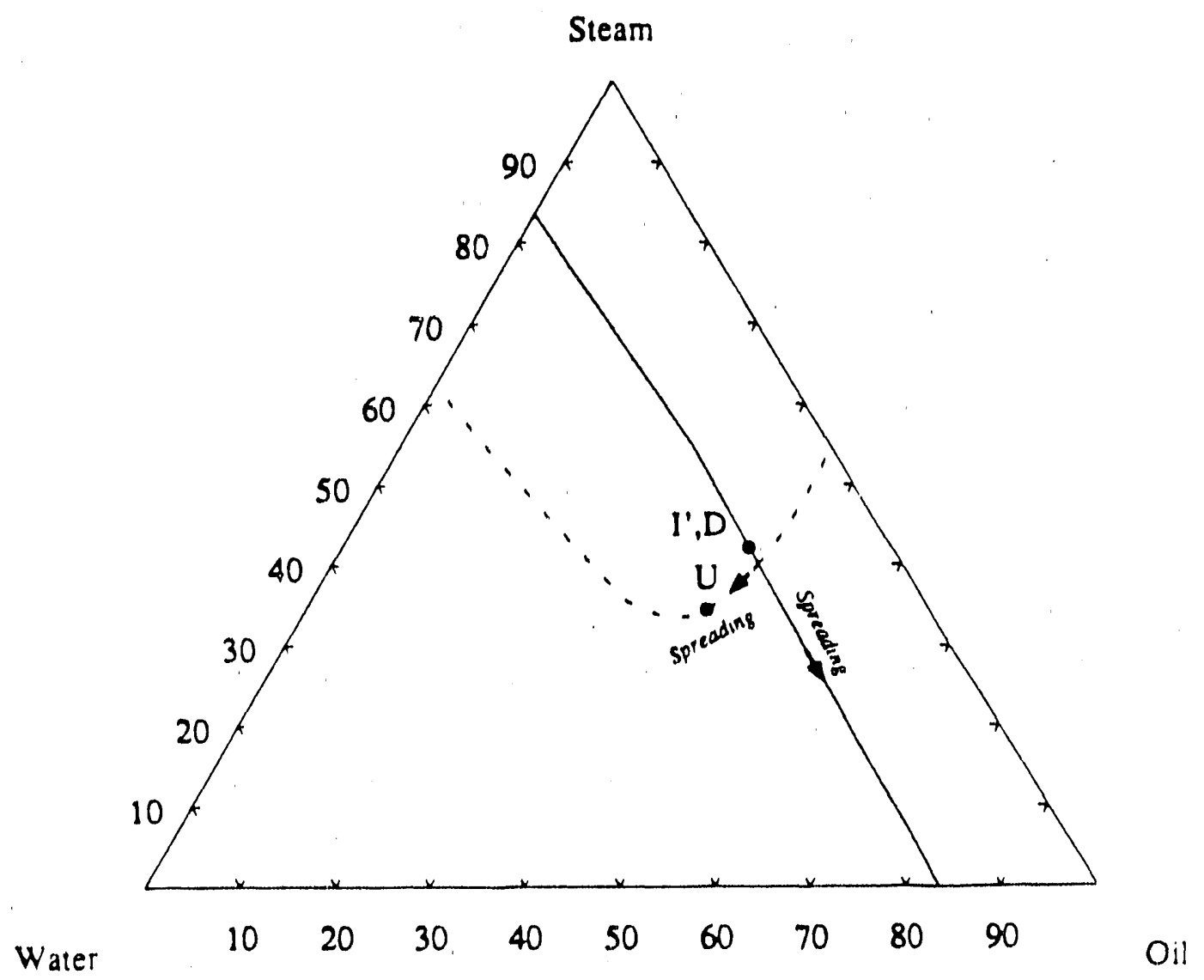

Figure 4.20: Adjusted Type 1 composition path that moves between two arbitrary points in the threephase region. 


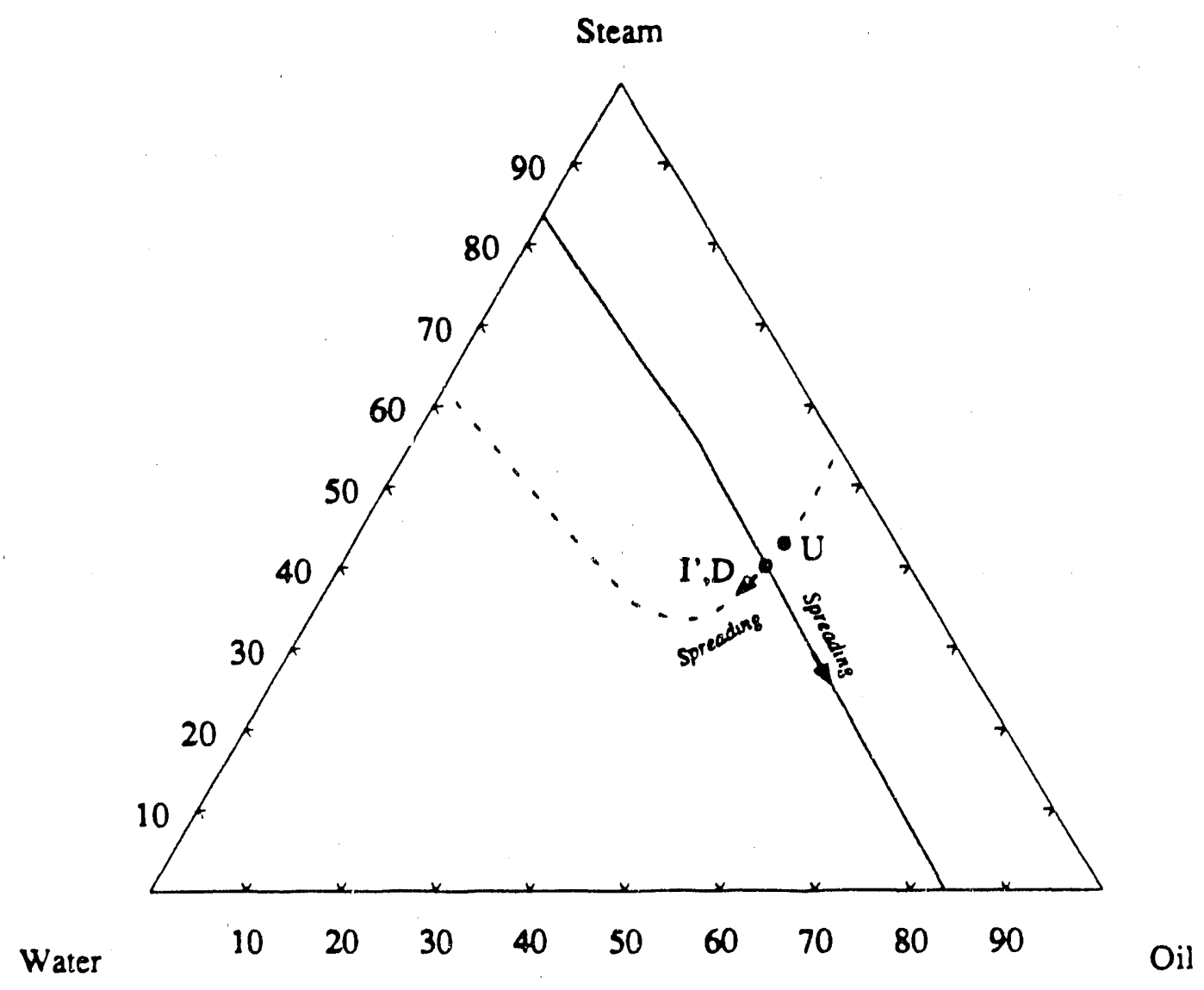

Figure 4.21: Adjusted Type 2 composition path that moves between two arbitrary points in the threephase region. 


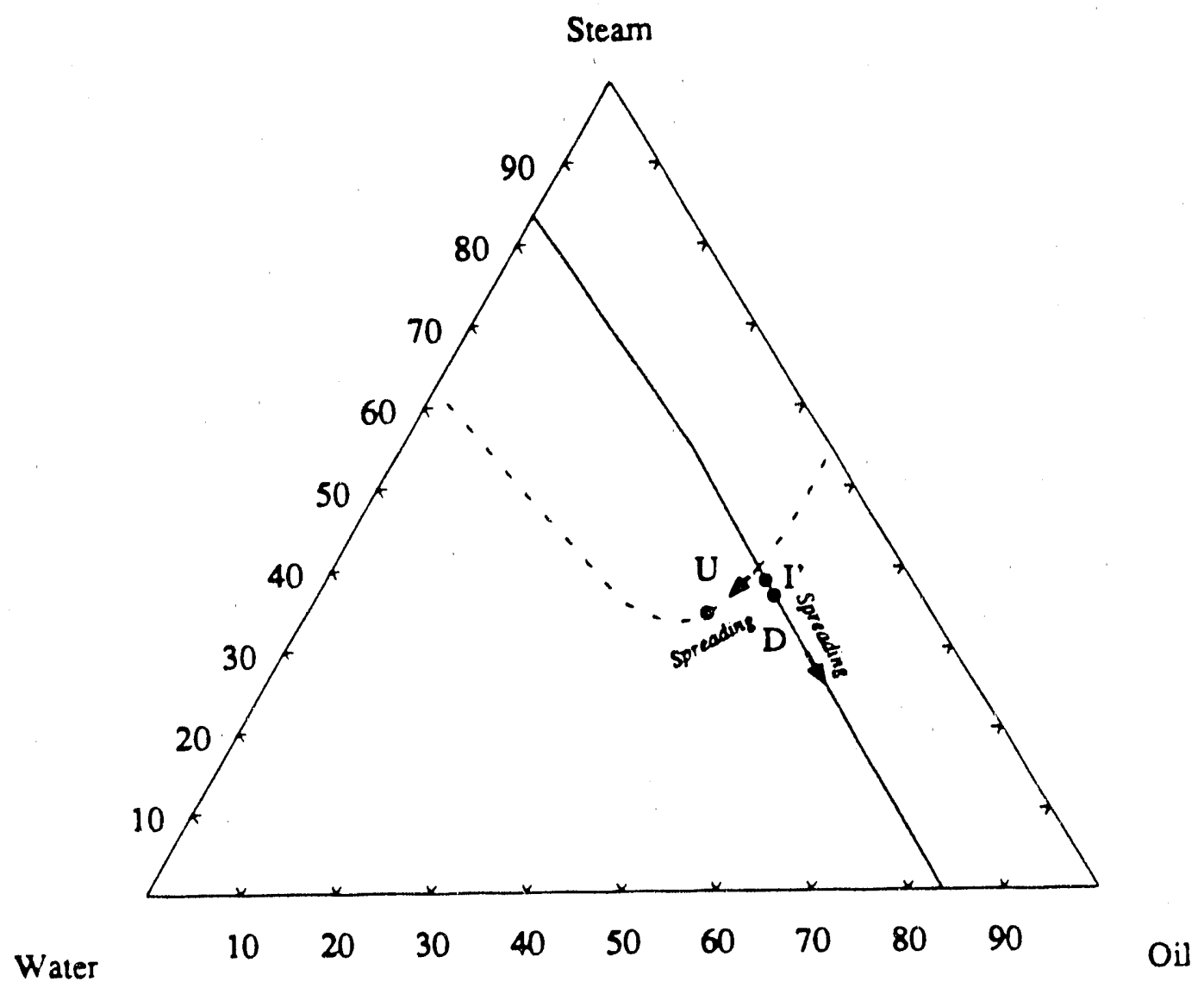

Figure 4.22: Adjusted Type 3 composition path that moves between two arbitrary points in the threephase region. 


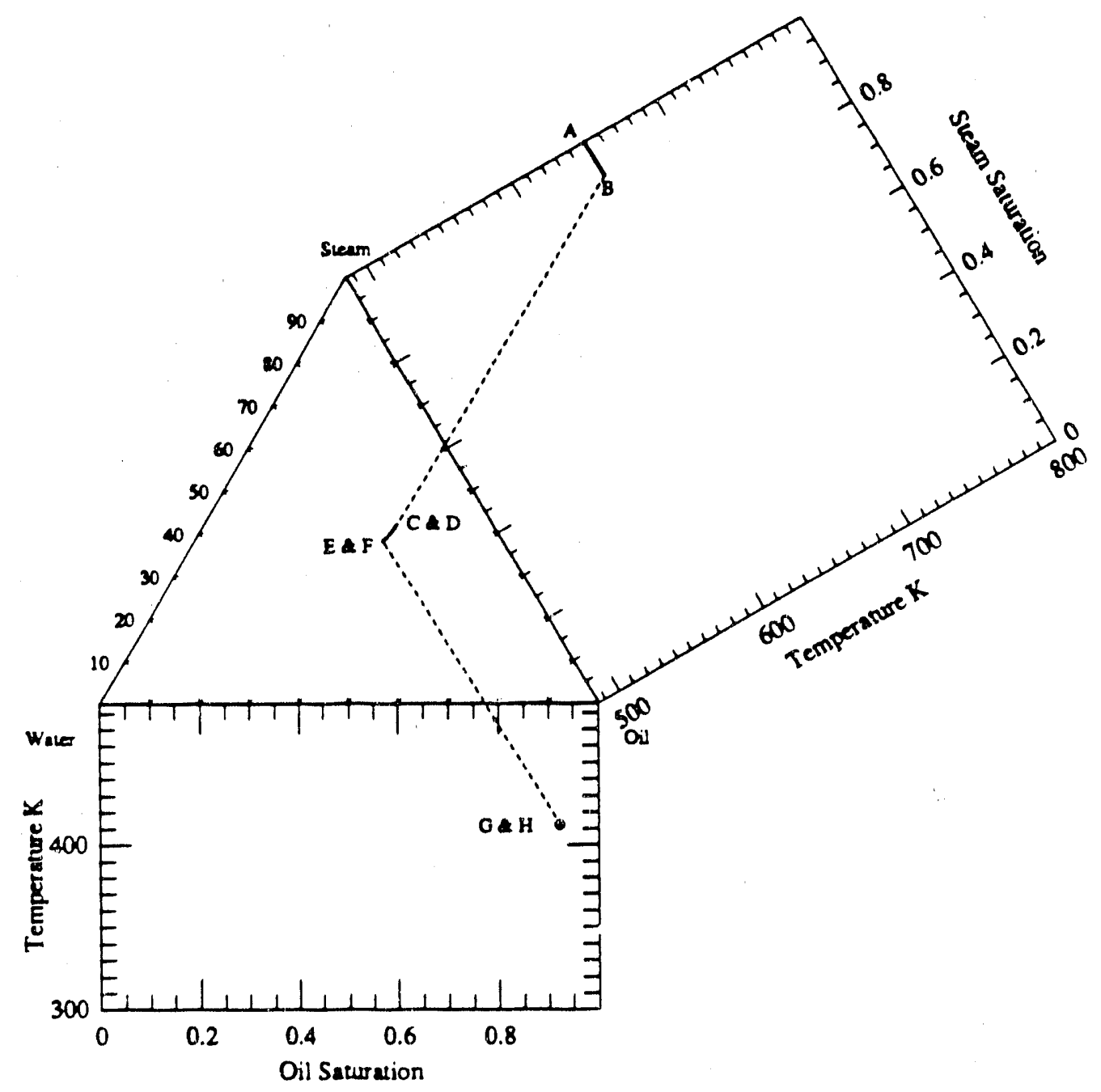

Figure 4.23: Composition path for the injection of $100 \%$ steam at $650 \mathrm{~K}$ into $92.4 \%$ oil and $7.6 \%$ water al $412.09 \mathrm{~K}$. 


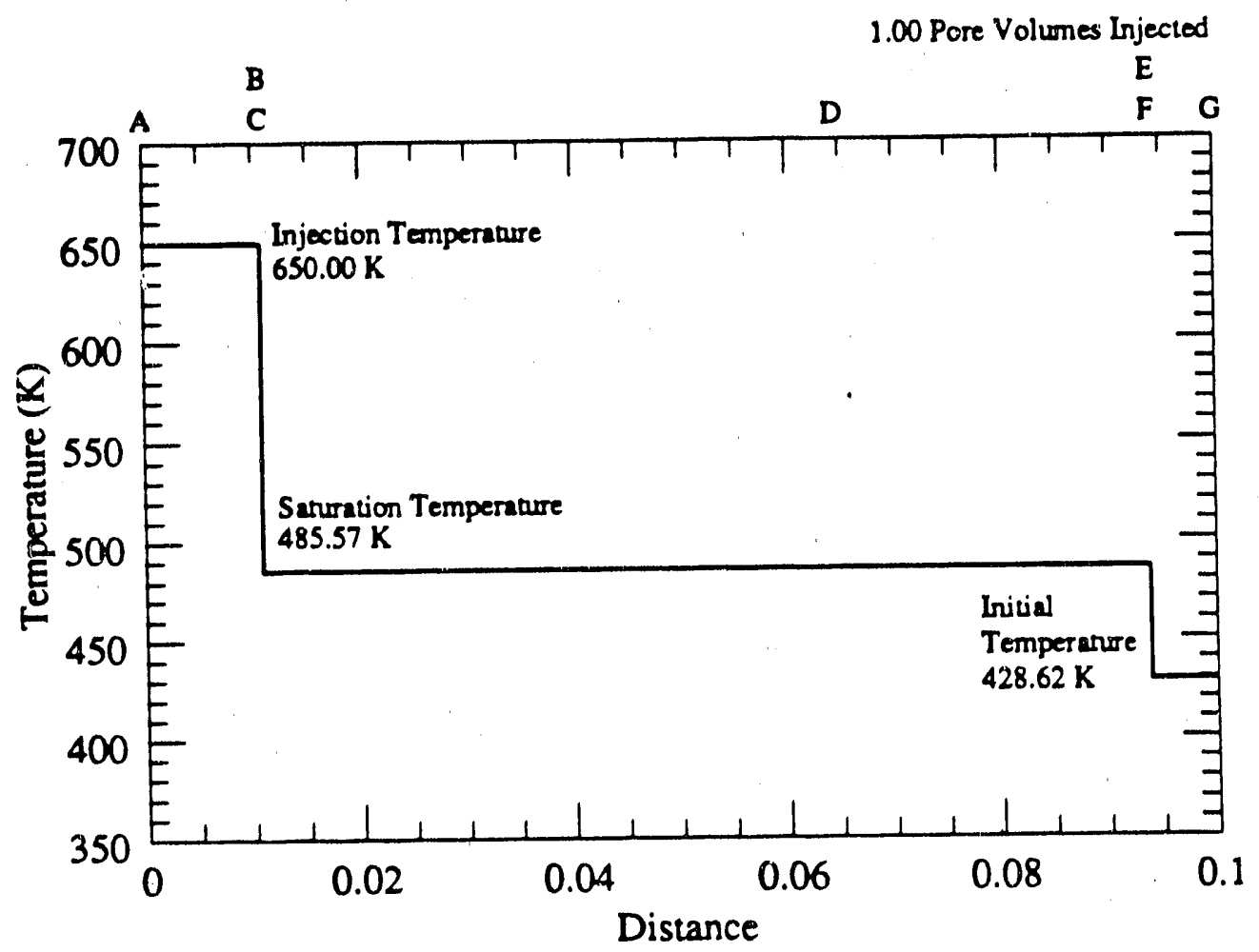

Figure 4.24: Temperature profile for the injection of 1.0 pore volumes of $100 \%$ steam at $650 \mathrm{~K}$ into $92.4 \%$ oil and $7.6 \%$ water at $412.09 \mathrm{~K}$.

The Type 2 temperature profile has a leading shock with a higher velocity and initial conditions at a higher temeprature. These two conditions are coupled by the heat balance across the shock. The higher temperature at the initial conditions require that less heat crosses the leading shock in the Type 2 displacement. Less heat crosses because the temperature difference is less in the Type 2 conditions. The result of the smaller heat content crossing the shock is that the shock travels with a higher velocity.

The flow velocity in the Type 2 displacement is shown in Figure 4.25. As in the Type 1 profile, the changes in velocity are a direct result the of density changes associated with the volume change of the water component as it condenses across both the leading and trailing shocks. The slightly higher velocity in the Type 2 case is the result of the slightly higher density of the liquid water at $412.09 \mathrm{~K}$ than at the $390.19 \mathrm{~K}$ in the Type 1 profile. 


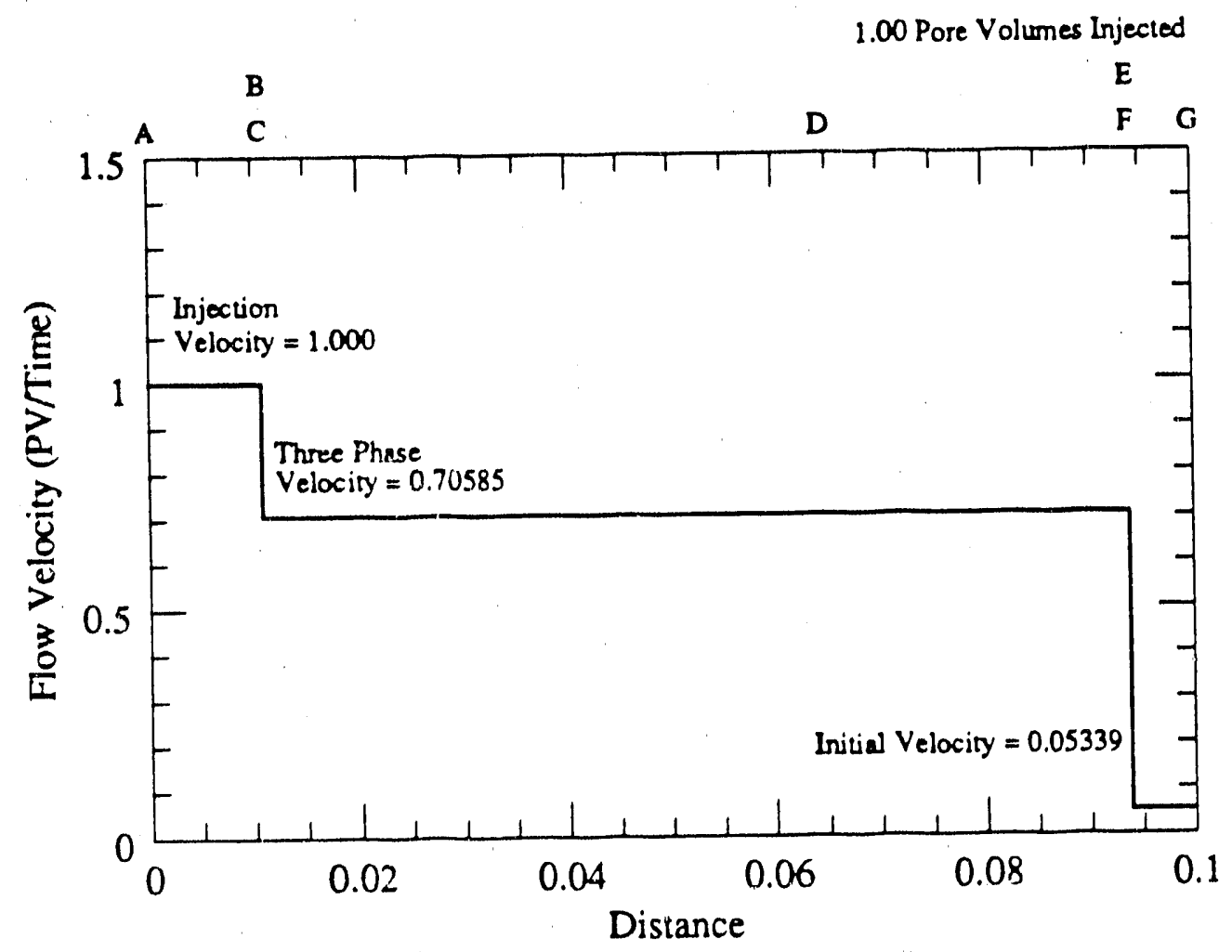

Figure 4.25: Flow velocity profile for the injection of 1.0 pore volumes of $100 \%$ steam at $650 \mathrm{~K}$ into $92.4 \%$ oil and $7.6 \%$ water at $412.09 \mathrm{~K}$.

The important differences are shown when the saturation profiles are compared. The saturation profile in the Type 2 displacement is illustrated in Figure 4.26. Comparing this saturation profile to the saturation profile of the Type 1 shown in Figure 4.11 shows that in the Type 2 saturation profile has a spreating wave that leads the central zone of constant state. This wave extends from Point D to Point D in Figure 4.26.

The solution path in composition space is shown in Figure 4.23. The solution path departs from the Type 1 solution path by in tegrating along the slow path from Point $\mathbf{D}$ to Point $\mathbf{E}$. The eigenvalues along this path increase, creating the spreading wave. The path roughly follows the contours of constant vapor fractional flow,

$$
\frac{\partial f_{v}}{\partial \eta_{1}}=0
$$

where $\eta_{1}$ is the distance along the slow path. 


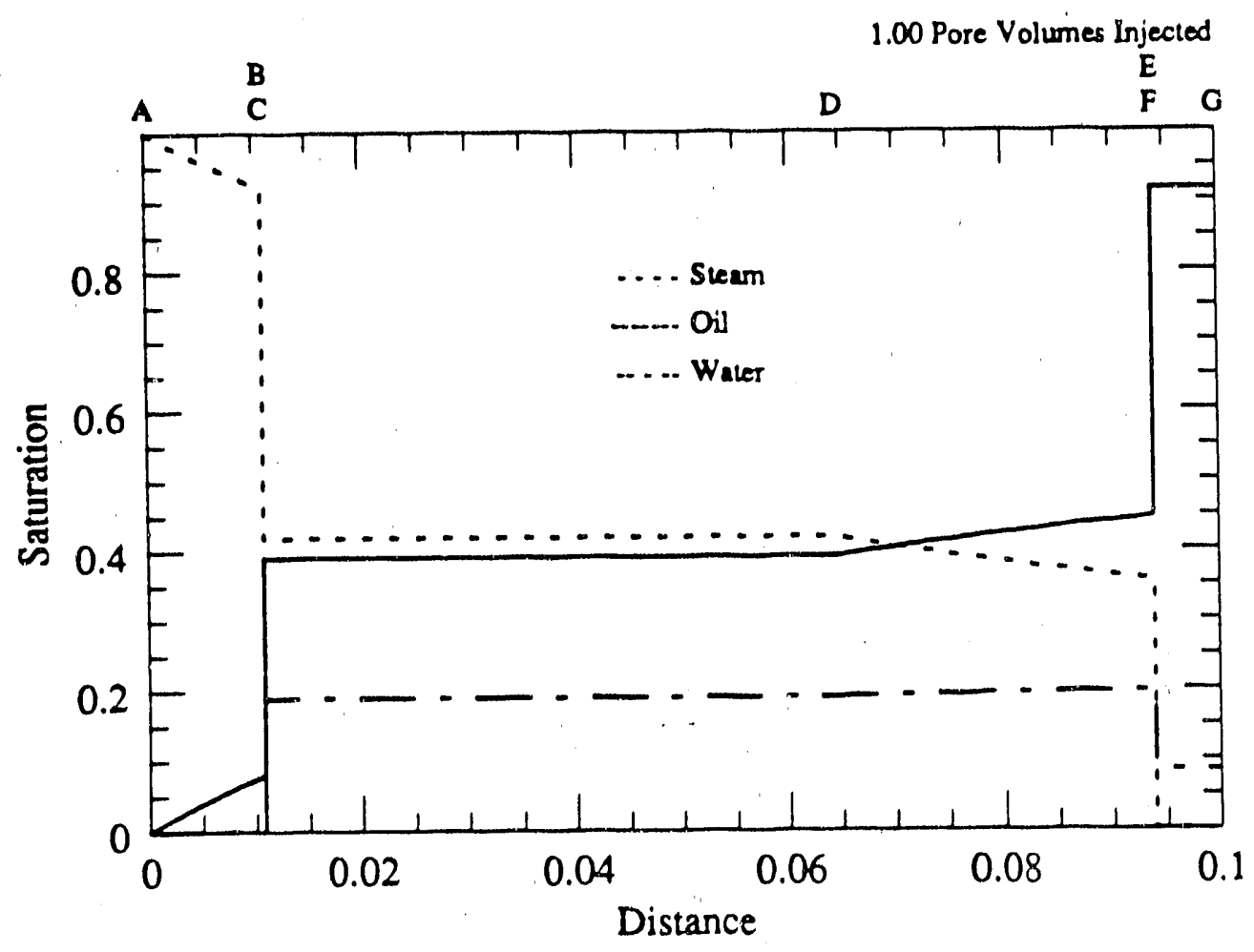

Figure 4.26: Saturation profile for the injection of 1.0 pore volumes of $100 \%$ steam at $650 \mathrm{~K}$ into $92.4 \%$ oil and $7.6 \%$ water at $412.09 \mathrm{~K}$.

At Point E, a path switches to the fast path and creates a zone of constant state ahead of the spreading wave. Point $\mathbf{E}$ is also the jumping point to the initial conditions via an upstream intermediate discontinuity. Because the large eigenvalue at Point $\mathbf{E}$ is higher than the jumping point in the base case, the initial temperature will also be higher than in the base case.

The spreading wave along the slow path changes the water saturation of the jumping point to a much larger extent than did integration along the fast path. This increase in water saturation at the initial conditions also increases the water saturation of the jumping point from the fast path. Physically the slow path represents the fractional flow relationship of the changing oil and water saturations at the trailing edge of the three phase region. In this region, the more mobile vapor phase has already moved ahead of these compositions and reached a steady value leaving only the oil and water saturations to change.

This increase in water saturation is shown along the example solution path. When the water saturation is higher at Point $\mathbf{E}$, the resulting waier saturation of the initial conditions, Point $\mathbf{F}$ is also 
higher. This increase in water saturation is accomplished by adding the spreading wave to the upstream end of the three-phase region.

\begin{tabular}{|c|c|c|c|c|c|c|c|}
\hline \multirow{3}{*}{ Label } & \multicolumn{5}{|c|}{ Composition Poinl } & \multirow{3}{*}{$\begin{array}{l}\text { Wave } \\
\text { Velocity }\end{array}$} & \multirow{3}{*}{$\begin{array}{l}\text { Type of } \\
\text { Flow } \\
\text { Region }\end{array}$} \\
\hline & \multicolumn{3}{|c|}{ Saturations } & \multirow[b]{2}{*}{$T(K)$} & \multirow{2}{*}{$\begin{array}{l}\text { Flow } \\
\text { Velocity }\end{array}$} & & \\
\hline & Steam & Oil & Water & & & & \\
\hline $\begin{array}{l}\mathrm{A} \\
\mathrm{B}\end{array}$ & $\begin{array}{l}1.00000 \\
0.92000\end{array}$ & $\begin{array}{l}0.00000 \\
0.08000 \\
\end{array}$ & $\begin{array}{l}0.00000 \\
0.00000 \\
\end{array}$ & $\begin{array}{l}650.00 \\
650.00 \\
\end{array}$ & $\begin{array}{l}1.00000 \\
1.00000 \\
\end{array}$ & $\begin{array}{l}0.000000 \\
0.010777 \\
\end{array}$ & $\begin{array}{l}\text { INJ } \rightarrow \text { SPW } \\
\text { SPW } \rightarrow \text { UID }\end{array}$ \\
\hline $\begin{array}{l}\text { C } \\
\text { D } \\
\text { E }\end{array}$ & $\begin{array}{l}0.41924 \\
0.41924 \\
0.35572\end{array}$ & \begin{tabular}{|l|}
0.39087 \\
0.39087 \\
0.44658 \\
\end{tabular} & $\begin{array}{l}0.18989 \\
0.18989 \\
0.19770 \\
\end{array}$ & $\begin{array}{l}485.57 \\
485.57 \\
485.57\end{array}$ & $\begin{array}{l}0.7058 \\
0.7058 \\
0.7058\end{array}$ & $\begin{array}{l}0.010777 \\
0.064505 \\
0.093985\end{array}$ & $\begin{array}{l}\text { UID } \rightarrow \mathrm{ZCS} \\
\mathrm{ZCS} \rightarrow \text { SPW } \\
\mathrm{SPW} \rightarrow \text { UID }\end{array}$ \\
\hline $\begin{array}{l}\bar{F} \\
G\end{array}$ & $\begin{array}{l}0.00000 \\
0.00000\end{array}$ & $\begin{array}{l}0.91566 \\
0.91566 \\
\end{array}$ & $\begin{array}{l}0.08434 \\
0.08434\end{array}$ & $\begin{array}{l}428.62 \\
428.62\end{array}$ & $\begin{array}{l}0.0534 \\
0.0534 \\
\end{array}$ & 0.093985 & $\underset{\mathrm{INI}}{\longrightarrow}$ \\
\hline
\end{tabular}

Table 4.6: Type 2 composition path for injection of $100 \%$ steam at $650 \mathrm{~K}$ into $92.4 \%$ oil and $7.6 \%$ water at $412.09 \mathrm{~K}$.

\section{Type 3 Displacement}

The Type 3 displacement attaches a spreading wave to the downstream portion of the three-phase region. Table 4.7 and Figure 4.27 outline the solution path for this displacement patem. The resulting hodograph profiles are shown in Figures $4.28-4.30$. The solution jumps immediately to the fast path from the three phase landing point as in the Type 1 wave pattern. Instead of immediately jumping to the initial conditions as with the Type 1 case, an integration along the fast path adds a spreading wave downstream of the zone of constant state before jumping to the initial conditions. This has the effect of increasing the oil saturation at the expense of the steam saturation. Another change is in the speed of the leading phase transition shock. As the integration proceeds along the fast path the eigenvalues must necessarily increase. Since the velocity of the phase transition shock matches the eigenvalue of the upstream conditions, this velocity must also increase.

The integration along the fast path from $10 \mathrm{E}$ is the new feature $c /$ the Type 3 displacement. This wave is highly spread over the zone preceeding the leading phase transition shock. The reason is that the increase in the wave velocity along the fast path is much larger than along the slow path seen in the Type 2 wave pattern. As the vapor saluration decreases, the derivative of the water phase fractional flow curve, $\left(\partial f_{w} / \partial S_{w}\right)$, increases, causing the eigenvalues along the fast path to rise rapidly. The end effect of an integration along this path is to force a change the initi thitions by significantly raising the initial temperature without appreciably affecting the initial wats: saludion.

\begin{tabular}{|c|c|c|c|c|c|c|c|}
\hline \multirow{3}{*}{ Label } & \multicolumn{5}{|c|}{ Composition Poinl } & \multirow{3}{*}{$\begin{array}{c}\text { Wave } \\
\text { Velocity }\end{array}$} & \multirow{3}{*}{$\begin{array}{l}\text { Type of } \\
\text { Flow } \\
\text { Rogion }\end{array}$} \\
\hline & \multicolumn{3}{|c|}{ Saturations } & \multirow[b]{2}{*}{$T(K)$} & \multirow{2}{*}{$\begin{array}{l}\text { Flow } \\
\text { Velocity }\end{array}$} & & \\
\hline & Steam & Oil & Water & & & & \\
\hline $\bar{A}$ & 1.00000 & 0.00000 & 0.00000 & 650.00 & 1.00000 & 0.000000 & $\begin{array}{l}\text { INJ } \rightarrow \text { SPW } \\
\text { SPW } \rightarrow \text { UID }\end{array}$ \\
\hline B & 0.92000 & 0.08000 & 0.00000 & 650.00 & $\frac{1.00000}{0.7050}$ & $\frac{0.010777}{0.010777}$ & $\frac{S P W \rightarrow \text { UID }}{\text { UID } \rightarrow \text { ZCS }}$ \\
\hline $\begin{array}{l}\text { C } \\
D\end{array}$ & $\begin{array}{l}0.41924 \\
0.41924\end{array}$ & $\begin{array}{l}0.39087 \\
0.39087\end{array}$ & $\begin{array}{l}0.18989 \\
0.18989\end{array}$ & $\begin{array}{l}485.57 \\
485.57\end{array}$ & $\begin{array}{l}0.7058 \\
0.7058\end{array}$ & 0.064505 & $\mathrm{ZCS} \rightarrow \mathrm{SPW}$ \\
\hline E & 0.35572 & 0.44658 & 0.19770 & 485.57 & 0.7058 & 0.093985 & SPW $\rightarrow$ UID \\
\hline F & 0.00000 & 0.91566 & 0.08434 & $\begin{array}{l}428.62 \\
428.62\end{array}$ & $\begin{array}{l}0.0534 \\
0.0534\end{array}$ & 0.093985 & $\underset{\mathbb{I N I}}{\mathrm{UID}} \rightarrow \mathrm{INI}$ \\
\hline
\end{tabular}

Table 4.7: Type 3 composition path for injection of $100 \%$ steam at $650 \mathrm{~K}$ into $91.6 \%$ oil and $8.4 \%$ water at $428.62 \mathrm{~K}$. 


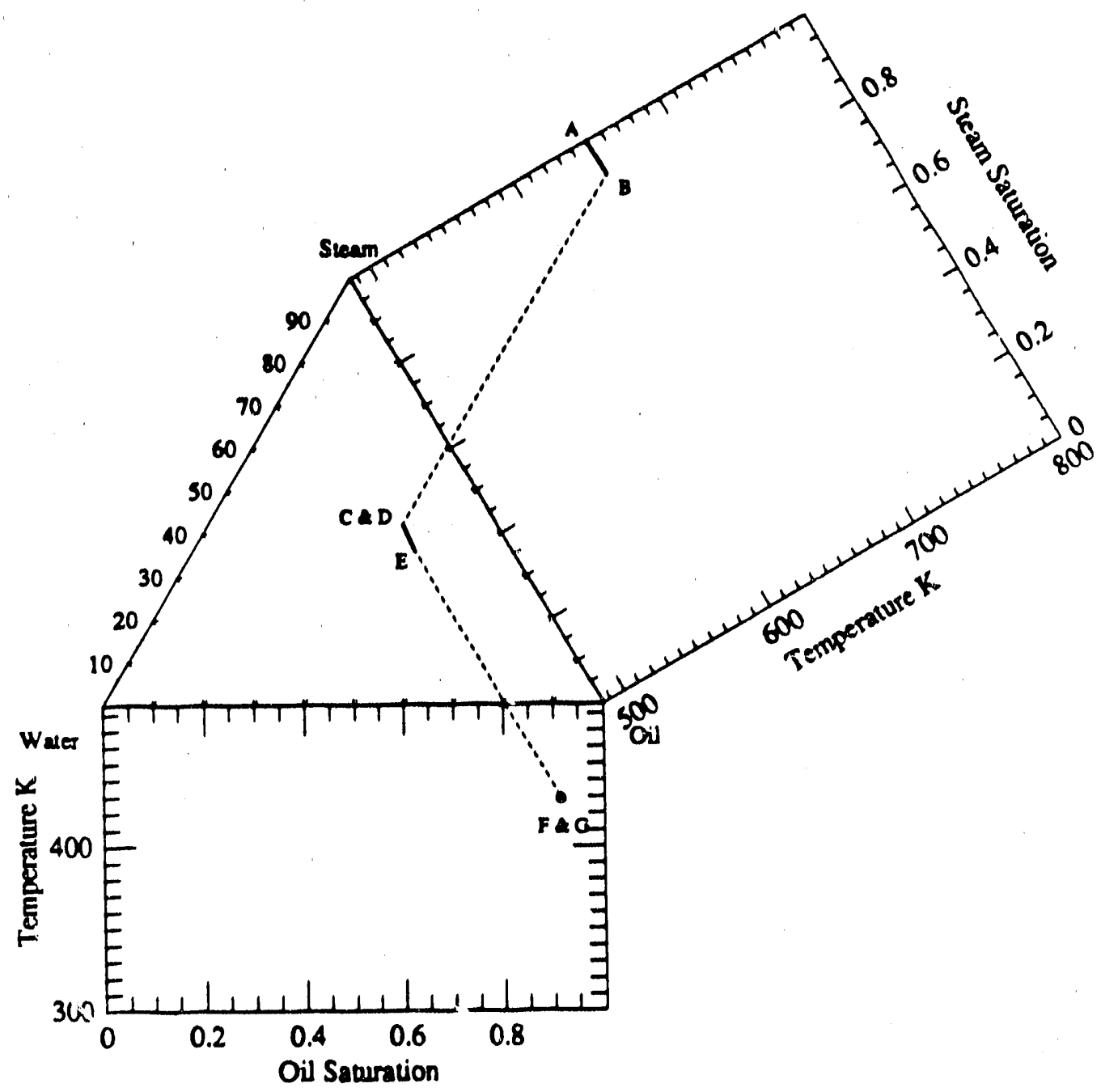

Figure 4.27: Composition path for the injection of $100 \%$ steam at $650 \mathrm{~K}$ into $91.6 \%$ oil and $8.4 \%$ water at $428.62 \mathrm{~K}$. 


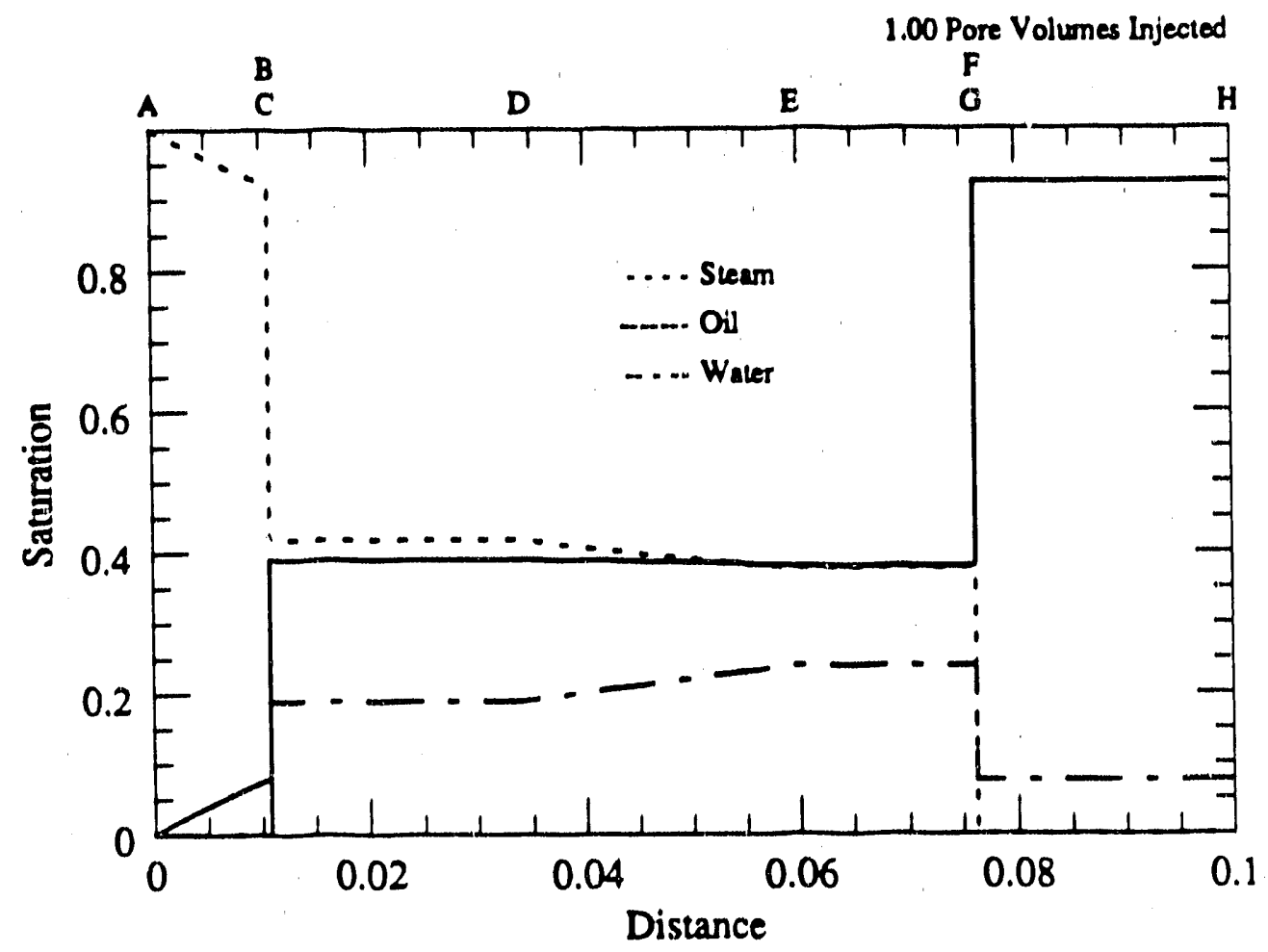

Figure 4.28: Saturation profile for the injection of $100 \%$ steam at $650 \mathrm{~K}$ into $91.6 \%$ oil and $8.4 \%$ water at $428.62 \mathrm{~K}$. 


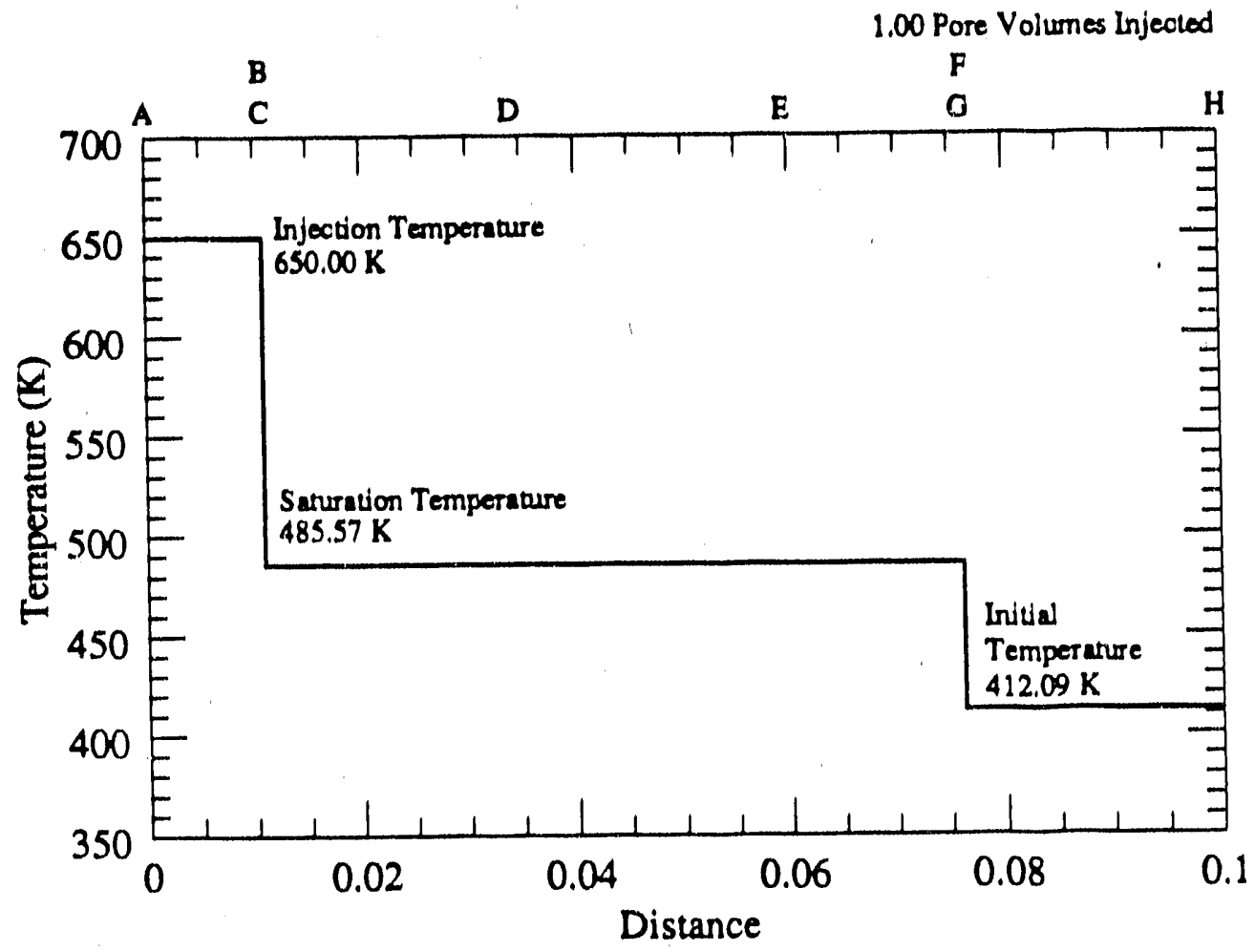

Figure 4.29: Temperature profile for the injection of $100 \%$ steam at $650 \mathrm{~K}$ into $91.6 \%$ oil and $8.4 \%$ water at $428.62 \mathrm{~K}$. 


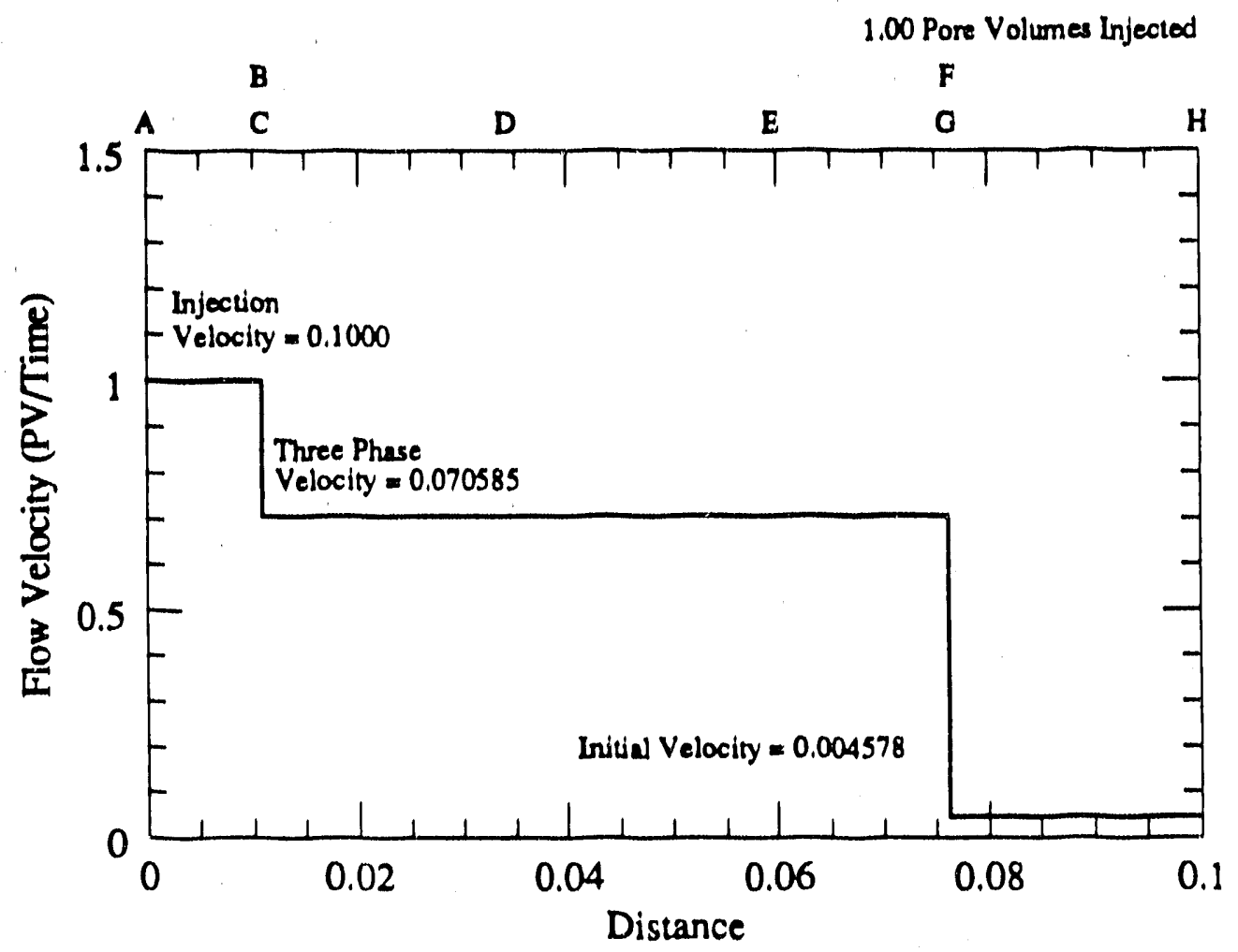

Figure 4.30: Flow velocity profile for the injection of $100 \%$ steam at $650 \mathrm{~K}$ into $91.6 \%$ oil and $8.4 \%$ water at $428.62 \mathrm{~K}$. 


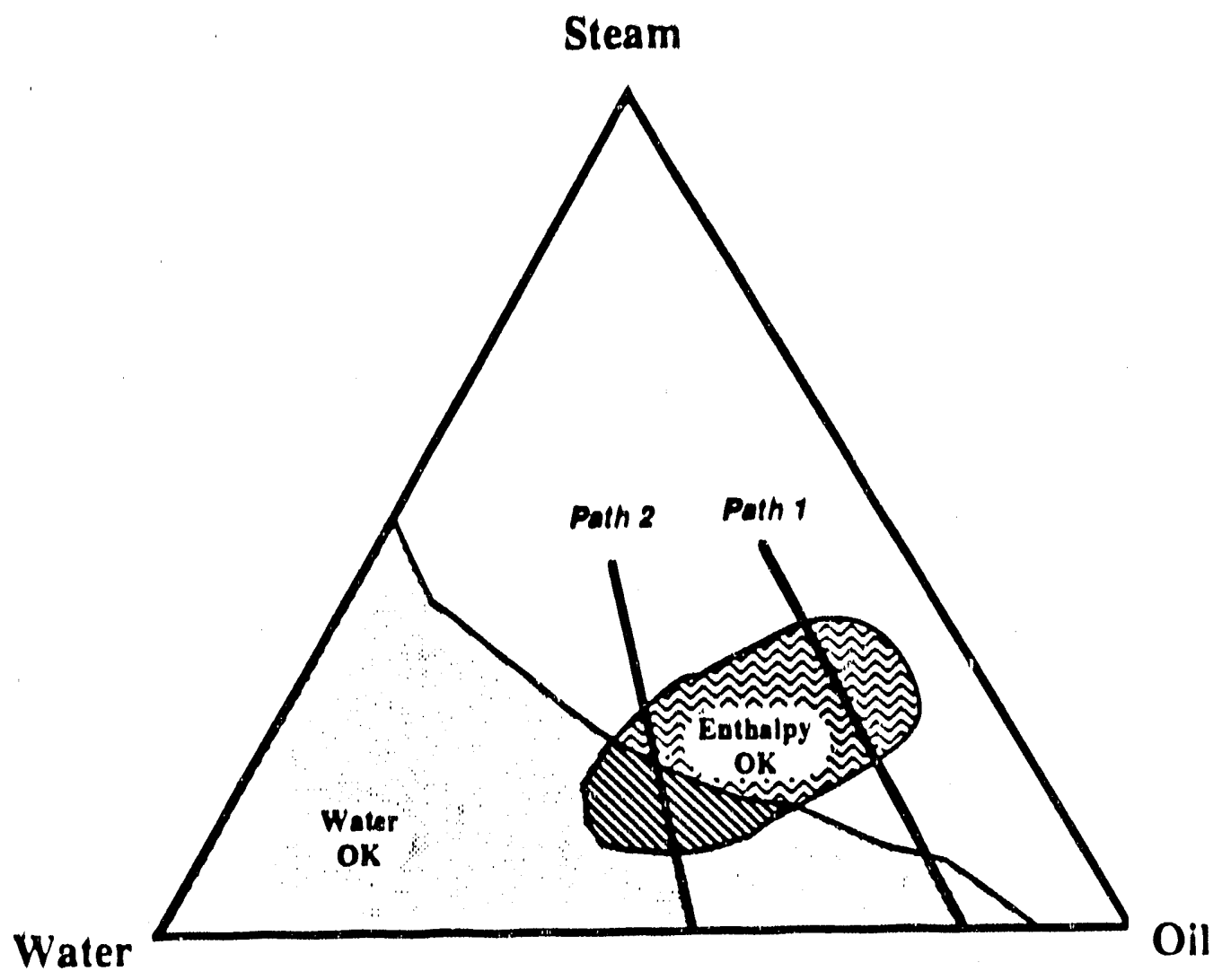

Figure 4.31: Three-phase region of the steam-oil-water displacements showing the regions where the fast path eigenvalues and water saturation permit a jump into the water-oil region.

The jumping condition from the fast path to the water-oil region is controlled by two factors; these are the enthalpy and water balances across the shock. Because the wave velocity changes rapidly, there is a small region along the fast composition path that can jump into the allowable hodograph space in the water oil region. In the three phase space there is a region where the eigenvalues are in the allowable range to satisfy the enthalpy balance. Another region has a large enough water saturation so that the steam and water are mobile enough to cross the phase change shock and form a liquid water phase. Only paths that cross the intersection of these two regions can jump into the water-oil region. These regions are illustrated in Figure 4.31. If the fast path is chosen as Path 1, there is no composition point along that path that satisfies both the enthalpy requirement and the water saturation for a jump into the wateroil region. Path 2 on the other hand does cross into the intersecting region and a solution for the phase transition shock can be found.

\section{Type 4 Displacement}

The Type 4 displacement is a combination of the Type 2 and Type 3 systems. A spreading wave is located before and after the zone of constant state. The Type 4 wave pauem has the fastest leading shock and hence requires the highest initial temperatures and water saturations. The composition path for the Type 4 wave patlern is illustrated in Figure 4.32 and listed in Table 4.8. 
The saturation, temperature and flow volocity profiles in Figures $4.33-4.35$ show the profiles that result from following the composition path in Figure 4.32. The oil saturation in the three phase region remains essentially constant over the entire region. An interesting feature of this path is that the oil saturation decreases along the path $\mathbf{D} \rightarrow \mathbf{E}$ upstream of the zone of constant state, then increases along $\mathbf{F} \rightarrow \mathbf{G}$ before jumping to the water-oil region.

The water bank has a lower saturation at the upstream end of the three-phase region and slowly increases over the length of the three-phase region. This is due to the spreading waves that follow increasing water saturation in the three-phase region.

\begin{tabular}{|c|c|c|c|c|c|c|c|}
\hline \multirow{3}{*}{ Label } & \multicolumn{5}{|c|}{ Composition Point } & \multirow{3}{*}{$\begin{array}{c}\text { Wave } \\
\text { Velocity }\end{array}$} & \multirow{3}{*}{$\begin{array}{l}\text { Type of } \\
\text { Flow } \\
\text { Region }\end{array}$} \\
\hline & \multicolumn{3}{|c|}{ Saturations } & \multirow[b]{2}{*}{$T(K)$} & \multirow{2}{*}{$\begin{array}{l}\text { Flow } \\
\text { Velocity }\end{array}$} & & \\
\hline & Steam & Oil & Water & & & & \\
\hline $\bar{A}$ & 1.00000 & 0.00000 & 0.00000 & 650.00 & 1.00000 & 0.000000 & $\mathrm{INJ} \rightarrow \mathrm{SPW}$ \\
\hline B & 0.92000 & 0.08000 & 0.00000 & 650.00 & 1.00000 & 0.010777 & SPW $\rightarrow$ UID \\
\hline $\mathrm{C}$ & 0.41924 & 0.39087 & 0.18989 & 485.57 & 0.7058 & 0.010777 & UID $\rightarrow$ ZCS \\
\hline D & 0.41924 & 0.39087 & 0.18989 & 485.57 & 0.7058 & 0.034233 & $\mathrm{ZCS} \rightarrow \mathrm{SPW}$ \\
\hline$E$ & 0.37839 & 0.38132 & 0.24029 & 485.57 & 0.7058 & 0.059471 & SPW $\rightarrow \mathrm{ZCS}$ \\
\hline $\mathbf{F}$ & 0.37839 & 0.38132 & 0.24029 & 485.57 & 0.7058 & 0.076318 & $\mathrm{ZCS} \rightarrow \mathrm{SPW}$ \\
\hline $\mathrm{O}$ & 0.34243 & 0.39977 & 0.25780 & 485.57 & 0.7058 & 0.098756 & SPW $\rightarrow$ UID \\
\hline $\mathrm{H}$ & 0.00000 & 0.88119 & 0.11881 & 433.79 & 0.0539 & 0.098756 & UID $\rightarrow$ INI \\
\hline 7 & 0.00000 & 0.8819 & 0.118 & 433.79 & 0.0539 & & \\
\hline
\end{tabular}

Table 4.8: Type 4 composition path for injection of $100 \%$ steam at $650 \mathrm{~K}$ into $88.1 \%$ oil and $11.9 \%$ water at $433.79 \mathrm{~K}$.

This section has presented example solutions for four different wave patterns seen in the steam-oilwater displacements. All the cases presented were limiting case examples where the velocity of the selfsharpening waves were matched to the tangent shocks that occurred at the phase transitions. Many other possible solutions exish all matching a given set of injection conditions to a specific set of initial conditions. The self-sharpening waves were calculated assuming that they were all upstrean intermediate discontinuities. Other solutions could involve one or two downstream intermediate discontinuities in place of the either upstream intermediate discontinuity. The effect of varying the injection conditions or the relative permeability model has not been discussed, but the tochniques described in the following section could be used to construct such solutions.

\subsubsection{Solution Procedure}

The complete solution to the steam-oll-water problem is obtained using the following steps:

1. Select the injection conditions. This requires choosing the injection composition, injection temperature and injection velocity.

2. Integrate along the slow path until some point, B, is reached. Point B can be completely arbitrary with one exception that is noted in the next step.

3. Calculate the composition point in the three-phase region that is reached by crossing an upstream intermediate discontinuity from Point $\mathbf{B}$, in the steam-oil region, into the three-phase region. This requires that the shock velocity as given by Eq. 2.35 be equal to the small eigenvalue at Point B. The landing point in the three-phase region, $\mathrm{C}_{\text {, is }}$ unique. 


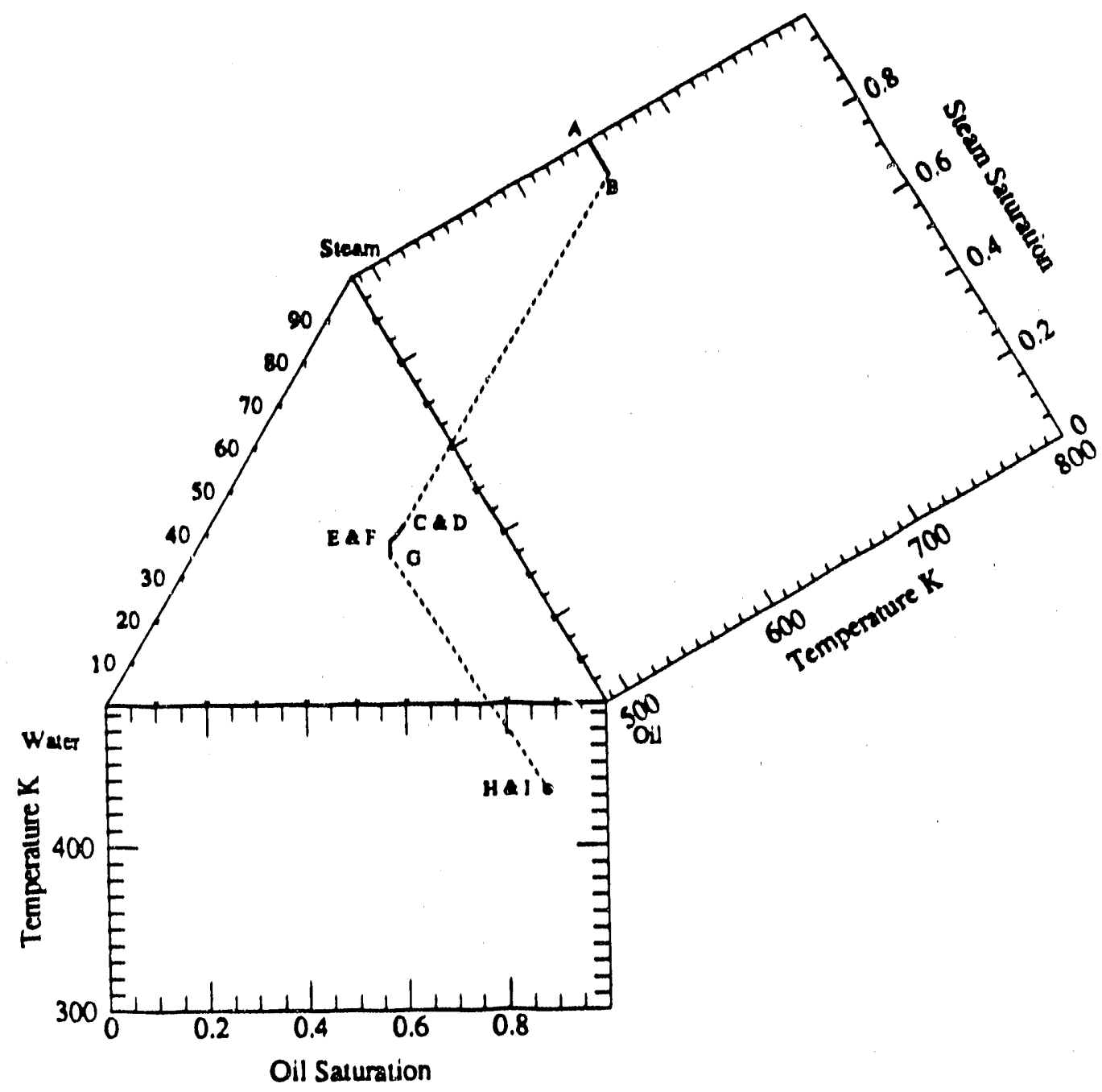

Figure 4.32: Composition path for the injection of $100 \%$ steam at $650 \mathrm{~K}$ into $88.1 \%$ oil and $11.9 \%$ water at $433.79 \mathrm{~K}$. 


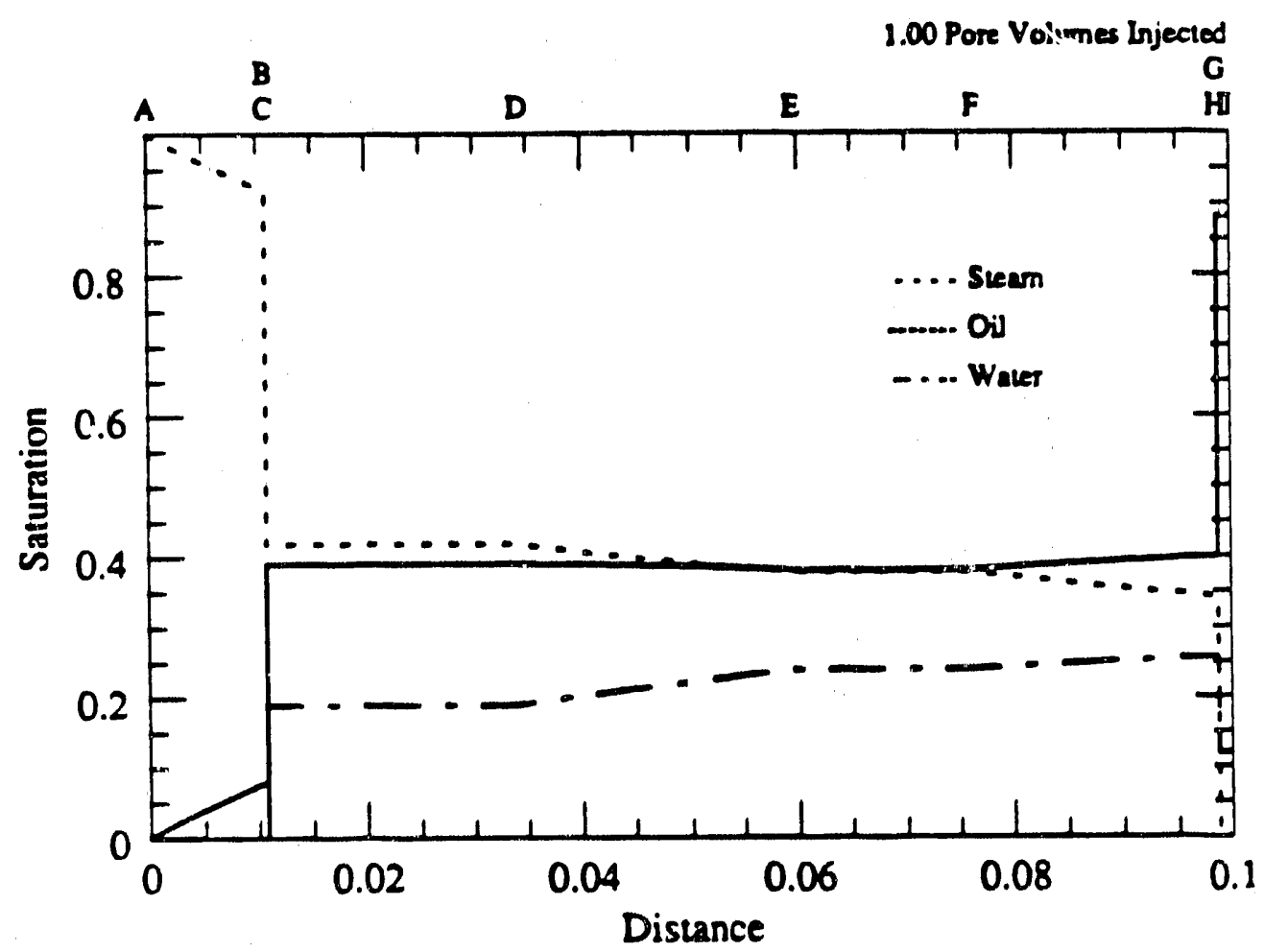

Figure 4.33: Saturation profile for the injection of 1.0 pore volumes of $100 \%$ steam at $6: \mathrm{K}$ into $88.1 \%$ oil and $11.9 \%$ water at $433.79 \mathrm{~K}$. 


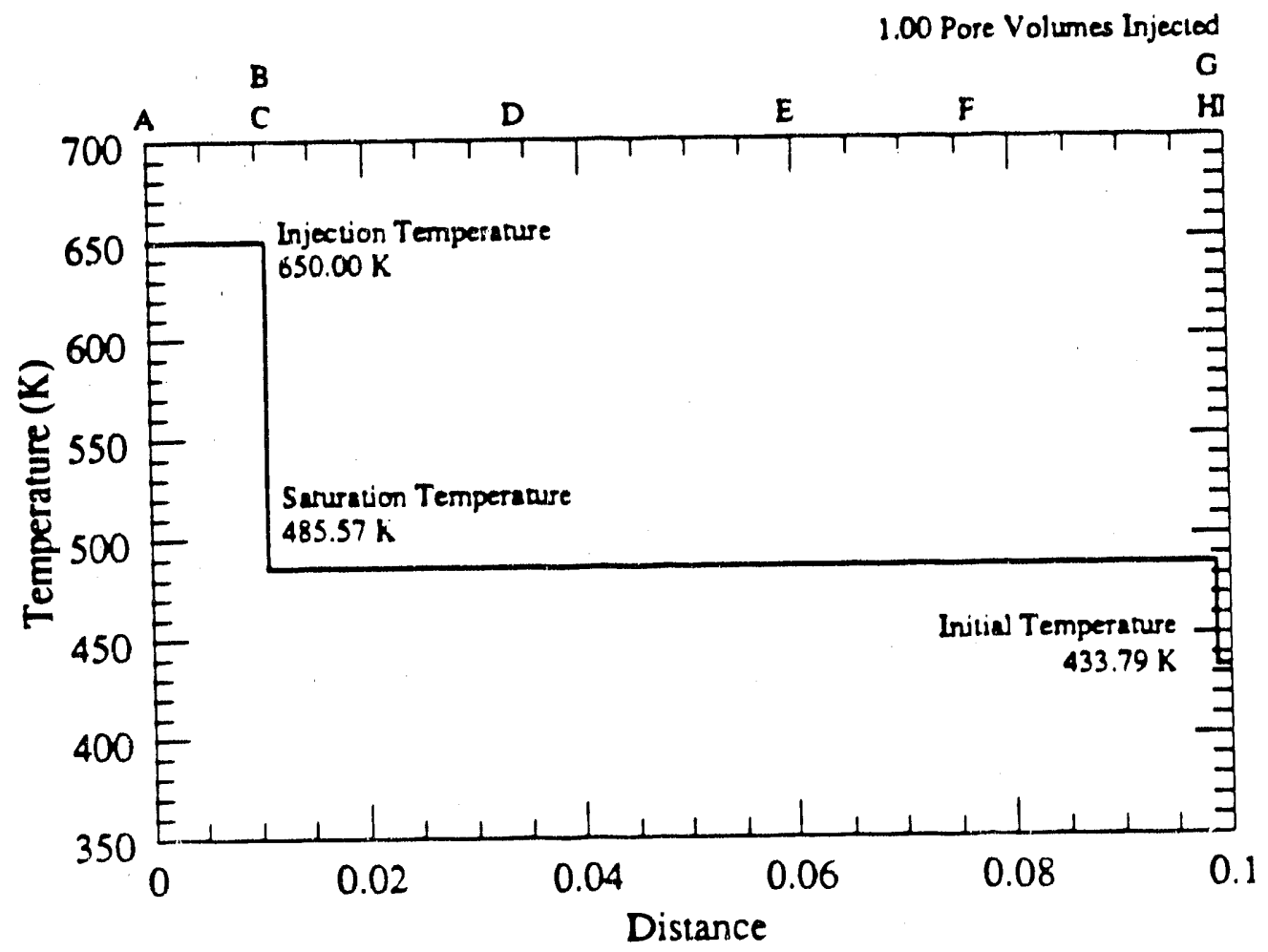

Figure 4.34: Temperature profile for the injection of 1.0 pore volumes of $100 \%$ steam at $650 \mathrm{~K}$ into

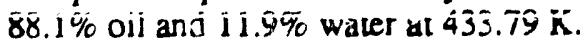




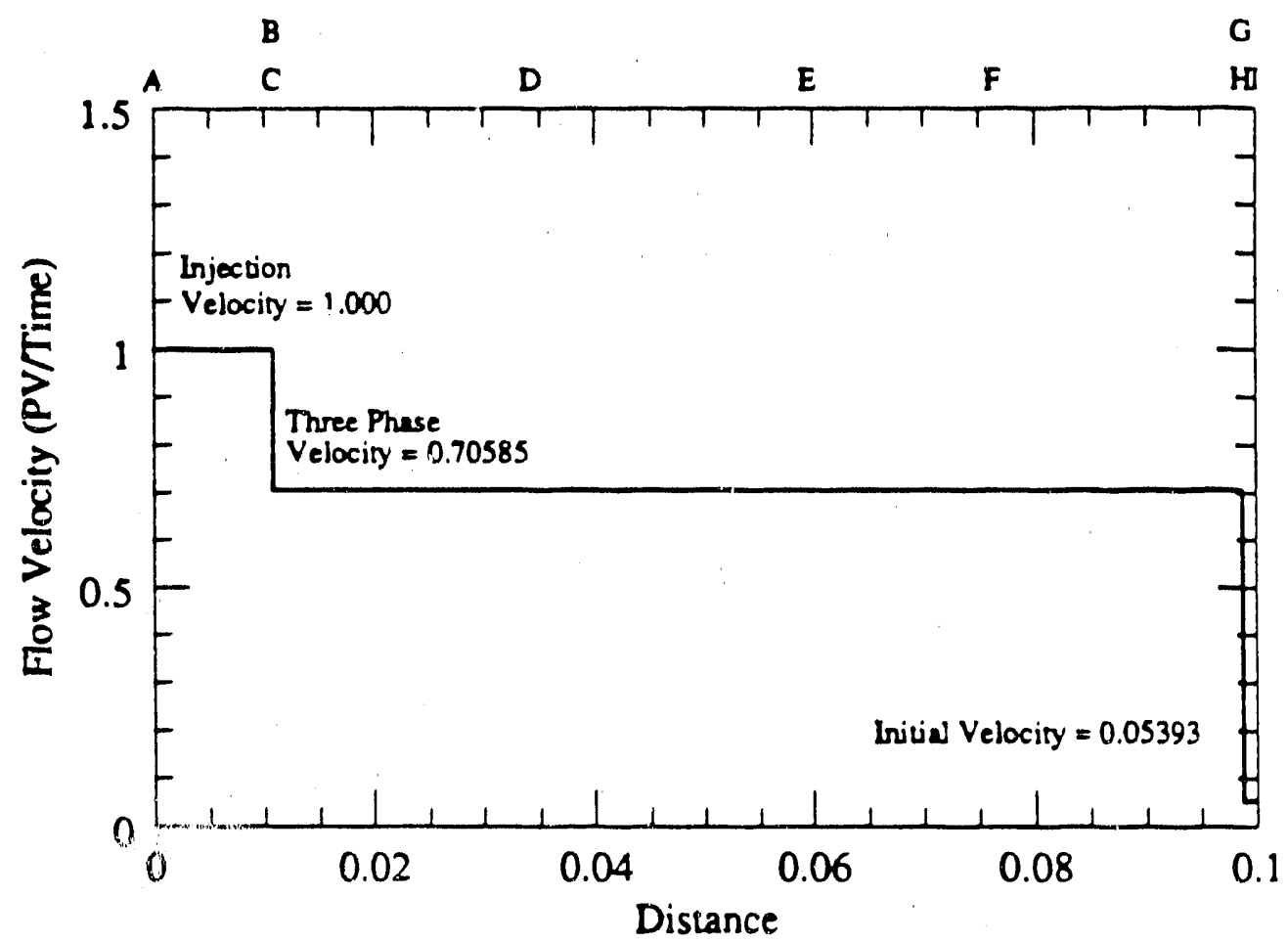

Figure 4.35: Flow velocity profile for the injection of 1.0 pore volumes of $100 \%$ steam at $650 \mathrm{~K}$ into $88.1 \%$ oil and $11.9 \%$ water at $433.79 \mathrm{~K}$.

If the velocity of Point B is 100 small, there will be no solution for Composition $\mathbf{C}$ in the allowable visdograph space. This is indicated by having one or more of the saturation values be less than zero or greater than one at the landing point. If this happens, the selected point, B, is not a valid jumping point and another composition point further along the injection path $(\mathbf{A} \rightarrow \mathbf{B})$ must be chosen.

4. Integrate along the slow path from $\mathbf{C}$ to a new point in the three-phase region, Composition $\mathbf{D}$. Composition D will be the point of intersection between the slow path and fast path. At this composition, the solution switches paths from the slow 10 the fast path, creating a zone of constant state at Point $\mathbf{D}$.

The velocities must increase as the integration moves from $\mathbf{C} \rightarrow \mathbf{D}$. If the velocities decrease, a self-sharpening wave is indicated. A point must be chosen on a spreading portion of the path as the upstream set of conditions. If the eigenvalutis immediately decrease in the desired direction, then the landing point must be at the upstream conditions. This is the limiting case presented in the example solutions. The self-sharpening wave is calculated by matching the shock velocity to the eigenvalue on either the upstream or downstream side of the shock. This has the effect of slightly shifting the intersection point $t$ the fast path because of fractional flow considerations. This adjustment is indicative of the Type 1 and Type 3 wave patterns discussed in Table 4.5.

5. Integrate from Point $\mathbf{D}$ to ar other composition point, $\mathbf{E}$, on the fast path. This large eigenvalue point cannot be so large that a material balance, or more importantly a heal balance, carries the shock solution out of the allowable hodograph space. 
The limitation on self-sharpening waves also applies as the integration travels from $\mathbf{D}$ to $\mathbf{E}$. The self-sharpening wave is resolved by jumping to the water-oil region directly from Point $\mathbf{D}$. The self-sharpening wave along the fast path is usually not seen beacuse this takes the solution away from the region where the solution can jump into the water-oil region. This kind of adjustment creates Type 1 or Type 2 displacements as mentioned in Table 4.5.

6. Point $\mathbf{E}$ is the jumping point into the water-oil region. Calculate the point in the water-oil region that satisfies the material balances and also matches the shock velocity to the large eigenvalue at Composition $\mathbf{E}$. The landing point in the water-oil region, $\mathbf{F}$, is the initial condition.

The nature of the eigenvalues in the water-oil region prevent the development of spreading waves in this region. All the eigenvalues in the water-oil region are slower than their three phase counterparts which necessarily lie upstream of the initial conditions. This situation often occurs in compressible gas dynamics where the leading shock travels faster than all the wave velocities in the region of initial conditions.

The initial temperature has a significant effect on the resulting velocity of the leading shock. The higher is the initial temperature, the less heat that must cross the shock and the faster its velocity must be. The Type 1 displacement has the minimum slowest leading shock for a given initial steam-oil jumping point (Point B). This type of displacement patten therefore, is a result of approaching the lower limit on the initial temperature and flow velocity at the initial conditions for a given initial jumping point, Point B.

The spreading directions on both the fast and especially the slow path in the three-phase region have increasing water siturations. This increase of the water saturation at the initial conditions results in an increase in water saturation on the upstream side of the leading shock.

So far a's the cases presented have followed the same path in the steam-oil region and have jumped into the thrie phase region from the same composition point. When the integration is allowed to continue farther along the slow path in the steam-oil region, a composition point is reached where the eigenvalues are equal. At this point, a path switch in accordance with Rule III, illustrated in Figure 3.2, takes the soiution from the isothermal path onto a path with temperature variations. Following this variable temperature path results in a temperature profile in the initial steam-oil region upstream of the trailing upstream intermediate discontinuity. The portion of the slow path that changes temperature is an example of a slightly spreading wave where the eigenvalues do not change significantly from the equal eigenvalue point to the saturation temperature. An example solution following this type of path is given in the rext section.

\subsubsection{Temperature Profile in the Steam-Oil Region}

Solutions with temperature profiles in the steam-oil region are presented in this section. By continuing along the injection path past the equal eigenvalue point, the solution follows a path that decreases in temperature as the eigenvalues increase. This forms a spreading wave where th' saturations, temperature and flow velocity are continuously changing upstream of the trailing ups ream intermediate discontinuity.

The composition path for the Type 1 displacement with a temperature profile in the steam-oil region is illustrated by Figure 4.36 and outlined in Table 4.9. The new solution path, (Figure 4.36) compared with the the solution path in the original Type 1 displacement, (Figure 4.5) appears very different on a first examination. The original solution path in the steam oil region is very short and the path in the new solution is much different. 


\begin{tabular}{|c|c|c|c|c|c|c|c|}
\hline \multirow{3}{*}{ Label } & \multicolumn{5}{|c|}{ Composition Point } & \multirow{3}{*}{$\begin{array}{l}\text { Wave } \\
\text { Velocity }\end{array}$} & \multirow{3}{*}{$\begin{array}{l}\text { Type of } \\
\text { Flow } \\
\text { Region }\end{array}$} \\
\hline & \multicolumn{3}{|c|}{ Saturations } & \multirow[b]{2}{*}{$T(K)$} & \multirow{2}{*}{$\begin{array}{c}\text { Flow } \\
\text { Velocity }\end{array}$} & & \\
\hline & Stcam & Oil & Water & & & & \\
\hline $\bar{A}$ & 100000 & 0.00000 & 0.00000 & 650.00 & 1.00000 & 0.000000 & INJ $\rightarrow$ SPW \\
\hline B & $\begin{array}{l}0.91681 \\
0.74335\end{array}$ & $\begin{array}{l}0.09319 \\
0.25665\end{array}$ & $\begin{array}{l}0.00000 \\
0.00000\end{array}$ & $\begin{array}{l}650.00 \\
525.00\end{array}$ & $\begin{array}{l}1.00000 \\
0.7712\end{array}$ & $\begin{array}{l}0.011266 \\
0.011916\end{array}$ & $\stackrel{\text { EEP }}{\text { SPW }} \rightarrow$ UID \\
\hline $\begin{array}{l}\text { D } \\
E\end{array}$ & $\begin{array}{l}0.39946 \\
0.39946\end{array}$ & $\begin{array}{l}0.39680 \\
0.39680\end{array}$ & $\begin{array}{l}0.20373 \\
0.20373\end{array}$ & $\begin{array}{l}485.57 \\
485.57\end{array}$ & $\begin{array}{l}0.6948 \\
0.6948\end{array}$ & $\begin{array}{l}0.040644 \\
0.069397\end{array}$ & $\begin{array}{l}\text { UID } \rightarrow \mathrm{ZCS} \\
\mathrm{ZCS} \rightarrow \text { UID }\end{array}$ \\
\hline F & $\begin{array}{l}0.00000 \\
0.00000\end{array}$ & $\begin{array}{l}0.94757 \\
0.94757\end{array}$ & $\begin{array}{l}0.52424 \\
0.52424\end{array}$ & $\begin{array}{l}402.13 \\
402.13\end{array}$ & $\begin{array}{l}0.0434 \\
0.0434\end{array}$ & 0.069397 & $\underset{\mathrm{INI}}{\rightarrow}$ \\
\hline
\end{tabular}

Table 4.9: Composition path for the injection of $100 \%$ steam at $650 \mathrm{~K}$ into $94.8 \%$ oil and $52.4 \%$ water at $402.13 \mathrm{~K}$ with a temperature wave in the steam-oil region.

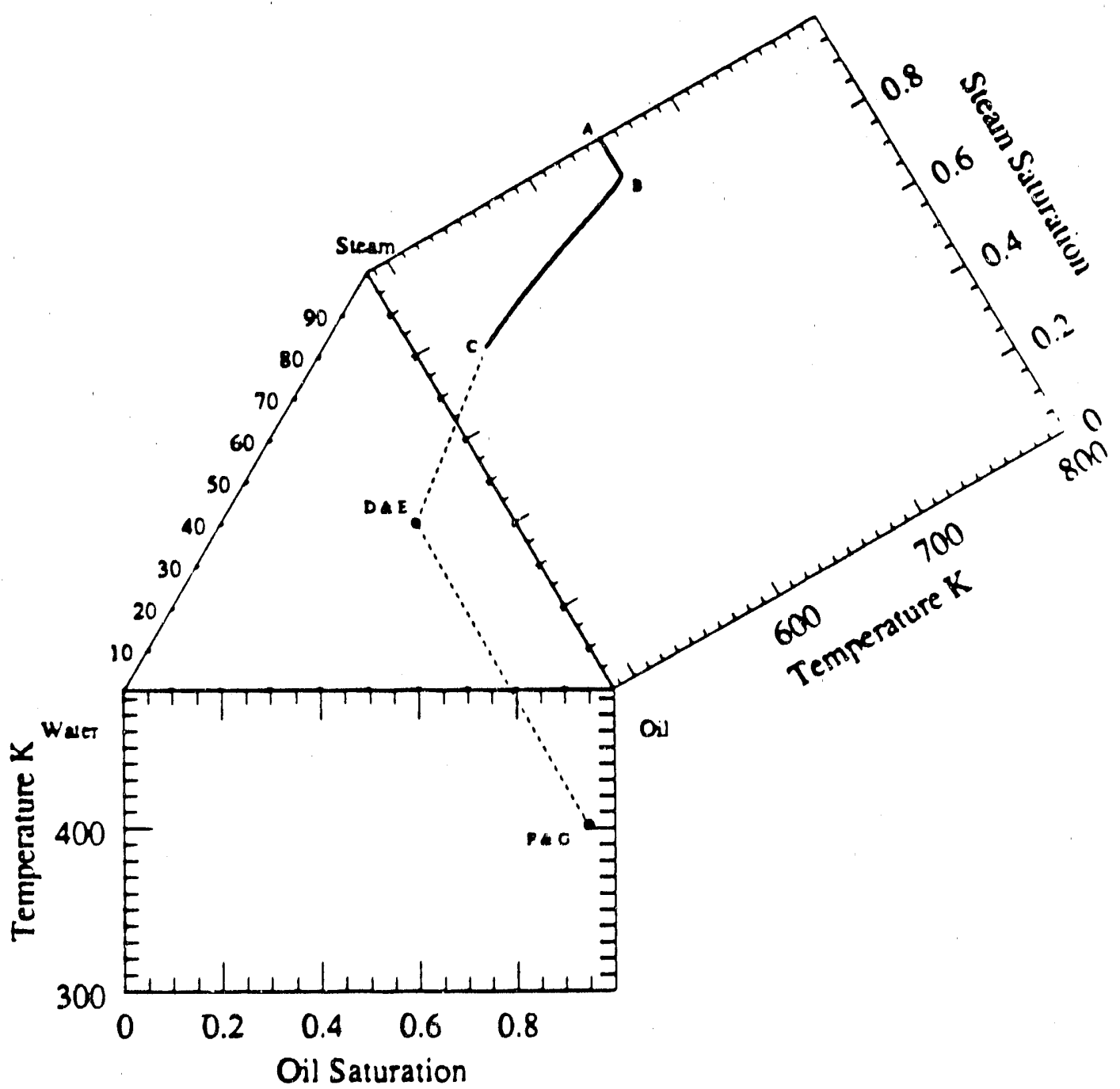

Figure 4.36: Composition path for the injection of $100 \%$ steam at $650 \mathrm{~K}$ into $94.8 \%$ oil and $5.24 \%$ water at $402.13 \mathrm{~K}$ with a temperature wave in the steam-oil region. 
The key points to compare are the two landing points in the three phase region; see Table 4.10.

The landing point the the thres-phase region in the original case is given by Point $\mathbf{C 1}$ and the landing point for the case whe ingtial temperature variation is Point D2. The variation between these points is very small. This stors to two inportant conclusions.

1. A small change in the three phase landing poist can have a large effect on the solution path in the steam-oil region.

2. The effect of a temperature variation in the steam-oil region has a small effect on the saturation and temperature profiles downstream of the steam-oil region.

\begin{tabular}{|c|c|c|c|c|c|c|c|}
\hline \multirow{3}{*}{ Label } & \multicolumn{5}{|c|}{ Composition Point } & \multirow{3}{*}{$\begin{array}{l}\text { Wave } \\
\text { Velocity }\end{array}$} & \multirow{3}{*}{$\begin{array}{l}\text { Type of } \\
\text { Flow } \\
\text { Region }\end{array}$} \\
\hline & \multicolumn{3}{|c|}{ Saturations } & \multirow[b]{2}{*}{$\mathrm{T}(\mathrm{K})$} & \multirow{2}{*}{$\begin{array}{l}\text { Flow } \\
\text { Velocily }\end{array}$} & & \\
\hline & Steam & Oil & Water & & & & \\
\hline \multicolumn{8}{|c|}{ Original Type 1 Composition Path } \\
\hline Al & 1.00000 & 0.00000 & 0.00000 & 6050.00 & 1.0000 & 0.000000 & $\mathrm{INJ} \rightarrow \mathrm{SPW}$ \\
\hline B1 & 0.92000 & 0.08000 & 0.00000 & 650.00 & 1.0000 & 0.010777 & SPW $\rightarrow$ UID \\
\hline $\mathrm{Cl}$ & 0.41924 & 0.39087 & 0.18989 & 485.57 & 0.7058 & 0.010777 & $\mathrm{UID} \rightarrow \mathrm{ZCS}$ \\
\hline \multicolumn{8}{|c|}{ Original Type 1 Composition Path } \\
\hline$\overline{A 2}$ & 1.00000 & 0.00000 & 0.00000 & 650.00 & 1.0000 & 0.00000 & INJ $\rightarrow$ SPW \\
\hline B2 & 0.91681 & 0.09319 & 0.00000 & 650.00 & 1.0000 & 0.011266 & EEP \\
\hline C2 & 0.74335 & 0.25665 & 0.00000 & 525.00 & 0.7712 & 0.011916 & SPW $\rightarrow$ UID \\
\hline D2 & 0.39946 & 0.39680 & 0.20373 & 485.57 & 0.6948 & 0.040644 & UID $\rightarrow \mathrm{ZCS}$ \\
\hline
\end{tabular}

Table 4.10: Comparison of composition paths for solutions with and without a temperature variation in the steam-oil region.

The reason that the temperature profile in the steam-oil region does not have a significant effect on the conditions in the three-phase region is seen clearly in the temperature and flow velocity profiles for the solution path in Figure 4.36. The temperature profile is shown in Figure 4.37. The portion of the temperature profile from Point B to Point $\mathbf{C}$ is the initial temperature variation. The temperature profile in the steam-oil region appears not as a continuous variation followed by a discontinuity, but as only a discontinuity from the injection temperature to the saturation temperature. This is because the eigenvalues along the spreading wave portion of the solution path do not significantly change along the solution from Point B to Point C.

The spreading wave in the steam-oil region is an excellent exumple of a slighty spreading wave. The eigenvalues from $B$ w $C$ increase only by $0.1 \%$. Because the w/ave from $10 C$ is slightly spreading, the combination of $\mathbf{A} \rightarrow \mathbf{D}$ in this example behaves almost exactly like the path $\mathbf{A} \rightarrow \mathbf{C}$ in the base case. Table 4.10 compares the three phase landing points for the base case arid the solution that includes an initial temperature variation.

The reason for the steep profile is the high volumetric heat capacity of the matrix relative to the steam and oil. The stationary matrix is the reservoir for most of the heat that is input by the injected steam. This locks the majority of the heat into a stationary phase, and the litue heat remaining in the fluids cannot flow downstrean very rapidly. Higher porosity or lower matrix heat capacity spreads the initial wave out over a longer portion of the steam-oil region. This also moves the location of the equal eigenvalue point further downstream for a given amount of steam injected. Figures 4.40 and 4.41 demonstrate how the composition path in the steam-oil region changes as a function of the porosity. The effect of porosity is not linear, bist closely follows the form found in the definition of the local heat content in Eq. 2.5. 


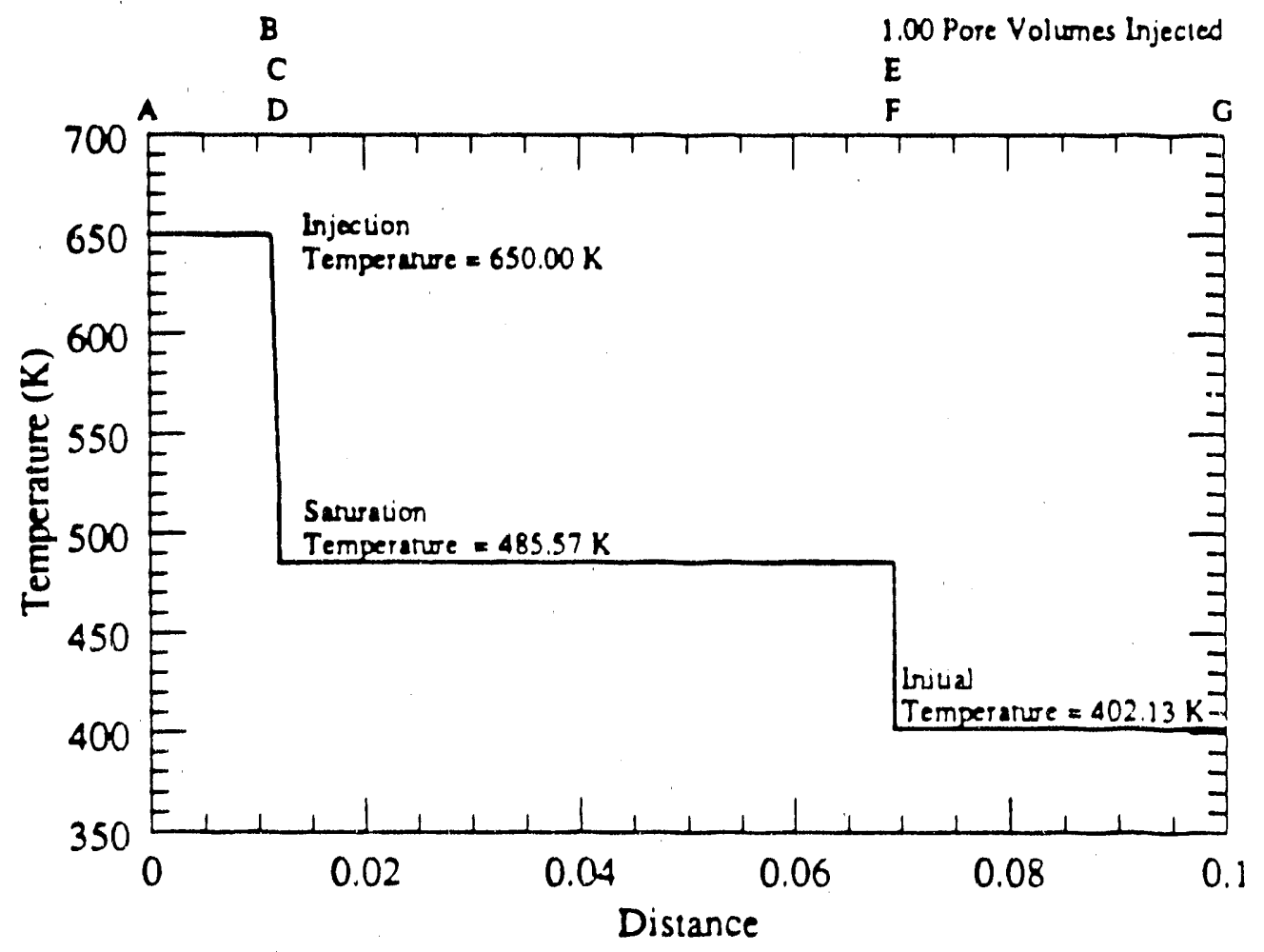

Figure 4.37: Temperature profile for the injection of 1.0 pore volumes of $100 \%$ steam at $650 \mathrm{~K}$ into $94.8 \%$ oil and $5.24 \%$ water at $402.13 \mathrm{~K}$ with a temperature wave in the steam-oil region. 


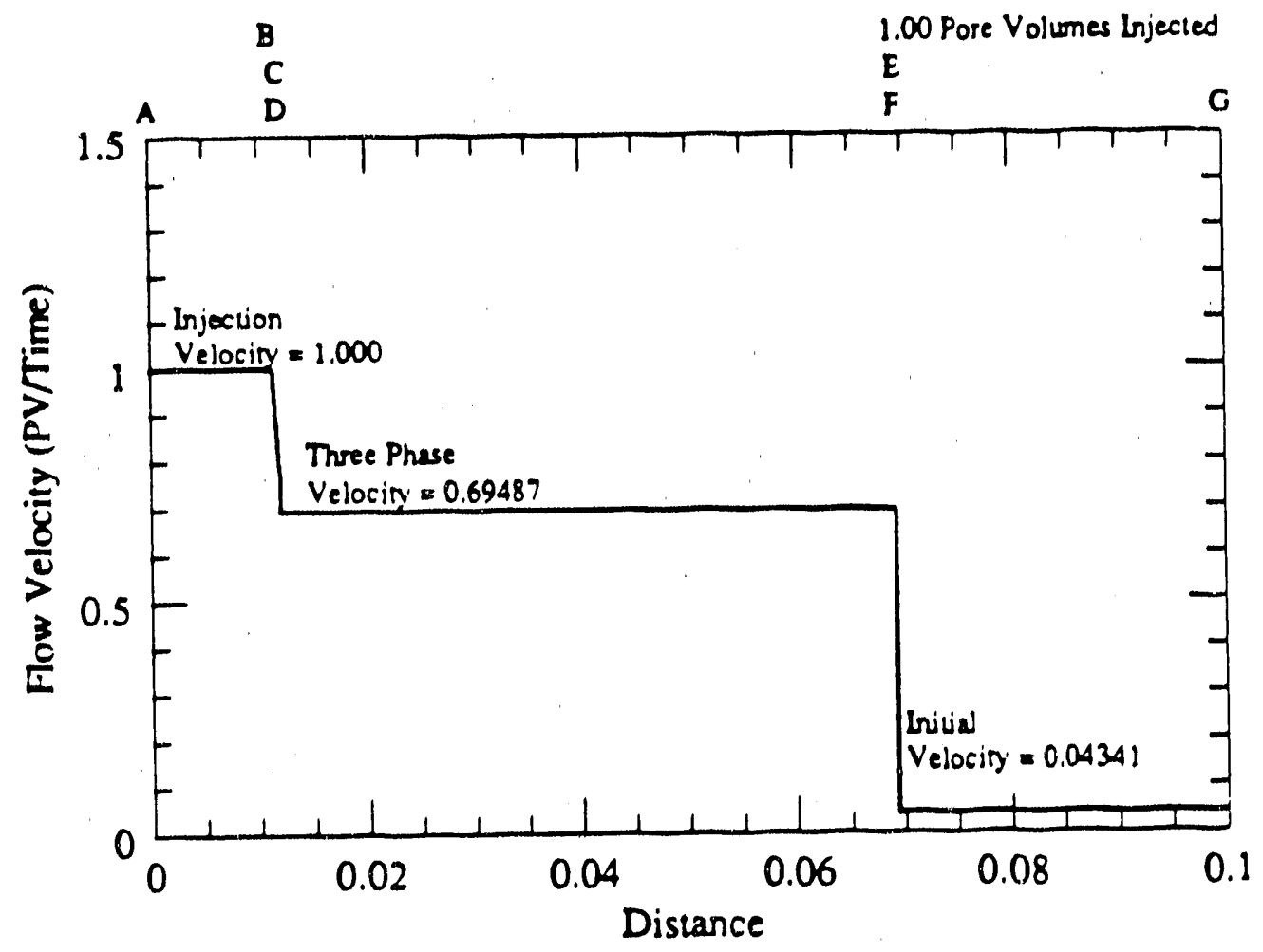

Figure 4.38: Flow velocity profile for the injection of 1.0 pore volumes of $100 \%$ steam at $650 \mathrm{~K}$ into $94.8 \%$ oil and $5.24 \%$ water at $402.13 \mathrm{~K}$ with a temperature wave in the steam-oil region. 


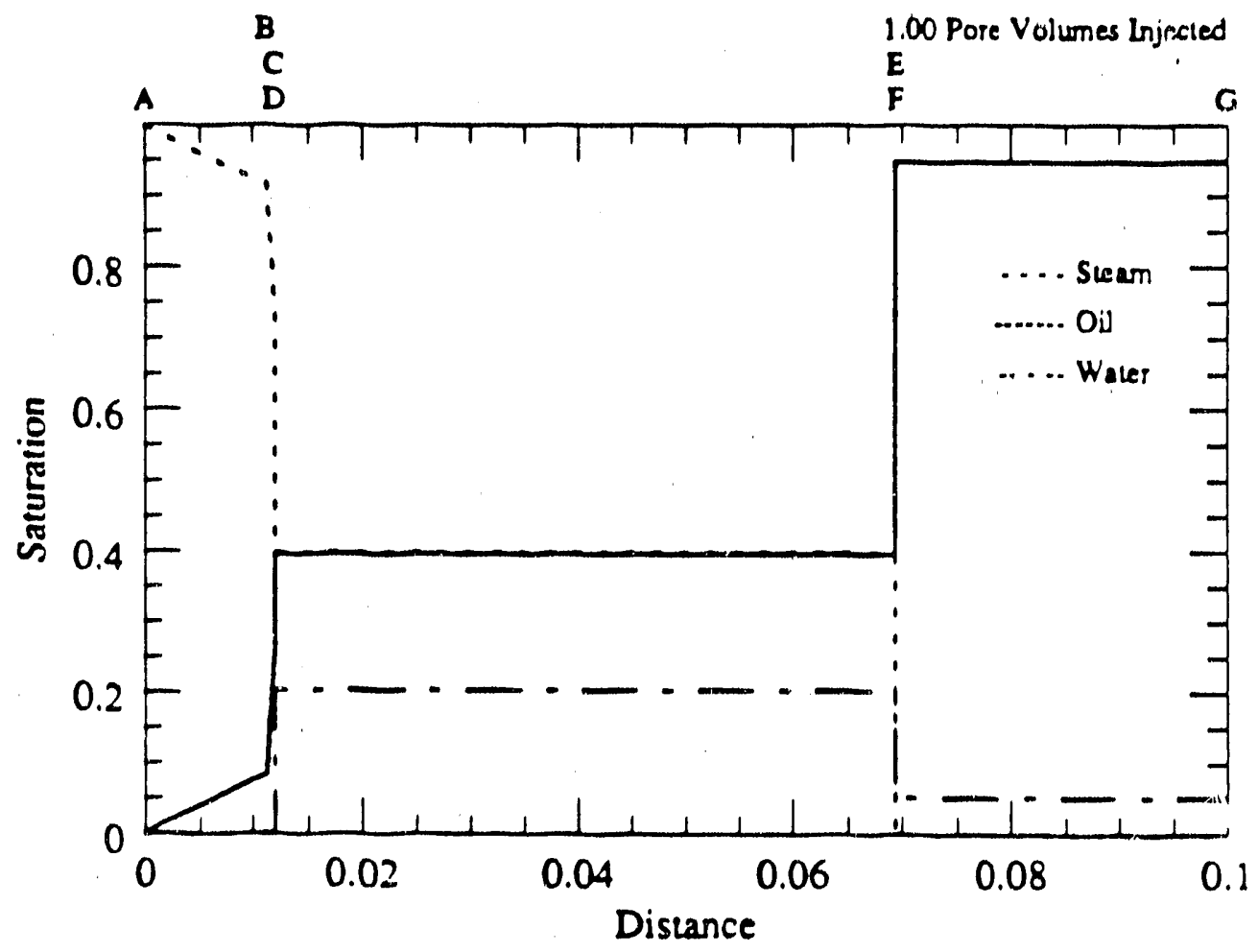

Figure 4.39: Saturation profile for the injection of 1.0 pore volumes of $100 \%$ steam at $650 \mathrm{~K}$ into $94.8 \%$ oil and $5.24 \%$ water at $402.13 \mathrm{~K}$ with a ternperawse wave in the steam-oil region. 


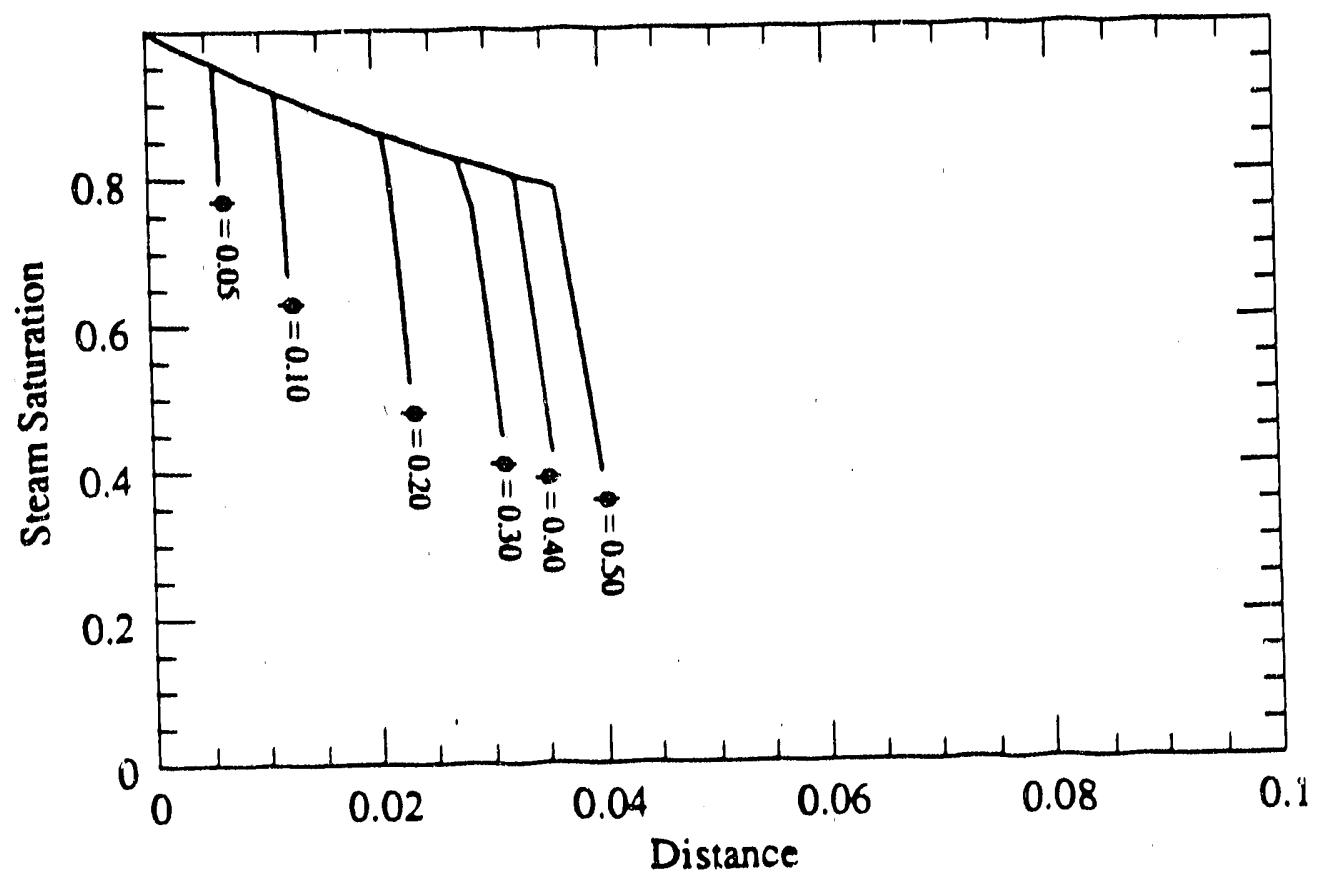

Figure 4.40: Saturation profiles in the steam-oil region for the injection of 1.0 pore volumes of $100 \%$ steam at $650 \mathrm{~K}$ into reservoirs with porosities from 0.05 to 0.5 . 


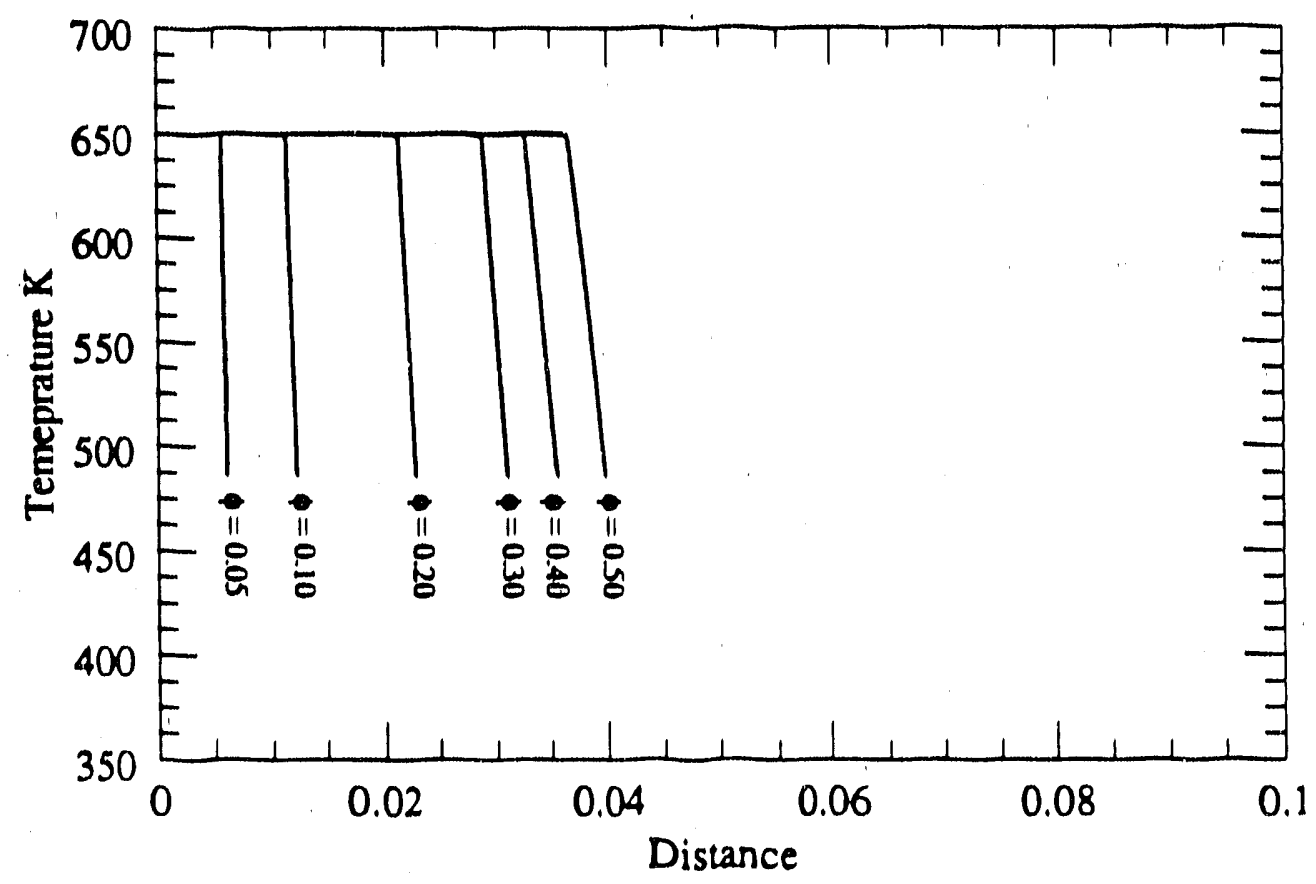

Figure 4.41: Temperature profiles in the steam-oil region for the injection of 1.0 pore volumes of $100 \%$ steam at $650 \mathrm{~K}$ into reservoirs with porosities from 0.05 to 0.5 .

In the three-phase region, the effect of the thermal properties is not seen. This is identical to the heat capacity effect in the steam-water problem in 84.1 . The thermal properties of the matrix did not effect the saturgtion profile in the two-phase, isothermal region. This is also the case in the three-phase region for this $;$ iem. Because there is no enthalpy transfer in the three-phase region, the thermal properties of the matrix such as density, heal capacity and porosity do not affect the solutions.

\subsubsection{Solutions with Viscous Oils}

Steam injection is extensively used in the recovery of heavy oils. The solutions for two "heavy" oils are presented in this section. The oils are modified by increasing the viscosity from the base case. Heavy Oil \#1 has a viscosity that is $\mathbf{2 0 0}$ times higher that the base case oil for the entire temperature range. Heavy Oil $\# 2$ has a higher viscosity at the initial conditions but a greater dependence on the temperature. This stronger temperature effect lowern the viscosity to near thict of the base oil at injection conditions. Figure 4.42 shows the viscosities of the base case oil, the two heavy oils and the two water phases against the temperature. The discontinuity in the steam-water curve occurs at the saturation temperature of $485.57 \mathrm{~K}$ at $2.0 \mathrm{MPa}$.

Composition points demarking the boundaries of the different flow regions are given in Table 4.11 and the composition path is shown in Figure 4.43. The composition path shown in Figure 4.43 is much more detailed than for the composition paths for the lighter oil (see Figures 4.9.4.23, 4.27, and 4.32). In the steam-oil region, there is a portion of the path that follows along the isothermal portion of the path from Point A to Point B. At Point B there is an equal eigenvalue point where the solution continues along the slow path. After Point B, continuing along the slow path to Point $\mathbf{C}$ lowers the temperature from the injection value of $650 \mathrm{~K} 1588 \mathrm{~K}$. 


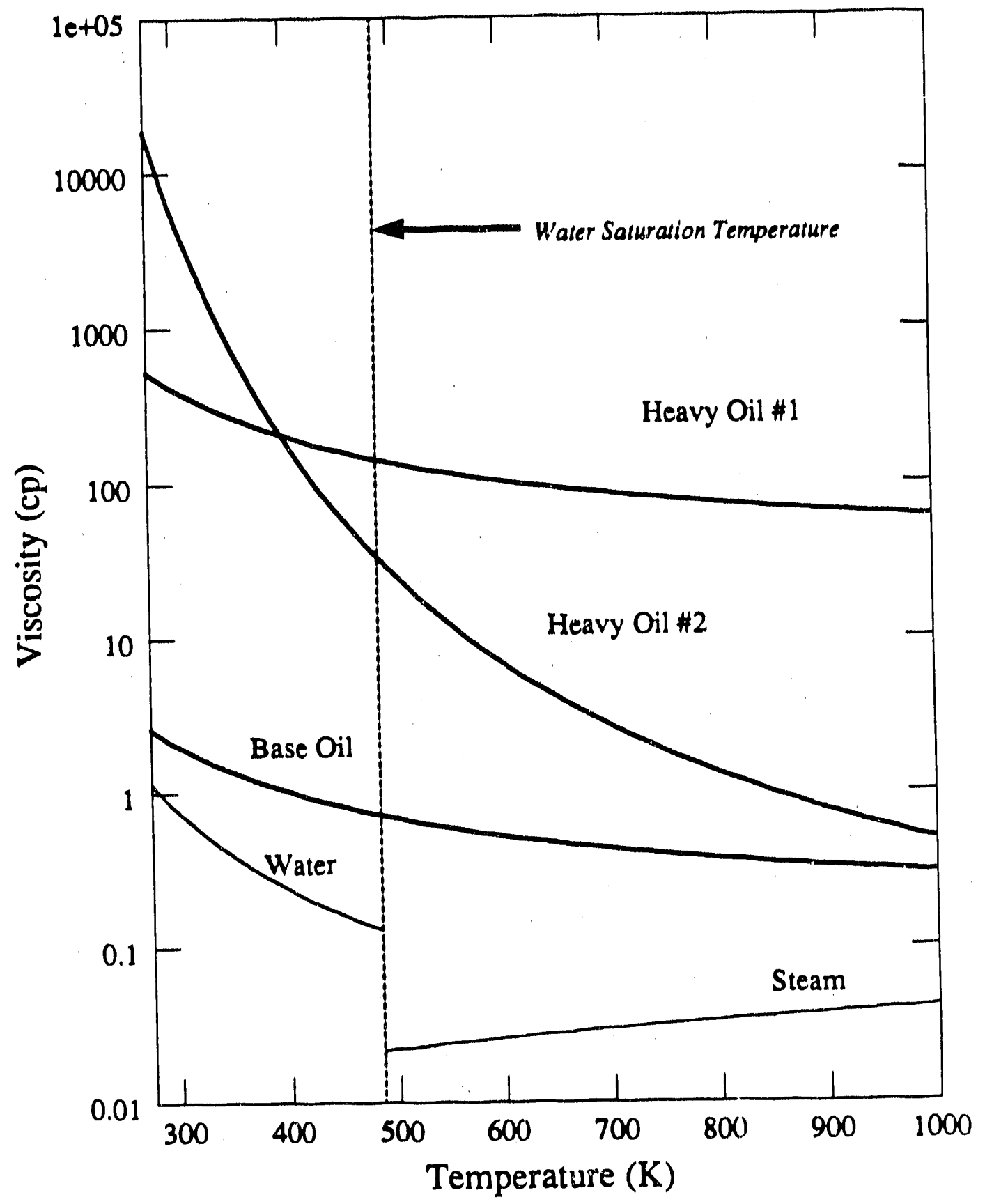

Figure 4.42: Viscosities for water, steam, and three different oils as a function of temperature from $275 \mathrm{~K}$ to $1000 \mathrm{~K}$. 


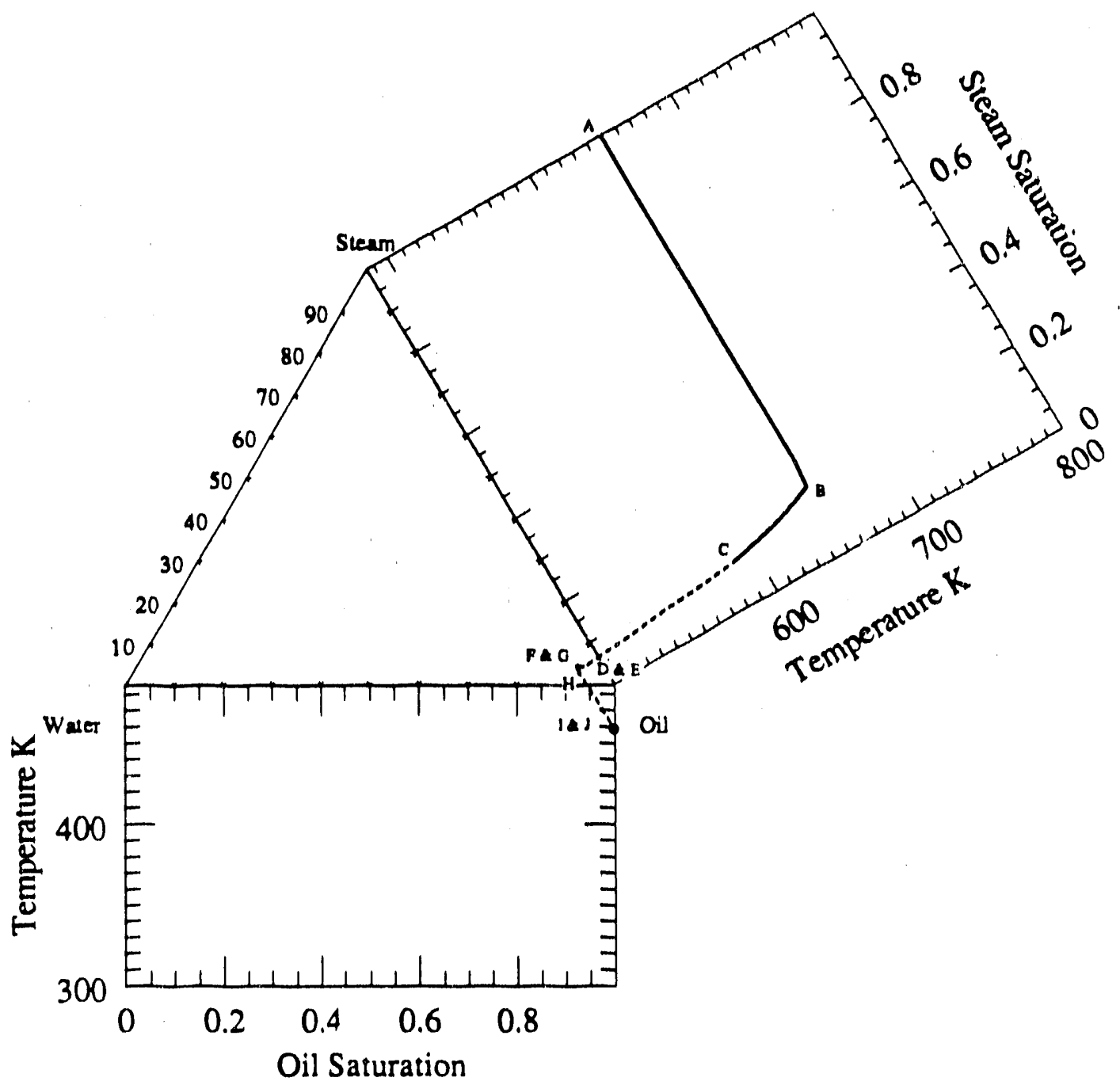

Figurc 4.43: Conıposition path for the injection of $100 \%$ stearn at $650 \mathrm{~K}$ into $99.9 \%$ Heavy Oil 1 and $0.1 \%$ water al $458.95 \mathrm{~K}$. 
Another difference is that the location of the equal eigenvalue point, represented by Point $B$ on Figure 4.43 and Table 4.11, has a much lower steam saiuration than with the light oils (see Trable 4.9 for example). This result is expected. The less favorable mobility ratio causes the steam to quickly override or channel through the heavier oil. For a given injection volume of steam, the steam saturation near the injection end will be much lower than in the case of the lighter oll. The steam will be "spread" over a larger volume of the system, reducing the local saturation.

\begin{tabular}{|c|c|c|c|c|c|c|c|}
\hline \multirow{3}{*}{ Label } & \multicolumn{5}{|c|}{ Composition Point } & \multirow{3}{*}{$\begin{array}{c}\text { Wave } \\
\text { Velocity }\end{array}$} & \multirow{3}{*}{$\begin{array}{l}\text { Type of } \\
\text { Flow } \\
\text { Region }\end{array}$} \\
\hline & \multicolumn{3}{|c|}{ Saturations } & \multirow[b]{2}{*}{$T(K)$} & \multirow{2}{*}{$\begin{array}{l}\text { Flow } \\
\text { Velocity }\end{array}$} & & \\
\hline & Steam & Oil & Water & & & & \\
\hline $\mathbf{A}$ & 1.00000 & 0.00000 & 0.00000 & 650.00 & 1.00000 & 0.000000 & $\mathrm{INJ} \rightarrow \mathrm{SPW}$ \\
\hline B & 0.22000 & 0.78000 & 0.00000 & 650.00 & 1.00000 & 0.00565 & SPW $\rightarrow$ UID \\
\hline C & 0.09651 & 0.90349 & 0.00000 & 588.00 & 0.88813 & 0.006054 & UID $\rightarrow \mathrm{ZCS}$ \\
\hline $\bar{D}$ & 0.043058 & 0.915853 & 0.04109 & 485.57 & 0.69682 & 0.006054 & $\mathrm{ZCS} \rightarrow \mathrm{SPW}$ \\
\hline $\mathrm{E}$ & 0.043058 & 0.915853 & 0.04109 & 485.57 & 0.69682 & 0.012700 & $\mathrm{SPW} \rightarrow \mathrm{ZCS}$ \\
\hline F & 0.047199 & 0.899429 & 0.053372 & 485.57 & 0.69682 & 0.016852 & $\mathrm{ZCS} \rightarrow \mathrm{SPW}$ \\
\hline G & 0.047199 & 0.899429 & 0.053372 & 485.57 & 0.69682 & 0.053628 & SPW $\rightarrow$ UID \\
\hline $\mathrm{H}$ & 0.027784 & 0.914043 & 0.058173 & 485.57 & 0.69682 & 0.156870 & $\mathrm{UID} \rightarrow \mathrm{INI}$ \\
\hline 1 & 0.00000 & 0.998536 & 0.001464 & $\begin{array}{l}458.95 \\
45805\end{array}$ & 0.01836 & 0.156870 & UDD $\rightarrow$ INI \\
\hline
\end{tabular}

Table 4.11: Composition path for the injection of $100 \%$ steam at $650 \mathrm{~K}$ into $99.9 \%$ Heavy Oil \#1 and $0.01 \%$ water at $458.95 \mathrm{~K}$.

In the three phase region, the heavy oil solution shows the Type 4 wave pattern discussed in Table 4.5. There is a spreading wave from Point $\mathbf{E}$ to Point $\mathbf{H}$ that trails the central zone of constant state. Another spreading wave precedes the central zone of constant state, given by the path from Point $\mathbf{G}$ to Point $\mathbf{H}$. The major difference from the light oil cases is that the composition changes along these two waves are very small. The changes in the hodograph variables along the spreading waves are more restrictive in the heavy oil solutions. Only a small corner of the three phase region has flow characteristics that permit passage from the stcam-oil region uhrough the three phase region and into the wateroil region.

The upstream spreading wave, Point $\mathbf{E}$ to Point $\mathbf{F}$, follows the slow path in the three phase region. The direction of the slow path causes a change in the oil saturation. Due to high oil viscosity, small changes in the oil saturation rapidly bring the composition to that of the central zone of constant state.

The downstream spreading wave, Point $\mathbf{G}$ to Point $\mathbf{H}$, follows the fast path. This path closely parallels the lines of constant oil saturation. The fast path is spread over a longer region for the same reasons that the trailing spreading wave is shortened. The changes in water and steam saturation have less effect on the fractional nows as the saturations of the steam and water change. This causes the spreading wave to take a longer time reaching the conditions at the leading shock than for the brse case.

The leading shock must be downstream of the initial spreading wave and must have enough water flowing across the shock. These two requirements tend to push the leading shock further and further downstrearm. As the the temperature at the initial conditions increase, the velocity of the leading shock must also increase. This leads to faster leading shocks for the displacement of Heavy Oil \#1.

The saturation profile for the case of Heavy Oil \#1, shown in Figure 4.44, is also very different from the light oil counterpar, Figure 4.33. In the case of the heavy oil, the initial steam-oil region, from Point $\mathbf{A}$ to Point $\mathbf{C}$, is much shorter and the saturation changes much greater than in the light oil case. The shape and size of the steam oil region verifies the observation made concerning the steam 
override with the heavy oils. With the high mobility ratio the steam quickly flows downstream, causing the shorter initial steam-oil region, and spreads out over a larger portion of the reservoir, causing a larger three phase region. ${ }^{3}$

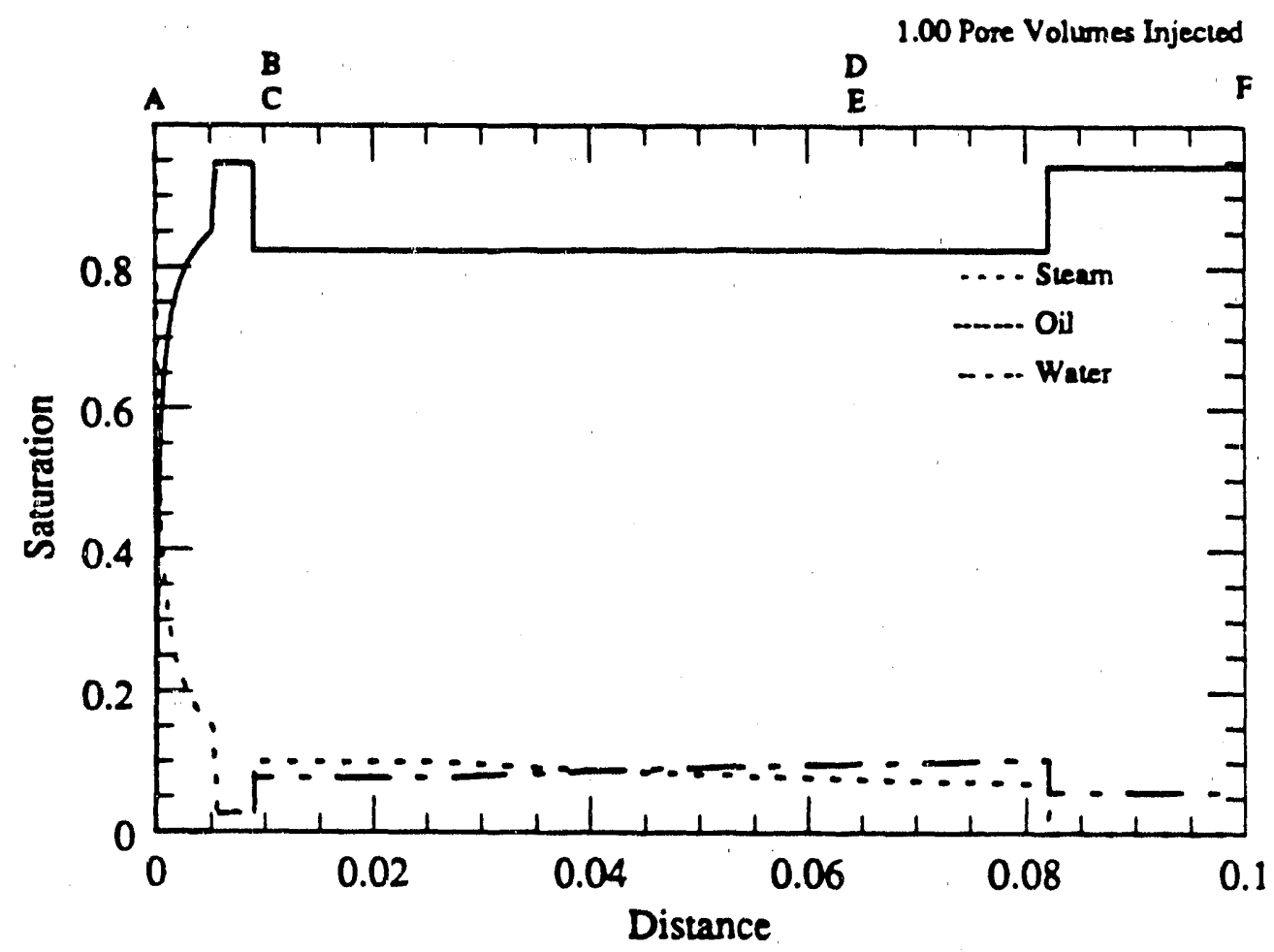

Figure 4.44: Saturation profile for the injection of $100 \%$ steam at $650 \mathrm{~K}$ into $99.9 \%$ Heavy Oil \#1 and $0.1 \%$ water at $458.95 \mathrm{~K}$.

On the other hand, the temperature profile, illustrated in Figure 4.45 , and the flow velocity profile shown in Figure 4.46 show the same behavior as the case of the light oils. The major difference is in the location of the leading and trailing temperature shocks. The leading shock has a higher velocity in the case of the heavy oil and the trailing shock a slower velocity. Again, the high shock velocity is attributable to the high mobility steam bypassing the steam-oil region and spreading over the three phase region.

As in the case of the light oils, the non-isothermal portion of the steam oil region, from Point B to Composition C in Figure 4.45 appears as an extension of the actual phase change shock between Point $\mathbf{C}$ and Point $\mathbf{D}$. The portion of the solution path that follows along the non-isothermal section in the steam-oil region is always seen in these solutions. The reason is that the wave velocity at the point where the two eigenvalues are equal is 100 small 10 satisfy the maierial and primarily the heat balance across the shock. The minimum velocity of an intermediate discontinuity is faster than the equal eigenvalue point. In order to satisfy the heat balance, the system must cool wwards the saturation temperature before a suitable heal balance across the trailing shosk is possible.

\footnotetext{
${ }^{3}$ Note the $X$-axis scale change between the figures given in the light oils $4.11,4.26,4.28$, and 4.33 to that in Figure 4.44 .
} 
The viscosity of Heavy Oil $\# 2$ is more dependent on the temperature than the base oil or Heavy Oil \#1. The composition path for a displacement of Heavy Oil \#2 with the same injection and initial conditions as the light oil, Type 4 displacement shown in Figures $4.32-4.35$ and outlined in Table 4.8 is presented in Figures $4.47-4.50$ and Table 4.12.

\begin{tabular}{|c|c|c|c|c|c|c|c|}
\hline \multirow{3}{*}{ Label } & \multicolumn{5}{|c|}{ Composition Point } & \multirow{3}{*}{$\begin{array}{c}\text { W'ave } \\
\text { Velocity }\end{array}$} & \multirow{3}{*}{$\begin{array}{l}\text { Type of } \\
\text { Flow } \\
\text { Reginn }\end{array}$} \\
\hline & \multicolumn{3}{|c|}{ Sapurations } & \multirow[b]{2}{*}{$\mathrm{T}(\mathrm{K})$} & \multirow{2}{*}{$\begin{array}{c}\text { Flow } \\
\text { Velocity }\end{array}$} & & \\
\hline & Steam & Oil & Waler & & & & \\
\hline $\begin{array}{l}\mathrm{A} \\
\mathrm{B} \\
\mathrm{C}\end{array}$ & $\begin{array}{l}1.00000 \\
0.65350 \\
0.11901\end{array}$ & $\begin{array}{l}0.00000 \\
0.34650 \\
0.88099\end{array}$ & $\begin{array}{l}0.00000 \\
0.00000 \\
0.00000\end{array}$ & $\begin{array}{l}650.00 \\
650.00 \\
526.00\end{array}$ & $\begin{array}{l}1.00000 \\
1.00000 \\
0.77235\end{array}$ & $\begin{array}{l}0.000000 \\
0.008822 \\
0.00961\end{array}$ & $\begin{array}{l}\mathrm{IiJ} \rightarrow \text { SPW } \\
\text { SPW } \rightarrow \text { UDD } \\
\mathrm{I} \longrightarrow \rightarrow \text { ZCS }\end{array}$ \\
\hline $\begin{array}{l}\text { D } \\
E \\
F \\
G \\
H \\
\text { I }\end{array}$ & $\begin{array}{l}0.052518 \\
0.05251 \\
0.05264 \\
0.13794 \\
0.13794 \\
0.13657\end{array}$ & $\begin{array}{l}0.88117 \\
0.88117 \\
0.87995 \\
0.70626 \\
0.70626 \\
0.70645\end{array}$ & $\begin{array}{l}0.06632 \\
0.06632 \\
0.06741 \\
0.15580 \\
0.15580 \\
0.15698\end{array}$ & $\begin{array}{l}485.57 \\
485.57 \\
485.57 \\
485.57 \\
485.57 \\
458.95\end{array}$ & $\begin{array}{l}0.69358 \\
0.69358 \\
0.69358 \\
0.69358 \\
0.69358 \\
0.69358\end{array}$ & $\begin{array}{l}0.009613 \\
0.027298 \\
0.028005 \\
0.028005 \\
0.086257 \\
0.088938\end{array}$ & $\begin{array}{l}\mathrm{ZCS} \rightarrow \mathrm{SPW} \\
\mathrm{SPW} \rightarrow \mathrm{ZCS} \\
\mathrm{ZCS} \rightarrow \mathrm{SPW} \\
\mathrm{SF}: \mathrm{V} \rightarrow \mathrm{UID} \\
\mathrm{UID} \rightarrow \mathrm{INI} \\
\mathrm{UID} \rightarrow \mathrm{INI}\end{array}$ \\
\hline $\begin{array}{l}J \\
K \\
L \\
M \\
N\end{array}$ & $\begin{array}{l}0.00000 \\
0.00000 \\
0.00000 \\
0.00000 \\
0.00000\end{array}$ & $\begin{array}{l}0.94420 \\
0.94420 \\
0.91321 \\
0.88123 \\
0.88123\end{array}$ & $\begin{array}{l}0.05579 \\
0.05579 \\
0.08679 \\
0.11876 \\
0.11876 \\
\end{array}$ & $\begin{array}{l}433.89 \\
433.89 \\
433.89 \\
433.85 \\
433.89 \\
\end{array}$ & $\begin{array}{l}0.02667 \\
0.02667 \\
0.02667 \\
0.02657 \\
0.02667\end{array}$ & $\begin{array}{l}0.088938 \\
0.119812 \\
0.197647 \\
0.197647\end{array}$ & $\begin{array}{c}\mathrm{INI} \\
\operatorname{UID} \rightarrow \mathrm{ZCS} \\
\mathrm{SPW} \rightarrow \mathrm{UID} \\
\mathrm{UID} \rightarrow \mathrm{NI} \\
\mathbb{I N I}\end{array}$ \\
\hline
\end{tabular}

Table 4.12: Composition path for the injection of $100 \%$ steam at $657 \mathrm{~K}$ i'jto $88.1 \%$ Heavy Oil \#2 and $11.9 \%$ water at $433.79 \mathrm{~K}$. 


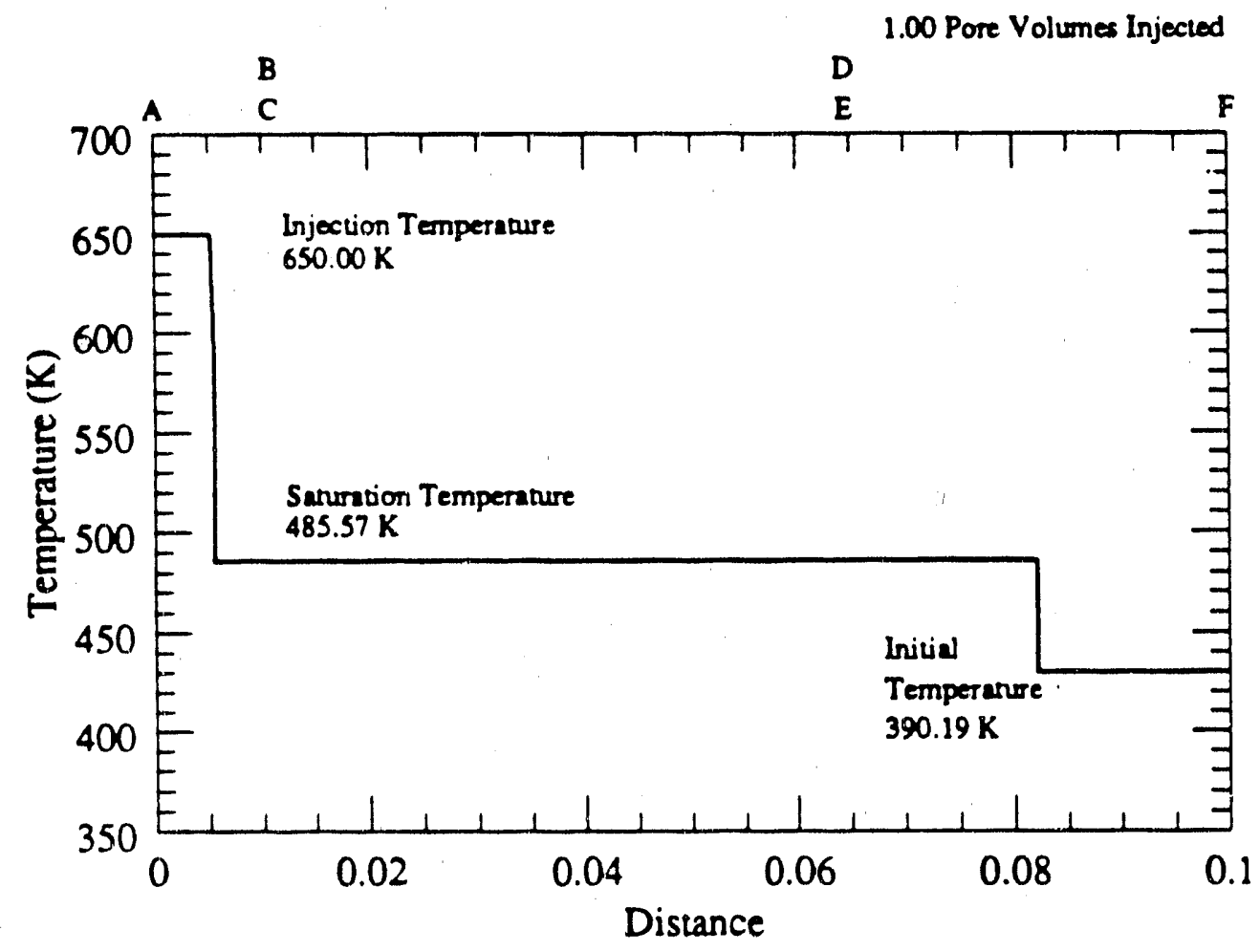

Figure 4.45: Temperature profile for the injection of $100 \%$ steam at $650 \mathrm{~K}$ into $99.9 \%$ Heavy Oil \#1 and $0.1 \%$ water at $458.95 \mathrm{~K}$. 


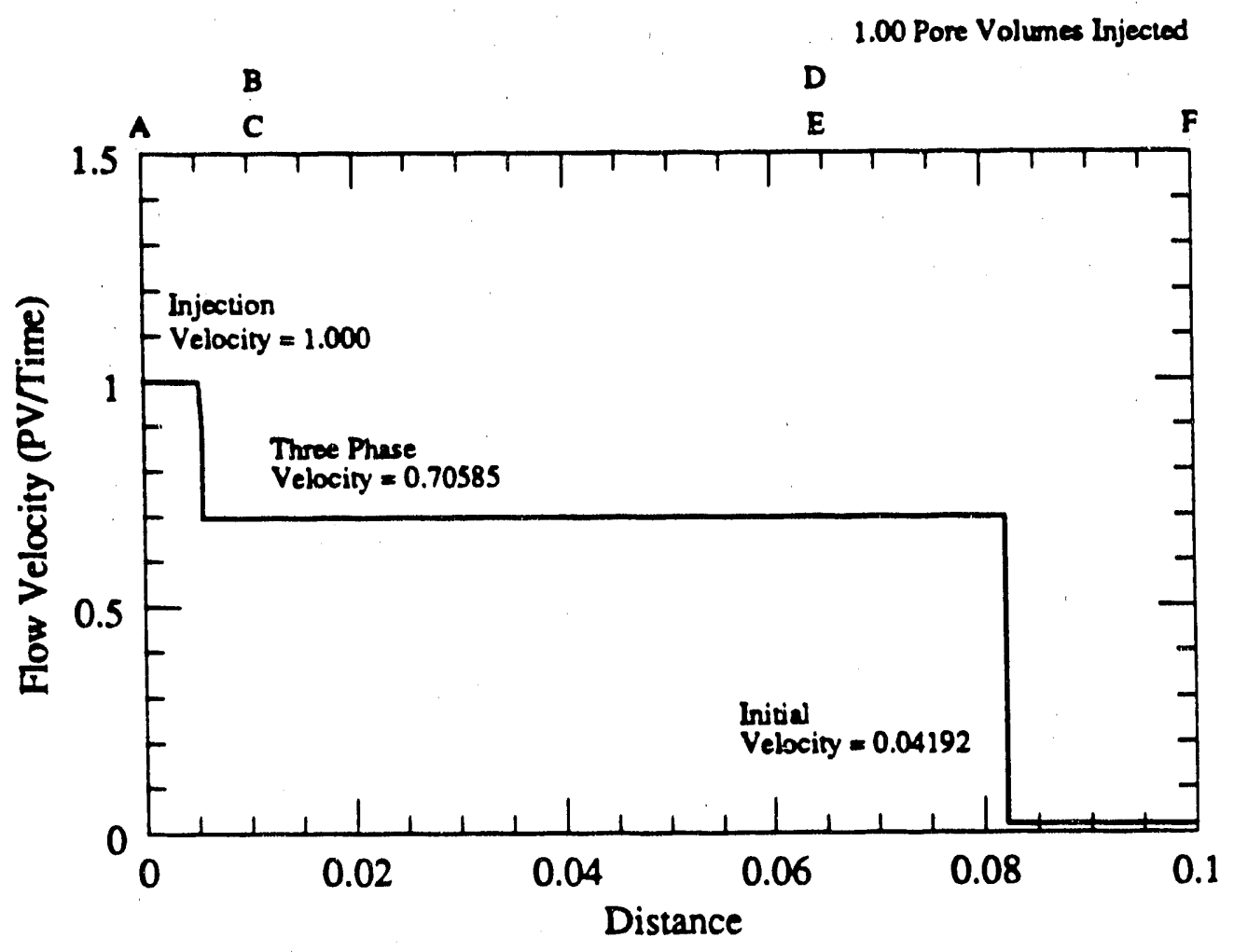

Figure 4.46: Fow velocity profile for the injection of $100 \%$ steam at $650 \mathrm{~K}$ into $99.9 \%$ Heavy Oil \#1 and $0.1 \%$ waler at $458.95 \mathrm{~K}$. 


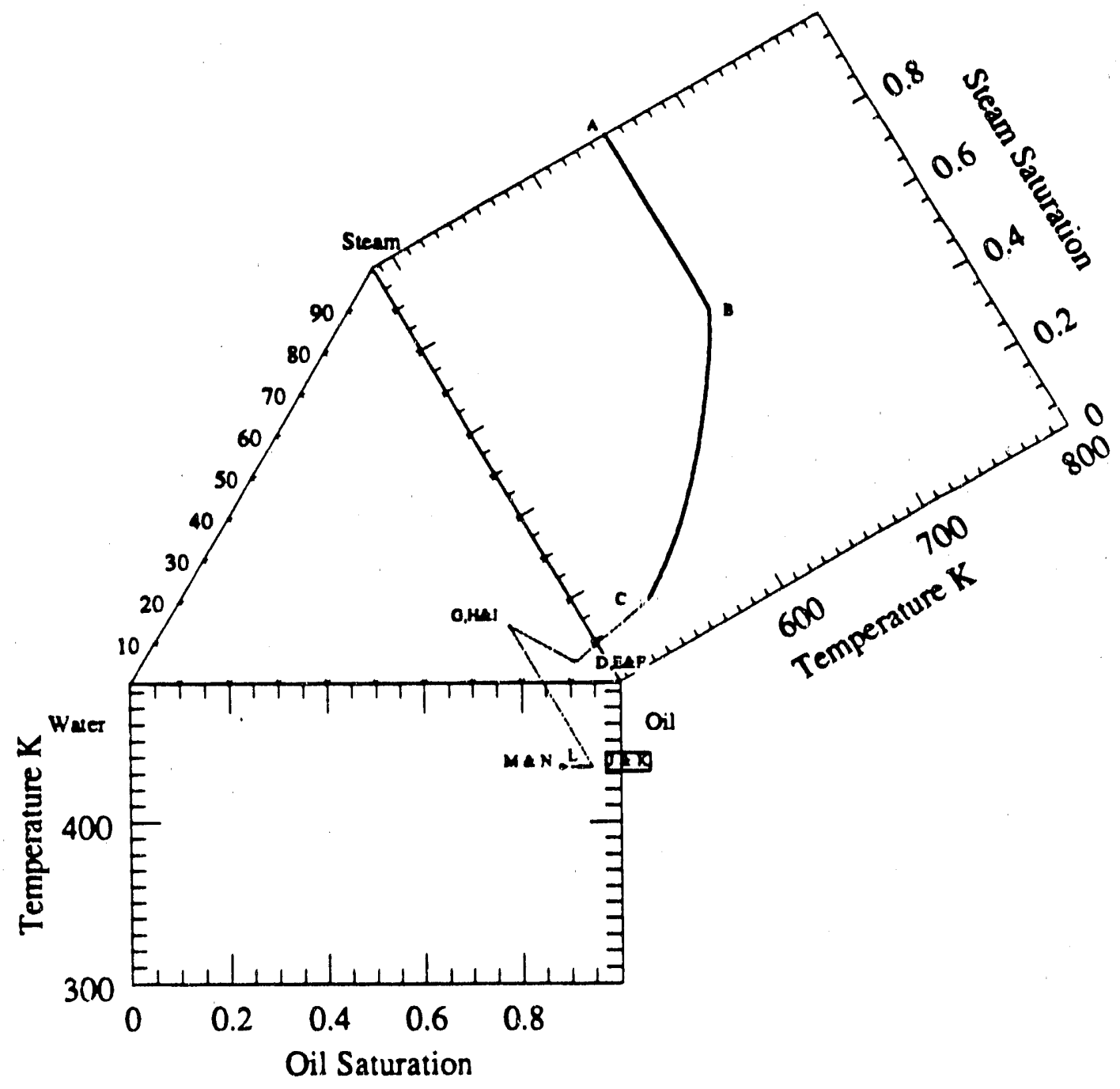

Figure 4.47: Composition path for the injection of $100 \%$ steam at $650 \mathrm{~K}$ into $88.1 \%$ Heavy Oil \#2 and $11.9 \%$ water at $433.79 \mathrm{~K}$. 


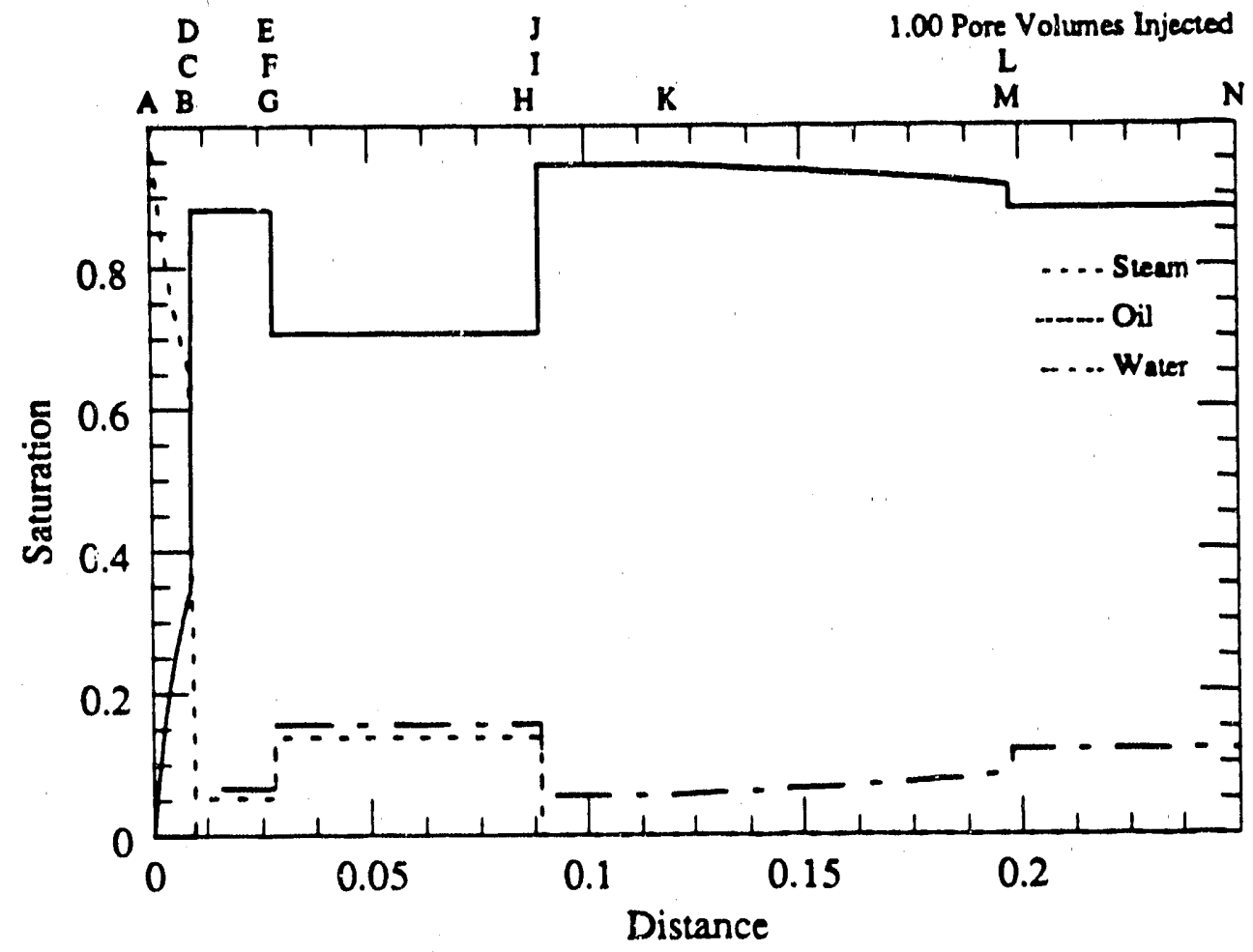

Figure 4.48: Saturation profile for the injection of $100 \%$ steam at $650 \mathrm{~K}$ into $88.1 \%$ Heavy Oil $\# 2$ and $11.9 \%$ water at $433.79 \mathrm{~K}$. 


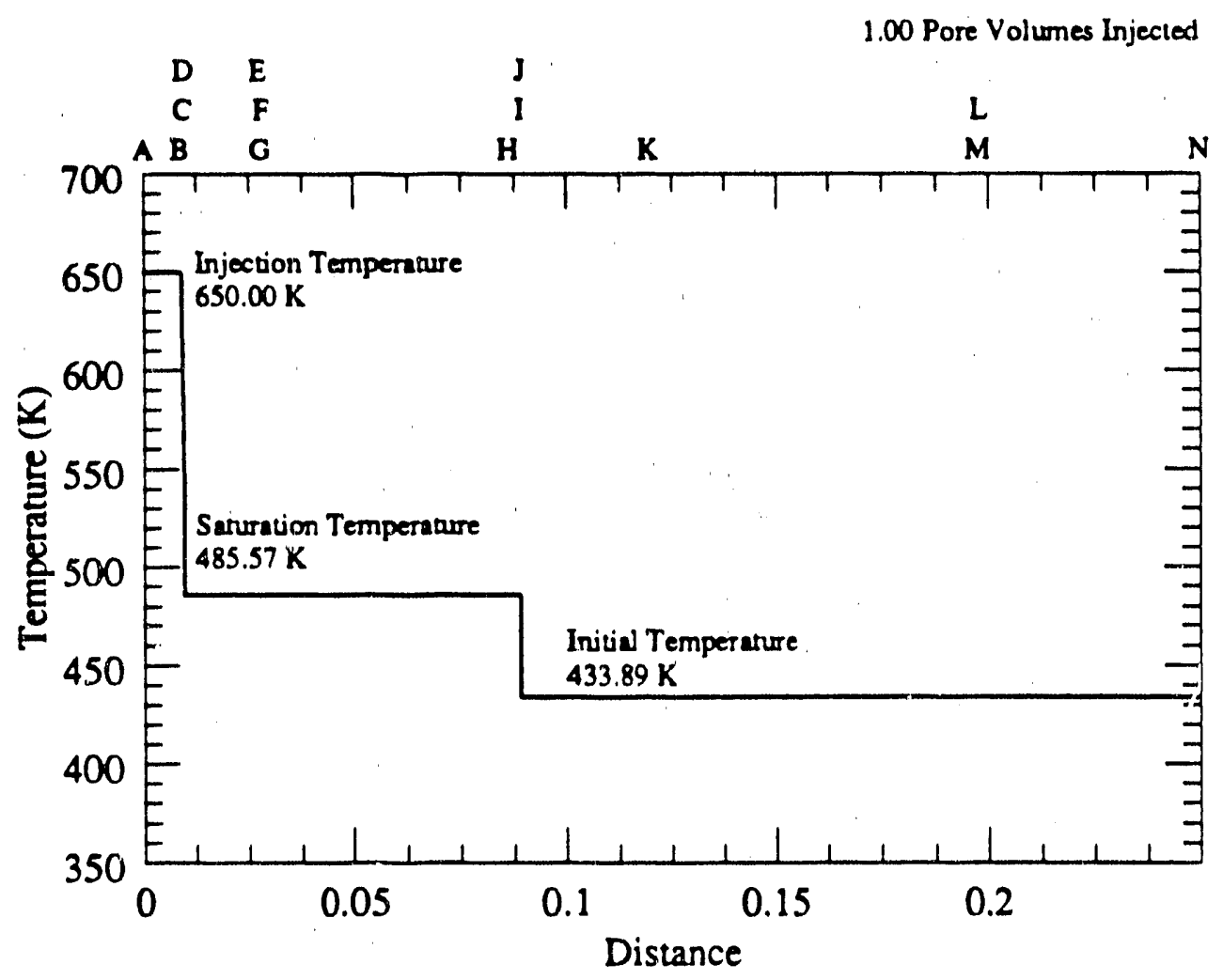

Figure 4.49: Temperature profile for the injection of $100 \%$ steam at $650 \mathrm{~K}$ into $88.1 \%$ Heavy Oil $\# 2$ and $11.9 \%$ waior at $433.79 \mathrm{~K}$. 


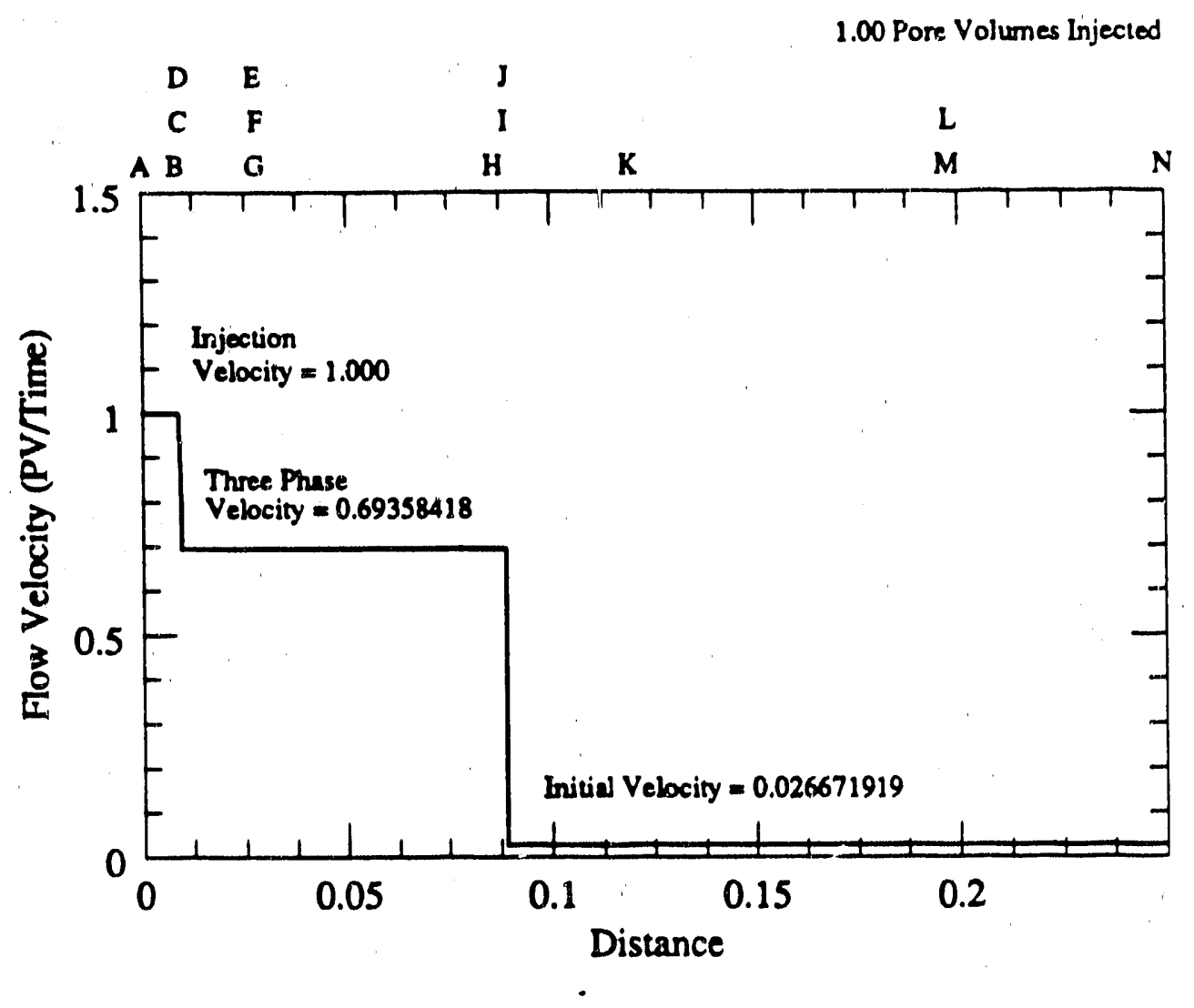

Figure 4.50: Flow velocity profile for the injection of $100 \%$ steam at $650 \mathrm{~K}$ into $88.1 \%$ Heavy Oil $\# 2$ and $11.9 \%$ water at $433.79 \mathrm{~K}$.

The most direct comparison between the heavy oil and light oil case can be made by comparing the saturation profiles of the light oil case, Figure 4.33 , and the heavy oil case, Figure 4.48. Beginning at the injection conditions and working downstream there is an immediate difference in the initial steam-oil region. Looking at Figure 4.48, the solution follows the slow path from the injection conditions at Composition A to the equal eigenvalue point, B. From Point B to Composition C, the solution continues along the slow path. This region is characterized by a slightly spreading wave carrying the solvtion from the injection temperature toward the saturation temperature. This path also changes steam saturation at the same time the temperature is changing. The rapidly changing oil viscosity helps to lengthen the section of the solution that travels along the non-isothermal path, from Point B to Point C. The increased oil viscosity also raises the oil saturation for a given velocity or temperature along the path from $B$ w $C$.

The shape of the steam-oil region resembles the stuam-oil region of the Heavy Oil \#1 displacement, Figure 4.44. The same analysis that applied in the case of Heavy Oil 11 also applies in this case. The high mobility steam rapidly moves downstream of the two phase region and spreads over the central three phase region that exists between Point D and Point I in Figure 4.48.

At Point $\mathbf{C}$, an upstream intermediate discontinuity carries the solution into the three-phase region at Point D. There are two restrictions that apply to the choice of the jumping point, $C$. If the eigenvalue at Point $C$ is 100 low, there will be no solution in the three-phase region that satifies the material and energy balances across the shock. A larger temperature jump across a shock results in a slower shock velocity; for some very slow shock velocities, the temperature jump is so large that the resulting tem- 
perature must be less than the saturation temperature. In these cases there is no solution in the threephase region.

The trailing phase transition shock is much slower than in the profiles with the base oil; as shown in Figures 4.11, 4.26, 4.28, and 4.33. The equal eigenvalue point represents the point where the Buckley-Leverett saturation velocity, $\left(\partial f_{v} / \partial S_{v}\right)$, is equal to the change in fractional increase in enthalpy transport. This is givon by,

$$
\frac{\partial f_{v}}{\partial S_{v}}=\frac{\partial}{\partial S_{v}}\left(\sum_{i=1}^{n} H_{i} f_{i}\right)
$$

The velocity of the non-isothermal path is relatively independent of the oil viscosity. At a given oil saturation, the velocity of the isothermal pach desreases as the oil viscosity increases. Therefore, to reach a given velocity, the oil saturation must be increased dramatically.

In the three-phase region, a zone of constant state, that extends from Point D to Point E, couples the shock velocity to the slow eigenvalue at the three-phase landing lPoint $\mathbf{D}$. The solution must be able to follow the slow path at the trailing end of the three-phase region. Were the velocity on the slow path, as represented by the smaller eigenvalue, to be less than the shock velocity, this would restrict the solution to only fast paths in the three-phase region. The problem with this is that in order to move between two arbitrary points in the three-phase region, the solution must first travel along the slow path. (See the discussion on page 52).

After the zone of constant state a spreading wave carries the solution from $\mathbf{E}$ to Composition $\mathbf{F}$. The change in composition along this portion of the solution is barely noticeable. Without this small change, the solution cannot reach the desired initial temperature. As the length of the spreading wave from $\mathbf{E}$ to $\mathbf{F}$ increases, lower and lower initial temperatures result for a fixed initial water saturation.

Continuing along the slow path past Composition $\mathbf{F}$, the eigenvalues will eventually reach a maximum value and begin to decline. The velocity constraint sets a limit on the distance that the integration along the slow path can continue without introducing a discontinuity in the form of a self-sharpr ning wave. This self-sharpening wave is found by selecting a point on the slow path before the small eigenvalue reaches its maximum value. This selected point is a Composition $\mathbf{F}$.

At Point $\mathbf{F}$ an upstream intermediate discontinuity carries the solution to Composition $\mathbf{G}$. The shock is necessary to keep the initial conditions in the allowabie hodograph space. For the solutions that switch to a fast pach before the shock, the velocity of the fast path or the oil saturation on the path is 100 large. When the oil saturation is too large, the shock solution results in a composition with a negative water saturation. Thus, when the eigenvalue is 100 fast, the solution results in a initial temperature that is not realizable due to physical considerations, e.g. below the freezing temperature of water.

The shock from $\mathbf{F}$ to $\mathbf{G}$ carries the solution to the other side of the maximum point, much like the a leading Buckley.Leveretl shock carries the solution past the saturation where $\left(\partial f_{w} / \partial S_{w}\right)$ is a maximum, to the initial conditions. On this side of the maximum point, the velocity of the fast path and the oil saturation are lower and give solutions that remain in the allowable hodograph space. The distance between the endpoints of the shock depends on how close the jumping point is to the maximum velocity point. The closer the jumping point, the closer the landing point is on the other side, and the closer the shock velocity is to the maximum. Because the self-sharpening wave is an upstream intermediate discontinuity, the shock velocity matches the eigenvalue of the upstream point, Composition $F_{\text {, and }}$ closely matches the eigenvalue of the downstream point.

At Point G, a second zone of constant state, often referred to as the "central" zone of constant state, couples the shock velocity to the wave velocity at $H$. The zone of constant state represents the path 
switch from the slow path at $\mathbf{G}$ to the fast path at $\mathbf{H}$. There must be a path switch at this point due to the behavior of the compositions that form a self-sharpening wave. Jeffrey (1976) showed that the velocity of the discontinuity formed by a self sharpening wave must be intermediate between the eigenvalues of the upstream and downstream composition points. Since we have a self-sharpening wave, the small eigenvalue at the downstream composition (Point $\mathbf{H}$ ) must be slower than the shock velocity. This requires that the solution, on arriving at Composition $\mathbf{H}$ switch to the fast path.

A spreading wave is attached ahead of the zone of constant state. This spreading wave is also small and does not noticably change the composition point. Along the path from $\mathrm{H}$ to $\mathbf{~}$, the spreading wave takes the solution to an upstream intermediate discontinuity that carries the solution out of the threephase region into the water-oil region. The eigenvalues along the fast path change very rapidly and the eigenvalue along this path must remain in a narrow range if the downstream temperature is to remain below the saturation temperature. As the solution turns out the length of the integration along this path is short and the eigenvalues change very little.

Comparing the three phase regions of Figure 4.33 and Figure 4.48 shows a striking difference in the shape of the saturation profiles in this region. The spreading wave in the heavy oil displacements are very small, both in real and hodograph space. The leading spreading wave, from Point $\mathbf{H}$ to Point I in Figure 4.48 has a wave velocity of 0.086357 at the trailing edge of the wave and only 0.088938 at the leading edge of the wave. This means that 387.5 pore volumes of steam would need to be injected before this wave occupied one pore volume of displacement space. In contrast, the leading spreading wave in the base oil case, Point $F$ to Point $G$ in Figure 4.33 has a wave velocity of 0.076318 at the trailing edge and a velocity of 0.098756 at the leading edge. This requires only 44.6 pore volumes of injected steam for this wave to occupy the same single pore volume.

The physical interpretation for the narrow spreading waves in the heavy oil case is founded in the three phase relative permeability model. In the region of three-phase space, where all phases can flow, the fractional flow derivatives, $\left(\partial f_{0} / \partial S_{8}\right)$ and $\left(\partial f_{0} / \partial S_{w}\right)$, are small and do not change much with saturation. In other words, because the oil is more viscous, it remains relatively immobile over a larger range of saturations than in the lighter oil case.

The upstream intermediate discontinuity at I takes the solution into the water-oil region, Point J. At Point $\mathbf{J}$ there is a path switch to the fast path in the water-oil region. This path switch follows Rule IV and is illustrated in Figure 3.3 This path switch takes the solution to Point $\mathbf{K}$. The solution then travels along the fast path, forming a spreading wave between Point $\mathbf{K}$ to Point $\mathbf{L}$. Finally, a leading upstream intermediate disc ontinuity carries the solution from the fast path at Point $L$ to the initial conditions at Point $\mathbf{M}$. This region of the solution resembles a traditinal Buckley-Levereu displacement system with an unfavorable mobility ratio. The saturation profile is long and stretched out and the leading shock is small.

The water-oil region in the heavy oil case can be compared to the same region in the light oil displacement as shown in Figure 4.33 from Point $\mathbf{H}$ to Point $\mathbf{1}$. There wave pattern is much more complex in the heavy oil case than the light oil case. As mentioned in \$4.2.3, the eigenvalues in the water-oil region are all slower than those in the three phase region. This forces the leading phase change shock $t$ jump directly to the initial conditions from the three-phase region. The highly adverse mobility ratio in the heavy oil case shown in Figure $\mathbf{4 . 4 8}$ does not have this restriction.

The eigenvalue on the fast past in the water-oil region is proportional to the derivative of the fractional flow of water with respect to the water phase saturation. This is the traditional BuckleyLeveretu saturation velocity. In the nomenclature of this dissertation this can be written as,

$$
\lambda_{3} \propto \frac{\partial f_{w}}{\partial S_{w}}
$$


When the mobility ratio is close to unity this derivative is small, but when the mobility ratio is high the derivative is also large. For the case of Heavy Oil $\$ 2$ the derivative is large enough to exist downstream of the leading phase change shock, between Point $I$ and Point $\mathbf{J}$.

The wave velocity on the fast path in the water-oil region reaches a maximum value before reaching the initial conditions. This is similar to the situation that created the self-sharpening wave between Compositions $\mathbf{F}$ and $\mathbf{G}$ in the three-phase region. The final portion of the solution is obtained by calculating an isothermal upstream intermediate discontinuity with downstream conditions that match the desired initial conditions. This shock was found at composition $\mathbf{L}$. Between $\mathbf{K}$ and $\mathbf{L}$, a spreading wave along the fast path moves the solution between the desired points.

Comparing the cemperature profiles shown in Figure 4.34 and the profile for the heavy oil case given in Figure 4.49 show the same basic pattern. The only differences are in the location of the phase change shocks. There is a portion of the path that remains at the injection conditions until the temperature shocks to the three-phase saturation temperature, $485.57 \mathrm{~K}$, and remains at that temperature throughout the three-phase region. The temperature jumps from the three-phase saturation temperature to the initiai conditions at the leading phase change shock.

In the heavy oil case, the steam cannot build up any saturation near the injection conditions because of its high mobility relative to the heavier oil phase. This causes the trailing phase change shock to be slower in the heavy oil solution. The leading phase change shock is only slightly faster in the light oil case. The difference in shock velocity is due primarily to the slower flow velocity on the two-phase side of the shock in the heavy oil case. The heavy oil case actually has less steam condensing across the shock, but the volume lost is a greater portion of the water phase in the heavy oil case. This volume change compensates for the increased energy transfer in the light oil case.

A closer examination of the solution paths in the steam-oil region reveals a subule difference between the light oil solution and the heavy oil case. In the light oil case, the temperature remains at the injection temperature from the injection conditions to the trailing phase change shock at Point $P$. In the heavy oil case, the path follows the isothermal slow path at the injection temperature between Point $A$ and Point B. At Point B a path switch at the equal eigenvalue point takes the solution along the nonisothermal path between Point $\mathbf{B}$ and Point $\mathbf{C}$. This portion of the path behaves mucit like the solutions described in \$4.2.5. The non-isothermal portion of the path is a "slightly" spreading wave and behaves much like the extension of the trailing phase change shock that is shown between Point $\mathbf{C}$ and Point $\mathbf{D}$.

The velocity profile for the heavy oil case is shown iff Figure 4.50. As in the other solutions, the velocity profile mimics the shape of the temeprature profile. The heavy oil solution follows this pattern. Again density changes due to temperature changes are the cause of the flow velocity variations and in the heavy oil case this relationship is maintained.

\section{Summary}

The steam-oil-water dispalacements presented in this section were calculated by applying the model to a different hodograph space in three separate regions. A central three-phase isothermal region is ahead of a trailing steam-oil region. A water-oil region, representing the initial conditions is ahead of the threephase region.

The three regions are connected by a pair of phase transition shocks. These shocks have cuincident temperature and saturation changes. Both shocts are intermediate discontinuities.

The trailing region is a steam-oil region. The solution path in this region follows slong the slow path until reaching a phase transition shock. This shock separates the two-nhase steam-oil region from the central three-phase region. The shock is an intermediate discontinuity with a velocity that matches the small eigenvalue on the upstream side of the shock. 
Tie solution path follows along the eigenvector associated with the small eigenvalue for the entire steam-oil region. At some point along this path, an equal eigenvalue point changes the nature of the slow path from an isothermal path to a path that also has a temperature gradient.

Downstream of the equal eigenvalue point, the wave becomes a slightly spreading wave. For this reason, the portion of the path with a temperature change behaves like an addition to the phase transition shock. As a result, the saturation profiles downstream of the trailing phase transition shock are not effected by the temperature profile in the steam-oil region.

In the central three-phase region, the solution proceeds along the slow path until a path switch carries the solution onto the fast path. This path switch is seen as a central zone of constant state. The solution then travels along the fast path until the phase transition shock into the water-oil region.

Four wave patterns describe the displacements in the three-phase regions. These patterns are distinguished by the waves that presede and follow the central zone of constant state. Each of the two waves may either be a spreading or a self-sharpening wave, creating the four wave patterns.

The leading region is a water-oil region that remains at the initial conditions until a phase transition shock carries the solution into the three-phase region. The low flow velocity reduces the wave velocities in the water-oil region below the wave velocities in the three phase region, 80 an immediate jump to the initial conditions is required.

The effect of increasing oil viscosity changes the pauterns in all three of the displacement regions. In the steam-oil region an increasing oil viscosity dramatically reduces the steam saluration on the upstream side of the phase transition shock. A reialed effect is to reduce the velocity of the phase transition shock and the equal eigenvalue point. This reduction is greater for the equal eigenvalue point. At very high mobility ratios no shocks between the steam-oil region and the three phase region are possible without a reduction in temperature, below the injection temperature.

In the urree-phase region, compositions do not exhibit a large variation. The increased oil viscosity causes the oil phase to be virually iminobile in the three-phase region. The trailing spreading waves are shortened and the leading spreading waves are lengthened as compared to the less viscous oil.

When the trailing spreading wave changes into a self-sharpening wave at very high oil viscosities, this new self-sharpening wave creates an oil bank at the trailing end of the three-phase region. Physically this bank is formed as the mobile water and vapor phases move ahead of the immobile oil phase.

The water-oil region is effected by the increase in mobility ratio. The increased oil viscosity compared to the water phase increases the fractional flow derivative. $\left(\partial \partial_{w} / \partial S_{w}\right)$, and hence, the isothermal wave increases velocity until it is greater than the waves in the three-phase region. This results in spreading and self-sharpening waves in the water-oil region.

Two different displacement systems that include temperature variations have been examined by the method of characteristics. These systems have included the effects of temperature and flew properties. The final and most intucate effect is that of phase behavior. A third component that is partially miscible with the oil phase is introduced into the system. The next section discusses a displacement of oil and water by $\mathrm{CO}_{2}$ and water at an elevated temperature. This represents a system that includes the interaction of phase behavior, temperature and flow properies in one series of displacements. 


\subsection{THREE COMPONENT - THREE PHASE SYSTEMS}

The final example solution combiner: the effects of phase behavior, teriperature variations sud flow properies olver the entire extent of the systcm. This problem is considerably more general that the previous systems. In this system, there is no natural temperature plateau that was present in the oil-water systems. Since the three components are allowed to exist in all three phases, the only phase restriction is that wi the three phase region, for a given tempirature, the compositions of the throe separate phases are fixed.

The section begins with a description of the problem and the formulation of the general eigenvalue probiem, given by Eq. 2.26, to this specific system. Preliminary results for a specific system are given along with the path topology. Final solution profiles and composition paths are not presented due to computational difficuities. The majority of the problems stem from the calculation of the phase transition shocks. The section concludes with a discussion of the problems trying to find solutions for a system where phase behavior, temperature variations and flow properties are always interacting over the length of the displacer:ent system.

\subsubsection{Problem Formulation}

The system exarnined in this section is the injection of $\mathrm{CO}_{2}$ and water into a reservoir filled with a heavy oil. The third system examined adds the effects of phase behavior to the interactions of temperature and flow properties studied in the previous sections. The phase behavior for this system is complex. The system has three distinct phases at low temperatures:

1. A vapor phase that contain mostly $\mathrm{CO}_{2}$, but can also contain water vapor and trace amounts of the oil component. As the temperature is increased, the concentration of water in the vapor phase increases until the vapor temperature of the water component is reached. At this temperature, the system becomes a two-phase system. A vapor phase containing $\mathrm{CO}_{2}$ and water coexisting with a liquid phase containing oil, water and small amounts of $\mathrm{CO}_{2}$.

2. An oleic or upper liquid phase. This phase contains mosuly the oil component. At lower temperatures the $\mathrm{CO}_{2}$ is able to dissolve into the cil and at higher temperatures significant amounts of the water are present in the hydrocarbon phase.

3. An aqueous phase that is almost pure water. Small amounts of $\mathrm{CO}_{2}$ sre also present in the water phase, but no oil enters the aqueous phase.

A typical phase diagram for the system at lower temperatures is shown in Figure 4.51.

At higher temperatures, the water moves into the vapor phase until the saturation temperature of the water is reachied. Above this temperature, the three phase triangle disappears and the vapor-upper liquid region extends across the entire diagram. The phase diagram for these conditions is shown in Figure 4.52 . 


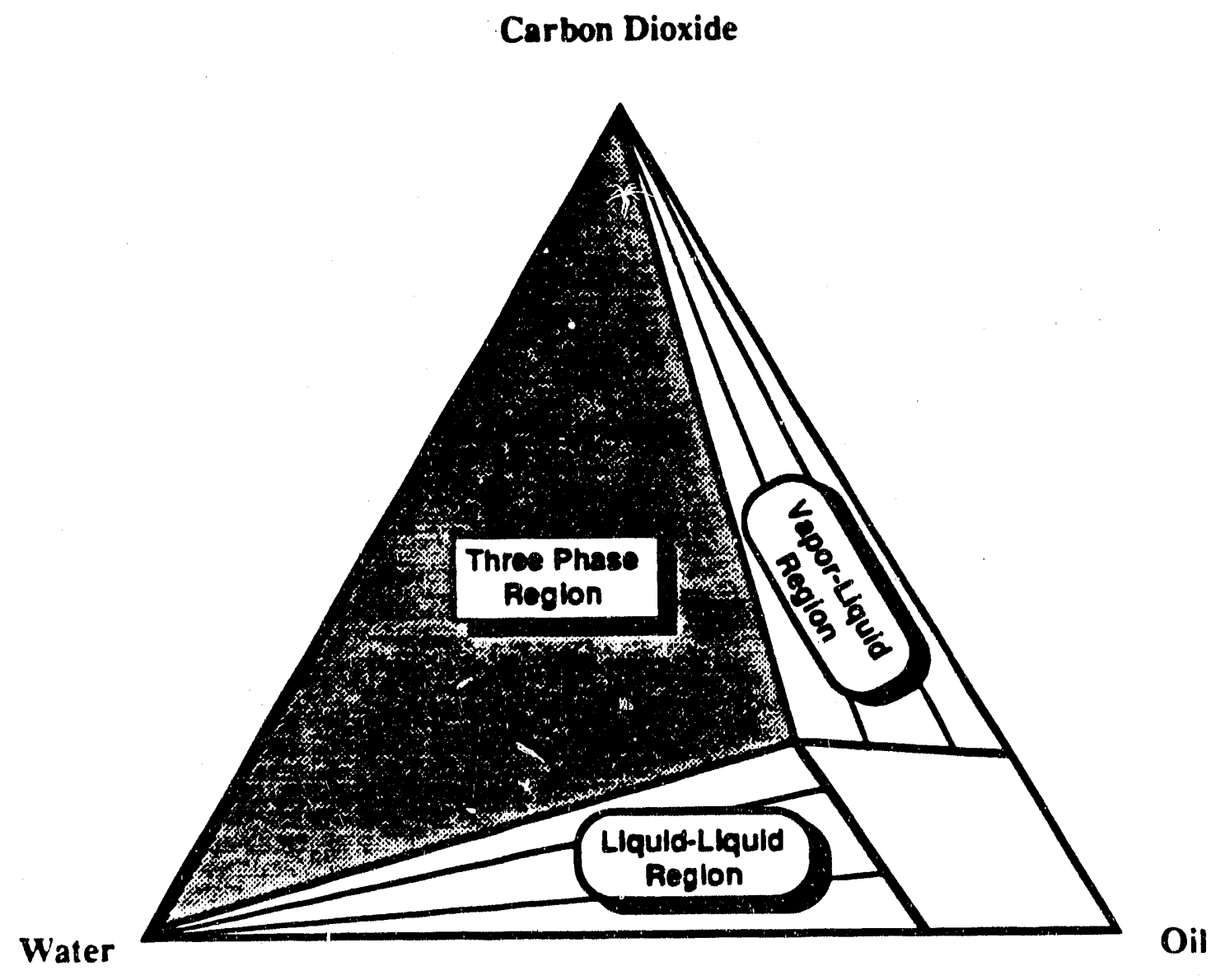

Figure 4.51: Phase diagram for a system of $\mathrm{CO}_{2}$-oil-water at temperatures below the saturation remperature of water. 


\section{Carbon Dioxide}

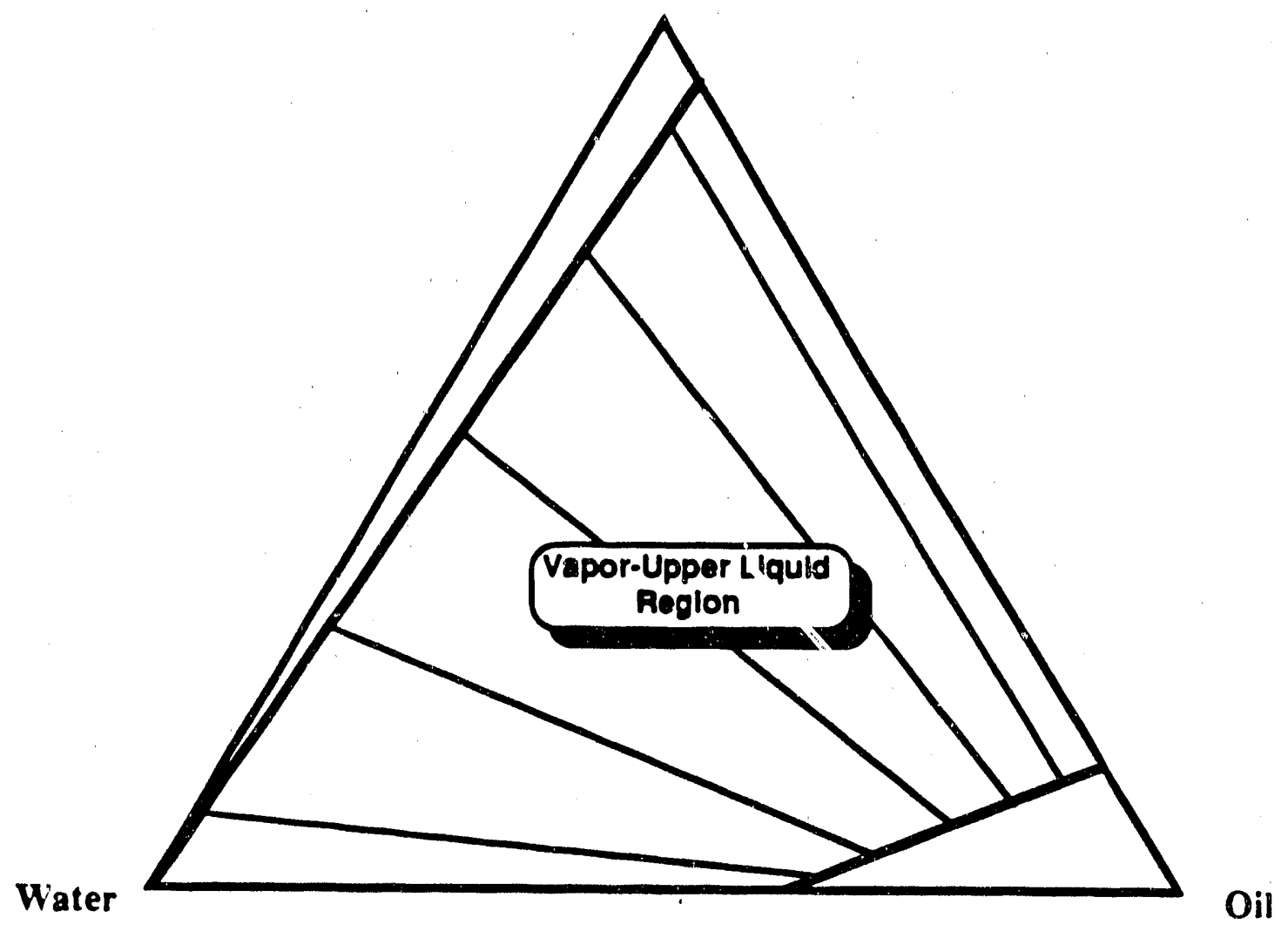

Figure 4.52: Phase diagram for a system of $\mathrm{CO}_{2}$-0il-water at temperatures below the saturation temperature of water.

The saturations of the phases cannot be used for the hodograph variables due to the phase behavior. When the components are free to transfer between phases, the amount of each component in a given phase changes over the length of the displacement. The hodograph variables for this system are mole fractions of the $\mathrm{CO}_{2}$ and the oil, the temperature and the flow velocity. The general eigenvalue problem described by Eq. 2.26 reduces to a $4 \times 4$ system given by, 


$$
\begin{aligned}
& {\left[\begin{array}{llll}
\frac{\partial F_{\mathrm{CO}_{2}}}{\partial C_{\mathrm{CO}_{2}}} & \frac{\partial F_{\mathrm{CO}}}{\partial C_{\text {oil }}} & \frac{\partial F_{\mathrm{CO}_{2}}}{\partial T} & \frac{\partial F_{\mathrm{CO}_{2}}}{\partial u} \\
\frac{\partial F_{\text {oil }}}{\partial C_{\mathrm{CO}_{2}}} & \frac{\partial F_{\text {oil }}}{\partial C_{\text {oil }}} & \frac{\partial F_{\text {oil }}}{\partial T} & \frac{\partial F_{\text {oil }}}{\partial u} \\
\frac{\partial F_{\mathrm{H}_{2} \mathrm{O}}}{\partial C_{\mathrm{CO}_{2}}} & \frac{\partial F_{\mathrm{H}_{2} \mathrm{O}}}{\partial C_{\text {oi. }}} & \frac{\partial F_{\mathrm{H}_{2} O}}{\partial T} & \frac{\partial F_{\mathrm{H}_{2} O}}{\partial u} \\
\frac{\partial \theta}{\partial C_{\mathrm{CO}_{2}}} & \frac{\partial \theta}{\partial C_{\text {oil }}} & \frac{\partial \theta}{\partial T} & \frac{\partial \theta}{\partial u}
\end{array}\right]\left[\begin{array}{c}
\frac{d C_{\mathrm{CO}_{2}}}{d \eta} \\
\frac{d C_{\text {oil }}}{d \eta} \\
\frac{d C_{\text {oil }}}{d \eta} \\
\frac{d u}{d \eta}
\end{array}\right]-} \\
& \lambda\left[\begin{array}{llll}
\frac{\partial G_{\mathrm{CO}_{2}}}{\partial C_{\mathrm{CO}_{2}}} & \frac{\partial G_{\mathrm{CO}_{2}}}{\partial C_{\text {oil }}} & \frac{\partial G_{\mathrm{CO}_{2}}}{\partial T} & 0 \\
\frac{\partial G_{\text {oil }}}{\partial C_{\mathrm{CO}_{2}}} & \frac{\partial G_{\text {oil }}}{\partial C_{\text {oil }}} & \frac{\partial G_{\text {oil }}}{\partial T} & 0 \\
\frac{\partial G_{\mathrm{H}_{2} O}}{\partial C_{\mathrm{CO}_{2}}} & \frac{\partial G_{\mathrm{H}_{2} O}}{\partial C_{\text {oil }}} & \frac{\partial G_{\mathrm{H}_{2} O}}{\partial T} & 0 \\
\frac{\partial T}{\partial C_{\mathrm{CO}_{2}}} & \frac{\partial T}{\partial C_{\text {oil }}} & \frac{\partial T}{\partial T} & 0
\end{array}\right]\left[\begin{array}{l}
\frac{d C_{\mathrm{CO}_{2}}}{d \eta} \\
\frac{d C_{\text {oil }}}{d \eta} \\
\frac{d T}{d \eta} \\
\frac{d u}{d \eta}
\end{array}\right]=0
\end{aligned}
$$

There are three eigenvalues and three corresponding eigenvectors representing the different comiposition path choices for a given composition point. At all times, at least one of the composition paths remains in the constant temperature plane. However since the heat of mixing is taken into account, the other two eigenvectors do not remain in the temperature plane.

The next section describes the topology of the composition paths in both the two and three phase regions for the $\mathrm{CO}_{2}$-oil-water system.

\subsubsection{Path Topology}

At a given temperature, the phase space can be divided into four distinct regions. The regions are illustrated in Figure 4.53. These four regions are:

Region 1 A vapor-upper liquid region. This region appears along the $\mathrm{CO}_{2}$-oil edge of the diagram. This region is small at low temperatures, but expands as the temperature increases. At high temperatures, the region expands and becomes a band that crosses the entire phase (see Figure 4.52).

Region 2 A two-phase liquid-liquid region. This region extends from the oil-water edge of the phase space to the towards the $\mathrm{CO}_{2}$ apex until it reaches the three phase boundary. This region is relatively small even at low temperatures. Increasing temperature moves the edge of the three-phase region towards the oil-water edge of the phase space, reducing the area of this two-phase region.

The next two regions are subsection of the three phase region. The size, shape and path topology in these two regions is primarily determined by the three-phase relative permeability model. The three-phase region is large at low temperatures and becomes smaller as the temeperature is increased.

Region 3 A three phase region where all three phases are able to flow simultaneously. This region begins at the $100 \%$ oleic comer of the three-phast region and extends towards the vapor-aqueous line. 


\section{Carbon Dioxide}

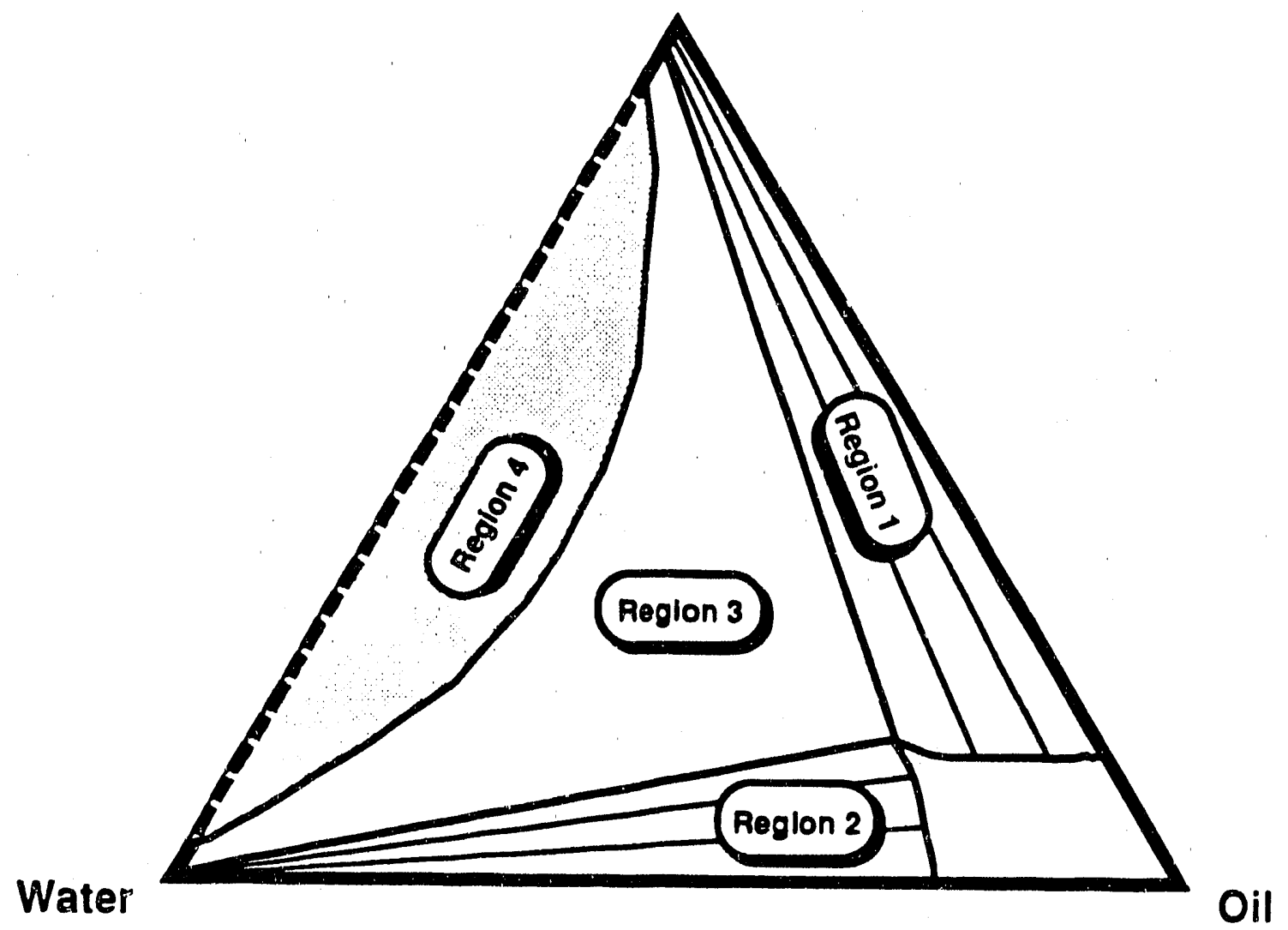

Figure 4.53: Four distinct flow regions in the $\mathrm{CO} 2$-oil-water system.

Region 4 A region where the fractional flow of the oleic phase is zero. For Stone's model II the shape of this region is shown as the shaded region in Figure 4.53. The size of this region depends only on the relative permeability relationships. As long as the relative permeabilities are assumed to be independent of temperature, then the size and shape of the region is also independent of the temperature.

The gradient of the fractional flow as the zero oil isoperm line is approached is dependent on the temperature. As the temperature is increased, the derivative of the fractional Mow is decreased. When the temperature is increased the viscosity of the oleic phase is reduced relative to the other two phases. The more favorable mobility ratio causes the fractional flow gradient to be reduced.

The composition paths in each of the four regions have a different topologies due to the different flow behavior in the different regions. In all the regions, there is one composition path that is the "cemperature" path. This path closely parallels the temperature axis in the hodograph space. Integration along this path results in a temperature change with litule change in composition. For most of the hodograph space, this path is the slow path.

The two remaining paths differ in direction, depending on the region of the phase space. These paths are shown in Figure 4.54. The faster of the two paths are shown by the dashed lines and the slower path by the solid lines. The tie line paths in the two two-phase regions are slow for the portions of the 
tieline where the saturation of the phase approaches zero or one. In the vapor-liquid region (Region 1), the portion of the tie-line path that is slow is very small and is difficult to show on the diagrams.

\section{Carbon Dioxide}

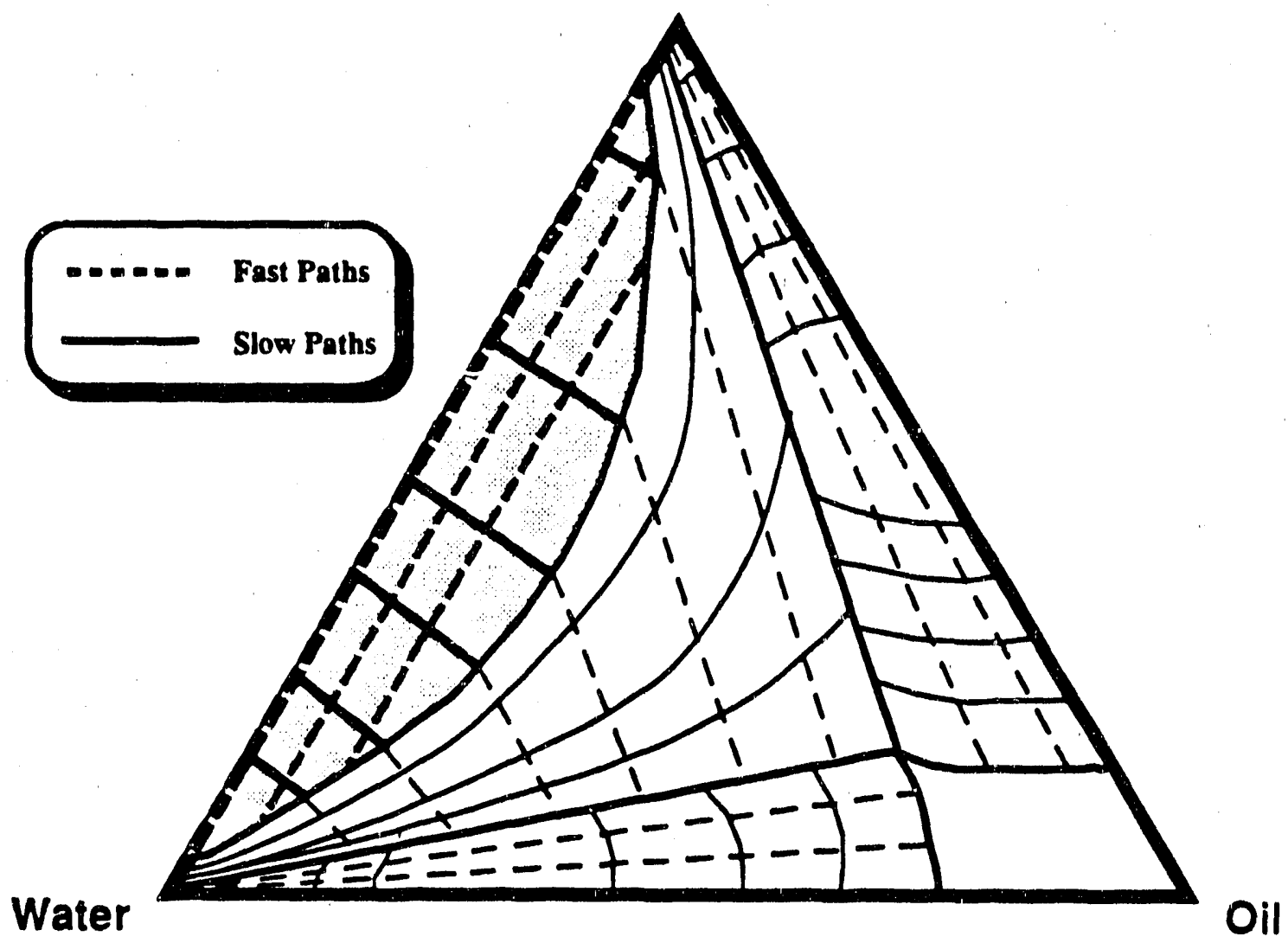

Figure 4.54: Composition paths for the $\mathrm{CO}_{2}$-oil-water system at a constant temperature.

The liquid-liquid tie lines (Region 2) have the same situation, but the regions where the tie line paths are slow are larger than the vapor-liquid tie ines. This is due to the lower mobility contrast on the liquid-liquid tie lines. The actual portion of the tie lines that are on the slow path depends on the particular relatively permeability model used.

In region 1, the vapor-oleic region, one path is constrained to follow along the tie lines in the region. The velocity of this path is proportional to the Buckley-Leveretl velocity, $\left(\partial_{v} / \partial S_{v}\right)$. As mentioned, for the majority of region 1, the tie line path is the fast path.

The third path in this region corresponds to the non-tie line path in the isothermal case. The eigenvector that corresponds to this path has a small change in temperature in addition to the composition change. This change in temperature is primarily due to the enthalpy of mixing. Unlike the tie line path, the composition of the two phases changes along this path. The heat capacity of the phases is a function of composition. This means that the total energy stored per degree of temperature changes with the total energy remaining constant, resulting in a small change in temperature along this mn-tie line path. 
The amount of temperature change along this path is relatively small for the $\mathrm{CO}_{2}$-oil-water system. A typical integration along the non-tie line path will have a composition change, $\Delta \mathrm{C}_{2} \mathrm{O}$, of 0.1 and a corresponding temperature change, $\Delta T$, that is approximately 3 degrees Kelvin. A typical path is given by 12 points in Table 4.13 .

\begin{tabular}{|c|c|c|c|c|}
\hline $\mathrm{CO}_{2}$ & Oil & Waler & Temperature & $\begin{array}{c}\text { Flow } \\
\text { Velocity }\end{array}$ \\
\hline \multicolumn{3}{|c|}{ Mole Fraction } & Kelvin & PVITime \\
\hline 0.300000 & 0.680000 & 0.020000 & 325.00 & 1.000000 \\
0.290389 & 0.667473 & 0.042137 & 324.34 & 0.998431 \\
0.281579 & 0.654384 & 0.064037 & 323.69 & 0.996837 \\
0.273422 & 0.640803 & 0.085776 & 323.03 & 0.995220 \\
0.265831 & 0.626795 & 0.107374 & 322.38 & 0.993582 \\
0.258745 & 0.612415 & 0.128840 & 321.72 & 0.991921 \\
0.252117 & 0.597713 & 0.150170 & 321.07 & 0.990238 \\
0.245910 & 0.582732 & 0.171358 & 320.41 & 0.988534 \\
0.240087 & 0.567508 & 0.192405 & 319.76 & 0.986809 \\
0.234630 & 0.552085 & 0.213285 & 319.10 & 0.985062 \\
0.229510 & 0.536490 & 0.234000 & 318.45 & 0.983293 \\
0.227974 & 0.531580 & 0.240447 & 318.24 & 0.982735 \\
\hline
\end{tabular}

Table 4.13: Non-tie line path in the vapor-liquid region of the $\mathrm{CO}_{2}$-oil-water system.

Region 2 is the liquid-liquid region. This region has two paths that are qualitatively similar to the two paths in the vapor-liquid region. A tie line path with an eigenvalue proportional to the fractional flow derivative is the fast path for the central portion of the tie lines. The fractional flow derivative apprnaches zero as the saturation of the phases approach zero and one. Al the ends of the tie lines, the tie line path is the slow path.

The non-tie line path in the liquid-liquid region behaves much like the same paths in the vaporliquid region. The enthalpy of mixing lerms have a lesser effect than along the vapor-liquid paths. This causes the temperature deviation 10 be smaller than along the paths in the vapor-liquid region. An example of a typical pach is shown in Table 4.14.

Region 3 has three phases, vapor, oleic, and aqueous, flowing simultaneously. The phase rule shows that for three components 10 partition into three phases at a constant pressure and temperature, the compositions of the phases cannot change. As the overall composition point changes in the three-phase region, only the amounts of each phase can change, while the $c$ mposition of each phase remains constant. 


\begin{tabular}{|c|c|c|c|c|}
\hline CO2 & Oil & Water & Temperature & $\begin{array}{c}\text { Flow } \\
\text { Velocity }\end{array}$ \\
\hline \multicolumn{3}{|c|}{ Mole Fraction } & Kelvin & PV/Time \\
\hline 0.300000 & 0.680000 & 0.020000 & 325.00 & 1.000000 \\
0.290389 & 0.667473 & 0.042137 & 324.34 & 0.998431 \\
0.281579 & 0.654384 & 0.064037 & 323.69 & 0.996837 \\
0.273422 & 0.640803 & 0.085776 & 323.03 & 0.995220 \\
0.265831 & 0.626795 & 0.107374 & 322.38 & 0.993582 \\
0.258745 & 0.612415 & 0.128840 & 321.72 & 0.991921 \\
0.252117 & 0.597713 & 0.150170 & 321.07 & 0.990238 \\
0.245910 & 0.582732 & 0.171358 & 320.41 & 0.988534 \\
0.240087 & 0.567508 & 0.192405 & 319.76 & 0.986809 \\
0.234630 & 0.552085 & 0.213285 & 319.10 & 0.985062 \\
0.229510 & 0.536490 & 0.234000 & 318.45 & 0.983293 \\
0.227974 & 0.531580 & 0.240447 & 318.24 & 0.982735 \\
\hline
\end{tabular}

Table 4.14: Typical composition path in the liquid-liquid region of the $\mathrm{CO}_{2}$-oil-water system.

Both the slow and fast paths in this region are isothermal. The composition of the three phases remains constant over the entire region so there is no enthalpy of mixing effect on the composition paths. The slow paths in this region parallel the contour of zero fractional flow of the oil phase near the boundary of regions 3 and 4 . These paths fan out toward the liquid-liquid boundary of the three-phase region until they parallel the boundary. ${ }^{4}$ The eigenvalues along this path increase towards the aqueous apex of the three-phase region.

The fast paths parallel the vapor-liquid boundary of the three-phase region. These paths do not change direction to a great extent over region 3 . The eigenvalues increase as you move along the fast paths away from the boundary between regions 3 and 4 and towards the liquid-liquid boundary.

\subsubsection{Solution Problems}

The major difficulty to obtaining a full solution to the $\mathrm{CO}_{2}$-oil-water system is the construction of the discontinuities. There are three major problems that make the calculation of the discontini:ies especially difficult. These are:

1. There are no tie line extensions that constrain the the location of the leading and trailing shocks.

2. There is no clear temperature plateau on one side of the discontinuities.

3. The material and enthalpy balances are extremely sensitive to the conditions on the unknown side of the discontinuity.

Each of these reasons make it much more difficult to isolate the physically correct shock from the many possible constructions.

\footnotetext{
${ }^{4}$ Becuuse the liquid-liquid boundary is also a tie line it must also be a path.
} 
When shocking from the single-phase into a two-phase region, it has been shown that the composition on the two-phase side of the shock must lie on the tie line extension that passes through the single-phase point (Dumore 1984). In the case where temperature is also a variable, an enthalpy balance across the discontinuity is not a linear combination of the total on the single-phase side of the shock. A mathematical description of the enthalpy balance across the discontinuity is discussed in Appendix $\mathrm{C}$.

A second effect that prevents any tie line considerations is the enthalpy of mixing. The overall heat capacity is diffe rent on the single-phase side of the shock than on the two-phase side. The compositional dependence of he phase heat capacities is especially important when the latent heat of the aqueous phase is considered.

The second difficulty is that there is no clear temperaturc plateau on one side of the shock. In the case of the steam-oil-water system, the three-phase region was constrained to a constant temperature. This physical constraint on the three-phase side of the discontinuity makes the shock construction much easier because temperature is not variable on that side of the discontinuity.

The addition of the third component that forms a partially miscible system allows the three-phase region exist over a wide range of temperatures. This fact, combined with the consideration of the compositional effects make the construction of the shocks in this system much more difficult.

The final reason that these shocks are especially difficult to construct is that the domain of convergence is very small. For the $\mathrm{CO}_{2}$-oil-water system the range of initial guesses that converges to a composition point that satisfies the material balances, the enthalpy balance, and also be tangent to one of the characteristic paths has proven to be the mosi difficult part of the solution. ${ }^{5}$

This concludes the presentation of the results for the three different example systems. The final section will discuss the conclusions and limitations of the model as applied to flow problems with temperature variations. The general conclusions about the model itself, apart from a specific problem or system, and the limitations are discussed first. The conclusions that apply to the three specific solutions are presented in turn, and finally, further applications of the method of characteristics are outlined.

\footnotetext{
In ract NO solutions have been found that are able to connect typical boundary condition to point in either the two-phase or three-phase region. This is primarily a failure of the enthalpy balance to converge in a reasonable temperature range.
} 


\section{CONCLUSIONS AND RECOMMENDATIONS}

This chapter is divided into three main sections. The first section presents general onclusions and observations about the model as a whole. The applications and limitations of tie model are emphasized.

The second section summarizes the results obtained from applying the model to the three specific types of displacement problems discussed in Chapter 4. Each of the three systems present different approaches and results. Practical application of each of the systems will be mentioned in these sections.

Finally, the third section will mention additional topics that need to be examined. Specifically, improvements on the solution technique and applications to flow problems not yet mentioned are discussed in this section.

\subsection{GENERAL CONCLUSIONS AND LIMITATIONS}

The section is divided into three subsections. The first are the conclusions. These are statements about the method of characteristics as applied to multiphase multicomponent flow in porous media. The second section are "observations." These are statements that seem to be generally true, br" sould not be proven or have some exceptions in the many solutions presented in this dissertation. The final subsection discusses the two major limitations that must be considered when applying the model to othar probleins.

\subsubsection{Conclusions}

A semi-analytical model capable of describing the complex interactions between the system temperature, phase behavior and flow properties has been presented. This model is an extension of the model presented by Dumore (1984) to include the interaction of system temperature with the phase behavior and flow properties. The solutions presented in this dissertation lead to the following general conclusions:

1. The mathematical model presented by Dumore can be extended to include temperature variations. The temperature of the system is found by treating the overall enthalpy as though it were another compunent that is transported in the flowing phases. Dispersive effects of conduction and heat gains or losses from the system are neglected.

2. The solutions to the model system are piecewise continuous in the weak sense. Specifically the solutions consist of waves that propagate with constant velocities. These waves are separated by zones of constant state or by jump discontinuities.

3. Temperature shocks are always accompanied by discontinuities in the other hodograph variables. These shocks are primarily the result of increasing or decreasing the number of phases.

4. The reverse of this is not true. Saturation or composition shocks are soen that do not have a coincident change in temperature.

5. Phase boundaries are al ways crossed by shocks. This is due to the discontinuities in the eigenvalues as the path crosses the phase boundaries. This discontinuity is present because of the discontinuity in the fractional now derivative as the path travels from a two phase to a three phase region.

6. In all cases there is at least one wave that moves in an isothermal plane. This is necessary for the limiting case of an isothermal injection to be possible. 


\subsubsection{Observations}

After studying many different displacement systems using the method of characteristics, a number of patterns and rules of thumb have emerged. These ideas are observations that have not been rigorously proven. They represent concepts that are useful to keep in mind as possible solutions are being constructed.

1. The solution always begins on the slowest path at the injection conditions.

2. The solution follows along the continuous composition path whenever possible. The discontinuities are only introduced when a continuous solution is not possible.

3. All the central shocks i.e., those that do not include the initial or injection conditions on one side of the shock, are intermediate discontinuities. Genuine shocks only appear when an earlier tangent shock has constrained the composition path to land on a tie-line extension of the initial or injection conditions. In order to travel from the laiding point to the boundary condition, the solution must cross a shock that does not match the wave velocity on either side of the shock. This is often the case when the boundary conditions are in the single phase region and there are no composition paths on that side of the shock.

4. At an equal eigenvalue point, where a path switch may take place, the solution leaves the point along the same eigenvector as the one on which it enteied. For example, when the solution arrives at an equal cigenvalue poini along the slow path, it also departs the equal eigenvalue point along the slow path. This is not a hard and fast rule, there are occasions where the solution leaves along a different path than the one it entered. The large majority of solutions however, do follow this maxim.

5. There is an ordering to the kinds of waves that are seen in the solutions. Continuously varying waves occur if they are possible. Discontinuities are only seen where necessary. The crossing of a phase boundary or a self-sharpening wave are examples where the discontinuities must be introduced into the solution. The discontinuicies also have a ranking. Genuine shocks are only seen when an intermediate discontinuity is not possible.

As the solution travels along a continuous variation, there must be some trigger that prevents it from continuing any further. This trigger is usually a malch between the characteristic velocity of the spreading wave and an intermediate discontinuity that is forced by the downstream conditions.

\subsubsection{Limitations}

There are a number of limitations that go along with every model. Some of these limits are not very restrictive, while others present major obstacles that need to be overcome. This section will discuss some of these limitations, both large and small, and attempt to suggest methods for dealing with some of the major restrictions.

The less restrictive limitations are inherent in the assumptions that are made in building the model. These are:

1. The system is restricted to one-dimensional displacements. The application of a method of characteristics to problems with more than two independent variables is the subject of considerable study by the mathematical community. The application of the concepts to flow problems in porous media has yet to be explored, but this restriction could be lifted.

2. The system is restricted to constant pressure. 
3. The system is adiabatic and enthalpy losses outside the system are neglected. This could be a severe limitation for practical reservoir applications if the injection rate is slow or the pay formation is not thick.

There are a number of major limitations that are inherent in the model. These are limitatious that severely limit the kinds of systems that can be solved by the model. The restrictions are primarily placed on the total number of components and/or phases the system can have.

One of the important considerations is the integration of a flash routine into the calculation of the eigenvalue problem. Flash programs for the calculation of two phase systems are well established and robust. This is not true for systems that exh, bit more than two phases. Three phase flash routines are not well behaved especially over a wide range of temperatures. In order to use the existing equations of state, a relatively quick and robust flash routine is needed that can internally determine the number and compositions of the phases over a wide range of temperatures.

The greatest hurdle to overcome is the calculation of the discontinuities that are present in all solutions. The solution of the Reimann problem described in Chapter 2 is not unique. The introduction of a physical limitation, such as the velocity constraint, should give the one physically correct solution to the problem. However, as more components are included in the systen, the complexity of the solutions increases dramatically. In the case of the viscous oil presented in \$4.2.6, solutions that in theory satisfied the velocity constraint but did not appear to be physically correct were rejected. Other solutions that also satisfied the velocity constraint and appeared to be physically consistent were later found. With each new component, a new dimension is added to the hodograph space, and the level of complexity increases dramatically. Practically, the number of comporents, including the enthalpy, is limited to a range from 3 to 5 .

\subsection{SPECIFIC APPLICATIONS}

This section presents the conclusions that pertain to a particular displacement system. Results for the steam-water, steam-oil-waler and throe phase-three component systems are discussed.

\section{シ.2.1 Single Component with Temperature Variation}

The system where a single component partitions into a vapor and liquid phase at the saturation represents one type of system that can be represented by the model. The following observations are made about the single component, two phase system:

1. The effects of temperature and flow behavior are separated by the physical requiremert that the twophase region exists only at the saturation temperature.

2. In the single-phase region, no separation of the local enthalpy content is possible due to the nature of the single phase flow. This prevents the formation of a temperature profile in the single-phase regions. In the trailing region, the temperature remains constant at the injection temperature. In the leading region, the temperature is at the initial temperature.

3. In the two-phase region, the system behaves as an two-phase immiscible displacement. Fractional flow considerations completely determine the saturation profile in the two-phase region.

4. The single-phase regions are coupled to the two-phase region by a trailing downstream intermediate discontinuity (tangent shock) and a leading upstream intermediate discontinuity. The velocity of the shock is dominated by the temperature change across the shock. The greater the temperature change, the lower the shock velocity. 
5. Chariges in certain parameters only affect the conditions downstream of the change. This means that changes in the injection conditions aller the entire displacement profile, but changes in the initial temperature onfy effect the leading shock. This has only been demonstrated for this case, but the result is due to the nature of the hyperbolic equations and should be true for all the cases presented.

6. The velocity of the trailing shock reaches a maxiuum and then decreases as the injection temperature is decreased. This is due to the competing naturo of the mass and enthalpy changes across the trailing shock.

7. The initial temperature only affects the velocity of the leading shock. As the initial temperature increases, the difference in temperature across the shock decreases. The smaller temperature change across the shock causes the shock velocity to increase.

8. The ability of the matrix to store heat is proportional to the quantity

$$
\left(\frac{1-\phi}{\phi}\right)\left(\rho_{m} C p_{m}\right)
$$

As matrix becomes less porous, more dense, or has a larger heat capacity, the velocity of the leading and trailing shocks is reduced. The change in velocity is much grealer for the leading sitock so the net effect is to decrease the size of the two-phase region.

\subsubsection{Two-Cumponent, Three-Phase Immiscible System}

1. The injection of high temperature steam into a cold immiscible oil phase (with some small initial water saturation also present) can be modeled using these tochniques.

2. The solution is divided into three regions. A leading liquid water-oil region, a central three-phase region where steam, oil and water flow simultaneously. A two-phase steam-oil region trails the three-phase region.

3. The three regions are connected by a pair of phase transition shocks. These shocks have a coincident change in temperature and saturations. These shocks are intermediate discontinuities with the matching eigenvalue on either the upstream or the downstream side of the shock.

4. The extent of the possible landing points in the three-phase region is very small. Greatly changing the steam-oil jumping point has only a small effect on the eventual landing point in the three-phase region. The opposite is also true, when searching for a point in the three-phase region that jumps into the steam-oil region, only a small part of the phase space will give an acceptable solution. Changing the three point greatly effects the landing point in the steam-oil region.

5. The central three-phase region is a strictly hyperbolic region where a path switch from the slow to the fast path creates a central zone of constant state.

6. The three-phase region can contain a spreading wave ahead or trailing the central zone of constant state. A self-sharpening wave cry also exist between the trailing phase transitions shock and the central zone of constant state.

7. The steam-oil region follows the slow path until an intermediate discontinuity takes the solution into the three-phase region. 
8. Increasing oil viscosity causes the highly mobile vapor phase to spread over the system to a much larger extent. This results in the vapor saturations being much lower than in the case of the less viscous oils.

9. The increased oil viscosity causes the formation of an oil bank at the trailing edge of the three-phase region.

10. Increased oil viscosity causes the saturation change across the phase transition shocks to be smaller than with less viscous oils.

11. The fast path follows lines of constant water saturation in the case with high oil viscosity. This is in contrast to the base case, where the fast path parallels lines of consiant oil saturation.

12. Spreading waves in the leading-water oil region are possible when the oil viscosity increases.

\subsubsection{Three-Component, Three-Phase System with Temperature Variations}

The three component system adds the effects of phase behavior to the model. At lower temperatures, the mass transfer of $\mathrm{CO}_{2}$ into the oleic phase alters the flowing properties of the phase by decreasing its density and viscosity. At higher temepratures, the increased temperature itself is responsible for reducing the viscosity of the oleic phase. The solutions presented for this section again represent only a smal' fraction of the possible systems and effects that can be studied using the method of characteristics. Some of the most important results are:

1. In the three-phase region, two paths remain in an isothermal plane and the third path follows a path that is only in the temperature direction.

2. In the two phase region, one path remains in the isothermal plane and two paths show a change in temperature. The isothermal path is the tie-line path and both nontie-line paths have a change in temperature. This temperature change is due to the enthalpy change of mixing. Along a tie-line, no mixing occurs and there is no enthalpy change because of mass transfer. Along the non-tie line paths the enthalpy of mixing changes the amount of enthalpy avaiulable as the components transfer phases influences the change in temperature.

\subsection{FUTURE RECOMMENDATIONS}

The solutions presented in this dissertation represent a fraction of the possible applications of the method of characteristics. Some of the projects mentioned here are direct extensions of the systems studied in chapter 4 . Other ideas are ideas that stem from practical considerations in reservoir engineering. Some final ideas represent systems that have a potential for study by the method of characteristics.

The solutions presented in this dissertation are only a part of a complete description to multiphase, multicomponent systems with temperature variations. Some of the immediate extensions that deserve attention are:

1. The effect of relative permeability models on the wave patterns for the three phase systems. The three phase relative permeability models represent the greatest uncertainty incorporated into the model. An application of this model could be to use three-phase slim tube displacement data to formulate, adjust and verify three phase relative permeability models.

2. The effect of adding a second oil component. This new component represents the light, volatile components which partition into the vapor phase at high temepratures or are extracted into a $\mathrm{CO}_{2}$ rich phasc. This addition would be a more accurate description of lighter oils that are traditionally candidates for miscible floods. 
3. The application of the model to one-dimensional flow in radial coordinates. Conceptually, this problem only requires the rewriting of the conservation equations into a cylindrical coordinate system. Practically, however, it changes the nature of the differential equations by grouping one of the dependent variables (the length) into the definitions of the primary variables ( $G, F, F$, and $\theta)$. 'This makes the coefficients of the $\bar{G}$ and $\bar{F}$ matrices functions of the inderendent variables, changing the rature differential equations from reducible to irreducible.

The application of a mathematical model to "practical" problems is always an important aspect of model development. The model by itself can be an aid to the understanding of how different phenomena. interact in a multiphase, multicomponent system with lemperature variations. However, these results are likely to remain in the academic arena unless a simple, practical use for the ideas is found. As an important area for future study, I feel that a concentration of effort on the application of the model to laboratory or field problems would have the greatest benefit in the long nun.

Some suggestions for practical problems that could be tackled by the method of characteristics are:

- Verifying and fine tuning three phase relative permeability models or equations ci state.

For the presentation in this dissertation, the task was to find the saturation or composition profiles of a displacement system given the phase behavior and relative permeability of the different components and phases. A good application of the model would be to study the reverse problem. In most actual circumstances, the composition profiles are the known data. The unknowns are the phase behavior and/or relative permeability models that apply to the fluiós. For example, by taking periodic fluid samples, an observation well in a field that is undergoing a miscible flood can supply the compositions of the fluids that pass the well at different points in time. This information can be assimilated to give the velocities and compositions as different fronts pass the observation well.

The task is to determine, given an initial reservoir composition and an injection history, what is the phase behavior and/or relative permeability model that results in the observed set of compositions and front velocities? Since the model has the ability to change directly both these factors and examine the results in a straightforward manner, it would be ideally suited for studying this problem.

The major difficulty would be constructing the initial solution path. Once that is completed, parameler studies are straightforward. The effect of alightly changing the phase behavior or relative perrneabilities is small. Because the composition path and shock values are the given data rather than the unknowns, the construction of the initial solution route is made much easier than if on'y the boundary conditions were apecified. 


\section{NOMENCLATURE}

\section{Roman Symbols}

$C_{i} \quad$ Concentration of component i. kgmol/m $\mathrm{m}^{3}$

$C_{P_{j}} \quad$ Specific Heal of phase j, J/kgmol $K$

$C_{p_{m}} \quad$ Specific Heal of matrix, J/kg $K$

$f_{j} \quad$ Fractional flow of phase $j$

$F_{i} \quad$ Overall flux of component $i, \mathrm{kgmol} / \mathrm{m}^{2} \mathrm{day}$

$G_{i} \quad$ O.erall local concentration of component $i, \mathrm{kgmol} / \mathrm{m}^{3}$

$H_{j} \quad$ Enthalpy of phase $j, J / k g m o l$

$H_{m} \quad$ Enthalpy of matrix, J/kg

$k_{r} \quad$ Relative permeability

$k_{r g} \quad$ Relative permeability of vapor phase

$k_{r l g}$ Two phase liquid relative permeability in liquid-vapor systern used in three phase relative permeability relations

kro Relative permeability of oil phase

krow Intermediate phase relative perneability at irreducible water saturation

krow Two phase oil relative permeability in oil-water system used in three phase relative permeability relations

$k_{\text {rw }}$ Relative permeability of aqueous phase

$n_{c} \quad$ Total Number of Components

$n_{p} \quad$ Total Number of Phases

$S_{j} \quad$ Saturation of phase $j$

$S_{l} \quad$ Liquid phase saturation

So Intermediate phase sauration

Sor Irreducible intermediate phase saturation

$S_{v} \quad$ Saturation of non wetting (vapor) phase

$S_{w} \quad$ Saturation of welling (aqueous) phase 
$\begin{array}{ll}S_{w c} & \text { Connate weting phase saturation } \\ t & \text { time, day } \\ T & \text { Temperature, } K \\ \vec{u} & \text { Local flow velocity, day } \\ u C_{i} & \text { Characteristic velocity of component } i \\ u H & \text { Characteristic velocity of the enthalpy } \\ x & \text { distance, } m \\ x_{i j} & \text { Mole Fraction of component } i \text { in phase } j\end{array}$

Greek Symbols

$\alpha_{g} \quad$ Gaseous phase Corey exponent

$\alpha_{\text {/g }}$ Corey exponent for liquid-gas system used in three phase relative permeability relations

$a_{\text {ow }}$ Core: exponent for oil-water system used in three phase relative permeability relations

$\alpha_{w} \quad$ Water phase Corey exponent

$\Gamma \quad$ Local enthalpy concentration, $J / m^{3}$

$\eta \quad$ Characteristic dummy variable

( Local enthalpy flux, $J / m^{2}$ day

$\Lambda \quad$ Shock Velocity, m/day

$\Lambda_{i} \quad$ Shock Velocity calculated using a material balance on component $i$, miday

$\Lambda^{k} \quad$ The velocity of a $k^{k h}$ discontinuity

$\Lambda_{s s} \quad$ Velocity of a discontinuity created by a self-sharpening wave m/day

$\Lambda_{\text {uid }}$ Velocity of a discontinuity created by an upstream intermediate discontinuity, m/day

$\lambda$ Characteristic velocity, m/day

$\lambda^{l} \quad$ Characteristic velocity in the single phase region, $m / d a y$

$\lambda^{\prime \prime} \quad$ Characteristic velocity in the two phase region, m/day

$\lambda^{\prime \prime \prime} \quad$ Characteristic velocity in the three phase region, $m / d a y$

$\lambda$ - Characteristic velocity downstream of a discontinuity, m/day 
$\lambda^{+} \quad$ Characteristic velocity upstream of a discontinuity, $m / d a y$

$\lambda_{1}$ Characteristic velocity along the slowest path, m/day

$\lambda_{2}$ Characteristic velocity along an inlermediate path, miday

$\lambda_{3}$ Characteristic velocity along the fastest path, $m / d a y$

$\mu_{j} \quad$ Viscosity of phase $j$, Pa.sec

Pj Molar density of phase j, kgmol// $\mathrm{m}^{3}$

Pm Mass density of matrix, $\mathrm{kg} / \mathrm{m}^{3}$.

$\sigma_{g} \quad$ End point relative permeability for non-wetuing phase

$\sigma_{l g}$ End Point relative permeability for intermediate phase in the liquid-gas system

ow End Point relative permeability for intermediate phase in the oil-water system

$\sigma_{w}$ End point relative permeability for wetting phase

Porosity of matrix 


\section{REFERENCES}

1. Aris, R. and Admundson, N.R.: Mathematical Methods in Chemical Engineering: Volume 2, First Order Partial Differensial Equations with Applications, Prentice-Hall, Englewood Cliffs, NJ (1973).

2. Aziz, K. and Setarri, A.: Petroleum Reservoir Simulation, Applied Science Publishers Ltd., London (1979).

3. Baker, L.E.: "Three-Phase Relative Permeability Correlations", SPE 17369, presented at the Sixth Symposium on Enhanced Oil Recovery, Tulsa, OK, April 17-20, 1988.

4. Bell, J.B., Trangenstein, J.A. and Shubin, G.: "Conservation Laws of Mixed Type Describing Three-Phase Flow In Porous Media", SIAM Journal of Applied Mathematics, 46(6):1000-1017 (December 1986).

5. Buckley, S.E. and Leverett N.C.: "Mechanisim of Fluid Displacement in Sands", Transactions of the AIME, 146:107 (1942).

6. Chung, F.T.H., Jones, R.A. and Nguyen, H.T.: "Measurements and Correlations of the Physical Properties of $\mathrm{CO}_{2}$-Heavy Oil Mixtures", SPE 15080, presented at the 56th California Regional Meeting, Ventura, CA, April, 1986.

7. Courant, R. and Friedrichs, K.O.: Supersonic Flow and Shock Waves, Springer-Verlag, New York (1948).

8. Dafermos, C.M.: "Hyberbolic Systems of Conservation Laws", In J.M Ball, editor: Systems of Nonlinear Partial Differential Equations, pages 25-71, D. Reidel Publishing Company, Dordrecht, Holland (1983).

9. Daripa, P., Glimm, J., Lindquist, B., and Maesumi, M.: On the Simulation of Heterogenous Petroleum Reservoirs, Technical Report DOE/ER/03077-279, Courant Mathematics and Computing Laboratory, New York, New York (April 1987).

10. de Pedr jsa, T., Escobar, E., and Rivas, O.: Research Program on Heavy Oil Recovery by $\mathrm{CO}_{2}$. Task $3 /$ of Annex IV. Technical Repon, INTEVEP, S.A., Los Teques, Edo. Miranda, Venezuela (Marcn 1986).

11. Dum rré, J.M., Hagoon, J., and Risseeuw, A.S.: "A Analytical Model for One Dimensional Three Comj onent and Vaporizing Gas Drives", SPEJ, 24:169-179 (1984).

12. Emarual, A.S.: "A Mixing Rule Method for Calculating the Viscosity of $\mathrm{CO}_{2}$ and Low-Gravity Reservoir O'l", SPE 14229, presented at the 60th Annual Technical Conference and Exhibition, Las Vegas, î̀, 1985.

13. Fayers, FJ.: "Extensions of Stone's Model I and Conditions for Real Characteristics in ThreePhace Flow", SPE 16965, presented at the 62nd Annual Technical Conference and Exhibition, Dallas, TX, September 27, 1987.

13. Fayers, F.J.: "Some Theoretical Results Concerning the Displacement of a Viscous Oil by a Hot Fluid in a Porous Medium", Jownal of Fluid Mechanics, 13:65-76 (1962).

14. Glimm, J.: The Interaction of Nonlinear Hyperbolic Waves: A Conference Report, Conference Reporh C urant Institute, New York University, New York, New York (1987). 
15. Gorell, S.B.: "Modeling the Effects of Trapping and Water Alternate Gas Injection on Tertiary Miscible Displacements", SPE 1734n, presented at the Sixth Symposium on Enhanced Oil Recovery, Tulsa, OK, April 17-20, 1988.

16. Hattori, H.: "The Reimann Problem for a van der Waals Fluid with Entropy Rate Admissibility Criterion-Nonisothermal Case", Journal of Differential Equations, 65:158-174 (1986).

17. Helfferich, F.G.: "General Theory of Multicomponent, Multiphase Displacement in Porous Media", SPEJ, 21:51-62 (1981).

18. Hirasaki, G.J.: "Application of the Theory of Multicomponent, Multiphase Displacement to ThreeComponent, Two-Phase Surfactant Flooding", SPEJ, 21:191-204 (1981).

19. Holden, H.: "On the Riemann Problem for a Prototype of a Mixed Type Conservation Law", Communications on Pure and Applied Mathematics, 40:229-264 (1987).

20. Holm, L.W. and Josendal, V.A.: "Effect of Oil Composition on Miscible-Type Displacement by Carbon Dioxide", SPEJ, 22:87-98 (1982).

21. Holm, L.W. and Josendal, V.A.: "Mechanisims of Oil Displacement by Carbon Dioxide", JPT, 16:1427-1436 (December 1982).

22. Hovdan, M.: "Vanninjeksjon - Inkompressibel Analytisk lфsning med Temperatureffekter" (1986).

23. Hutchinson, C.A., Jr, and Braun, P.H.: "Phase Relations of Miscible Displacement and Oil Recovery", A. I. Ch. E. Journal, 7:64-72 (1961).

24. Jeffrey, A.: "The Propagation of Weak Discontinuities in Quasilinear Hyperbolic Systems with Discontinuous Coefficients Pan ! - Fundamental Theory", Applicable Analysis, 3:79-100 (1973).

24. Jeffrey, A.: Quasilinear Hyperbolic Systems and Waves, Pirman Publishing, London (1976).

25. Jossi, J.A., Stiel, L.I., and Thodos, G.: "The Viscosity of Pure Substances in the Dense Gaseous and Liquid Phases", AJ.Ch.E. Journal, 8(1):59-62 (1962).

26. Karakas, M., Saneie, S., and Yortsos, Y.: "Displacement of a Viscous Oil by the Combined Injection of Hot Water and Chemical Additive", SPE Reservoir Engineering, 1:391-402 (July 1986).

27. Keyfitz, B.L. and Kranzer, H.C.: "A System of Non-Strictly Hyperbolic Conservation Laws Arising in Elasticity Theory", Archive for Rational Mechanics and Analysis, 72:220-241 (1980).

28. Lawal, A.S.: "Prediction of Vapor and Liquid Viscosities From the Lawal-Lake-Silberberg Equation of State". SPE 15677, presented at the Fifth Symposium on Enhanced Oil Recovery, Tulsa, OK, April, 1986.

29. Lax, P.D.: "Hyperbolic Systems of Conservation Laws: Par II", Cornmunications in Pure and Applied Mathematics, 10:537-566 (1957).

30. Leach, M.P. and Yellig, W.F.: "Compositional Model Studies - $\mathrm{CO}_{2}$ Oil-Displacement Mechanisms", SPEJ, 21:89-97 (1981).

31. Litue, J.E. and Kennedy, H.T.: "A Correlation of the Viscosity of Hydrocarbon Systems with Pressure, Temperature, and Composition", SPEJ, 57-161 (June 1968). 
32. Leob, V.M.: A Model for the Viscosity of Liquid-Liquid Mixsures, PhD thesis, University of Rochester (1973).

33. Lohrenz, J., Bray, B.C., and Clark, C.R.: "Calculation Viscosities of Reservoir Fluids from their Composition", JPT, 6:1171-1176 (1964).

34. Metcalf, R.S. and Yarborough, L.: "The Effect of Phase Equilibria on the $\mathrm{CO}_{2}$ Displacement Mechanism", SPEJ, 19:242-252 (1979).

35. Moler, C.B. and Stewart, G.W.: "An Ngorithm for Generalized Matrix Eigenvalue Problems", SIAM Journal of Numerical Analysis, 10:241-256 (1973).

36. Monroe, W.W.: The Effect of Dissolved Methane on Composition Paths in Quaternary $\mathrm{CO}_{2}$ Hydrocarbon Systems, Master's thesis, Stanford University, Stanford, CA (September 1986).

37. Monroe, W.W., Silva, M.K., Larsen, L.L., and Orr, F.M., Jr.: "Composition Paths in FourCompsnent Systems: The Effect of Dissolved Methane on $\mathrm{CO}_{2}$ Flood Perfo:mance in One Dimension", SPE 16172, presented at the 62nd Annual Technical Conference and Exhibition, Dallas, TX, September 27-30, 1987.

38. Nilson, R.H. and Romero, L.A.: "Self-Similar Condensing Flows in Porous Media", International Journal of Heat and Mass Transfer, 23:1461-1470 (1980).

39. Nutakki, R.: Phase Behavior Calculations for Systems Containing Hydrocarbons and Water, PhD thesis, Stanford University, Stanford, CA (September 1988).

40. Nutakki, R., Wong, T., Firoozabadi, A., and Aziz, K.: Development of General Purpose Simulacors Part 1: Vapor-Liquid Equilibrium Calculations Using Three Equations of State and Accelerated Successive Substitution, Technical Report, Stanford University, Department of Petroleum Engineering (1985).

41. Orr, F.M., Jr.: Simulation of the One-Dimensional Convection of Four-Phase, Four-Component Mixtures, Topical Report DOE/ET/12082-8, New Mexico Petroleum Recovery Research Center, Socorro, NM (Oclober 1980).

42. Pande, K.K.: An Anayltical Method of Describing Crossflow in Layered Systems using the Method of Characteristics, PhD thesis, Stanford University, Deparment of Petroleum Engineering (1988).

42. Peng, D. and Robinson, D.: "A New Two-Constant Equation of State", Industrial and Engineering Chemistry Fundamentals, 15(1):59-64 (1970).

44. Prats, M.: Thermal Recovery, Society of Petroleum Engineers, Dallas, TX (1982).

45. Rathmell, J.J., Stalkup, F.I., and Hassinger, R.C.: “A Laboratory Investigation of Miscible Displacement by Carbon Dioxide", Proceeding of the 46ih Annual Conference (October 3-6 1971).

46. Rauch, J. and Reed, M.C.: "Propagation of Singularities in Non-Strictly Hyperbolic Semilinear Systems: Examples", Communications on Pure and Applied Mathematics, 35:555-565 (1982).

47. Reid, R.C., Prausnitz, J.M., and Sherwood, T.K.: The Properties of Gases and Liquids, McGraw Hill, New York, thind edition (1977).

48. Reynolds, W.C.: Thermodynamic Properties in SI, Department of Mechanical Enginecring at Stanford University, Stanford, CA (1979). 
49. Rhee, H.K., Aris, R., and Amundson, N.R., "On the Theory of Multicomponent Chromatography", Philosophical Transactions of the Royal Society of London, Series A, 267:419-455 (19'70).

50. Schaeffer, D.G. and Shearer, M.: "The Classification of $2 \times 2$ Systems of Non-Strictly Hyperbolic Conservation Laws, With Applications to Oil Recovery", Communications on Pure and Applied Mathematics, 40:1 (1987).

51. Scheidegger, A.E.: Physics of Flow Through Porous Media, MacMillian, London (1957).

52. Schmidt, G. and Wenzel, H.: "A Modified Van der Waals Type Equation of State" Chemical Engineering Science, 35:1503-12 (1980).

53. Sillen, L.G.: Arkive Kemi Mineral Geology, 2:477 (1950).

54. Silva, M.K., Larsen, L.L., and Orr, F.M., Jr.: $\mathrm{CO}_{2}$ Hydrocarbon Phase Behavior in Model Systems, Part 1: $\mathrm{CO}_{2}-C_{1}-C_{4}-C_{10}$, Technical Report 85-31, New Mexico Petroleum Recovery Research Center (1985).

55. Simon, R. and Graue, D.J.: "Generalized Correlation for Predicting Solubility, Swelling and Viscosity Behavior of $\mathrm{CO}_{2}$-Crude Oil Systems", JPT, 1:102-106 (January 1965).

56. Slobod, R.L. and Koch, H.A., Jr.: "High-Pressure Gas Injection-Mechanism of Recovery Increase", Oil and Gas Journal, 84 (1953).

57. Stone, H.L.: "Probability Model for Fstimating Three-Phase Relative Permeability", JPT, 22:214218 (February 1970).

58. Stone, H.L. and Crump, J.S.: "The Effect of Gas Composition upon Oil Recovery by Gas Drive", Trans. AIME, 146:91 (1956).

59. Temple, B.: "Global Solution of the Cauchy Problem for a Class of $2 \times 2$ Nonstrictly Hyperbolic Conservation Laws", Advances in Applied Mathematics, 3:335-375 (1982).

60. Torabezedeh, S.J. and Handy, L.L.: "The Effect of Temperature and Interfacial Tension on Water/ Oil Relative Permeabilities of Consolidated Sands", SPE 12689, presented at the SPE/DOE Fourth Symposium on Enhanced Oil Recovery, Tulsa, OK, April 15-18, 1984.

61. Vargaftik, N.B.: Handbook of Physical Properties of Liquids and Gases, Pure Substances and Mixtures, Hemisphere Publishing Corporation, Washington, 2nd edition (1975).

62. Walas, S.M.: Phase Equilibria in Chemical Engineering, Butterworth Publishers, Boston (1985).

63. Welge, H.J.: "The Linear Displacement of Oil from Porous Media by Enriched Gas", JPT, 3:787. 796 (1961).

64. Welge, H.J.: "A Simplified Method for Computing Oil Recovery by Gas and Water Drive", Trans. $A J M E, 146: 91$ (1952).

65. Yellig, W.F. and Metcalfe, R.S.: "Determination and Prediction of $\mathrm{CO}_{2}$ Minimum Miscibility Pressures", JPT, 32:160-168 (1980).

66. Zapata, V.J. and Lake, L.W.: "A Theoretical Analysis of Viscous Crossflow", SPE 10111, presented at the 56th Annual Conference, San Antonio, Texas, October 5-7, 1981. 


\section{APPENDIX A \\ FLUID PROPERTIES}

\section{A.1 STEAM-OIL-WATER PROBLEM}

The fluid properties for this section were calculated using equations from various sources. The density and enthalpy equations for the water were obtained by fitting a $3^{\text {rd }}$ order polynomial through data obtained from Reynolds (1979) at 2.0 MPa. These results of the curve fit are for the liquid phase,

$$
\begin{aligned}
P_{w}=\left[\begin{array}{ll}
6.94310^{-4} & 2.19810^{-6} t-6.40110^{-9} t^{2}+ \\
& \left.8.06610^{-12} t^{3}\right]^{-1}
\end{array}\right. \\
H_{w}=-1.34053610^{6}+6041210^{3} t-5.7 t^{2}+5.69910^{-3} \beta^{3}
\end{aligned}
$$

and for the vapor phase

$$
\begin{aligned}
& p_{s} \quad=\left[-0.078+4.62210^{-4} t-2.34310^{-7} t^{2}+7.95810^{-11} t^{3}\right]^{-1} \\
& H_{s}=1.311911710^{6}+3.780110^{3} t-1.9 t^{2}+7.88110^{-4} t^{3}
\end{aligned}
$$

The equations for the oil component were taken from a Reid et al. (1977) using the values for $n-C_{20} \mathrm{H}_{42}$. The equations are,

$$
\begin{gathered}
\rho_{0}=1132.59=0.750946 t+3.3739110^{-4} \imath^{2} \\
H_{0}=2.08884510^{5}-79.9557 t+3.4318 t^{2}- \\
1.317110^{-3} \imath^{3}+2.737110^{-7} A^{4}
\end{gathered}
$$

The viscosity equations were taken from the ISCOM commercial simulator supplied by the Computer Modelling Group. The curve for the viscosity of the water vapor is from the internal viscosity tables from the ISCOM simulator. The $0 \%$ salinity brine data is used for the liquid water viscosity. The curve for the base oil is the equation used by ISCOM to model the viscosity of $n-C_{20} \mathrm{H}_{42}$.

$$
\begin{array}{ll}
\mu_{w} & =0.00752 e^{(1384.86 /) t} \\
\mu_{s} & =0.0032+3.810^{-5} t+3.92510^{-13} t^{2}- \\
\mu_{\text {base oil }} & =0.00910^{-16 / 3} \\
\mu_{\text {heavy oil \#1 }}= & 26.5366 \mathrm{e}^{(821.29 / t)} \\
\mu_{\text {heavy oil \#2 }}=0.009 \mathrm{e}^{(4000.0 / t)} \text { (A.11) }
\end{array}
$$




\section{A.2 $\mathrm{CO}_{2}$-OIL-WATER PROBLEM}

\section{A.2.1 Flash Calculations}

Flash calculations for the problem described in $\$ 4.3$ require a three phase flash routine that is robust and relatively quick. Such a routine using existing equations of state is not readily available. An alternative solution is to input phase diagrams at varying temperatures and interpolate points that lie between the input values.

The flash routine is essentially the one used by Orr (1980). For the system studied in this dissertation, an isothermal phase diagram is divided into five regions as illustrated in Figure A.1. The regions are described by entering 15 values for the boundaries of the single phase regions.

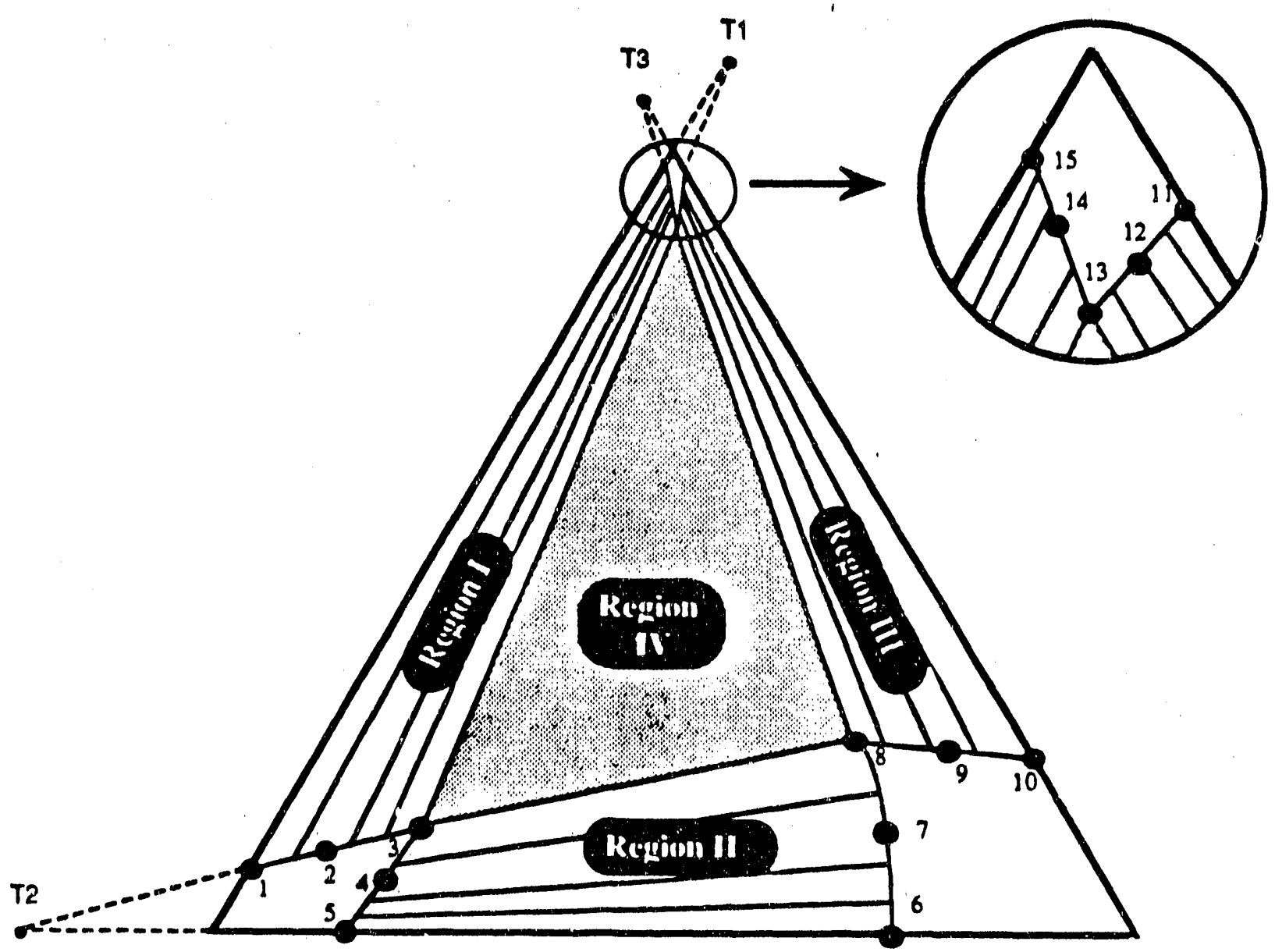

Figure A.1: Isothermal temary phase diagram showing the five regions and the 15 composition points.

The bubble and dew point curves for each region are found by fiting a quadratic equation through the three points representing the boundary. The flash routine assumes that the tie line extensions for the two-phase regions (region I, II, and III) meet at a common point for each region ( $\mathrm{T}_{\mathrm{I}}, \mathrm{T}_{\mathrm{I}}$, and $\left.\mathrm{T}_{\mathrm{II}}\right)$.

The flash calculations are done by performing a linear interpolation between the 15 points on the two phase diagrams surrounding the flash temperature. Once a new set of points is found, the phase boundaries and the tie line extension points are calculated at the new temperature. This new phase dia- 
gram is stored for future use. When one of the existing phase diagrams is at the current temperature no calculation of the phase boundaries is needed. The final flash is carried out using the procedure described by Orr on the interpolated phase diagram.

The flash routine uses a set of 9 phase diagrams ranging from $300 \mathrm{~K}-475 \mathrm{~K}$. All the diagrams were constructed by using a three phase flash routine developed by Nutakkj (1988) to find the boundaries of the three phase region. Additional two phase flashes in regions II and III help to locite points 3.13 on the phase diagrams. A plot of the phase diagrams and the listing of the 15 composition points used to construct these diagrams are shown in Figures A.2-A.10 and Tables A.1-A.9.

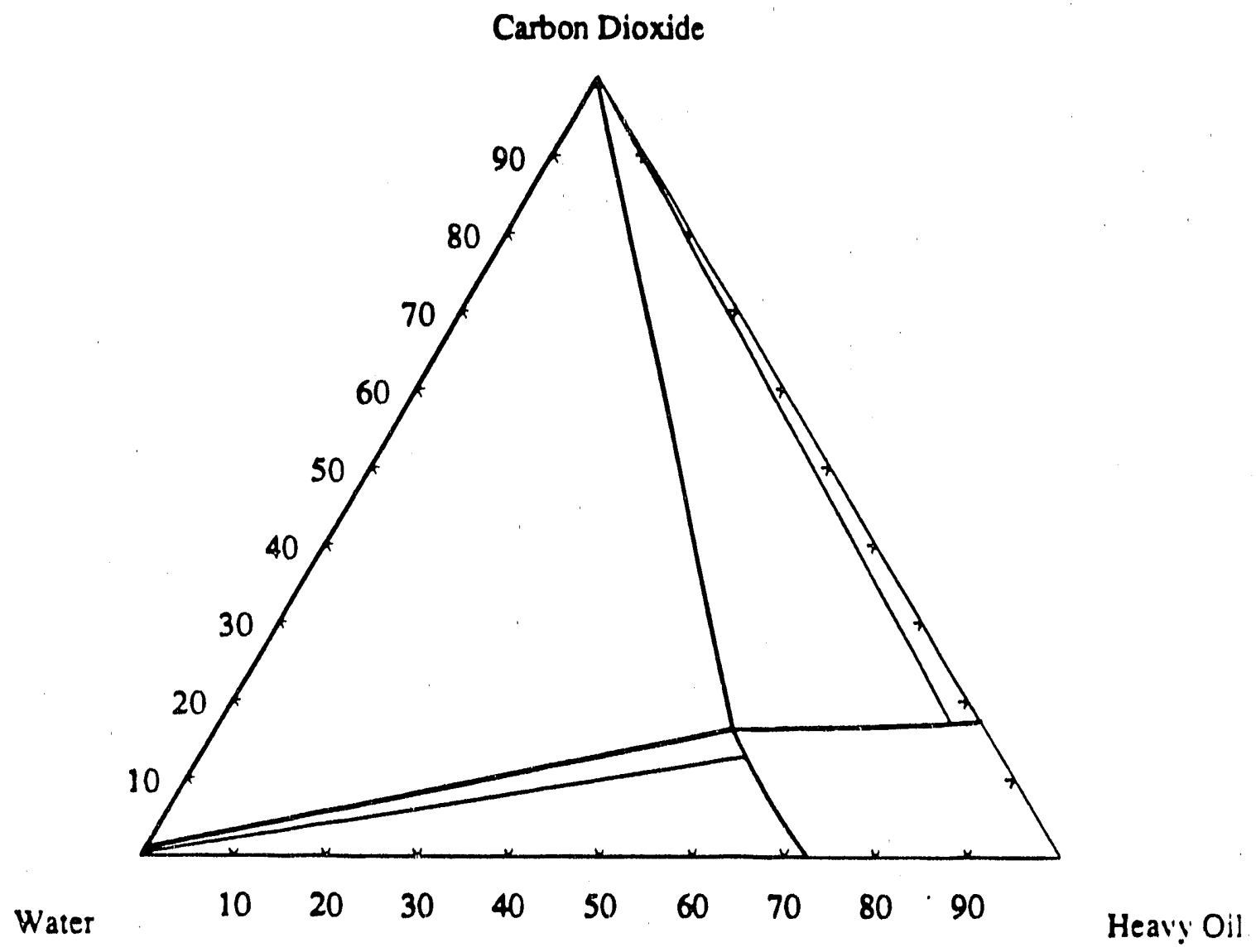

Figure A.2 Isothermal slice of the complete ternary phase diagram for the $\mathrm{CO}_{2}$-oil-water system at $300 \mathrm{~K}$ 


\begin{tabular}{|c|c|c|c|}
\hline D.la & \multicolumn{3}{|c|}{ Composition } \\
\hline Point & CO2 & Oil & Water \\
\hline 1 & 0.01000000 & 0.00000000 & 0.99000000 \\
2 & 0.01008750 & 0.00000005 & 0.98991995 \\
3 & 0.01017500 & 0.00000010 & 0.98982490 \\
4 & 0.00417500 & 0.00000007 & 0.99582493 \\
5 & 0.00000000 & 0.00000060 & 0.99999940 \\
\hline 6 & 0.00000000 & 0.72500000 & 0.27500000 \\
7 & 0.12796600 & 0.59411400 & 0.27792000 \\
8 & 0.16270100 & 0.59411400 & 0.27431800 \\
9 & 0.17009000 & 0.79758700 & 0.03232300 \\
10 & 0.17500000 & 0.82500000 & 0.00000000 \\
\hline 11 & 0.99995900 & 0.00000250 & 0.00003850 \\
12 & 0.99975900 & 0.00000200 & 0.00023900 \\
13 & 0.99793200 & 0.00000100 & 0.00206700 \\
14 & 0.99893200 & 0.00000050 & 0.00106750 \\
15 & 0.99943200 & 0.00000000 & 0.00056800 \\
\hline
\end{tabular}

Table A.1: Input data for $\mathrm{CO}_{2}$-oil-water phase diagram at $300 \mathrm{~K}$ 
$-126$.

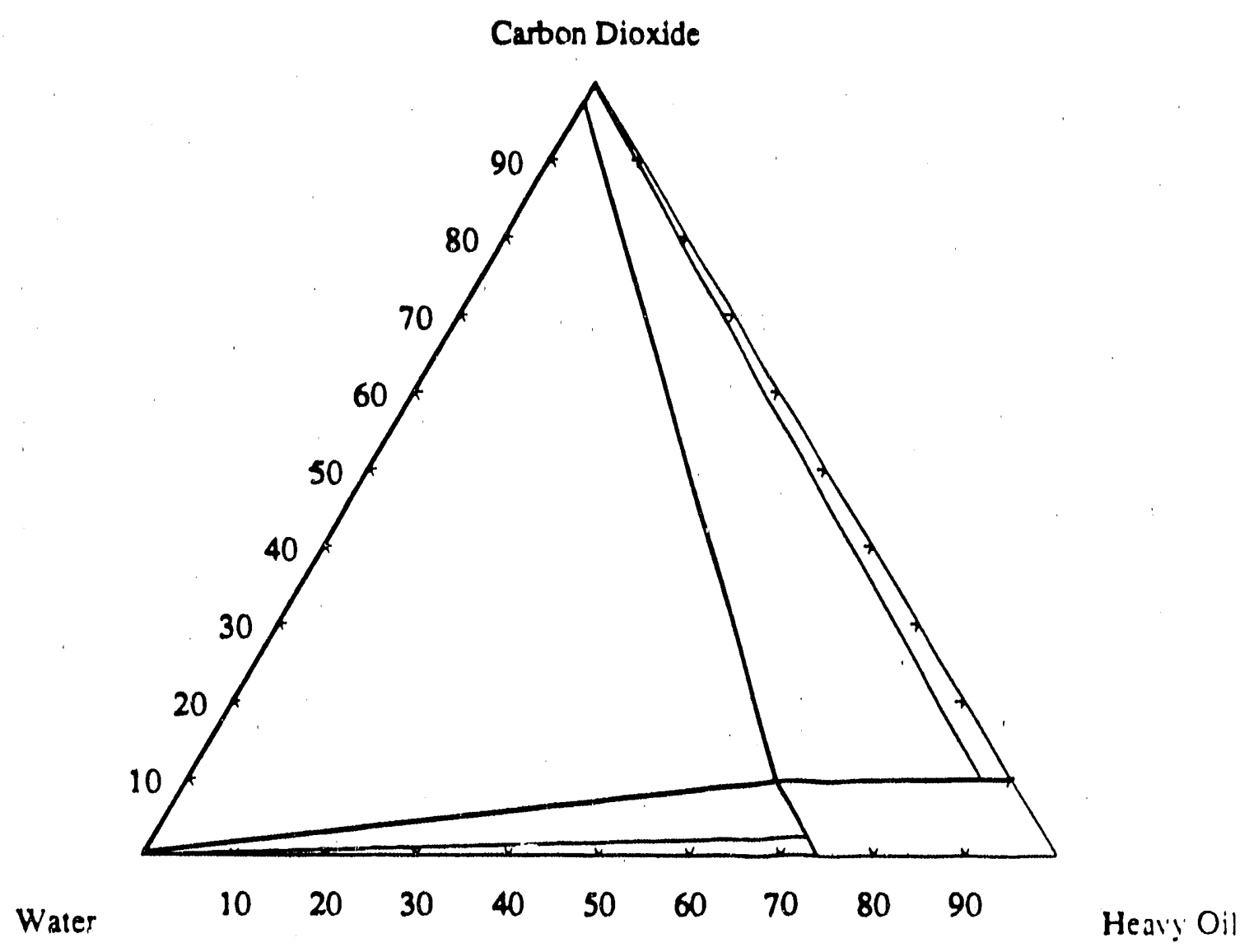

Figure A.3: Isothermal slice of the complete ternary phase diagram for the $\mathrm{CO}_{2}$-oil-water system at $350 \mathrm{~K}$. 


\begin{tabular}{|c|c|c|c|}
\hline Data & \multicolumn{3}{|c|}{ Composition } \\
\hline Point & CO2 & Oil & Water \\
\hline 1 & 0.00300000 & 0.00000000 & 0.99900000 \\
2 & 0.00340550 & 0.00000005 & 0.99899195 \\
3 & 0.00381100 & 0.00000010 & 0.99618899 \\
4 & 0.00093300 & 0.00000003 & 0.99906697 \\
5 & 0.00000000 & 0.00000003 & 0.99999997 \\
\hline 6 & 0.00000000 & 0.73880000 & 0.26120000 \\
7 & 0.02369800 & 0.71636400 & 0.25993800 \\
8 & 0.09623000 & 0.64769400 & 0.25607600 \\
9 & 0.10202200 & 0.86517700 & 0.03280100 \\
10 & 0.10100000 & 0.89900000 & 0.00000000 \\
\hline 11 & 0.99992322 & 0.00007678 & 0.00000000 \\
12 & 0.99664200 & 0.00007400 & 0.00328400 \\
13 & 0.97424200 & 0.00005500 & 0.02570300 \\
14 & 0.97434200 & 0.00005100 & 0.02560700 \\
15 & 0.97440200 & 0.00000000 & 0.2559800 \\
\hline
\end{tabular}

Table A.2: Input data for CO2-oil-water phase diagram at 350K 


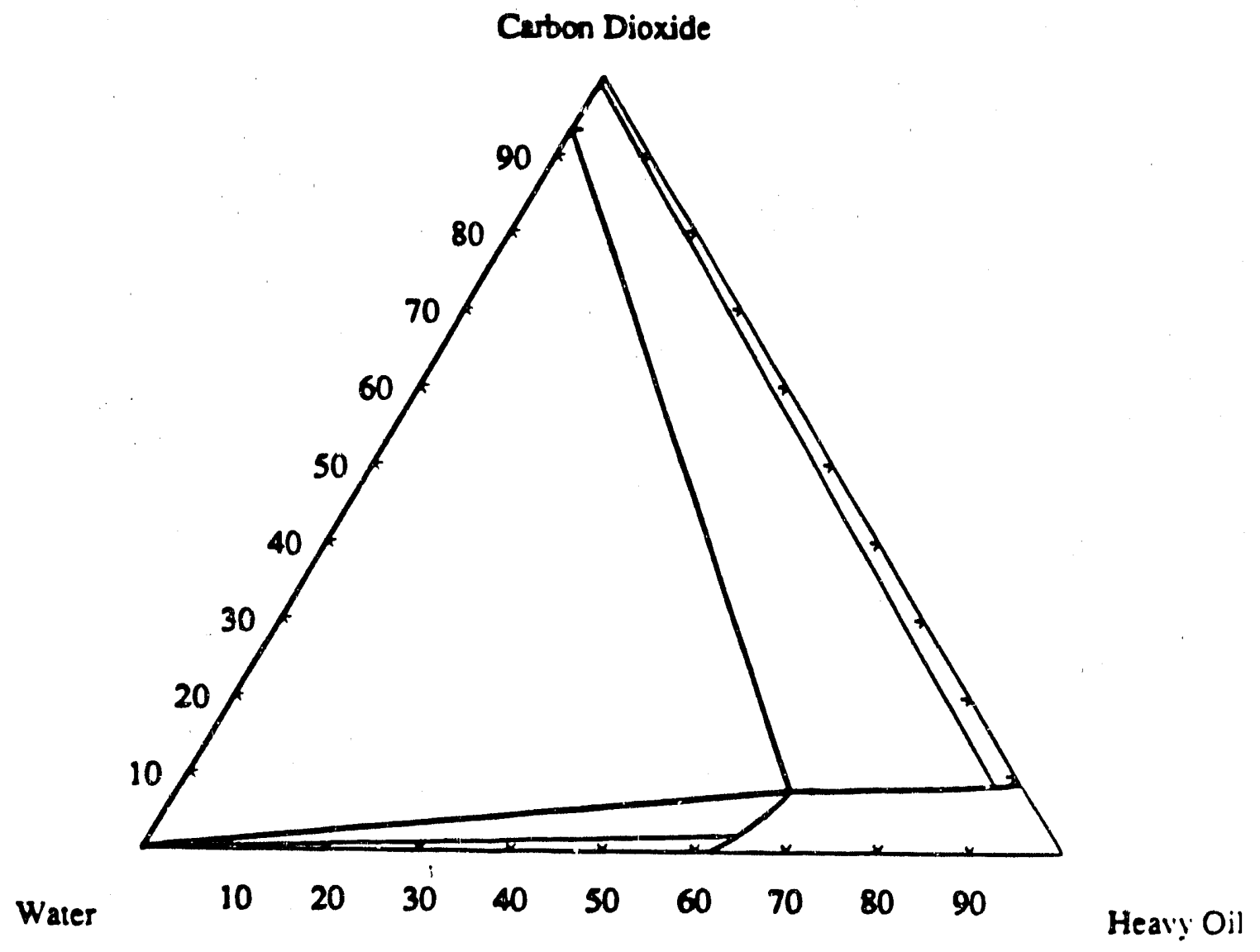

Figure A.4 Isothermal slice of the complete temary phase diagram for the $\mathrm{CO}_{2}$-0il-water system at $375 \mathrm{~K}$. 


\begin{tabular}{|c|c|c|c|}
\hline Dala & \multicolumn{3}{|c|}{ Composition } \\
\hline Point & CO2 & Oil & Water \\
\hline 1 & 0.02000000 & 0.00000000 & 0.99800000 \\
2 & 0.00241100 & 0.00000005 & 0.99858895 \\
3 & 0.00282200 & 0.00000010 & 0.99717790 \\
4 & 0.00193350 & 0.00000003 & 0.99806647 \\
5 & 0.00000000 & 0.00000003 & 0.99999997 \\
\hline 6 & 0.00000000 & 0.61780000 & 0.38220000 \\
7 & 0.02106600 & 0.63760000 & 0.34133400 \\
8 & 0.07809400 & 0.66556200 & 0.25634400 \\
9 & 0.08546900 & 0.88474700 & 0.02978400 \\
10 & 0.08892300 & 0.91106700 & 0.00000000 \\
\hline 11 & 0.99969800 & 0.00030200 & 0.00000000 \\
12 & 0.99184200 & 0.00029500 & 0.00786300 \\
13 & 0.93248500 & 0.00022200 & 0.06729300 \\
14 & 0.93249900 & 0.00009200 & 0.06740900 \\
15 & 0.93250400 & 0.00000000 & 0.06749600 \\
\hline
\end{tabular}

Table A.3: Inpur data for $\mathrm{CO}_{2}$-oil-water phase diagram at 375K 


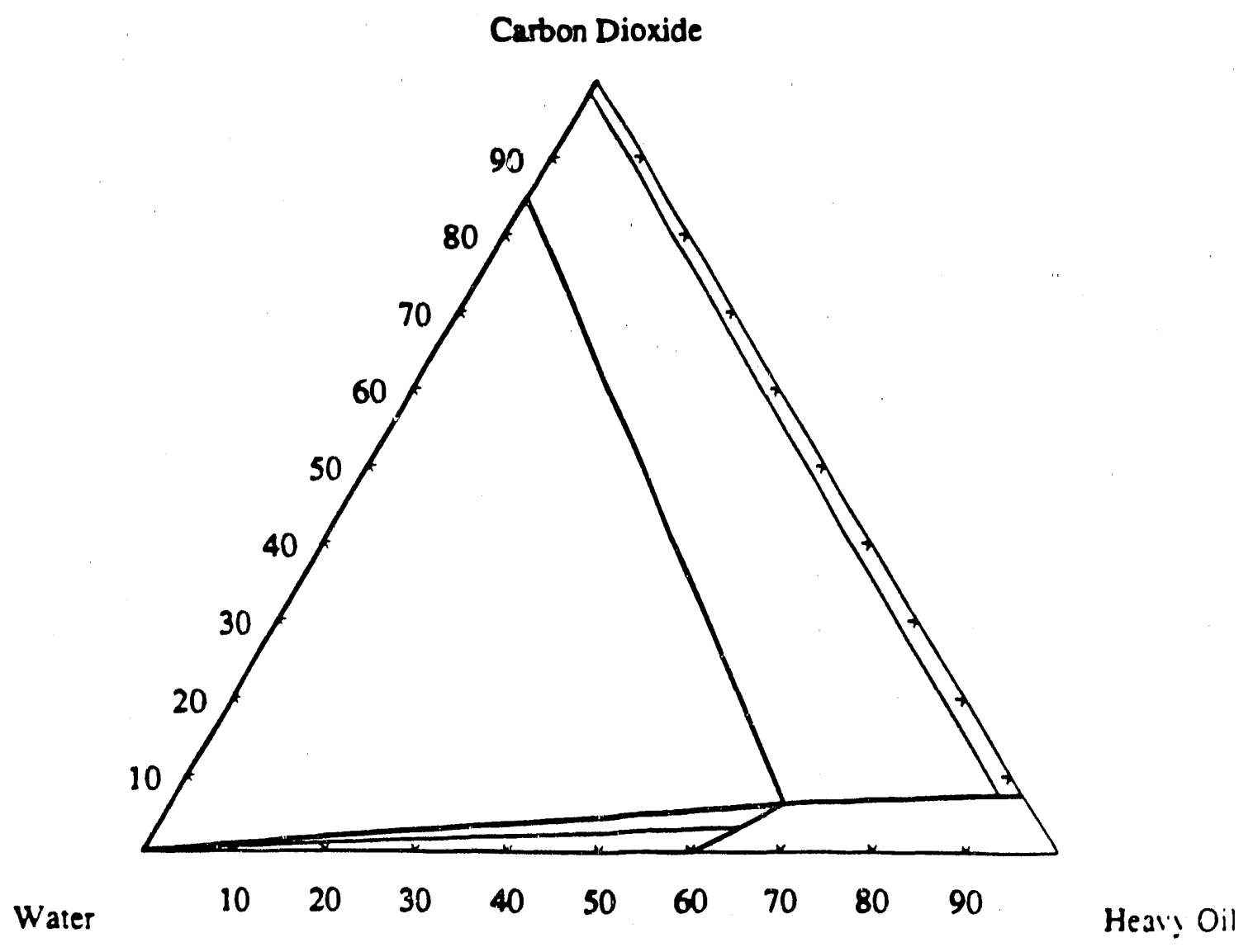

Figure A.5 Isothermal slice of the complete ternary phase diagram for the $\mathrm{CO}_{2}$-oil-water system at $400 \mathrm{~K}$. 


\begin{tabular}{|c|c|c|c|}
\hline Data & & \multicolumn{3}{c|}{ Composition } \\
\hline Poini & CO2 & Oil & Wale \\
\hline 1 & 0.00200000 & 0.00000000 & 0.99800000 \\
2 & 0.00209650 & 0.00000005 & 0.99890345 \\
3 & 0.00219300 & 0.00000010 & 0.99780690 \\
4 & 0.00193100 & 0.00000003 & 0.99806897 \\
5 & 0.00000000 & 0.00000003 & 0.99999997 \\
\hline 6 & 0.00000000 & 0.60232100 & 0.39767900 \\
7 & 0.03262700 & 0.64173600 & 0.32563700 \\
8 & 0.06255600 & 0.67354600 & 0.26389800 \\
9 & 0.07422800 & 0.90110200 & 0.02467000 \\
10 & 0.07532200 & 0.92467800 & 0.00000000 \\
\hline 11 & 0.99900100 & 0.00099900 & 0.00000000 \\
12 & 0.98459700 & 0.00097200 & 0.01443100 \\
13 & 0.84687900 & 0.00071500 & 0.15240600 \\
14 & 0.84691400 & 0.0035500 & 0.15273100 \\
15 & 0.84726800 & 0.00000000 & 0.15273200 \\
\hline
\end{tabular}

Tabis A.4: Inpul daea for $\mathrm{CO}_{2}$-oil-water phase dingram a $400 \mathrm{~K}$ 


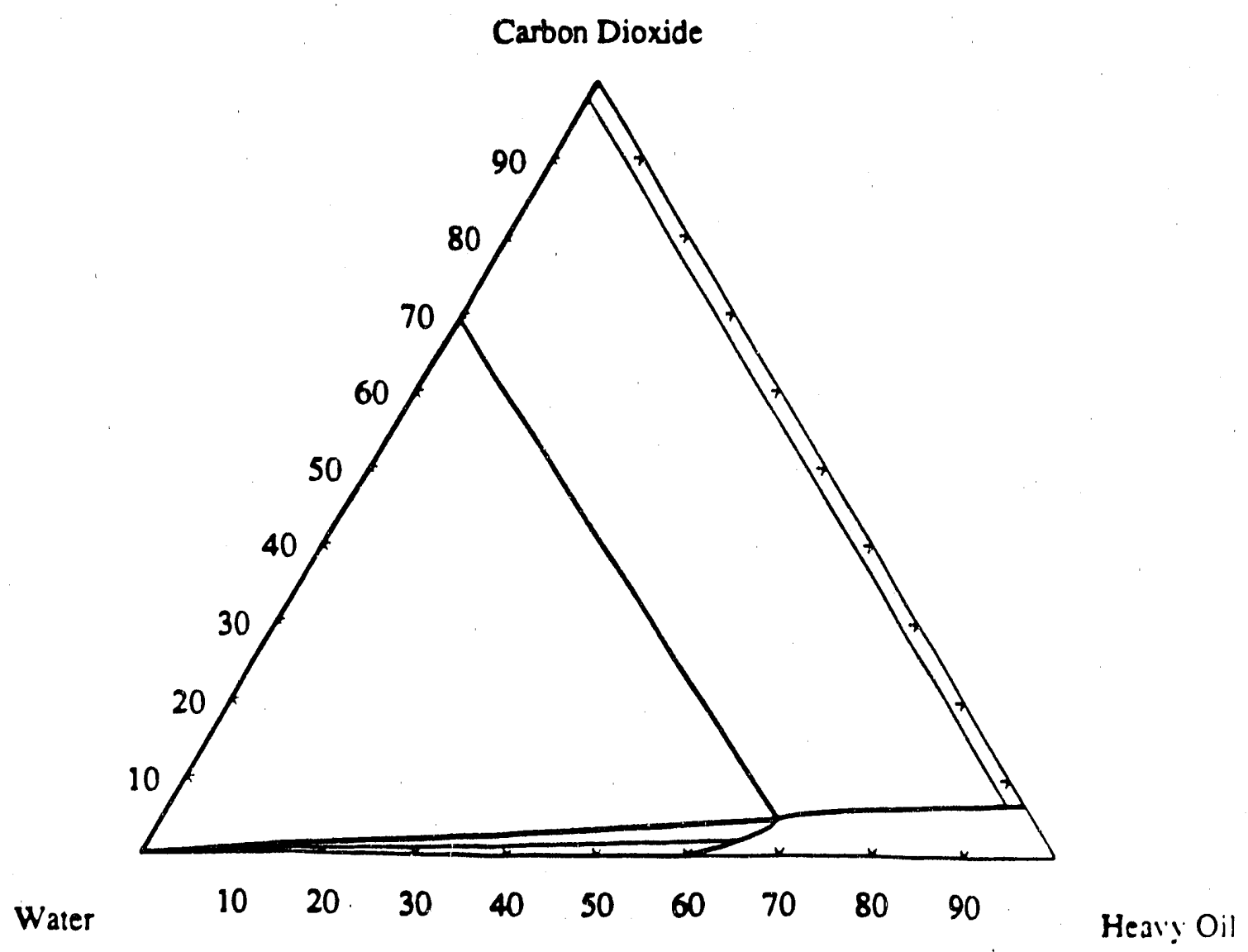

Figure A.6 Isothermal slice of the complete temary phase diagram for the $\mathrm{CO}_{2}$-oil water system at $425 \mathrm{~K}$. 


\begin{tabular}{|c|c|c|c|}
\hline Data & \multicolumn{3}{|c|}{ Composition } \\
\hline Point & CO2 & Oil & Water \\
\hline 1 & 0.00160000 & 0.00000000 & 0.99840000 \\
2 & 0.00165800 & 0.00000002 & 0.99834198 \\
3 & 0.00171600 & 0.00000010 & 0.99828390 \\
4 & 0.00126200 & 0.00000003 & 0.99873797 \\
5 & 0.00000000 & 0.00000003 & 0.99999997 \\
\hline 6 & 0.00000000 & 0.59676200 & 0.40323800 \\
7 & 0.02000000 & 0.64838000 & 0.33162000 \\
8 & 0.04661500 & 0.67525500 & 0.27813000 \\
9 & 0.06641100 & 0.91472800 & 0.01886100 \\
10 & 0.06713700 & 0.98286300 & 0.00000000 \\
\hline 11 & 0.99832050 & 0.00167950 & 0.00000000 \\
12 & 0.97606400 & 0.00262800 & 0.02130800 \\
13 & 0.69094600 & 0.00193500 & 0.30711900 \\
14 & 0.69782100 & 0.00040300 & 0.30177600 \\
15 & 0.69822500 & 0.00000000 & 0.30177500 \\
\hline
\end{tabular}

Tabie A.5: Input data for $\mathrm{CO}_{2}$-oil-water phase diagram a $425 \mathrm{~K}$ 


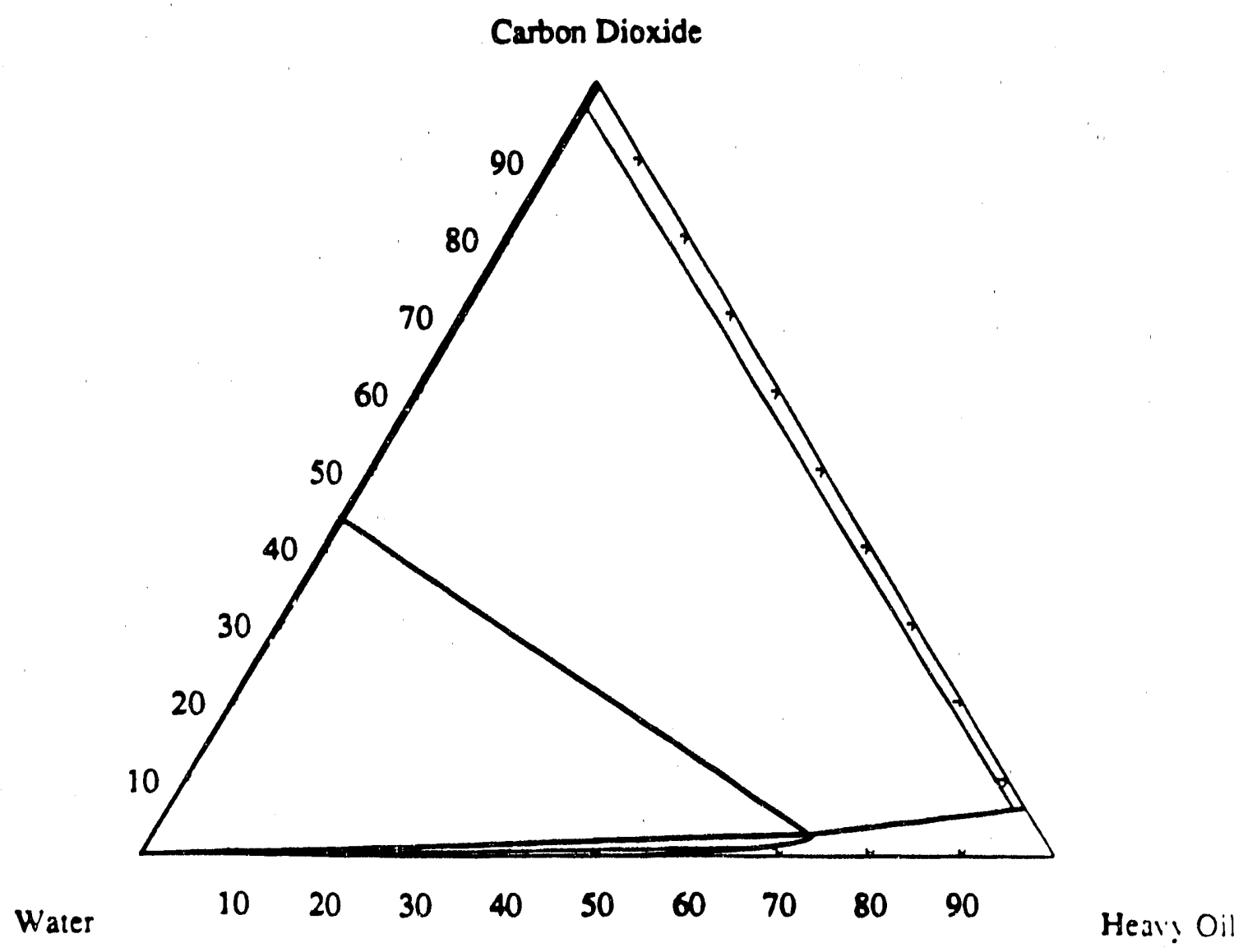

Figure A.7 Isothermal slice of the complete ternary phase diagram for the $\mathrm{CO}_{2}$-oil-water system at $450 \mathrm{~K}$. 


\begin{tabular}{|c|c|c|c|}
\hline Data & \multicolumn{3}{|c|}{ Composition } \\
\hline Point & CO2 & Oil & Waler \\
\hline 1 & 0.00100000 & 0.00000000 & 0.99900000 \\
2 & 0.00106650 & 0.00000002 & 0.99893348 \\
3 & 0.00113300 & 0.00000010 & 0.99886690 \\
4 & 0.00089980 & 0.00000003 & 0.99910017 \\
5 & 0.00000000 & 0.00000003 & 0.99999997 \\
\hline 6 & 0.00000000 & 0.55435000 & 0.44565000 \\
7 & 0.10000000 & 0.66564000 & 0.32436000 \\
8 & 0.02791900 & 0.71998800 & 0.25209300 \\
9 & 0.06084800 & 0.92685900 & 0.01228300 \\
10 & 0.06273000 & 0.93727000 & 0.00000000 \\
\hline 11 & 0.99369200 & 0.00630800 & 0.00000000 \\
12 & 0.96517000 & 0.00628500 & 0.02854500 \\
13 & 0.43347800 & 0.00486200 & 0.56166000 \\
14 & 0.43485670 & 0.00243100 & 0.56271230 \\
15 & 0.43623530 & 0.00000000 & 0.56376470 \\
\hline
\end{tabular}

Table A.6: Input data for $\mathrm{CO}_{2}$-oil-water phase diagram at 450K 


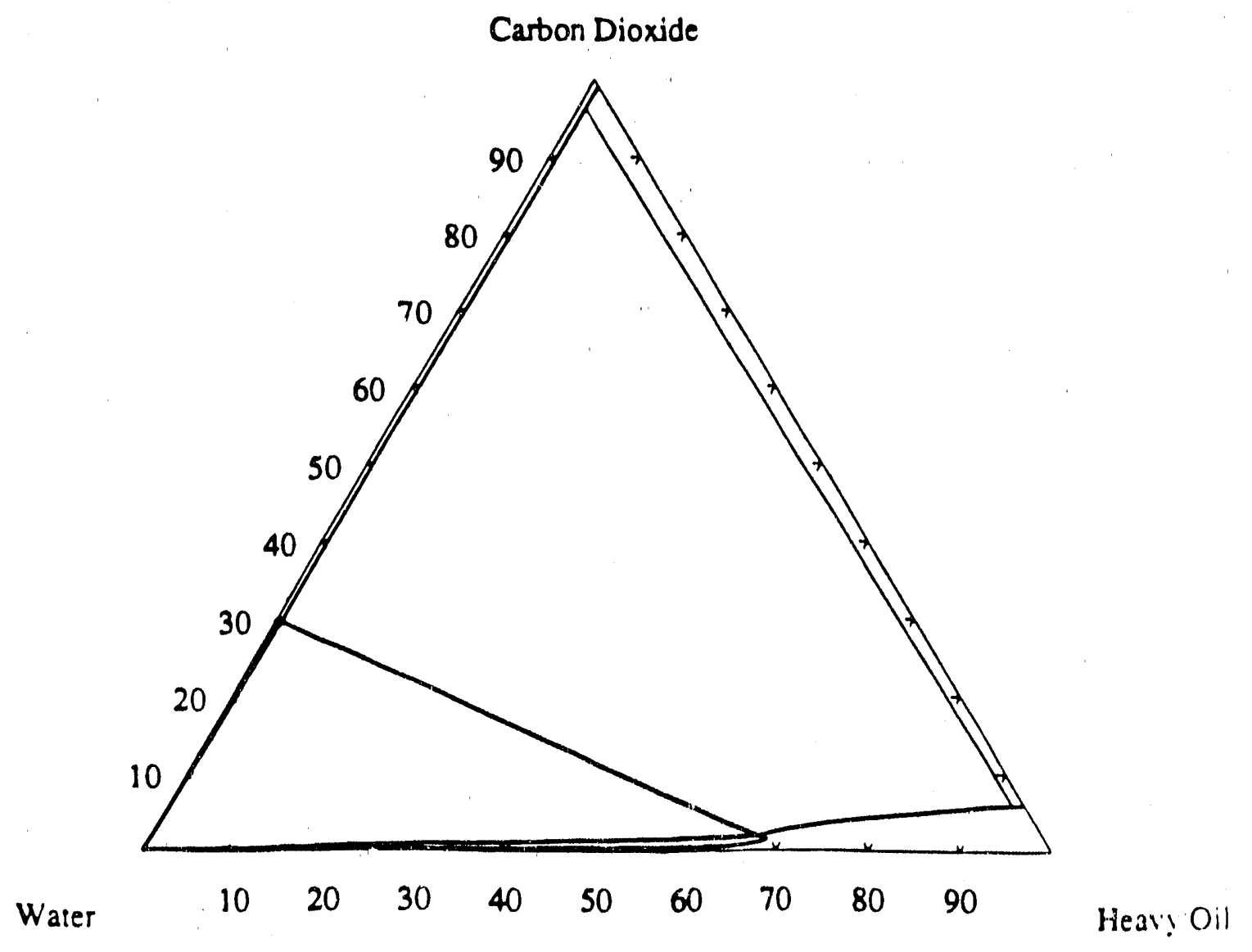

Figure A.8 Isothermal slice of the complete ternary phase diagram for the $\mathrm{CO}_{2}$-oil-water system at $460 \mathrm{~K}$. 


\begin{tabular}{|c|c|c|c|}
\hline Data & \multicolumn{3}{|c|}{ Composition } \\
\hline Point & CO2 & Oil & Water \\
\hline 1 & 0.00069000 & 0.00000000 & 0.99931000 \\
2 & 0.00074350 & 0.00000002 & 0.99256498 \\
3 & 0.00079700 & 0.00000010 & 0.99920290 \\
4 & 0.00038470 & 0.00000003 & 0.99961527 \\
5 & 0.00000000 & 0.00000003 & 0.99999997 \\
\hline 6 & 0.00000000 & 0.57335000 & 0.42665000 \\
7 & 0.00110000 & 0.65621000 & 0.33669000 \\
8 & 0.01855500 & 0.67200400 & 0.30944100 \\
9 & 0.05921500 & 0.92862000 & 0.01216500 \\
10 & 0.06028200 & 0.93971300 & 0.00000500 \\
\hline 11 & 0.99129640 & 0.00870360 & 0.00000000 \\
12 & 0.96280000 & 0.00860000 & 0.02860000 \\
13 & 0.29577900 & 0.00618200 & 0.69803900 \\
14 & 0.29581000 & 0.00301200 & 0.70117800 \\
15 & 0.29583200 & 0.00000000 & 0.70416800 \\
\hline
\end{tabular}

Table A.7: Input data for $\mathrm{CO}_{2}$-oil-water phase diagram at $460 \mathrm{~K}$ 


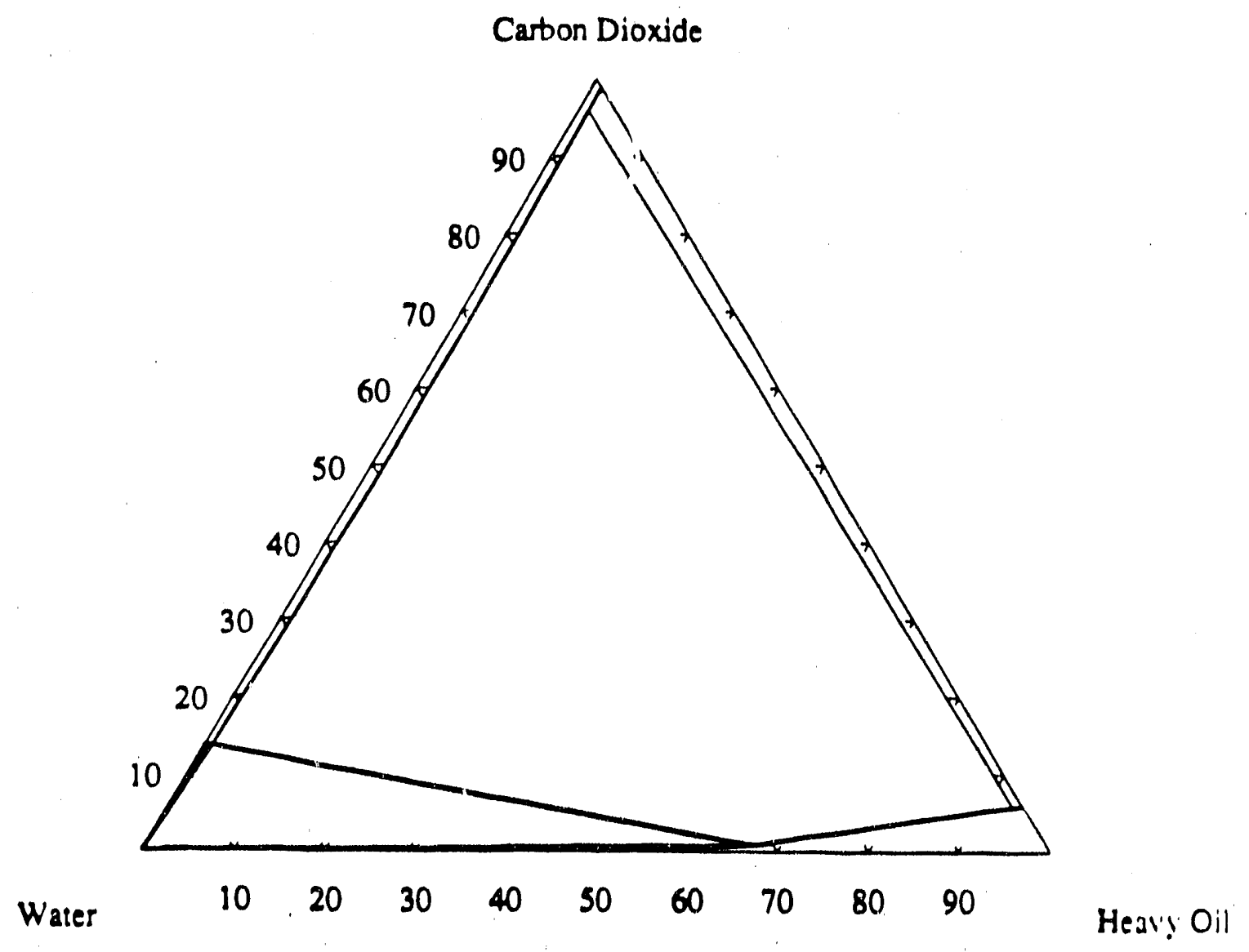

Figure A.9 Isothermal slice of the complete ternary phase diagram for the $\mathrm{CO}_{2}$-oil-water system at $470 \mathrm{~K}$. 


\begin{tabular}{|c|c|c|c|}
\hline Data & \multicolumn{3}{|c|}{ Composition } \\
\hline Point & CO2 & Oil & Water \\
\hline 1 & 0.00030000 & 0.00000000 & 0.99970000 \\
2 & 0.00034050 & 0.00000002 & 0.99965948 \\
3 & 0.00038100 & 0.00000010 & 0.99961900 \\
4 & 0.00023200 & 0.00000003 & 0.39976797 \\
5 & 0.00000000 & 0.00000003 & 0.99999997 \\
\hline 6 & 0.00000000 & 0.49994000 & 0.50006000 \\
7 & 0.00400000 & 0.58750000 & 0.40850000 \\
8 & 0.00841300 & 0.67054100 & 0.32104600 \\
9 & 0.05772300 & 0.93155300 & 0.01072400 \\
10 & 0.05937300 & 0.94062700 & 0.00000000 \\
\hline 11 & 0.99893600 & 0.01106400 & 0.00000000 \\
12 & 0.95835500 & 0.01159400 & 0.03005100 \\
13 & 0.13530800 & 0.00826400 & 0.85642800 \\
14 & 0.13536000 & 0.00543600 & 0.85920400 \\
15 & 0.13538900 & 0.00000000 & 0.86461100 \\
\hline
\end{tabular}

Table A.8: Input data for $\mathrm{CO}_{2}$-oil-water phase diagram at 470K 


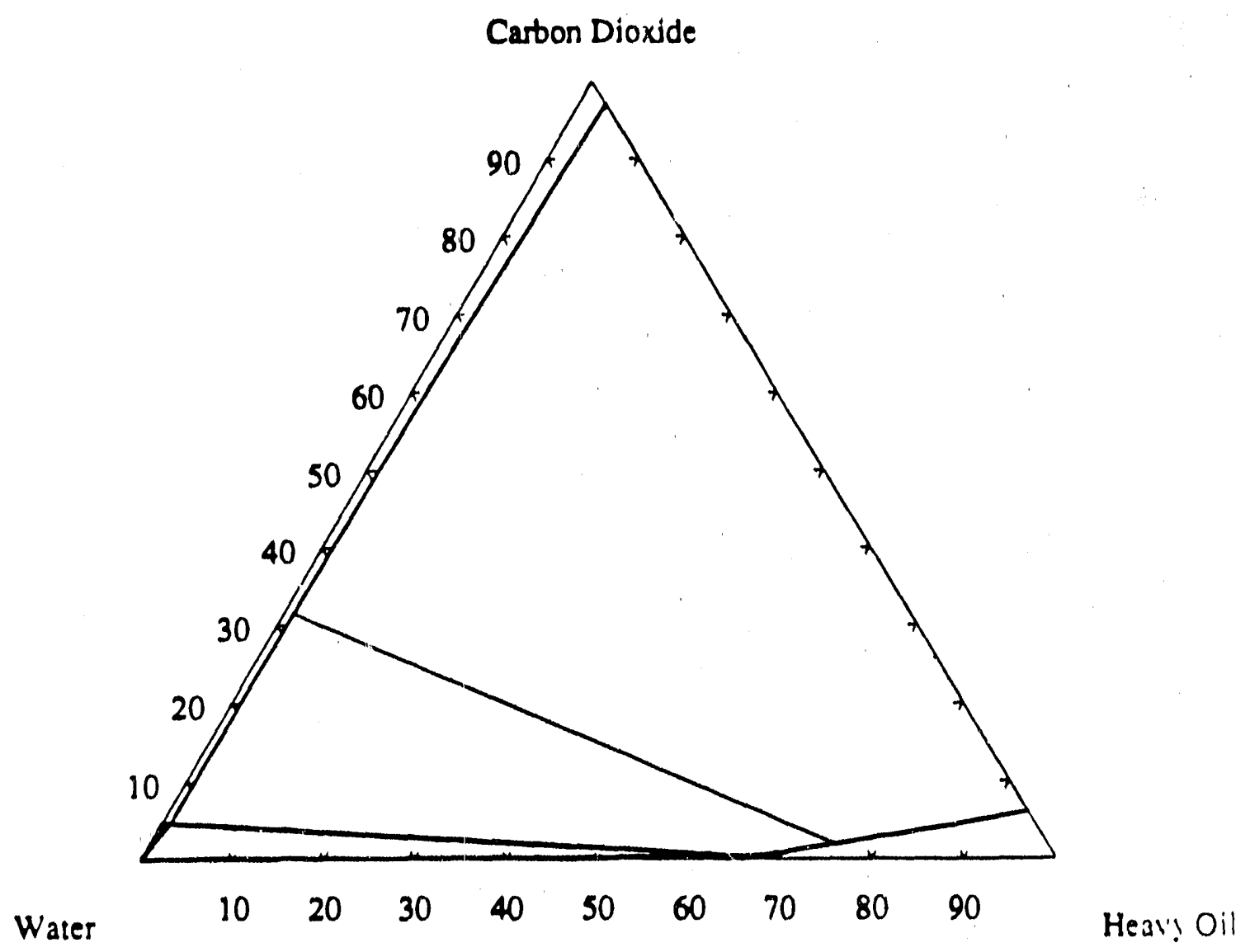

Figure A.10 Isothermal slice of the complete ternary phase diagram for the $\mathrm{CO}_{2}$-oil-water system at $475 \mathrm{~K}$. 


\begin{tabular}{|c|c|c|c|}
\hline Dala & \multicolumn{3}{|c|}{ Composition } \\
\hline Point & CO2 & Oil & Watcr \\
\hline 1 & 0.00008600 & 0.00000000 & 0.99991400 \\
2 & 0.00011050 & 0.00000002 & 0.99988948 \\
3 & 0.00013500 & 0.00000010 & 0.99986490 \\
4 & 0.00007500 & 0.00000003 & 0.99992497 \\
5 & 0.00000000 & 0.00000001 & 0.99999999 \\
\hline 6 & 0.00000000 & 0.43540000 & 0.56460000 \\
7 & 0.00173000 & 0.60839300 & 0.38987700 \\
8 & 0.00287500 & 0.66879200 & 0.32833300 \\
9 & 0.01927700 & 0.75207100 & 0.22865200 \\
10 & 0.06062700 & 0.93937300 & 0.00000000 \\
\hline 11 & 0.96995600 & 0.03004400 & 0.00000000 \\
12 & 0.31565800 & 0.01068700 & 0.67365500 \\
13 & 0.04636200 & 0.00948100 & 0.94415700 \\
14 & 0.04636100 & 0.00474050 & 0.94889850 \\
15 & 0.04636000 & 0.00000000 & 0.95364000 \\
\hline
\end{tabular}

Table A.9: Input data for $\mathrm{CO}_{2}$-oil-water phase diagram at $475 \mathrm{~K}$ 


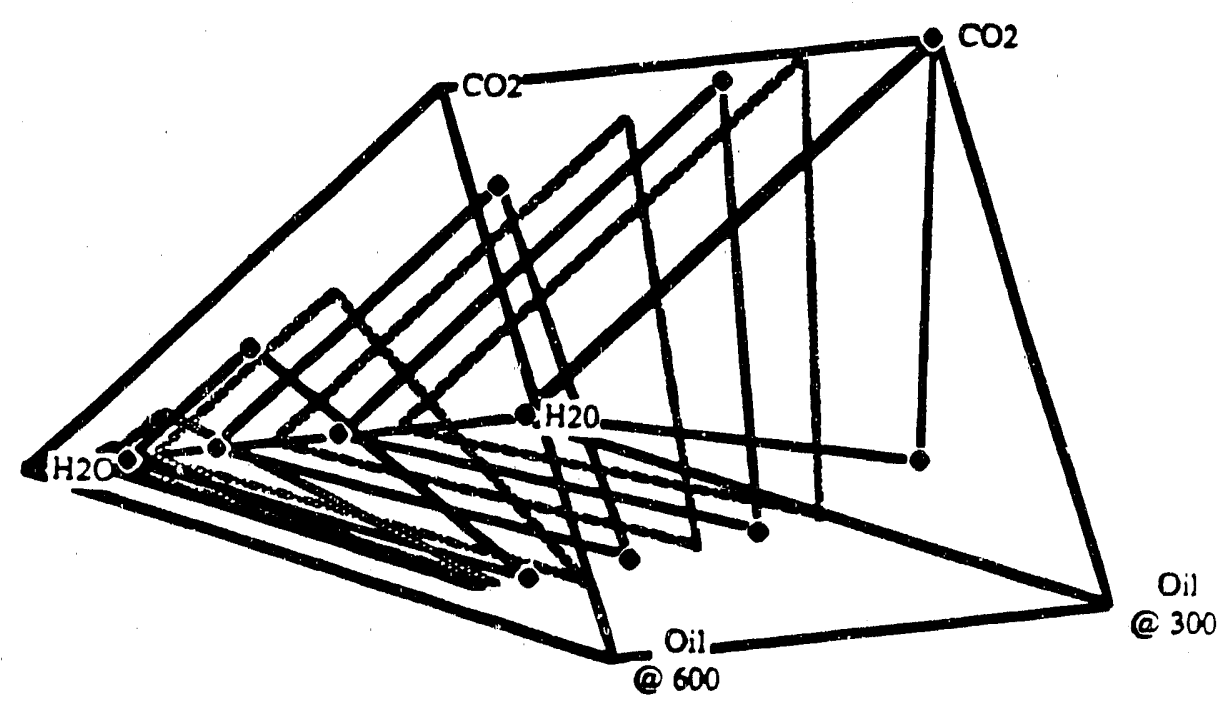

Figure A.11: Stacked temary phase diagrams for the $\mathrm{CO}_{2}$-oil-water system from $300-600 \mathrm{~K}$.

\section{A.2.2 Fluid Properties}

The flash calculation only gives the composition of each phase. The properties of the existing phases are found by applying the Schmidt-Wenzel equation of sate to the phases found by the flash calculation. The Schmidi-Wenzel equation gives improved density calculations compared to the more familiar Peng-Robinson equation of state. The density, saburations and specific molar enthalpies of all the phases are calculated from the phase composition, temperature and pressure.

The viscosity correlations commonly used for calculating viscusities of hydrocarbon mixtures are not very accurate for heavy oils. Specific correlations for mixtures of $\mathrm{CO}_{2}$ and heavy oils have been presented, but none of these correlations take into account a water component. The formula used is an adaptation of the one given by Loeb and is valid when high viscosity contrasts between components are present The mixing rule is given by,

$$
\ln \mu_{\operatorname{mix}}=\sum_{i=1}^{n_{i}} x_{i} \ln \mu_{i}
$$

The equations used to model the pure component viscosities of the water and oil phases are the same as in the steam-oil-water case. The $\mathrm{CO}_{2}$ viscosities are taken from Vargafik (1975) at a pressure of 17.23 MPa [250 psia]. The equations used for the pure components are:

$$
\begin{aligned}
& \mu_{\mathrm{CO}_{2}, v}=-0.14658+0.0017199 \sqrt{ } t \\
& \mu_{\mathrm{CO}_{2}, l}=0.0441259 e^{(578.08 / t)} \\
& \mu_{O i l, v}=0.80+0.0035 \sqrt{ } \mathrm{l} \\
& \mu_{O i l, l}=0.132836 e^{(821.29 / t)}
\end{aligned}
$$




$$
\begin{aligned}
\mu H_{2} O . v & =0.0032+3.810^{-5} t+3.92510^{-13} t^{2}- \\
3.00910^{-16} t^{3} & \\
\mu_{H_{2} O . l}= & 0.00752 e^{(1384.86 / t)}
\end{aligned}
$$




\section{APPENDIX B \\ ENTHALPY BALANCE ACROSS A DISCONTINUITY}

The transition from the single phase to the two-phase region must occur via a shock as well as the transition from a two-phase to a three-phase region. This is because the velocities as given by the eigenvalues are discontinuous at the phase boundary. The material balance tells us that shocks entering or exiting a two phase region from a single phase boundary condition (Nem must) lie along the tie-line extension that passes through the single phase composition (Dumort et al. 1984).

Applying the same procedure to the enthalpy balance indicates how the temperawure changes as the solution jumps from the single phase to the two phase region. Consider a system with a single phase on the downstream side of a shock and two phases on the upstream side. The two phases will be gas and oil. Since there can be no enthalpy accumulation inside the shock, the enthalpy on the downstream side must equal the enthalpy on the two-phase side of the shock. Writing an enthalpy balance yields,

$$
\begin{aligned}
& \rho_{0}^{-} S_{0}^{-} H_{0}^{-}+\rho_{g}^{-} S_{g}^{-} H_{g}^{-}+\left(\frac{1-\phi}{\phi}\right) \rho_{m} H_{m}^{-}-\rho^{+} H^{+}-\left(\frac{1-\phi}{\phi}\right) \rho_{m} H_{m}^{+}= \\
& \frac{u^{-}}{\phi}\left[\rho_{0}^{-} f_{0}^{-} H_{0}^{-}+\rho_{g}^{-} f_{g}^{-} H_{l}^{-}\right]-\frac{u^{+}}{\phi} \rho^{+} H^{+}
\end{aligned}
$$

where superscript $(+)$ refers to the downstream side of the shock, superscript $(--)$ refers to the upstream side, and the subscripts $O$ and $\&$ refer to the oil and gas phases, respectively.

Equation B.l can be rearranged and solved for the upstream enthalpy content $\rho^{+} H^{+} \omega$ give,

$$
\begin{gathered}
\frac{1}{\left(1-\frac{u^{+}}{\phi}\right)}\left\{\left[S_{0}^{-}-\frac{U^{-}}{\phi} f_{0}^{-}\right] \rho_{0}^{-} H_{0}^{-}+\left[S_{e}^{-}-\frac{u^{-}}{\phi} f_{g}^{-}\right] \rho_{i}^{-} H_{g}^{-}+\right. \\
\left.\left(\frac{1-\phi}{\phi}\right) \rho_{m} C_{m}\left(T^{-}-T^{+}\right)\right\}=\rho^{+} H^{+}
\end{gathered}
$$

The final term on the left side of Eq. B.2 represents the extra contribution of the matrix. As the porosity goes $t 0$ unity or the heat capacity of the matrix goes 10 zero, the enthalpy balance is similar to the component balances. The enthalpy in the two phase region is found from linear combination of the single phase enthalpy.

When the matrix is allowed to take up or release enthalpy across a shock, the speed of the shock is slowed or increased depending on the relationship of the upstream to downstream conditions. The flash that calculates the conditions on the two phase side of the shock is not a strictly isenthalpic nash. The wotal enthalpy of the flashed nuids plus the matrix must remain constant during the flash. The deviation from the traditional isenthalpic flash is quantified by the $p_{m} C_{p_{m}} \Delta T$ term in Eq. B.2. 


\section{APPENDIX C COMPLEX EIGENVALUES}

Complex eigenvalues have been observed by Fayers (1987) and later, Gorell (1988) in the phase space where three phase flow is present. The complex eigenvalues indicate that the problem is no longer hyperbolic, but elliptic. Fayers reports that the elliptic regions cover only a small area of the three phase space. The complex regions are shown to occur when the oil viscosity is close to that of the aqueous. phase. When the oil viscosity is much greater, no elliptic regions appear.

Gorell noted the existence of complex eigenvalues in regions where trapping occurs. The complex eigenvalues were attributed to the assumption that only oil is trapped, which is not always physically correct. The paths in this region were integrated using only the real parts of the eigenvalues and eigenvectors.

Glimm (1986) argues that the elliptic region introduces a linear instability which is stabilized by the non-linear considerations. A solution that is forced to begin in the elliptic region will exit the region via a shock. The solution will not retum unless the boundary conditions are force the solution to return. He further argues that a solution not in the elliptic region will not enter the region unless the initial conditions are in the elliptic region.

Bell et.al. (1986) conducted a numerical study of the elliptic region. By adjusting the fractional flow relationships in a three phase immiscible system, they maximized the elliptic region in the saturation space. One dimensional finite difference simulations were conducted with boundary conditions that were in various locations surrounding and within the elliptic region. The results indicate that unless one of the boundary conditions was inside the elliptic region, the solution path avoided this region altogether. This even occured when the initial and injection conditions were located near, but on opposite sides of the elliptic region.

Figures $C .1$ and C. 2 indicate the regions in three phase space where complex eigenvalues were found for two different relative permeability models and two sets of permeability exponents. The complex regions are confined to regions near the zero oil isoperm. The solutions presented in this model avoid these elliptic regions allogether. 


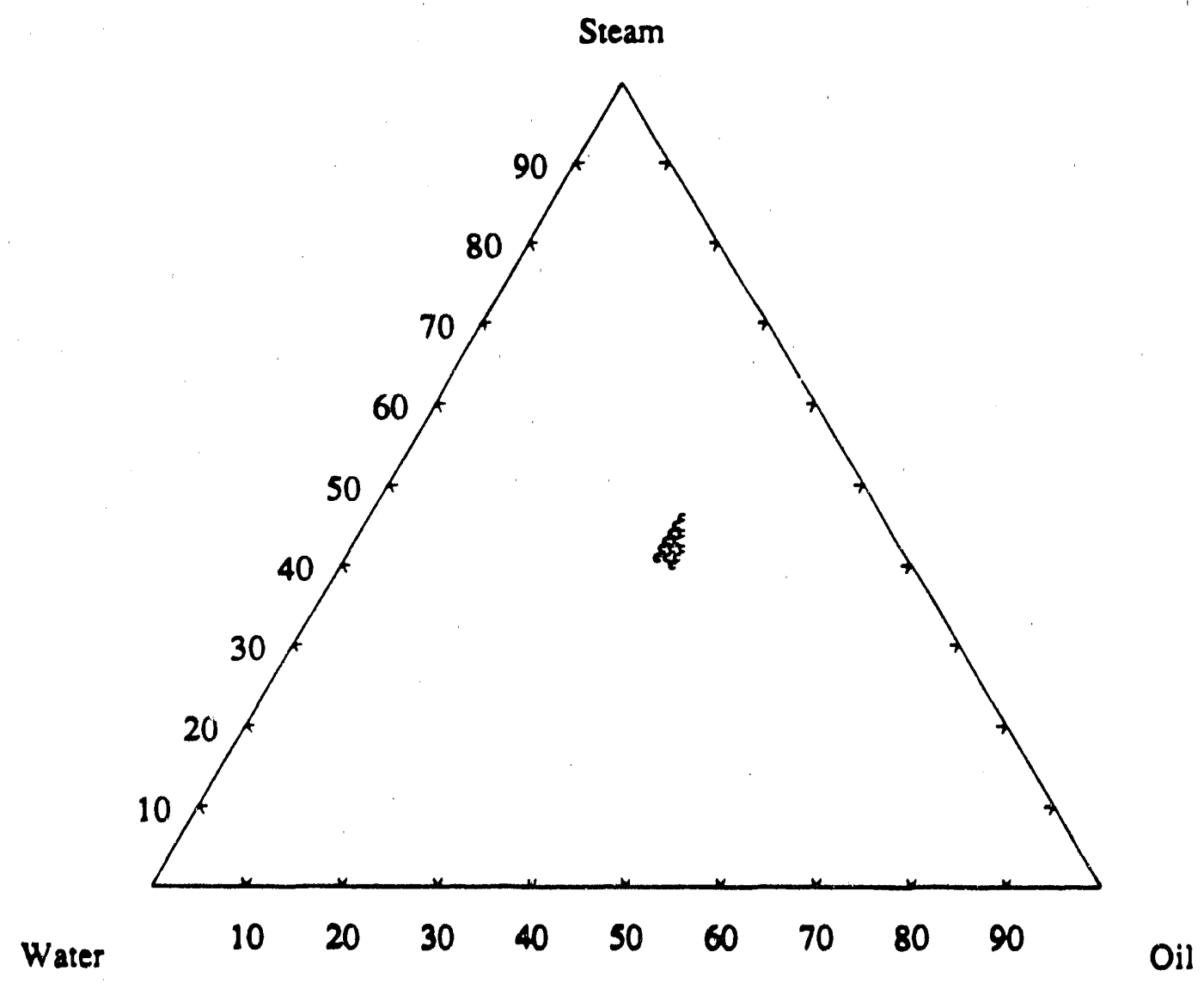

Figure C.1: Region of complex eigenvalues using SLone's model II with $\alpha_{g}=1.0, \alpha_{w}=3.0, \alpha_{\text {ow }}=$ 2.0 , and $\alpha_{l g}=2.0$. 


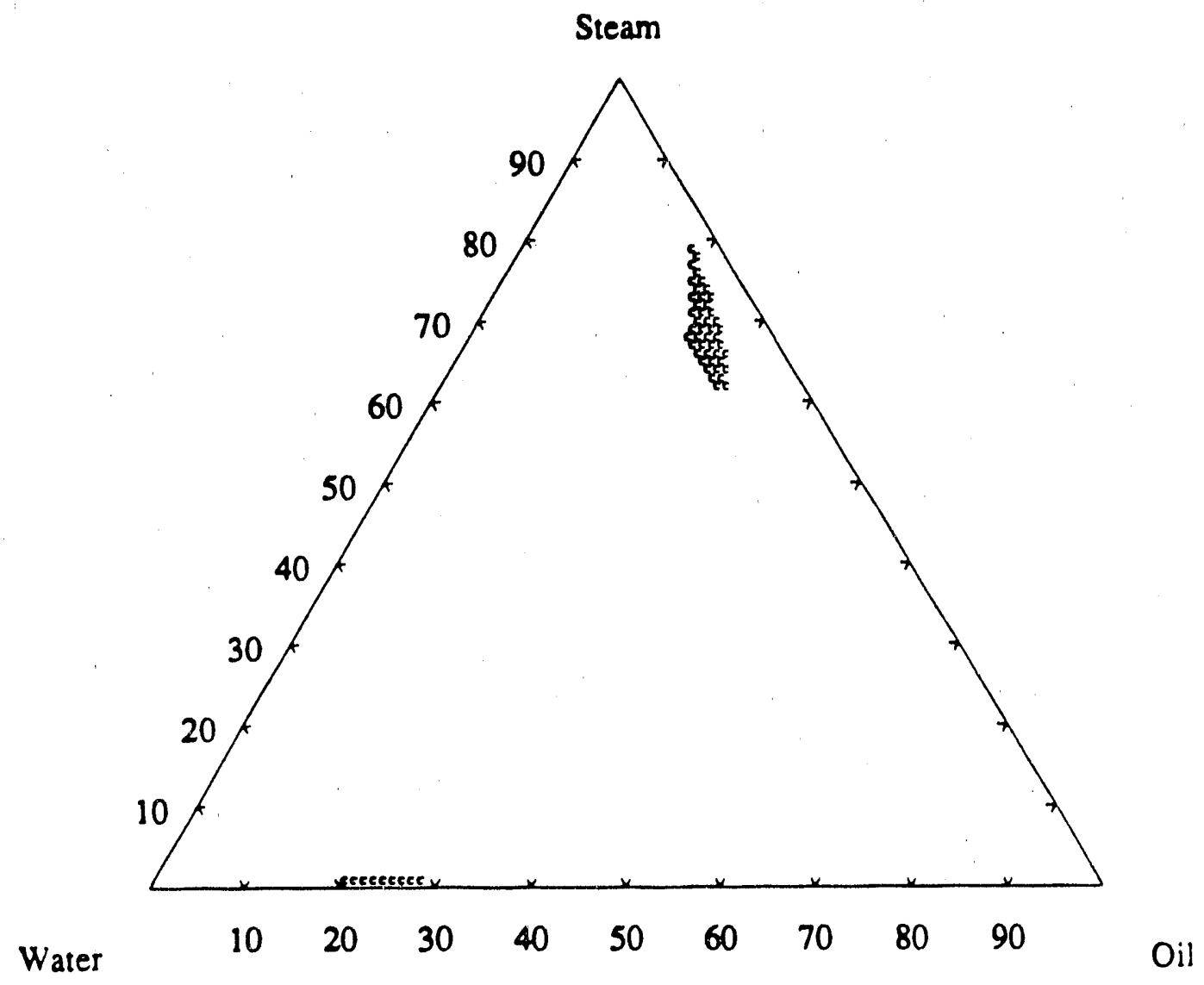

Figure C.2: Region of complex eigenvalues using Stone's model II with $\alpha_{g}=1.0, \alpha_{w}=2.0, \alpha_{o w}=$ 2.0. and $\alpha_{18}=2.0$. 


\section{APPENDIX D \\ EXCEL SPREADSHEETS}

This appendix contains the EXCEL spreadsheets used to calculate the steam-water profiles found in 84.1. The spreadsheet is divided into four sections. The upper section, rows $1-25$ contain the important information on the system. The matrix properties and boundary conditions are entered in this section. Results of the shock velocities and heights are reponted in this area of the worksheet.

The second section calculates the saturation profiles using the relationship given by Eq. 4.7. The saturation profile is divided into fifty equally spaced points between the saturations at the trailing and leading shocks.

The third and fourth sections contain details on the calculation of the trailing and leading shocks, respectively. The calculation is done by 25 bisections over a user specified interval. The final value of the objective function, $\lambda^{I I}-\Lambda_{S}$, is reported under the shock conditions.

\section{D.1 Cell Formulas}

This section of the appendix contains a cell by cell listing of the four different areas of the spreadsheet. This information should be enough to reconstruct the worksheet from scratch. The initial area is the input and output area. This area is used to enter the parameters and read the resulting conditions for the trailing and leading shocks.

Numbers appear in the cells that are used as inputs to the spreadsheet. The cells with formulas are either calculated input conditions or results.

\section{D.2 Changes in Injection Temperature}

These worksheets examine the effect of injection temperature on the saturation profiles. The injection temperature is entered in cell B22. The section contains 11 worksheets with the injection temperature ranging from $500-1000 \mathrm{~K}$, in $50 \mathrm{~K}$ intervals. 
Spreadsheet for Calculation of Stream-Water Saturation Profiles

Injection Temperature $500^{\circ} \mathrm{K}$
Inlibl Temperature

$350 \cdot \mathrm{K}$
Matrix Beat Capacily

1.0000 (kJ/kg)

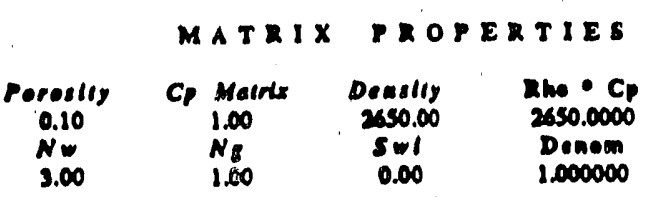

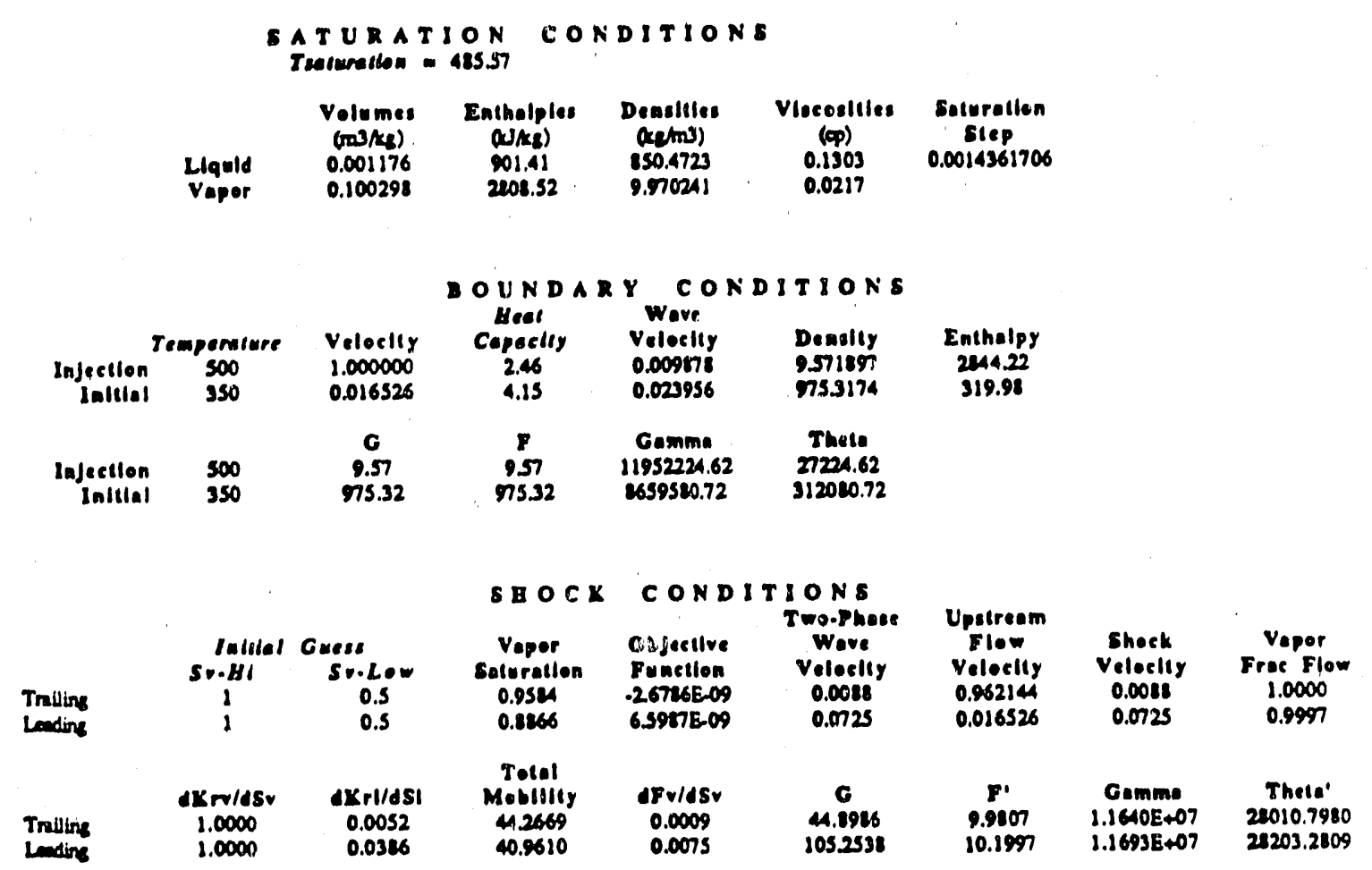

MATERIAL BALANCES

\begin{tabular}{|c|c|c|c|c|c|c|}
\hline Mase & $\begin{array}{l}\text { Inside } \\
90.985248 \\
890325527\end{array}$ & $\begin{array}{c}\text { Inllial } \\
\text { n.531740 } \\
\text { 265958.0720 }\end{array}$ & $\begin{array}{c}\text { Injected } \\
9.571597 \\
27204.6208\end{array}$ & $\begin{array}{l}\text { Preduced } \\
16.118375 \\
\text { 5157.S3S4 }\end{array}$ & $\begin{array}{l}\text { Delence } \\
0.0000 \\
.7 .3953\end{array}$ & $\begin{array}{c}\text { Error } \\
0.0000 \% \\
0.0008 \%\end{array}$ \\
\hline
\end{tabular}


Spreadsheet for Calculatinn of Stream-Water Saturation Profiles

Injectlon Tumperalure

$500 \cdot \mathrm{K}$
Inlical Temperalure

$350 \cdot \mathrm{K}$
Motrix Beas Copacily

1.0000 (kJ/kg)

\section{SATURATION PROPILE}

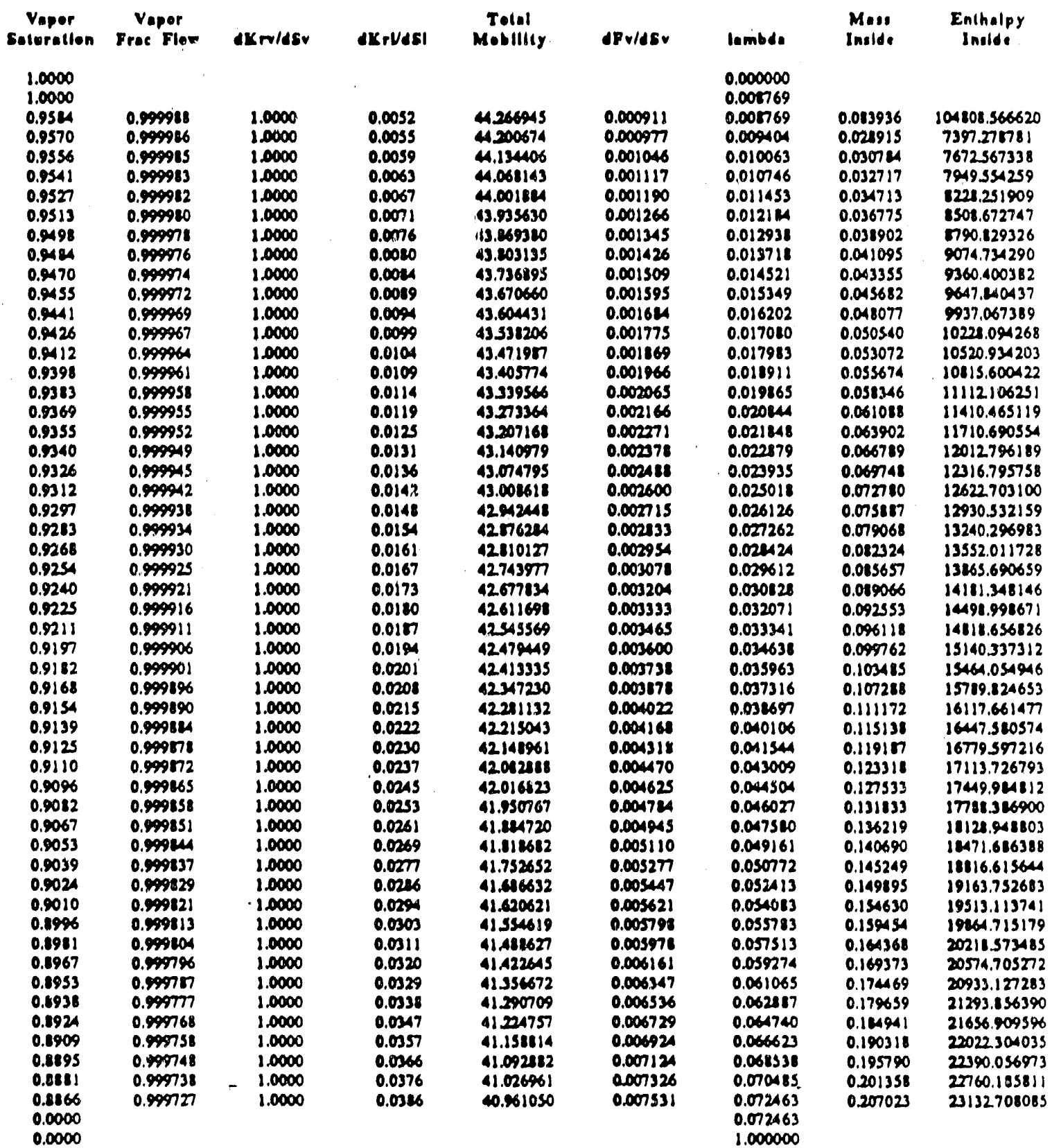


Spreadsheet for Calculation of Stream-Water Saturation Proftles

Injection Temperalure $500 \cdot \mathbf{K}$
Inlibl Temperalure

sso $\cdot \mathbf{K}$
Molrlx Heat Capucily

$1.0000\left(\mathrm{~kJ} / \mathrm{k}_{\mathrm{g}}\right)$

TRAILING SBOCK CALCULATIONS

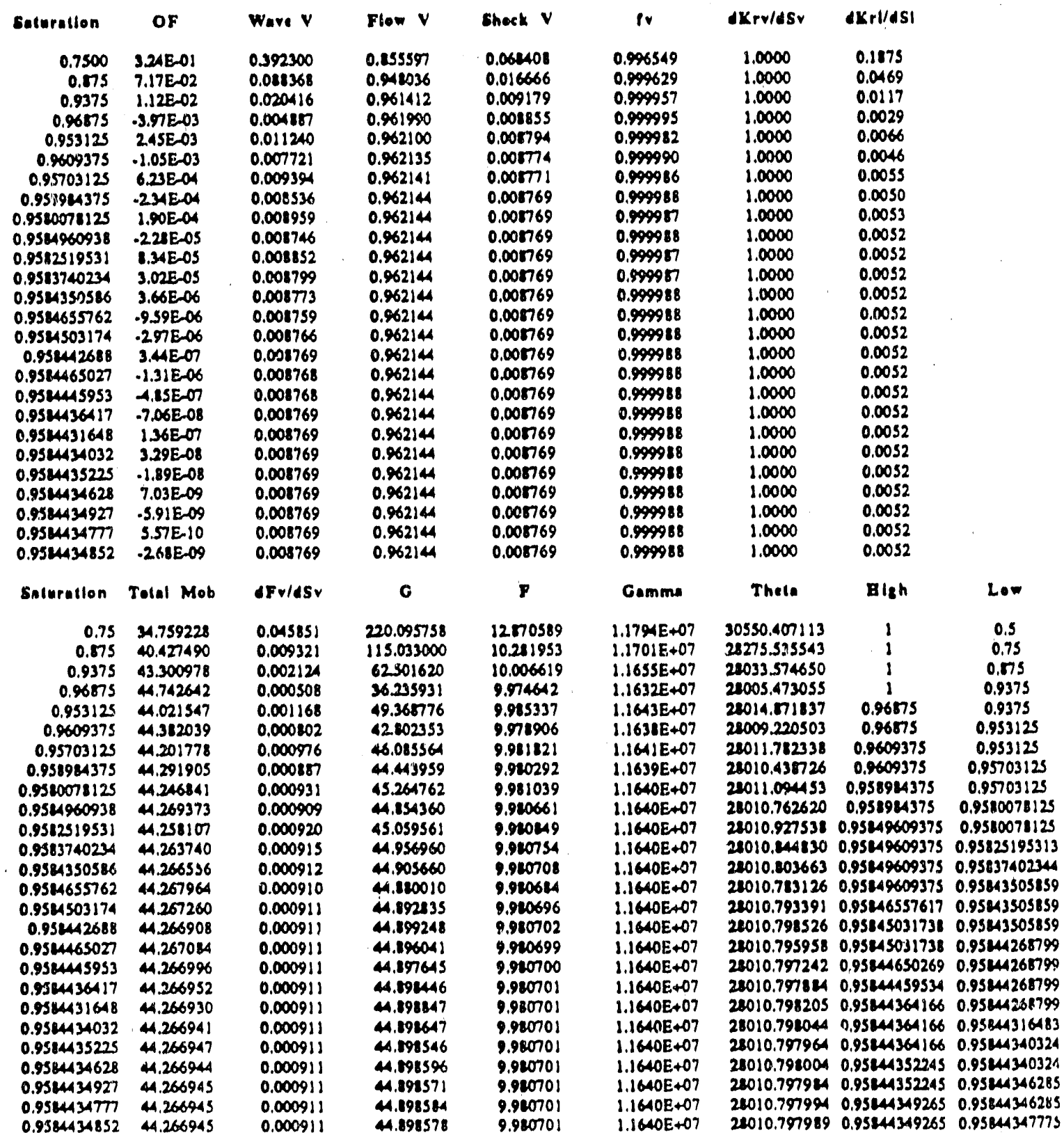


Spreadsheet for Calculation of Stream-Water Saturation Profiles

Injoction Temperoture $\operatorname{son} \bullet \mathbf{K}$
Inllial Temperalure 380 K
Matrix Bout Capoelty

$1.0000\left(\mathrm{~kJ} / \mathrm{k}_{\mathrm{t}}\right)$

LEADING SIOCK CALCULATIONS

\begin{tabular}{|c|c|c|c|c|c|}
\hline Ceturatlen & op & Wove V & Flow V & Shoek V & iv \\
\hline 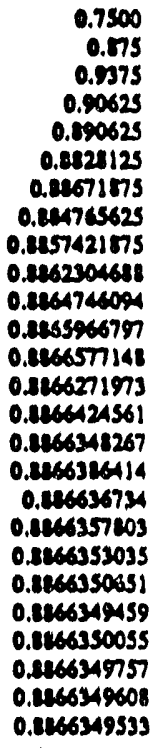 & 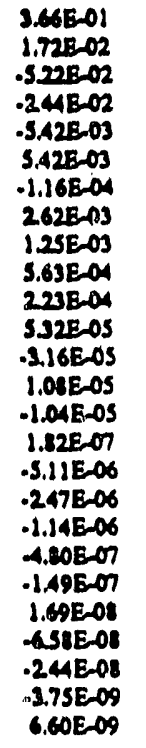 & $\begin{array}{l}0.411153 \\
0.089613 \\
0.020432 \\
0.041117 \\
0.067045 \\
0.077644 \\
0.072447 \\
0.075046 \\
0.073709 \\
0.073026 \\
0.072646 \\
0.072516 \\
0.072432 \\
0.072474 \\
0.072453 \\
0.072463 \\
0.072458 \\
0.072461 \\
0.072462 \\
0.072463 \\
0.072463 \\
0.072463 \\
0.072463 \\
0.072463 \\
0.072463 \\
0.072463\end{array}$ & $\begin{array}{l}0.012531 \\
0.016536 \\
0.016673 \\
0.016551 \\
0.016527 \\
0.016527 \\
0.016526 \\
0.016527 \\
0.016526 \\
0.016526 \\
0.016526 \\
0.016526 \\
0.016526 \\
0.016526 \\
0.016526 \\
0.016526 \\
0.016526 \\
0.016526 \\
0.016526 \\
0.016526 \\
0.016526 \\
0.016526 \\
0.016526 \\
0.016526 \\
0.016526 \\
0.016526\end{array}$ & $\begin{array}{l}0.07540 \\
0.072477 \\
0.072674 \\
0.072491 \\
0.072465 \\
0.072465 \\
0.072463 \\
0.072444 \\
0.072463 \\
0.072463 \\
0.072463 \\
0.072463 \\
0.072463 \\
0.072463 \\
0.072463 \\
0.072463 \\
0.072463 \\
0.072463 \\
0.072463 \\
0.072463 \\
0.072463 \\
0.072463 \\
0.072463 \\
0.072463 \\
0.072463 \\
0.072463\end{array}$ & 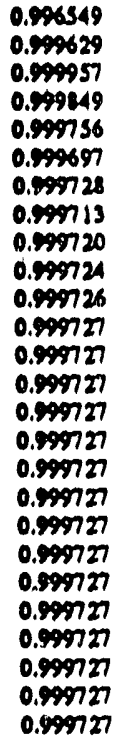 \\
\hline Saturation & Total Mab & APV/dSr & $\boldsymbol{C}$ & $\mathbf{P}$ & Gamma \\
\hline 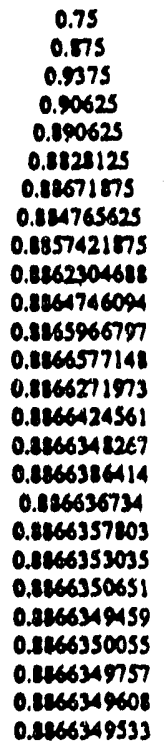 & 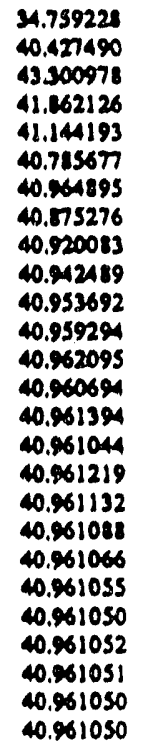 & $\begin{array}{l}0.045151 \\
0.009321 \\
0.002121 \\
0.005001 \\
0.006968 \\
0.008095 \\
0.007519 \\
0.007201 \\
0.007661 \\
0.007590 \\
0.007555 \\
0.007537 \\
0.007521 \\
0.007533 \\
0.007530 \\
0.007531 \\
0.007531 \\
0.007531 \\
0.007531 \\
0.007531 \\
0.007531 \\
0.007531 \\
0.007531 \\
0.007531 \\
0.007531 \\
0.007531\end{array}$ & $\begin{array}{l}220.095751 \\
115.033000 \\
62501620 \\
18.167310 \\
101.900155 \\
101.466577 \\
105.183366 \\
106.124972 \\
106.004169 \\
105.593767 \\
105.385567 \\
105.245966 \\
105.234666 \\
105.260316 \\
105.247491 \\
105.253901 \\
105.250691 \\
105.252301 \\
105.253102 \\
105.253503 \\
105.253703 \\
105.253101 \\
105.253753 \\
105.253779 \\
105.253791 \\
105.253797\end{array}$ & $\begin{array}{l}12.770599 \\
10.281953 \\
10.006619 \\
10.097238 \\
10.175426 \\
10.224828 \\
10.199203 \\
10.211779 \\
10.209432 \\
10.20203 \\
10.200749 \\
10.199975 \\
10.199519 \\
10.199782 \\
10.199615 \\
10.199733 \\
10.19709 \\
10.199721 \\
10.199727 \\
10.199730 \\
10.199732 \\
10.199733 \\
10.199732 \\
10.199732 \\
10.199133 \\
10.199733\end{array}$ & $\begin{array}{l}1.1794 E+07 \\
1.1701 E+07 \\
1.1635 E+07 \\
1.1676 E+07 \\
1.1690 E+07 \\
1.1695 E+07 \\
1.1693 E+07 \\
1.1694 E+07 \\
1.1693 E+07 \\
1.1693 E+07 \\
1.1693 E+07 \\
1.1693 E+07 \\
1.1693 E+07 \\
1.1693 E+07 \\
1.1693 E+07 \\
1.1693 E+07 \\
1.1693 E+07 \\
1.1693 E+07 \\
1.1693 E+07 \\
1.1693 E+07 \\
1.1693 E+07 \\
1.1693 E+07 \\
1.1693 E+07 \\
1.1693 E+07 \\
1.1693 E+07 \\
1.1693 E+07\end{array}$ \\
\hline
\end{tabular}

$\begin{array}{cc}\text { dKrv/dsv } & d K r V d s 1 \\ & \\ 1.0000 & 0.1875 \\ 1.0000 & 0.0469 \\ 1.0000 & 0.0117 \\ 1.0000 & 0.0264 \\ 1.0000 & 0.0359 \\ 1.0000 & 0.0412 \\ 1.0000 & 0.0385 \\ 1.0000 & 0.0398 \\ 1.0000 & 0.0392 \\ 1.0000 & 0.0388 \\ 1.0000 & 0.0387 \\ 1.0000 & 0.0316 \\ 1.0000 & 0.0385 \\ 1.0000 & 0.0586 \\ 1.0000 & 0.0385 \\ 1.0000 & 0.0386 \\ 1.0000 & 0.0386 \\ 1.0000 & 0.0386 \\ 1.0000 & 0.0386 \\ 1.0000 & 0.0386 \\ 1.0000 & 0.0386 \\ 1.0000 & 0.0386 \\ 1.0000 & 0.0386 \\ 1.0000 & 0.0386 \\ 1.0000 & 0.0386 \\ 1.0000 & 0.0316\end{array}$

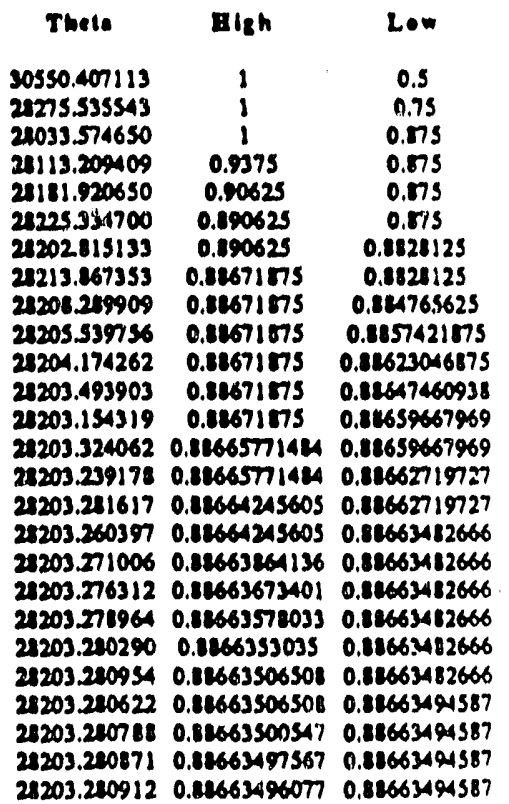


Spreadsheet for Calculation of Stream-Water Saturation Profiles

Infectlon Temperalure $580 \cdot \mathcal{K}$
Inillal Temperalurt

350 "K
Mairlx Heal Capoclty

$1.0000(\mathrm{~kJ} / \mathrm{kg})$
MATRIX PROPERTIES

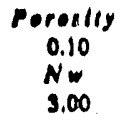

$\begin{array}{cc}\text { Densily } & \text { Rho } \bullet C p \\ 2650.00 & 2650.0000 \\ s w 1 & \text { Denem } \\ 0.00 & 1.000000\end{array}$

SATURATION CONDITIONS Tearumtion -485.57

\begin{tabular}{|c|c|c|c|c|c|}
\hline & $\begin{array}{c}\text { Volvmes } \\
\text { (m3/k8) } \\
0.001176 \\
0.100298\end{array}$ & $\begin{array}{c}\text { Enthalples } \\
(2 \mathrm{k} / \mathrm{s}) \\
901.41 \\
2008.52\end{array}$ & $\begin{array}{c}\text { Denaltles } \\
\text { (ae/mis) } \\
850.4723 \\
9.970241\end{array}$ & $\begin{array}{c}\text { Vlisestitles } \\
\text { (cp) } \\
0.1903 \\
0.0217\end{array}$ & $\begin{array}{c}\text { Soturatlon } \\
\text { Step } \\
0.0014037937\end{array}$ \\
\hline
\end{tabular}

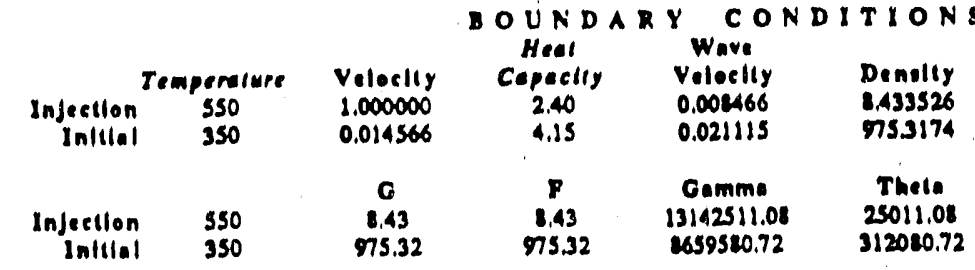

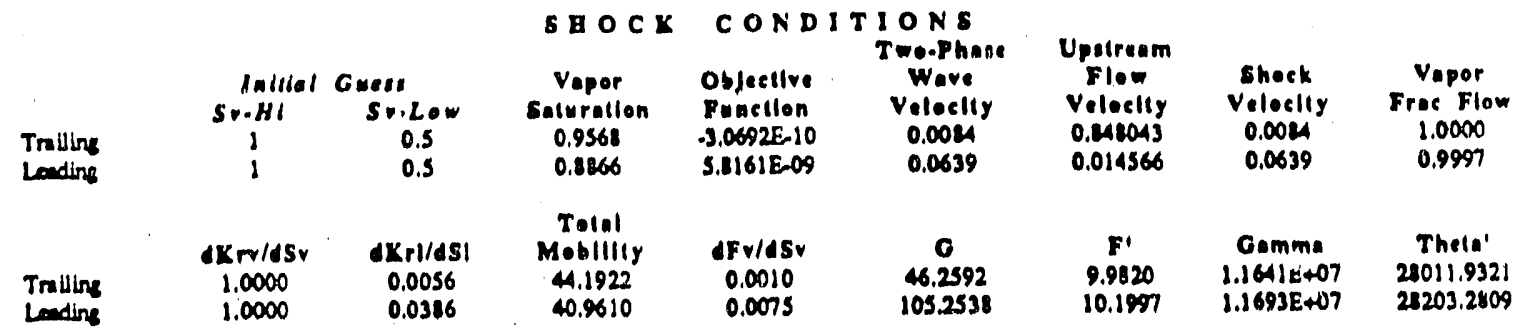

MATERIALDALANCES

\begin{tabular}{|c|c|c|c|c|c|c|}
\hline $\begin{array}{l}\text { Mass } \\
\text { Entholpy }\end{array}$ & $\begin{array}{c}\text { Instde } \\
91.758372 \\
0 \times 429.7368\end{array}$ & $\begin{array}{c}\text { InItial } \\
97.531740 \\
055958.0720\end{array}$ & $\begin{array}{c}\text { Injected } \\
8,433526 \\
25011.0798\end{array}$ & $\begin{array}{l}\text { Produced } \\
14.206881 \\
4545.0913\end{array}$ & $\begin{array}{c}\text { Dolanee } \\
0.0000 \\
6.4834\end{array}$ & $\begin{array}{c}\text { Errer } \\
0.00005 \\
0,00075\end{array}$ \\
\hline
\end{tabular}


Spreadsheet for Calculation of Stream-Water Saturation Proftles

Injecllon Temperalure

$350 \cdot \mathrm{K}$
Inllial Temperalure

$3300^{\circ} \mathrm{K}$
Moirlx Beal Capacley

$1.0000(\mathrm{~kJ} / \mathrm{kB})$

SATURATION PROFILE

\begin{tabular}{|c|c|c|c|c|c|c|c|c|}
\hline $\begin{array}{l}\text { Vapor } \\
\text { salurallon }\end{array}$ & $\begin{array}{l}\text { Vopor } \\
\text { Prac Flow }\end{array}$ & $d K r v / d S v$ & $\| K r V d S I$ & $\begin{array}{c}\text { Tolal } \\
\text { Mobllliy }\end{array}$ & $d F v / d s v$ & lambda & $\begin{array}{l}\text { Mole } \\
\text { Inalde }\end{array}$ & $\begin{array}{l}\text { Enlhalpy } \\
\text { Inside }\end{array}$ \\
\hline $\begin{array}{l}1.0000 \\
1.0000\end{array}$ & & & & & & $\begin{array}{l}0.000000 \\
0.008362\end{array}$ & & \\
\hline 0.9568 & 0.999986 & 1.0000 & 0.0056 & 44.192244 & 0.000986 & 0.001362 & 0.070520 & \\
\hline 0.9554. & 0.909915 & 1.0000 & 0.0060 & $\begin{array}{r}4.127471 \\
4.062702\end{array}$ & $\begin{array}{l}0.001053 \\
0.001123\end{array}$ & $\begin{array}{l}0.008932 \\
0.009522\end{array}$ & $\begin{array}{l}0.026712 \\
0.028345\end{array}$ & $\begin{array}{l}6377.799000 \\
6871.204397\end{array}$ \\
\hline $\begin{array}{l}0.9540 \\
0.9526\end{array}$ & & & $\begin{array}{l}0.0063 \\
0.0067\end{array}$ & $\begin{array}{r}4.062702 \\
43.997938\end{array}$ & $\begin{array}{l}0.001123 \\
0.001195\end{array}$ & $\begin{array}{l}0.009522 \\
0.010132\end{array}$ & 0.030031 & \\
\hline $\begin{array}{l}0.9526 \\
0.9512\end{array}$ & $\begin{array}{l}0.999981 \\
0.999980\end{array}$ & $\begin{array}{l}1.0000 \\
1.0000\end{array}$ & 0.0071 & 43.933177 & 0.001269 & 0.010763 & 0.031770 & $\begin{array}{l}7106,018903 \\
7342.252548\end{array}$ \\
\hline 0.9498 & 0.999978 & 1.0000 & 0.0076 & 43.26421 & 0.001346 & 0.011414 & 0.033564 & 7579.915436 \\
\hline 0.9484 & 0.999976 & 1.0000 & 0.0020 & 43.103670 & 0.001425 & 0.012015 & 0.035811 & 7819.017744 \\
\hline 0.9170 & 0.999974 & 1.0000 & 0.0084 & 43.738923 & 0.001507 & 0.012777 & 0.037314 & 3059.569725 \\
\hline 0.9456 & 0.999972 & 1.0000 & $\begin{array}{l}0.0089 \\
0.0003\end{array}$ & 43.674111 & $\begin{array}{l}0.001591 \\
0.001677\end{array}$ & $\begin{array}{l}0.013490 \\
0.014223\end{array}$ & $\begin{array}{l}0.039272 \\
0.041285\end{array}$ & $\begin{array}{l}8301.581705 \\
8543.064089\end{array}$ \\
\hline $\begin{array}{l}0.9442 \\
0.9428\end{array}$ & $\begin{array}{l}0.999969 \\
0.999967\end{array}$ & $\begin{array}{l}1.0000 \\
1.0000\end{array}$ & $\begin{array}{l}0.0093 \\
0.0098\end{array}$ & 43544712 & 0.001766 & 0.014978 & 0.043355 & $\begin{array}{l}7959.06027356 \\
7790.026\end{array}$ \\
\hline 0.9414 & 0.999964 & 1.0000 & 0.0103 & 43.479915 & 0.001858 & 0.015753 & 0.045412 & 9036,482063 \\
\hline 0.9400 & 0.999962 & 1.0000 & 0.0108 & 43.415264 & 0.001952 & 0.016550 & 0.047666 & 224.49864 \\
\hline 0.9386 & 0.999939 & 1.0000 & 0.0113 & 43.350548 & 0.002048 & 0.017368 & 0.019909 & 9593.00413 \\
\hline 0.9372 & 0.999956 & 1.0000 & 0.0118 & 43.285837 & 0.002147 & 0.011207 & 0.052207 & 774.901562 \\
\hline 0.9358 & 0.999953 & 1.0000 & 0.0124 & 43.221133 & 0.002249 & 0.019068 & 0.054565 & 10037.429162 \\
\hline 0.8344 & 0.999950 & 1.0000 & 0.0129 & 43.156434 & 0.002353 & 0.019951 & 0.056913 & 10291.502166 \\
\hline 0.9330 & 0.99946 & 1.0000 & 0.0135 & 43.091741 & 0.002459 & 0.020156 & 0.059460 & 10547.131606 \\
\hline 0.9316 & $0.9999 \times 13$ & 1.0000 & 0.0141 & 43.027054 & 0.002569 & 0.021783 & 0.061998 & 10804.328597 \\
\hline 0.9302 & 0.999939 & 1.0000 & 0.0146 & $42 \% 2373$ & 0.002680 & 0.022731 & 0.064397 & 11063.104337 \\
\hline 0.9287 & 0.999939 & 1.0000 & 0.0152 & 12897699 & 0.002795 & 0.023702 & 0.067257 & 11322.470105 \\
\hline 0.9273 & 0.999931 & 1.0000 & 0.0158 & 42833031 & 0.002912 & 0.024696 & 0.069979 & J1S15.437265 \\
\hline 0.9259 & 0.999927 & 1.0000 & 0.0163 & 12.768370 & 0.003032 & 0.025711 & 0.072763 & 11449.017204 \\
\hline 0.9245 & 0.999923 & 1.0000 & 0.0171 & $\$ 2703715$ & 0.003154 & 0.026750 & 0.075810 & 12114.221636 \\
\hline 0.9231 & 0.999918 & 1.0000 & 0.0177 & 12.639068 & 0.003279 & 0.028111 & 0.078321 & 12311.061998 \\
\hline 0.9217 & 0.999914 & 1.0000 & 0.014 & 12.574127 & 0.003407 & 0.028195 & 0.011495 & 12649.550058 \\
\hline $\begin{array}{l}0.9203 \\
0.9199\end{array}$ & $\begin{array}{l}0.999909 \\
0.999904\end{array}$ & $\begin{array}{l}1.0000 \\
1.0000\end{array}$ & $\begin{array}{l}0.0190 \\
0.0197\end{array}$ & $\begin{array}{l}42509793 \\
42445167\end{array}$ & $\begin{array}{l}0.003538 \\
0.003671\end{array}$ & $\begin{array}{l}0.030003 \\
0.031133\end{array}$ & $\begin{array}{l}0.044535 \\
0.07639\end{array}$ & $\begin{array}{l}12919.697605 \\
13191.516521\end{array}$ \\
\hline 0.9175 & 0.999898 & 1.0000 & 0.0204 & 42380548 & 0.003107 & 0.032287 & 0.090809 & $13665.018 \mathrm{~ms}$ \\
\hline 0.9161 & 0.999193 & 1.0000 & 0.0211 & 42.315937 & 0.003946 & 0.033464 & 0.094046 & 13740.216123 \\
\hline 0.9147 & $0.9998 \mathrm{n}$ & 1.0000 & 0.0218 & 12251333 & 0.004086 & 0.034665 & 0.007330 & 14017.121614 \\
\hline 0.9133 & 0.999881 & 1.0000 & 0.0223 & 12.186737 & 0.001232 & 0.035890 & 0.100721 & 14295.746588 \\
\hline 0.9119 & 0.999875 & 1.0000 & 0.0233 & 42122148 & 0.004379 & 0.037138 & 0.104160 & 14576.103673 \\
\hline 0.9105 & 0.999169 & 1.0000 & 0.0240 & 12057561 & 0.004529 & 0.034111 & 0.107667 & 14851.205293 \\
\hline 0.9091 & 0.999863 & 1.0000 & 0.0218 & 41.992996 & 0.004682 & 0.039708 & 0.11124 & 15142063964 \\
\hline 0.9077 & 0.999856 & 1.0000 & 0.0256 & 41.92432 & 0.004838 & $0 .(41029$ & 0.114891 & 19427.692294 \\
\hline 0.9063 & 0.999449 & 1.0000 & 0.0263 & 41.263876 & 0.004997 & 0.042374 & 0.112600 & 15715.102977 \\
\hline 0.9049 & 0.99942 & 1.0000 & 0.0271 & 41.799329 & 0.005158 & 0.043744 & 0.122397 & 16004.30144 \\
\hline 0.9035 & 0.999835 & 1.0000 & 0.0279 & 41.734791 & 0.005323 & 0.045140 & 0.126231 & 16295.322759 \\
\hline 0.9021 & 0.999127 & 1.0000 & 0.0281 & 41.670261 & 0.005490 & 0.046560 & 0.130190 & 16511.157725 \\
\hline 0.9007 & 0.999119 & 1.0000 & 0.0296 & 41.605741 & 0.005661 & 0.042005 & 0.13196 & 16812826832 \\
\hline 0.8993 & 0.999811 & 1.0000 & 0.0304 & 41.51229 & 0.005834 & 0.049475 & 0.131276 & 17179.343269 \\
\hline 0.8979 & 0.999103 & 1.0000 & 0.0313 & 41.476726 & 0.006010 & 0.050971 & 0.142429 & 17477.720325 \\
\hline 0.8965 & 0.09979 & 1.0000 & 0.0322 & 41.412233 & 0.006190 & 0.052492 & 0.146658 & 1777.971316 \\
\hline 0.8951 & 0.999715 & 1.0000 & 0.0330 & 41.347749 & 0,006372 & 0.054039 & 0.150062 & 12010.109944 \\
\hline 0.8937 & 0.999776 & 1.0000 & 0.0339 & 41.243275 & 0.006551 & 0.053612 & $0 . ! 35343$ & In:4.149588 \\
\hline 0.0923 & 0.999767 & 1.0000 & 0.0341 & 41.211810 & 0.006746 & 0.057211 & 0.159100 & 18690.104014 \\
\hline 0.0908 & 0.999757 & 1.0000 & 0.0357 & 41.154353 & 0.006938 & 0.051836 & 0.164335 & 19997.987018 \\
\hline 0.8194 & 0.999748 & 1.0000 & 0.0367 & 11.019910 & 0.007133 & 0.060487 & 0.161949 & 19307.812503 \\
\hline 0.8880 & 0.999737 & 1.0000 & 0.0376 & 11.025475 & 0.007330 & 0.062165 & 0.173641 & 19019.594476 \\
\hline 0.0366 & 0.999727 & 1.0000 & 0.0386 & 40.961050 & 0.007531 & 0.063870 & 0.17414 & 19933.347051 \\
\hline $\begin{array}{l}0.0000 \\
0.0000\end{array}$ & & & & & & 0.053870 & & \\
\hline 0.0000 & & & & & & 1,000000 & & \\
\hline
\end{tabular}


Injecllon Temperature 850 "K
Inlibal Temperature $350 \cdot \mathrm{K}$
Matrix Beat Capacily

$1.0000\left(w_{J} / k_{8}\right)$

TRAILING SBOCK CALCULATIONE

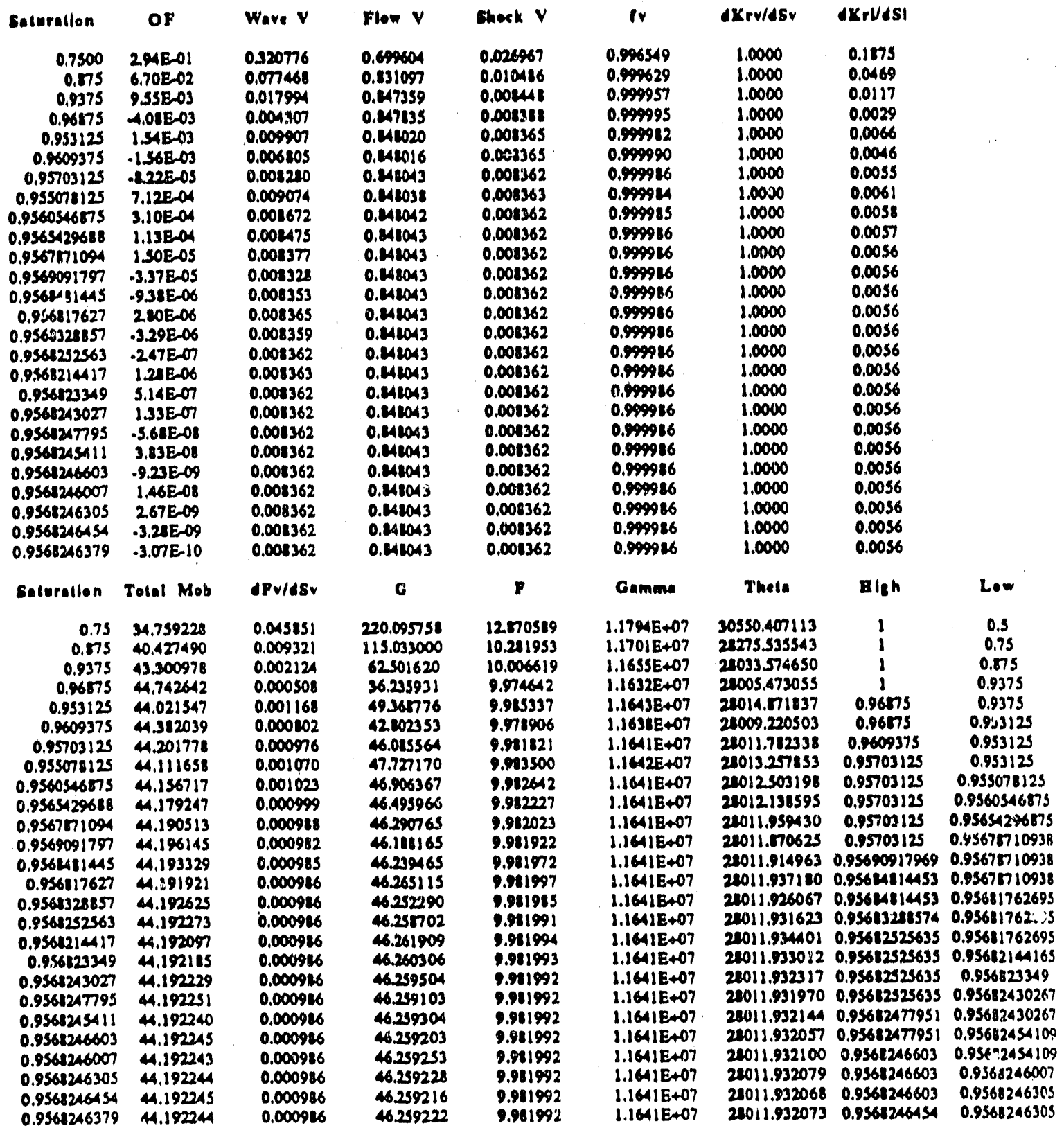


Spreadsheet for Calculation of Stream-Water Saturation Profiles

10jection Temperature $5 s 0 \cdot \boldsymbol{X}$
Inlilat Temperature $350 \cdot k$
Motrix Beat Caperity $1.0000\left(k J / k_{e}\right)$

LEADING SBOCK.CALCULATIONS

\begin{tabular}{|c|c|c|c|c|c|c|c|c|}
\hline Seteration & OP & Wave V & Flew V & Shock V & Iv & dKrv/dSV & dXrVdSI & \\
\hline 0.7500 & $3202 E-01$ & 0.341836 & 0.016333 & 0.068405 & 0.996549 & 1.0000 & 0.1875 & \\
\hline 0.075 & $152 E-02$ & 0.079047 & 0.014575 & 0.063882 & 0.999629 & 1.0000 & 0.0469 & \\
\hline 0.9375 & $-4.60 \mathrm{E}-02$ & 0.018009 & 0.014698 & $0.06405 s$ & 0.999957 & 1.0000 & 0.0117 & \\
\hline 0.90625 & $-215 E-02$ & 0.042411 & 0.014588 & 0.063901 & 0.999849 & 1.0000 & 0.0264 & \\
\hline 0.090625 & - $.76 E-03$ & 0.059094 & 0.014567 & 0.063871 & 0.999756 & 1.0000 & 0.0359 & \\
\hline 0.8828125 & $4.78 E-03$ & 0.068648 & $0.0: 4567$ & 0.063871 & 0.999657 & 1.0000 & 0.0412 & \\
\hline 0.24671875 & -1.03504 & 0.063767 & 0.014566 & 0.063570 & 0.999728 & 1.0000 & 0.0385 & \\
\hline 0.84765625 & $2315-03$ & 0.066181 & 0.014567 & 0.063870 & 0.999113 & 1.0000 & 0.0398 & \\
\hline 0.8857421875 & $1.10 E-03$ & 0.064968 & 0.014566 & 0.063670 & 0.999720 & 1.0000 & 0.0392 & \\
\hline $0 \$ 862304688$ & 4.96E- 1 & 0.064366 & 0.014566 & 0.063870 & 0.999724 & 1.0000 & 0.0388 & \\
\hline 0.8164746094 & $1.965-04$ & 0.064066 & 0.014566 & 0.083570 & 0.999726 & 1.0000 & 0.0387 & \\
\hline 0.8165966797 & 4.69E-05 & 0.063917 & 0.014566 & 0.063570 & $0.9 \% 727$ & 1.0000 & 0.0386 & \\
\hline 0.2466577148 & $-278 E-0 S$ & 0.063242 & 0.014566 & 0.063570 & 0.999727 & 8.0000 & 0.0385 & \\
\hline 0.8166271973 & 9.5OE-O6 & 0.063879 & 0.014566 & 0.063870 & 0.999727 & 1.0000 & 0.0386 & \\
\hline 0.2866424561 & $-9.1 \mathrm{E}-06$ & 0.063261 & 0.014566 & 0.063570 & 0.999127 & .0000 & 0.0385 & \\
\hline 0.8866348267 & $1.61 \mathrm{EN}$ & 0.063670 & 0.014566 & 0.063870 & 0.999727 & 1.0000 & 0.0386 & \\
\hline 0.81663164414 & $\triangle 51 E-06$ & 0.063865 & 0.014566 & 0.063870 & 0.999727 & 1.0000 & 0.0386 & \\
\hline 0.826636734 & $-217 \mathrm{E}-06$ & 0.063268 & 0.014566 & 0.063570 & 0.999727 & 1.0000 & 0.0386 & \\
\hline 0.2166357203 & $-1.01 E-06$ & 0.063269 & 0.014566 & 0.063870 & 0.999727 & $2.00 \mathrm{CO}$ & 0.0326 & \\
\hline 0.8466353035 & A23EO & 0.063269 & 0.014566 & 0.063870 & 0.999727 & 1.0000 & 0.0326 & \\
\hline 0.2466350651 & $-131 E-O 7$ & 0.063170 & 0.014566 & 0.063570 & 0.999727 & 1.0000 & 0.0386 & \\
\hline 0.2466349459 & 1.49EDO8 & 0.063170 & 0.014566 & 0.063570 & 0.999727 & 1.0000 & 0.0386 & \\
\hline 0.8466350055 & $-5.20 \mathrm{E}-08$ & 0.063870 & 0.014566 & 0.063870 & 0.999727 & 1.0000 & 0.0386 & \\
\hline 0.8466349757 & -215E-08 & C.063870 & 0.014566 & 0.063870 & 0.999727 & 1.0000 & 0.0386 & \\
\hline 0.8166349608 & $-3.30 \mathrm{E}-\mathrm{OS}$ & 0.063870 & 0.014566 & 0,063870 & 0.999727 & $\$ .0000$ & 0.0386 & \\
\hline 0.2866349533 & $5.82 E-09$ & 0.063870 & 0.014566 & 0.063570 & 0.999727 & 1.0000 & 0.0386 & \\
\hline Seteration & Tolal Mob & dFv/dSV & G & $\mathbf{P}$ & Gemme & There & Algh & Lew \\
\hline 0.75 & 34.759228 & 0.045851 & 220.095758 & 12870589 & 1.1794E+07 & 30550.407113 & 1 & 0.5 \\
\hline 0.875 & 40.427490 & 0.009321 & 115.033000 & 10.281953 & $1.1701 E+07$ & 22275535543 & $i$ & 0.75 \\
\hline 0.9375 & 43.300978 & 0.002124 & 62501620 & 10.006619 & $1.1655 E+07$ & 28033.574650 & 1 & 0.075 \\
\hline $\begin{array}{l}0.90625 \\
0.090625\end{array}$ & $\begin{array}{l}41.862126 \\
41.14193\end{array}$ & $\begin{array}{l}0.005001 \\
0.006 \% 88\end{array}$ & $\begin{array}{r}81.767310 \\
101.900155\end{array}$ & $\begin{array}{l}10.097238 \\
10.175426\end{array}$ & $\begin{array}{l}1.1678 E+07 \\
1.1690 E+07\end{array}$ & $\begin{array}{l}28113.200409 \\
28181.90050\end{array}$ & $\begin{array}{l}0.9375 \\
0.90625\end{array}$ & $\begin{array}{l}0.575 \\
0.775\end{array}$ \\
\hline 0.8223125 & 40.785677 & 0.008095 & 108.466577 & 10.220828 & 1.1695E+07 & $22225.33 \times 700$ & 0.090625 & 0.875 \\
\hline 0.86671575 & 40.964895 & $\operatorname{con} 519$ & 105.1103366 & 10.199203 & $1.1693 E+07$ & 28202815133 & 0.890625 & 0.8828125 \\
\hline 0.244765625 & 40.575276 & 0.007104 & $106 . \$ 21972$ & 10.211779 & 1.1694E+07 & 28213.667353 & 0.286711575 & 0.8828125 \\
\hline 0.1857421875 & 40.920083 & 0.007661 & 108.004169 & 10.205432 & $1.1693 E \propto 07$ & 28208.289909 & 0.86671875 & 0.844765625 \\
\hline $\begin{array}{l}0.1462304688 \\
0.1261746094\end{array}$ & $\begin{array}{l}40.942489 \\
40.953692\end{array}$ & $\begin{array}{l}0.007590 \\
0.007555\end{array}$ & $\begin{array}{l}105.593767 \\
105.381567\end{array}$ & $\begin{array}{l}10.202303 \\
10.200749\end{array}$ & $\begin{array}{l}1.1693 E+07 \\
1.1693 E+07\end{array}$ & $\begin{array}{l}23205.539756 \\
23204.174262\end{array}$ & $\begin{array}{l}0.82671875 \\
0.28671875\end{array}$ & $\begin{array}{c}0.8857421875 \\
0.82623046875\end{array}$ \\
\hline $\begin{array}{l}0.866596797 \\
0.8465577141\end{array}$ & $\begin{array}{l}40.959294 \\
40.962095\end{array}$ & $\begin{array}{l}0.007537 \\
0.007528\end{array}$ & $\begin{array}{l}105.245966 \\
105.234666\end{array}$ & $\begin{array}{l}10.199975 \\
10.199589\end{array}$ & $\begin{array}{l}1.1693 E+07 \\
1.1693 E+07\end{array}$ & $\begin{array}{l}28203.493903 \\
28203.154319\end{array}$ & $\begin{array}{l}0.86671875 \\
0.82671675\end{array}$ & $\begin{array}{l}0.82647460938 \\
0.82659667969\end{array}$ \\
\hline $\begin{array}{l}\text { a } 1266271973 \\
0.3166424561\end{array}$ & $\begin{array}{l}40.960694 \\
40.961394\end{array}$ & $\begin{array}{l}0.007533 \\
0.007530\end{array}$ & 105.260316 & 10.199782 & $1.1693 E+07$ & 28203.324062 & 0.886657 iac4 & 0.18659667969 \\
\hline & $\begin{array}{l}0.961394 \\
40.9104\end{array}$ & & $\begin{array}{l}105.247491 \\
105.253904\end{array}$ & 10.199685 & 1.1693E +07 & 20203.239178 & 0.86665771484 & 0.18662719727 \\
\hline $\begin{array}{l}0.0666348267 \\
0.84663864 / 4\end{array}$ & $\begin{array}{l}0.9104 \\
40.961219\end{array}$ & $\begin{array}{l}0.007531 \\
0.007531\end{array}$ & $\begin{array}{l}105.253904 \\
105.250698\end{array}$ & 10.199733 & $1.1693 E+07$ & 25203.281617 & 0.16004245805 & 0.86562719727 \\
\hline $\begin{array}{c}0.8106356414 \\
0.816636734\end{array}$ & $\begin{array}{l}40.961219 \\
40.961132\end{array}$ & 0.007531 & $\begin{array}{l}105.250698 \\
105.252301\end{array}$ & 10.199709 & $1.1693 E+07$ & 21203.260397 & 0.86661245605 & 0.82663482666 \\
\hline $\begin{array}{l}0.120636734 \\
0.2166357203\end{array}$ & $\begin{array}{l}40.961132 \\
40.961088\end{array}$ & 0.007531 & $\begin{array}{l}105.252301 \\
105.253102\end{array}$ & 10.199721 & $1.1693 E+07$ & 28203.271006 & 0.81663164136 & 0.18663482666 \\
\hline 0.2866353035 & 40.961066 & 0.007531 & $\begin{array}{l}105.253102 \\
105.253503\end{array}$ & 10.199727 & $1.1693 E+07$ & 28203.276312 & 0.8253673401 & 0.88663482666 \\
\hline 0.246635005: & 40.961055 & 0.007531 & $\begin{array}{l}105.253503 \\
105.253703\end{array}$ & 10.199730 & 1.1693E+07 & 28203.278964 & 0.12663578033 & 0.186663422666 \\
\hline 0.8466349459 & $\infty 0 \% 1350$ & 0.007531 & $\begin{array}{l}105.253703 \\
105.253204\end{array}$ & 10.199732 & $1.1693 E+07$ & 28203.280290 & 0.8566353035 & 0.86663412666 \\
\hline 0.2466350055 & 40.001052 & 0.007531 & $\begin{array}{l}105.253104 \\
105.253753\end{array}$ & 10.199733 & $1.1693 E+07$ & 21203.200954 & 0.22663506508 & 0.81663482166 \\
\hline 0.2866349757 & 40.961051 & 0.007531 & $\begin{array}{l}105.253753 \\
105.253779\end{array}$ & 10.199732 & $1.1693 E+07$ & 21203.280622 & 0.82663505508 & 0.88663494587 \\
\hline 0.1466349608 & 40.961050 & 0.007531 & $\begin{array}{l}105.253779 \\
105.253791\end{array}$ & 10.199732 & $1.1693 E+07$ & 28203.280788 & 0.82663500547 & 0.88663494587 \\
\hline 0.2266349533 & 40.961050 & 0.007531 & $\begin{array}{l}105.253791 \\
105.253797\end{array}$ & 10.199733 & $1.1693 E+07$ & 28203.280871 & 0.1663497567 & 0.86663494587 \\
\hline & & & 105.253797 & 10.199733 & $1.1693 E+07$ & 28203.280912 & 0.86663496077 & 0.88663494587 \\
\hline
\end{tabular}


Spreadsheet for Calculation of Stream-Water Saturation Profiles

Injectlon Temperalure 600 'K
Inllial Temperature

$330^{\circ} \mathrm{K}$
Moirix Beal Capactiy

$1.0000\left(\mathrm{~kJ} / \mathrm{k}_{\mathrm{E}}\right)$

\section{MATRIX PROPERTIES}

Cp Mardx Densliy Rho $-\mathrm{C}_{p}$ $1.00 \quad 2650.00 \quad 2650.0000$ $\begin{array}{lll}N_{8} & S=1 & \text { Denom } \\ 1.00 & 0.00 & 1.000000\end{array}$

$N$

3.00
1.000000

CONDITIONS

SA TURATION

Volumes $(003 / 48)$ 0.001176

Enthalples Eastheles Densllies (a)ike) (xetrus)

Vincenteles

2208.52

150.4723

(op)
0.1303

0.1303

0.0217
Saturation
siep

Vapor 0.100298

DOUNDARY CONDITIONS

Heal wave

Capectiy velocily 0.007420

Deasily

2.34

0.018946

7.565513

Entholpy

1.000000

4.15

975.3174

304.04

F Camme Thet: $\begin{array}{lll}975.32 & 659580.72 & \end{array}$

$\begin{array}{ll} & 600 \\ \text { Injection } & 350\end{array}$

7.57

975.32

\section{S BOCX}

CONDITIONS

Initial Cuess

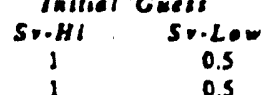

Tralling

Londine

Tnilins

$\underset{1.0000}{1.0000}$

vapor

Salurallon

0.9568

0.8166

Objectlve

Functlon

3.17KE 10

S.21 LEE-09

Two.Phase
Wave
Veloclly

0.0075

0.0573

Tolol

Mobllity

4.1921

IFv/dSr
0.0010

0.0010

G
0.2613

105.2538

\section{Upstream}

Flow

Velecily

0.760925

0.013070

Shock

Velecliy

$\begin{array}{ll}0.0573 & 1.0000 \\ 0.9997\end{array}$

MATERIAL BaLANCES

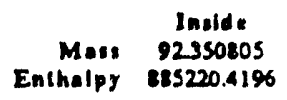

Inllial

en 531740

265951.0720
Injecled
7566513

23335.4376
Produced 12.747437 4078.9074

$\begin{array}{ll}\text { Dolence } & \text { Error } \\ 0.0000 & 0.0000 \% \\ .5 .8174 & 0.0007\end{array}$


Spreadsheet for Calculation of Stream-Water Saturation Profiles

Injectlon Temperature $\cos { }^{\circ} \mathrm{K}$
Inltul Temperalure $350 \cdot x$
Metrix Eeat Capocity

$8.0000\left(k J / k_{E}\right)$

SATURATION PROFILE

\begin{tabular}{|c|c|c|c|c|c|c|c|c|}
\hline $\begin{array}{c}\text { Vaper } \\
\text { saterstien }\end{array}$ & $\begin{array}{l}\text { Vaper } \\
\text { Frac Flow }\end{array}$ & eXrr/dSr & IX rVASI & $\begin{array}{c}\text { Telal } \\
\text { Moblilty }\end{array}$ & $d F v / d S v$ & lembda & $\begin{array}{l}\text { Moss } \\
\text { Inside }\end{array}$ & $\begin{array}{l}\text { Enthalpy } \\
\text { Inside }\end{array}$ \\
\hline $\begin{array}{l}1.0000 \\
1.0000\end{array}$ & & & & & & $\begin{array}{l}0.000000 \\
0.007504\end{array}$ & & \\
\hline $\begin{array}{l}0.9568 \\
0.9554\end{array}$ & $\begin{array}{l}0.999986 \\
0.999885\end{array}$ & $\begin{array}{l}1.0000 \\
2.0000\end{array}$ & $\begin{array}{l}0.0056 \\
0.0060\end{array}$ & $\begin{array}{l}4.192132 \\
4.12736 !\end{array}$ & $\begin{array}{l}0.000986 \\
0.001053\end{array}$ & $\begin{array}{l}0.007504 \\
0.002015\end{array}$ & $\begin{array}{l}0.056777 \\
0.023969\end{array}$ & $\begin{array}{c}107553.776798 \\
5956.062891\end{array}$ \\
\hline $\begin{array}{l}0.9540 \\
0.9526\end{array}$ & $\begin{array}{l}0.999983 \\
0.999981\end{array}$ & $\begin{array}{l}1.0000 \\
1.0000\end{array}$ & $\begin{array}{l}0.0062 \\
0.0067\end{array}$ & $\begin{array}{l}4.062595 \\
43.997132\end{array}$ & $\begin{array}{l}0.001123 \\
0.001195\end{array}$ & $\begin{array}{l}0.008545 \\
0.009092\end{array}$ & $\begin{array}{l}0.025435 \\
0.026948\end{array}$ & $\begin{array}{l}6165.478665 \\
6376.158678\end{array}$ \\
\hline $\begin{array}{l}0.9512 \\
0.9498\end{array}$ & $\begin{array}{l}0.999920 \\
0.999078\end{array}$ & $\begin{array}{l}1.0000 \\
1.0000\end{array}$ & $\begin{array}{l}0.0071 \\
0.0076\end{array}$ & $\begin{array}{l}43.933074 \\
43.268320\end{array}$ & $\begin{array}{l}0.001269 \\
0.001346\end{array}$ & $\begin{array}{l}0.009658 \\
0.010242\end{array}$ & 0.021508 & $\begin{array}{l}6518.111928 \\
6801.347478\end{array}$ \\
\hline $\begin{array}{l}0.9424 \\
0.9470\end{array}$ & $\begin{array}{l}0.999976 \\
0.999974\end{array}$ & $\begin{array}{l}1.0000 \\
1.0000\end{array}$ & $\begin{array}{l}0.0020 \\
0.0024\end{array}$ & $\begin{array}{l}43.803571 \\
43.738827\end{array}$ & $\begin{array}{l}0.001425 \\
0.001507\end{array}$ & $\begin{array}{l}0.010845 \\
0.011465\end{array}$ & $\begin{array}{l}0.031775 \\
0.033482\end{array}$ & $\begin{array}{l}7015.774461 \\
7231.702073\end{array}$ \\
\hline $\begin{array}{l}0.9456 \\
0.942\end{array}$ & $\begin{array}{l}0.999972 \\
0.949969\end{array}$ & $\begin{array}{l}1.0000 \\
1.0000\end{array}$ & $\begin{array}{l}0.0089 \\
0.0093\end{array}$ & $\begin{array}{l}43.674087 \\
43.609352\end{array}$ & $\begin{array}{l}0.001591 \\
0.001677\end{array}$ & $\begin{array}{l}0.012105 \\
0.012763\end{array}$ & $\begin{array}{l}0.035239 \\
0.037046\end{array}$ & $\begin{array}{l}7448.839580 \\
7667.296316\end{array}$ \\
\hline $\begin{array}{l}0.9428 \\
0.9414\end{array}$ & $\begin{array}{l}0.999967 \\
0.999964\end{array}$ & $\begin{array}{l}1.0000 \\
1.0000\end{array}$ & $\begin{array}{l}0.0098 \\
0.0103\end{array}$ & $\begin{array}{l}43544622 \\
43.479898\end{array}$ & $\begin{array}{l}0.001766 \\
0.001858\end{array}$ & $\begin{array}{l}0.013440 \\
0.014136\end{array}$ & $\begin{array}{l}0.038903 \\
0.020811\end{array}$ & $\begin{array}{l}7187.081683 \\
1108.20515 !\end{array}$ \\
\hline $\begin{array}{l}0.9400 \\
0.9386\end{array}$ & $\begin{array}{l}0.999962 \\
0.999959\end{array}$ & $\begin{array}{l}1.0000 \\
1.0000\end{array}$ & $\begin{array}{l}0.0108 \\
0.0113\end{array}$ & $\begin{array}{l}43.415179 \\
43.350465\end{array}$ & $\begin{array}{l}0.001952 \\
0.002048\end{array}$ & $\begin{array}{l}0.014851 \\
0.015585\end{array}$ & $\begin{array}{l}0.042770 \\
0.024781\end{array}$ & $\begin{array}{l}1330.676261 \\
\text { ess4.50s626 }\end{array}$ \\
\hline $\begin{array}{l}0.9372 \\
0.9358\end{array}$ & $\begin{array}{l}0.999956 \\
0.999953\end{array}$ & $\begin{array}{l}1.0000 \\
1.0000\end{array}$ & $\begin{array}{l}0.0118 \\
0.0124\end{array}$ & $\begin{array}{l}43.285757 \\
43.221054\end{array}$ & $\begin{array}{l}0.002147 \\
0.002249\end{array}$ & $\begin{array}{l}0.016338 \\
0.0171111\end{array}$ & $\begin{array}{l}0.046845 \\
0.048961\end{array}$ & $\begin{array}{l}779.699927 \\
9006.271918\end{array}$ \\
\hline $\begin{array}{l}0.9344 \\
0.9330\end{array}$ & $\begin{array}{l}0.999950 \\
0.999946\end{array}$ & $\begin{array}{l}1.0000 \\
1.0000\end{array}$ & $\begin{array}{l}0.0129 \\
0.0135\end{array}$ & $\begin{array}{l}43.156358 \\
43.091607\end{array}$ & $\begin{array}{l}0.002353 \\
0.002459\end{array}$ & $\begin{array}{l}0.017903 \\
0.018714\end{array}$ & $\begin{array}{l}0.051130 \\
0.053353\end{array}$ & $\begin{array}{l}9234.230424 \\
9463.585344\end{array}$ \\
\hline $\begin{array}{l}0.9316 \\
0.9302\end{array}$ & $\begin{array}{l}0.999943 \\
0.999939\end{array}$ & $\begin{array}{l}1.0000 \\
1.0000\end{array}$ & $\begin{array}{l}0.0141 \\
0.0146\end{array}$ & $\begin{array}{l}43.026912 \\
42962304\end{array}$ & $\begin{array}{l}0.002569 \\
0.002681\end{array}$ & $\begin{array}{l}0.019546 \\
0.020397\end{array}$ & $\begin{array}{l}0.055630 \\
0.057962\end{array}$ & $\begin{array}{l}964.346650 \\
9926.524386\end{array}$ \\
\hline $\begin{array}{l}0.9287 \\
0.9273\end{array}$ & $\begin{array}{l}0.999935 \\
0.999931\end{array}$ & $\begin{array}{l}1.0000 \\
1.0000\end{array}$ & $\begin{array}{l}0.0152 \\
0.0158\end{array}$ & $\begin{array}{r}12897632 \\
42832 \% 66\end{array}$ & $\begin{array}{l}0.002795 \\
0.002912\end{array}$ & $\begin{array}{l}0.021268 \\
0.022160\end{array}$ & 0.060348 & $\begin{array}{l}10160.128673 \\
10395.169706\end{array}$ \\
\hline $\begin{array}{l}0.9259 \\
0.9245\end{array}$ & $\begin{array}{l}0.999927 \\
0.999923\end{array}$ & $\begin{array}{l}1.0000 \\
1.0000\end{array}$ & $\begin{array}{l}0.0165 \\
0.0171\end{array}$ & $\begin{array}{l}127618307 \\
42703655\end{array}$ & $\begin{array}{l}0.003032 \\
0.003154\end{array}$ & $\begin{array}{l}0.023071 \\
0.024003\end{array}$ & $\begin{array}{l}0.065288 \\
0.067443\end{array}$ & $\begin{array}{l}10631.657755 \\
10269.603167\end{array}$ \\
\hline 0.9231 & 0.999918 & 1.0000 & 0.0177 & 12639010 & 0.003210 & 0.024955 & 0.070454 & 11109.016365 \\
\hline 0.9217 & 0.999914 & 1.0000 & $0.01 \mu$ & 42574371 & 0.003407 & 0.025928 & 0.073123 & 11349.907852 \\
\hline $\begin{array}{l}0.9203 \\
0.9189\end{array}$ & $\begin{array}{l}0.999909 \\
0.999904\end{array}$ & $\begin{array}{l}1.0000 \\
1.0000\end{array}$ & $\begin{array}{l}0.0190 \\
0.0197\end{array}$ & $\begin{array}{l}12 \operatorname{sog} 10 \\
12451: 6\end{array}$ & $\begin{array}{l}0.003538 \\
0.003671\end{array}$ & $\begin{array}{l}0.026921 \\
0.027936\end{array}$ & $\begin{array}{l}0.075850 \\
0.07836\end{array}$ & $\begin{array}{l}11592281206 \\
11836.168085\end{array}$ \\
\hline $\begin{array}{l}0.9175 \\
0.9161\end{array}$ & $\begin{array}{l}0.999898 \\
0.999893\end{array}$ & 1.0000 & $\begin{array}{l}0.0204 \\
0.0211\end{array}$ & $\begin{array}{l}42.380499 \\
42315890\end{array}$ & $\begin{array}{l}0.003807 \\
0.003946\end{array}$ & $\begin{array}{l}0.028971 \\
0.030027\end{array}$ & $\begin{array}{l}0.0814100 \\
0.084344\end{array}$ & $\begin{array}{l}12011.538228 \\
12328.469452\end{array}$ \\
\hline $\begin{array}{l}0.9147 \\
0.9133\end{array}$ & $\begin{array}{l}0.999887 \\
0.999881\end{array}$ & $\begin{array}{l}1.0000 \\
1.0000\end{array}$ & $\begin{array}{l}0.0218 \\
0.0225\end{array}$ & $\begin{array}{l}42231288 \\
42186694\end{array}$ & $\begin{array}{l}0.004088 \\
0.004232\end{array}$ & $\begin{array}{l}0.031105 \\
0.032204\end{array}$ & $\begin{array}{l}0.077348 \\
0.090373\end{array}$ & $\begin{array}{l}12576.912656 \\
12826.898819\end{array}$ \\
\hline $\begin{array}{l}0.9119 \\
0.9105\end{array}$ & $\begin{array}{l}0.999875 \\
0.999869\end{array}$ & $\begin{array}{l}1.0000 \\
1.0000\end{array}$ & $\begin{array}{l}0.0233 \\
0.0240\end{array}$ & $\begin{array}{l}42122108 \\
42057530\end{array}$ & $\begin{array}{l}0.004379 \\
9.004529\end{array}$ & $\begin{array}{l}0.033324 \\
0.034466\end{array}$ & $\begin{array}{l}0.093458 \\
0.096606\end{array}$ & $\begin{array}{l}13078.439004 \\
13331.544357\end{array}$ \\
\hline 0.9091 & $\begin{array}{l}0.999863 \\
0.999856\end{array}$ & & 0.0248 & 41.992960 & 0.004682 & 0.035629 & 0.099815 & 13586.226104 \\
\hline 0.907 & $\begin{array}{l}0.999156 \\
0.999449\end{array}$ & $\begin{array}{l}1.0000 \\
1.0000\end{array}$ & $\begin{array}{l}0.0256 \\
0.0263\end{array}$ & 41.928398 & 0.004838 & 0.036814 & 0.103087 & 1342495559 \\
\hline $\begin{array}{l}0.5063 \\
0.5049\end{array}$ & $\begin{array}{l}0.999449 \\
0.999142\end{array}$ & $\begin{array}{l}1.0000 \\
1.0000\end{array}$ & $\begin{array}{l}0.0263 \\
0.0271\end{array}$ & $\begin{array}{l}1.1263445 \\
41.799300\end{array}$ & $\begin{array}{l}0.004997 \\
0.005158\end{array}$ & $\begin{array}{l}0.032022 \\
0.039251\end{array}$ & $\begin{array}{l}0.106422 \\
0.109822\end{array}$ & $\begin{array}{l}14100.361119 \\
14359.843267\end{array}$ \\
\hline $\begin{array}{l}0.9035 \\
0.9021\end{array}$ & $\begin{array}{l}0.999835 \\
0.999827\end{array}$ & $\begin{array}{l}1.0000 \\
1.0000\end{array}$ & $\begin{array}{l}0.0279 \\
0.0288\end{array}$ & $\begin{array}{l}41.734764 \\
41.670237\end{array}$ & $\begin{array}{l}0.005323 \\
0.005490\end{array}$ & $\begin{array}{l}0.040503 \\
0.041777\end{array}$ & $\begin{array}{l}0.113285 \\
0.116814\end{array}$ & $\begin{array}{l}14620.94573 \\
14883.679692\end{array}$ \\
\hline 0.9007 & 0.999819 & 1.0000 & 0.0296 & 41.605718 & 0.005661 & 0.043074 & 0.120408 & 15148.060369 \\
\hline 0.8993 & 0.999811 & 1.0000 & 0.0304 & 41.541200 & 0.005834 & 0.044393 & 0.124068 & 15414.092435 \\
\hline $\begin{array}{l}0.8979 \\
0.0965\end{array}$ & $\begin{array}{l}0.999803 \\
0.999794\end{array}$ & $\begin{array}{l}1.0000 \\
1.0000\end{array}$ & $\begin{array}{l}0.0313 \\
0.0322\end{array}$ & $\begin{array}{l}41.476708 \\
11.412217\end{array}$ & $\begin{array}{l}0.006010 \\
0.006190\end{array}$ & $\begin{array}{l}0.045735 \\
0.047100\end{array}$ & $\begin{array}{l}0.127794 \\
0.131588\end{array}$ & 15681.805812 \\
\hline 0.8951 & 0.999785 & 1.0000 & 0.0330 & 4134736 & 0.006372 & 0.048488 & $\begin{array}{l}0.131588 \\
0.835450\end{array}$ & $\begin{array}{l}15951.194512 \\
16222276637\end{array}$ \\
\hline 0.8937 & 0.999776 & 1.0000 & 0.0339 & 41.283263 & 0.006558 & 0.049899 & 0.139381 & 16495.064379 \\
\hline 0.16922 & 0.999767 & 1.0000 & 0.0348 & 41.218201 & 0.006746 & 0.051334 & 0.143380 & 16769.570025 \\
\hline 0.0908 & 0.999757 & 1.0000 & 0.0357 & 41.154348 & 0.006938 & $0.05 \% 782$ & 0.147449 & 17045.805953 \\
\hline 0.8894 & 0.999748 & 1.0000 & 0.0367 & 11.089905 & 0.007133 & 0.094274 & 0.151588 & 17323.744635 \\
\hline 0.8880 & 0.999737 & 1.0000 & 0.0376 & 41.025473 & 0.007330 & 0.055779 & 0.155798 & 17603.518638 \\
\hline 0.8866 & 0.999727 & 1.0000 & 0.0366 & 40.961050 & 0.007531 & $\begin{array}{l}0.057309 \\
0.057309\end{array}$ & 0.160080 & 17885.020624 \\
\hline 0.0000 & & & & & & 1.000000 & & \\
\hline
\end{tabular}


Spreadsheet for Calculation of Stream-Water Saturation Profiles

Injection Temperature $600 \cdot x$
Iniliet Temperature sso $\bullet \mathbf{x}$
Motrlx Eeat Capedily 1.0000 (kJ/kg)

TRAILING BEOCK CALCULATIONS

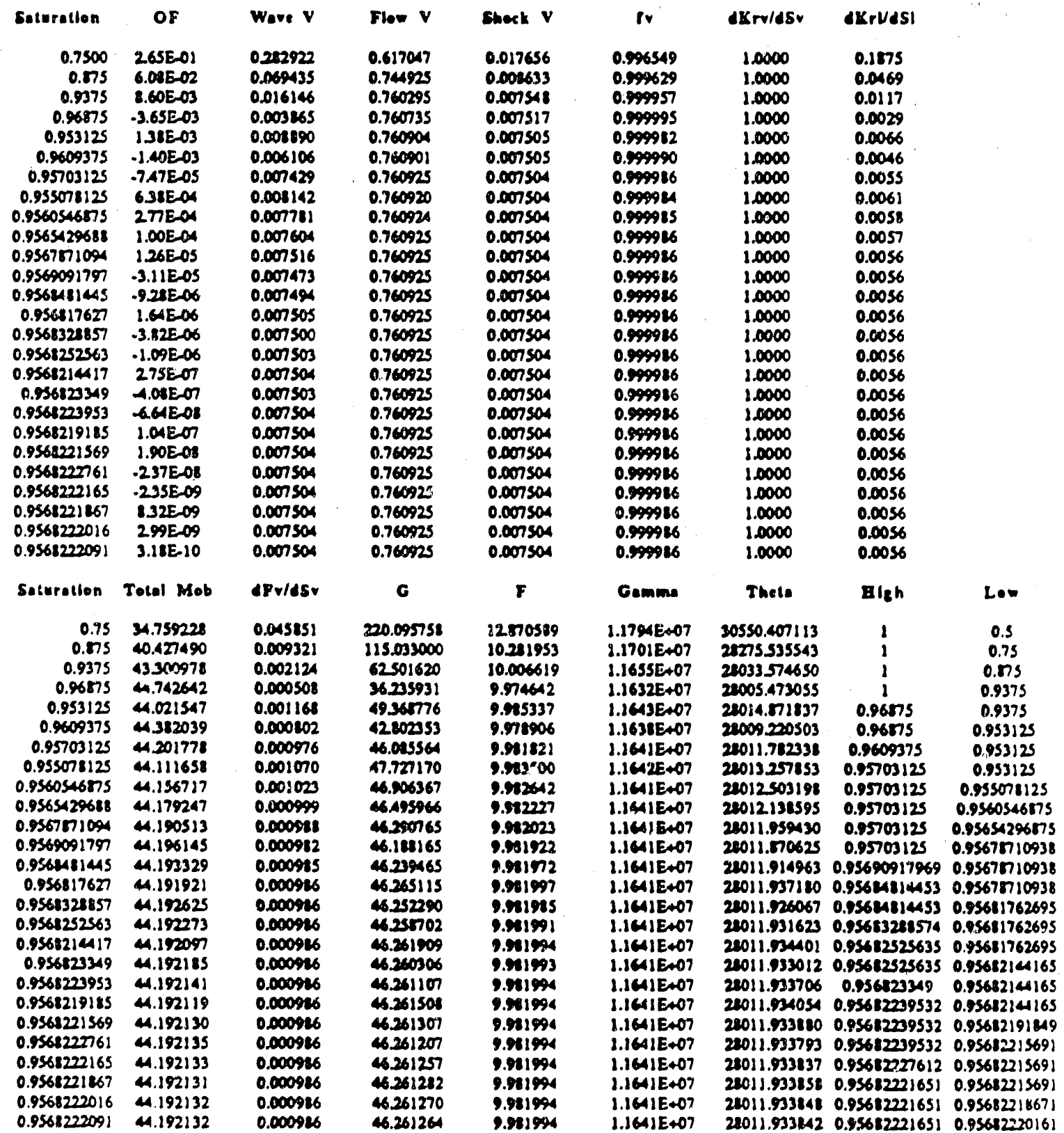


Spreadshet for Calculation of Stream-Water Saturation Profiles

Injection Temperature $600 \cdot \mathrm{K}$
Inltibl Temperature

$350 \cdot x$
Motrlx Beat Copacity

$1.0000\left(\mathrm{~kJ} / \mathrm{kg}_{\mathrm{E}}\right)$

\section{LEADING BOCK CALCULATIONS}

\begin{tabular}{|c|c|c|c|c|c|}
\hline Saturetlo: & OF & Wove $v$ & Fher V & abeck $V$ & Iv \\
\hline 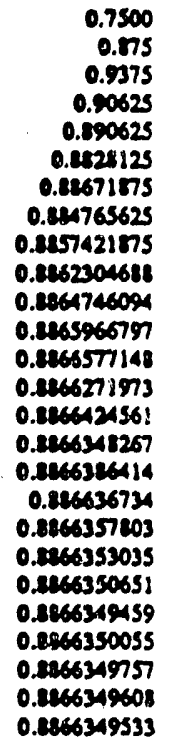 & 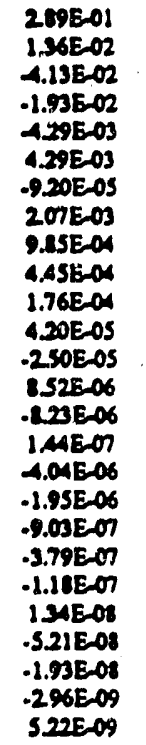 & $\begin{array}{l}0.348992 \\
0.070927 \\
0.016159 \\
0.058054 \\
0.053024 \\
0.061596 \\
0.057217 \\
0.059313 \\
0.058294 \\
0.057754 \\
0.057419 \\
0.057351 \\
0.057244 \\
0.057317 \\
0.057300 \\
0.057309 \\
0.057304 \\
0.057307 \\
0.057308 \\
0.057308 \\
0.057308 \\
0.057309 \\
0.057308 \\
0.057309 \\
0.057309 \\
0.057309\end{array}$ & $\begin{array}{l}0.014655 \\
0.013078 \\
0.013126 \\
0.013019 \\
0.013071 \\
0.013071 \\
0.013070 \\
0.013070 \\
0.013070 \\
0.013070 \\
0.013070 \\
0.013070 \\
0.013070 \\
0.013070 \\
0.013070 \\
0.013070 \\
0.013070 \\
0.013070 \\
0.013070 \\
0.013070 \\
0.013070 \\
0.013070 \\
0.013070 \\
0.013070 \\
0.013070 \\
0.013070\end{array}$ & $\begin{array}{l}0.059513 \\
0.057320 \\
0.057475 \\
0.057336 \\
0.057310 \\
0.057310 \\
0.057309 \\
0.057309 \\
0.057309 \\
0.057309 \\
0.057309 \\
0.057309 \\
0.057309 \\
0.057309 \\
0.057309 \\
0.057309 \\
0.057309 \\
0.057309 \\
0.057309 \\
0.057309 \\
0.057309 \\
0.057309 \\
0.057309 \\
0.057309 \\
0.057309 \\
0.057309\end{array}$ & 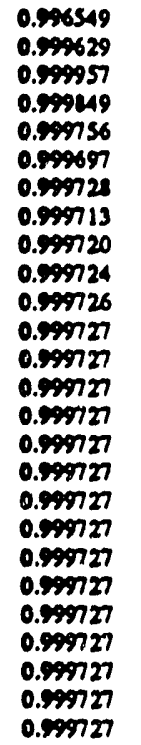 \\
\hline Enturetion & Total Mob & dPr/dSr & $\boldsymbol{6}$ & $F$ & Comans \\
\hline 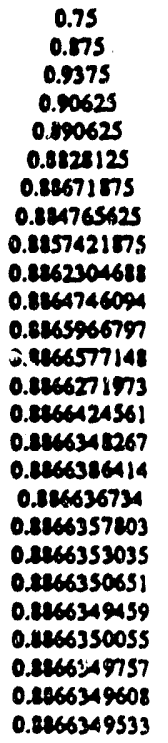 & 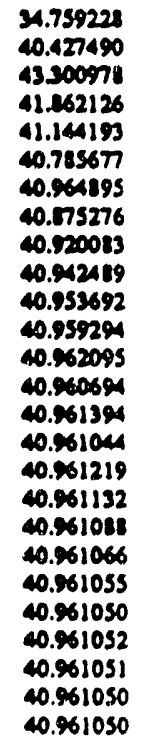 & $\begin{array}{l}0.045151 \\
0.009321 \\
0.002124 \\
0.005001 \\
0.006958 \\
0.007095 \\
0.007519 \\
0.007501 \\
0.007661 \\
0.007590 \\
0.007555 \\
0.007537 \\
0.007521 \\
0.007533 \\
0.007530 \\
0.007531 \\
0.007531 \\
0.007531 \\
0.007531 \\
0.007531 \\
0.007531 \\
0.007531 \\
0.007531 \\
0.007531 \\
0.007531 \\
0.007531\end{array}$ & $\begin{array}{c}20.095758 \\
115.033000 \\
62501620 \\
18.767310 \\
101.500155 \\
101.466577 \\
105.183366 \\
106.624972 \\
105.004169 \\
105.593767 \\
105.310567 \\
105.285966 \\
105.244666 \\
105.260316 \\
105.247491 \\
105.253901 \\
105.250698 \\
105.252301 \\
105.253102 \\
105.253503 \\
105.253703 \\
105.253104 \\
105.253753 \\
105.253779 \\
105.253791 \\
105.253797\end{array}$ & $\begin{array}{l}12.70519 \\
10.211953 \\
10.004619 \\
10.097238 \\
10.175426 \\
10224828 \\
10.199203 \\
10.21179 \\
10.205132 \\
10.202303 \\
10.20979 \\
10.19975 \\
10.199519 \\
10.199712 \\
10.199615 \\
10.199733 \\
10.197109 \\
10.199721 \\
10.199727 \\
10.199730 \\
10.199732 \\
10.199733 \\
10.199732 \\
10.199732 \\
10.199733 \\
10.199733\end{array}$ & 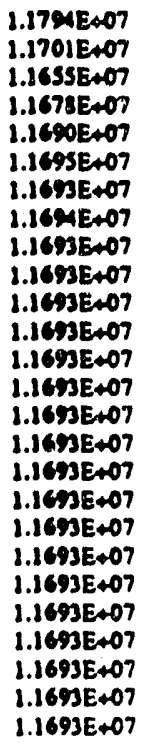 \\
\hline
\end{tabular}

$\begin{array}{ll}\text { IKrvidsr } & \text { dKrVdSI } \\ 1.0000 & 0.1875 \\ 1.0000 & 0.0465 \\ 1.0000 & 0.0117 \\ 1.0000 & 0.0264 \\ 1.0000 & 0.0359 \\ 1.0000 & 0.0412 \\ 1.0000 & 0.0315 \\ 1.0000 & 0.0398 \\ 1.0000 & 0.0392 \\ 1.0000 & 0.0388 \\ 1.0000 & 0.0387 \\ 1.0000 & 0.0386 \\ 1.0000 & 0.0385 \\ 1.0000 & 0.0386 \\ 1.0000 & 0.0385 \\ 1.0000 & 0.0386 \\ 1.0000 & 0.0316 \\ 1.0000 & 0.0386 \\ 1.0000 & 0.0316 \\ 1.0000 & 0.0316 \\ 1.0000 & 0.0316 \\ 1.0000 & 0.0316 \\ 1.0000 & 0.0386 \\ 1.0000 & 0.0386 \\ 1.0000 & 0.0386 \\ 1.0000 & 0.0386\end{array}$

rhete Bigh

$\begin{array}{lll}30550.407113 & 1 & 0.5\end{array}$

$2202535543 \quad 0.75$

$2033574650 \quad 0.775$

$211132094090.9375 \quad 0.875$

$28181.920650 \quad 0.00625 \quad 0.775$

$2225.334700 \quad 0.090625 \quad 0.175$

$242028151330.890625 \quad 0.8128125$

2213.2673530 .866718750 .8128125

2420828909 0.4671675 0.84765625

21205539786 0.c6671575 0.8357421675

28204.174262 0.846716750 .12622046875

22203.4939030 .166717750 .80647460938

24203.154319 0.84671675 0.8465\%667969 33203.324062 0.46665771444 0.26659667969 28203.2391780 .28665771440 .82662719727 22203.2816170 .286642456050 .18662719727 28203.2603970 .86642456050 .24634182666 22203.2710060 .146632641360 .86663412606 232032763120.846636734010 .04663482666 23203278964 0.166635720330 .86663482666 2203.210290 0.8266353035 0.82663482666 282032.00540 .866635065080 .82663412666 21203.2206220 .186535065080 .82663494587 282032807880.86635005470 .88663444587 22203.2208710 .126634975670 .28663494387 21203.2809120 .826634960770 .86663494587 
-njection Temperature $6.90 \cdot \mathrm{K}$
Inllial Temperature

$350 \cdot \mathrm{K}$
Moirlx Beat Capactiy

1.0000 (kJ/kg)
MATRIX POFERTIES

Cp Matrix Densliy Rho $-C_{p}$ $1.00 \quad 2650.00 \quad 2650.0000$

NE SWI Denom

$\begin{array}{lll}N_{\ell} & 5.1 & \text { Denom } \\ 1.00 & 0.00 & 1.000000\end{array}$

SATURATION CONDITIONS Traimation - a1s

\begin{tabular}{|c|c|c|c|c|c|}
\hline & $\begin{array}{c}\text { Volumes } \\
(\text { mis/48) } \\
0.001176\end{array}$ & $\begin{array}{c}\text { Entmelpleo } \\
\text { (a)kE) } \\
901.41\end{array}$ & $\begin{array}{c}\text { Densliles } \\
\text { ayem3) } \\
\text { sso.4723 }\end{array}$ & $\begin{array}{c}\text { Vlscesllles } \\
\text { (Fp) } \\
0.1303\end{array}$ & $\begin{array}{c}\text { Solurallon } \\
\text { S1ep } \\
0.0014063597\end{array}$ \\
\hline & 0.100298 & 2208.52 & 9.970241 & 0.0217 & \\
\hline
\end{tabular}

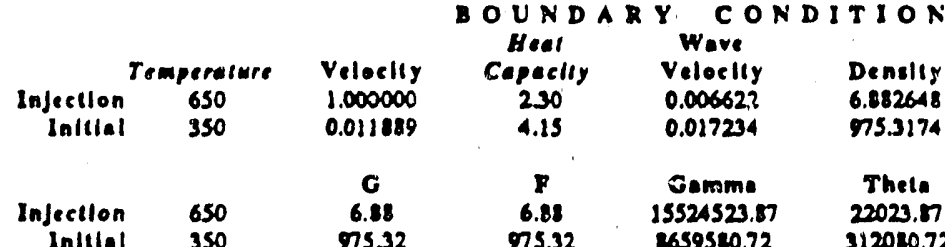

SEOCX CONDITIONS

\begin{tabular}{|c|c|c|c|c|c|c|c|c|}
\hline $\begin{array}{l}\text { Trailing } \\
\text { Lendine }\end{array}$ & $\begin{array}{c}\text { Iabial } \\
\text { So-HI } \\
1 \\
1\end{array}$ & $\begin{array}{c}\text { Gwers } \\
\text { Sv.Lew } \\
0.5 \\
0.5\end{array}$ & $\begin{array}{l}\text { Vapor } \\
\text { Saluratlon } \\
0.9570 \\
0.8666\end{array}$ & $\begin{array}{l}\text { Objeellve } \\
\text { Funellon } \\
\text { l.4356E-09 } \\
4.7472 E-09\end{array}$ & $\begin{array}{l}\text { Two-phase } \\
\text { Wave } \\
\text { veloclly } \\
0.0068 \\
0.0521\end{array}$ & $\begin{array}{l}\text { Upstresm } \\
\text { Flow } \\
\text { Velocliy } \\
0.692182 \\
0.011889\end{array}$ & $\begin{array}{c}\text { Shock } \\
\text { Velecliy } \\
0.0068 \\
0.0521\end{array}$ & $\begin{array}{l}\text { Vepor } \\
\text { Frec Flow } \\
1.0000 \\
0.9947\end{array}$ \\
\hline adins & $\begin{array}{l}d K r v / d S v \\
1.0000 \\
1.0000\end{array}$ & $\begin{array}{c}d \mathrm{Kr} I / \mathrm{dSI} \\
0.0056 \\
0.0386\end{array}$ & $\begin{array}{c}\text { Total } \\
\text { Mobllily } \\
M .1982 \\
40.9610\end{array}$ & $\begin{array}{l}\text { dPv/dSv } \\
0.0010 \\
0.007 s\end{array}$ & $\begin{array}{c}\mathbf{G} \\
46.1514 \\
105.2538\end{array}$ & $\begin{array}{c}F^{\prime} \\
9.9819 \\
10.1997\end{array}$ & $\begin{array}{l}\text { Gamms } \\
1.1641 E+07 \\
1.1693 E+07\end{array}$ & $\begin{array}{c}\text { Thele' } \\
28011.8389 \\
28203.28(19\end{array}$ \\
\hline
\end{tabular}

MATERJAL BALANCES

\begin{tabular}{|c|c|c|c|c|c|}
\hline $\begin{array}{c}\text { Mess } \\
\text { nthalpy }\end{array}$ & $\begin{array}{c}\text { Inslde } \\
\text { 9281855s } \\
\text { 24276.8231 }\end{array}$ & $\begin{array}{c}\ln \mid 11 \text { al } \\
97.531740 \\
665951.0720\end{array}$ & $\begin{array}{l}\text { Injected } \\
6.882648 \\
22023.0721\end{array}$ & $\begin{array}{l}\text { Produced } \\
11.595824 \\
3710.4157\end{array}$ & $\begin{array}{c}\text { Bolance } \\
0.0000 \\
.5 .2947\end{array}$ \\
\hline
\end{tabular}


Injeclion Temperalure

$650 *$
Intibl Temperalure

$350 \%$
Malrix Beal Cepocliy

$1.0000(\mathrm{~kJ} / \mathrm{ke})$

SATURATION PROFILE

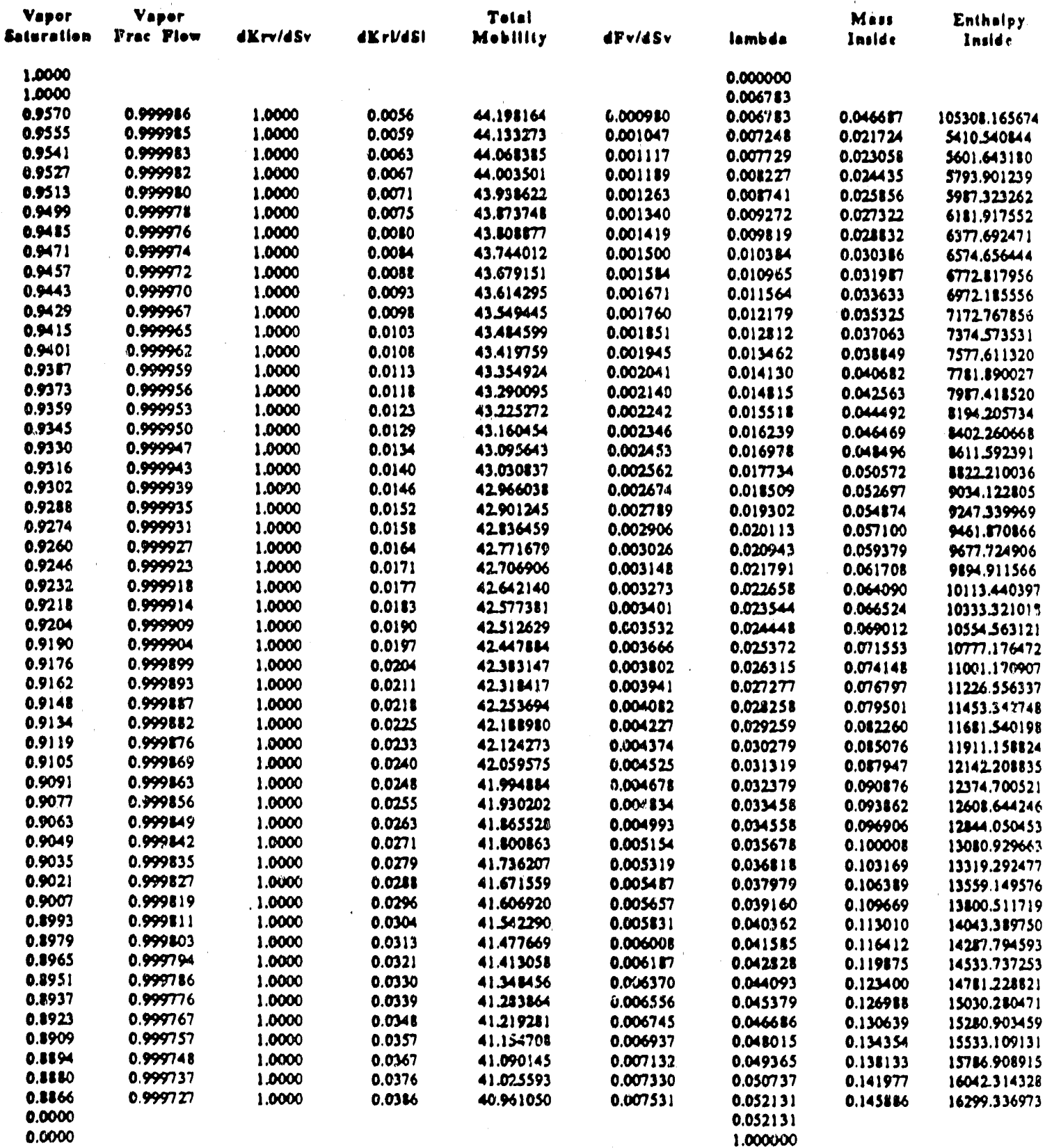


Injectlon Tamperacure $650^{\circ} \mathrm{K}$
Inlliel Temperature sso $\mathcal{K}$
Matrlx Beat Capacity

$1.0000\left(\mathrm{hJ} / \mathrm{k}_{\mathrm{E}}\right)$

TRAILING SBOCX CALCULATIONS

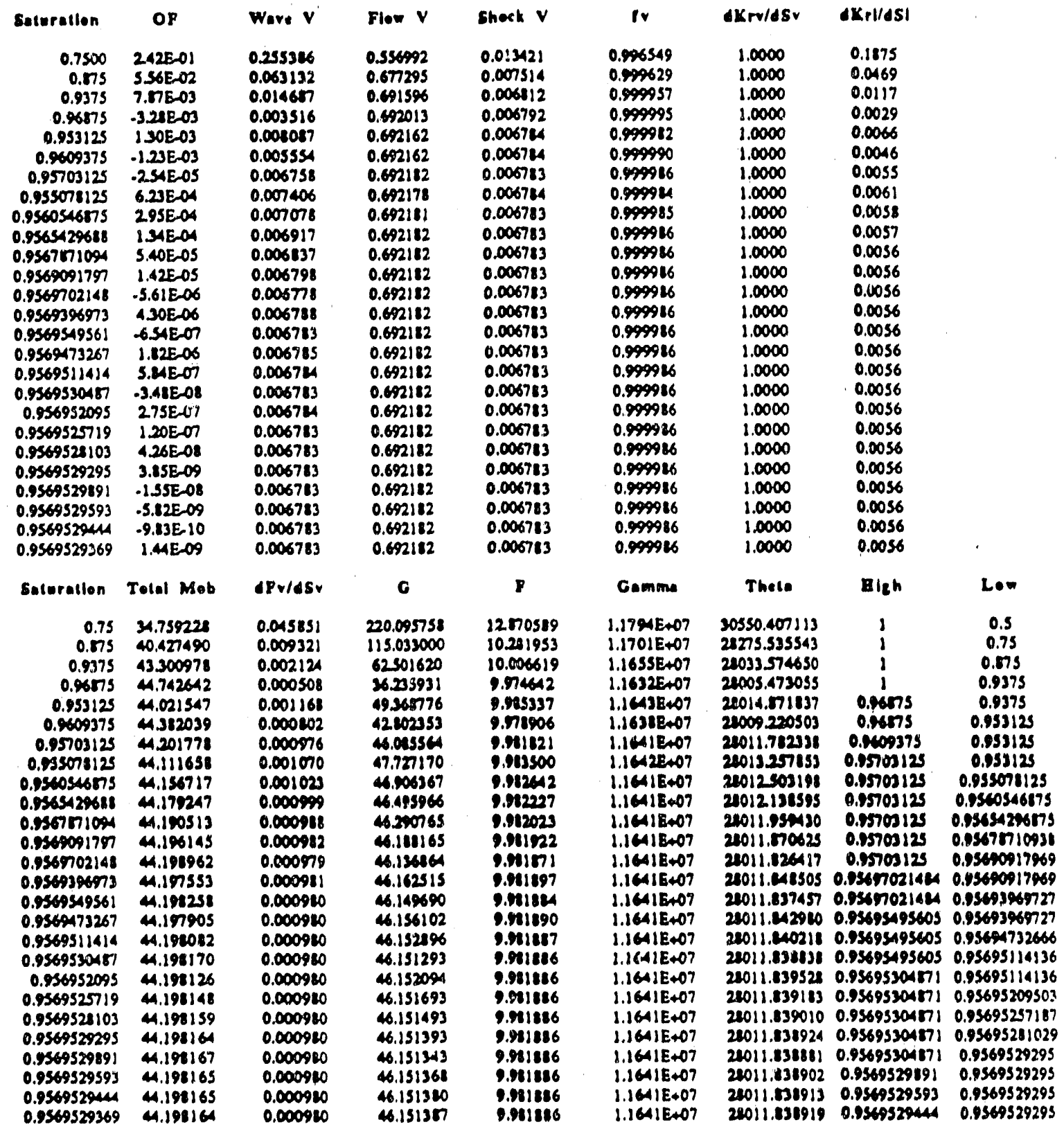


Spreadsheet for Calculation of Stream-Water Saturation Proftles

Injection Temperalure

$650 \cdot \mathcal{K}$
Inllial Temperalure

$350 \cdot x$
Matrix Beat Capacliy

$1.0000\left(k J / k_{R}\right)$

LEADING SHOCK CALCULATIONS

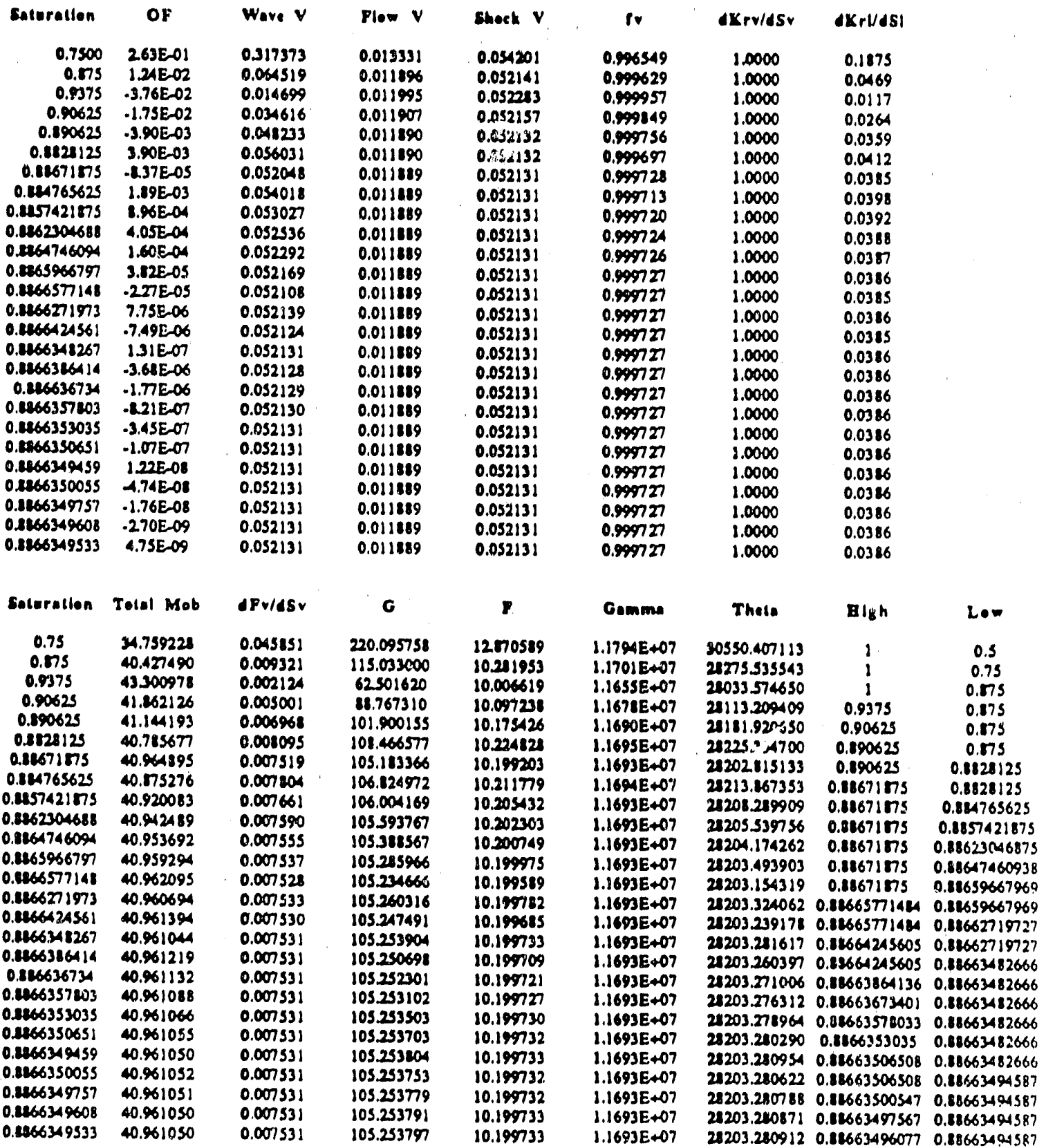


Spreadsheet for Calculation of Stream-Water Saturation Profilea

Infection Temperature $700^{\circ} \mathrm{x}$
Inlilal Temperature

$380 \cdot \mathbf{K}$
Matrix Deat Cepactly 1.0006 (WJ/kB)
MATRIX PROPERTIES

Pereolly
0.10
$N=0$
3.00

Cp Matrix 1.00

$N_{8}$

1.00

Dencily
2650.00
$S=1$

0.00
Rhe $-C_{p}$

2650.0000

Denem

1.000000

\section{SATURATION riciuraten - Ales.s7}

\begin{tabular}{|c|c|c|c|c|c|}
\hline . & $\begin{array}{c}\text { Velomes } \\
\text { (minsike } \\
0.001176\end{array}$ & $\begin{array}{c}\text { Eotholples } \\
\text { (0)ke) } \\
01.41\end{array}$ & $\begin{array}{c}\text { Denaliles } \\
\text { argin3) } \\
2.00 .4723 \\
0.09021\end{array}$ & $\begin{array}{c}\text { Vloceslites } \\
\text { (cp) } \\
0.1303\end{array}$ & $\begin{array}{c}\text { Saturation } \\
\text { Step } \\
0.0014093366\end{array}$ \\
\hline
\end{tabular}

DOUNDADY CONDITIONS Hoir Wave

Cepertily Velocliy Denally

0.006003 Enthalpy

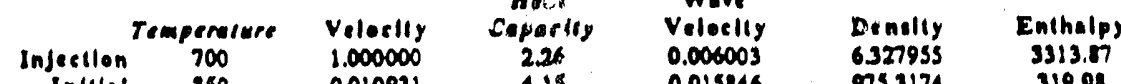

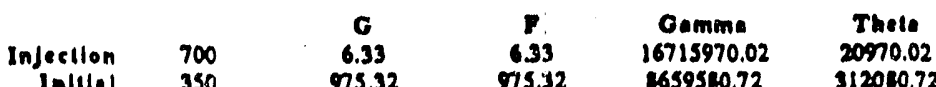

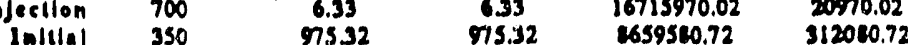

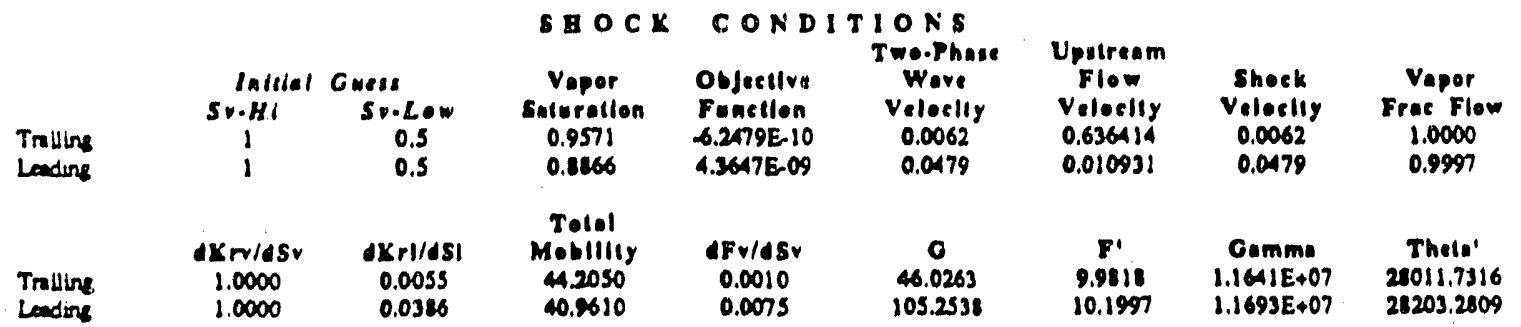

MATERIAL DALANCES

\begin{tabular}{|c|c|c|c|c|c|c|}
\hline - & $\begin{array}{c}\text { Inilde } \\
93.198119 \\
833521.4811\end{array}$ & $\begin{array}{c}\text { InIIIal } \\
\text { n.S31740 } \\
\text { ess958.0720 }\end{array}$ & $\begin{array}{c}\text { Injecled } \\
6.327955 \\
20070.0198\end{array}$ & $\begin{array}{l}\text { Produced } \\
10.661567 \\
\text { M11.4735 }\end{array}$ & $\begin{array}{c}\text { Dolunce } \\
0.0000 \\
4.0688\end{array}$ & $\begin{array}{c}\text { Error } \\
0.0000 \% \\
0.0005 \%\end{array}$ \\
\hline
\end{tabular}


Injection Temperalure $700 \cdot x$
Inllial Temperature sso $\bullet \mathrm{K}$
Matrix Beat Capaclly $1.0000(k J / k t)$

SATURATION PROFILE

\begin{tabular}{|c|c|c|c|c|c|c|c|c|}
\hline $\begin{array}{l}\text { Vaper } \\
\text { soturation }\end{array}$ & $\begin{array}{c}\text { Vapor } \\
\text { Frec Flow }\end{array}$ & $d K r / d s v$ & $d x p l d S \mid$ & $\begin{array}{c}\text { Telal } \\
\text { Mobllity }\end{array}$ & dFv/dev & Lambda & $\begin{array}{l}\text { Maes } \\
\text { Inside }\end{array}$ & $\begin{array}{l}\text { Entholpy } \\
\text { Inolde }\end{array}$ \\
\hline $\begin{array}{l}1.0000 \\
1.0000\end{array}$ & & & & & & $\begin{array}{l}0.000000 \\
0.006192\end{array}$ & & \\
\hline 0.9571 & 0.999986 & 1.0000 & 0.0055 & 44.205033 & 0.000973 & 0.006192 & 0.039186 & 103513.160498 \\
\hline 0.9557 & 0.999985 & 1,0000 & 0.0059 & 44.140003 & 0.001040 & 0.006619 & 0.018819 & 4965.764438 \\
\hline 0.9543 & 0.999983 & 1.0000 & 0.0063 & 44.074978 & 0.001109 & 0.007061 & 0.0211117 & 5143.104365 \\
\hline 0.9529 & 0,999982 & 1.0000 & 0.0067 & 44.009956 & 0.001181 & 0.007518 & 0.02234 & 5320.512883 \\
\hline 0.9515 & 0.999980 & 1.0000 & 0.0071 & 43.944939 & 0.001255 & 0.007990 & 0.023693 & 5498.997628 \\
\hline 0.9501 & 0.999978 & 1.0000 & 0.0075 & 43.879927 & 0.001332 & 0.00478 & 0.025042 & 5678.566292 \\
\hline 0.9186 & 0.999976 & 1.0000 & 0.0079 & 43.814919 & 0.001411 & 0.008981 & 0.026432 & 5859.226625 \\
\hline 0.9172 & 0.999974 & 1.0000 & 0.004 & 43.749916 & 0.001493 & 0.009499 & 0.027164 & 6040.916431 \\
\hline 0.9458 & 0.999972 & 1.0000 & 0.0088 & 43.644917 & 0.001577 & 0.010034 & 0.029337 & 6223.853575 \\
\hline 0.944 & 0.999970 & 1.0000 & 0.0093 & 43.619924 & 0.001663 & 0.01054 & 0.030853 & 6407.835977 \\
\hline 0.9430 & 0.999967 & 1.0000 & 0.0097 & 43.554936 & 0.001752 & 0.011149 & 0.032112 & 6592.941617 \\
\hline 0.9416 & 0.999965 & 1.0000 & 0.0102 & 43.489952 & 0.00143 & 0.011731 & 0.034014 & 6779.178539 \\
\hline 0.9402 & 0.999962 & 1.0000 & 0.0107 & 43.424975 & 0.001937 & 0.012329 & 0.035659 & 6966.554828 \\
\hline 0.9988 & 0.999959 & 1.0000 & 0.0112 & 43.360002 & 0.002034 & 0.012943 & 0.037348 & 7155.074655 \\
\hline 0.9374 & 0.999956 & 1.0000 & 0.0118 & 43.295035 & 0.002133 & 0.019373 & 0.039081 & 7344.798234 \\
\hline 0.9360 & 0.999953 & 1.0000 & 0.0123 & 43.230074 & 0.002234 & 0.014220 & 0.040858 & 7535.001244 \\
\hline 0.9346 & 0.999950 & 1.0000 & 0.0129 & 43.165119 & 0.002338 & 0.014883 & 0.042831 & 7727.617227 \\
\hline 0.9331 & $\ln 999947$ & 1.0000 & 0.0134 & 43.100170 & 0.002445 & 0.015562 & 0.044549 & 7920.814585 \\
\hline 0.9317 & 0.999913 & 1.0000 & 0.0140 & 43.035227 & 0.002555 & 0.016258 & 0.046462 & 8115.200582 \\
\hline 0.9303 & 0.999940 & 1.0000 & 0.0146 & 42970290 & 0.002667 & 0.016971 & $0.04 \times 422$ & 8310.74349 \\
\hline 0.9289 & 0.999936 & 1.0000 & 0.0152 & 42005360 & 0.002781 & 0.017700 & 0.050428 & 8507.574474 \\
\hline 0.9275 & 0.999932 & 1.0000 & 0.0158 & 42840436 & 0.002399 & 0.01447 & 0.052412 & $805.57 \% 616$ \\
\hline 0.9261 & 0.999928 & 1.0000 & 0.0164 & 12775sis & 0.003018 & 0.019210 & 0.054582 & 9904.208492 \\
\hline 0.9247 & 0.999923 & 1.0000 & 0.0170 & 42710608 & 0.003141 & 0.019991 & 0.056730 & 9105.269888 \\
\hline 0.9233 & 0.999919 & 1.0000 & 0.0177 & 12445704 & 0.003266 & 0.020788 & 0.055927 & 9306.972656 \\
\hline 0.9219 & 0.999914 & 1.0000 & 0.0183 & 42560008 & 0.003395 & 0.021604 & 0.061172 & 9509.925710 \\
\hline 0.9205 & 0.999909 & 1.0000 & 0.0190 & 42515918 & 0.003525 & 0.022436 & 0.063466 & 914.132035 \\
\hline 0.9190 & 0.999904 & 1.0000 & 0.019 & 12451036 & 0.003659 & 0.022286 & 0.055809 & 9919.611680 \\
\hline 0.9176 & 0.999899 & 1.0000 & 0.0203 & 42386161 & 0.003795 & 0.024154 & 0.068203 & 10126.376765 \\
\hline 0.9162 & 0.999893 & 1.0000 & 0.0211 & 4232129 & 0.003934 & 0.025039 & 0.070647 & 10334.421476 \\
\hline 0.9148 & 0,999888 & 1.0000 & 0.0218 & 12256434 & 0.004076 & 0.025943 & 0.073141 & 10543.762067 \\
\hline 0.9134 & 0.999812 & 1.0000 & 0.0225 & 42.191512 & 0.004221 & 0.026864 & 0.075687 & 10754.407863 \\
\hline 0.9120 & 0.949576 & 1.0000 & 0.0232 & 12.126739 & 0.004369 & 0.027803 & 0.07824 & $10966.36826 n$ \\
\hline 0.9106 & 0.999870 & 1.0000 & 0.0240 & 42061903 & 0.004519 & 0.028761 & 0.080934 & 11179.652723 \\
\hline 0.9092 & 0.999263 & 1.0000 & 0.0247 & 41.997075 & 0.004673 & 0.029737 & 0.083636 & 11394.270788 \\
\hline 0.9078 & 0.999856 & 1.0000 & 0.0255 & 41.932256 & 0.004829 & 0.030731 & 0.046391 & 18610.232063 \\
\hline 0.9064 & 0.99949 & 1.0000 & 0.0263 & 41.667445 & 0.004988 & 0.031743 & 0.089200 & 11127.546229 \\
\hline 0.9050 & $0.999 \times 42$ & 1.0000 & 0.0211 & 41.202642 & 0.005150 & 0.032775 & 0.082062 & 12046.223040 \\
\hline 0.9035 & 0.999835 & 1.0000 & 0.0279 & 41.737249 & 0.005315 & 0.033825 & 0.094910 & 12266.272322 \\
\hline 0.9021 & 0.999827 & 1.0000 & 0.0287 & 41.673064 & 0.005483 & 0.034894 & 0.097952 & 12477.703978 \\
\hline 0.9007 & 0.999820 & 1.0000 & $0.02 \%$ & 48.608208 & 0.005654 & 0.035982 & 0.100979 & 12710.527982 \\
\hline 0.8993 & 0.999811 & 1.0000 & 0.0304 & 41.543521 & 0.005822 & 0.037089 & 0.104062 & 12934.754388 \\
\hline 0.0979 & 0.999803 & 1.0000 & 0.0313 & 41.470764 & 0.006005 & 0.038215 & 0.107202 & 13160.393321 \\
\hline 0.0965 & 0.99979 & 1.0000 & 0.0321 & 41.414015 & 0.008185 & 0.039361 & 0.110399 & 13307454987 \\
\hline 0.0951 & 0.999786 & 1.0000 & 0.0330 & 4134927 & 0.006368 & 0.040526 & 0.283653 & 13615.949667 \\
\hline 0.8937 & $0.999 m 7$ & 1.0000 & 0.0339 & 41.214547 & 0.006554 & 0.041710 & 0.116965 & 13845.8R7720 \\
\hline 0.0923 & 0.999767 & 1.0000 & 0.0341 & 41.219328 & 0.006743 & 0.042915 & 0.120336 & 14077.279584 \\
\hline 0.1909 & 0.999758 & 1.0000 & 0.0357 & 41.155118 & 0.006936 & 0.041139 & 0.123765 & 14310.135778 \\
\hline 0.8195 & 0.999748 & 1.0000 & 0.0367 & 41.090419 & 0.007131 & 0.045383 & 0.127254 & 14544.466197 \\
\hline 0.8880 & 0.999737 & 1.0000 & 0.0376 & 41.025729 & 0.007330 & 0.046647 & 0.130803 & 14780.283620 \\
\hline 0.8866 & 0.999727 & 1.0000 & 0.0386 & 40.961050 & 0.007531 & 0.047931 & 0.134112 & 15017.596707 \\
\hline $\begin{array}{l}0.0000 \\
0.0000\end{array}$ & & & & & & 0.047931 & & \\
\hline 0.0000 & & & & & & 1.000000 & & \\
\hline
\end{tabular}


Spreadsheet for Calculation of Stream-Water Saturation profiles

Injectlon Temperalure $700-\mathrm{K}$
Inlital Temperalure

$350 \cdot \mathrm{K}$
Matrlx Beal Capacliy

1.0000 (kJ/kE)

TRAILING SHOCK CALCULATIONS

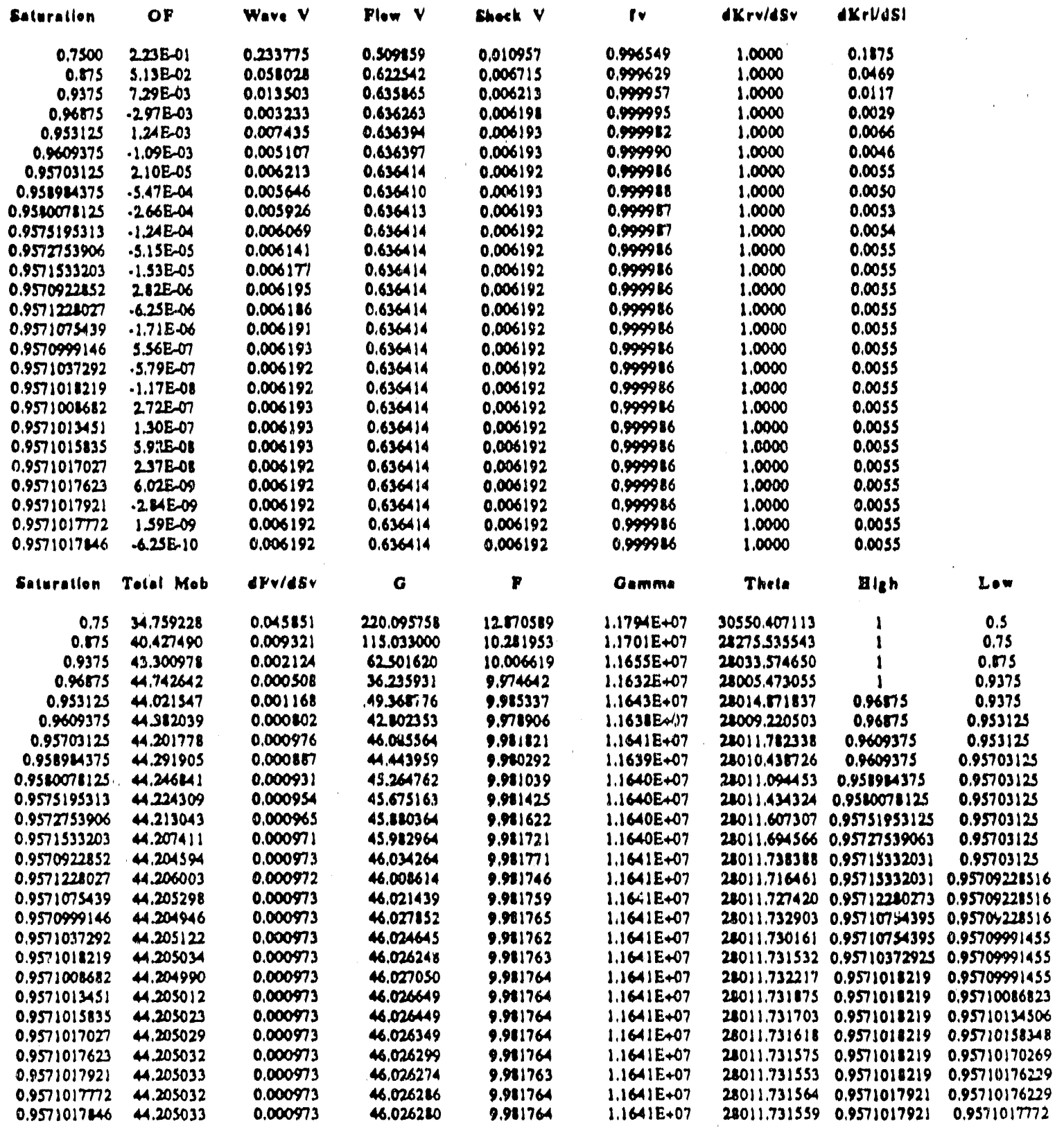


$-168$.

Spreadsheet for Calculation of Stream-Water Saturation Profiles

Injecllon Timperuture $700 \cdot \%$
Inllial Temperature

$350 \cdot \mathrm{K}$
Malrix Beal Capucliy

$1,0000\left(k J / k_{B}\right)$

LEADINO SUOCX. CALCULATIONS

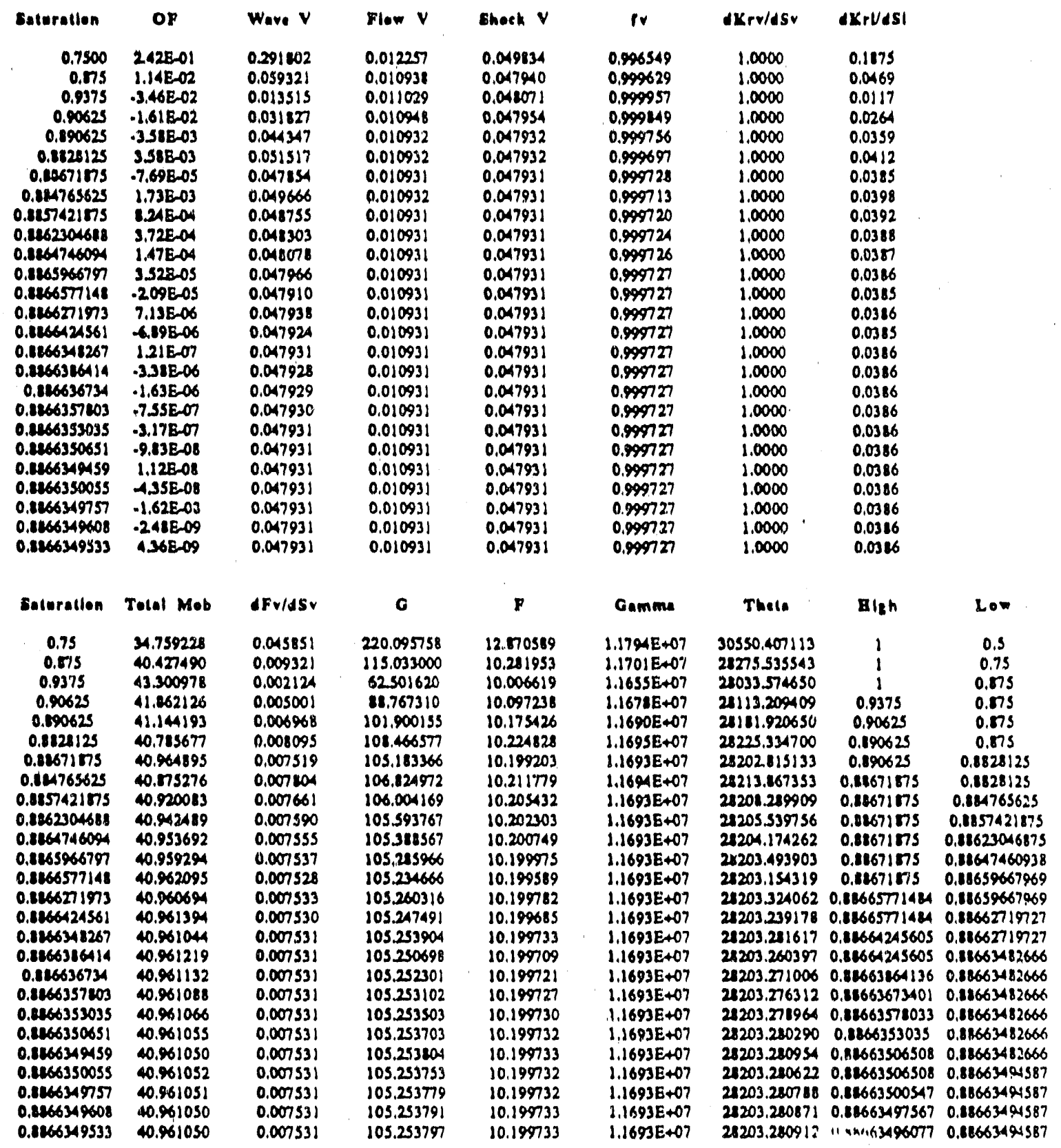


Spreadsheet for Calculation of Stream-Water Saturation Profiles

Iajection Temperalure $750^{\circ} \mathrm{K}$
Inlial Temperature

350. $\bullet$
Matrix Beul Capocliy

$1.0000(\mathrm{~kJ} / \mathrm{kg})$

MATRIX PROPERTIES

$\underset{\substack{\text { Peresily } \\ 0.10 \\ 3.00}}{ }$

Cp Mards

Densily
2650.00
$5=1$
0.00

Rho $\cdot \mathrm{Cp}_{\mathrm{p}}$

2650.0000

Denom

$\begin{array}{lll}00 & 0.00 & 1.000000\end{array}$

SATURATION CONDITIONS Trecumichen 485.57

\begin{tabular}{|c|c|c|c|c|c|}
\hline $\begin{array}{l}\text { Llauld } \\
\text { Vapor }\end{array}$ & $\begin{array}{c}\text { Volumes } \\
\text { (misks) } \\
0.001176 \\
0.100298\end{array}$ & $\begin{array}{c}\text { Entliniples } \\
(21 / 4) \\
901.41 \\
2008.52\end{array}$ & $\begin{array}{c}\text { Densitles } \\
\text { (ce/m3) } \\
050.4723 \\
9.970241\end{array}$ & $\begin{array}{c}\text { Vlscoslities } \\
\text { (Fp) } \\
0.1303 \\
0.0217\end{array}$ & $\begin{array}{c}\text { Soluratlon } \\
\text { siep } \\
0.0014120916\end{array}$ \\
\hline
\end{tabular}

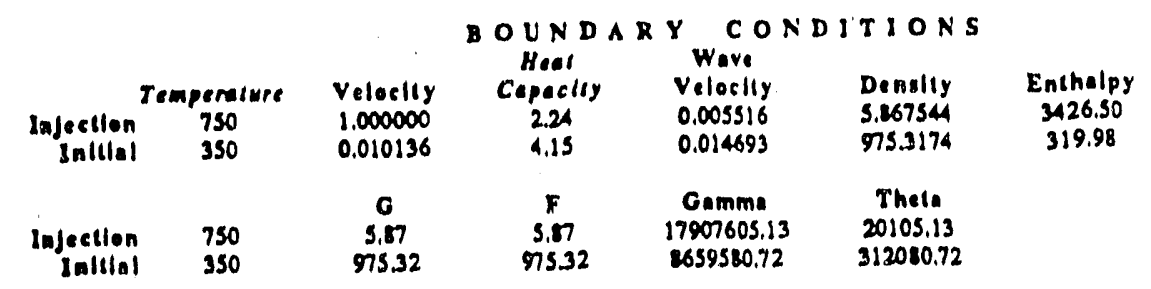

\begin{tabular}{|c|c|c|c|c|c|c|c|c|}
\hline Triling & $\begin{array}{c}\text { Inllial } \\
\text { Sy.Hi } \\
1 \\
1\end{array}$ & $\begin{array}{c}\text { Guess } \\
\text { srolew } \\
0.5 \\
0.5\end{array}$ & $\begin{array}{c}\text { S B O C } \mathrm{K} \\
\text { Vapor } \\
\text { saturatlon } \\
0.9572 \\
0.1266\end{array}$ & $\begin{array}{l}\text { C O N D } \\
\text { Objectlre } \\
\text { Funetlon } \\
1.92 \% 0 \mathrm{E}-09 \\
4.0472 \mathrm{E}-09\end{array}$ & $\begin{array}{c}\text { IO NS } \\
\text { Two.Phase } \\
\text { Wave } \\
\text { Velocliy } \\
0.0057 \\
0.0444\end{array}$ & $\begin{array}{l}\text { Upotream } \\
\text { F tow } \\
\text { Velocity } \\
0.590121 \\
0.010136\end{array}$ & $\begin{array}{c}\text { Shock } \\
\text { veloctly } \\
0,0057 \\
0.0444\end{array}$ & $\begin{array}{c}\text { Vopor } \\
\text { Frac Flaw } \\
1.0000 \\
0.9997\end{array}$ \\
\hline $\begin{array}{l}\text { Trulline } \\
\text { Lendine }\end{array}$ & $\begin{array}{l}\text { dKrv/dSv } \\
1.0000 \\
1.0000\end{array}$ & $\begin{array}{c}\text { KrI/dSI } \\
0.0055 \\
0.0316\end{array}$ & $\begin{array}{c}\text { Tolal } \\
\text { Moblllty } \\
4.21114 \\
40.9610\end{array}$ & $\begin{array}{c}\text { dFv/dSv } \\
0.0010 \\
0.0075\end{array}$ & $\begin{array}{c}\sigma \\
4 \$ .9105 \\
105.2538\end{array}$ & $\begin{array}{c}F^{\prime} \\
9.9817 \\
10.1997\end{array}$ & $\begin{array}{c}\text { Commi } \\
1.1640 \mathrm{E}+07 \\
1.1693 \mathrm{E}+07\end{array}$ & $\begin{array}{c}\text { Thete' } \\
28011.6329 \\
2 \$ 203.2109\end{array}$ \\
\hline
\end{tabular}

MATERIAL BALANCES

\begin{tabular}{|c|c|c|c|c|c|c|}
\hline $\begin{array}{l}\text { Mass } \\
\text { inchalpy }\end{array}$ & $\begin{array}{c}\text { Inside } \\
93.513236 \\
182904.3953\end{array}$ & $\begin{array}{c}\ln |t| a \mid \\
97531740 \\
65958.0720\end{array}$ & $\begin{array}{l}\text { Infecled } \\
\text { s.267544 } \\
20: 05.1252\end{array}$ & $\begin{array}{l}\text { Produced } \\
9.186039 \\
3163.3211\end{array}$ & $\begin{array}{c}\text { Dalence } \\
0.0000 \\
4.5192\end{array}$ & $\begin{array}{c}\text { Errer } \\
0.0000 \% \\
0.0005 \%\end{array}$ \\
\hline
\end{tabular}


Spreadsheet for Calculation of Stream-Water Saturation Profiles

Injectlon Tempet lart $780 \cdot \mathrm{K}$
Inllial Temperature

$350 \cdot x$
Matrix Beat Capodity $1.0000(\mathbf{K J} / \mathbf{k} \mathbf{t})$

SATURATION PROFILE

\begin{tabular}{|c|c|c|c|c|c|c|c|c|}
\hline $\begin{array}{l}\text { Vapor } \\
\text { Saturatloo }\end{array}$ & $\begin{array}{c}\text { Vaper } \\
\text { Prae Flow }\end{array}$ & $d K r v / d S r$ & AXrVASt & $\begin{array}{c}\text { Total } \\
\text { Mobillty }\end{array}$ & AFviesv & lombde & $\begin{array}{l}\text { Mass } \\
\text { Inside }\end{array}$ & $\begin{array}{l}\text { Enthalpy } \\
\text { Inaslde }\end{array}$ \\
\hline 1.0000 & & & & & & $\begin{array}{l}0.000000 \\
0.0 x^{2} 704\end{array}$ & & \\
\hline 0.9572 & 0. einges & 1.0000 & $0.005 s$ & 4.211399 & 0.000967 & 0.00s7un & 0.033469 & 102146.953944 \\
\hline 0.9558 & 0.899985 & 1.0000 & 0.0059 & 44.146232 & 0.001034 & 0.006099 & 0.018370 & 4598.682045 \\
\hline 0.954 & $0 . \operatorname{sing} 4$ & 1.0000 & 0.0062 & 44.011079 & 0.001103 & 0.006508 & 0.019509 & 4762739893 \\
\hline 0.9530 & 0.999912 & 1.0000 & 0.0066 & 4.015930 & 0.001175 & 0.006931 & 0.020886 & 4927.793751 \\
\hline 0.9516 & 0.990920 & 1.0000 & 0.0070 & 13.950726 & 0.001249 & 0.007369 & 0.021900 & 5093.150752 \\
\hline 0.9502 & 0.999978 & 1.0000 & 0.0074 & 43.815646 & 0.001325 & 0.007121 & 0.023153 & 5260.912078 \\
\hline 0.9488 & $0.9 \sin 6$ & 1.0000 & 0.0079 & 43.220510 & 0.001404 & 0.008287 & 0.024443 & 5429.002967 \\
\hline 0.9474 & 974 & 1.0000 & 0.0083 & 43.755379 & 0.001486 & 0.005767 & 0.025773 & 5598.112708 \\
\hline 0.9459 & 0.99972 & 1.0000 & 0.0088 & 43.690254 & 0.001570 & 0.009263 & 0.027141 & 5768.254645 \\
\hline 0.9445 & 0.9 .9770 & 1.0000 & 0.0092 & 43.625133 & 0.001656 & 0.009772 & 0.022549 & 5939.436177 \\
\hline 0.9431 & o.spinss & 1.0000 & 0.0097 & 43560017 & 0.001745 & 0.010297 & 0.029997 & 6111.661757 \\
\hline 0.9417 & 0.999965 & 1.0000 & 0.0102 & 43.494908 & 0.001836 & 0.010836 & 0.031485 & 624.947892 \\
\hline 0.9403 & $0.9 x p e 2$ & 1.0000 & 0.0107 & 43.429101 & 0.001930 & 0.011391 & 0.033013 & 459.293147 \\
\hline U.9389 & 0.999960 & 1.0000 & 0.0122 & 43344701 & 0.002027 & 0.011960 & 0.034582 & 6634.708110 \\
\hline 0.9375 & 0.999957 & 8.0000 & 0.0117 & 43299607 & 0.002126 & 0.01254 & 0.036193 & 6111.200548 \\
\hline 0.9361 & o.giness & 1.0000 & 0.0123 & 43224519 & 0.002227 & 0.013144 & 0.037 4s & 6988.778106 \\
\hline 0.9346 & $0 . \operatorname{logs50}$ & 1.0000 & 0.0121 & 43.169436 & 0.002331 & 0.013759 & 0.039538 & 7167.48602 \\
\hline 0.9332 & o.sivint & 1.0000 & 0.0134 & 43.104360 & 0.002431 & 0.014389 & 0.041274 & 7347.219886 \\
\hline 0.9318 & 043 & 1.0000 & 0.0139 & 43.039229 & 0.002541 & 0.025035 & 0.043053 & 7528.099165 \\
\hline 0.5304 & 940 & 1.0000 & 0.0145 & 42974225 & 0.002660 & $0.0156 \%$ & 0.044874 & 7710.096505 \\
\hline 0.9290 & 0.999936 & 1.0000 & 0.0151 & 42909167 & 0.002774 & 0.016373 & 0.046739 & 7093.217830 \\
\hline 0.9276 & 0.999932 & 1.0000 & 0.0157 & 4284116 & 0.002892 & 0.017065 & 0.042648 & $\operatorname{com} .471928$ \\
\hline 0.9262 & 0.499928 & 1.0000 & 0.0164 & 12779071 & 0.003012 & 0.017774 & 0.050601 & 1062866942 \\
\hline 0.9248 & $0.99 x_{23}$ & 1.0000 & 0.0170 & 19714034 & 0.003135 & 0.016498 & 0.052598 & 249.411079 \\
\hline 0.9233 & $0.9 \sin 18$ & 1.0000 & 0.0176 & 42449003 & 0.003260 & 0.018238 & 0.054640 & 2637.112607 \\
\hline 0.9219 & $0 . \cos 114$ & 1.0000 & 0.0123 & 42583979 & 0.003388 & 0.019995 & 0.056728 & 1825.97985s \\
\hline 0.9205 & $0 . \operatorname{senses}$ & 1.0000 & 0.01189 & 42516962 & 0.003519 & 0.020767 & 0.058861 & 5016.021216 \\
\hline 0.9191 & $0.9 \sin 0$ & 1.0000 & $0.01 \%$ & 42453953 & 0.003653 & 0.021556 & 0.061041 & 8207.245144 \\
\hline 0.917 & 0.999899 & 1.0000 & 0.0203 & 42385951 & 0.003789 & 0.022362 & 0.063267 & 9399.660157 \\
\hline 0.9163 & 6894 & 1.0000 & 0.0210 & 42323957 & 0.003929 & 0.02314 & 0.065539 & 0593.274838 \\
\hline 0.9149 & 0.99288 & 1.0000 & 0.0217 & 42.258970 & 0.004071 & 0.024022 & 0.067860 & ก18.097135 \\
\hline 0.9135 & 0.1908s2 & 1.0000 & 0.0225 & 42198901 & 0.004216 & 0.024878 & 0.070228 & Fed.137854 \\
\hline 0.9121 & 0.790876 & 1.0000 & 0.0232 & 42.120020 & 0.004363 & 0.025750 & 0.07244 & 10161.403677 \\
\hline 0.0106 & 0.79870 & 1.0000 & 0.0240 & 42064057 & 0.004584 & 0.024339 & 0.075108 & 10379.904147 \\
\hline 0.0078 & 0. कoust & $\begin{array}{l}1.0000 \\
1.0000\end{array}$ & $\begin{array}{l}0.0247 \\
0.0255\end{array}$ & 01.00102 & 0.00688 & 0.027545 & 0.077622 & 10579.48172 \\
\hline 0.004 & o.runso & 10000 & $\begin{array}{l}0.0253 \\
0.0263\end{array}$ & at.134iss & 0.004124 & 0.021468 & 0.000185 & 10720.44731 \\
\hline 0.1050 & & 1.0000 & 0.0271 & 41.004289 & 0.005146 & $\begin{array}{l}0.024409 \\
0.000365\end{array}$ & $\begin{array}{l}0.042798 \\
0.055462\end{array}$ & $\begin{array}{l}10012902367 \\
11126.431693\end{array}$ \\
\hline 0.0036 & 835 & 1.0000 & 0.0279 & 41.739369 & 0.005311 & 0.031341 & 0.021176 & 11391.240392 \\
\hline 0.02022 & & 10000 & 0.0277 & 91.674457 & $\operatorname{cocos} 479$ & 0.037334 & oscosil & 11597338214 \\
\hline 0.1008 & $0 . \cos 320$ & 10000 & 0.0295 & ai.cooss & 0.005450 & 0.033345 & 0.078759 & 11204.7344t2 \\
\hline 0.5003 & $0 . \operatorname{mos} 12$ & 1.0000 & 0.0304 & atsanes! & 0.005125 & 0.084373 & $0.0 \times 6628$ & 12013.438587 \\
\hline 0.0979 & o.smoves & 8.0000 & 0.0313 & 41.479776 & 0.004002 & 0.035419 & 0.020550 & 12023.459991 \\
\hline 0.0965 & o.siviss & 1.0000 & 0.0321 & 41A14901 & 0.005182 & o.osentis & 0.102525 & 12434.808231 \\
\hline 0.0951 & o.sings & $1.000 n$ & 0.0330 & 41.350036 & 0.006366 & 0.037565 & $0.10555 ?$ & 12617.492913 \\
\hline 0.0937 & $0.994 m$ & 1.0000 & 0.0339 & 41225120 & 0.006552 & 0.038666 & $0.10: 636$ & 12861523717 \\
\hline 0.5923 & $0.9 \sin 67$ & 1.0000 & 0.0348 & 41.220334 & 0.006742 & 0.03974 & 0.111773 & 12076.910398 \\
\hline 0.0904 & $0.99 \% 5$ & 1.0000 & 0.0357 & 41.155498 & 0.000934 & 0.040921 & 0.114965 & 13293.662715 \\
\hline 0.8985 & o.sinst & 1.0000 & 0.0367 & 41.090672 & 0.007130 & 0.04207 & 0.118212 & 13511.790775 \\
\hline 0.8310 & $0 . \sin 37$ & 1.0000 & 0.0376 & 41.025856 & 0.001329 & 0.013251 & 0.121515 & 13731.304153 \\
\hline $\begin{array}{l}0.8166 \\
0.0000\end{array}$ & 0.4ing? & 1.0000 & 0.0366 & $\omega 0.861050$ & 0.007531 & $\begin{array}{l}0.0 \mathrm{ma4} 5 \\
0.0 \mathrm{~m} 45\end{array}$ & $0.12487 \%$ & 13952213571 \\
\hline 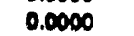 & & & & & & mons & & \\
\hline
\end{tabular}


Spreadsheet for Calculation of Stream-Water Saturation Profiles

Injection Temperature Tso "X
Inillat Temperature $350 \cdot \mathbf{K}$
Metrix Beat Capacity

$1.0000\left(k J / k_{2}\right)$

TRAILING SAOCK CALCULATIONS

\begin{tabular}{|c|c|c|c|c|c|c|c|c|}
\hline Saturatlon & or & Were $v$ & Plow V & Check $V$ & Pv & dKrr/dSr & dXrVdSI & \\
\hline 0.7500 & 2078-01 & 0.216148 & 0.471414 & 0.009321 & 0.996549 & 1.0000 & 0.1875 & \\
\hline 0.775 & A.TIE-O2 & $0.0537 \%$ & 0.577141 & 0.006100 & $0.99 \% 629$ & 1.0000 & 0.0169 & \\
\hline 0.9375 & 6.20E-03 & 0.012521 & $0.58 \% 604$ & 0.005720 & 0.999957 & 1.0000 & 0.0117 & \\
\hline 0.96775 & $-271 E-03$ & 0.002997 & 0.589944 & 0.005708 & 0.999995 & 1.0000 & 0.0029 & \\
\hline 0.953125 & $1.19 E .03$ & 0.006194 & 0.590101 & 0.005705 & 0.999982 & 1.0000 & 0.0066 & \\
\hline 0.9609375 & -9.60E-OA & 0.004736 & 0.590106 & 0.005705 & 0.999990 & 1.0000 & 0.0046 & \\
\hline 0.95703125 & S.74E-OS & 0.005761 & 0.590121 & 0.005704 & 0.999966 & 1.0000 & 0.0055 & \\
\hline 0.958944375 & $-4.69 \mathrm{E}-04$ & 0.005235 & 0.590118 & 0.005704 & 0.999988 & 1.0000 & 0.0050 & \\
\hline 0.9520071125 & -20OEOA & 0.005495 & 0.590121 & 0.005704 & 0.999987 & 2.0000 & 0.0053 & \\
\hline 0.9575195313 & $-7.67 \mathrm{E}-\mathrm{OS}$ & 0.005627 & 0.590121 & 0.005704 & 0.999987 & 1.0000 & 0.0054 & \\
\hline 0.9572753906 & 9.15EE-OS & 0.005694 & 0.590121 & 0.005704 & 0.999986 & 1.0000 & 0.0055 & \\
\hline 0.9571533203 & 237E-OS & 0.005728 & 0.590121 & 0.005704 & 0.999986 & 1.0000 & 0.0055 & \\
\hline 0.9572143555 & $6.92 E-06$ & 0.005711 & 0.590121 & 0.005704 & 0.999986 & 1.0000 & 0.0055 & \\
\hline 0.957244873 & $-1,47 E-06$ & 0.005703 & 0.590121 & 0.005704 & 0.999986 & 1.0000 & 0.0055 & \\
\hline $0.95722 \% 6143$ & 273E-06 & 0.005707 & 0.590121 & 0.005704 & 0.999986 & 1.0000 & 0.0055 & \\
\hline 0.9572372437 & $6.30 E-07$ & 0.005705 & 0.590121 & 0.005704 & 0.999986 & 1.0000 & 0.0055 & \\
\hline 0.9572410583 & 4.18EOT & 0.005704 & 0.590121 & 0.005704 & 0.999986 & 1.0000 & 0.0055 & \\
\hline 0.957239151 & $1.06 \mathrm{EDT}$ & 0.005704 & 0.590121 & 0.005704 & 0.999916 & 1.0000 & 0.0055 & \\
\hline 0.9572401047 & $.156 E-07$ & 0.005704 & 0.590121 & 0.005704 & 0.999986 & 1.0000 & 0.0055 & \\
\hline 0.9572396278 & $-2475-08$ & 0.005704 & 0.590121 & 0.005704 & 0.999986 & 1.0000 & 0.0055 & \\
\hline 0.9572393894 & 4.08E-08 & 0.005704 & $0.5 \% 0121$ & 0.005704 & 0.999986 & 1.0000 & 0.0055 & \\
\hline 0.9572395086 & 8.07E-09 & 0.005704 & 0.590121 & 0.005704 & 0.999986 & 1.0000 & 0.0055 & \\
\hline 0.9572395682 & $.231 E-09$ & 0.005704 & 0.590121 & 0.005704 & 0.599986 & 1.0000 & 0.0055 & \\
\hline 0.9572395384 & $-1.14 E-10$ & 0.005704 & 0.590121 & 0.005704 & 0.949986 & 1.0000 & 0.0055 & \\
\hline 0.9572395235 & 3.912009 & 0.005704 & 0.590121 & 0.005704 & 0.999986 & 1.0000 & 0.0055 & \\
\hline 0.957239531 & $1.93 E-09$ & 0.005704 & 0.590121 & 0.005704 & 0.999926 & 1.0000 & 0.0055 & \\
\hline Soturatlon & Total Mob & AFvidSv & G & $\mathbf{F}$ & Gamma & Theres & Bleh & LeW \\
\hline $\begin{array}{r}0.75 \\
0.575\end{array}$ & $\begin{array}{l}34.759228 \\
40.427490\end{array}$ & $\begin{array}{l}0.045851 \\
0.009321\end{array}$ & $\begin{array}{l}220.095758 \\
115.033000\end{array}$ & $\begin{array}{l}12.770589 \\
10.211953\end{array}$ & $\begin{array}{l}1.179 E A 07 \\
1.1701 E \wedge 07\end{array}$ & $\begin{array}{l}30550.407113 \\
28275535543\end{array}$ & $\begin{array}{l}1 \\
1\end{array}$ & $\begin{array}{l}0.5 \\
0.75\end{array}$ \\
\hline $\begin{array}{r}0.875 \\
0.9375\end{array}$ & $\begin{array}{r}40.427490 \\
43,300978\end{array}$ & $\begin{array}{l}0.009321 \\
0.002124\end{array}$ & $\begin{array}{r}115.033000 \\
62501620\end{array}$ & $\begin{array}{l}10.281953 \\
10.006619\end{array}$ & $\begin{array}{l}1.1701 E=07 \\
1.1655 E \div 07\end{array}$ & $\begin{array}{l}22275535543 \\
22033.574650\end{array}$ & $i$ & $\begin{array}{l}0.75 \\
0.175\end{array}$ \\
\hline 0.96875 & 4.742642 & 0.000508 & $36.235 \% 31$ & 0.97462 & $1.1632 E+07$ & 22005.473055 & 1 & 0.9375 \\
\hline 0.953125 & 4.021547 & 0.001168 & 49.360776 & 9.025337 & 1.1643E+07 & 28014.571137 & 0.06775 & 0.9375 \\
\hline 0.9609375 & 44312039 & 0.000102 & $42 \cos 53$ & 9.710906 & $1.1838 E+07$ & 22009.220503 & 0.06775 & 0.953125 \\
\hline 0.95703125 & 4.201778 & 0.000976 & 46.025564 & ext 1821 & $1.10418+07$ & & 0.900375 & 0.953125 \\
\hline $\begin{array}{r}0.95894375 \\
0.9580078125\end{array}$ & $\begin{array}{l}4.241905 \\
4246241\end{array}$ & $\begin{array}{l}0.000857 \\
0.000031\end{array}$ & $\begin{array}{r}4.443950 \\
4524162\end{array}$ & $\begin{array}{l}0.02482 \\
9.0811 \text { i9 }\end{array}$ & $\begin{array}{l}1.16305407 \\
1.1640 E \times 07\end{array}$ & $\begin{array}{l}22010.43 n 726 \\
22011.094533\end{array}$ & $\begin{array}{c}0.9609375 \\
0.050944375\end{array}$ & $\begin{array}{l}0.05703125 \\
0.05703125\end{array}$ \\
\hline 0.9575195313 & 4.224309 & 0.000054 & 45.675163 & 9.04) < & 1.16405007 & 22011.43432 & 0.0510073125 & 0.95703125 \\
\hline 0.9572753906 & 4.213043 & 0.0000 & 45.teos 4 & -.151622 & $1.16405 \times 07$ & 22011.607307 & 0.95751953125 & 0.95703125 \\
\hline 0.9571533203 & 4207411 & 0.0009 & 45.912964 & 9.011721 & $1.10405+07$ & 22011.604566 & 0.95727539063 & 0.95703125 \\
\hline $\begin{array}{c}0.9572143555 \\
0.957244773\end{array}$ & $\begin{array}{r}4.210227 \\
4.211635\end{array}$ & $\begin{array}{l}0.000 \% \\
0.000 \% 6\end{array}$ & $\begin{array}{l}45.531664 \\
45.506014\end{array}$ & $\begin{array}{l}9.011672 \\
9.811647\end{array}$ & $\begin{array}{l}1.16405407 \\
1.16405407\end{array}$ & $\begin{array}{l}22011.650172 \\
25011.629074\end{array}$ & $\begin{array}{l}0.95727539013 \\
0.957275390 ;\end{array}$ & $\begin{array}{l}0.55715332031 \\
0.95721435547\end{array}$ \\
\hline $0.95722 \% 143$ & 44210931 & 0.000967 & 45.918839 & 9.981659 & $1.1640 \mathrm{E} 407$ & 22011.639969 & 0.9572448705 & 0.95721435547 \\
\hline 0.9572372437 & 44211283 & 0.000967 & 45.912420 & 9.941653 & $2.16405+07$ & 22012.634520 & 0.95724477305 & 0.95722961426 \\
\hline 0.9572410583 & 4.211459 & 0.000967 & 45.509220 & 9.41650 & $1.1640 E 407$ & 28011.631797 & 0.95724477305 & 0.95723724365 \\
\hline 0.957239151 & 44.211371 & 0.000967 & 45.910823 & 9.901652 & $1.1600 E+07$ & 22011.633158 & 0.95724105835 & 0.95723724365 \\
\hline 0.9572401047 & M.211a1s & 0.000967 & 45.910022 & 9.981651 & $1.1640 E+07$ & 28011.632178 & 0.95724105835 & 0.957239151 \\
\hline $0.95722 \% 6278$ & 4.211393 & 0.000967 & 45.910422 & 9.981651 & $1.1640 E-07$ & 28011.632818 & 0.95724010468 & 0.957239151 \\
\hline 0.9372393894 & -4211382 & 0.000967 & 45.910623 & $9.9116 \$ 1$ & $1.1640 E+07$ & 28011.632988 & 0.95723962724 & 0.957238151 \\
\hline 0.9572395086 & 4211388 & 0.000967 & 48.810522 & 9.911651 & $1.1640 E A 07$ & 28011.632903 & 0.9572396274 & 0.95723938942 \\
\hline 0.9572395682 & 4.211390 & 0.000967 & 45.910472 & 9.911651 & $1.1640 E \wedge 07$ & 28011.632861 & 0.95723962784 & 0.95723950863 \\
\hline 0.9572395384 & 44.211389 & 0.000967 & 45.910497 & 9.911651 & 1.16405407 & 23011.632882 & 0.95723956823 & 0.95723950863 \\
\hline $\begin{array}{r}0.0572395235 \\
0.95729531\end{array}$ & $\begin{array}{r}4.211388 \\
4.211389\end{array}$ & $\begin{array}{l}0.000967 \\
0.000967\end{array}$ & $\begin{array}{l}45.910510 \\
45.910504\end{array}$ & $\begin{array}{l}9.01651 \\
0.021651\end{array}$ & $\begin{array}{l}1.1640 E-07 \\
1.16405407\end{array}$ & $\begin{array}{l}22011.632893 \\
22011.632587\end{array}$ & $\begin{array}{l}0.9572395343 \\
0.0572995343\end{array}$ & $\begin{array}{l}0.95723950863 \\
0.95723952353\end{array}$ \\
\hline
\end{tabular}


Injection Temperafure $750 \cdot \mathbf{K}$
Inllial Temperature

$350 \cdot \mathcal{Y}$
Metrix Beal Cepecliy

$1.0000\left(\mathrm{~kJ} / \mathrm{k}_{\mathrm{B}}\right)$

LEADING SBOCE CALCULATIONS

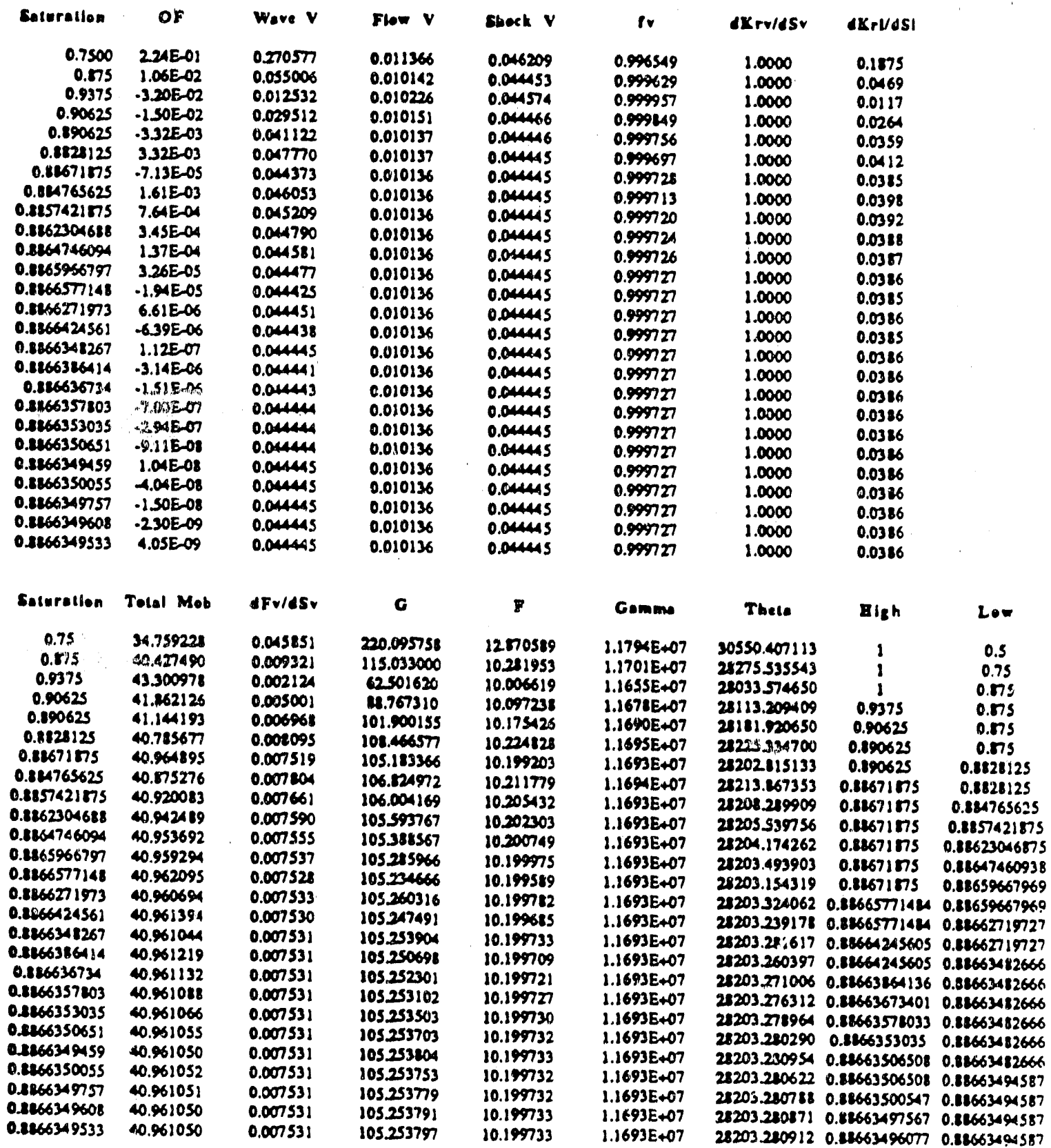


Spreadsheet for Calculation of Stream-Water Saturation Profiles

Injecllon Temperalure $300 \times$
Inlibal Temperature $360 \cdot \mathrm{K}$
Metrix Beol Capacliy

$1.0000\left(k J / k_{k}\right)$

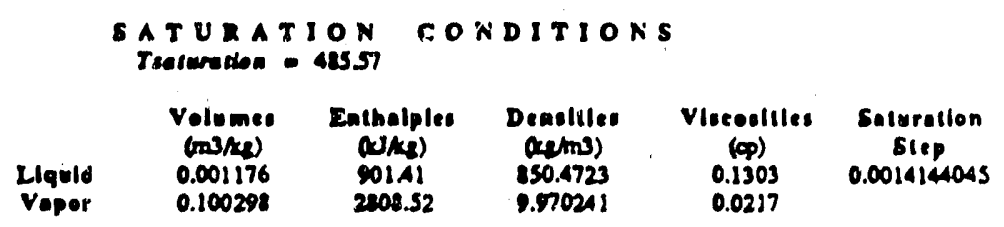

MATIIX PROPERTIES

Peresilty

0.10

$N=$

Cp Matrly Deasley

$1.00 \quad 2450.00$

no

$5 \times 1$
0.00

2650.0000

Denom

1.000000

0.100298

2008.52

0.0217

DOUNDARY CONDITIONE

Heel

Capecily

223

4.15

Were

Vofocliy Denally

$0.005129 \quad 5.477162$ $0.013717 \quad 75.3174$

Enthalpy

1.000000

0.009463

Inlilal

350

C

Injectlon

Ialial

800

350

$$
\underset{9.48}{5.32}
$$

F

5.48

Gamme

1 coseses275

6659510.72

These

19382.75

312020.72
3538.38

319.98

\section{SEOCX CONDITIONS}

\begin{tabular}{|c|c|c|}
\hline & Ialtial & Guess \\
\hline Truiline & So-HI & $\begin{array}{c}\text { Sr-Low } \\
0.5\end{array}$ \\
\hline & $\begin{array}{l}d K r v / d S v \\
1.0000 \\
1.0000\end{array}$ & $\begin{array}{c}\text { dKrl/dsi } \\
0.0055 \\
0.0326\end{array}$ \\
\hline
\end{tabular}

\section{Vaper}

Gateration

0.9574

0.2266

Orjeetlve

Puacllon

7.6573E-10

3.7กESE-09

Tolal

Mobliliy

4.2167

$40 . \% 10$

dFvidsv

0.0010

0.0075

$\begin{array}{cc}\text { Two.Phase } & \text { Upotream } \\ \text { Wove } & \text { Flow } \\ \text { Veloclty } & \text { Velecliy } \\ 0.0053 & 0.550838 \\ 0.015 & 0.009463\end{array}$

G

45.8133 105.2538
5

9.9116

10.1997

MATEIAL DALANCES

Mass
Inolde
93.779969
Enibolpy
$\mathbf{2 8 2 3 9 1 . 7 6 2 3}$

$\underset{\text { Inlti.l }}{\text { n.531740 }}$

IN reeled

S.A77R62

Produced

9229625

265938.0720

193827528

2953.2427

Balance
0.0000

Vapor

Shock Velecily 0.0053

0.0415

Frac Flow 1.0000 0.0997

Thero'

28011.5505 2203.2109 
Spreadsheet for Calculation of Stream-Water Saturation Profiles

Injecllon Temperature $800^{\circ} \mathrm{K}$
Inltbal Temperalure

$350 \cdot x$
Moirlx Eear Copacliy

$1.0000\left(k J / k_{E}\right)$

SATURATION PROPILE

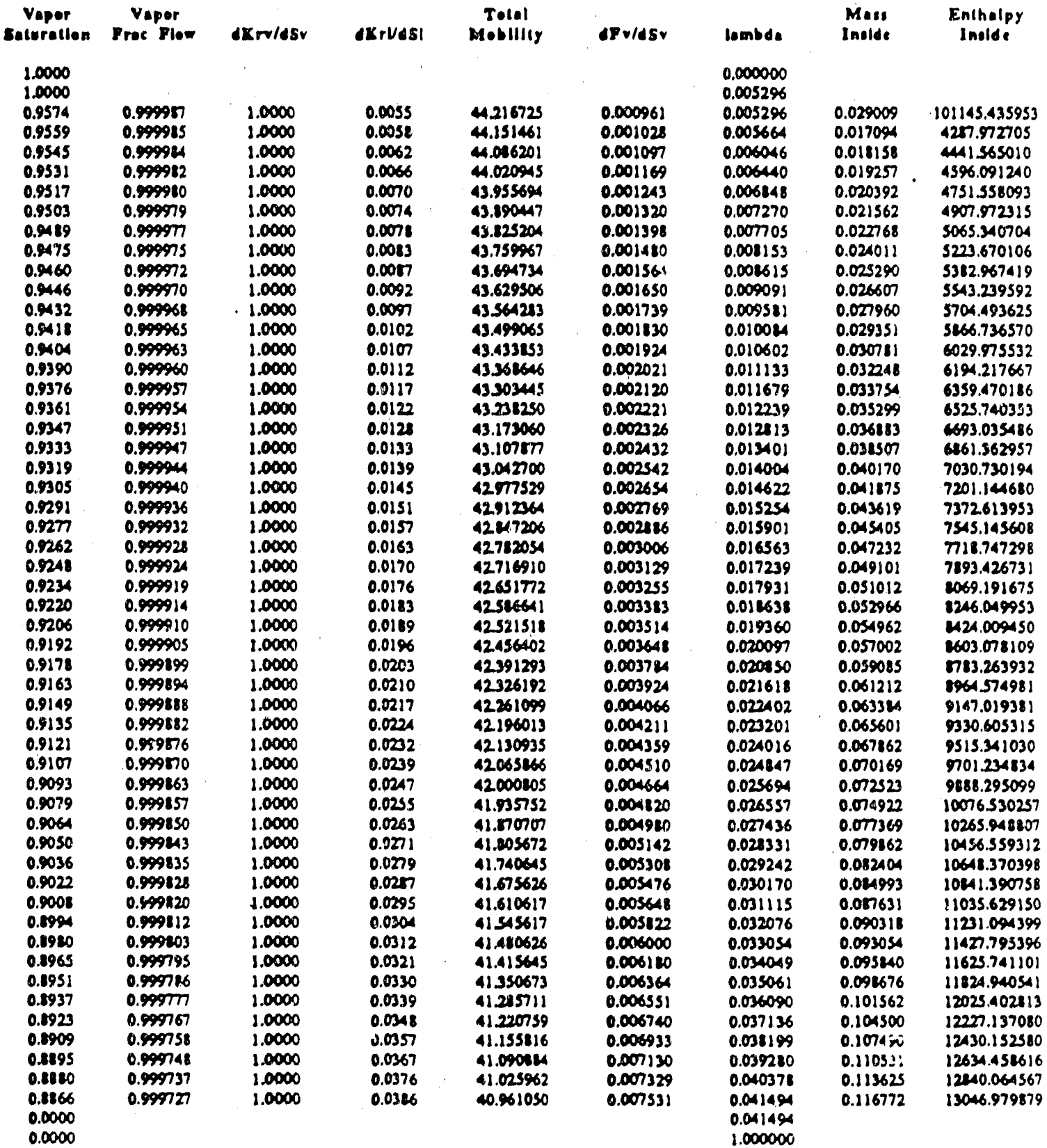


Spreadsheet for Calculation of Stream-Water Saturation Profiles

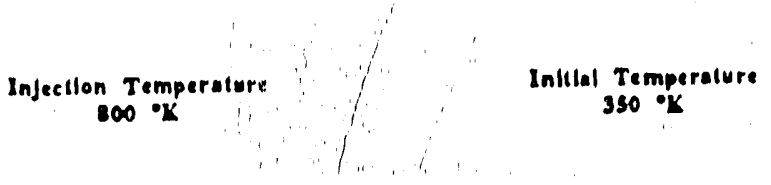

Malrix tieal Cepacliy

1.0000 (kJ/ke)

TRAILING SBOCKCALCULATIONS

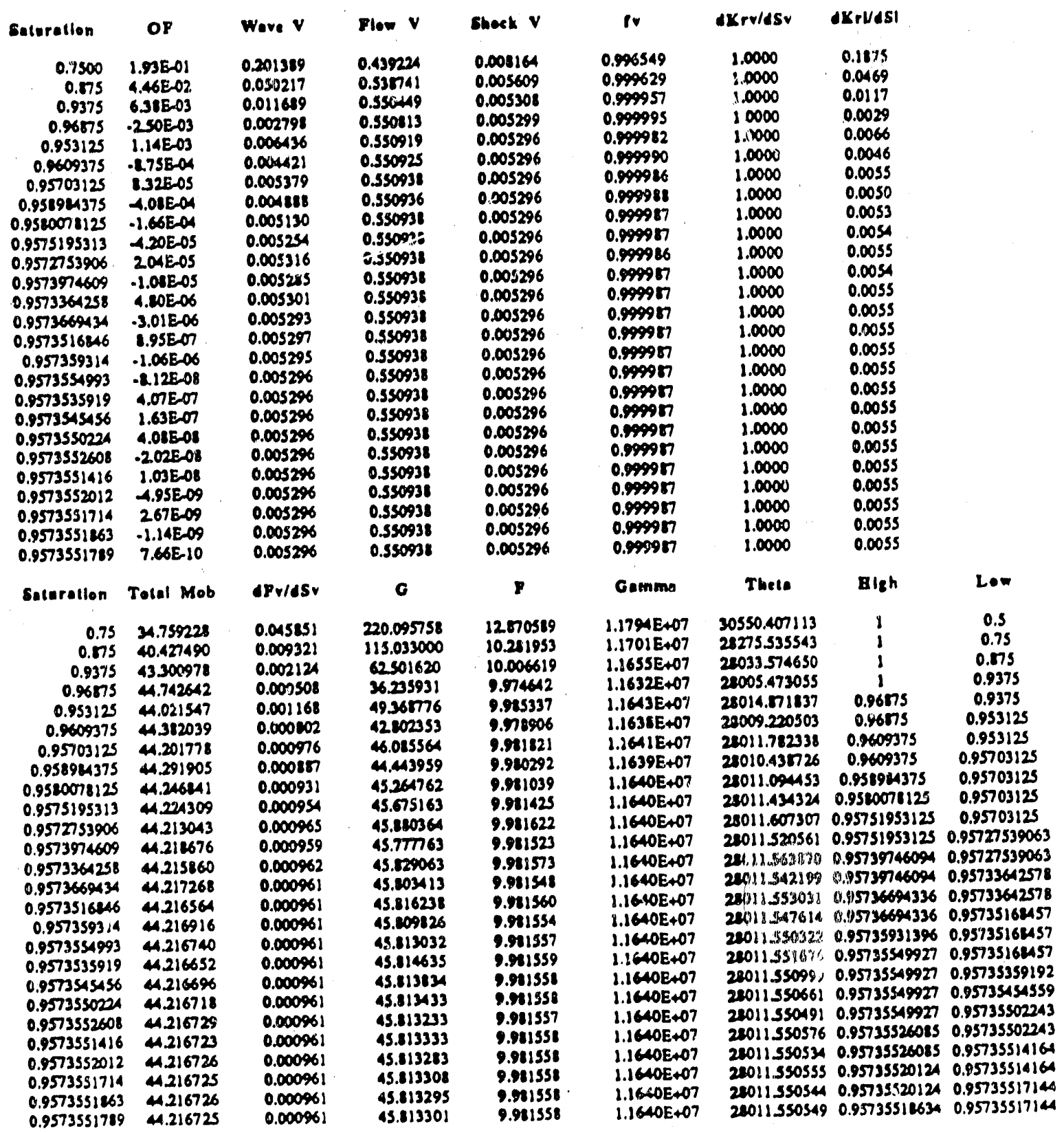


Spreadsheet for Calculation of Stream-Water Saturation Profiles

Injection Temperalure $100 \cdot \mathrm{K}$
Inltial Temperature

$390 \cdot \mathrm{X}$
Matrix Beat Capaclly

$1.0000(\mathrm{~kJ} / \mathrm{kB})$

LEADING BOCK CALCULATIONS

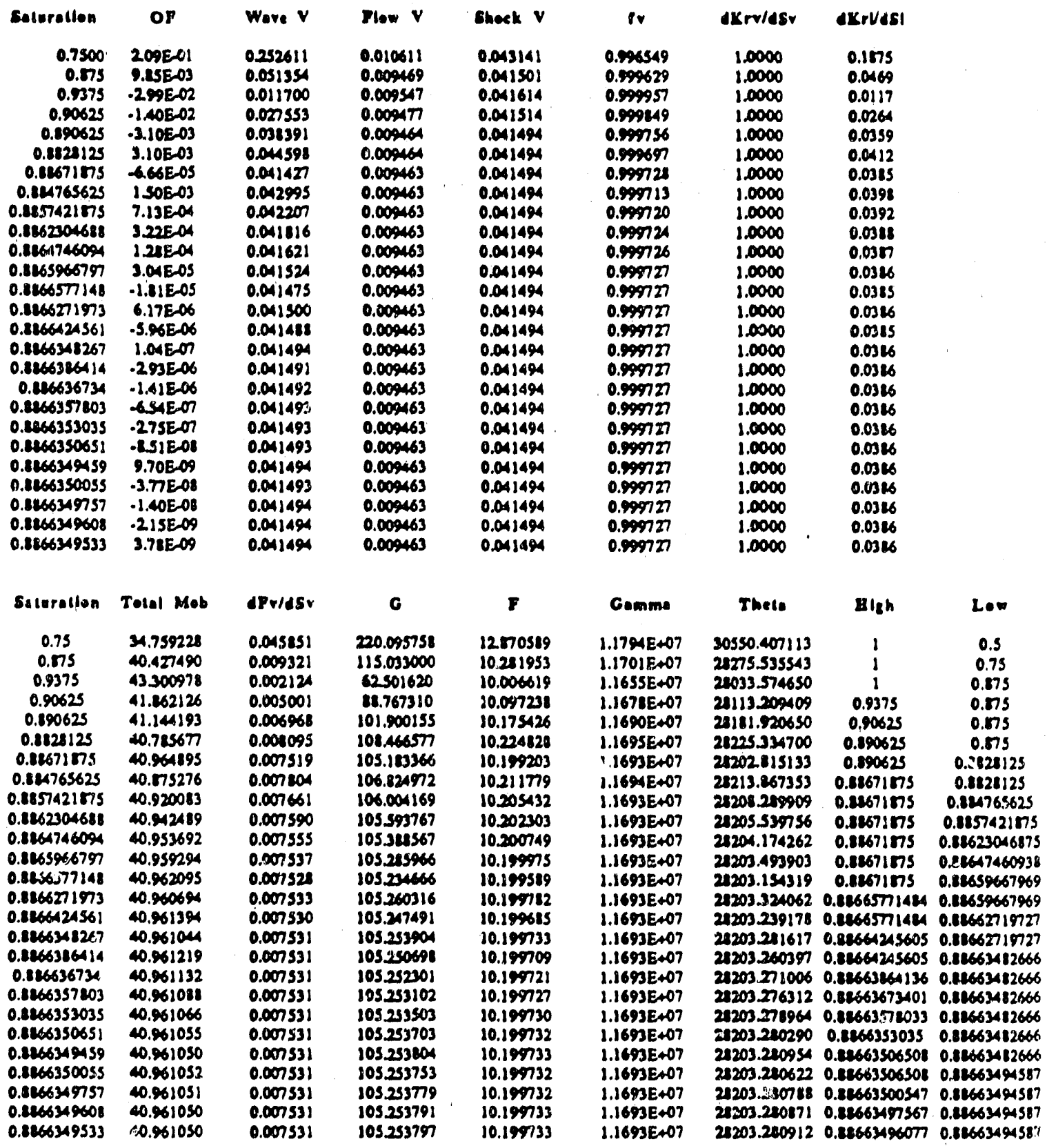


Spreadsheet for Calculation of Stream-Water Saturation profiles

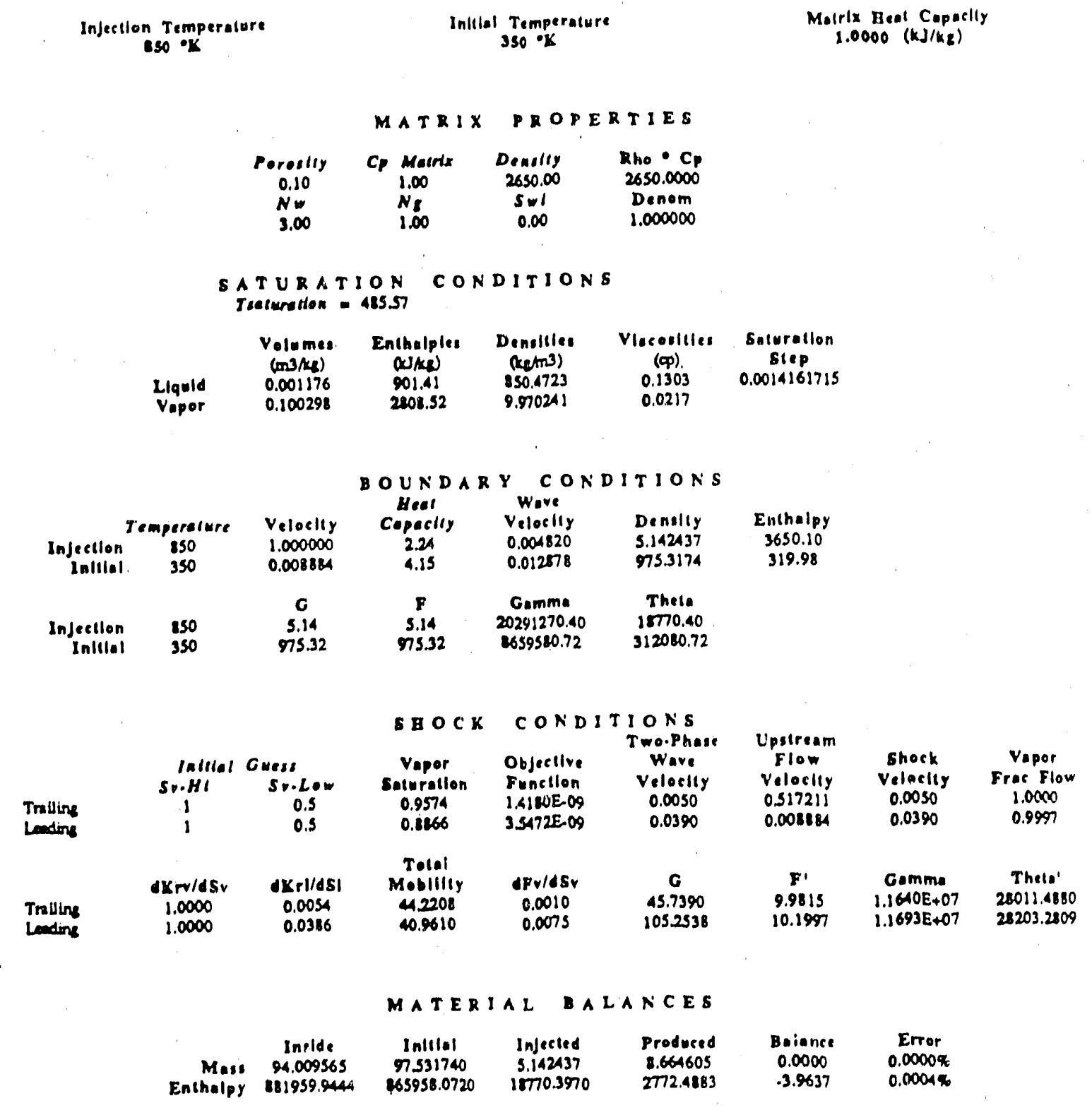


Spreadsheet for Calculation of Stream-Water Saturation Profiles

Injectlon Temperalure 180 ' $\mathrm{K}$.
Inllial Temperature

380 \%
Matrlx Beaf Capacily

1.0000 (kJ/kg)

SATURATION PROFILE

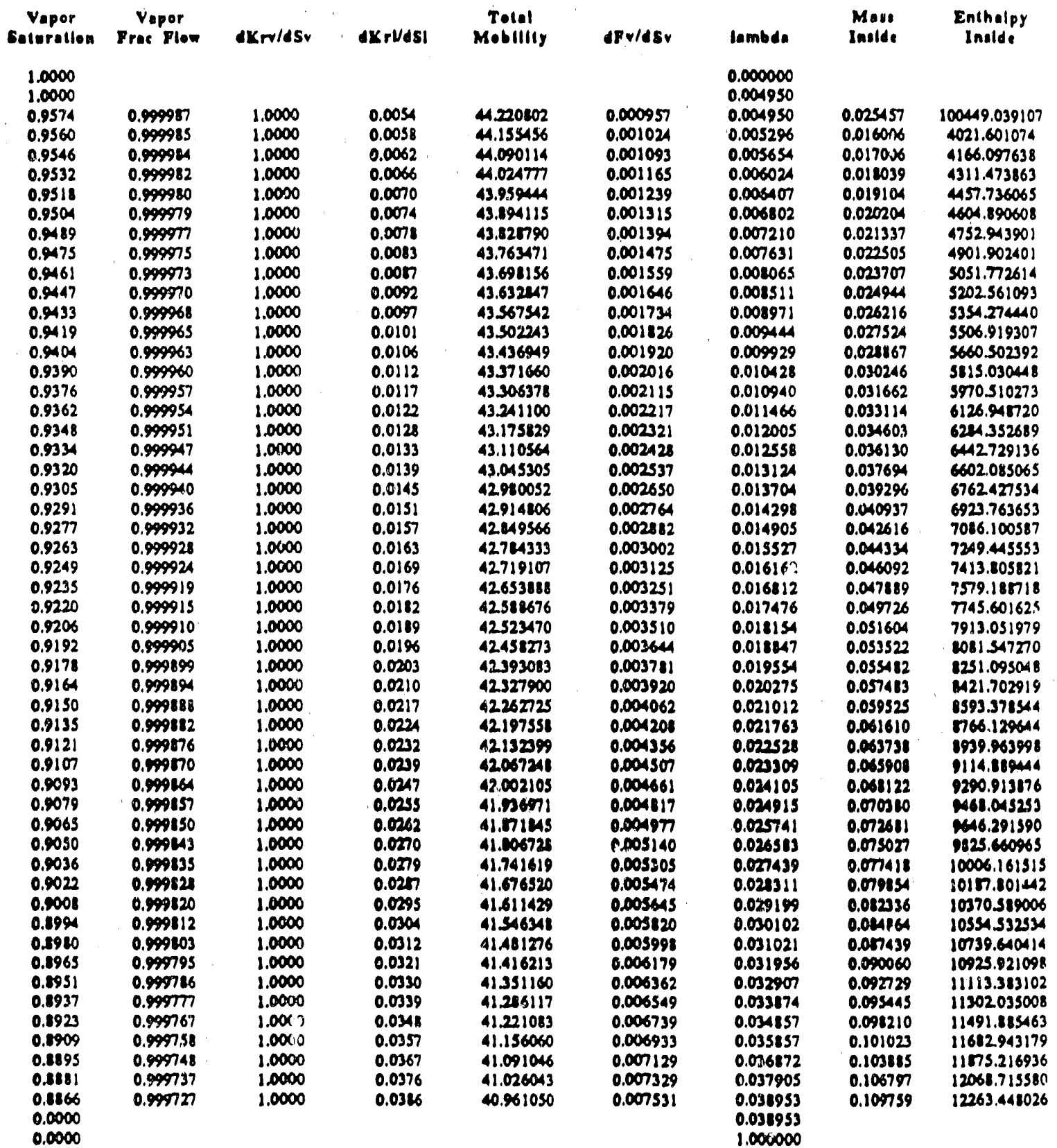


Spreadsheet for Calculation of Stream-Water Saturation Profiles

Injerllen Temperalure' eso or
Inltial Temperature

$\mathbf{3 s 0} 0^{\circ} \mathrm{K}$
Matrix Aleal Capocily

1.0000 (KJ/kg)

TRAILING SEOCK CALCULATIONE

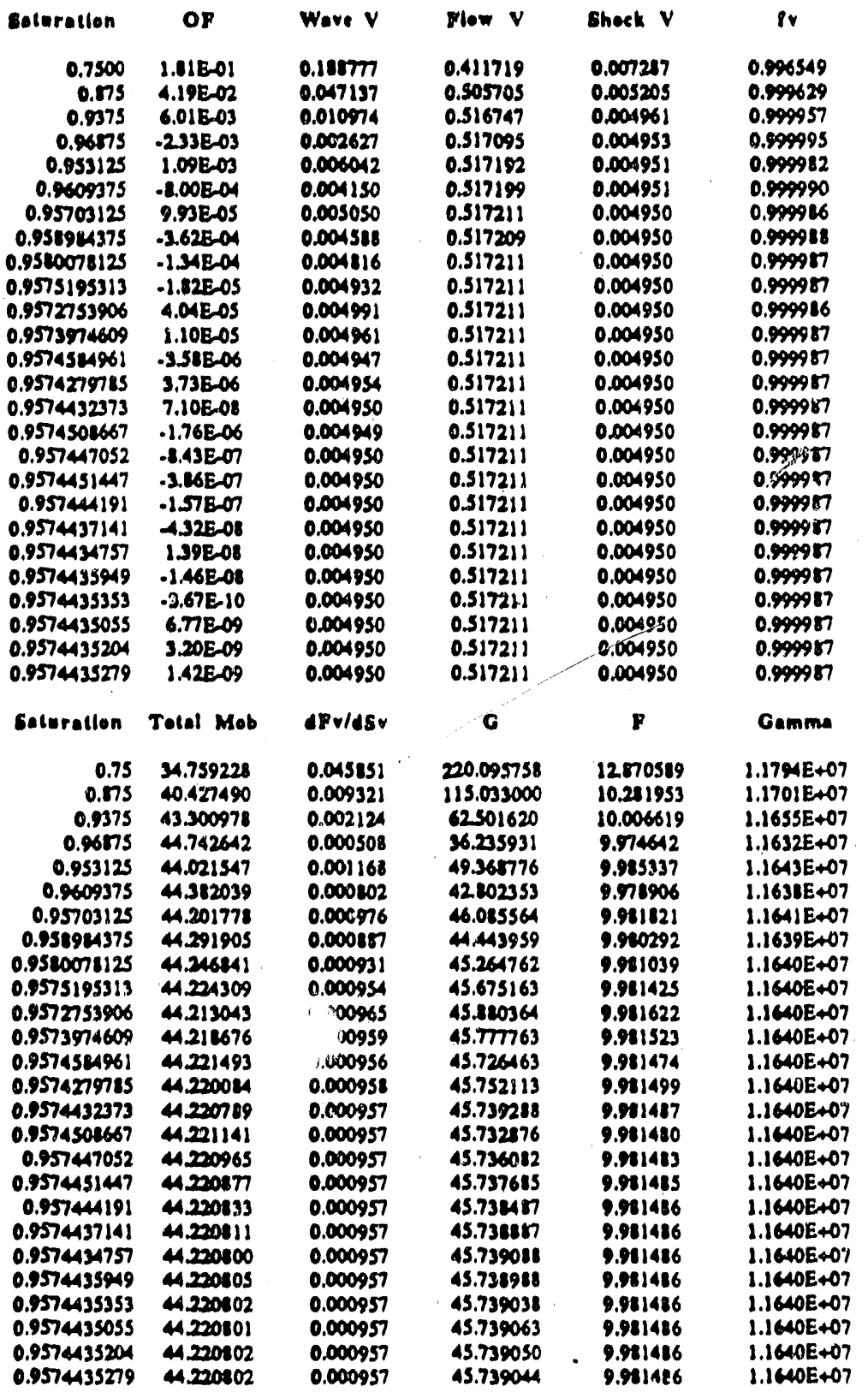

\begin{tabular}{|c|c|}
\hline $\mathrm{Krv} / \mathrm{dSv}$ & $\Delta K r V d S \mid$ \\
\hline 1.0000 & 0.1879 \\
\hline 1.0000 & 0.0469 \\
\hline 1.0000 & 0.0117 \\
\hline 1.0000 & 0.0029 \\
\hline 1.0000 & 0.0066 \\
\hline 1.0000 & 0.0046 \\
\hline 1.0000 & 0.00 .55 \\
\hline 1.0000 & 0.0050 \\
\hline 1.0000 & 0.0053 \\
\hline 1.0000 & 0.0054 \\
\hline 1.0000 & 0.0055 \\
\hline 1.0000 & 0.0054 \\
\hline 1,0000 & 0.0054 \\
\hline 1.0000 & 0.0054 \\
\hline 1.0000 & 0.0054 \\
\hline 1.0000 & 0.005 \\
\hline 1.0000 & 0.0054 \\
\hline 1.0000 & 0.0054 \\
\hline 1.0000 & 0.0054 \\
\hline 1.0000 & 0.0054 \\
\hline 1.0000 & 0.0054 \\
\hline 1.0000 & 0.0054 \\
\hline 1.0000 & 0.0054 \\
\hline 1.0000 & 0.0054 \\
\hline 1.0000 & $0 . \cos 4$ \\
\hline 1.0000 & 0.0054 \\
\hline Thets & Bleh \\
\hline
\end{tabular}

30550.407113

22275.535543

22033.574650

22005.473055

2014.071837

28009.220503

.2011 .712331

$2 \times 010.437736$

20011.43324 0.958007125

$28011.007307 \quad 0.95751953125 \quad 0.95703125$

$\begin{array}{llll}22011.520561 & 0.95751953125 & 0.95127539063\end{array}$

28011.4773790 .957519331250 .95739746094

28011.4989540 .95745496090 .95739746094 28011.488162 0.9574 .5496090 .95742797132 $28011.412770 \quad 0.9574549608 \quad 0.9574432373$ 2801 i.485466 $0.957450 .8667 \quad 0.9574432373$ 28011 A26814 $0.957447052 \quad 0.9574432373$ $28011.4874880 .957 \div 45144650.9574432373$ $28011.4878250 .9574419098 \quad 0.9574432373$ $25011.4779940 .95744371414 \quad 0.96 / 4432373$ 22011.4879090 .95744371414 n 95704347572 $28011.417952 \quad 0.9574439493 \quad 0.957437572$ 28011.4579730 .95744335330 .9574447572 28011.4679620 .957443535330 .9574350553 22011.4799570 .957443535330 .95744352043

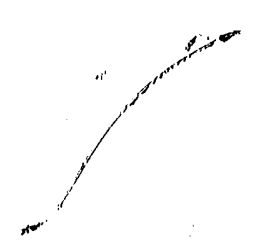


.180.

Spreadsheet: for Calculation of stream-Water Saturation profiles

Indecllon Temperalure oso $\mathrm{K}$
Inllial Temperalure

$350 \cdot \mathrm{K}$
Malrix Beat Capactily

1.0000 (hJ/ke)

LEADINO SHOCK CALCULATIONS

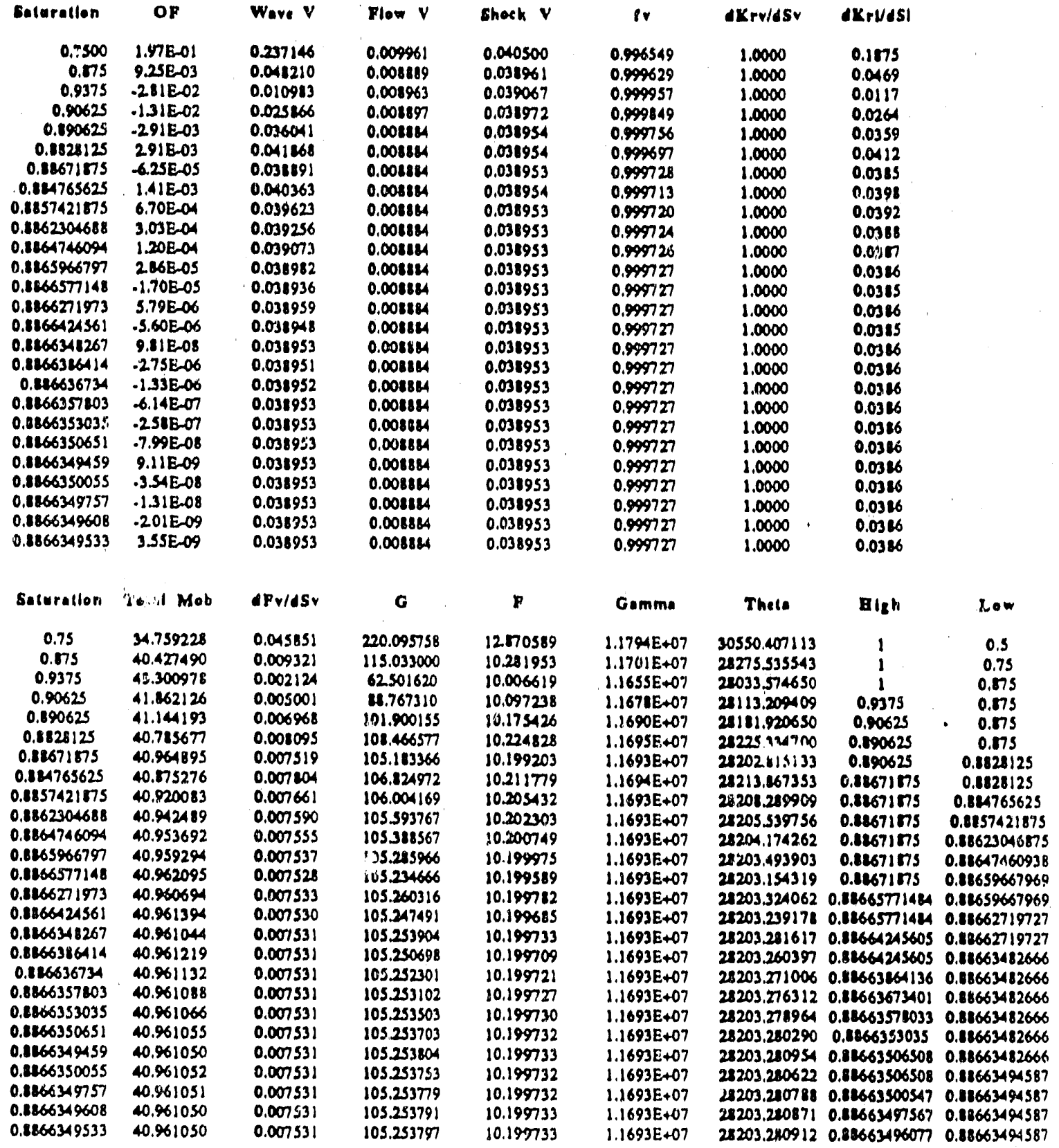


Spreadsheet for Calculation of Stream-Water Saturation Profiles

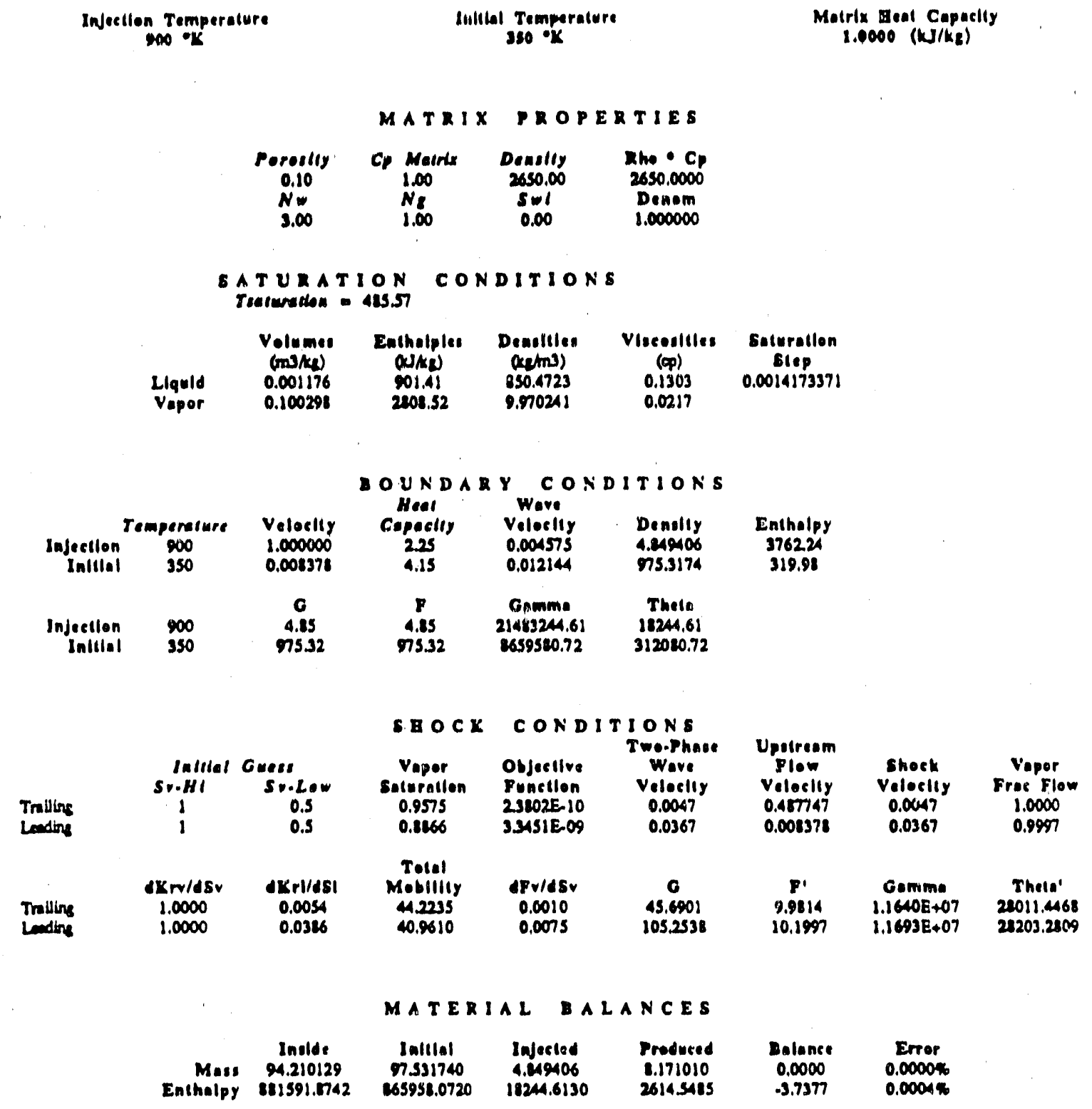


Spreadsheet for Calculation of Stream-Water Saturation Profiles

Injection Temperalure $900 \cdot \mathrm{K}$
Inlilal Temperature $390 \cdot \mathrm{K}$
Matrix Beal Capacily

$1.0000(k J / k E)$

EATURATION PROPILE

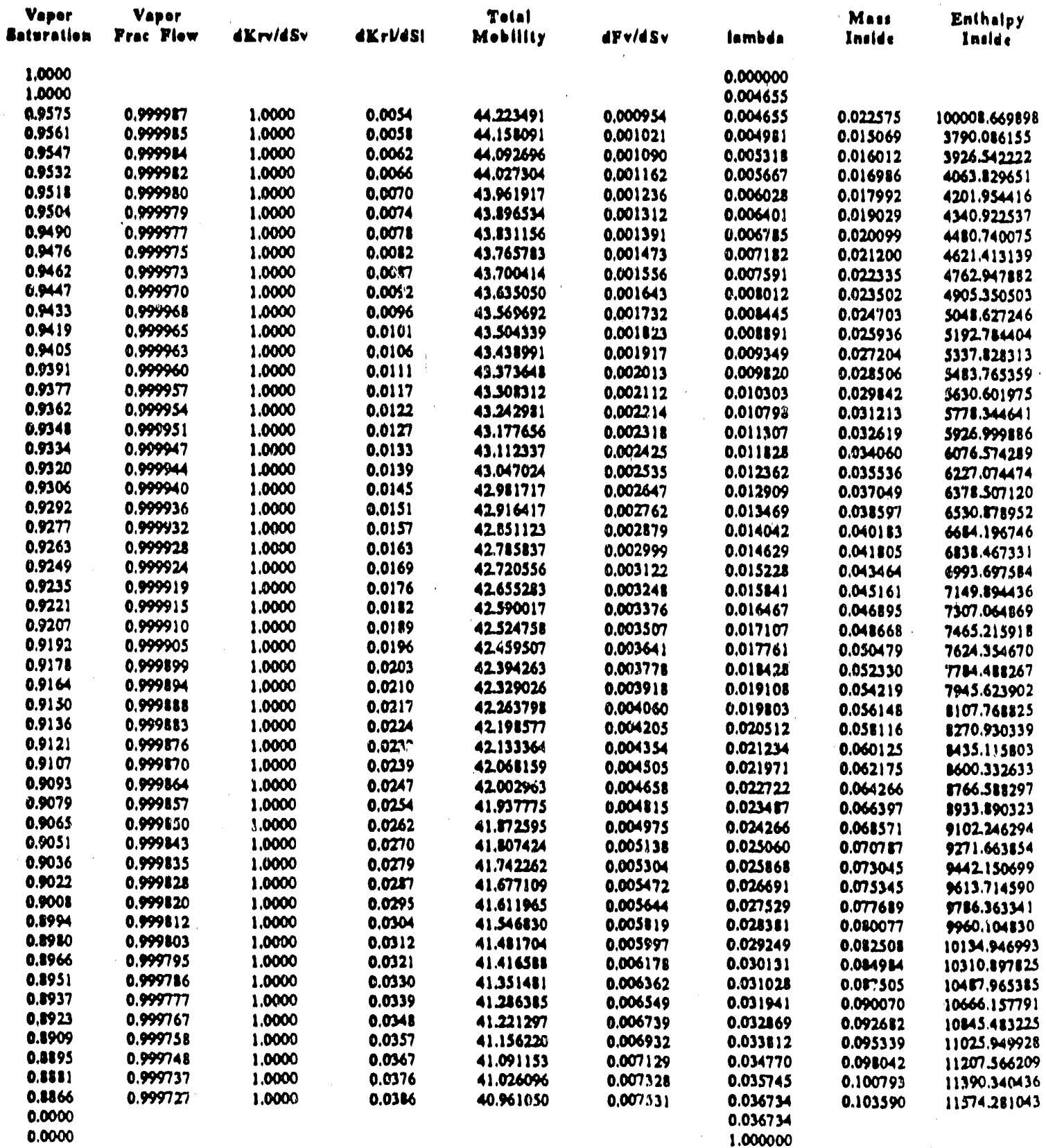


Injectlon Temperalure $900 \mathrm{~K}$
Inlilal Teinperalure yso $x$
Matrix Bend Copactily

1.0000:(kJ/ke)

TRAIIING SIOCK CALCULATIONS

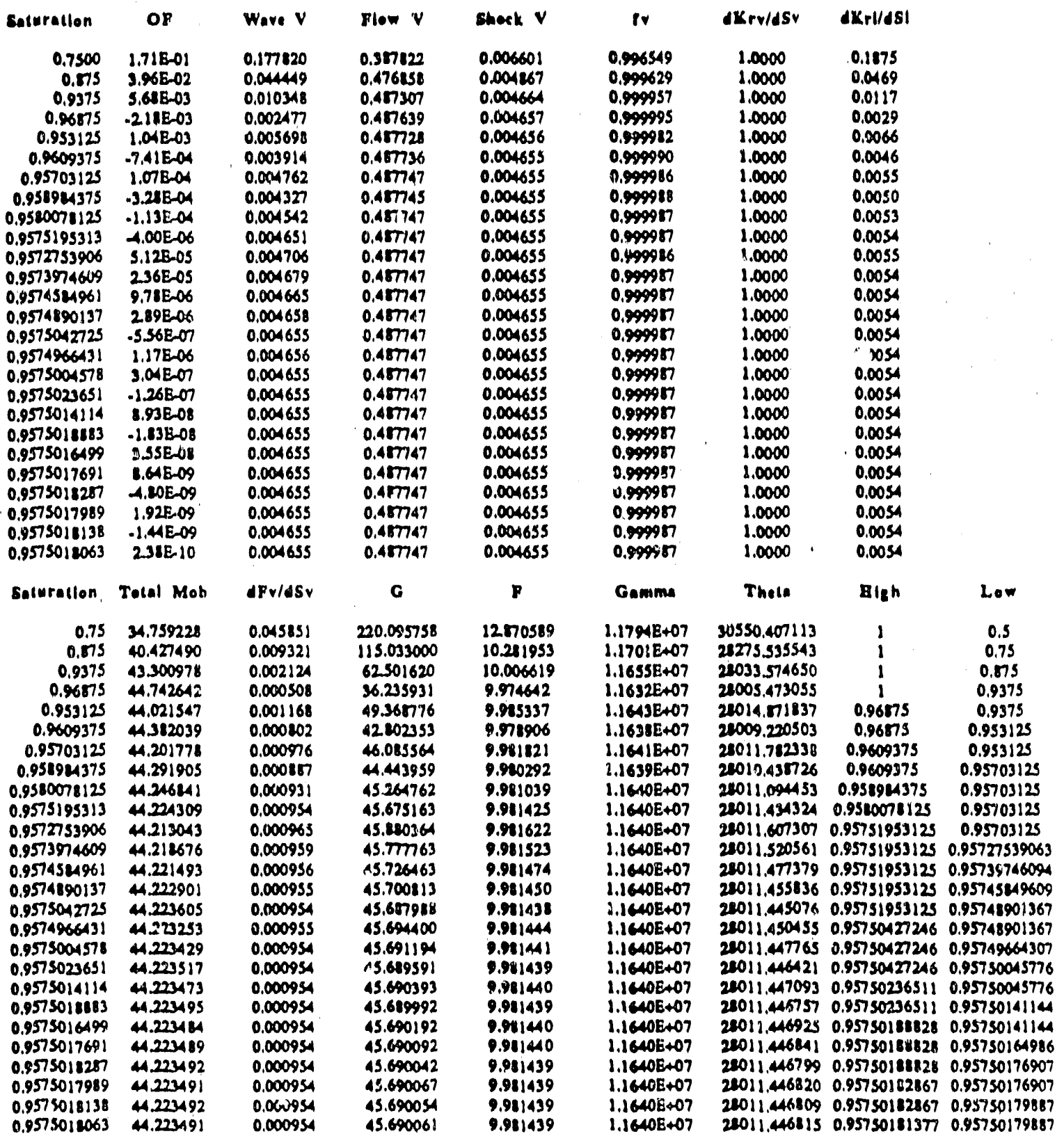


Injectlon Temperalure $\infty \mathrm{x}$
Inllal Temperalere

$350 \cdot \mathrm{K}$
Matrix Beat Copacliy

1.0000 (thJ/kg)

LEADING SROCE CALCULATIONE

\begin{tabular}{|c|c|c|c|c|c|}
\hline Coturation & $O P$ & Wave $v$ & How V & Cheek $V$ & Iv \\
\hline 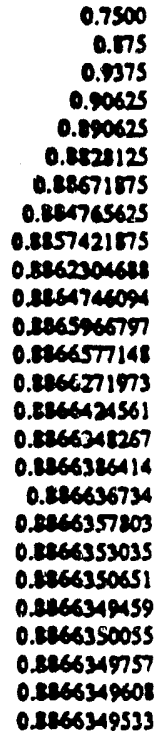 & 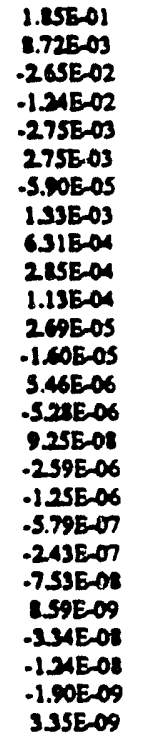 & 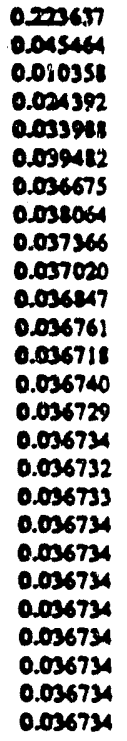 & 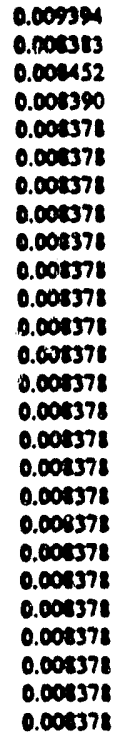 & $\begin{array}{l}0.038193 \\
0.036741 \\
0.036441 \\
0.036752 \\
0.036735 \\
0.036735 \\
0.036734 \\
0.036735 \\
0.036734 \\
0.036734 \\
0.036734 \\
0.036734 \\
0.036734 \\
0.036734 \\
0.036734 \\
0.036734 \\
0.036734 \\
0.036734 \\
0.036734 \\
0.036734 \\
0.006734 \\
0.036734 \\
0.036734 \\
0.036734 \\
0.036734 \\
0.036734\end{array}$ & 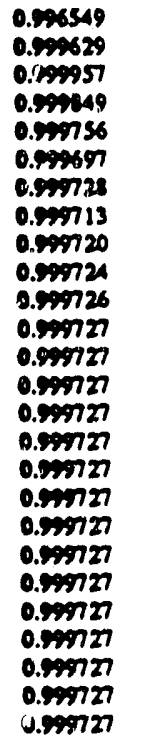 \\
\hline Saturation & Total Mab & dPv/ASV & $\mathbf{6}$ & $\boldsymbol{F}$ & Gemeno \\
\hline 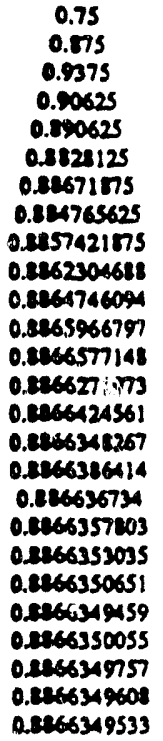 & 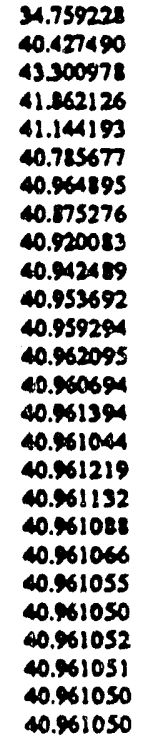 & $\begin{array}{l}0.005251 \\
0.009321 \\
0.002121 \\
0.005001 \\
0.006961 \\
0.001095 \\
0.007519 \\
0.007501 \\
0.007661 \\
0.007590 \\
0.007555 \\
0.007537 \\
0.007528 \\
0.007533 \\
0.007530 \\
0.007531 \\
0.007531 \\
0.007531 \\
0.007531 \\
0.007531 \\
0.007531 \\
0.007531 \\
0.007531 \\
0.007531 \\
0.007531 \\
0.007531\end{array}$ & $\begin{array}{c}220.095758 \\
115.033000 \\
62501620 \\
18.767310 \\
101.50155 \\
101.466577 \\
105.163366 \\
105.824972 \\
106.004169 \\
105593767 \\
105389567 \\
105.225966 \\
105.23466 \\
105.20316 \\
105.257491 \\
105.253904 \\
105250698 \\
105.252301 \\
105.253102 \\
105.251503 \\
105.253703 \\
105225304 \\
105253753 \\
105253779 \\
105.253791 \\
105.253797\end{array}$ & 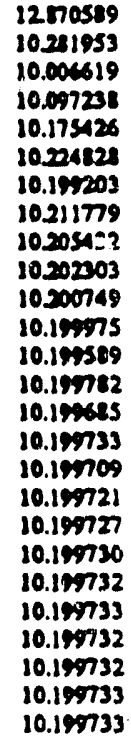 & 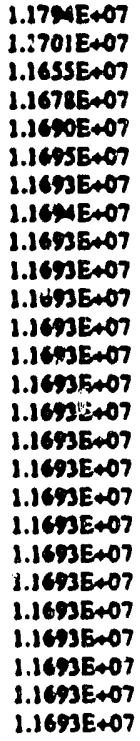 \\
\hline
\end{tabular}

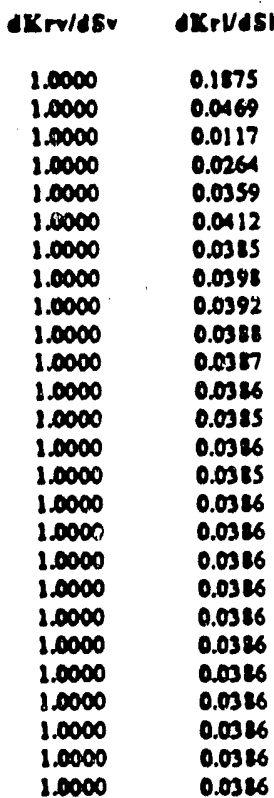

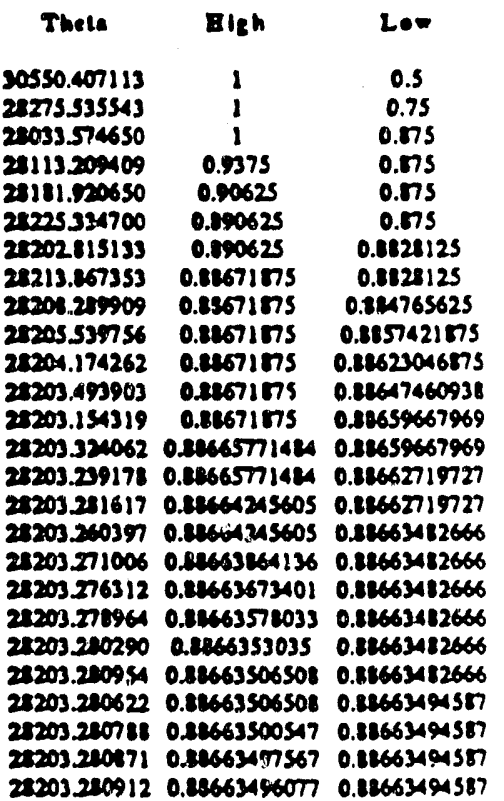


$-185-$

Spreadsheet for Calculation of Stream-Water Saturation Profiles

Injection Temperature

oso $\bullet \mathrm{K}$
Inllial Temperacure

$350^{\circ} \mathrm{K}$
Motrix Beat Cepocily

1.0000 (kJ/ke)
MATRIX PROPERTIES

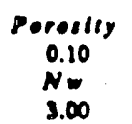

Cp Matrix

$\mathrm{N} \& \mathrm{~S}=1$

1.00

0.00

1.000000

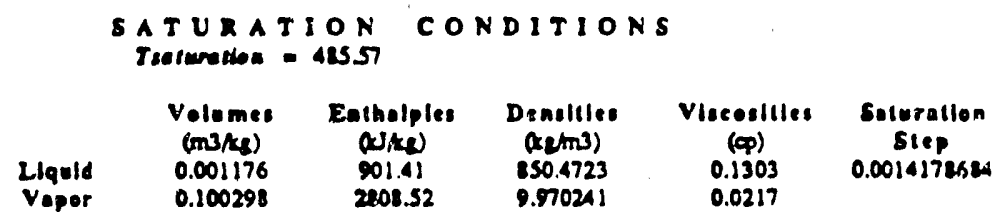

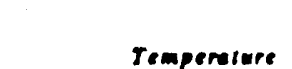

Iojectlen
Inltiol

Tempereture

950
350

Velecity

1.000000

0.007930

c

Injectlon

inltiel

950
350

4.59

975.32
8

O UN DAR

Capacity

228

.4 .15

T

459
9532

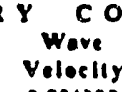

$0.004 \times 80$
0.011495

Gamm.

23675288.06

C659520.72
Denstiy 4390016 975.3174

Enthelpy

3675.38

$\$ 19.98$

\begin{tabular}{|c|c|c|c|c|c|c|c|c|}
\hline \multirow[b]{2}{*}{$\begin{array}{l}\text { Truiling } \\
\text { Leding }\end{array}$} & \multirow[b]{2}{*}{$\begin{array}{c}\ln \mid f i a l \\
5 v+H i \\
1 \\
1\end{array}$} & \multirow[b]{2}{*}{$\begin{array}{c}\text { Geess } \\
\text { So.Lew } \\
0.5 \\
0.5\end{array}$} & \multirow{2}{*}{$\begin{array}{c}5 \text { a O C x } \\
\text { Vepor } \\
\text { soteratlon } \\
0.9575 \\
0.4666\end{array}$} & \multicolumn{2}{|c|}{ CONDITIONS } & Upatream & \multirow[b]{2}{*}{$\begin{array}{l}\text { Shock } \\
\text { Veleclly } \\
0.0044 \\
0.0348\end{array}$} & \multirow[b]{2}{*}{$\begin{array}{c}\text { Vapor } \\
\text { Frar Flow } \\
1.0600 \\
0.999 ?\end{array}$} \\
\hline & & & & $\begin{array}{l}\text { Orjectlve } \\
\text { Fuactlon } \\
7.7304 E_{-10} \\
3.1062 E_{0} 09\end{array}$ & $\begin{array}{l}\text { Wove } \\
\text { Velority } \\
\text { o.0044 } \\
0.0348\end{array}$ & $\begin{array}{c}\text { Flev } \\
\text { Velecily } \\
\text { O. } \$ 61667 \\
0.007920\end{array}$ & & \\
\hline $\begin{array}{l}\text { Tniline } \\
\text { Lending }\end{array}$ & $\begin{array}{l}\text { IXrv/dSV } \\
1.0000 \\
1.0000\end{array}$ & $\begin{array}{l}\text { AKrl/dSI } \\
0.0054 \\
0.0326\end{array}$ & $\begin{array}{c}\text { Total } \\
\text { Mobliliy } \\
4.2247 \\
40.9610\end{array}$ & $\begin{array}{l}\text { Grv/dSV } \\
0.0010 \\
0.0075\end{array}$ & $\begin{array}{c}\text { G } \\
45.6677 \\
105.2538\end{array}$ & $\begin{array}{c}F \\
9.9114 \\
10.1997\end{array}$ & $\begin{array}{c}\text { Gamme } \\
1.1640 E \leftrightarrow 07 \\
1.1693 E+07\end{array}$ & $\begin{array}{l}\text { These' } \\
22011.4281 \\
28203.2809\end{array}$ \\
\hline
\end{tabular}

MATERIAL BALANCES

\begin{tabular}{|c|c|c|c|c|c|c|}
\hline 1 & $\begin{array}{c}\text { Inside } \\
\text { M.37764s } \\
28 ! 274.9209\end{array}$ & $\begin{array}{c}\text { Inlilal } \\
\text { 97531740 } \\
\text { 265958.0720 }\end{array}$ & $\begin{array}{l}\text { INected } \\
\text { IS90016 } \\
17188.0580\end{array}$ & $\begin{array}{l}\text { Produced } \\
7.734104 \\
2474.74100\end{array}$ & 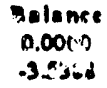 & $\begin{array}{l}\text { Errer } \\
0.00004 \\
0.0004 \pi\end{array}$ \\
\hline
\end{tabular}


Spreadsheet for Calculation of Stream-Water Saturation Profiles

Infecllos Temperature

so $\cdot \mathrm{K}$
Inlibel Temperalure

$350^{\circ} \mathrm{K}$
Matrix Beat Copocliy

$1.0000\left(\mathrm{~kJ} / \mathrm{kE}_{\mathrm{E}}\right)$

SATURATION PROFILE

\begin{tabular}{|c|c|c|c|c|c|c|c|c|}
\hline $\begin{array}{c}\text { Vaper } \\
\text { Eaturatlen }\end{array}$ & $\begin{array}{c}\text { Vaper } \\
\text { Prac Plew }\end{array}$ & dKr/dSr & AKrVISI & $\begin{array}{c}\text { Total } \\
\text { Moblility }\end{array}$ & APr/dSv & lambda & $\begin{array}{l}\text { Moses } \\
\text { Inside }\end{array}$ & $\begin{array}{l}\text { Enthalpy } \\
\text { Inolde }\end{array}$ \\
\hline $\begin{array}{l}1.0000 \\
1.0000\end{array}$ & & & & & & $\begin{array}{l}0.000000 \\
0.00401\end{array}$ & & \\
\hline $\begin{array}{l}0.9575 \\
0.9561\end{array}$ & 0.99997 & $\begin{array}{l}1.0000 \\
1.0000\end{array}$ & $\begin{array}{l}0.0054 \\
0.0058\end{array}$ & $\begin{array}{r}4.224717 \\
4.159293\end{array}$ & $\begin{array}{l}0.000953 \\
0.001020\end{array}$ & $\begin{array}{l}0.004401 \\
0.004709\end{array}$ & $\begin{array}{l}0.020199 \\
0.011253\end{array}$ & $\begin{array}{l}\text { 9nes.059391 } \\
3526.383911\end{array}$ \\
\hline $\begin{array}{l}0.9547 \\
0.9533\end{array}$ & $\begin{array}{l}0.999944 \\
0.999982\end{array}$ & $\begin{array}{l}1.0000 \\
1.0000\end{array}$ & $\begin{array}{l}0.0062 \\
0.0065\end{array}$ & $\begin{array}{r}4.093872 \\
4.024456\end{array}$ & $\begin{array}{l}0.001089 \\
0.001161\end{array}$ & $\begin{array}{l}0.005028 \\
0.005358\end{array}$ & $\begin{array}{l}0.015145 \\
0.016067\end{array}$ & $\begin{array}{l}3715.626122 \\
345.655996\end{array}$ \\
\hline $\begin{array}{l}0.9519 \\
0.9504\end{array}$ & $\begin{array}{l}0.999981 \\
0.999979\end{array}$ & $\begin{array}{l}1.0000 \\
1.0000\end{array}$ & $\begin{array}{l}0.0070 \\
0.0074\end{array}$ & $\begin{array}{l}43.963045 \\
43.897637\end{array}$ & 0.001235 & $\begin{array}{l}0.005700 \\
0.005052\end{array}$ & & $\begin{array}{l}3976.479224 \\
4108.101511\end{array}$ \\
\hline $\begin{array}{l}0.9504 \\
0.9490\end{array}$ & $\begin{array}{l}0.999979 \\
0.99997\end{array}$ & $\begin{array}{l}1.0000 \\
1.0000\end{array}$ & $\begin{array}{l}0.0074 \\
0.0078\end{array}$ & $\begin{array}{l}43.897637 \\
43.832234\end{array}$ & $\begin{array}{l}0.001311 \\
0.001390\end{array}$ & $\begin{array}{l}0.006052 \\
0.006416\end{array}$ & $\begin{array}{l}0.018002 \\
0.019014\end{array}$ & \\
\hline 0.9476 & 0.999975 & 1.0000 & 0.0082 & 43.766836 & 0.001471 & 0.006792 & 0.020057 & 4373.766291 \\
\hline 0.9462 & 0.999913 & 1.0000 & 0.0087 & 43.701443 & 0.001555 & 0.007179 & 0.021131 & 4507.120405 \\
\hline 0.948 & 0.999910 & 1.0000 & 0.0082 & 43.636055 & 0.001641 & $0.0075 n$ & 0.022236 & 4642696822 \\
\hline 0.9433 & 0.999968 & 1.0000 & 0.0096 & 43570672 & 0.001730 & 0.007918 & 0.023373 & 4778.401461 \\
\hline 0.9419 & 0.999965 & 1.0000 & 0.0101 & 43.50529 & 0.001822 & 0.004409 & 0.024341 & 4914.940284 \\
\hline 0.9405 & 0.999963 & 1.0000 & 0.0106 & 43.439922 & 0.001915 & 0.001243 & 0.025742 & 5032319300 \\
\hline 0.9391 & 0.999960 & 1.0000 & 0.0111 & 43.374555 & 0.002012 & 0.009288 & 0.026974 & 5190.54560 \\
\hline $0.93 T^{\prime}$ & 0.99957 & 1.0000 & 0.0117 & 43309194 & 0.002111 & 0.009746 & 0.022239 & 5329.622161 \\
\hline 0.9363 & 0.999954 & 1.0000 & 0.0122 & 42.243838 & 0.002213 & 0.010215 & 0.029537 & 5464.558246 \\
\hline 0.9348 & 0.99951 & 1.0000 & 0.0127 & 43.17428 & 0.002317 & 0.010696 & 0.030869 & $5610.35900 s$ \\
\hline 0.9334 & 0.999047 & 1.0000 & 0.0133 & 43.113145 & 0.002424 & 0.011190 & 0.032233 & 5752030670 \\
\hline $\begin{array}{l}0.9320 \\
0.9306\end{array}$ & $\begin{array}{l}0.99944 \\
0.999940\end{array}$ & $\begin{array}{l}1.0000 \\
1.0000\end{array}$ & $\begin{array}{l}0.0139 \\
0.0145\end{array}$ & $\begin{array}{l}13.047807 \\
42912476\end{array}$ & $\begin{array}{l}0.002533 \\
0.002645\end{array}$ & $\begin{array}{l}0.011695 \\
0.012213\end{array}$ & $\begin{array}{l}0.033631 \\
0.033064\end{array}$ & $\begin{array}{l}5894579524 \\
\cos 1.011895\end{array}$ \\
\hline 0.9292 & 0.999936 & 1.0000 & 0.0151 & 42917151 & 0.002760 & 0.012743 & 0.036530 & 6182334158 \\
\hline 0.9278 & 0.999932 & 1.0000 & 0.0157 & 42851833 & 0.002871 & 0.013216 & 0.031031 & 6327552736 \\
\hline 0.9263 & 0.999928 & 1.0000 & 0.0163 & 42716522 & 0.002998 & $0.01344 !$ & 0.039568 & 0473.674102 \\
\hline 0.9249 & 0.999924 & 1.0000 & 0.0169 & 42721217 & 0.003121 & 0.014408 & 0.041139 & 620.704775 \\
\hline 0.9235 & 0.999919 & 1.0000 & 0.0176 & 42655920 & 0.003247 & 0.019918 & 0.042746 & 6768.651324 \\
\hline 0.9221 & 0.999915 & 1.0000 & 0.0112 & 12590629 & 0.003375 & 0.015581 & 0.04338 & 0917520367 \\
\hline 0.920 ? & 0.999910 & 1.0000 & 0.0189 & 12525346 & 0.003506 & 0.01617 & 0.046088 & 7067318573 \\
\hline 0.9192 & 0.999905 & 1.0000 & $0.01 \%$ & 42460070 & 0.003640 & 0.016106 & 0.047783 & 7211.052659 \\
\hline 0.9178 & 0.999900 & 1.0000 & 0.0203 & 12394101 & 0.003777 & 0.017437 & 0.049535 & 7369.729394 \\
\hline 0.9164 & 0.999894 & 1.0000 & 0.0210 & 42329540 & 0.003917 & 0.012082 & 0.051324 & 7322353599 \\
\hline 0.9150 & 0.999888 & 1.0000 & 0.0217 & 42.264287 & 0.004059 & 0.018739 & 0.053151 & 7675.938143 \\
\hline 0.9136 & 0.999813 & 1.0000 & 0.0224 & 42.199041 & 0.004204 & 0.01910 & 0.055016 & 7830.483951 \\
\hline 0.9122 & $0.999 m$ & 1.0000 & 0.0231 & 12133804 & 0.004353 & 0.020094 & 0.056919 & 7915.999998 \\
\hline 0.9107 & 0.999870 & 10000 & 0.0239 & $420 \times 8575$ & 0.004504 & 0.020791 & 0.058160 & 8142493312 \\
\hline 0.9093 & 0.999164 & 1.0000 & 0.0217 & 22.003354 & 0.004658 & 0.021502 & 0.060840 & 8299.91093 \\
\hline 0.9079 & 0.99157 & .0000 & 0.0254 & 41.938141 & 0.004814 & 0.022227 & 0.062859 & 2451.40117 \\
\hline 0.9065 & 0.999850 & 1.0000 & 0.0262 & 41.82937 & 0.004974 & 0.022965 & 0.064917 & 617.907933 \\
\hline $0.905 !$ & 0.99943 & 1.0000 & 0.0270 & 41.007742 & 0.005137 & 0.023716 & 0.067016 & I771.381664 \\
\hline 0.9036 & 0.999836 & 1.0000 & 0.0238 & 41.742555 & 0.005303 & 0.024481 & 0.069155 & 0939.166609 \\
\hline 0.9022 & 0.999328 & 1.0000 & 0.025 & 41.677378 & 0.005472 & 0.025261 & 0.071334 & 9102376120 \\
\hline 0.9008 & 0.999820 & 1.0000 & 0.0295 & $\$ 1.612009$ & 0.005643 & 0.020044 & 0.073554 & $=265911609$ \\
\hline 0.099 & 0.999112 & 1.0000 & 0.0304 & 41547050 & 0.005811 & 0.026861 & 0.075815 & 930.482540 \\
\hline 0.8980 & 0.999803 & 1.0000 & 0.0312 & 41.411900 & $0.0059 \%$ & 0.027612 & 0.071118 & $95 \% .096437$ \\
\hline 0.8966 & 0.999795 & 1.0000 & 0.0321 & 41.416759 & $0.0061 n$ & 0.028518 & 0.050464 & 9762760879 \\
\hline 0.0951 & 0.599726 & 1.0000 & 0.0330 & 41.351628 & 0.006361 & 0.029367 & 0.002451 & $\$ 930.483505$ \\
\hline 0.8937 & $0.999 m$ & 1.0000 & 0.0339 & 41286507 & 0.006548 & 0.030231 & 0.085281 & 10099.272010 \\
\hline 0.0923 & 0.999767 & 1.0000 & 0.0348 & 41221395 & 0.006739 & 0.031110 & $0.00775 s$ & $10269.134 / 50$ \\
\hline 0.0909 & 0.99758 & 1.0000 & 0.0357 & 41.156293 & 0.006932 & 0.032003 & 0.090272 & 10440.077739 \\
\hline 0.2895 & $0.9 \% 94$ & 1.0000 & 0.0367 & 41.091202 & 0.007129 & 0.032911 & 0.092832 & 10612110651 \\
\hline 0.8881 & 0.999737 & 1.0000 & 0.0376 & 41.026121 & 0.007328 & 0.033933 & 0.095437 & 10785.240818 \\
\hline 0.2166 & 0.99927 & 1.0000 & 0.0386 & .50 .961050 & 0.007331 & $0.03 \times 770$ & 0.092017 & 10959476238 \\
\hline 0.0000 & & & & & & 0.034770 & & \\
\hline 0.0000 & & & & & & 1.000000 & & \\
\hline
\end{tabular}


Spreadsheet for Calculation of Stream-Water Saturation Profiles

Injectlon Temperature $950 \cdot \mathbf{K}$
Inllal Temperalure $350 \cdot \mathbf{x}$
Matrlx Beal Capacliy $1.0000(\mathrm{~kJ} / \mathrm{kE})$

TRAILING SBOCE CALCULATIONS

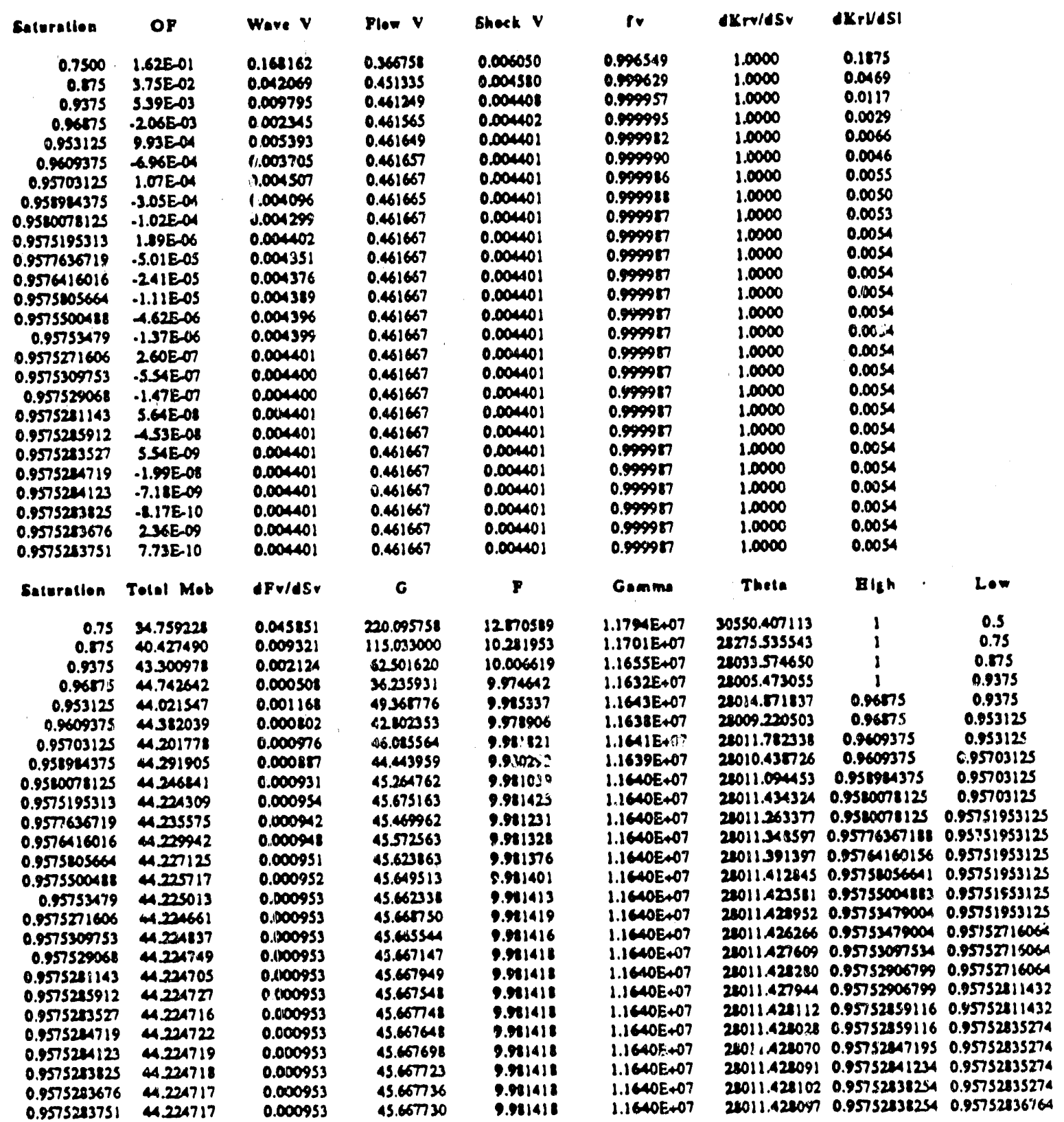


.188.

Spreadsheet for Calculation of Stream-Water Saturation Profiles

Injection Temperalure 950 ०K
Inlilel Tempersture $350^{\circ} \mathrm{K}$
Matrix Beal Copacily 1.0000 (tu/ke)

LEADING SBOCKCALCULATIONS

\begin{tabular}{|c|c|c|c|c|c|}
\hline Saturation & OF & Wave $v$ & Plow V & Shock V & Iv \\
\hline 0.7500 & $1.76 \mathrm{E}-01$ & 0.211679 & 0.001292 & 0.036150 & $0.9 \% 549$ \\
\hline $\begin{array}{r}0.775 \\
0.9375\end{array}$ & $\begin{array}{r}.26 \mathrm{E}-03 \\
.251 \mathrm{E}-02\end{array}$ & $\begin{array}{l}0.043033 \\
0.009204\end{array}$ & $\begin{array}{l}0.007934 \\
0.001000\end{array}$ & $\begin{array}{l}0.034777 \\
0.034871\end{array}$ & $\begin{array}{l}0.999629 \\
0.999957\end{array}$ \\
\hline $\begin{array}{r}0.90625 \\
0.890625\end{array}$ & $\begin{array}{l}-1.17 E-{ }^{2} 2 \\
-260 \mathrm{E}-03\end{array}$ & $\begin{array}{l}0.023088 \\
0.032170\end{array}$ & $\begin{array}{l}0.007942 \\
0.007930\end{array}$ & $\begin{array}{l}0.034787 \\
0.034771\end{array}$ & $\begin{array}{l}0.999849 \\
0.999756\end{array}$ \\
\hline $\begin{array}{r}0.8828125 \\
0.86671875\end{array}$ & $\begin{array}{l}260 \mathrm{E}-03 \\
-5.5 R E-05\end{array}$ & $\begin{array}{l}0.037371 \\
0.034714\end{array}$ & $\begin{array}{l}0.007930 \\
0.007930\end{array}$ & $\begin{array}{l}0.034771 \\
0.034770\end{array}$ & $\begin{array}{l}0.999697 \\
0.999728\end{array}$ \\
\hline $\begin{array}{c}0.84765625 \\
0.8457421875\end{array}$ & $\begin{array}{l}1.26 E-03 \\
5.98 E-04\end{array}$ & $\begin{array}{l}0.036029 \\
0.035368\end{array}$ & $\begin{array}{l}0.007930 \\
0.007930\end{array}$ & $\begin{array}{l}0.034770 \\
0.034770\end{array}$ & $\begin{array}{l}0.199713 \\
0.999720\end{array}$ \\
\hline $\begin{array}{l}0.8162304688 \\
0.8264746094\end{array}$ & $\begin{array}{l}270 \mathrm{E}-0 \mathrm{~A} \\
1.07 \mathrm{E}-04\end{array}$ & $\begin{array}{l}0.035040 \\
0.034877\end{array}$ & $\begin{array}{l}0.007930 \\
0.007930\end{array}$ & $\begin{array}{l}0.034770 \\
0.034770\end{array}$ & $\begin{array}{l}0.999724 \\
0.599726\end{array}$ \\
\hline 0.8865966797 & 25SE-OS & 0.034796 & 0.007930 & 0.034770 & 0.999127 \\
\hline 0.8266577148 & $-152 E-05$ & 0.034755 & 0.007930 & 0.034770 & 0.999727 \\
\hline 0.0866271973 & $5.17 \mathrm{E}-06$ & 0.034775 & 0.007930 & 0.034770 & 0.999727 \\
\hline 0.8166424561 & $-5.00 E-06$ & 0.034765 & 0.007930 & 0.034770 & 0.999727 \\
\hline 0.0166348267 & 8.76E-08 & 0.034770 & 0.007930 & 0.034770 & 0.999727 \\
\hline 0.8266386414 & $-245 E-06$ & 0.034768 & 0.007930 & $0.034: 10$ & 0.999727 \\
\hline 0.186636734 & 1.125006 & 0.034769 & 0.007930 & 0.034770 & 0.999727 \\
\hline 0.8866357203 & $-5.48 E D O T$ & 0.034770 & 0.007930 & 0.03477 & 0.999727 \\
\hline 0.1866353035 & $230 E 07$ & 0.034770 & 0.007930 & 0.034770 & 0.999127 \\
\hline 0.8466350651 & $-7.13 \mathrm{E}-08$ & 0.034770 & 0.007930 & 0.034770 & 0.999727 \\
\hline 0.2266349459 & $8.135-09$ & $0.03477 D$ & 0.007930 & 0.034770 & 0.999127 \\
\hline 0.8466350055 & $-3.16 \mathrm{E}-08$ & 0.034770 & 0.007930 & 0.034770 & 0.999727 \\
\hline 0.8666349757 & $-1.17 E-08$ & 0.034770 & 0.007930 & 0.034770 & 0.999727 \\
\hline 0.1866349608 & $-1.20 E-09$ & 0.034770 & 0.007930 & 0.034770 & 0.999727 \\
\hline 0.8166349533 & $3.17 \mathrm{E}-09$ & 0.034770 & 0.007930 & 0.034770 & 0.999727 \\
\hline Saturation & Tolal Mob & dFv/dSr & $\mathbf{G}$ & $F$ & Goinms \\
\hline 0.75 & 34.759228 & 0.045851 & 220.095758 & 12770589 & 1.1794E+07 \\
\hline 0.875 & 40.427490 & 0.009321 & 115.033000 & 10.281953 & $1.1701 E+07$ \\
\hline 0.9375 & 43.300978 & 0.002124 & 62501620 & 10.006619 & $1.1695 \mathrm{E} A 07$ \\
\hline 0.90625 & 41.262126 & 0.005001 & $\mathbf{8 8 . 7 6 7 3 1 0}$ & 10.097238 & $1.1678 E+07$ \\
\hline 0.890625 & 41.144193 & 0.006968 & 101.900155 & 20.175426 & $1.1690 E+07$ \\
\hline 0.8128125 & 40.785677 & 0.008095 & 108.466577 & 10.224128 & $1.16955+07$ \\
\hline $0.82671 \mathrm{ks}$ & 10.964895 & 0.007519 & 105.183366 & 10.199203 & $1.1693 \mathrm{E}+07$ \\
\hline 0.884765625 & 40.875276 & 9.007804 & 106.824972 & 10211779 & $1.1694 E+07$ \\
\hline 0.8857421875 & 40.920083 & 0.007661 & $106.00 \times 169$ & 10.203432 & $1.1693 E+07$ \\
\hline 0.8262304688 & 40.942489 & 0.007590 & $20 \$ 59276 \%$ & 10.202303 & $1.1693 \mathrm{E} 407$ \\
\hline $0.826474 \cos 4$ & 40.953692 & 0.007555 & 105.388567 & 10.200149 & $1.1693 E+07$ \\
\hline $\begin{array}{l}0.1865966797 \\
0.8266577148\end{array}$ & $\begin{array}{l}40.959294 \\
40.962095\end{array}$ & $\begin{array}{l}0.007537 \\
0.007528\end{array}$ & $\begin{array}{l}105.225466 \\
105.234666\end{array}$ & 10.199975 & $1.1693 \mathrm{E}+07$ \\
\hline 0.1865271973 & 40.960694 & 0.007533 & $\begin{array}{l}105.234666 \\
105.260316\end{array}$ & $\begin{array}{l}10.1995199 \\
10.199782\end{array}$ & $\begin{array}{l}1.1699 \mathrm{E}+07 \\
1.1693 \mathrm{E}+07\end{array}$ \\
\hline 0.2866424561 & 4.95139 & 0.007530 & $10 \$ 247491$ & 10.199605 & $1.1693 E+07$ \\
\hline 0.8866348267 & 40.961044 & 0.007531 & 205.253904 & 10.199733 & $1.1693 \mathrm{E}+07$ \\
\hline 0.8266386414 & 40.961219 & 0.007531 & $105.25069 \mathrm{n}$ & 10.199109 & $1.1693 \mathrm{E}+07$ \\
\hline 0.058636734 & 40.961132 & 0.007531 & 105252301 & 10.199721 & 1.16935007 \\
\hline 0.1266357203 & 40.961088 & 0.007531 & 105.253102 & 0.199727 & $1.1693 E+07$ \\
\hline 0.1866353035 & 40.961066 & 0.007531 & 105.253503 & 10.199730 & $1.1693 \mathrm{E}+07$ \\
\hline 0.8266350651 & 40.961055 & 0.007531 & 105.253703 & 10.198732 & $1.1693 E+07$ \\
\hline 0.1866349459 & $40.9 \times 1050$ & 0.007531 & 105.253804 & 10.199733 & $1.1693 E+07$ \\
\hline 0.8666350055 & 40.961052 & 0.007531 & 105.253753 & 10.199732 & $1.1693 E+07$ \\
\hline 0.8266349757 & 40.951051 & 0.007531 & 105.253779 & 10.199732 & $1.1693 \mathrm{~K}+07$ \\
\hline 0.2866349608 & 40.961050 & 0.007531 & 105.253791 & 10.199733 & $1.16938+07$ \\
\hline 0.2866349533 & 40.961050 & 0.007531 & 105.253797 & 30.197733 & $1.1693 E+07$ \\
\hline
\end{tabular}

$\begin{array}{cc}\text { dKrv/dSr } & \text { dKrU/dSI } \\ 1.0000 & 0.1875 \\ 1.0000 & 0.0169 \\ 1.0000 & 0.0117 \\ 1.0000 & 0.0264 \\ 1.0000 & 0.0359 \\ 1.0000 & 0.0412 \\ 1.0000 & 0.0385 \\ 1.0000 & 0.0398 \\ 1.0000 & 0.0392 \\ 1.0000 & 0.0388 \\ 1.0000 & 0.0387 \\ 1.0000 & 0.0386 \\ 1.0000 & 0.0385 \\ 1.0000 & 0.0386 \\ 1.0000 & 0.0385 \\ 1.0000 & 0.0386 \\ 1.0000 & 0.0386 \\ 1.0000 & 0.0386 \\ 1.0000 & 0.0386 \\ 1.0000 & 0.0386 \\ 1.0000 & 0.0386 \\ 1.0000 & 0.0386 \\ 1.0000 & 0.0386 \\ 1.0000 & 0.0386 \\ 1.0000 & 0.0386 \\ 1.0000 & 0.0386\end{array}$

\begin{tabular}{|c|c|c|}
\hline - & Ish & 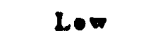 \\
\hline 20550.407113 & 1 & 0.5 \\
\hline 2275.535543 & $i$ & 0.75 \\
\hline 2033.574650 & i & 0.775 \\
\hline 1113.209409 & 0.937 & 0.075 \\
\hline 1111.920550 & 0.90825 & 0.875 \\
\hline 225334700 & 0.890625 & 0.875 \\
\hline 202815133 & 0.390825 & 0.2128125 \\
\hline 1213.167353 & 0.82671875 & 0.0828125 \\
\hline 208.229909 & 0.86671875 & 0.844765625 \\
\hline 205.539756 & 0.88671875 & 0.8157421875 \\
\hline 1262 & 371875 & 46875 \\
\hline 203.493902 & 0.82671875 & 0.82647460938 \\
\hline 203.154319 & 0.86671 175 & $0.12659667 \% 59$ \\
\hline 1203.324062 & 0.86665771424 & 0.82659667969 \\
\hline 1203.239178 & 0.82665771 .484 & 0.11632719727 \\
\hline 2203.281617 & 0.12564245605 & 0.81662719727 \\
\hline 203260397 & 0.82664245605 & 0.216663482666 \\
\hline 1203.271006 & 0.82663164136 & 0.21663412666 \\
\hline 203276312 & 0.22663673401 & 0.82663422666 \\
\hline 203.278964 & 0.82663578033 & 0.88663482666 \\
\hline 203.280290 & 0.1266353035 & 0.82663482666 \\
\hline $\begin{array}{l}1203.280954 \\
203.210622\end{array}$ & $\begin{array}{l}0.86663506508 \\
0.8 \% 663506508\end{array}$ & $\begin{array}{l}0.82663482666 \\
0.88663494587\end{array}$ \\
\hline 203.280788 & 3500547 & 0.88663494587 \\
\hline 203.280871 & 3497567 & 0.8866349458 \\
\hline & & \\
\hline
\end{tabular}


Spreadsheet for Calculation of Stream-Water Saturation Profiles

Injection Temperature $1000 \cdot \mathrm{K}$
Inllial Temperature

$350 \cdot \mathrm{K}$
Motrix Best Capeclly

$1.0000(\mathrm{~kJ} / \mathrm{kg})$
MATRIX PROPERTIES

Peresily
0.10
$N=$
3.00
Cp $\operatorname{Mathix}_{1.00}$

1.00

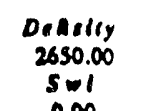

0.00
Rho $-\mathrm{CP}_{\mathrm{p}}$ 2650.0000

Denom 1.000000

SATURATION CONDITIONS Traturaton $-1,65.57$

\begin{tabular}{|c|c|c|c|c|c|}
\hline $\begin{array}{l}\text { Llquid } \\
\text { Vepor }\end{array}$ & $\begin{array}{c}\text { Volvines } \\
\text { (ms/4s) } \\
0.001176 \\
0.100298\end{array}$ & $\begin{array}{c}\text { Enthulples } \\
\text { (a)ke! } \\
901.4 ! \\
2008.52\end{array}$ & $\begin{array}{c}\text { Dencllles } \\
\text { (xemis) } \\
850.4723 \\
9.970241\end{array}$ & $\begin{array}{c}\text { Vlucesitles } \\
\text { (qp) } \\
0.1303 \\
0.0217\end{array}$ & $\begin{array}{c}\text { Salurallon } \\
\text { Eiep } \\
0.0014177462\end{array}$ \\
\hline
\end{tabular}

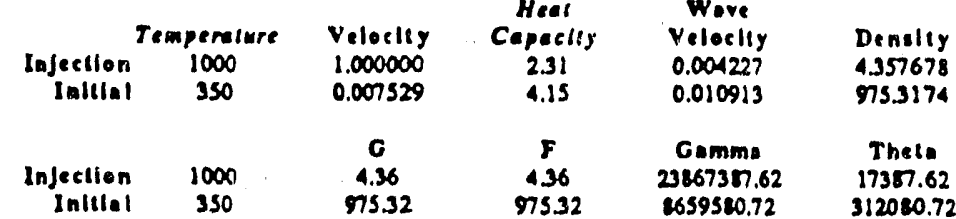

\begin{tabular}{|c|c|c|c|c|c|c|c|c|}
\hline $\begin{array}{l}\text { Trailing } \\
\text { Ladins }\end{array}$ & $\begin{array}{c}\text { Ialial } \\
\text { So-Hi } \\
1 \\
1\end{array}$ & $\begin{array}{c}\text { Geest } \\
\text { Sr.Lew } \\
0.5 \\
0.5\end{array}$ & $\begin{array}{c}\text { S BOCX } \\
\text { Vepor } \\
\text { Saturatlon } \\
0.9575 \\
0.8266\end{array}$ & $\begin{array}{l}\text { COND } \\
\text { Objecllve } \\
\text { Functlon } \\
1.0026 \mathrm{E}-09 \\
3.0060 \mathrm{E}-09\end{array}$ & $\begin{array}{l}10 \mathrm{~N} S \\
\text { Two.Phase } \\
\text { Kure } \\
\text { Velocliy } \\
0.0042 \\
0.0330\end{array}$ & $\begin{array}{l}\text { Upstreain } \\
\text { Flow } \\
\text { Velocliy } \\
0.438309 \\
0.007529\end{array}$ & $\begin{array}{l}\text { Shock } \\
\text { Veloclly } \\
0.0042 \\
0.0330\end{array}$ & $\begin{array}{l}\text { Vapor } \\
\text { Frae Flow } \\
1.0000 \\
0.9997\end{array}$ \\
\hline $\begin{array}{l}\text { Truiling } \\
\text { Leading }\end{array}$ & $\begin{array}{l}\text { dKrv/dSV } \\
1.0000 \\
1.0000\end{array}$ & $\begin{array}{l}A \mathrm{Krl} / \mathrm{ASI} \\
0.005 \mathrm{~A} \\
0.0326\end{array}$ & $\begin{array}{c}\text { Tolal } \\
\text { Mobllily } \\
4.2244 \\
40.9610\end{array}$ & $\begin{array}{l}d F v / d S \\
0.0010 \\
0.0075\end{array}$ & $\begin{array}{c}G \\
45.6729 \\
105.2538\end{array}$ & $\begin{array}{c}51 \\
9.9814 \\
10.2997\end{array}$ & $\begin{array}{c}\text { Gomma } \\
1.1640 E+07 \\
1.1693 E+07\end{array}$ & $\begin{array}{l}\text { Thele' } \\
28011.4324 \\
28203.2809\end{array}$ \\
\hline
\end{tabular}

MATERIAL. DALANCES

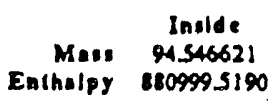

$\ln |6| a \mid$

n.531740

Injected

4357678

73279

c65958.0720

17387.6229

2449.5350

Balence

0.0000

.$\$ 3601$

Error

$0.0000 \%$

0.0004 \%

3990.11

319.98

28203.2809 
Spreadsheet for Calculation of Stream-Water Saturation Profiles

Infectlon Temperature $1000^{\circ} \mathrm{K}$
Inllat Temperature $350^{\circ} \mathbf{K}$
Matrix Beal Capneliy

$1.0000(\mathrm{~kJ} / \mathrm{kL})$

SATURATION PROFILE

\begin{tabular}{|c|c|c|c|c|c|c|c|c|}
\hline $\begin{array}{c}\text { Vaper } \\
\text { Eeterallen }\end{array}$ & $\begin{array}{c}\text { Vepor } \\
\text { Fre: Flow }\end{array}$ & $d K r v / d S v$ & dKrVdSI & $\begin{array}{c}T \bullet 101 \\
\text { Mobllily }\end{array}$ & $d F v / d S v$ & lambde & $\begin{array}{l}\text { Muse } \\
\text { Invide }\end{array}$ & $\begin{array}{l}\text { Enthalpy } \\
\text { Inolde }\end{array}$ \\
\hline $\begin{array}{l}1.0000 \\
1.0000\end{array}$ & & & & & & $\begin{array}{l}0.000000 \\
0.004179\end{array}$ & & \\
\hline $\begin{array}{l}0.9575 \\
0.9561\end{array}$ & $\begin{array}{l}0.999987 \\
0.999985\end{array}$ & $\begin{array}{l}1.0000 \\
1.0000\end{array}$ & $\begin{array}{l}0.0054 \\
0.0058\end{array}$ & $\begin{array}{l}4.224435 \\
4.159017\end{array}$ & $\begin{array}{l}0.000953 \\
0.001020\end{array}$ & $\begin{array}{l}0.004179 \\
0.004472\end{array}$ & $\begin{array}{l}0.018212 \\
0.013534\end{array}$ & $\begin{array}{l}99746.431124 \\
305.156115\end{array}$ \\
\hline $\begin{array}{l}0.9547 \\
0.5533\end{array}$ & $\begin{array}{l}0.999984 \\
0.999982\end{array}$ & $\begin{array}{l}1.0000 \\
1.0000\end{array}$ & $\begin{array}{l}0.0062 \\
0.0066\end{array}$ & $\begin{array}{l}4.093602 \\
4.028191\end{array}$ & $\begin{array}{l}0.001089 \\
0.001161\end{array}$ & $\begin{array}{l}0.004775 \\
0.005088\end{array}$ & $\begin{array}{l}0.014381 \\
0.015257\end{array}$ & $\begin{array}{l}3527.41176 \\
3651.273903\end{array}$ \\
\hline 0.5555 & 0.999981 & 1.0000 & 0.0070 & 43.962718 & 0.001235 & 0.005413 & 0.016161 & 3775.459671 \\
\hline 0.9504 & 0.999979 & 1.0000 & 0.0074 & $43.8973 \mathrm{M}$ & 0.001311 & 0.005747 & 0.017093 & 3900.403895 \\
\hline 0.9190 & 0.999977 & 1.0000 & 0.0078 & 43.831957 & 0.001390 & 0.006093 & 0.011054 & 4026.112029 \\
\hline 0.9476 & 0.999975 & 1.0000 & 0.0082 & 43.766594 & 0.001471 & 0.006450 & 0.019044 & 4152589567 \\
\hline 0.9462 & 0.999973 & 1.0000 & 0.0087 & 43.701207 & 0.001535 & 0.006817 & 0.020064 & 4279.42044 \\
\hline 0.9448 & 0.999970 & 1.0000 & 0.0092 & 43.635824 & 0.001642 & 0.007195 & 0.021113 & 407.875038 \\
\hline 0.9433 & 0.999968 & $\begin{array}{l}1.0000 \\
1.0000\end{array}$ & $\begin{array}{l}0.00 \% 6 \\
0.0101\end{array}$ & $\begin{array}{l}43.57047 \\
43.505075\end{array}$ & $\begin{array}{l}0.001730 \\
0.001122\end{array}$ & $\begin{array}{l}0.007585 \\
0.007985\end{array}$ & $\begin{array}{l}0.022192 \\
0.023301\end{array}$ & \\
\hline $\begin{array}{l}0.9405 \\
0.9391\end{array}$ & $\begin{array}{l}0.999963 \\
0.999960\end{array}$ & 1.0000 & 0.0111 & 43.374346 & 0.002012 & 0.008820 & 0.025611 & 4927.925160 \\
\hline 0.937 & 0.999957 & 1.0000 & 0.0117 & 43.308991 & 0.002111 & 0.009254 & 0.026812 & 5059.94542 \\
\hline 0.9363 & 0.999954 & 1.0000 & 0.0122 & 13.243641 & 0.002213 & 0.009699 & 0.022044 & 5192781380 \\
\hline 0.9348 & 0.999951 & 1.0000 & 0.0127 & 43.171297 & 0.002317 & 0.010156 & 0.029308 & 5326.437647 \\
\hline 0.9334 & 0.999947 & 1.0000 & 0.0133 & 43.112959 & 0.002424 & 0.010825 & 0.030603 & 5460.920562 \\
\hline 0.9320 & 0.99994 & 1.0000 & 0.0139 & 43.047627 & 0.002334 & 0.0111105 & 0.031931 & 5596.236084 \\
\hline 0.9306 & $0.999 \times 40$ & 1.0000 & 0.0145 & 42912302 & 0.002646 & 0.011590 & 0.033290 & 5732390222 \\
\hline 0.9292 & 0.999936 & 1.0000 & 0.0151 & 42916983 & 0.002761 & 0.012100 & 0.034683 & 5869.319025 \\
\hline $0.92 n$ & 0.999932 & 1.0000 & 0.0157 & 42851670 & 0.002878 & 0.012615 & 0.036108 & $\cos 238590$ \\
\hline 0.9263 & 0.999928 & 1.0000 & 0.0163 & 42786364 & 0.002998 & 0.013142 & 0.037566 & 6145.945058 \\
\hline 0.9249 & 0.999924 & 1.0000 & 0.0169 & 12721065 & 0.003121 & 0.013680 & 0.039058 & 6285.514619 \\
\hline 0.9235 & 0.999919 & 1.0000 & 0.0176 & 42655773 & 0.003247 & 0.014231 & 0.040583 & 425.953506 \\
\hline 0.9221 & 0.999915 & 1.0000 & 0.0182 & 42590488 & 0.003375 & 0.014794 & 0.042143 & 6567.268001 \\
\hline 0.9207 & 0.999910 & 1.0000 & 0.0189 & 42525211 & 0.003500 & 0.015369 & 0.043736 & 6709.464133 \\
\hline 0.9192 & 0.999905 & 1.0000 & 0.0196 & 42459940 & 0.003640 & 0.015956 & 0.045365 & 6152549177 \\
\hline 0.9178 & 0.999900 & 1.0000 & 0.0203 & 42394677 & 0.00377 & 0.016556 & 0.047028 & 6996.528658 \\
\hline 0.9164 & 0.999894 & 1.0000 & 0.0210 & 12329422 & 0.003917 & 0.017168 & 0.048727 & 7141.409349 \\
\hline 0.9150 & 0.999888 & 1.0000 & 0.0217 & 12264174 & 0.004059 & 0.017792 & 0.050461 & 7257.197770 \\
\hline 0.9136 & 0.999883 & 1.0000 & 0.0224 & 42198935 & 0.004205 & 0.01429 & 0.052231 & 7433900493 \\
\hline 0.9122 & 0.999876 & 1.0000 & 0.0232 & 42.133703 & 0.004353 & 0.019078 & 0.054037 & 7581.524138 \\
\hline 0.9107 & 0.999870 & 1.0000 & 0.0239 & 42062479 & 0.004504 & 0.19740 & 0.055880 & 7730.075374 \\
\hline 0.9093 & 0.999864 & 1.0000 & 0.0247 & 42003264 & 0.004658 & 0.020415 & 0.057759 & 7779.560923 \\
\hline 0.9079 & 0.999857 & 1.0000 & 0.0254 & 41.932057 & 0.004815 & 0.021103 & 0.059676 & 2029.987556 \\
\hline 0.9065 & 0.999850 & 1.0000 & 0.0262 & 41.872859 & 0.004974 & 0.021803 & 0.061630 & 8111.362096 \\
\hline 0.9051 & 0.999443 & 1.0000 & 0.0270 & 41.807669 & 0.005137 & 0.022517 & 0.063622 & 8333.691418 \\
\hline 0.9036 & 0.999835 & 1.0000 & 0.0279 & 41.742488 & 0.005303 & 0.02324 & 0.065652 & 2486.912449 \\
\hline 0.9022 & 0.999828 & 1.0000 & $0.0, n$ & 41.677316 & 0.005472 & 0.023983 & 0.067721 & 641242167 \\
\hline 0.9008 & 0.999820 & 1.0000 & 0.02 is & 41.612153 & 0.0056 & 0.024736 & 0.069828 & 7796.477605 \\
\hline 0.8994 & 0.999812 & 1.0000 & $0.03 M$ & 41546999 & 0.005118 & 0.025502 & 0.071975 & c952695249 \\
\hline 0.1980 & 0.993203 & 1.0000 & 0.031 : & 41.481855 & 0.005996 & 0.026282 & 0.074161 & 9109.904039 \\
\hline 0.8966 & 0.999795 & 1.0000 & 0.0321 & 41.416720 & 0.00617 & 0.027075 & 0.076387 & 9268.109369 \\
\hline 0.8951 & 0.99978 & 1.0000 & 0.0330 & 41.351594 & 0.006361 & 0.027812 & 0.078654 & 9427319087 \\
\hline 0.8937 & 0.9997 & 1.0000 & 0.0339 & 41286479 & 0.006548 & 0.026702 & 0.080960 & 9577.540499 \\
\hline 0.1923 & 0.999767 & 1.0000 & 0.0348 & 41.221373 & 0.006739 & 0.029536 & 0.083308 & 9748.780963 \\
\hline 0.5909 & 0.999758 & 1.0000 & 0.0357 & $41.1562 \mathrm{~m}$ & 0.006932 & 0.03034 & 0.085698 & 9911.047897 \\
\hline 0.8895 & 0.999748 & 1.0000 & 0.0367 & 41.091191 & 0.007129 & 0.031246 & 0.085128 & 10074.348773 \\
\hline 0.8881 & 0.999737 & 1.0000 & 0.0376 & $41.02611 \mathrm{~s}$ & 0.007328 & 0.032121 & 0.090601 & 10238.691122 \\
\hline $\begin{array}{l}0.8866 \\
0.0000\end{array}$ & 0.999727 & 1.0000 & 0.0368 & 40.961050 & 0.007531 & 0.033011 & 0.093117 & 10404.082531 \\
\hline
\end{tabular}


Spreadsheet for Calculation of Stream-Water Saturation Profiles

Infection Temperature $1000 \mathrm{~K}$
Inltial Temperature

390 '
Matrix Beat Capacliy

1.0000 (kJ/kE)

TRAILING EROCKCALCULATIONE

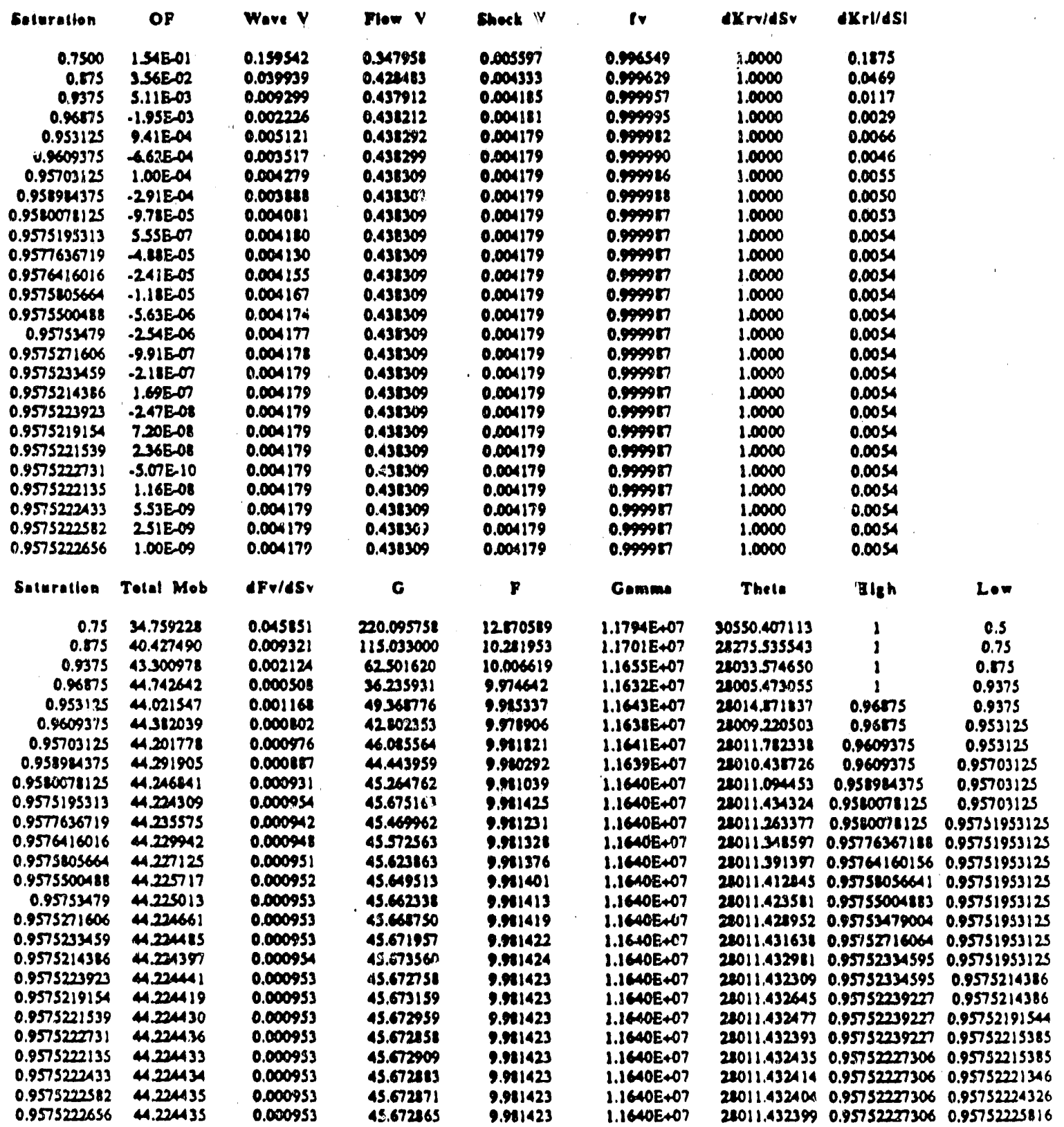


Spreadsheet for Calculation of Stream-Water Saturation Profiles

Injection Temperature

$1000 \cdot \mathrm{K}$
Inlilal Temperalure

350 " $\mathrm{K}$
Motrlx Beat Capoctly

2.0000 (tuJ/ke)

\section{LEADING SIDCK CALCULATIONE}

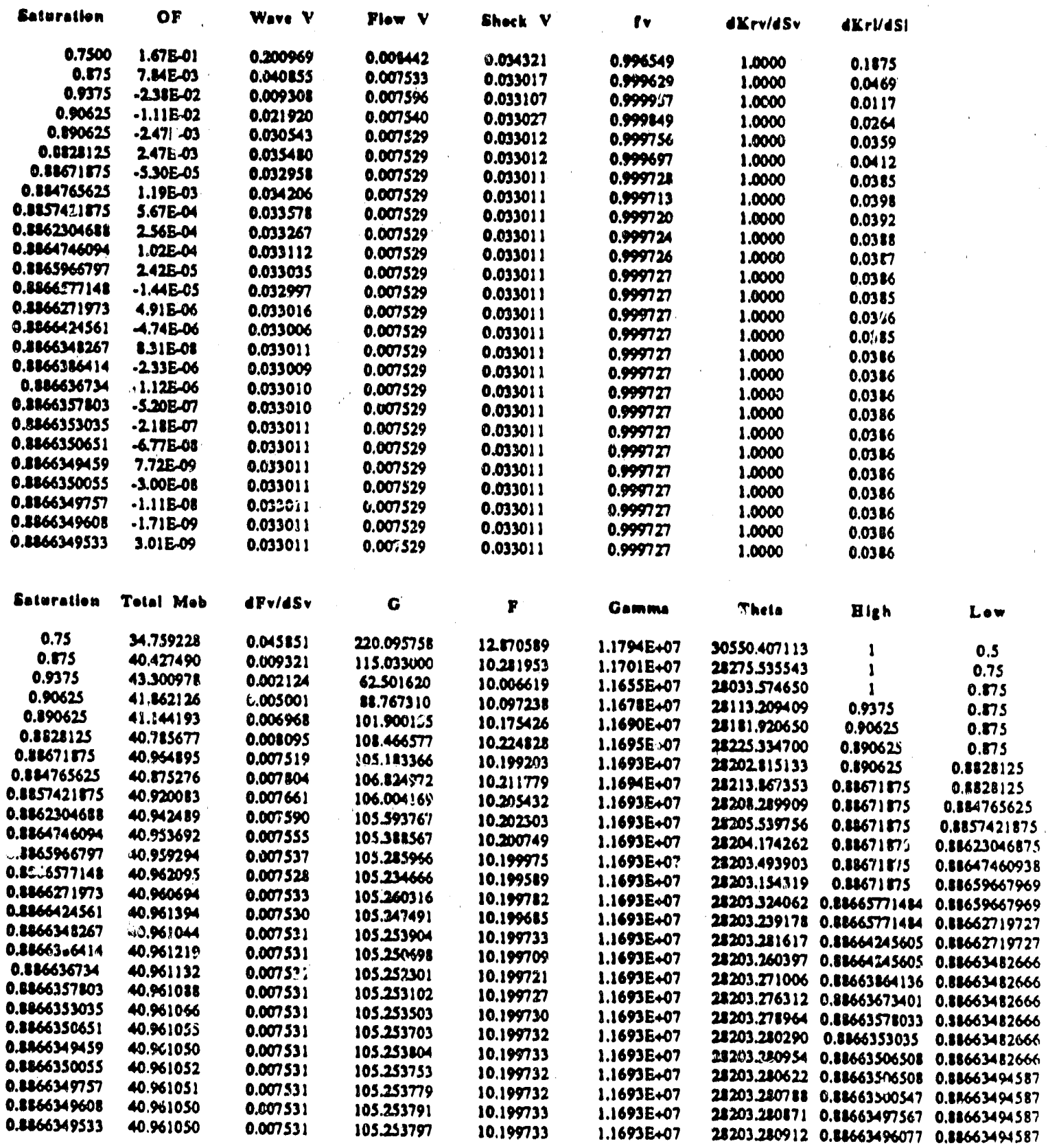




\section{- 193 .}

\section{D.3 Changes in Initial Temperature}

The next eight worksheets examine the effect of initial temperature. The initial temperature is entered in cell B23. The section contains worksheets with the injection temperature ranging from 300 -.. $475 \mathrm{~K}$, in $25 \mathrm{~K}$ intervals. 
Spreadsheet for Calculation of Stream-Water Saturation Profiles

Injectlon Tempersture $650 \cdot \mathrm{K}$
Inllial Temperolure $300 \bullet \mathrm{K}$
Matrix Beat Copactity $1.0000(\mathrm{~kJ} / \mathrm{kg})$

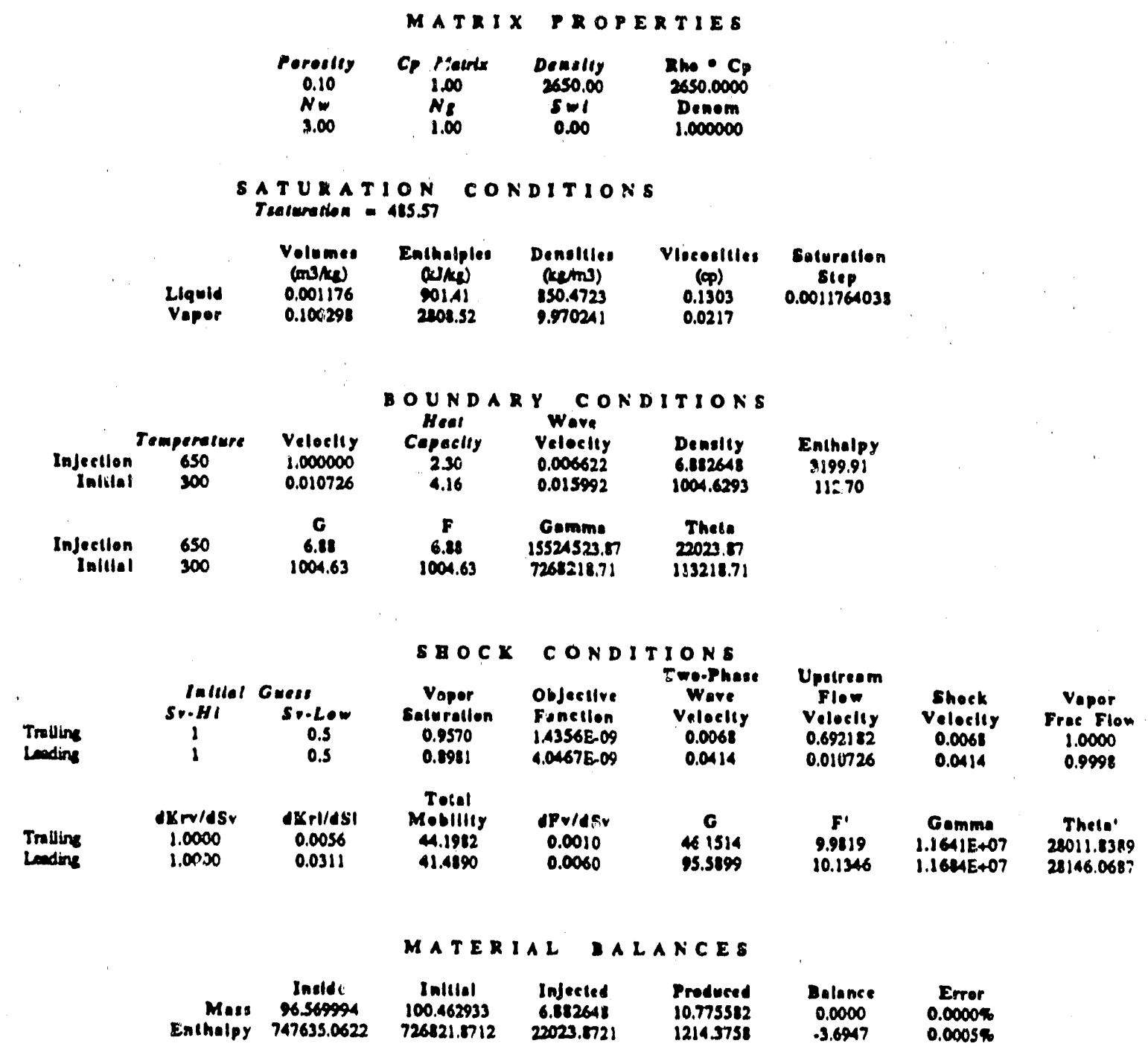


$-195$.

Spreadsheet for Calculation of Stream-Water Saturation Profiles

Injectlon Temperalure $650^{\circ} \mathrm{K}$
Inlilal Temperature

$300 \cdot \mathrm{K}$
Marifix Beal Capactiy

$1.0000\left(\mathrm{~kJ} / \mathrm{k}_{\mathrm{g}}\right)$

SATURATION PROFIRE

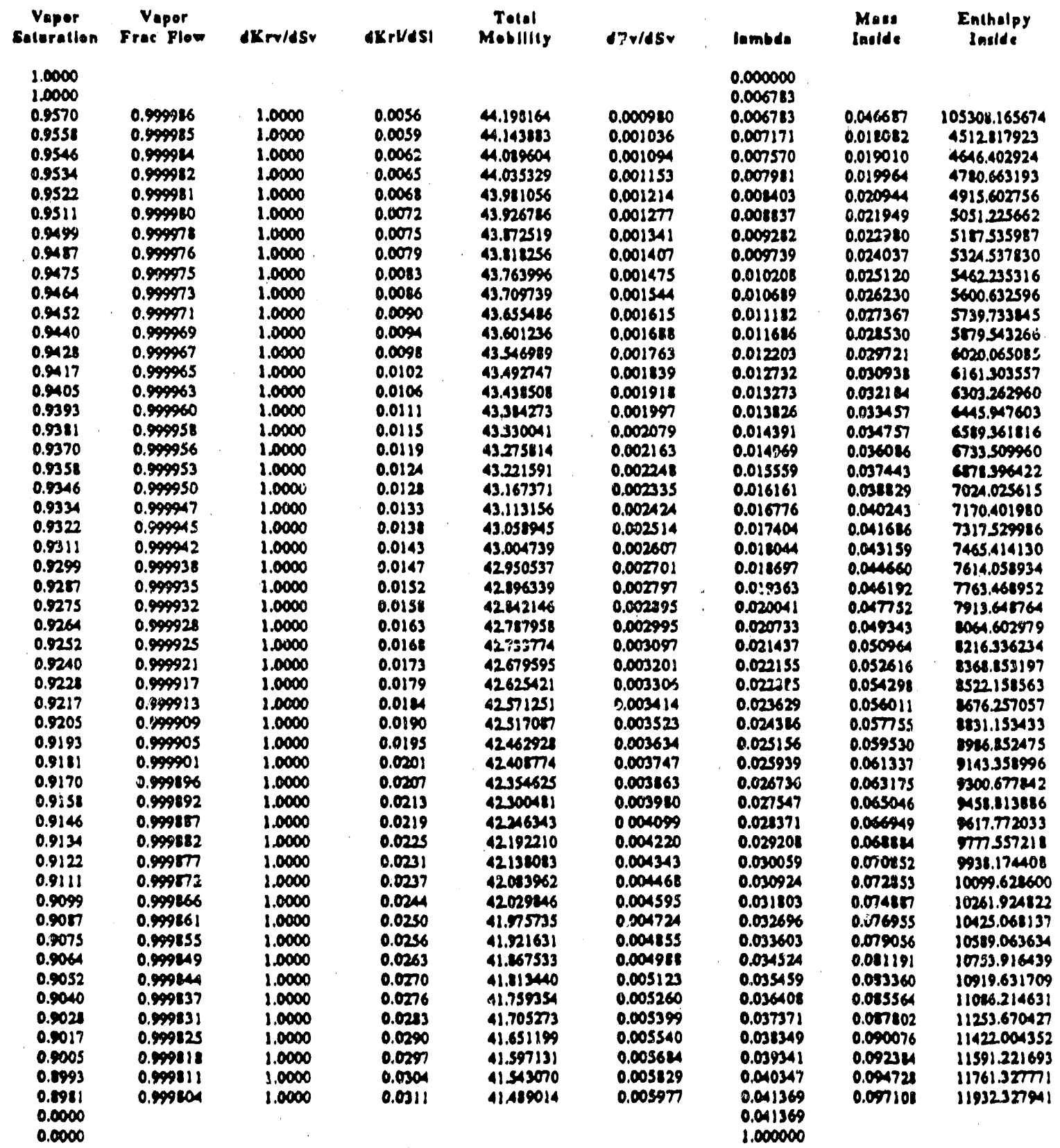


-196 -

Spreadsheet for Calculation of Stream-Water Saturation Profiles

Injection Temperature GSO 'x
Inthlal Temperature

$300^{\circ} \mathrm{K}$
Motrix Beal Capacliy

$8.0000\left(\mathrm{~kJ} / \mathrm{k}_{2}\right)$

TRAILING SBOCX CALCULATIONB

\begin{tabular}{|c|c|c|c|c|c|c|c|c|}
\hline Soturation & OP & Wave $v$ & Fhow $v$ & shock V & rv & dKrviesv & Rirl/ss! & \\
\hline 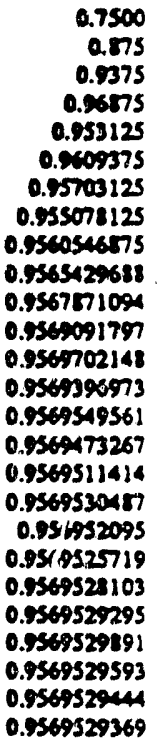 & 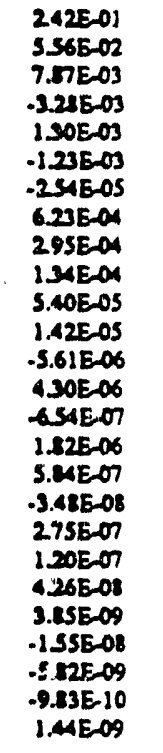 & $\begin{array}{l}0.255386 \\
0.063132 \\
0.011687 \\
0.0051516 \\
0.008677 \\
0.005554 \\
0.006758 \\
0.007406 \\
0.007078 \\
0.006917 \\
0.006837 \\
0.006798 \\
0.006778 \\
0.006788 \\
0.006783 \\
0.006785 \\
0.066784 \\
0.006783 \\
0.006784 \\
0.006783 \\
0.006783 \\
0.006783 \\
0.006783 \\
0.066783 \\
0.006783 \\
0.006783\end{array}$ & 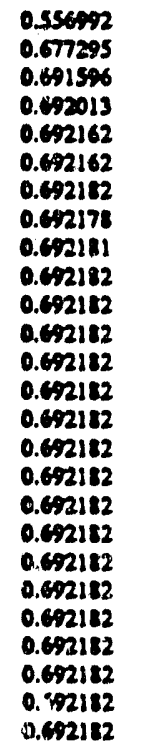 & 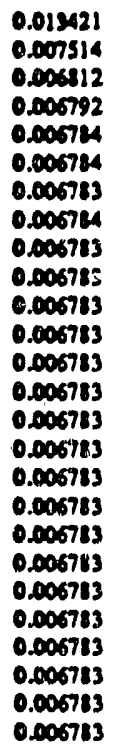 & 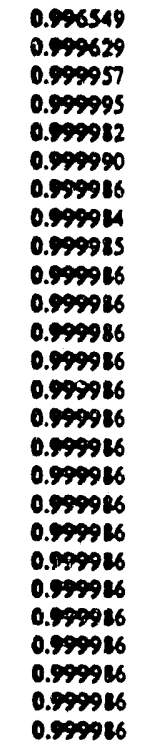 & $\begin{array}{l}1.0000 \\
1.0000 \\
1.0000 \\
1.0000 \\
1.0000 \\
1.0000 \\
1.0000 \\
1.0000 \\
1.0000 \\
1.0000 \\
1.0000 \\
1.0000 \\
1.0000 \\
1.0000 \\
1.0000 \\
1.0000 \\
1.0000 \\
1.0000 \\
1.0000 \\
1.0000 \\
1.0000 \\
1.0000 \\
1.0000 \\
1.0900 \\
1.0000 \\
1.0000\end{array}$ & $\begin{array}{l}0.1875 \\
0.0469 \\
0.0117 \\
0.0029 \\
0.0066 \\
0.0056 \\
0.0055 \\
0.0061 \\
0.0056 \\
0.0057 \\
0.0056 \\
0.0056 \\
0.0056 \\
0.0056 \\
0.0056 \\
0.0056 \\
0.0056 \\
0.0056 \\
0.0056 \\
0.0056 \\
0.0056 \\
0.0056 \\
0.0056 \\
0.0056 \\
0.0056 \\
0.0056\end{array}$ & \\
\hline Eoturation & Tolal Mob & efvlasv & C & 7 & Gommen & Thete & Algh & Low \\
\hline 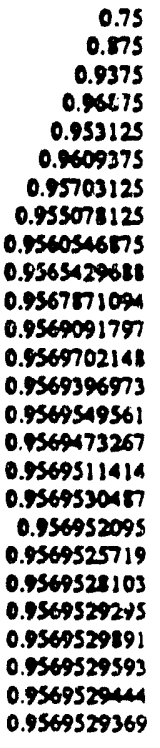 & 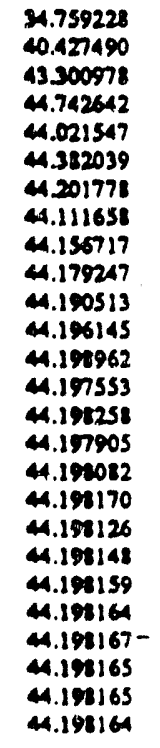 & 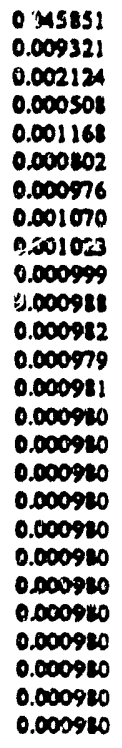 & 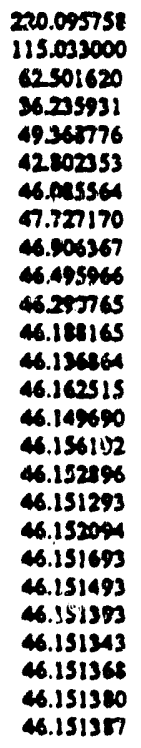 & 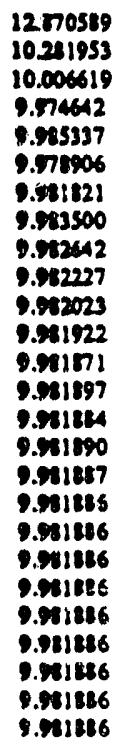 & 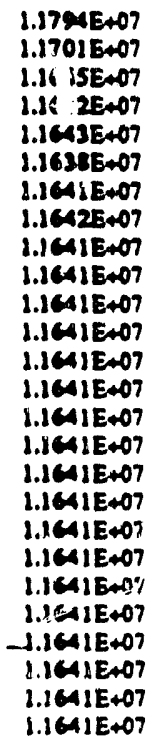 & 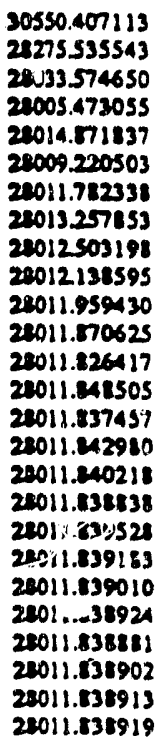 & 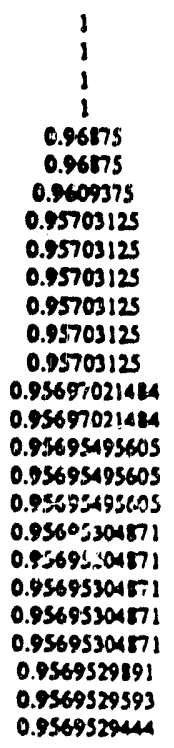 & $\begin{array}{c}0.5 \\
0.75 \\
0.075 \\
0.0375 \\
0.9375 \\
0.953125 \\
0.953125 \\
0.953125 \\
0.955078125 \\
0.9560546175 \\
0.95651296875 \\
0.95671710938 \\
0.95690917969 \\
0.95690917969 \\
0.95693969727 \\
0.95693969727 \\
0.95691732666 \\
0.95655114136 \\
0.95695114136 \\
0.95695209503 \\
0.95695257187 \\
0.95695281029 \\
0.9569529295 \\
0.9569529295 \\
0.9569529299 \\
0.9569529295\end{array}$ \\
\hline
\end{tabular}


Spreadsheet for Calculation of Stream-Water Saturation Profiles

Infectlen Temperatere $690 \cdot \mathrm{K}$
Inllibl Tenperatere

$300 \cdot K$
Matrlx Beat Capacliy

$1.0000\left(\mathrm{~kJ} / \mathrm{kg}_{\mathrm{g}}\right)$

\section{LEADING SEOCKCALCULATLONS}

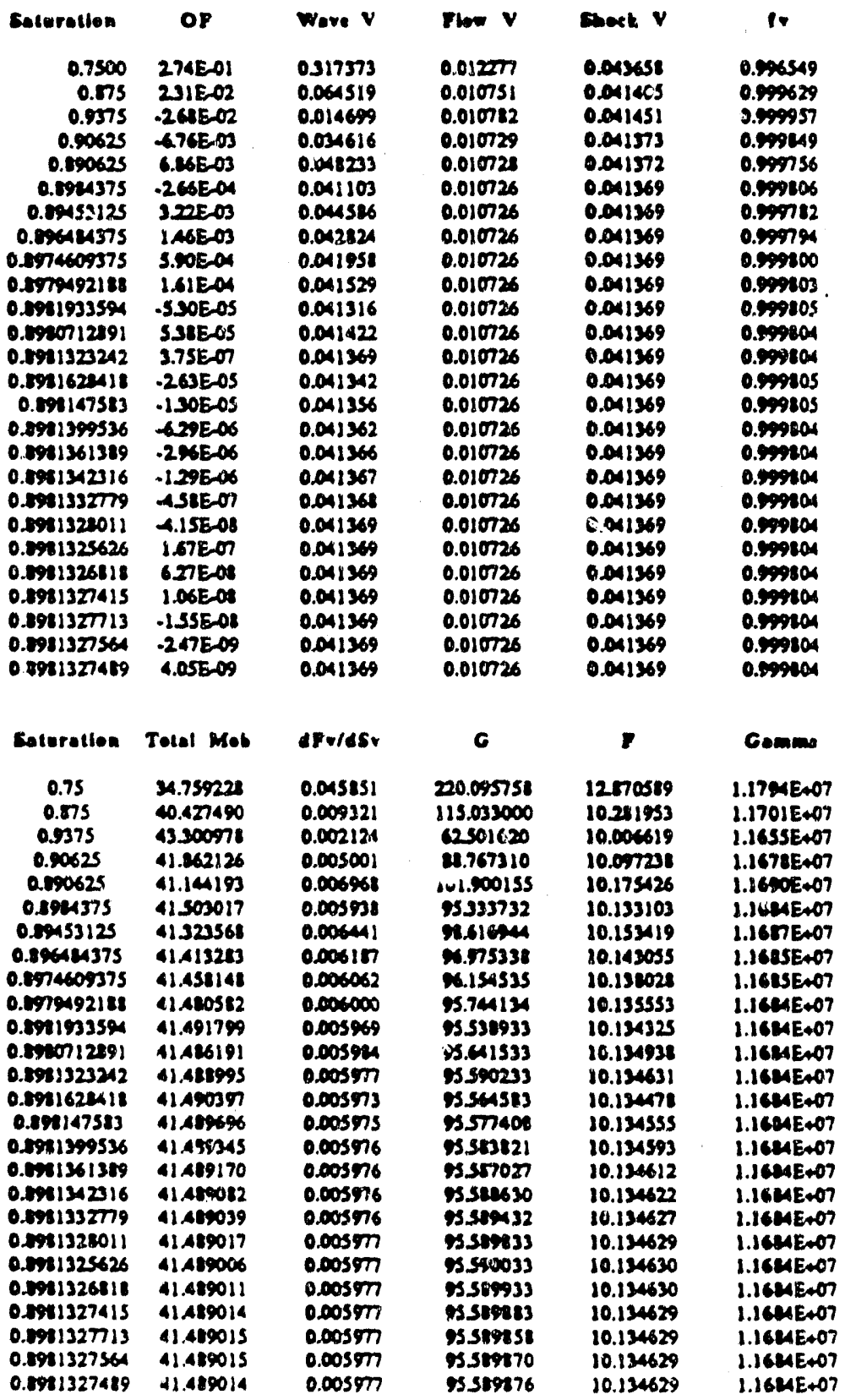

$\begin{array}{ll}\text { IKroldsr } & \text { UKrVASI } \\ 1.0000 & 0.1875 \\ 1.0000 & 0.0469 \\ 1.0000 & 0.01 ! ? \\ 1.0000 & 0.0261 \\ 1.0000 & 0.0359 \\ 1.0000 & 0.0309 \\ 1.0000 & 0.0331 \\ 1.0000 & 0.0321 \\ 1.0000 & 0.0315 \\ 1.0000 & 0.0312 \\ 1.0000 & 0.0311 \\ 1.0000 & 0.0312 \\ 1.0000 & 0.0311 \\ 1.0000 & 0.0311 \\ 1.0000 & 0.0311 \\ 1.0000 & 0.0311 \\ 1.0000 & 0.0311 \\ 1.0000 & 0.0311 \\ 1.0000 & 0.0311 \\ 1.0000 & 0.0311 \\ 1.0000 & 0.0311 \\ 1.0000 & 0.0311 \\ 1.0000 & 0.0311 \\ 1.0000 & 0.0311 \\ 1.0000 & 0.0311 \\ 1.0000 & 0.0311\end{array}$

30550 A07 113

20275s35s4

22033574650

2113200409

28181.920650

214.72756

24162581158

2153.472957

28149.053510

2816.80045

0.0x41s7s 0.0974609375

2146.enocos 0.0919335938 0.89601122906 21145.eas932 o.seties359s: 0.09113232428 $211460032590.2816244180 .091323 \% 422$ 28146.0369310 .091147513010 .09113212422 28) 46.0537680 .04134953610 .091323 .2422 28146.0621880 .03136138920 .00113232422 $21146.0663980 .0913423157 \quad 0.09813232423$ 20146.068503 0.01413327789 0.09813232422 20146.069535 0.49132101060 .49132232422 2146.0590290 .09132601060 .0981325624 28146.0677660 .091132901060 .09113261185 21146.0686340 .851132801060 .89113274145 $24146.068700 \quad 0.09113271250 .09813274145$ 28146.0687330 .891132756350 .09813274145 
Spreadsheet for Calculation of Stream-Water Saturation Profiles

Injectlon Temperalure cso $\mathrm{K}$ inlital Temperalure

$325 x$
Meirlx Beat Copaclity $1.0000(\mathrm{~kJ} / \mathrm{k} E)$
MATRIXTROPERTIES

Perasily

0.10
$N=0$
Cp Matrix

$$
\begin{aligned}
& 1.00 \\
& N_{8} \\
& 1.00
\end{aligned}
$$

Deacily

2050.00

0.00
Rine - Cp

2650.0000

Denem

1.000000

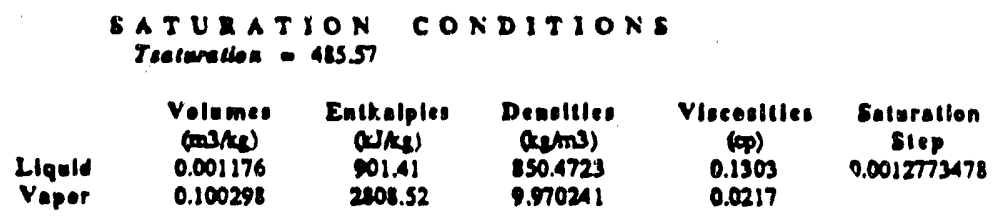

DOUNDARY CONDITIONE

Heal Wove

Copactiy Velectiy Denalty

Denolity Enthalpy

Tempensiare Velecliy

1.000000

2.30

0.006622

6.052648

10.6532

Inthelpy

0.011229

0.016483

$\begin{array}{ccc}6.88 & \text { Gemme } & \text { Timela } \\ 6524523.87 & 23023.87\end{array}$

$\begin{array}{lll}60.65 & 7 \% 5654.57 & 2140457\end{array}$

G.88

990.65
S B C K

Vapor

Saturation

0.9570

0.0931

Tetal

Moblility

44.1982

4.2572
CONDITIONS

Objectlve Tre-Phase Upstream

Function Veloelty

$1.4356 \mathrm{E}-09 \quad 0.0068$

3.0045-09 0.0459

$\begin{array}{ll}\text { Lrv/dSV } & \text { Lipl/ASI } \\ 1.0000 & 0.0056 \\ 1.0000 & 0.0343\end{array}$

Trailing
Lading

\section{dFv/dSv \\ 0.0010}

0.0066
G

46.1514

\%.8321
MATERIAL DALANCES

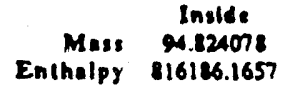

Ialtiol 9.065316 796565.4571

Infected 6.822648 20023.0721
Prodeced 11.123879 24075132
Dalunce 0.0000

4.397
Sheck Velosily 0.0068 0.0459 Vepor Froc Flow 1.0000 0.9998

Thela' 28011.8389 28169.5618 
-199-

Spreadshet for Calculation of Stream-Water Saturation Profiles

Injecilon Tempeinature

$650 \cdot \mathrm{K}$
Inllial Temperature $323^{\circ} \mathrm{X}$
Motrlx Beat Capoelly

$1.0000\left(k J / k_{E}\right)$

SATURATION PROFILE

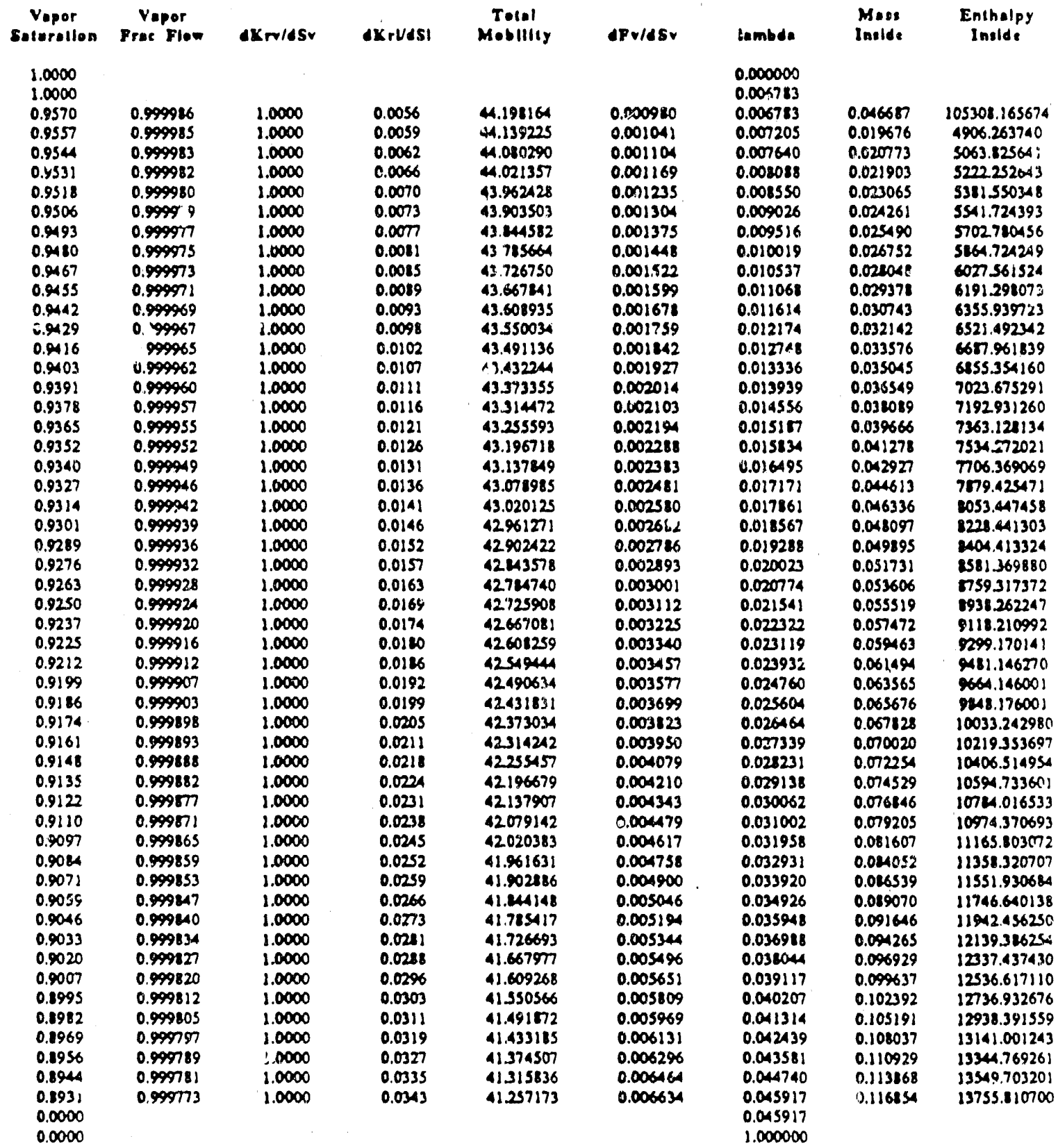


Injection Temperature $650^{\circ} \mathrm{K}$
Inlelal Temperature

$325 \cdot \mathrm{K}$
Mairlx Beal Capacliy

$1.0000(\mathrm{~kJ} / \mathrm{kg})$

TRAILING SBOCK CALCULATIONE

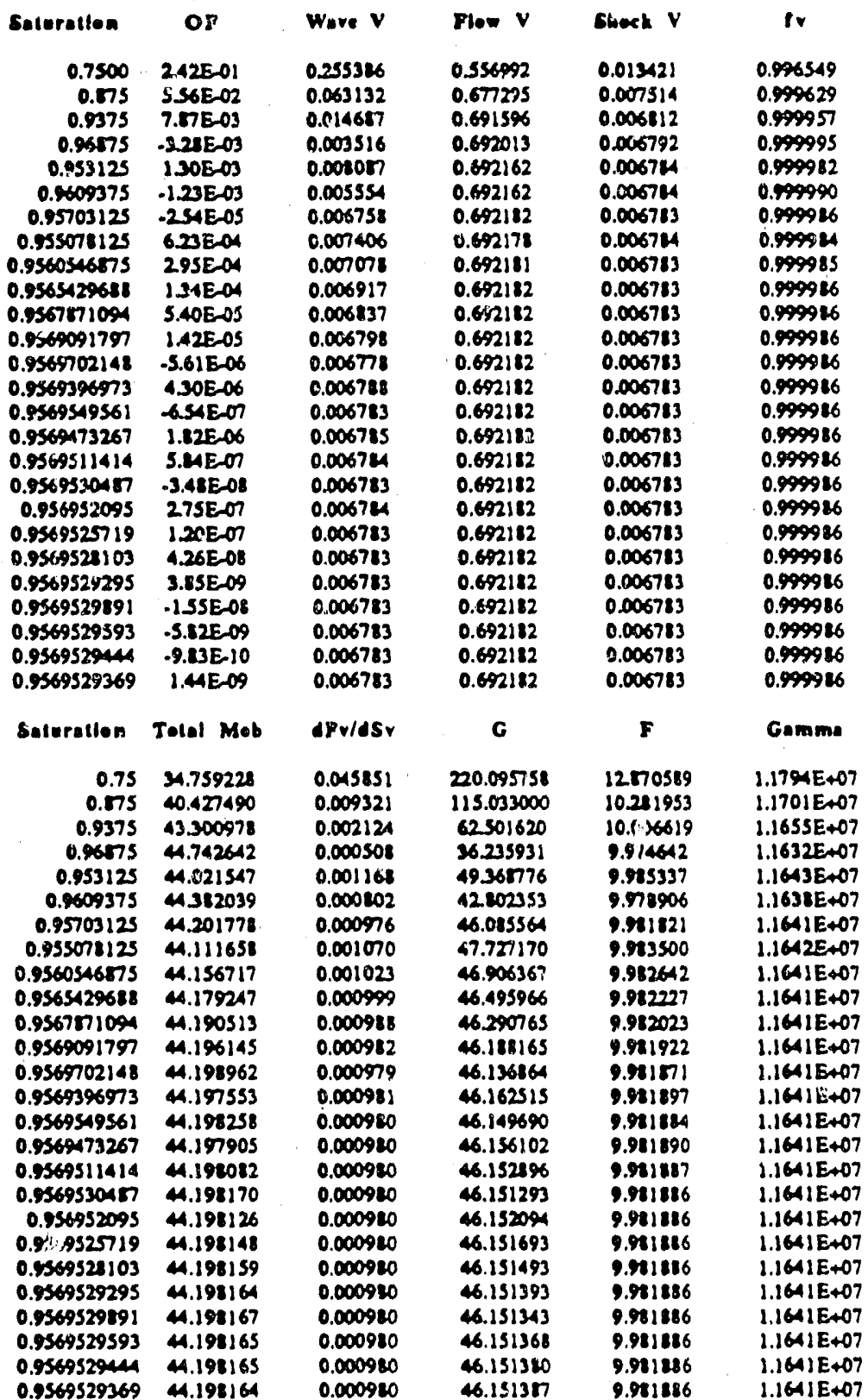

\begin{tabular}{|c|c|}
\hline Krv/eSv & LYrVISI \\
\hline 1.0000 & 0.1875 \\
\hline 1.0000 & 0.0469 \\
\hline 1.0000 & 0.0117 \\
\hline 1.0000 & 0.0029 \\
\hline 1.0000 & 0.0066 \\
\hline 1.0000 & 0.0046 \\
\hline 1.0000 & 0.0055 \\
\hline 1.0000 & 0.0061 \\
\hline 1.0000 & 0.0058 \\
\hline 1.0000 & 0.0057 \\
\hline 1.0000 & 0.0056 \\
\hline 1.0000 & 0.0056 \\
\hline 1.0000 & 0.0056 \\
\hline 3,0000 & 0.0056 \\
\hline 1.0000 & 0.0056 \\
\hline 1.0000 & 0.0056 \\
\hline 1.0000 & 0.0056 \\
\hline 1.0000 & 0.0056 \\
\hline 1.0000 & 0.0056 \\
\hline 1.0000 & 0.0056 \\
\hline 1.0000 & 0.0056 \\
\hline 1.0000 & 0.0056 \\
\hline 1.0000 & 0.0056 \\
\hline 1.0000 & 0.0056 \\
\hline 1.0000 & 0.0056 \\
\hline 1.0000 & 0.0056 \\
\hline Theta & Blgh \\
\hline
\end{tabular}

30550.407113

21275.535543

20033574650

22005.473055

28014.071837

22009.220503

22011.712338

28013.257153

20012503198

28012138595

22011.959430

28011.07062

28011.24850s 0.956970214240 .95690917969 28011.0374570 .956970214440 .95693969727 28011.429800 .956954956050 .95693969727 20011.2402180 .956954956050 .95694732666 28011.0388380 .956954956050 .95695114136 22011.8395280 .956953048710 .95695114136 28011.8391830 .956953048710 .95695209503 $28011.839010 \quad 0.956953048710 .95695257187$ 28011.8389240 .956953048710 .95695281029 25011.038881 0.95695304710 .9569529295 $28011.836902 \quad 0.9569529891 \quad 0.9569529295$ $\begin{array}{lll}22011.038913 & 0.9569529593 & 0.9569529295\end{array}$ $\begin{array}{lll}28011.238913 & 0.9569529593 & 0.9569529295 \\ 28011.138919 & 0.9569529444 & 0.9569529295\end{array}$ 
Spreadsheet for Calculation of Stream-Water Saturation Profiles

Injection Temperalure sso $\cdot \mathbf{K}$
Inllial Temperature $325 \cdot \mathrm{K}$
Matrlx Beat Copacliy

$1,0000\left(\mathrm{~kJ} / \mathrm{k}_{\mathrm{E}}\right)$

LEADING SBOCX CALCULATIONS

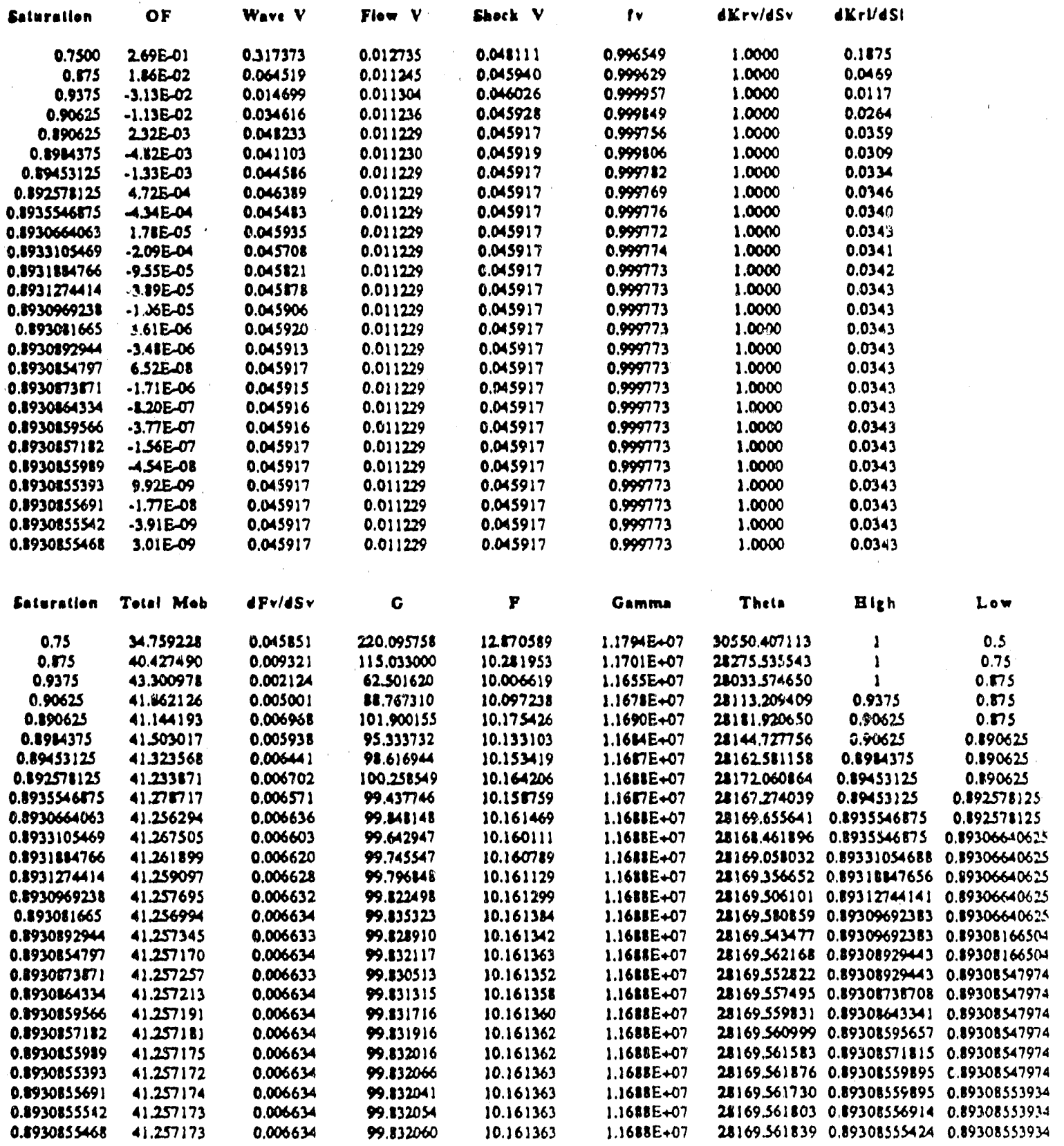


Spreadsheet for Calculation of Stream-Water Saturation Profiles

Injeclion Temperolure

$650 \cdot \mathbf{K}$
Inllial Temperolure

$350 \cdot x$
Malrix Eeat Capocliy

$1.0000\left(\mathbf{J} / \mathrm{kE}_{\mathrm{E}}\right)$

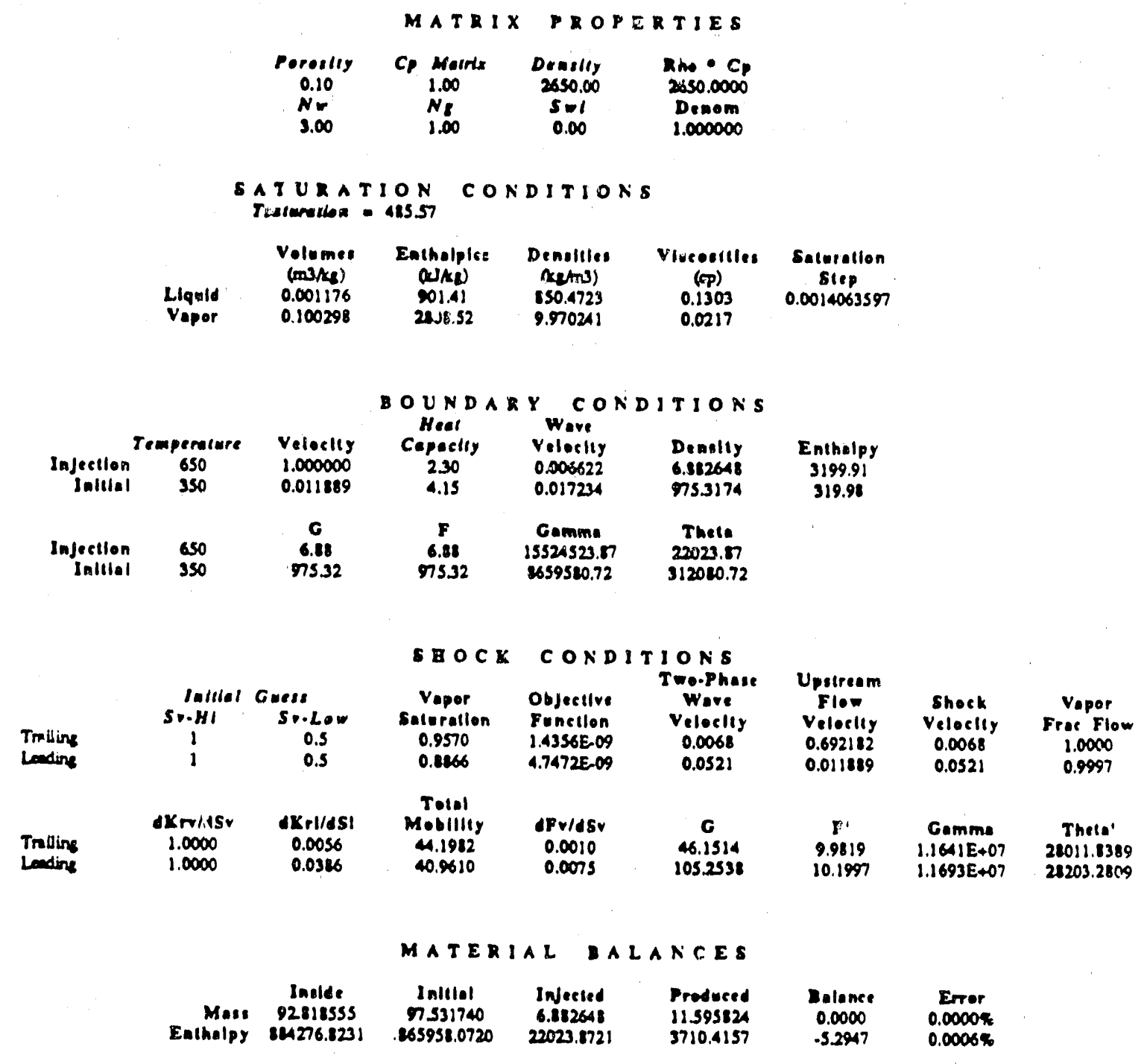


Injectlon Temperatupe $650 \mathrm{~K}$
Inlilal Temperature

$350^{\circ} \mathrm{K}$
Molrix Beet Capacley

$1.0000(\mathrm{~kJ} / \mathrm{kg})$

SATURATION PROPILE

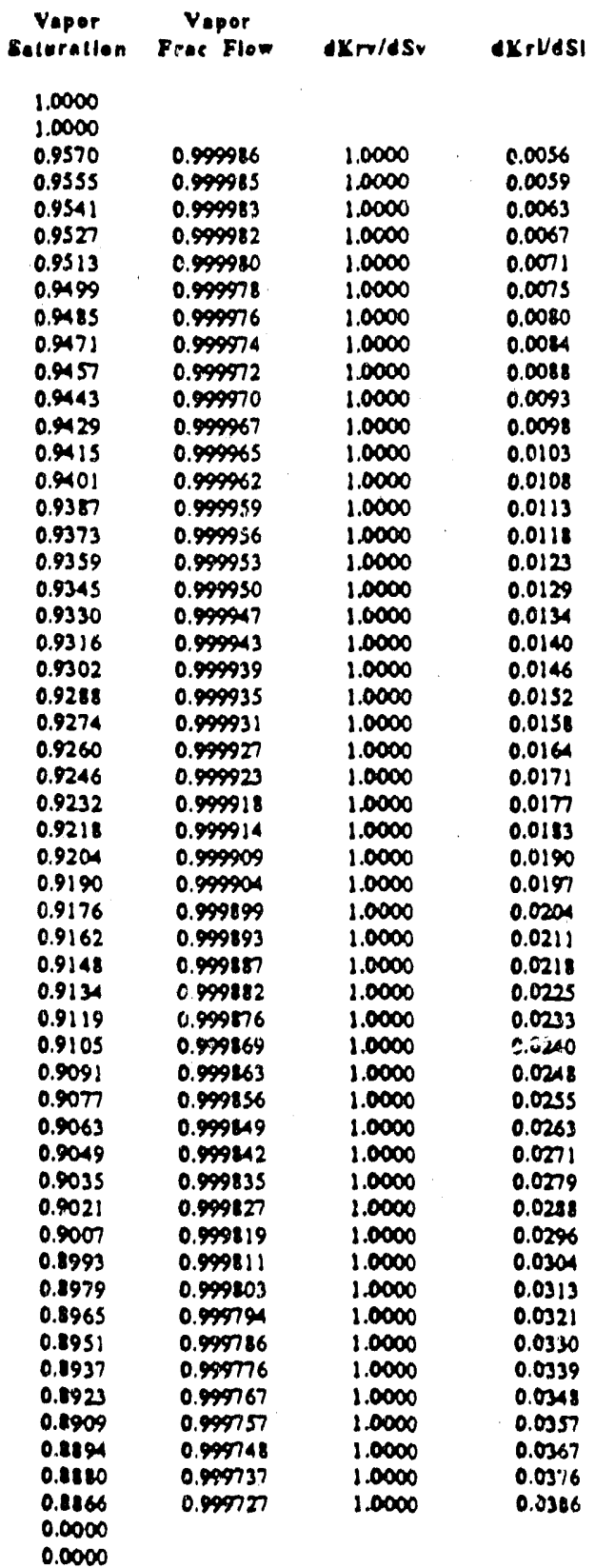

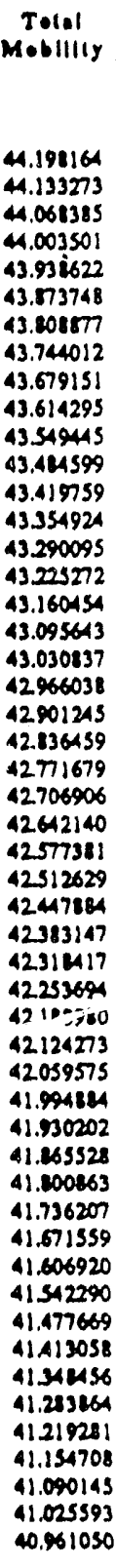

\begin{tabular}{|c|c|}
\hline \multirow[t]{2}{*}{ AP $\nabla / A S r$} & Sambde \\
\hline & $\begin{array}{l}0.000000 \\
0.006783\end{array}$ \\
\hline $\begin{array}{l}0.000980 \\
0.001047\end{array}$ & $\begin{array}{l}0.006783 \\
0.007248\end{array}$ \\
\hline 0.0011117 & 0.007729 \\
\hline 0.001189 & 0.008227 \\
\hline 0.001263 & 0.008741 \\
\hline 0.001340 & 0.009272 \\
\hline 0.001419 & 0.009819 \\
\hline 0.001500 & 0.010384 \\
\hline 0.001584 & 0.010965 \\
\hline 0.001671 & 0.011564 \\
\hline 0.001760 & 0.012179 \\
\hline 0.001851 & 0.012812 \\
\hline 0.001945 & 0.013462 \\
\hline 0.002041 & 0.014130 \\
\hline 0.002140 & 0.014815 \\
\hline 0.002242 & 0.015518 \\
\hline 0.002346 & 0.016239 \\
\hline 0.002153 & $0.0169^{\circ} 78$ \\
\hline 0.002562 & 0.017734 \\
\hline 0.00267 & 0.018509 \\
\hline 0.002719 & 0.019302 \\
\hline 0.002906 & 0.020113 \\
\hline 0.003026 & 0.020943 \\
\hline$(0.003) 48$ & 0.021791 \\
\hline 0.003273 & 0.020658 \\
\hline 0.003401 & 0.02354 \\
\hline 0.003532 & 0.024448 \\
\hline 0.003666 & 0.025372 \\
\hline $\begin{array}{l}0.003802 \\
0.003941\end{array}$ & $\begin{array}{l}0.026315 \\
n .021277\end{array}$ \\
\hline 0.004082 & 0.028258 \\
\hline 0.004227 & 0.029259 \\
\hline 0.004374 & 0.030279 \\
\hline 0.004525 & 0.031319 \\
\hline 0.004678 & 0.032379 \\
\hline 0.004834 & 0.033458 \\
\hline 0.004993 & 0.034558 \\
\hline 0.005154 & 0.035678 \\
\hline 0.005319 & 0.036818 \\
\hline 0.00547 & 0.037979 \\
\hline 0.003657 & 0.039160 \\
\hline 0.005831 & 0.040362 \\
\hline 0.006008 & 0.041585 \\
\hline 0.006187 & 0.042828 \\
\hline 0.006370 & 0.044093 \\
\hline 0.006956 & 0.045379 \\
\hline 0.006745 & 0.046616 \\
\hline 0.006937 & 0.042013 \\
\hline 0.007132 & 0.049365 \\
\hline 0.007330 & 0.050737 \\
\hline 0.007331 & 0.052131 \\
\hline & $\begin{array}{l}0.052131 \\
1000000\end{array}$ \\
\hline & 1.000000 \\
\hline
\end{tabular}

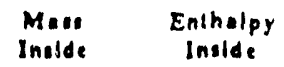


Injection Temperatur: $6 s 0 \cdot \mathbf{K}$
Inltial Temperalure $350 \cdot \mathrm{K}$
Matrix Beat Copacliy

$1.0000\left(k J / k_{e}\right)$

TRAILING SBOCK CALCULATIONS

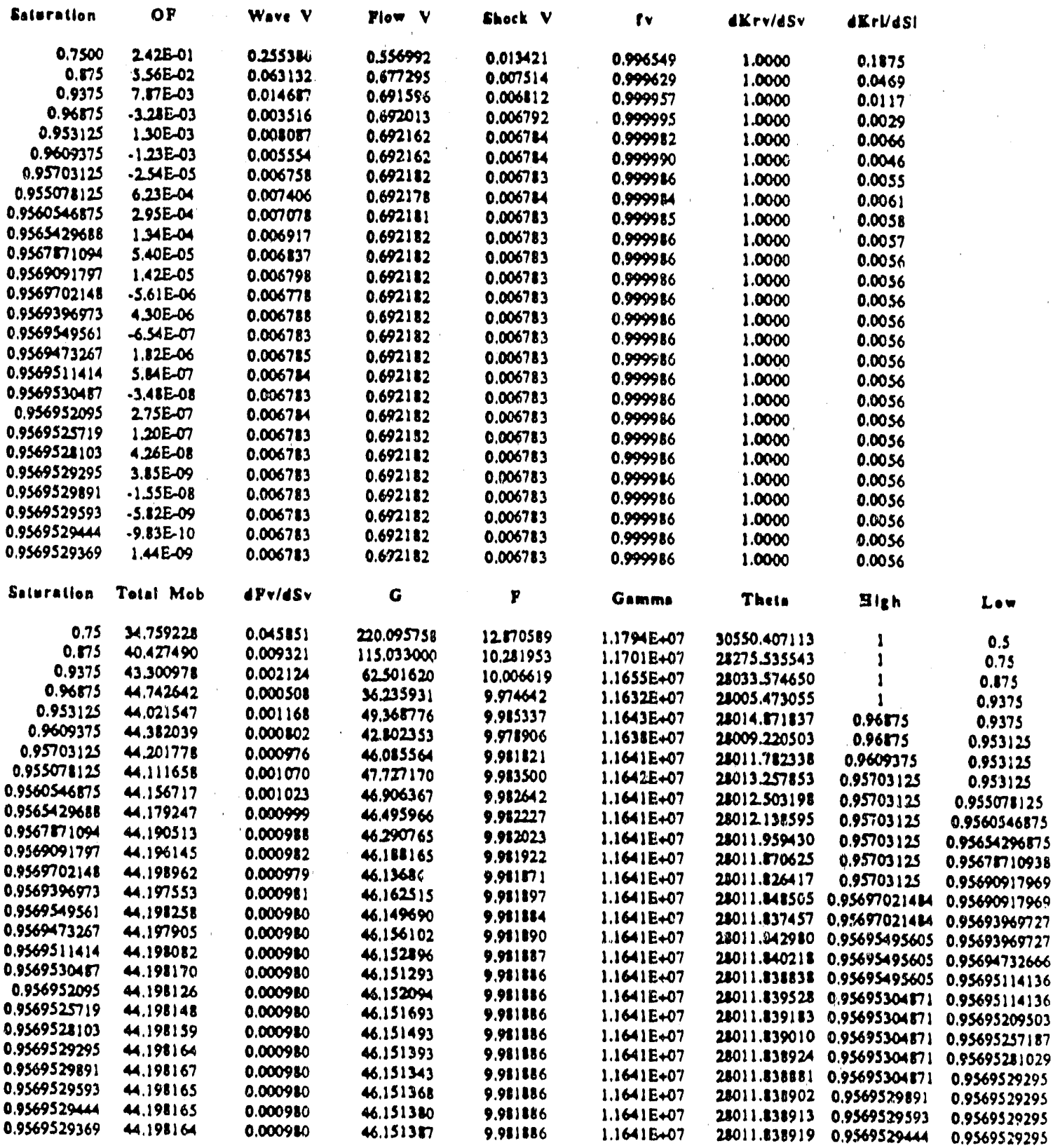


Spreadsheet for Calculation of stream-Water Saturation Profiles

Injectlon Temperature $650 \cdot \mathrm{K}$
Inlilal Temperature

$350 \cdot \boldsymbol{K}$
Matrlx Eeat Cepocliy

$1.0000(k J / k 8)$

LEADING SDOCK CALCULATIONE

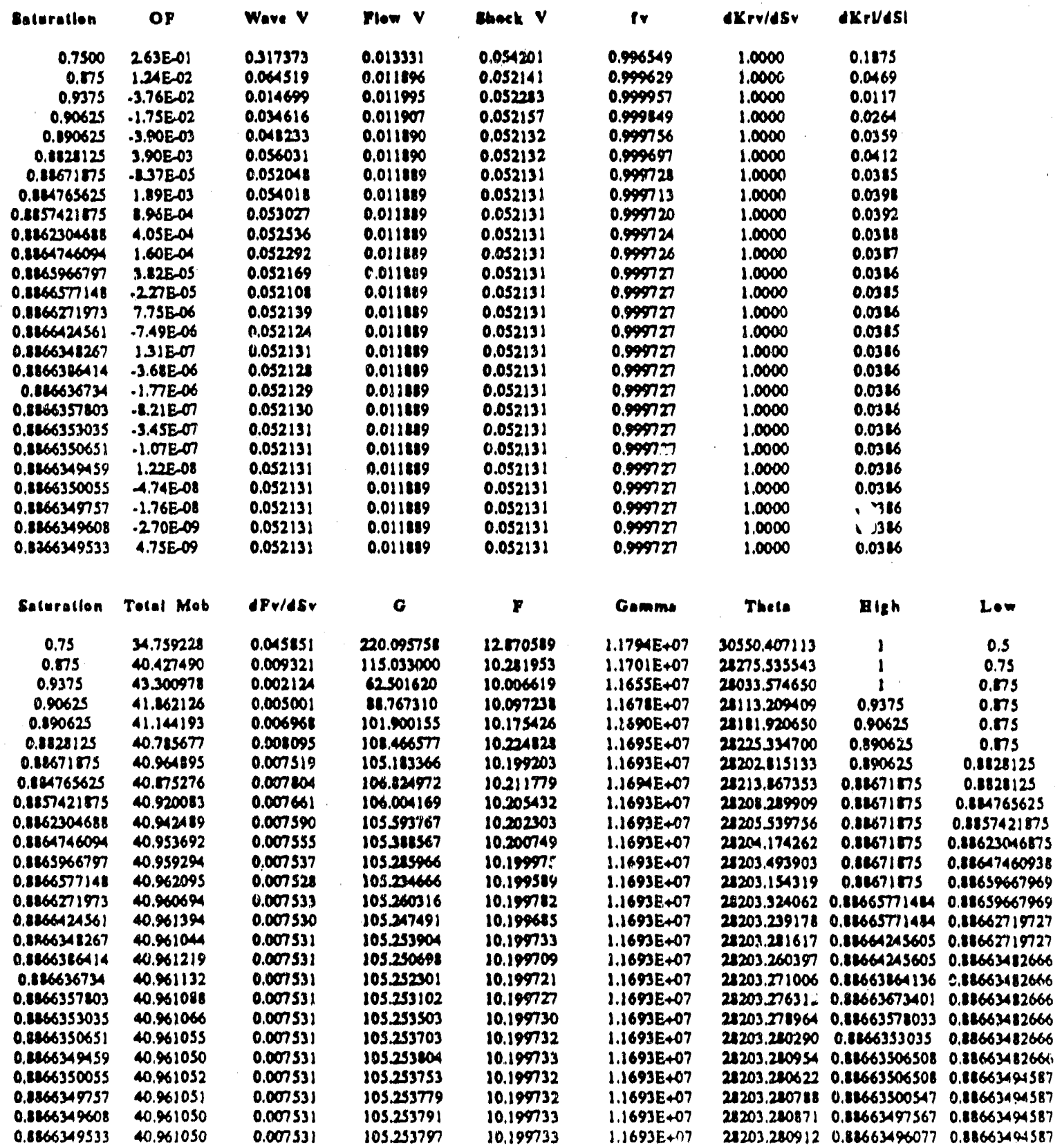


Spreadsheet for Calculation of Stream-Water Saturation Profiles

Injectlen Temperature $650 \cdot \mathrm{K}$
Inlibl Temperalure

$37 \mathbf{S}^{\circ} \mathrm{K}$
Matrlx Beat Cupacliy 1.0000 (kJ/ke)
MATRIX PROPERTIES

Pereslly

0.10

$+\infty$

C) Mard:

1.00

N8

$1 . \infty$

2650.00

$s=1$

tho $\mathrm{CP}_{\mathrm{P}}$

2650.0000

1.000000

SATURATION CONDITIONS

Treicuration - ARs.57

Volumes

(mink)

0.001176

0.100298
Demallies

(achims)

850.4723

9.970241

9.97024

$$
91.41
$$

Vlocoollles
(pp)
0.1303
0.0217 0.0217
Saturation

slep

0.0015779915

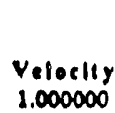

1.000000

0.012106

G

6.18
958.07

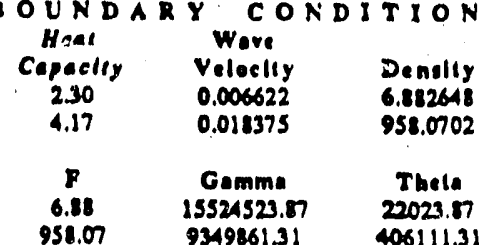

958.07

9349861.31

406111.31

Inthalpy

3199.91

423.88
SAOCK CONDITIONS

$\begin{array}{cc}\text { Vapor } & \text { Objective } \\ \text { saluratlon } & \text { Functlon } \\ 0.9570 & 1.4356 E-09\end{array}$

0.8781

$12943 \mathrm{E}-09$

Two.phase

Ware

Veloclly

0.0068

0.0611

Tetal

Mobllity

4.1982

40.5674

dPv/dSv

0.0010

0.0088 c

46.1514
Upstream

Flow

Velocily

0.692182

0.012806

Shock Vapor

Veloclly Frac Flow

$0.0068 \quad 1.0000$

$\begin{array}{ll}0.0611 & 0.9997\end{array}$

F'

9.9819

10.2587
Camma 1.1641E+07 $1.1699 \mathrm{E}+07$
Thels' 28011.8389

28255.0707

MATERIAL BALANCES

$\begin{array}{cc}\text { Inside } \\ \text { Mass } & 90.420616 \\ \text { Entholpy } & 951816.0963\end{array}$

Inlilal

95.207021

934986.1305

Injected

6.282648
22023.8721

Produced

12.269040
5200.6583

Belence

0.0000

Error

$0.0000 \%$

$0.0007 \%$ 
Injecllon Temperalure $650^{\circ} \mathrm{K}$
Inlikel Temperalure $375 \cdot \mathbf{x}$
Motrix Bent Copacliy

$1.0000(\mathrm{~kJ} / \mathrm{ke})$

SATURATION PROFILE

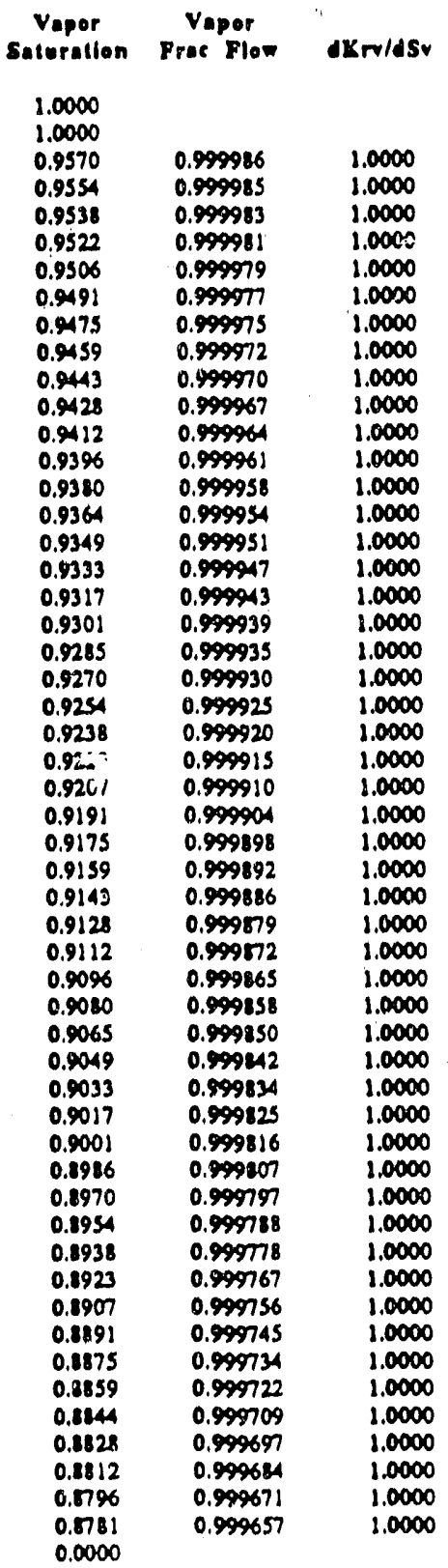

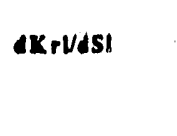

0.0056

0.0060

0.0004

0.0068

0.0073

0.0078

0.0013

0.0018

0.0093

0.0098

0.0104

0.0109

0.0115

0.0121

0.0127

0.0134

0.0140

0.0146

0.0153

0.0160

0.0167

0.0174

0.0181

0.0189

0.0196

0.0204

0.0212

0.0220

0.0228

0.0237

0.0245

0.025

0.0263

0.027

0.0281

0.0290

0.0299

0.0309

0.0318

0.0328

0.0338

0.0348

0.0359

0.0369

0.0310

0.0390

0.0401

0.0412

0.0423

0.0435
Total

mobliliy

44.191164

4.125354

4.052541

43.979747

43.906953

43.834163

43.761320

43.682602

43.615831

43543066

43.470308

43.397557

43.324812

43.252075

43.179344

43.106622

43.033907

42961199

42812500

42815809

42.743127

42670453

42597717

42525131

42452413

42379445

42307217

42234598

42161949

42019390

42016201

41.944222

41.7165

41.799097

41.726551

41.634015

41581491

41.505979

A1.43647

41.363989

4).291512

41.219047

41.146594

41.074154

41.00172

40.929312

40.856911

40.784523

40.712148

40.63975

40.567440 drvidsv

0.000910

0.001055

0.00113

0.001215

0.001300

0.001387

0.001478

0.001572

0.001669

0.001768

0.001871

0.001971

0.002017

0.002200

0.002315

0.002435

0.002557

0.002682

0.002811

0.002944

0.003079

0.003218

0.003361

0.003507

0.003656

0.003809

0.003965

0.00125

0.004288

0.004455

0.004626

0.004100

0.004977

0.005159

0.005344

0.005533

0.005725

0.005922

0.006122

0.006326

0.00653

0.006745

0.006961

0.007181

0.007404

0.007632

0.007863

0.002098

0.008338

0.008582

0.008830

\begin{tabular}{|c|c|c|}
\hline lambda & $\begin{array}{l}\text { Mese } \\
\text { Inside }\end{array}$ & $\begin{array}{l}\text { Enthalpy } \\
\text { Inside }\end{array}$ \\
\hline $\begin{array}{l}0.000000 \\
0.006783\end{array}$ & & \\
\hline 0.006783 & 0.046687 & PUS308.165674 \\
\hline 0.007308 & 0.024465 & 6083.902275 \\
\hline 0.007149 & 0.026151 & 6324.672234 \\
\hline 0.008413 & 0.027198 & 6567.076916 \\
\hline 0.008998 & 0.029708 & 6811.129407 \\
\hline 0.009604 & 0.031510 & 7056.42901 \\
\hline 0.010231 & 0.033516 & 7304.530701 \\
\hline 0.010879 & 0.035515 & 7553.306219 \\
\hline 0.011549 & 0.037579 & 704.032978 \\
\hline 0.012241 & 0.039708 & $\cos 6.574612$ \\
\hline 0.012954 & 0.041903 & 1310.794870 \\
\hline 0.013689 & 0.044164 & 8566.757614 \\
\hline 0.014446 & 0.044492 & 8824,476820 \\
\hline 0.015226 & 0.048818 & 9083.966580 \\
\hline 0.016027 & 0.051352 & 9345.241104 \\
\hline 0.016851 & 0.053815 & 9601.314720 \\
\hline 0.017698 & 0.056488 & 9873.201875 \\
\hline 0.011568 & 0.059162 & 10139.917134 \\
\hline 0.019460 & 0.061906 & 10408.475188 \\
\hline 0.020376 & 0.064722 & 10678.99047 \\
\hline 0.021315 & 0.067611 & 10951.179045 \\
\hline 0.022277 & 0.070573 & 11225.354241 \\
\hline 0.023263 & 0.073609 & 18501.433422 \\
\hline 0.024272 & 0.076719 & 11779.430099 \\
\hline 0.025306 & 0.079905 & 12059.360313 \\
\hline 0.026363 & 0.023167 & 12341.239634 \\
\hline 0.027445 & 0.086506 & 12625.013763 \\
\hline 0.028551 & 0.089922 & 12910.908531 \\
\hline $0.02 \% 82$ & 2.093417 & 13198.729905 \\
\hline 0.030837 & 0.098991 & 13488.563964 \\
\hline 0.032017 & 0.100649 & 13720.427004 \\
\hline 0.033222 & 0.104310 & 14074,335336 \\
\hline 0.034453 & $0.1081 \%$ & 14370.305492 \\
\hline 0.035709 & 0.112094 & 14664.354120 \\
\hline 0.036990 & 0.116076 & 14968.498011 \\
\hline 0.038297 & 0.120142 & 15270.754097 \\
\hline 0.039631 & 0.124292 & 15575.139453 \\
\hline 0.040990 & 0.128528 & 15181.671300 \\
\hline 0.042375 & 0.132550 & 16190.367003 \\
\hline 0.043787 & 0.137260 & 16591.244076 \\
\hline 0.045226 & 0.141758 & 16814.320181 \\
\hline 0.046691 & 0.146343 & 17129.613130 \\
\hline 0.04814 & 0.151022 & 17447.140846 \\
\hline 0.049703 & 0.155790 & 17766.921567 \\
\hline 0.051250 & 0.160650 & 12088.973443 \\
\hline 0.052825 & 0.165602 & IMI3.314940 \\
\hline 0.054427 & 0.170648 & 18739.964643 \\
\hline 0.056057 & 0.175788 & 19068.911294 \\
\hline 0.057716 & 0.181024 & $19400.2637 \%$ \\
\hline 0.059403 & 0.186356 & 19733.951214 \\
\hline 0.0611118 & 0.191716 & 20070.022776 \\
\hline
\end{tabular}


Spreadsheet for Calculation of Stream-Water Saturation Proflles

Injetilon Temperature $630 \bullet$
Inlilal Temperalure $373 \cdot x$
Matrlx Beal Capacliy $1.0000(\mathrm{~kJ} / \mathrm{kg})$

TRAILING SBOCK CALCULATIONS

\begin{tabular}{|c|c|c|c|c|c|c|c|c|}
\hline Soluratlon & OP & Wave $v$ & Plow V & Sheck V & fr & dKrv/dSV & AKrVASI & \\
\hline $\begin{array}{r}0.7500 \\
0.075 \\
0.9375 \\
0.9675 \\
0.953125 \\
0.9509375 \\
0.95703175 \\
0.955074125 \\
0.9560546175 \\
0.9565429618 \\
0.9587571094 \\
0.9569091797 \\
0.9569702141 \\
0.9569396973 \\
0.9569549561 \\
0.9569473267 \\
0.9569511414 \\
0.9569530477 \\
0.956952095 \\
0.9569525719 \\
0.9569521103 \\
0.9569524295 \\
0.9569529191 \\
0.9569529593 \\
0.9569529444 \\
0.9569529369\end{array}$ & 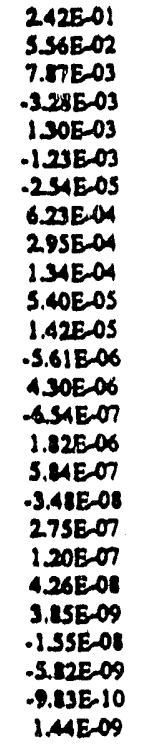 & $\begin{array}{l}0.255316 \\
0.063132 \\
0.014677 \\
0.005516 \\
0.001077 \\
0.005554 \\
0.006758 \\
0.007406 \\
0.007078 \\
0.006917 \\
0.006837 \\
0.006798 \\
0.006778 \\
0.0067188 \\
0.006783 \\
0.006785 \\
0.006744 \\
0.006713 \\
0.006744 \\
0.006783 \\
0.006713 \\
0.006783 \\
0.006783 \\
0.006713 \\
0.006713 \\
0.006783\end{array}$ & $\begin{array}{l}0.559912 \\
0.677295 \\
0.691596 \\
0.692013 \\
0.692162 \\
0.492162 \\
0.692182 \\
0.692178 \\
0.692181 \\
0.692182 \\
0.692182 \\
0.692182 \\
0.692112 \\
0.692182 \\
0.692182 \\
0.692182 \\
0.692112 \\
0.692112 \\
0.692182 \\
0.692182 \\
0.692182 \\
0.692112 \\
0.692182 \\
0.692112 \\
0.692182 \\
0.692182\end{array}$ & $\begin{array}{l}0.01421 \\
0.007514 \\
0.006812 \\
0.006792 \\
0.006784 \\
0.006784 \\
0.006783 \\
0.006744 \\
0.006783 \\
0.006783 \\
0.006783 \\
0.0067133 \\
0.0067113 \\
0.006713 \\
0.006713 \\
0.006783 \\
0.006783 \\
0.006783 \\
0.0067113 \\
0.006713 \\
0.0017713 \\
0.001783 \\
0.0015783 \\
0.001783 \\
0.006783 \\
0.001713\end{array}$ & 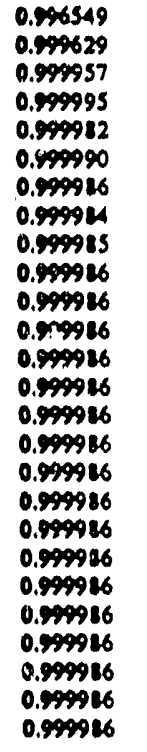 & $\begin{array}{l}1.0000 \\
1.0000 \\
1.0000 \\
1.0000 \\
1.0000 \\
1.0000 \\
1.0000 \\
1.0000 \\
1.0000 \\
1.0000 \\
1.0000 \\
1.0000 \\
1.0000 \\
1.0000 \\
1.0000 \\
1.0000 \\
1.0000 \\
1.0000 \\
1.0000 \\
1.0000 \\
1.0000 \\
1.0000 \\
1.0000 \\
1.0000 \\
1.0000 \\
1.0000\end{array}$ & $\begin{array}{l}0.1875 \\
0.0469 \\
0.0117 \\
0.0029 \\
0.0066 \\
0.0046 \\
0.0055 \\
0.0061 \\
0.0058 \\
0.0057 \\
0.0056 \\
0.0056 \\
0.0056 \\
0.0056 \\
0.0056 \\
0.0056 \\
0.0056 \\
0.0056 \\
0.0056 \\
0.0056 \\
0.0056 \\
0.0056 \\
0.0056 \\
0.0056 \\
0.0056 \\
0.0056\end{array}$ & \\
\hline Soteration & Tolal Mob & dFrldsr & 6 & It & Gemerar: & Thete & Aleh & Leळ \\
\hline $\begin{array}{r}0.75 \\
0.175 \\
0.9375 \\
0.9675 \\
0.953125 \\
0.9609375 \\
0.95703125 \\
0.955071125 \\
0.9560546775 \\
0.9565429688 \\
0.9567071094 \\
0.9569091797 \\
0.9569702148 \\
0.956939973 \\
0.9569549561 \\
0.956943267 \\
0.95695114114 \\
0.9569530477 \\
0.956952095 \\
0.9569525719 \\
0.956952103 \\
0.9569529295 \\
0.9569529191 \\
0.9569529593 \\
0.9569529444 \\
0.9569529369\end{array}$ & 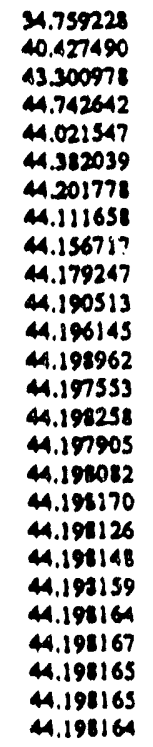 & $\begin{array}{l}0.045851 \\
0.009321 \\
0.002124 \\
0.000501 \\
0.001168 \\
0.000202 \\
0.000916 \\
0.001010 \\
0.001023 \\
0.000999 \\
0.000981 \\
0.000982 \\
0.000979 \\
0.000981 \\
0.000990 \\
0.000910 \\
0.000920 \\
0.000920 \\
0.000920 \\
0.000980 \\
0.000920 \\
0.000910 \\
0.000910 \\
0.000910 \\
0.000920 \\
0.000980\end{array}$ & $\begin{array}{l}220.095758 \\
115.033000 \\
62501620 \\
36.295931 \\
49.367776 \\
42.02353 \\
46.015564 \\
47.727170 \\
46.906367 \\
46.495966 \\
46.290765 \\
46.1416165 \\
46.136464 \\
46.162515 \\
46.149690 \\
46.156102 \\
46.152196 \\
46.151293 \\
46.152094 \\
46.151693 \\
46.151493 \\
46.151393 \\
46.151343 \\
46.151368 \\
46.151310 \\
46.151387\end{array}$ & 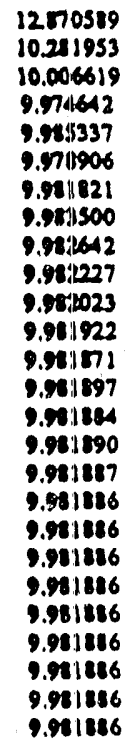 & $\begin{array}{l}1.1794 E+07 \\
1.1701 E+07 \\
1.1655 E+07 \\
1.1632 E+07 \\
1.1643 E+07 \\
1.1631 E+07 \\
1.1641 E+07 \\
1.1442 E+07 \\
1.1641 E+07 \\
1.164 \mid E+07 \\
1.141 E+07 \\
1.1641 E+07 \\
1.1641 E+07 \\
1.1641 E+07 \\
1.1441 E+07 \\
1.141 E+07 \\
1.141 E+07 \\
1.1641 E+07 \\
1.141 E+07 \\
1.1641 E+07 \\
1.1641 E+07 \\
1.1641 E+07 \\
1.1641 E+07 \\
1.141 E+07 \\
1.164 \mid E+07 \\
1.141 E+07\end{array}$ & $\begin{array}{l}30550.407113 \\
22275.535543 \\
22033.574650 \\
22005.473055 \\
22014.571837 \\
22009.220503 \\
22011.782338 \\
22013.257853 \\
22012503198 \\
22012138595 \\
22011.959430 \\
22011.570625 \\
22011.226117 \\
22011.848505 \\
22011.237457 \\
22011.242920 \\
22011.240218 \\
22011.031831 \\
22011.039528 \\
22011.839183 \\
22011.839010 \\
22011.838924 \\
22011.838881 \\
22011.838902 \\
22011.838913 \\
22011.038919\end{array}$ & $\begin{array}{c}1 \\
1 \\
1 \\
1 \\
0.96775 \\
0.96575 \\
0.9609375 \\
0.95703125 \\
0.95703125 \\
0.95703125 \\
0.95703125 \\
0.95703125 \\
0.95703125 \\
0.95697021444 \\
0.95697021414 \\
0.95695495605 \\
0.95695495605 \\
0.95695495605 \\
0.95695304771 \\
0.95693304771 \\
0.95695304871 \\
0.95695304871 \\
0.95695304871 \\
0.9569529891 \\
0.9569529593 \\
0.9569529444\end{array}$ & $\begin{array}{c}0.5 \\
0.75 \\
0.775 \\
0.9375 \\
0.9375 \\
0.953129 \\
0.953125 \\
0.953125 \\
0.955078125 \\
0.9560546875 \\
0.95654296875 \\
0.95675710938 \\
0.95690917969 \\
0.95690917969 \\
0.95693969727 \\
0.95693969727 \\
0.95694732666 \\
0.95695114136 \\
0.95695114136 \\
0.95695209503 \\
0.95695257187 \\
0.95695281029 \\
0.9569529295 \\
0.9569529295 \\
0.9569529295 \\
0.9569529295\end{array}$ \\
\hline
\end{tabular}


Spreadsheet for Calculation of Stream-Water Saturation Profiles

Injecllon Temperature $650 \cdot \mathbf{K}$
Inlilal Temperalure

$375^{\circ} \mathrm{K}$
Matrlx Beat Capacliy

1.0000 (kJ/ke:

LEADING SBOCK CALCULATIONS

\begin{tabular}{|c|c|c|c|c|c|c|c|c|}
\hline Saturallen & OF & Weve $V$ & Plow V & Shock V & ev & dKrv/dSV & AKVASI & \\
\hline 0.9373 & $4.66 \mathrm{E}-02$ & 0.014699 & $\begin{array}{l}0.0120101 \\
0.012963\end{array}$ & 0.0611119 & $\begin{array}{l}0.999629 \\
0.999957\end{array}$ & 1.0000 & 0.0117 & \\
\hline $\begin{array}{r}0.90625 \\
0.090625\end{array}$ & $-266 E-02$ & 0.034616 & 0.012846 & 0.061174 & 0.999249 & 1.0000 & 0.0264 & \\
\hline $\begin{array}{r}0.190625 \\
0.1828125\end{array}$ & $.129 \mathrm{E}-02$ & 0.041233 & 0.012814 & 0.061130 & 0.999756 & 1.0000 & 0.0359 & \\
\hline $\begin{array}{r}0.1828125 \\
0.87890625\end{array}$ & $.5 .095-03$ & 0.050031 & 0.012207 & 0.061120 & 0.999697 & 1.0000 & 0.0412 & \\
\hline $\begin{array}{r}0.87890625 \\
0.176953125\end{array}$ & $.9 .31 \mathrm{E}-0 \mathrm{~d}$ & 0.060187 & 0.012206 & 0.061111 & 0.999664 & 1.0000 & 0.0440 & \\
\hline $\begin{array}{r}0.176953125 \\
0.1779296875\end{array}$ & $121 \mathrm{E}-03$ & 0.062331 & 0.012206 & 0.061118 & 0.999647 & 1.0000 & 0.0454 & \\
\hline $\begin{array}{l}0.577929675 \\
0.574179688\end{array}$ & $1,36 \mathrm{E}-04$ & 0.061254 & 0.012206 & 0.061118 & 0.999536 & 1.0000 & 0.0447 & \\
\hline 0.874417968 & $.3 .99 E-04$ & 0.060719 & 0.012806 & 0.061118 & 0.999660 & 1.0000 & 0.0443 & \\
\hline 0.6781738281 & $.132 E-O M$ & 0.060966 & 0.012206 & 0.061118 & $0.99 \times 558$ & 1.0000 & 0.0415 & \\
\hline 0.8720517578 & $1.76 \mathrm{E}-06$ & 0.081120 & 0.012805 & 0.061118 & 0.999657 & 1.0000 & 0.0446 & \\
\hline 0.871112793 & GSIE-OS & C.061053 & 0.012806 & 0.0611118 & 0.999857 & 1.0000 & 0.0446 & \\
\hline 0.6720122754 & $.3 .17 \mathrm{E}-05$ & 0.061086 & 0.012206 & 0.061118 & 0.999657 & 1.0000 & 0.0446 & \\
\hline 0.1710670166 & $-1.50 \mathrm{E}-05$ & 0.061103 & 0.012106 & 0.061118 & 0.999657 & 1.0000 & 0.0446 & \\
\hline 0.8710593872 & $6.61 \mathrm{E}-06$ & 0.061112 & 0.012806 & 0.0611118 & 0.999657 & 1.0000 & 0.0446 & \\
\hline $0.8720555 i 25$ & $.242 E-06$ & 0.061116 & 0.012806 & 0.0611118 & 0.099657 & 1.0000 & 0.0446 & \\
\hline 0.7720536652 & $.3 .34 \mathrm{E}-07$ & 0.061118 & 0.012806 & 0.061116 & 0.999657 & 1.0000 & 0.0446 & \\
\hline $0.07 \cos 27115$ & $7.12 \mathrm{E}-07$ & 0.061119 & 0.012806 & 0.0611118 & 0.999657 & 1.0000 & 0.0416 & \\
\hline $0.87 \cos 31883$ & $2.09 E-07$ & 0.061118 & 0.012208 & 0.051118 & 0.999657 & 1.0000 & 0.0446 & \\
\hline $0.87 \cos 34267$ & $.1202 \mathrm{E}-08$ & 0.061118 & 0.012206 & 0.061111 & 0.999657 & 1.0000 & 0.0446 & \\
\hline 0.8720533075 & S.8SE-OB & 0.0611118 & 0.012806 & 0.0611118 & 0.999657 & 1.0000 & 0.0446 & \\
\hline 0.8720533671 & $6.77 \mathrm{E}-09$ & 0.061118 & 0.012806 & 0.0611118 & 0.999657 & 1.0000 & 0.0446 & \\
\hline 0.8720533373 & 258E-08 & 0.061118 & 0.012806 & 0.061118 & 0.999657 & 1.0000 & 0.0446 & \\
\hline 0.8720533522 & $9,46 \mathrm{E}-09$ & 0.0611118 & 0.012806 & 0.061118 & 0.999657 & 1.0000 & 0.0416 & \\
\hline $0.8 / 70533597$ & $129 \mathrm{E}-09$ & 0.061118 & 0.012206 & 0.061118 & 0.999657 & 1.0000 & 0.0446 & \\
\hline Seturation & Total Mob & $d F v / d S v$ & C & $\mathbf{P}$ & Gamma & Thele & algh & $L \circ w$ \\
\hline 0.75 & 34.759228 & 0.045851 & 220.095758 & 12870589 & 1.179EE +07 & 30550.407113 & 1 & 0.5 \\
\hline 0.075 & 40.427490 & 0.009321 & 115.033000 & 10.281953 & $1.1701 \mathrm{E}+07$ & 28275.535543 & 1 & 0.75 \\
\hline 0.9375 & 13.300971 & 0.002124 & 62.501620 & 10.006819 & $1.1655 E+07$ & 28033.574650 & 1 & 0.175 \\
\hline $\begin{array}{l}0.90625 \\
0.890625\end{array}$ & $\begin{array}{l}41.262126 \\
41.144193\end{array}$ & $\begin{array}{l}0.005001 \\
0.006968\end{array}$ & $\begin{array}{r}88.767310 \\
101.900155\end{array}$ & $\begin{array}{l}10.097238 \\
10.173426\end{array}$ & $\begin{array}{l}1.1671 E+07 \\
1.1690 E+07\end{array}$ & $\begin{array}{l}22113.200409 \\
28181.920650\end{array}$ & $\begin{array}{l}0.9375 \\
0.90625\end{array}$ & $\begin{array}{l}0.873 \\
0.875\end{array}$ \\
\hline 0.8528125 & 40.715677 & 0.001095 & 108.466577 & 10.224128 & $1.1695 E+07$ & 22225.334700 & 0.090625 & 0.875 \\
\hline 0.87290625 & 40.606541 & 0.008695 & 211.749788 & 10.25234 & $1,1698 E+07$ & 28249.550656 & 0.21428125 & 0.075 \\
\hline 0.876953123 & 40.517005 & 0.009005 & 113.391394 & 10.2669112 & $1.1700 E+07$ & 22262317400 & 0.07890625 & 0.075 \\
\hline 0.8779296775 & 40.561770 & 0.001249 & 812570591 & 10.25958 & $1.1699 E+07$ & 25255.718179 & 0.07190625 & 0.076953125 \\
\hline $0.87417 \% 88$ & 40.564155 & 0.008772 & 112160190 & 10.255968 & $1.1699 E+07$ & 28252700527 & 0.07890825 & 0.8779296875 \\
\hline 0.8781738281 & 40.572963 & 0.008811 & 112365391 & 10.257772 & $1.1699 E+07$ & 25254.2458720 & 0.5741796875 & 0.57792 .96875 \\
\hline 0.8720517578 & 40.567367 & 0.008830 & 112.467991 & $\quad 10.258677$ & $1.1699 \mathrm{E}+07$ & 28235.0811440 & 0.87817382813 & 0.5779296875 \\
\hline 0.578112793 & 40.570165 & 0.008820 & 112116691 & 10.251225 & $1.1699 E+07$ & 28254.683295 & 0.07817382813 & 0.07805175781 \\
\hline 0.6720822754 & 40.568766 & 0.008825 & 112442341 & 10.25451 & $1.1699 E+07$ & 28254.1121700 & $\begin{array}{l}0.87811279297 \\
0.5750 .227539\end{array}$ & $\begin{array}{l}0.87205175781 \\
0.5705175781\end{array}$ \\
\hline 0.8780670166 & 40.561066 & 0.008828 & 112455166 & $10.25 \pm 564$ & $1.1699 E+07$ & 28254.981648 & $\begin{array}{l}0.87801227539 \\
0.5710670166\end{array}$ & $\begin{array}{l}0.87105173781 \\
0.87205175781\end{array}$ \\
\hline $\begin{array}{l}0.8720593872 \\
0.8720555725\end{array}$ & $\begin{array}{l}40.567716 \\
40.567541\end{array}$ & $\begin{array}{l}0.008829 \\
0.008829\end{array}$ & $\begin{array}{l}112461578 \\
112464785\end{array}$ & & & $\begin{array}{l}28255.031398 \\
28255.056275\end{array}$ & $\begin{array}{l}0.8720570166 \\
0.87805938721\end{array}$ & $\begin{array}{l}0.07205175781 \\
0.07805175781\end{array}$ \\
\hline $\begin{array}{l}0.8720555725 \\
0.87 \cos 36652\end{array}$ & $\begin{array}{l}40.567541 \\
40567454\end{array}$ & $\begin{array}{l}0.008829 \\
0.008130\end{array}$ & $\begin{array}{l}112464785 \\
112466318\end{array}$ & $\begin{array}{l}10.251649 \\
10.254663\end{array}$ & $\begin{array}{l}1.1699 \mathrm{E}+07 \\
1.1699 \mathrm{E}+07\end{array}$ & 28255.068714 & 0.57805557251 & 0.57805175781 \\
\hline $\begin{array}{l}0.8720536652 \\
0.2720527115\end{array}$ & $40.567+10$ & 0.008130 & 112467189 & 10.258570 & $1.1699 \mathrm{E}+07$ & 21255.074934 & 0.07805366516 & 0.87805175781 \\
\hline 0.8780531883 & 40.567432 & 0.008830 & 112466789 & 10.251667 & 1.16995 .07 & 28255.071824 & 0.57805366516 & $0.87 \cos 271149$ \\
\hline 0.8720534267 & 40.567443 & 0.008830 & 112466588 & 10.252665 & $1.1699 \mathrm{E}+07$ & 28255.070269 & 0.87805366516 & $0.87 \cos 316832$ \\
\hline 0.8720533075 & 40.567438 & 0.008830 & 112466688 & 10.251666 & $1.1699 \mathrm{E}+07$ & 22255.071047 & 0.57805342674 & 0.87805311832 \\
\hline 0.5710533671 & $\$ 0.567440$ & 0.008830 & 112466638 & 10.258665 & $1.1699 \mathrm{E}+07$ & 28255.070658 & 0.87805342674 & $0.0720533 \mathrm{ms3}$ \\
\hline $0.177 \cos 33373$ & 40.567439 & 0.008830 & 112466663 & 10.258666 & $1.1699 \mathrm{E}+07$ & 28255.070852 & 0.87805336714 & $\begin{array}{l}0.87805330753 \\
0.7805333734\end{array}$ \\
\hline $0.77 \cos 33522$ & 40.567440 & 0.008830 & 112466651 & 10.2516666 & $1.1699 \mathrm{E}+07$ & 28255.070753 & 0.07805336714 & 0.87805333734 \\
\hline 0.8720533597 & 40.567440 & 0.008830 & 112.46664 .5 & 10.258666 & $1.1699 E+07$ & 28235.070707 & 0.67805336714 & 0.67805335224 \\
\hline
\end{tabular}


Spreadsheet for Calculation of Stream-Water Saturation Profiles

Injection semperature cso $\mathrm{K}$
Inllal Temperature

$400 \cdot \mathrm{K}$
Matrix Beal Capacity

1.0000 (WJ/kg)

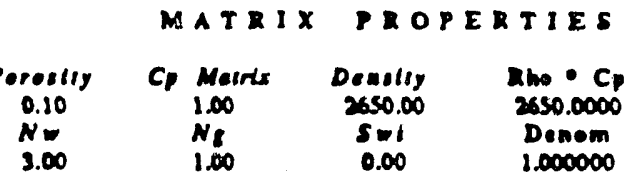

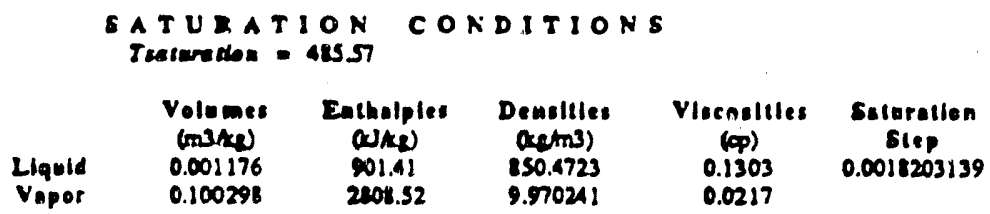

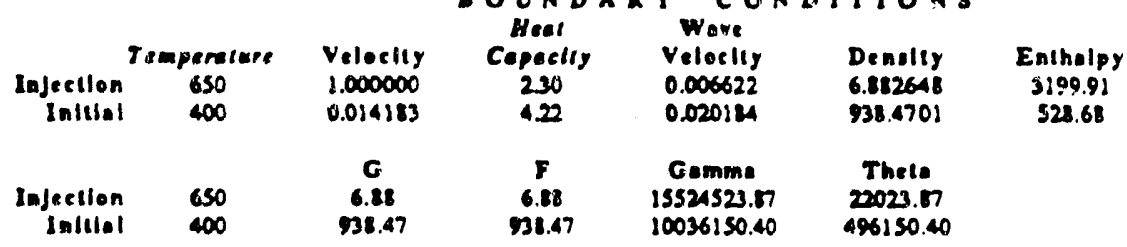

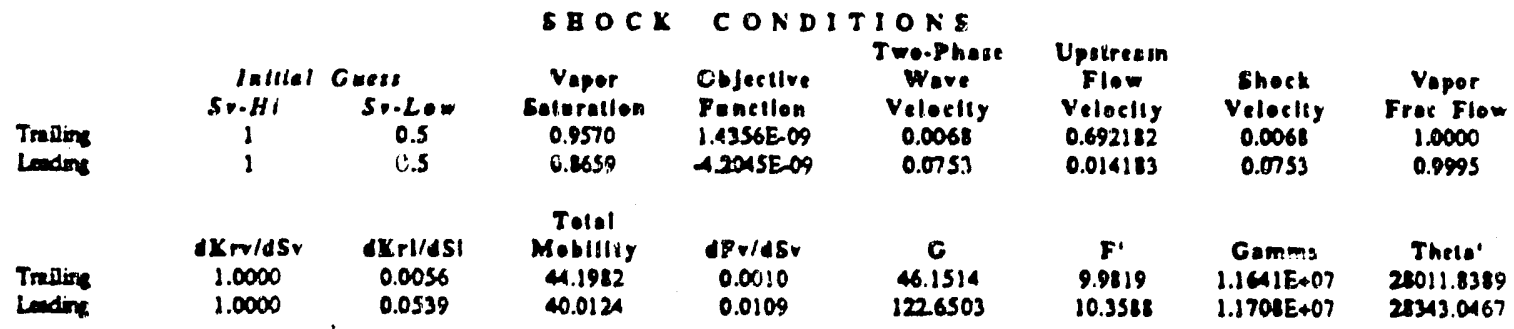

MATERIAL BALANCES

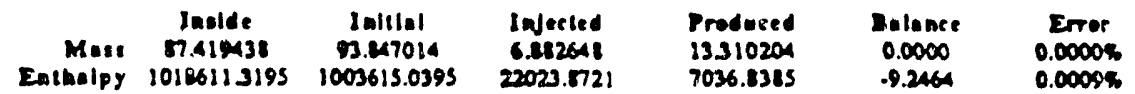


Spreadsheet for Calculation of Stream-Water Saturation Profiles

Injection Temperalure $650 \bullet \mathcal{X}$
Inlilel Temperature $400 \cdot \mathrm{K}$
Meirlx Beal Copediy 1.0000 (t]/ke)

SATURATION PROPILE

\begin{tabular}{|c|c|c|c|c|c|c|c|c|}
\hline $\begin{array}{c}\text { Yeper } \\
\text { ceteration }\end{array}$ & $\begin{array}{l}\text { Veper } \\
\text { Prec Fitor }\end{array}$ & dKrw/GSv & AErVASI & $\begin{array}{c}\text { Tolal } \\
\text { Mobliliy }\end{array}$ & dPv/dSV & lombde & $\begin{array}{l}\text { is zas } \\
\text { Inalde }\end{array}$ & $\begin{array}{l}\text { Entholpy } \\
\text { Inolde }\end{array}$ \\
\hline $\begin{array}{l}1.0000 \\
1.0000\end{array}$ & & & & & & $\begin{array}{l}0.000000 \\
0.006783\end{array}$ & & \\
\hline $\begin{array}{l}0.9570 \\
0.9551\end{array}$ & $\begin{array}{l}0.999946 \\
0.999944\end{array}$ & $\begin{array}{l}1.0000 \\
1.0000\end{array}$ & $\begin{array}{l}0.0056 \\
0.0060\end{array}$ & $\begin{array}{l}4.198164 \\
4.114173\end{array}$ & $\begin{array}{l}0.0009120 \\
0.001067\end{array}$ & $\begin{array}{l}0.006783 \\
0.007318\end{array}$ & $\begin{array}{l}0.046687 \\
0.028369\end{array}$ & $\begin{array}{c}105308.165574 \\
7039.452627\end{array}$ \\
\hline $\begin{array}{l}0.9533 \\
0.9515\end{array}$ & $\begin{array}{l}0.999982 \\
0.999910\end{array}$ & $\begin{array}{l}1.0000 \\
1.0000\end{array}$ & $\begin{array}{l}0.0065 \\
0.0071\end{array}$ & $\begin{array}{l}44.030188 \\
43.946211\end{array}$ & $\begin{array}{l}0.001139 \\
0.001254\end{array}$ & $\begin{array}{l}0.001020 \\
0.008680\end{array}$ & $\begin{array}{l}0.030625 \\
0.032976\end{array}$ & $\begin{array}{l}7360.180838 \\
7683.423065\end{array}$ \\
\hline $\begin{array}{l}0.497 \\
0.9479\end{array}$ & $\begin{array}{l}0.999978 \\
0.999975\end{array}$ & $\begin{array}{l}1.0000 \\
1.0000\end{array}$ & $\begin{array}{l}0.0076 \\
0.0082\end{array}$ & $\begin{array}{l}43.262241 \\
43.773278\end{array}$ & $\begin{array}{l}0.001353 \\
0.001457\end{array}$ & $\begin{array}{l}0.009368 \\
0.010044\end{array}$ & $\begin{array}{l}0.035122 \\
0.037965\end{array}$ & $\begin{array}{l}0009.202536 \\
8337.512705\end{array}$ \\
\hline $\begin{array}{l}0.9463 \\
0.542\end{array}$ & $\begin{array}{l}0.99972 \\
0.999969\end{array}$ & $\begin{array}{l}1.0000 \\
1.0000\end{array}$ & $\begin{array}{l}0.0017 \\
0.0093\end{array}$ & $\begin{array}{l}43.491324 \\
43.610378\end{array}$ & $\begin{array}{l}0.001564 \\
0.001676\end{array}$ & $\begin{array}{l}0.010828 \\
0.011600\end{array}$ & $\begin{array}{l}0.040608 \\
0.043345\end{array}$ & $\begin{array}{l}2661.467246 \\
9002000061\end{array}$ \\
\hline $\begin{array}{l}0.9429 \\
0.9406\end{array}$ & $\begin{array}{l}0.999 \times 66 \\
0.999963\end{array}$ & $\begin{array}{l}1.0000 \\
1.0000\end{array}$ & $\begin{array}{l}0.0100 \\
0.0106\end{array}$ & $\begin{array}{l}43.526440 \\
43.442511\end{array}$ & $\begin{array}{l}0.001792 \\
0.001912\end{array}$ & $\begin{array}{l}0.012402 \\
0.013232\end{array}$ & $\begin{array}{l}0.046185 \\
0.049126\end{array}$ & $\begin{array}{l}9338.165279 \\
9576.917258\end{array}$ \\
\hline $\begin{array}{l}0.9377 \\
0.9369\end{array}$ & $\begin{array}{l}0.999959 \\
0.999955\end{array}$ & $\begin{array}{l}1.0000 \\
1.0000\end{array}$ & $\begin{array}{l}0.0113 \\
0.0119\end{array}$ & $\begin{array}{l}43.352591 \\
43274681\end{array}$ & $\begin{array}{l}0.002036 \\
0.002164\end{array}$ & $\begin{array}{l}0.014092 \\
0.014981\end{array}$ & $\begin{array}{l}0.052168 \\
0.055315\end{array}$ & $\begin{array}{l}10018.49593 \\
10362700110\end{array}$ \\
\hline 0.9351 & 0.999951 & 1.0000 & 0.0126 & 43.190720 & 0.002297 & 0.015900 & 0.052566 & 10709.640876 \\
\hline 0.9333 & 0.999947 & 1.0000 & 0.0134 & 43.106889 & 0.002434 & 0.016448 & 0.061922 & 11059338195 \\
\hline $\begin{array}{l}0.9315 \\
0.9296\end{array}$ & $\begin{array}{l}0.991943 \\
0.994938\end{array}$ & $\begin{array}{l}1.0000 \\
1.0000\end{array}$ & $\begin{array}{l}0.0141 \\
0.0148\end{array}$ & $\begin{array}{l}43.023008 \\
42939138\end{array}$ & $\begin{array}{l}0.002575 \\
0.002721\end{array}$ & $\begin{array}{l}0.017827 \\
0.018836\end{array}$ & $\begin{array}{l}0.065316 \\
0.061957\end{array}$ & $\begin{array}{l}11411.817619 \\
11767.104940\end{array}$ \\
\hline 0.9278 & 0.999933 & 1.0000 & 0.0156 & 42135278 & 0.002871 & 0.019176 & 0.072638 & 12125226201 \\
\hline 0.9260 & 0.999827 & 1.0000 & 0.0164 & 42711429 & 0.003026 & 0.020946 & 0.076430 & 12446.207696 \\
\hline 0.9242 & 0.999922 & 1.0000 & 0.0172 & 42647592 & 0.003185 & 0.022048 & 0.020333 & 12450.075972 \\
\hline 0.9224 & 0.999916 & 1.0000 & 0.0181 & 42603766 & 0.003349 & 0.023181 & 0.044350 & 13216.857132 \\
\hline 0.9205 & 0.999909 & 1.0000 & 0.0189 & 12519952 & 0.003517 & 0.024345 & oosersi & 13566.510336 \\
\hline 0.9117 & 0.999903 & 1.0000 & 0.0198 & 42436151 & 0.003690 & 0.025541 & 0.092727 & 13959270809 \\
\hline 0.9169 & 0.999196 & 1.0000 & 0.0207 & 42352361 & 0.003867 & 0.026770 & 0.097091 & 14334.9541838 \\
\hline 0.9151 & 0.999819 & 1.0000 & 0.0216 & 42261585 & 0.004050 & 0.022030 & 0.101573 & 14713.666278 \\
\hline 0.9133 & & $\begin{array}{l}1.0000 \\
1.0000\end{array}$ & 0.0226 & 4214421 & 0.004236 & $\begin{array}{l}0.029323 \\
0.030649\end{array}$ & 0.106175 & \\
\hline 0.9114 & $\begin{array}{l}0.999573 \\
0.099165\end{array}$ & $\begin{array}{l}1.0000 \\
1.0000\end{array}$ & $\begin{array}{l}0.0235 \\
0.0245\end{array}$ & 42101071 & $\begin{array}{l}0.00428 \\
0.004624\end{array}$ & $\begin{array}{l}0.030649 \\
0.032008\end{array}$ & 0.110497 & $\begin{array}{l}15400.261165 \\
15868.217682\end{array}$ \\
\hline $\begin{array}{l}0.9096 \\
0.9078\end{array}$ & & $\begin{array}{l}1.0000 \\
1.0000\end{array}$ & $\begin{array}{l}0.0245 \\
0.0255\end{array}$ & $\begin{array}{l}42017334 \\
41.833610\end{array}$ & $\begin{array}{l}0.004624 \\
0.004225\end{array}$ & $\begin{array}{l}0.032008 \\
0.033401\end{array}$ & $\begin{array}{l}0.115743 \\
0.120112\end{array}$ & \\
\hline 0.9060 & $0.999 e^{2}$ & 1.0000 & 0.0265 & 41.49901 & 0.005031 & 0.034827 & 0.125806 & 16653.556633 \\
\hline 0.9042 & 0.999638 & 1.0000 & 0.0276 & 41.766207 & 0.005242 & 0.03627 & 0.131027 & 17053.001820 \\
\hline 0.9023 & 0.999828 & 1.0000 & 0.0286 & $41.63252 \pi$ & 0.005458 & 0.037781 & 0.136375 & 17451.685127 \\
\hline 0.8005 & 0.990818 & 1.0000 & 0.0297 & 41598261 & 0.005679 & 0.039309 & 0.141854 & 17855.617654 \\
\hline 0.0917 & 0.999208 & 1.0000 & 0.0308 & 41315211 & 0.005905 & 0.040872 & 0.147463 & 18262136792 \\
\hline 0.8969 & 0.99979 & 1.0000 & 0.0319 & 41.431577 & 0.006136 & 0.042470 & 0.133206 & 18673.373231 \\
\hline 0.8951 & 0.999785 & 1.0000 & 0.0330 & 41347958 & 0.006372 & 0.044103 & 0.159082 & 19087.257961 \\
\hline 0.5932 & 0.999774 & 1.0000 & 0.0422 & 41.264355 & 0.006613 & 0.045772 & 0.165093 & 19504322776 \\
\hline 0.0914 & 0.999761 & 1.0000 & 0.0354 & 41.120769 & 0.006159 & 0.047476 & 0.171242 & 19925.19776 \\
\hline 0.2896 & 0.999749 & 1.0000 & 0.0366 & 11.097199 & 0.007110 & 0.049217 & 0.177530 & 20349.316372 \\
\hline 0.2878 & $0 . \operatorname{sen} 35$ & 10000 & 0.0378 & 41.013646 & 0.007367 & 0.050994 & 0.113957 & 20776.910290 \\
\hline 0.2860 & 0.999722 & 1.0000 & 0.0390 & $\$ 0.930110$ & 0.007629 & 0.052209 & 0.190527 & 21208.012071 \\
\hline oser) & 0.99908 & 1.0000 & 0.0403 & 40.46591 & 0.007196 & 0.054658 & 0.197240 & 21642654577 \\
\hline 0.2523 & 0.999693 & 1.0000 & 0.0415 & 40.763090 & 0.008169 & 0.056546 & 0.204099 & 22020.870995 \\
\hline 0.2805 & 0.999678 & 1.0000 & 0.0428 & $40.67 \% 607$ & 0.004447 & 0.058471 & 0.211104 & 22522694138 \\
\hline 0.578 & 0.990682 & 1.0000 & 0.0412 & 0.596143 & 0.008731 & 0.060434 & 0.218258 & 20968.159952 \\
\hline 0.0769 & 0.999646 & 1.0000 & 0.0455 & $\$ 0.512696$ & 0.009020 & 0.052435 & 0.225562 & 2417.300515 \\
\hline 0.750 & 0.999629 & 1.0000 & 0.0468 & 40.429269 & 0.009315 & 0.064475 & 0.233018 & 23570.151046 \\
\hline 0.7732 & 0.999612 & 1.0000 & 0.0462 & 40345861 & $0.00 \% 615$ & 0.066554 & 0.210627 & 24326.746404 \\
\hline 0xI14 & 0.949594 & 1.0000 & 0.0496 & 10262472 & 0.009921 & 0.062672 & 0.248392 & 24787.121795 \\
\hline 0.2656 & 0.999576 & 1.0000 & 0.0510 & $\$ 0.179102$ & 0.010233 & 0.070829 & 0.256315 & $25251.312 \mathrm{ma}$ \\
\hline 0.8678 & 0.999557 & 1.0000 & 0.0525 & 40.095753 & 0.010550 & 0.073025 & 0.264396 & 25719.353248 \\
\hline 0.5659 & 0.999538 & 1.0000 & 0.0539 & 40.012424 & 0.010873 & 0.075262 & 0.272638 & 26191.28544 \\
\hline $\begin{array}{l}0.0000 \\
0.0000\end{array}$ & & & & & & 0.075262 & & \\
\hline
\end{tabular}


Injection Temperature $650 \cdot \mathrm{K}$
Inlibal Temperalure

$400 \cdot \mathrm{K}$
Malrix Beal Copacity

$1.0000(k J / k E)$

TRAILING SBOCK CALCULATIONS

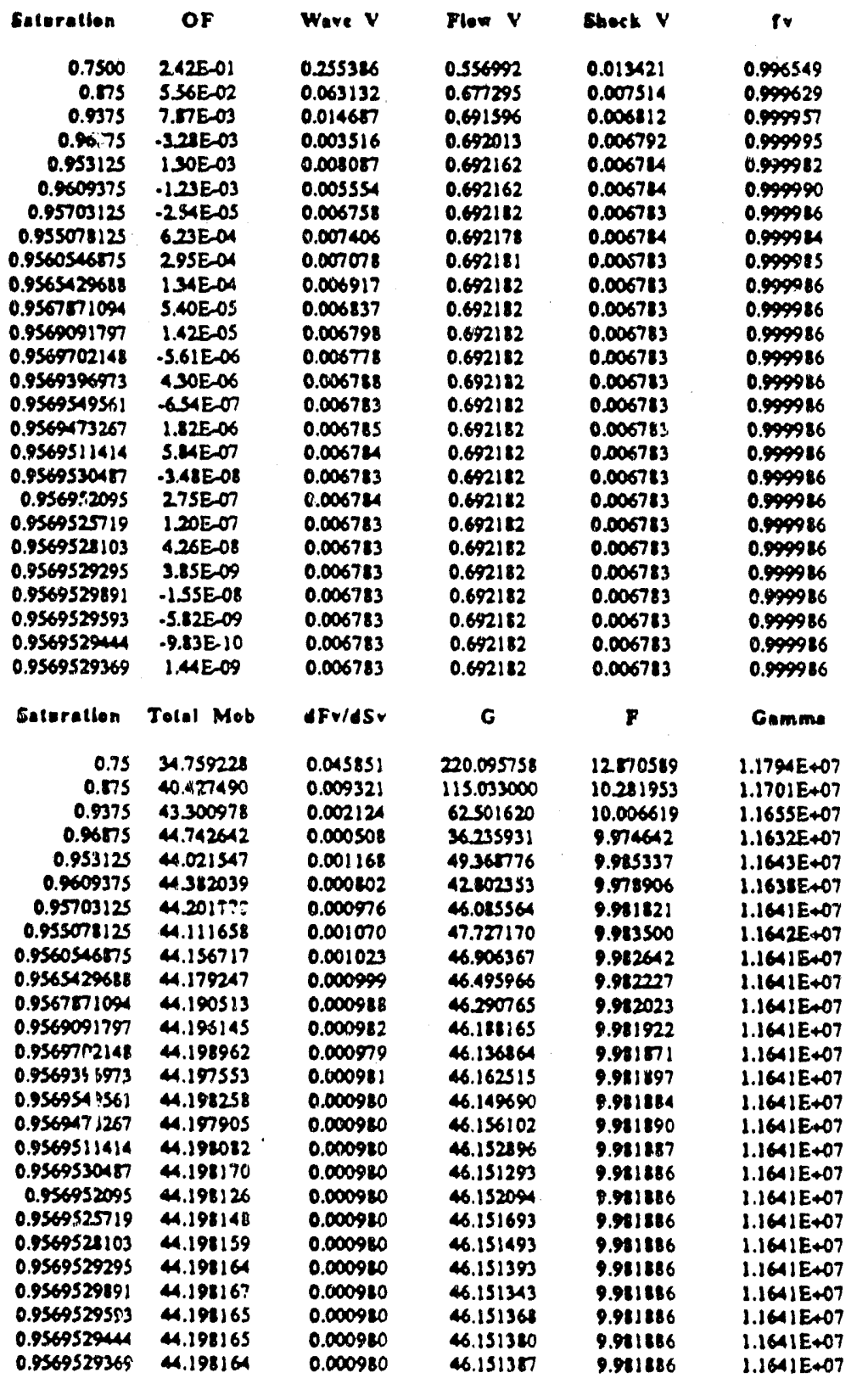

\begin{tabular}{|c|c|}
\hline dKrv/dSV & $d K r V / d S I$ \\
\hline 1.0000 & 0.1875 \\
\hline 1.0000 & 0.0469 \\
\hline 1.0000 & 0.0117 \\
\hline 1.0000 & 0.0029 \\
\hline 1.0000 & 0.0066 \\
\hline 1.0000 & 0.0046 \\
\hline 1.0000 & 0.0055 \\
\hline 1.0000 & 0.0061 \\
\hline 1.0000 & 0.0058 \\
\hline 1.0000 & 0.0057 \\
\hline 1.0000 & 0.0056 \\
\hline 1.0000 & 0.0056 \\
\hline 1.0000 & 0.0056 \\
\hline 1.0000 & 0.0056 \\
\hline 1.0000 & 0.0056 \\
\hline 1.0000 & 0.0056 \\
\hline 1.0000 & 0.0056 \\
\hline 1.0000 & 0.0056 \\
\hline 1.0000 & 0.0056 \\
\hline 1.0000 & 0.0056 \\
\hline 1.0000 & 0.0056 \\
\hline 1.0000 & 0.0056 \\
\hline 1.0000 & 0.0056 \\
\hline 1.0000 & 0.0056 \\
\hline 1.0000 & 0.0056 \\
\hline 1.0000 & 0.0056 \\
\hline Thete & Blgh \\
\hline
\end{tabular}

30550.407113

28275.535543

28033574650

22005.473055

28014.871837

22000.220503

20011.762338

28013.257853

28012503198

28012138595

$22011.95 \% 30$

28011.870625

0.95703125

0.95690917969

$25011.437457 \quad 0.956970214840 .95693969727$

$28011.42980 \quad 0.95695455605 \quad 0.95693969727$

22011.2402180 .956954956050 .9569472666

28011.1388380 .956954956050 .956951141136

$25011.6395280 .956953048710953351: 4136$

28011.839183 $0.9569530487: 0.95695209503$

$28011.839010 \quad 0.9569530167110 .95695257187$

28011.8389240 .956953448710 .95695281029

28011.0388810 .956953048710 .9569529295

$28011.838902 \quad 0.9569529891 \quad 0.9569529295$

$28011.1389130 .9569529593 \quad 0.9569529295$

28011.8389190 .9569529440 .9569529295 
Spreadsheet for Calculation of Stream-Water Saturation Profiles

Injectlon Temperature $650 \cdot \mathbf{x}$
Inllial Temperature

$100 \cdot \mathrm{K}$
Matrlx Beal Capeclty

$1.0000(\mathrm{~kJ} / \mathrm{k} E)$

LEADING SBOCK CALCULATIONS

\begin{tabular}{|c|c|c|c|c|c|c|c|c|}
\hline Eotoratian & OP & Ware $\mathbf{V}$ & Flow $v$ & Shoek $V$ & Pr & dKrv/dSr & $d K r \mid / d S I$ & \\
\hline 0.7500 & 240E-01 & 0.317373 & 0.015320 & 0.016909 & 0.996549 & 1.0000 & 0.1875 & \\
\hline 0.875 & $-1.07 \mathrm{E}-02$ & 0.064519 & 0.014188 & 0.075269 & 0.99629 & 1.0000 & 0.0469 & \\
\hline 0.8125 & 8.48E-02 & 0.160394 & 0.014395 & 0.075554 & 0.992653 & 1.0000 & 0.1055 & \\
\hline 0.24375 & 3.06E-02 & 0.105872 & 0.014216 & 0.075308 & 0.999249 & 10000 & 0.0732 & \\
\hline 0.259375 & B.40E-03 & 0.083664 & 0.014126 & 0.075266 & 0.999462 & 1.0000 & 0.0593 & \\
\hline 0.8671875 & - 1SAE-03 & 0.073722 & 0.014183 & 0.075262 & 0.999551 & 1.0000 & 0.0529 & \\
\hline 0.86328125 & 33AE-03 & 0.078598 & 0.014183 & 0.075253 & 0.999508 & 1.0000 & $0.056 !$ & \\
\hline 0.865234375 & $8.74 E-04$ & 0.076137 & $0.014: 13$ & 0.075262 & 0.99530 & 1.0000 & 0.0545 & \\
\hline 0.26621179375 & $.3395-04$ & C.074923 & 0.014183 & 0.075262 & 0.999541 & 1.0000 & 0.0537 & \\
\hline 0.2657226563 & 266E-OA & 0.075529 & 0.014183 & 0.075262 & 0.999535 & 1.0000 & 0.0541 & \\
\hline 0.2659667969 & $-3.66 E_{-05}$ & 0.075226 & 0.014183 & 0.075262 & 0.999538 & 1.0000 & 0.0539 & \\
\hline 0.265447266 & 1.15E-OA & 0.075377 & 0.014183 & 0.075262 & 0.999537 & 1.0000 & 0.0540 & \\
\hline 0.6659057617 & $3.00 E-05$ & 0.075301 & 0.014183 & 0.073262 & 0.999537 & 1.0000 & 0.0539 & \\
\hline 0.2659362793 & 1.19E-D6 & 0.075263 & 0.014183 & 0.075262 & 0.999538 & 1.0000 & 0.0539 & \\
\hline 0.2659525381 & -1.77 E-0S & 0.075244 & 0.014183 & 0.075262 & 0.999538 & 1.0000 & 0.0539 & \\
\hline $\begin{array}{r}0.2639439007 \\
0.265940094\end{array}$ & $\begin{array}{l}.227 E-06 \\
.35 A E-06\end{array}$ & $\begin{array}{l}0.075254 \\
0.075259\end{array}$ & $\begin{array}{l}0.014183 \\
0.014183\end{array}$ & $\begin{array}{l}0.073262 \\
0.075262\end{array}$ & $\begin{array}{l}0.999538 \\
0.99538\end{array}$ & $\begin{array}{l}1.0000 \\
1.0000\end{array}$ & $\begin{array}{l}0.0539 \\
0.0539\end{array}$ & \\
\hline - osspusiass & $-1.14 E-06$ & 0.075261 & 0.014183 & 0.075262 & 0.999538 & 1.0000 & 0.0539 & \\
\hline 0.265937233 & 5.03E-09 & 0.075262 & 0.014183 & 0.075262 & 0.999538 & 1.0000 & 0.0539 & \\
\hline $\begin{array}{l}0.2659377098 \\
0.2659374714\end{array}$ & $\begin{array}{l}.5 .86 \mathrm{E}-09 \\
.291 \mathrm{E}-07\end{array}$ & $\begin{array}{l}0.075262 \\
0.075262\end{array}$ & $\begin{array}{l}0.014183 \\
0.014183\end{array}$ & $\begin{array}{l}0.075262 \\
0.075262\end{array}$ & $\begin{array}{l}0.999538 \\
0.999538\end{array}$ & $\begin{array}{l}1.0000 \\
1.0000\end{array}$ & $\begin{array}{l}0.0539 \\
0.0539\end{array}$ & \\
\hline 0.2659373522 & - -ASE-OI & 0.075262 & 0.014183 & 0.075262 & 0.999538 & 1.0000 & 0.0539 & \\
\hline 0.2659372926 & -6.89E-08 & 0.075262 & 0.014183 & 0.075262 & 0.999538 & 1.0000 & 0.0539 & \\
\hline 0.2659372628 & $-3.19 E-08$ & 0.075262 & 0.014183 & 0.075262 & 0.999538 & 1.0000 & 0.0539 & \\
\hline 0.2659372479 & -1.34E-08 & 0.075262 & 0.014183 & 0.073262 & 0.999538 & 1.0000 & 0.0539 & \\
\hline 0.8659372404 & $-20 E-09$ & 0.075262 & $0.01<183$ & 0.073262 & 0.999538 & 1.0000 & 0.0539 & \\
\hline Saturacton & Tolal Mob & APv/dSr & G & $\mathbf{P}$ & Gommo & Thete & yleh & Low \\
\hline 0.75 & 34.759228 & 0.045851 & 220.095738 & 12570589 & $1.1794 E+07$ & 30550.407113 & 1 & 0.5 \\
\hline 0.075 & 40.427490 & 0.009321 & 115.033000 & 10.241953 & $1.1701 E+07$ & $22275.5355 \times 3$ & 1 & 75 \\
\hline 0.8125 & 37.576492 & 0.023172 & 167.564379 & 11.102088 & 1.1747E+07 & 24996.265468 & 0.875 & 0.75 \\
\hline 0.4375 & 38.99477 & 0.015295 & 141.294689 & 10.601362 & $1.1724 \mathrm{E}+07$ & 22556.229528 & 0.773 & 0.8125 \\
\hline 0.159375 & 39.712193 & 0.01207 & 128.165445 & 10.422059 & 1.1713Eथ07 & 28391.659931 & 0.875 & 0.24375 \\
\hline 0.8671875 & 40.069655 & 0.010651 & 121.599422 & 10.347467 & $1.1707 E+07$ & 28333.106609 & 0.875 & 0.259375 \\
\hline 0.26328125 & 39.590876 & 0.011335 & 124.882633 & 10.38354 & $1.17105 \times 07$ & $28364.2412 B 4$ & 0.8671875 & 0.259375 \\
\hline 0.865234375 & 39.960254 & 0.011000 & 123.241028 & 10.363236 & 1.1704E\&07 & 25348.724340 & 0.8671875 & 0.46328125 \\
\hline 0.2662109375 & 40.024952 & 0.010824 & 122420225 & 10356240 & 1.1708E407 & 22340.253555 & 0.8671875 & 0.865234375 \\
\hline 0.2657226563 & $\$ 0.002602$ & 0.010912 & 122830626 & 10.360740 & $1.1708 E+07$ & 21344.773142 & 0.1662109375 & 0.265234375 \\
\hline 0.8659667969 & 40.013777 & 0.010268 & 122625426 & 10.358506 & $1.1708 E+07$ & 28342800407 & 0.2662109375 & 0.26572265625 \\
\hline $\begin{array}{l}0.265241266 \\
0.2659057617\end{array}$ & $\begin{array}{r}40.008189 \\
40.010983\end{array}$ & $\begin{array}{l}0.010890 \\
0.010879\end{array}$ & $\begin{array}{l}122721026 \\
122676726\end{array}$ & $\begin{array}{l}10359622 \\
10.359064\end{array}$ & $\begin{array}{l}1.1708 E+07 \\
1.1708 E \div 07\end{array}$ & $\begin{array}{l}28343.790288 \\
28343.299601\end{array}$ & $\begin{array}{l}0.26596679688 \\
0.26596679688\end{array}$ & $\begin{array}{l}0.86572265625 \\
0.0652472656\end{array}$ \\
\hline 0.6659362793 & 40.012360 & 0.010873 & 122651076 & 10.358785 & 1.1700 E.07 & 28343.054442 & 0.86596679681 & 0.26590576172 \\
\hline 0.2659515381 & 40.013078 & 0.010071 & 122638251 & 10.358645 & $1.1708 E+07$ & 25342931909 & 0.26596679681 & 0.0659362793 \\
\hline $\begin{array}{l}0.2659439007 \\
0.865940094\end{array}$ & $\begin{array}{l}40.012729 \\
40.012554\end{array}$ & $\begin{array}{l}0.010772 \\
0.010873\end{array}$ & $\begin{array}{l}12264663 \\
122647669\end{array}$ & $\begin{array}{l}10.358715 \\
10.358750\end{array}$ & $\begin{array}{l}1.1701 E+07 \\
1.1700 E+07\end{array}$ & $\begin{array}{l}22342993172 \\
28343.023806\end{array}$ & $\begin{array}{l}0.86595153809 \\
0.86594390869\end{array}$ & $\begin{array}{l}0.0659362793 \\
0.0659362793\end{array}$ \\
\hline 0.1659381866 & 40.012467 & 0.010873 & 122649473 & 10.358767 & $1.1704 E+07$ & 28343.039124 & 0.86594009399 & 0.8659362793 \\
\hline 0.865937233 & 40.012123 & 0.010873 & $12265027 \mathrm{~A}$ & 10.358776 & 1.1708E+07 & 28343.046783 & 0.16593818665 & 0.2659362793 \\
\hline 0.2659377098 & 40.012445 & 0.010573 & 122649873 & 10.35571 & $1.1704 E+07$ & 20343.042953 & 0.165593811665 & 0.26593723297 \\
\hline 0.2659374714 & 40.012134 & 0.010573 & 122650074 & $10.351 \mathrm{ma}$ & $1.1703 E+07$ & 25343.044868 & 0.2659370981 & 0.26593723297 \\
\hline 0.2659373522 & $40.01 r 129$ & 0.010873 & 122650174 & $10.35 \mathrm{~ms}$ & $1.1708 E+07$ & 28343.045825 & 0.26593747139 & $\begin{array}{l}0.06593723297 \\
0.26593723297\end{array}$ \\
\hline $\begin{array}{l}0.2659372926 \\
0.2659372628\end{array}$ & $\begin{array}{l}40.012426 \\
40.012125\end{array}$ & & $\begin{array}{l}122650224 \\
122650249\end{array}$ & $\begin{array}{l}10.355775 \\
10.358776\end{array}$ & $\begin{array}{l}1.1703 E+07 \\
1.1708 E+07\end{array}$ & $\begin{array}{l}28343.046304 \\
28343.046543\end{array}$ & 0.26593735218 & $\begin{array}{l}0.86593723297 \\
0.26593723297\end{array}$ \\
\hline $\begin{array}{l}0.1655372628 \\
0.2659372479\end{array}$ & $\begin{array}{l}40.012225 \\
40.012124\end{array}$ & $\begin{array}{l}0.010873 \\
0.010873\end{array}$ & $\begin{array}{l}1226550249 \\
122650262\end{array}$ & $\begin{array}{l}10.335776 \\
10.358776\end{array}$ & $\begin{array}{l}1.1708 E+07 \\
1.1708 E+07\end{array}$ & $\begin{array}{l}28343.006513 \\
20343.046663\end{array}$ & $\begin{array}{l}0.65593729258 \\
0.16593726277\end{array}$ & $\begin{array}{l}0.86593723297 \\
0.86593723297\end{array}$ \\
\hline 0.2659372404 & 40.012424 & 0.010873 & 122650268 & 10.358776 & $1.1708 E+07$ & 28343.046723 & 0.66593724787 & 0.186593723297 \\
\hline
\end{tabular}




$$
.214 .
$$

Spreadsheet for Calculation of Stream-Water Saturation Profiles

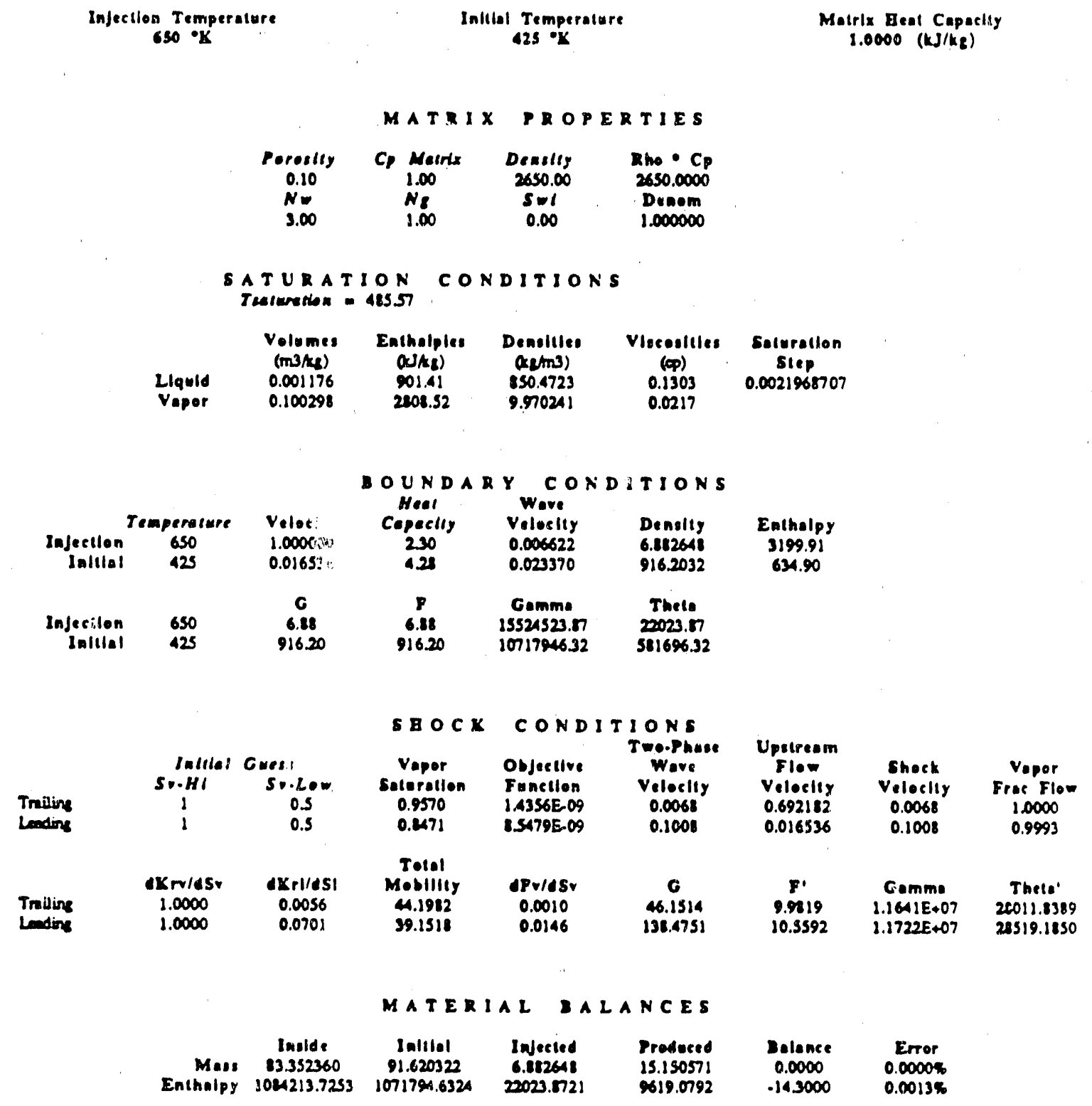


Iajection Temperature $650 \cdot \mathrm{K}$
Inllal Temperalure

$425^{\circ} \mathrm{K}$
Malrix Beat Capadity

$1.0000(\mathrm{~kJ} / \mathrm{kg})$

SATURATION PROFILE

\begin{tabular}{|c|c|c|c|c|c|c|c|c|}
\hline $\begin{array}{c}\text { Vapor } \\
\text { Soturation }\end{array}$ & $\begin{array}{c}\text { Vapor } \\
\text { Frac Flom }\end{array}$ & $d x r / d S r$ & dKrVdSI & $\begin{array}{c}\text { Tolal } \\
\text { Mobllity }\end{array}$ & dFv/dSr & lambda & $\begin{array}{l}\text { Mois } \\
\text { Inside }\end{array}$ & $\begin{array}{l}\text { Enthalpy } \\
\text { Inside }\end{array}$ \\
\hline $\begin{array}{l}1.0000 \\
1.0000\end{array}$ & & & & & & $\begin{array}{l}0.000000 \\
0.006783\end{array}$ & & \\
\hline $\begin{array}{l}0.9570 \\
0.9548\end{array}$ & $\begin{array}{l}0.999926 \\
0.99994\end{array}$ & $\begin{array}{l}1.0000 \\
1.0000\end{array}$ & $\begin{array}{l}0.0056 \\
0.0061\end{array}$ & $\begin{array}{r}4.198164 \\
4.096799\end{array}$ & $\begin{array}{l}0.050910 \\
0.001026\end{array}$ & $\begin{array}{l}0.0067113 \\
0.007517\end{array}$ & $\begin{array}{l}0.046687 \\
0.034514\end{array}$ & $\begin{array}{c}105308.165674 \\
8535.609228\end{array}$ \\
\hline $\begin{array}{l}0.9526 \\
0.9504\end{array}$ & $\begin{array}{l}0.999981 \\
0.999979\end{array}$ & $\begin{array}{l}1.0000 \\
1.0000\end{array}$ & $\begin{array}{l}0.0068 \\
0.0074\end{array}$ & $\begin{array}{l}43.995444 \\
43.194099\end{array}$ & $\begin{array}{l}0.001198 \\
0.001315\end{array}$ & $\begin{array}{l}0.008290 \\
0.009103\end{array}$ & $\begin{array}{l}0.037828 \\
0.041310\end{array}$ & $\begin{array}{l}9003.512125 \\
475.446160\end{array}$ \\
\hline $\begin{array}{l}0.9482 \\
0.9460\end{array}$ & $\begin{array}{l}0.999976 \\
0.999972\end{array}$ & $\begin{array}{l}1.0000 \\
1.0000\end{array}$ & $\begin{array}{l}0.0081 \\
0.0088\end{array}$ & $\begin{array}{l}43.792765 \\
43.691443\end{array}$ & $\begin{array}{l}0.001439 \\
0.001568\end{array}$ & $\begin{array}{l}0.009958 \\
0.010854\end{array}$ & $\begin{array}{l}0.044960 \\
0.048782\end{array}$ & $\begin{array}{l}9952.662911 \\
10434.010322\end{array}$ \\
\hline $\begin{array}{l}0.9438 \\
0.9416\end{array}$ & $\begin{array}{l}0.999969 \\
0.999965\end{array}$ & $\begin{array}{l}1.0000 \\
1.0000\end{array}$ & $\begin{array}{l}0.0095 \\
0.0102\end{array}$ & $\begin{array}{l}43.590132 \\
43.488835\end{array}$ & $\begin{array}{l}0.001703 \\
0.001445\end{array}$ & $\begin{array}{l}0.011791 \\
0.012770\end{array}$ & $\begin{array}{l}0.052777 \\
0.056948\end{array}$ & $\begin{array}{l}10919.939716 \\
11410.502299\end{array}$ \\
\hline $\begin{array}{l}0.9394 \\
0.9372\end{array}$ & $\begin{array}{l}0.999961 \\
0.999956\end{array}$ & $\begin{array}{l}1.0000 \\
1.0000\end{array}$ & $\begin{array}{l}0.0110 \\
0.0118\end{array}$ & $\begin{array}{l}43.387350 \\
43.286279\end{array}$ & $\begin{array}{l}0.001993 \\
0.002146\end{array}$ & $\begin{array}{l}0.013792 \\
0.014856\end{array}$ & $\begin{array}{l}0.061298 \\
0.065829\end{array}$ & $\begin{array}{l}11905.749868 \\
12405.7 \mathrm{M} 18\end{array}$ \\
\hline $\begin{array}{l}0.9350 \\
0.9328\end{array}$ & $\begin{array}{l}0.999951 \\
0.999946\end{array}$ & $\begin{array}{l}1.0000 \\
1.0000\end{array}$ & $\begin{array}{l}0.0127 \\
0.0136\end{array}$ & $\begin{array}{l}43.185021 \\
43.083778\end{array}$ & $\begin{array}{l}0.002306 \\
0.002473\end{array}$ & $\begin{array}{l}0.015964 \\
0.017115\end{array}$ & $\begin{array}{l}0.070542 \\
0.075442\end{array}$ & $\begin{array}{l}12910.510149 \\
13420.129478\end{array}$ \\
\hline $\begin{array}{l}0.9306 \\
0.9284\end{array}$ & $\begin{array}{l}0.999940 \\
0.999934\end{array}$ & $\begin{array}{l}1.0000 \\
1.0000\end{array}$ & $\begin{array}{l}0.0145 \\
0.0154\end{array}$ & $\begin{array}{l}42.912551 \\
42411338\end{array}$ & $\begin{array}{l}0.002645 \\
0.002824\end{array}$ & $\begin{array}{l}0.018310 \\
0.019549\end{array}$ & $\begin{array}{l}0.020530 \\
0.025809\end{array}$ & $\begin{array}{l}13934.647041 \\
14454.117705\end{array}$ \\
\hline $\begin{array}{l}0.9262 \\
0.9240\end{array}$ & $\begin{array}{l}0.999928 \\
0.999921\end{array}$ & $\begin{array}{l}1.0000 \\
1.0000\end{array}$ & $\begin{array}{l}0.0163 \\
0.0173\end{array}$ & $\begin{array}{l}42780141 \\
42678961\end{array}$ & $\begin{array}{l}0.003010 \\
0.003202\end{array}$ & $\begin{array}{l}0.020834 \\
0.022163\end{array}$ & $\begin{array}{l}0.091281 \\
0.086949\end{array}$ & $\begin{array}{l}14978.596974 \\
15508.140998\end{array}$ \\
\hline $\begin{array}{l}0.9218 \\
0.91 \%\end{array}$ & $\begin{array}{l}0.999914 \\
0.999906\end{array}$ & $\begin{array}{l}1.0000 \\
1.0000\end{array}$ & $\begin{array}{l}0.0183 \\
0.0194\end{array}$ & $\begin{array}{l}42577798 \\
42476652\end{array}$ & $\begin{array}{l}0.003401 \\
0.003606\end{array}$ & $\begin{array}{l}0.023538 \\
0.024959\end{array}$ & $\begin{array}{l}0.102817 \\
0.108816\end{array}$ & $\begin{array}{l}16012.006583 \\
16512651195\end{array}$ \\
\hline $\begin{array}{l}0.9174 \\
0.9152\end{array}$ & $\begin{array}{l}0.999898 \\
0.999889\end{array}$ & $\begin{array}{l}1.0000 \\
1.0000\end{array}$ & $\begin{array}{l}0.0205 \\
0.0216\end{array}$ & $\begin{array}{l}42375524 \\
2274414\end{array}$ & $\begin{array}{l}0.003818 \\
0.004037\end{array}$ & $\begin{array}{l}0.026427 \\
0.027942\end{array}$ & $\begin{array}{l}0.115159 \\
0.121639\end{array}$ & $\begin{array}{l}17127.732973 \\
17678.110736\end{array}$ \\
\hline $\begin{array}{l}0.9130 \\
0.9108\end{array}$ & $\begin{array}{l}0.599880 \\
0.999871\end{array}$ & $\begin{array}{l}1.0000 \\
1.0000\end{array}$ & $\begin{array}{l}0.0227 \\
0.239\end{array}$ & $\begin{array}{l}12173324 \\
42072252\end{array}$ & $\begin{array}{l}0.004262 \\
0.004495\end{array}$ & $\begin{array}{l}0.020504 \\
0.031: 13\end{array}$ & $\begin{array}{l}0.123329 \\
0.135233\end{array}$ & $\begin{array}{l}18233.43991 \\
17794.992942\end{array}$ \\
\hline $\begin{array}{l}0.9086 \\
0.9064\end{array}$ & $\begin{array}{l}0.999860 \\
0.999850\end{array}$ & $\begin{array}{l}1.0000 \\
1.0000\end{array}$ & 0.0250 & 11.971200 & 0.004735 & 0.032772 & 0.142351 & 19361.618501 \\
\hline 0.9064 & $\begin{array}{l}0.999850 \\
0.999839\end{array}$ & $\begin{array}{l}1.0000 \\
1.0000\end{array}$ & 0.0263 & 41.870169 & 0.004981 & 0.034478 & 0.149689 & 19933.712295 \\
\hline $\begin{array}{l}0.9012 \\
0.9020\end{array}$ & $\begin{array}{l}0.999839 \\
0.999827\end{array}$ & $\begin{array}{l}1.0000 \\
1.0000\end{array}$ & $\begin{array}{l}0.0275 \\
0.0288\end{array}$ & $\begin{array}{l}41.769158 \\
41.668169\end{array}$ & $\begin{array}{l}0.005235 \\
0.005496\end{array}$ & $\begin{array}{l}0.036235 \\
0.038040\end{array}$ & $\begin{array}{l}0.157249 \\
0.165033\end{array}$ & $\begin{array}{l}20511.546676 \\
21094.974729\end{array}$ \\
\hline $\begin{array}{l}0.8998 \\
0.8976\end{array}$ & $\begin{array}{l}0.999814 \\
0.999801\end{array}$ & $\begin{array}{l}1.0000 \\
1.0000\end{array}$ & $\begin{array}{l}0.0301 \\
0.0314\end{array}$ & $\begin{array}{l}41567202 \\
41.466257\end{array}$ & $\begin{array}{l}0.005764 \\
0.006039\end{array}$ & $\begin{array}{l}0.039896 \\
0.041803\end{array}$ & $\begin{array}{l}0.173045 \\
0.181288\end{array}$ & $\begin{array}{l}21644.130286 \\
20799.077930\end{array}$ \\
\hline 0.8954 & 0.999788 & 1.0000 & 0.0321 & A1.365334 & 0.006322 & 0.043761 & 0.189766 & 22779.813008 \\
\hline 0.1932 & 0.999774 & 1.0000 & 0.0342 & 41264435 & 0.006612 & 0.045770 & 0.19441 & 22426.611641 \\
\hline 0.8910 & 0.999759 & 1.0000 & 0.0358 & 41.163539 & 0.008910 & 0.047832 & 0.207436 & 24009330734 \\
\hline o.eses & 0.999743 & 1.0000 & 0.0371 & 41 262708 & 0.007216 & 0.049946 & 0.216336 & 24718.107983 \\
\hline $\begin{array}{l}0.8867 \\
0.8245\end{array}$ & $\begin{array}{l}0.999727 \\
0.999710\end{array}$ & $\begin{array}{l}1.0000 \\
1.0000\end{array}$ & $\begin{array}{l}0.0385 \\
0.0201\end{array}$ & $\begin{array}{l}40.961882 \\
40.261090\end{array}$ & 0.007529 & 0.052113 & 0.226044 & 25343.011190 \\
\hline 0.1823 & 0.999693 & $\begin{array}{l}1.0000 \\
1.0000\end{array}$ & $\begin{array}{l}0.0001 \\
0.0416\end{array}$ & $\begin{array}{l}40.861020 \\
\$ 0.760304\end{array}$ & $\begin{array}{l}0.007185 \\
0.005178\end{array}$ & $\begin{array}{l}0.054334 \\
0.056609\end{array}$ & $\begin{array}{l}0.235712 \\
0.245734\end{array}$ & $\begin{array}{l}25814.111772 \\
26611.177770\end{array}$ \\
\hline $0.280_{2}$ & 0.999674 & 10000 & 0.0432 & 40.659555 & 0.008515 & 0.051939 & 0.255945 & 27255.120861 \\
\hline 0.8779 & $0.99 \% 655$ & 1.0000 & 0.0448 & 0.558232 & 0.002860 & 0.061324 & 0.266417 & 27905.292869 \\
\hline 0.8757 & 0.999635 & 1.0000 & 0.0464 & 40.458136 & 0.009212 & 0.063765 & $0.2 \mathrm{mis}$ & 24561.086477 \\
\hline $\begin{array}{l}0.8735 \\
0.8713\end{array}$ & $\begin{array}{l}0.999615 \\
0.999593\end{array}$ & $\begin{array}{l}1.0000 \\
1.0000\end{array}$ & $\begin{array}{l}0.0480 \\
0.0497\end{array}$ & $\begin{array}{l}40.357468 \\
40.256828\end{array}$ & $\begin{array}{l}0.009573 \\
0.009912\end{array}$ & $\begin{array}{l}0.066262 \\
0.068816\end{array}$ & $\begin{array}{l}0.258160 \\
0.299438\end{array}$ & $\begin{array}{l}29255.035237 \\
29094.813581\end{array}$ \\
\hline C.269! & 0.999571 & 1.0000 & 0.0514 & 40.156217 & 0.010319 & 0.071428 & 0.310993 & 30571.296833 \\
\hline 0.2669 & 0.999548 & 1.0000 & 0.0532 & 40.035635 & 0.010705 & 0.074097 & 0.322828 & 31254561223 \\
\hline 0.2647 & 0.999524 & 1.0000 & 0.0549 & 39.955012 & 0.011099 & 0.076825 & 0.334947 & 3194.683896 \\
\hline 0.2625 & 0.999499 & 1.0000 & 0.0567 & 39.854560 & 0.0111502 & 0.079612 & 0.347353 & 32641.742925 \\
\hline 0.2603 & 0.999473 & 1.0000 & 0.0586 & 39.754068 & 0.011913 & 0.012459 & 0.360052 & 33345.817325 \\
\hline 0.8581 & 0.999447 & 1.0000 & 0.0604 & $39.653 \times 01$ & 0.012333 & 0.085366 & 0.373047 & 3056.957060 \\
\hline 0.1559 & 0.999419 & 1.0000 & 0.0623 & 39.553177 & 0.012762 & 0.081334 & 0.346343 & 37ns.333065 \\
\hline 0.5537 & 0.999391 & 1.0000 & 0.0642 & 39.452780 & 0.013199 & 0.091364 & 0.399942 & 35500.937249 \\
\hline 0.2515 & 0.999361 & 1.0000 & 0.0662 & 39.352415 & 0.013646 & 0.09456 & 0.413851 & 36233.682513 \\
\hline 0.4993 & 0.499331 & 1.0000 & 0.0681 & 39.252083 & 0.014102 & 0.097610 & 0.422072 & 36974.252765 \\
\hline $\begin{array}{l}0.4471 \\
0.0000\end{array}$ & 0.999299 & 1.0000 & 0.0701 & 39.151725 & 0.014567 & $\begin{array}{l}0.100828 \\
0.100828\end{array}$ & 0.42610 & 3722132929 \\
\hline 0.0000 & & & & & & 1.000000 & & \\
\hline
\end{tabular}


Spreadsheet for Calculation of Stream-Water Saturatior Profiles

Injectuen Temperature 650
Inlical Temperalure

$429 \times$
Matrix Deat Capoclty 1.0000 (kJ/kg)

TRAILING SBOCK.CALCULATIONG

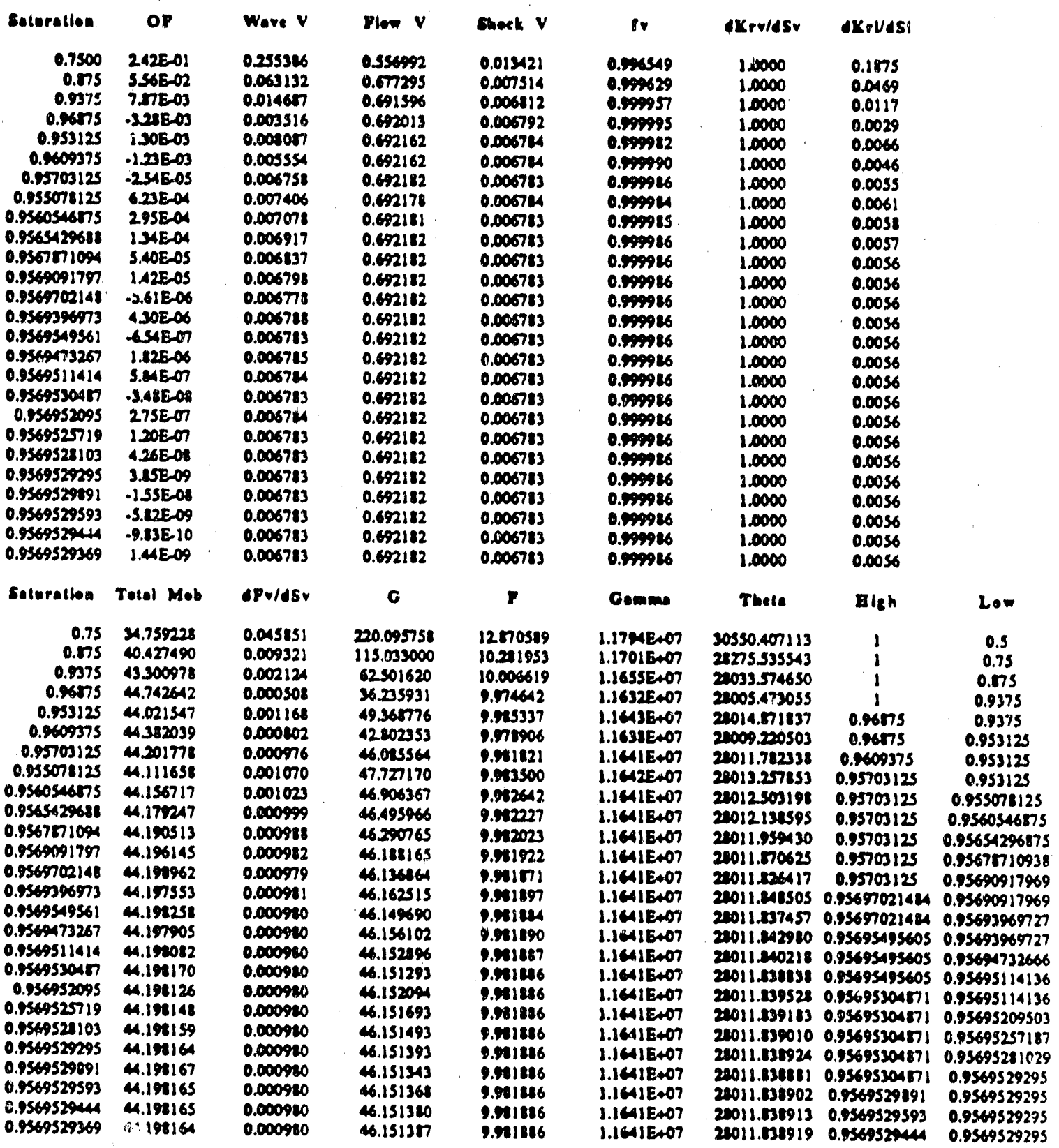


Injectlon Temperature $650^{\circ} \mathrm{X}$
Inllial Temperalure

$425^{\circ} \mathrm{K}$
Matrix Bani Capocliy

$1.0000\left(\mathrm{~kJ} / \mathrm{k}_{\mathrm{E}}\right)$

LEADING SBOCK CALCULATIONS

\begin{tabular}{|c|c|c|c|c|c|}
\hline Colurallon & OP & Wave $v$ & Flow $V$ & Shoek V & er \\
\hline $\begin{array}{l}0.7500 \\
0.075\end{array}$ & $\begin{array}{l}215 E-01 \\
.3 .64 E-02\end{array}$ & $\begin{array}{l}0.317373 \\
0.064519\end{array}$ & $\begin{array}{l}0.017479 \\
0.016591\end{array}$ & $\begin{array}{l}0.102077 \\
0.100901\end{array}$ & $\begin{array}{l}0.996549 \\
0.99629\end{array}$ \\
\hline 0.8125 & S.94E-02 & 0.16039 & 0.016637 & 0.100962 & 0.998653 \\
\hline 0.4375 & $5.04 E-03$ & 0.105172 & 0.016537 & 0.100129 & 0.999249 \\
\hline 0.859375 & $-1.72 \mathrm{E}-02$ & 0.083664 & 0.016547 & 0.10043 & 0.999162 \\
\hline 0.1515625 & $-6.46 E-03$ & 0.094371 & 0.016538 & 0.100830 & 0.999362 \\
\hline 0.4465625 & $.07 E-O A$ & 0.100021 & 0.016536 & 0.100128 & 0.999307 \\
\hline $\begin{array}{r}0.45703125 \\
0.466796775\end{array}$ & $\begin{array}{l}209 E-03 \\
6.37 E-04\end{array}$ & $\begin{array}{l}0.102921 \\
0.101465\end{array}$ & $\begin{array}{l}0.016536 \\
0.016536\end{array}$ & $\begin{array}{l}0.100828 \\
0.100828\end{array}$ & $\begin{array}{l}0.999279 \\
0.999293\end{array}$ \\
\hline 0.471679688 & $-266 \mathrm{E}-05$ & 0.100741 & 0.016536 & 0.100828 & 0.999300 \\
\hline 0.469238281 & 275E-OA & 0.101103 & $0.01 \times 536$ & 0.100828 & 0.999291 \\
\hline 0.470458984 & $9.40 E-05$ & 0.100922 & 0.016536 & 0.100828 & 0.999298 \\
\hline 0.2471069336 & $3.66 \mathrm{E}-06$ & 0.100832 & 0.016536 & 0.100828 & 0.999299 \\
\hline 0.471374512 & $4.15 E-05$ & 0.100786 & 0.016536 & 0.100828 & 0.999300 \\
\hline 0.471221924 & $-1.89 \mathrm{E}-05$ & 0.100809 & 0.016536 & 0.200828 & 0.999299 \\
\hline 0.47114563 & $-7.63 \mathrm{E}-06$ & 0.100820 & 0.016536 & 0.100828 & 0.999299 \\
\hline 0.471107483 & $-1.99 E-06$ & 0.100826 & 0.016536 & 0.100828 & 0.999299 \\
\hline 0.471082409 & 1.35E-OT & 0.100829 & 0.016536 & 0.100828 & 0.999299 \\
\hline 0.471097946 & $.5 .76 \mathrm{E}-07$ & 0.100127 & 0.016536 & 0.100828 & 0.999299 \\
\hline 0.471093178 & $1.30 \mathrm{E}-07$ & 0.100128 & 0.016536 & 0.100828 & 0.999299 \\
\hline 0.4471005562 & $.223 \mathrm{E}-07$ & 0.100128 & 0.016536 & 0.100828 & 0.999299 \\
\hline 0.47109437 & $-4.66 \mathrm{E}-08$ & 0.100828 & 0.016536 & 0.100828 & 0.999299 \\
\hline 0.471093774 & $4.16 E-08$ & 0.100828 & 0.016536 & 0.100828 & 0.999299 \\
\hline 0.4771094072 & $-247 \mathrm{E}-09$ & 0.100128 & 0.016536 & 0.100828 & 0.999299 \\
\hline 0.471093923 & 1.96E-08 & 0.100828 & 0.016536 & 0.100128 & 0.999299 \\
\hline 0.4471093997 & 8.55E-O9 & 0.100828 & 0.016536 & 0.100828 & 0.999299 \\
\hline Saluration & Total Mob & AFr/dSV & c & $\boldsymbol{F}$ & Gamma \\
\hline 0.75 & 34.759228 & 0.043851 & 220.095758 & $12 n 0589$ & 1.1794E+07 \\
\hline o.ns & 40.427490 & 0.009321 & 115.033000 & 10.281953 & $1.1701 E+07$ \\
\hline 0.8125 & 37.576492 & 0.023172 & 167.564379 & 11.102018 & $1.1747 E+07$ \\
\hline 0.4375 & 38.99477 & 0.015295 & 141.291689 & 10.601362 & $1.172 A E+07$ \\
\hline 0.859375 & 39.712193 & 0.012087 & 128.16545 & 10.422059 & $1.1713 E+07$ \\
\hline 0.8515625 & 39.355127 & 0.013634 & 134.732267 & 10.50645 & $1.1718 E+07$ \\
\hline 0.4765625 & 39.176748 & 0.014450 & 138.015478 & 10.552540 & $1.1721 E+07$ \\
\hline 0.455703125 & 39.017399 & 0.014269 & 139.657024 & 10.576604 & $1.1723 \mathrm{E}+07$ \\
\hline 0.466796875 & 39.132170 & 0.014659 & 138.836281 & $10 \operatorname{sen} 16$ & $1.1722 E+07$ \\
\hline 0.471679688 & 39.154459 & 0.014554 & 238.425880 & $10.55 \mathrm{~kg} 91$ & $1.17222 \mathrm{E}+07$ \\
\hline 0.2469238281 & 39.143314 & 0.014606 & 138.631080 & 10.561483 & $1.1722 E+07$ \\
\hline O.W704589B & 39.148286 & 0.014580 & 138.524480 & 10.559986 & $1.1722 E+07$ \\
\hline 0.4471069336 & 39.151672 & 0.014567 & 138.47120 & 10.539238 & $1.1722 E+07$ \\
\hline 0.441374512 & 39.153066 & 0.014561 & 138.451530 & 10.5stess & $1.1722 E+07$ \\
\hline 0.471221824 & 39.152369 & 0.014564 & 138.464355 & 10.599052 & $1.1722 E+07$ \\
\hline 0.497114563 & 39.152021 & 0.014566 & 138.470767 & 10.559145 & $1.1722 E+07$ \\
\hline 0.471107483 & 39.151847 & 0.014566 & 138.473973 & 10.559192 & $1.1722 E+07$ \\
\hline 0.47108409 & 39.151760 & 0.014567 & 138.475577 & 10.559215 & $1.1722 E+07$ \\
\hline 0.471097946 & 39.151803 & 0.014567 & 138.474775 & 10.559203 & $1.1722 E+07$ \\
\hline 0.4471093178 & 39.151711 & 0.014567 & 138.475176 & 10.559209 & $1.17222 E+07$ \\
\hline 0.4771095562 & 39.191792 & 0.014567 & 138.474975 & 10.559206 & $1.1722 E+07$ \\
\hline 0.44109437 & 39.151787 & 0.014567 & 138.479076 & 10.559208 & $1.1722 E+07$ \\
\hline 0.471093774 & 39.15174 & 0.014567 & 131.475126 & 10.539208 & $1.1722 E+07$ \\
\hline 0.8471094072 & 39.15178s & 0.014567 & 238.475101 & 10.559208 & $1.1722 E+07$ \\
\hline 0.471093923 & 39.151785 & 0.014567 & 138.475113 & 10.559208 & $1.1722 E+07$ \\
\hline 0.471093997 & 39.151785 & 0.014567 & 138.475107 & 10.559208 & $1.1722 E+07$ \\
\hline
\end{tabular}

$\begin{array}{cc}\text { dKrv/dSv } & \text { dKrVISI } \\ 1.0000 & 0.1175 \\ 1.0000 & 0.0169 \\ 1.0000 & 0.1055 \\ 1.0000 & 0.0732 \\ 1.0000 & 0.0593 \\ 1.0000 & 0.0661 \\ 1.0000 & 0.0696 \\ 1.0000 & 0.0714 \\ 1.0000 & 0.0705 \\ 1.0000 & 0.0701 \\ 1.0000 & 0.0703 \\ 1.0000 & 0.0702 \\ 1.0000 & 0.0701 \\ 1.0000 & 0.0701 \\ 1.0000 & 0.0701 \\ 1.0000 & 0.0101 \\ 1.0000 & 0.0101 \\ 1.0000 & 0.0701 \\ 1.0000 & 0.0701 \\ 1.0000 & 0.0701 \\ 1.0000 & 0.0701 \\ 1.0000 & 0.0701 \\ 1.0000 & 0.0701 \\ 1.0000 & 0.0701 \\ 1.0000 & 0.0701 \\ 1.0000 & 0.0701\end{array}$

\begin{tabular}{|c|c|c|}
\hline Thele & Blzh & Low \\
\hline 0550.407113 & f & 0.5 \\
\hline 2275.5355 & $i$ & 0.75 \\
\hline 2996.263468 & 0.875 & 0.7 \\
\hline 1556.229528 & 0.75 & 0.81 \\
\hline 8391.659931 & 0.875 & 0.4 \\
\hline $1472: 16977$ & 0.059375 & 0.43 \\
\hline 513.324807 & 0.8515625 & 0.4 \\
\hline 34.472287 & 0.4765625 & 0.43 \\
\hline
\end{tabular}

$\begin{array}{lll}28523.822989 & 0.4465625 & 0.45503125\end{array}$ 28511.555091 $0.24765625 \quad 0.466676875$ $28521.14328 \quad 0.4716796875 \quad 0.4466796875$ 28519.8685330 .47167968750 .4692382813 23519.2115180 .47167968750 .4704589844 $28511.883231 \quad 0.44716796875 \quad 0.4710693399$ 28519.0473560 .47137451170 .4710693359 $28519.129432 \quad 0.4712219238 \quad 0.4710693359$ $24519.170474 \quad 0.4711456299 \quad 0.4710693359$ 28519.190996 0.47711074129 0.4710693359 22519.120735 0.4711074829 0.471084094 $28519.185865 \quad 0.8471097962 \quad 0.471084094$ $22519.183300 \quad 0.4710979462 \quad 0.4710931778$ 22519.144583 $0.471095562 \quad 0.4710931778$ 28519.185224 $0.44710943699 \quad 0.47710931778$ $28519.14903 \quad 0.47109436990 .4710937738$ 28519.145064 0.47100407190 .4710937738 $\begin{array}{llll}28519.184983 & 0.4710940719 & 0.4710939220\end{array}$ 
$-218$.

Spreadsheet for Calculation of Stream-Water Saturation Profiles

Injection Temperature $650 \cdot \mathrm{K}$
Inlilal Temperalure 450 "K
Matrlx Beal Capacily $1.0000\left(\mathrm{~kJ} / \mathrm{k}_{\mathrm{E}}\right)$

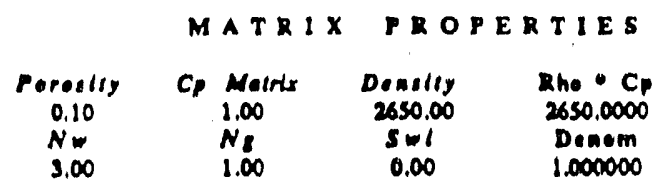

SATURATION CONDITIONS Tselurvion - 485.57

Volumes (m) $3 / 40)$

0.001176

0.100298

Enihaiples
Qute)

Qitel

Densities

(acins)

850.4723

Viseceitile
(cop)

(cp)

2201.52

$\begin{array}{ll}9.970241 & 0.0217\end{array}$
Saturatlon

siep

0.0028981477

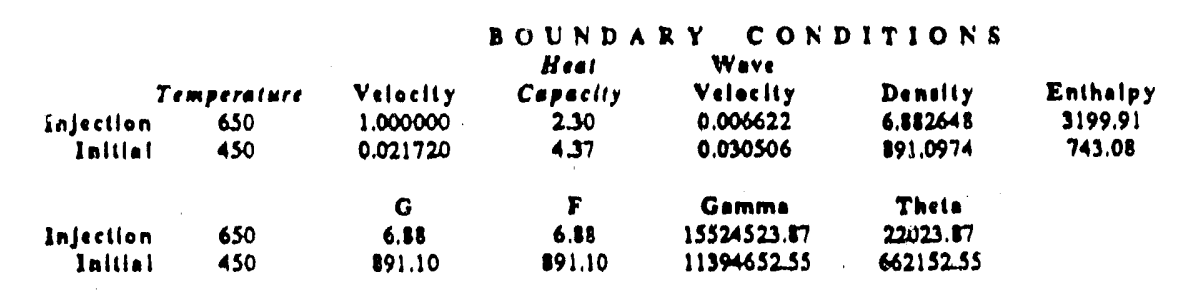

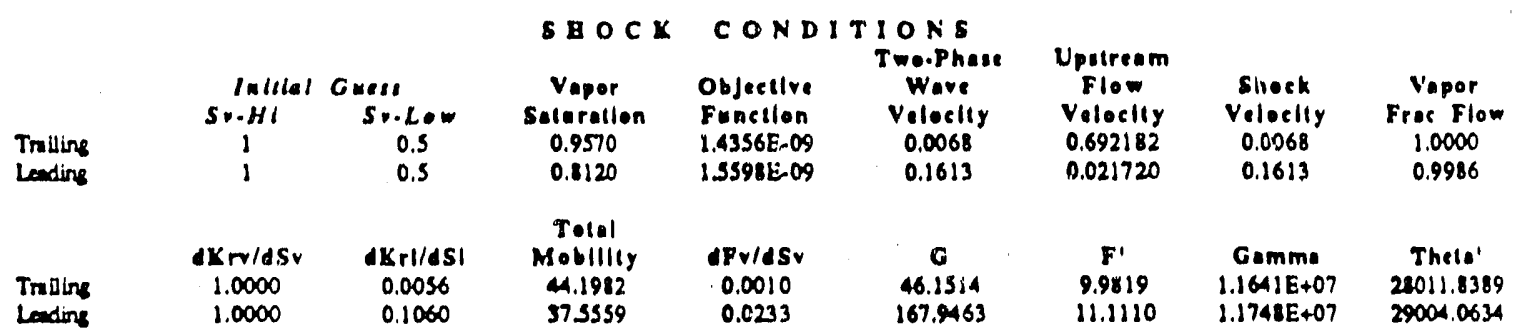

MATERIAL BALANCES

$\begin{array}{ccccccc} & \text { Inside } & \text { Inllial } & \text { Injecled } & \text { Prodeced } & \text { Aalance } & \text { Error } \\ \text { Mass } & 76.637490 & 89.109742 & 6.882648 & 19.354803 & 0.0001 & 0.0001 \% \\ \text { Enthalpy } & 11471355437 & 1139465.2553 & 23023.0721 & 143820777 & .28 .4941 & 0.0025 \%\end{array}$


.219.

Spreadsheet for Calculation of Stream-Water Saturation lrofiles

Indectlon Temperalure

$630^{\circ} \mathrm{K}$
Inltial Temperature

$450 \cdot \mathrm{K}$
Mulrix Beal Capocliy

$1,0000(\mathrm{~kJ} / \mathrm{kg})$
Vaper

Vaper

Caturallon Frac Flow

1.0000

1.0000

0.9570

0.9541

0.9512

0.9483

0. 454

0.9425

0.9396

0.9367

0.9338

0.9309

0.9280

0.9251

0.9222

0.9193

0.9164

0.9135

0.9106

0.9077

0.9048

0.0019

0.0990

0.8961

0.8932

0.0903

0.0874

0.8845

0.1816

0.1787

0.8758

0.0729

0.8700

0.2671

0.2642

0.2613

0.854

0.8555

0.8526

0.497

0.2468

0.0439

0.410

0.8381

0.2352

0.1323

0.1294

0.8265

0.8236

0.8207

0.1178

0.1149

0.1120

0.0000

0.0000

\subsection{6}

0.999980

0.999971

0.999966

0.999687

0.999663

0.999637

0.999609

0.999581

0.999550

0.999519

0.999486

0.999451

0.999414

0.999376

0.999337

0.999295

0.999252

0.999201

0.999160

0.999111

0.999060

0.999007

0.998952

0.998894

0.992835

0.998773

0.998709

0.991643

\section{SATURATION PROBILE}

dKroldsv

dKrVIAst

Tolal

AFv/ASv

Mobllity

(n)

1.0000
1.0000

0.0056

0.0063

0.0072

1.0000

1.0000

1.0000

0.0040

0.0090

0.0099

0.0110

0.0120

0.0132

0.0143

$\begin{array}{ll}0.999948 & 1.0000\end{array}$

$\begin{array}{ll}0.999941 & 1.0000 \\ 0.99933 & 1.0000\end{array}$

$0.999924 \quad 1.0000$

$0.999915 \quad 1.0000$

$0.999905 \quad 1.0000$

$0.999894 \quad 1.0000$

$0.999882 \quad 1.0000$

$0.999870 \quad 1.0000$

$0.999856 \quad 1.0000$

$0.99941 \quad 1.0000$

$0.999826 \quad 1.0000$

$0.999809 \quad 1.0000$

$0.999792 \quad 1.0000$

$0.999773 \quad 1.0000$

$0.999754 \quad 1.0000$

$0.999733 \quad 1.0000$

$0.999711 \quad 1.0000$

1.0000

1.0000

1.0000

1.0000

1.0000

1.0000

1.0000

1.0000

1.0000

1.0000

1.0000

1.0000

1.0000

$\cdot 1.0000$

1.0000

1.0000

1.0000

1.0000

1.0000

1.0000

1.0000

1.0000

1.0000

1.0000

0.0168

0.0182

0.0195

0.0210

0.0225

0.0240

0.0256

0.0272

0.0289

0.0308

0.032

0.0342

0.0361

0.0320

0.0400

0.0421

0.0441

0.0463

0.0485

0.0507

0.0530

0.0553

0.057

0.0601

0.0626

0.0652

0.0678

0.0704

0.0731

0.0758

$0.07 \times 6$

0.0814

0.0443

0.0773

0.0903

0.0933

0.0964

0.0995

0.1027

4.198164

0.000980

44.06444

0.001821

13.930740

0.001272

43.797056

0.001433

43.663392

43.529749

0.001605

0.001787

$43.396128 \quad 0.001980$

43.262531

0.002183

43.122951

0.002398

299511

0.002623

$42 \times 61891$

42728398

42594934

0.002859

0.003107

0.003366

42.461501

42325048

4219428

0.003637

0.003920

0.004214

0.004520

0.004839

41.79402

41.61595

0.005170

0.005513

0.005869

0.006238

0.006619

0.007014

41.262139

0.007422

0.007243

0.001278

0.008727

0.009190

0.009667

0.010158

0.010684

0.011185

0.011720

0.012271

0.012237

0.013418

0.014015

0.014628

0.015257

0.015902

0.016564

0.017243

0.017938

0.012651

0.019311

0.020129

0.020895

0.021679

0.022481

37.819006

37555872

0.023302

\begin{tabular}{|c|c|c|}
\hline lambdo & $\underset{\text { Inotde }}{\text { Mute }}$ & $\begin{array}{l}\text { Enthalpy } \\
\text { Inoide }\end{array}$ \\
\hline \multicolumn{3}{|l|}{0.000000} \\
\hline $\begin{array}{l}0.006783 \\
0.007759\end{array}$ & $\begin{array}{l}0.046687 \\
0.046215\end{array}$ & $\begin{array}{c}105308.165674 \\
11358.614465\end{array}$ \\
\hline 0.008805 & 0.052076 & 12175.378329 \\
\hline 0.009921 & 0.058322 & 13002371478 \\
\hline 0.011109 & 0.064961 & 13839.744867 \\
\hline 0.012370 & 0.071998 & 14687.631745 \\
\hline 0.013704 & 0.079412 & 15546.247699 \\
\hline 0.0151112 & 0.087300 & 16415.690688 \\
\hline 0.016596 & 0.095580 & 17296.141087 \\
\hline 0.018156 & 0.104218 & 18177.761725 \\
\hline 0.019793 & 0.113432 & 19090.717929 \\
\hline 0.021508 & 0.123021 & 20005.177563 \\
\hline 0.023302 & 0.133063 & 20931.311076 \\
\hline 0.025176 & 0.143565 & 21169.291542 \\
\hline 0.027131 & 0.154537 & 22119.294704 \\
\hline 0.029169 & 0.165986 & 23711.499023 \\
\hline 0.031289 & 0.177921 & 24756.085723 \\
\hline 0.033494 & 0.190351 & 25743.231838 \\
\hline 0.03574 & 0.203285 & 26743.145256 \\
\hline 0.031160 & 0.216733 & 27755.994778 \\
\hline 0.040623 & 0.230703 & 2711.980156 \\
\hline 0.043175 & 0.245205 & 29821.297154 \\
\hline 0.045816 & 0.260249 & 30874,144591 \\
\hline 0.048549 & 0.275845 & 31940.724401 \\
\hline 0.051373 & 0.292002 & 33021.241683 \\
\hline 0.054290 & 0.308732 & 34115.904754 \\
\hline 0.057302 & 0.326044 & 35224,925210 \\
\hline 0.060409 & 0.343950 & 3634517976 \\
\hline 0.063612 & 0.362460 & 37416.901372 \\
\hline 0.066914 & 0.381585 & SAMO 297162 \\
\hline 0.070315 & 0.401336 & 39108.930623 \\
\hline 0.073916 & 0.421726 & 40993,030601 \\
\hline 0.077419 & 0.442765 & 42192.129574 \\
\hline 0.081126 & 0.464486 & 43008.563716 \\
\hline 0.044936 & $0.4(6884)$ & 44640.472962 \\
\hline 0.098853 & 0.509903 & astes.801074 \\
\hline 0.092877 & 0.533663 & 47193.795706 \\
\hline 0.097009 & 0.558135 & 44435.702473 \\
\hline 0.101232 & 0.583333 & 49734.795025 \\
\hline 0.105606 & 0.609268 & 51051.315111 \\
\hline 0.110073 & 0.635956 & 52385.532655 \\
\hline 0.114654 & 0.663410 & 53737.715831 \\
\hline 0.119351 & 0.691644 & 55108.137134 \\
\hline 0.124166 & 0.720673 & 5497.073461 \\
\hline 0.129100 & 0.750512 & 57904.106187 \\
\hline 0.134155 & 0.781174 & 59331.621243 \\
\hline 0.139331 & 0.312677 & 60777.809198 \\
\hline 0.144632 & 0.843034 & 62243.665344 \\
\hline 0.150058 & 0.878263 & 63729.489777 \\
\hline 0.155612 & 0.912379 & 65235.577483 \\
\hline 0.161294 & 0.947400 & 66762.268427 \\
\hline 0.161294 & & \\
\hline
\end{tabular}


Injecllon Tumperature. sso $\mathrm{K}$
Inlical Temperalure

$450 \cdot K$
Malrlx Beal Copacity

$1.0000\left(k J / K_{E}\right)$

TRAILING SHOCK CALCULATIONE

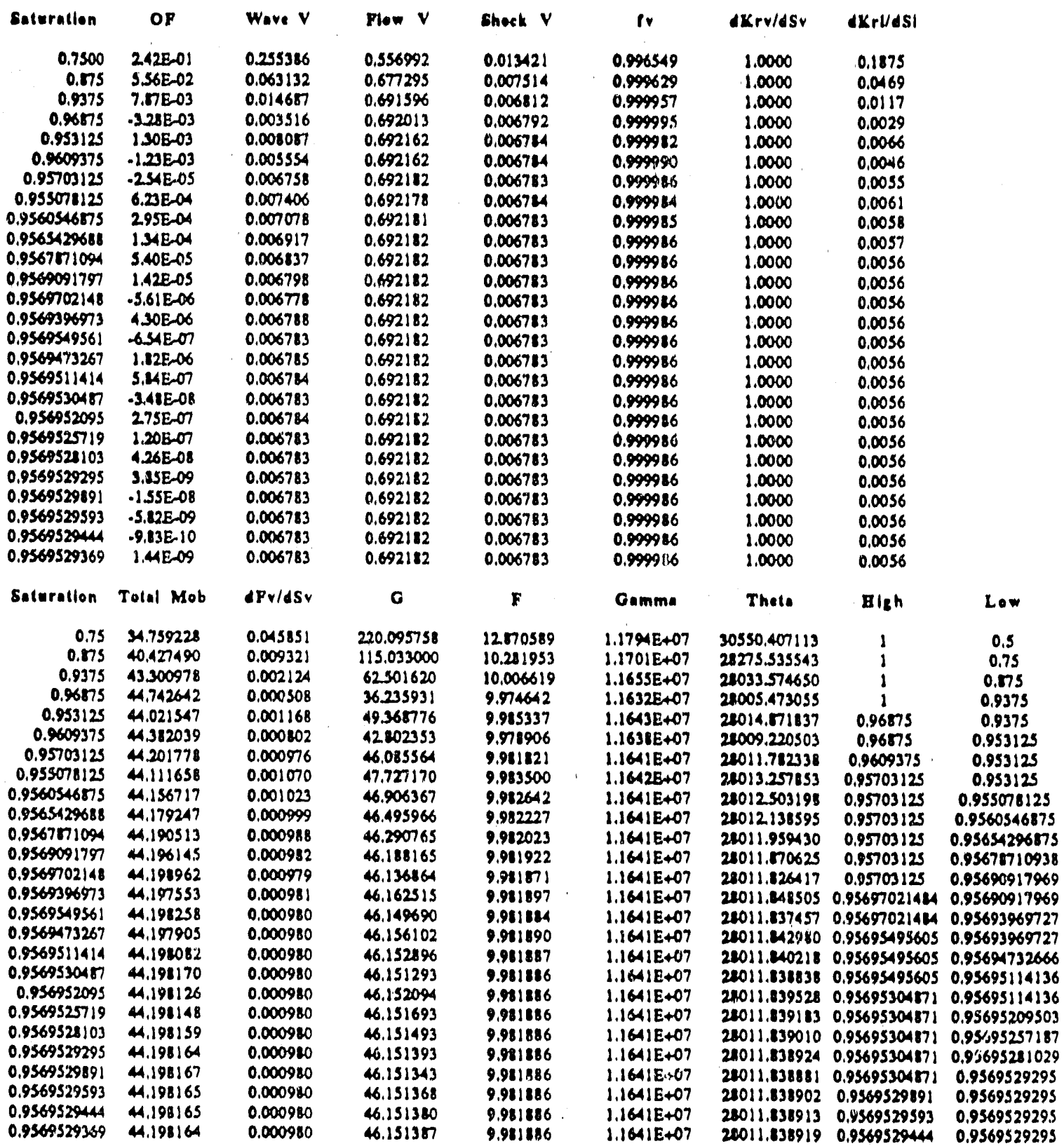


.221

Spreadsheet for Calculation of stream-Water Saturation Profiles

Injectlon Temperalure $6 \$ 0$ "K
Inlilal Temperalure

$450 \cdot x$
Matrix Beat Copocity

1,0000 (kJ/ke)

LEADING SHOCK CALCULATIONS

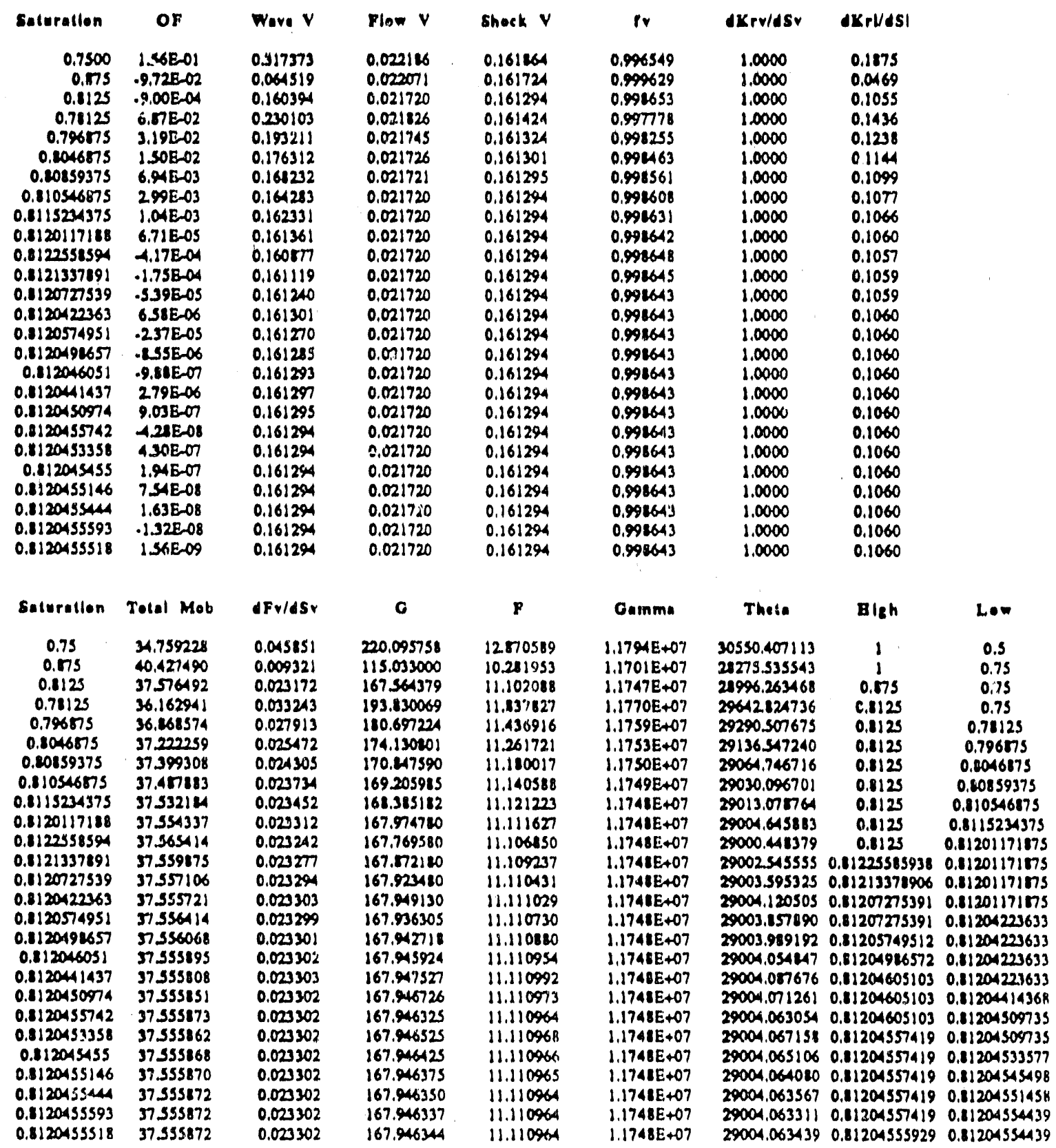


Spreadsheet for Calculation of Stream-Water Saturation Proflles

Injection Temperalure 650 " $\mathrm{K}$
Inllbil Temperature $473^{\circ} \mathrm{K}$
Matrix Beal Capacliy $1.0000\left(k_{\mathrm{J}} / \mathrm{k}_{\mathrm{B}}\right)$
MATRIX PROPERTIES

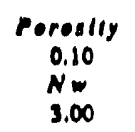

Cp Mart

1.00
$N B$
1.00

Dewelly
2650.00
$5 w 1$
0.00

Rhe $\bullet C$ 2650.0000 Denem 1.000000

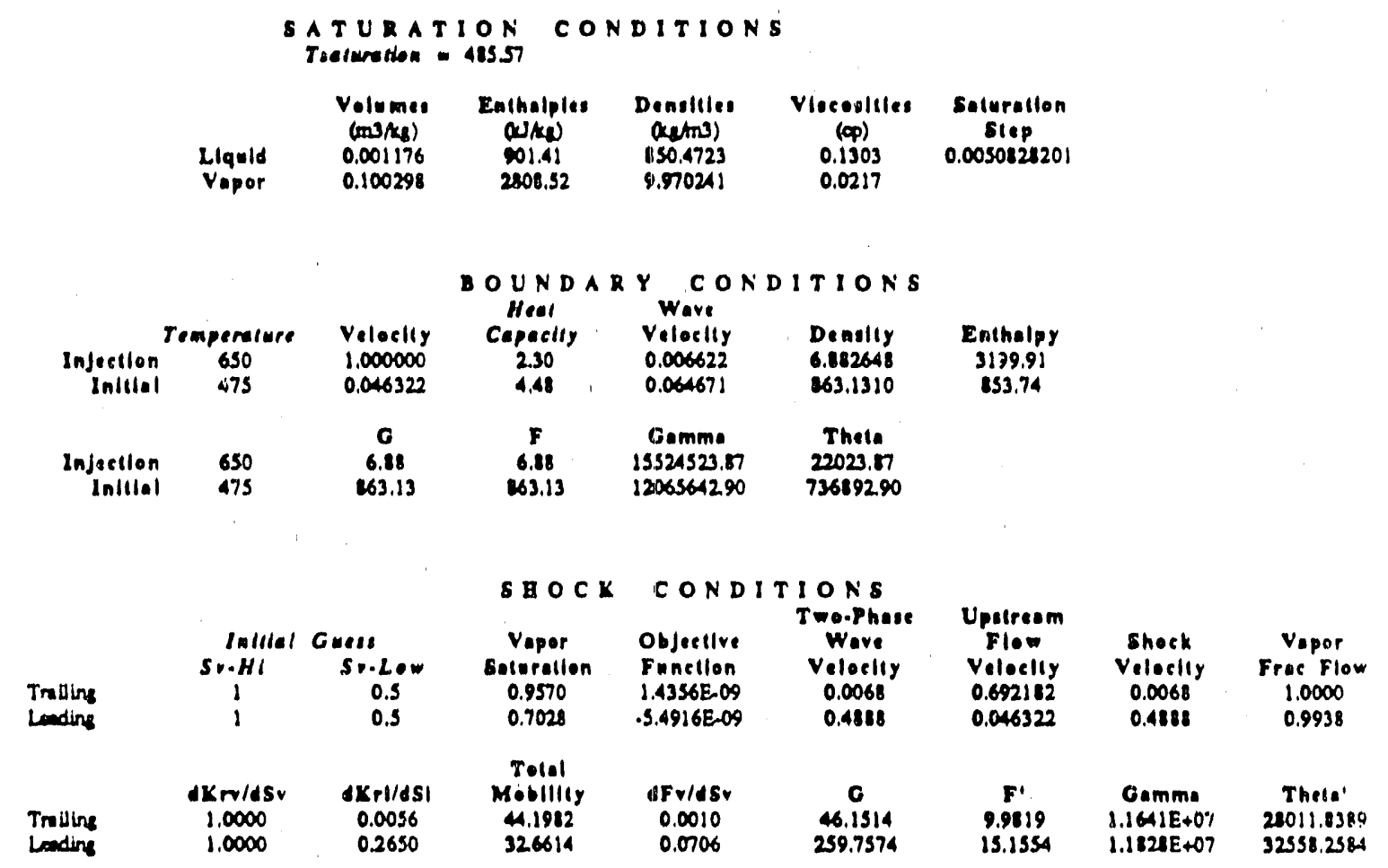

MATERIAL BALANCES

Mass
Inolde
53.213192
Enthalpy
1194593.1371

Inlllal

06313097

Injecled

6.882648

Preduced

39.981852

34134.2668

Bulence
0.0007

Error

12065642904

92023,8721

.139 .2414

$0.0113 \%$ 
Injection Temperalure $650 \cdot \mathbf{K}$
Iallat Temperalure $479 \cdot \mathrm{K}$
Matrix Beat Capacity 1.0000 (kJ/kg)

SATURATION PROPILE

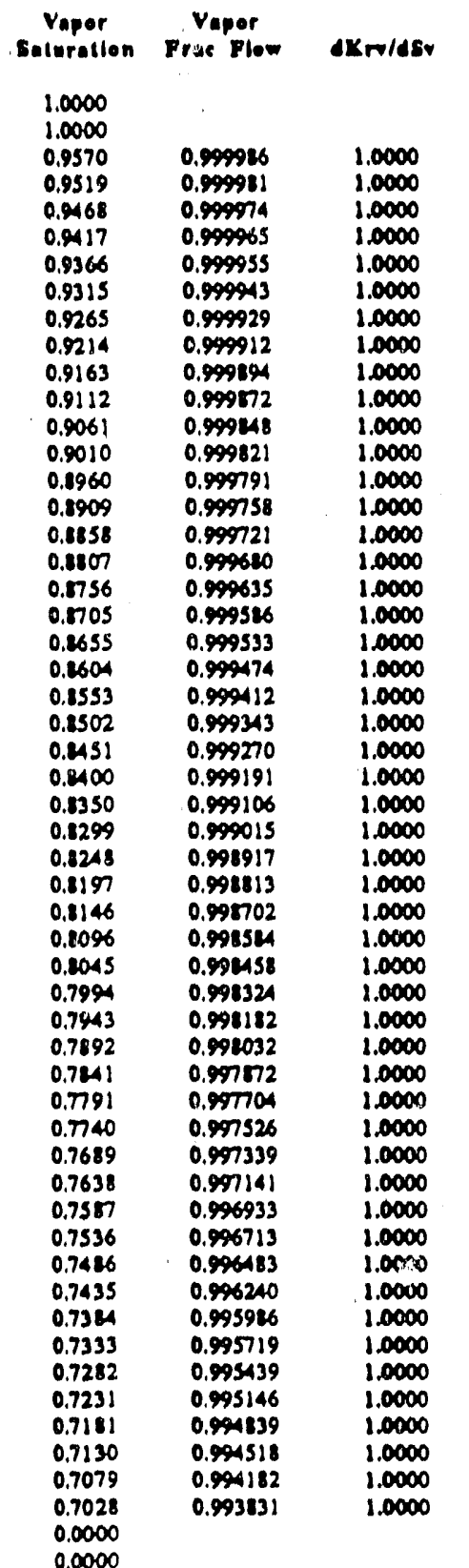

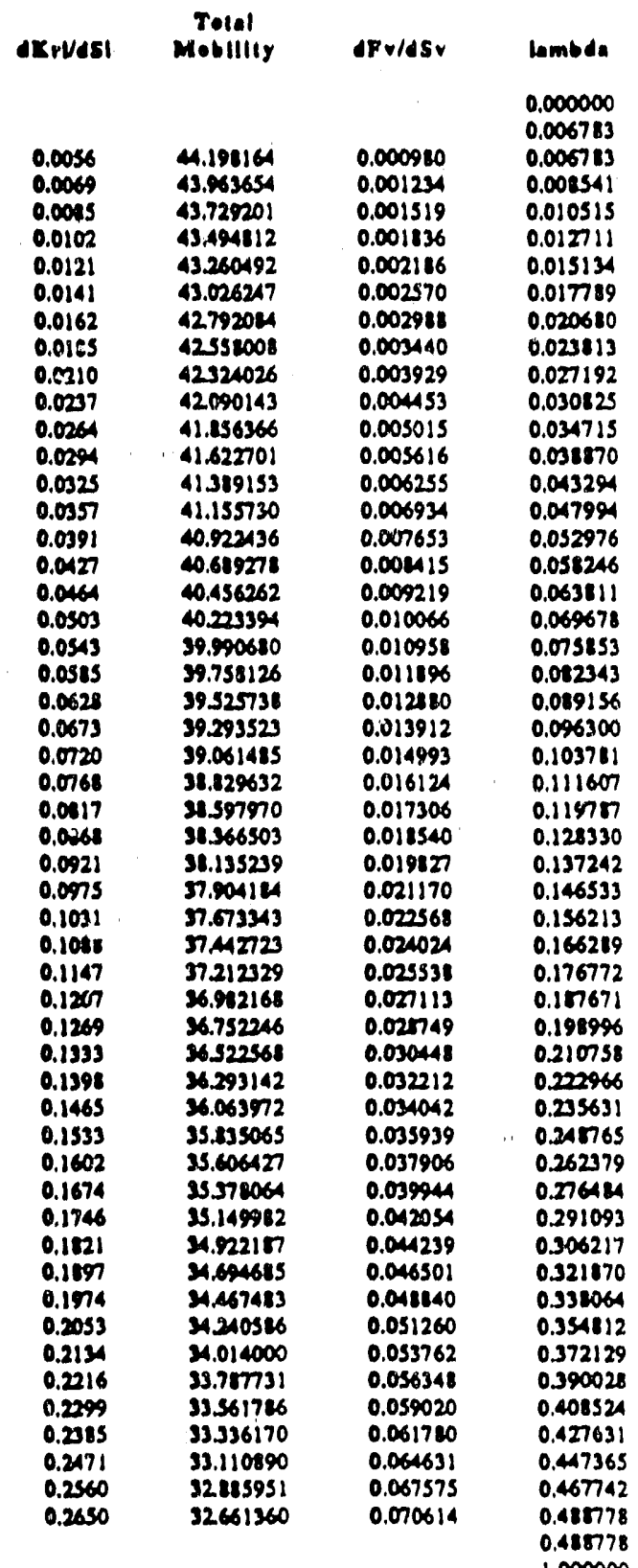

Moss Enthalpy

Inolde Inolde

$0.046687 \quad 105308.165674$

0.04485420460 .207161

$0.103776 \quad 22996.220295$

0.12481825381 .340310

$0.141049 \quad 21238.024402$

$0.173538 \quad 30946.785096$

$0.201356 \quad 33716.151411$

$0.231579 \quad 36547.71007$

$0.264282 \quad 3944.086781$

$0.299346 \quad 42403.951455$

$0.337452 \quad 45432019610$

$0.372086 \quad 48529.053708$

$0.421535 \quad 51696.164582$

$0.467189 \quad 54937312902$

$0.517243 \quad 58252310690$

$0.56 \% 94 \quad 61643.82217$

$0.625341 \quad 65113.861926$

0.64288 68664.524494

0.74664272297 .923157

$0.812514 \quad 76016.250189$

0.82201879821 .724403

$0.955273 \quad 83716.220051$

$1.032400 \quad$ m03.748788

$1.113527 \quad 91785.021700$

$1.197783 \quad 95963.159403$

1218304 100240.75214

$1382229 \quad 104620.472389$

iscorar 109105.056440

$1.583876 \quad 113697327534$

$1.691901 \quad 11400.187969$

1.c04931 123216.623732

$1.023151 \quad 128149.707155$

$2046713 \quad 1332025 \% 48$

$2175799 \quad 138378.554536$

2310591 1A3680.919988

$2451279 \quad 149113.142050$

$2596057 \quad 154678.767782$

$2751127 \quad 160381.4150$

$2910699 \quad 166224.943149$

$3.076988 \quad 172213.12174$

$3.250216 \quad 178349.969002$

$3.430617 \quad 14639.581043$

$3.61427 \quad 191016.201248$

$3.113896 \quad 197694.16924$

$4.017277 \quad 204467.96503$

$4.221837 \quad 211412201257$

4 41848 218531.654648

$4.67595 \quad 225131.203546$

$4.915369 \quad 233315.00736$

$5.162473 \quad 240990.953276$

S.419227 248861.714624 
Injection Temperature $650 \cdot \mathbf{K}$
Inllbl Temperature

$475 \%$
Matrix Beat Copacity

$1.0000(\mathrm{~kJ} / \mathrm{kg})$

TRAILING SBOCKCALCULATIONS

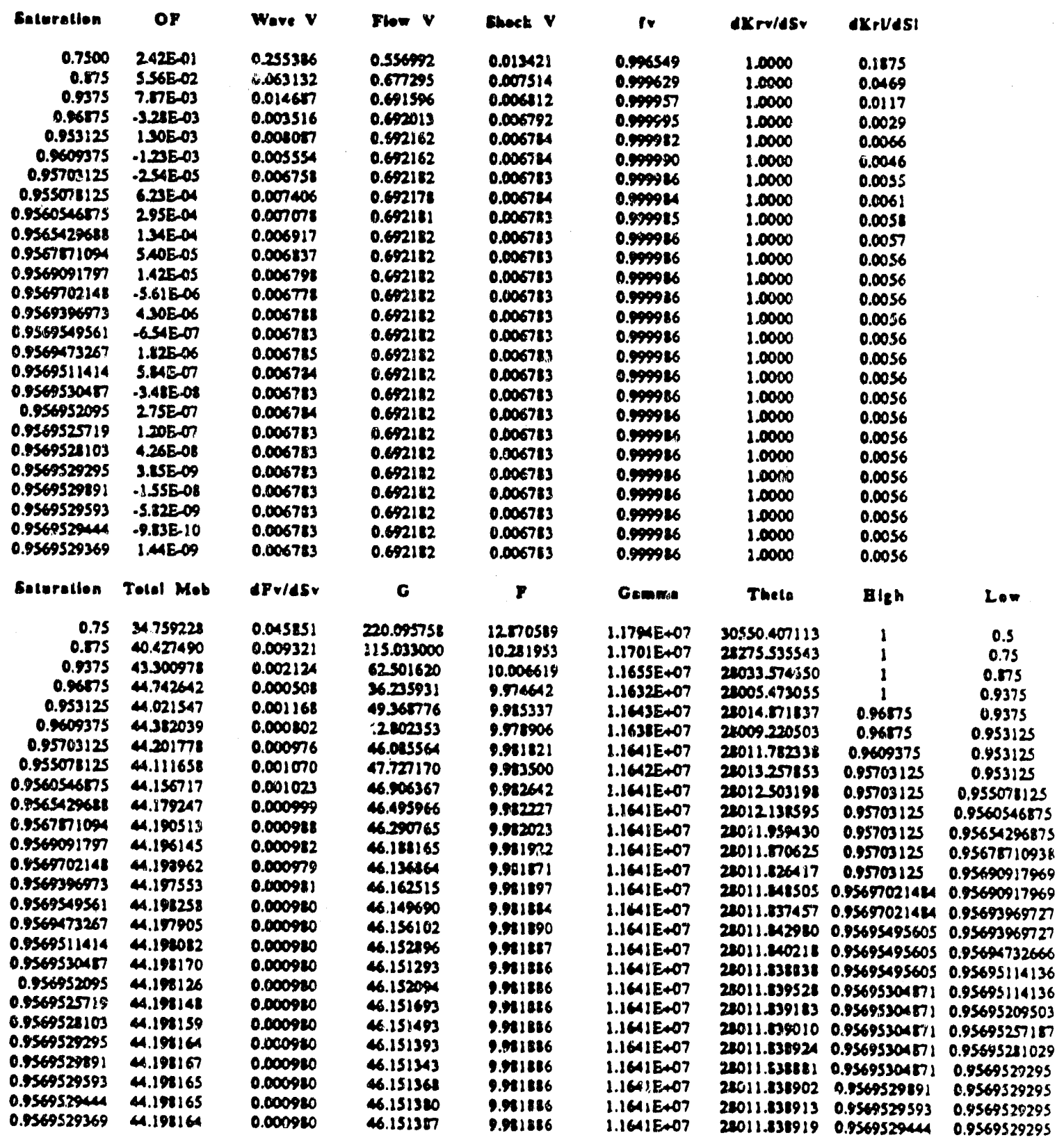


Injection Temperature $690^{\circ} \mathrm{K}$
Inlibl Temperature

$475 \cdot \mathrm{K}$
Matrix Beal Cepacily

$1.0000\left(\mathrm{~kJ} / \mathrm{k}_{\mathrm{Z}}\right)$

LEADING SBOCI CALCULATIONE

\begin{tabular}{|c|c|c|c|c|c|c|c|c|}
\hline Seturatien & OP & Wave $\mathbf{V}$ & Plow V & Chock V & Iv & dKrv/ASV & dKrVASI & \\
\hline 0.7500 & $-1.72 \mathrm{E}-01$ & $0 y 17373$ & 0.046760 & 0.409101 & 0.966549 & 1.0000 & 0.1875 & \\
\hline $\begin{array}{r}0.625 \\
0.6775\end{array}$ & $4.16 \mathrm{E}-01$ & 0.906281 & 0.047852 & 0.409907 & 0.916170 & 1.0000 & 0.4219 & \\
\hline $\begin{array}{r}0.6875 \\
0.71875\end{array}$ & 6.7SE-02 & 0.556298 & 0.046374 & 0.488816 & 0.992676 & 1.0000 & 0.2730 & \\
\hline $\begin{array}{r}0.71875 \\
0.703125\end{array}$ & $-6.38 E-02$ & 0.425002 & 0.046375 & 0.488817 & 0.994882 & 1.0000 & 0.2373 & \\
\hline 0.703125 & $-1.32 E-03$ & 0.487463 & 0.046322 & 0.42577 & 0.993853 & 1.0000 & 0.264 & \\
\hline $\begin{array}{r}0.6953125 \\
0.69921175\end{array}$ & $3.23 E-02$ & 0.521053 & 0.046334 & 0.485787 & 0.99324 & 1.0000 & 0.2785 & \\
\hline $\begin{array}{l}0.69921175 \\
0.7011711775\end{array}$ & 153E-02 & 0.504055 & 0.046325 & 0.487780 & 0.993573 & 1.0000 & 0.2714 & \\
\hline $\begin{array}{r}0.701171875 \\
0.7021484375\end{array}$ & $6.93 E-03$ & 0.495709 & 0.046322 & 0.485778 & 0.993714 & 1.0000 & 0.2679 & \\
\hline $\begin{array}{l}0.7021444375 \\
0.7026367188\end{array}$ & $2 \angle 0 E 03$ & 0.491573 & 0.046322 & 0.48778 & 0.993784 & 1.0000 & 0.2661 & \\
\hline $\begin{array}{l}0.7026367188 \\
0.702201594\end{array}$ & 7.37E-04 & 0.409515 & 0.046322 & $0.48 \pi 778$ & 0.993519 & 1.0000 & 0.2653 & \\
\hline $\begin{array}{l}0.7022208594 \\
0.7027587891\end{array}$ & -290E-OA & 0.482488 & 0.046322 & 0.428778 & 0.993836 & 1.0000 & 0.2648 & \\
\hline 0.7027587891 & 223E-04 & 0.419001 & 0.046322 & 0.48578 & 0.993827 & 1.0000 & 0.2651 & \\
\hline 0.7028191242 & $.3 .32 E-05$ & 0.485745 & 0.046322 & 0.486778 & 0.993131 & 1.0000 & 0.2649 & \\
\hline 0.7027893066 & OSIEOS & 0.428573 & 0.046322 & 0.485778 & 0.993829 & 1.0000 & 0.2650 & \\
\hline 0.7028045654 & 3.10E-OS & 0.488209 & 0.046322 & 0.48578 & 0.993830 & 1.0000 & 0.2650 & \\
\hline 0.7028121948 & $-1.10 E-06$ & $0.4887 m$ & 0.046322 & 0.48078 & $0.99383 !$ & 1.0000 & 0.2650 & \\
\hline 0.7028083801 & 1.49E-OS & 0.488793 & 0.046322 & 0.48678 & 0.993131 & 1.0000 & 0.2650 & \\
\hline 0.7028102775 & $6.925-06$ & 0.488785 & 0.046322 & 0.48578 & 0.993131 & 1.0000 & 0.2650 & \\
\hline 0.7028112411 & 291E-06 & 0.485781 & 0.046322 & $0.48 m$ ( & 0.993831 & 1.0000 & 0.2650 & \\
\hline 0.702831718 & $9.03 E-07$ & 0.488779 & 0.046322 & 0.486778 & 0.993831 & 1.0000 & 0.2650 & \\
\hline 0.7028119564 & $-9.95 E-08$ & 0.488778 & 0.046322 & 0.42778 & 0.993831 & 1.0000 & 0.2650 & \\
\hline 0.70281118372 & $0.02 E-07$ & 0.485778 & 0.046322 & $0.45,0,78$ & 0.953431 & 1.0000 & 0.2650 & \\
\hline 0.7028118968 & $131 \mathrm{EO}$ & 0.488778 & 0.046322 & 0.48578 & 0.993131 & 10000 & 0.2650 & \\
\hline 0.7028119266 & 258E-08 & 0.48877 & 0.046322 & 0.485778 & 0.093831 & 1.0000 & 0.2650 & \\
\hline 0.7028119415 & $.3 .68 E-08$ & 0.488778 & 0.046322 & 0.485778 & 0.993831 & 1.0000 & 0.2650 & \\
\hline 0.7028119341 & $-5.49 E-09$ & 0.488778 & 0.046322 & OAET78 & 0.993831 & 1.0000 & 0.2050 & \\
\hline Seteratlen & Tolal Mob & dFr/dsv & G & $\boldsymbol{P}$ & Gumme & Thele & Bigh & $L \bullet \pi$ \\
\hline 0.75 & 34.759228 & 0.045851 & 200.095758 & 12870589 & 1.1794E 407 & 30550.407113 & 1 & 0.5 \\
\hline 0.625 & 29.270883 & 0.130931 & 325.152517 & $21 . \operatorname{sen} 311$ & 1.1826E+07 & 31216.739851 & 0.75 & 0.5 \\
\hline 0.6875 & 31.92694 & 0.020369 & 272627138 & 16.125943 & 1.1840E+07 & 34111.144085 & 0.75 & 0.625 \\
\hline 0.71875 & 33.366761 & 0.061400 & 246.361448 & 14272175 & 1.1817E+07 & 31712109202 & 0.75 & 0.6875 \\
\hline 0.703125 & 32675183 & 0.070424 & 259.494293 & 15.136120 & 1.1822E 407 & 32541.951705 & 0.71875 & 0.6875 \\
\hline 0.6953125 & 32330635 & 0.07527 & 266.060715 & 15.615059 & J.1834EA07 & $32 \% 2223587$ & 0.703125 & 0.6875 \\
\hline 0.69921575 & 32502803 & 0.072821 & $262 \mathrm{~m}$ 504 & 15.371956 & $1.1831 E+07$ & 32746507401 & 0.703125 & 0.6953125 \\
\hline $\begin{array}{l}0.701171175 \\
0.70214<4375\end{array}$ & 32581967 & 0.071615 & 261.135898 & $\begin{array}{l}15.253405 \\
15.144268\end{array}$ & $\begin{array}{l}1.1830 E-07 \\
1.1829 E-07\end{array}$ & $\begin{array}{l}32644,405041 \\
32592933553\end{array}$ & $\begin{array}{l}0.703125 \\
0.703125\end{array}$ & $\begin{array}{c}0.59921875 \\
0.201171875\end{array}$ \\
\hline 0.7026367188 & $\begin{array}{l}32632068 \\
32653624\end{array}$ & 0.070721 & $\begin{array}{l}200.3150 \% 4 \\
259.904694\end{array}$ & 25.165783 & 1.1828E.07 & 32567.404087 & 0.703125 & 0.7021484375 \\
\hline 0.702860859 & 3266403 & 0.070572 & 259.69949 & 15.151246 & 1.1828E 407 & 32554.664530 & 0.703125 & 0.702636711875 \\
\hline 0.7027587891 & 32659014 & 0.070646 & 259.102094 & 15.158531 & 1.1222EE+07 & 32561.030965 & 0.70282085938 & 0.702636711575 \\
\hline 0.7022196242 & 32001708 & 0.070609 & 259.750794 & 15.154908 & 1.182SE -07 & 32557.446912 & 0.70281085938 & 0.70275878506 \\
\hline 0.7027893066 & 32060361 & 0.070628 & $259.7 \mathrm{~cm}$ & 15.156719 & 1.182SE+07 & 32559.437729 & 0.70281982422 & 0.70275878906 \\
\hline 0.7028045654 & 32661035 & 0.070619 & 259.763619 & 15.155813 & $1.1822 E+07$ & 32558.42768 & 0.70211912422 & 0.70278930664 \\
\hline 0.7028121948 & 32661372 & 0.070614 & 259.757206 & 15.155361 & 1.1823E+07 & 32558.24487 & 0.70281912422 & 0.70220456543 \\
\hline 0.7028083201 & 32661203 & 0.070616 & 259.760412 & 15.155587 & 1.182SE +07 & 32558.443794 & 0.70281219482 & 0.70220456543 \\
\hline 0.7021102875 & 32661287 & 0.070615 & 259.758209 & 15.155474 & $1.1828 E-07$ & 3255834310 & 0.70281219482 & 0.70280838013 \\
\hline 0.7028112411 & 32661329 & 0.070614 & 289.751008 & 15.155417 & 1.18211E+07 & 32558.294568 & 0.70211219482 & 0.70281028748 \\
\hline 0.702811718 & 32661350 & 0.070614 & 259.757607 & 15.155389 & 1.1821EA07 & 32558.269698 & 0.70281219482 & 0.70281124115 \\
\hline 0.7028119564 & 32661361 & 0.070814 & 259.757407 & 15.155375 & $1.1821 E+07$ & 32558.257262 & 0.70281219482 & 0.70281171799 \\
\hline 0.70281118372 & 32661356 & 0.070814 & 259.757507 & 15.155382 & 1.1824E+07 & 32558.263410 & 0.70281195641 & 0.70281171799 \\
\hline 0.70281118968 & 32661358 & 0.070614 & 259.757457 & 15.155378 & $1.1828 E+07$ & 32538.260371 & 0.70281195641 & 0.7028118372 \\
\hline 0.7028119266 & 32661360 & 0.070614 & 259.757432 & 15.155377 & $1.1828 E-07$ & 32558.258317 & 0.70281195641 & 0.70281118968 \\
\hline 0.702211194 .5 & 32661360 & 0.070614 & 259.757419 & 15.155376 & $1.1822 E+07$ & 32551.258040 & 0.70261195641 & 0.7021119264 \\
\hline 0.7028119341 & 32661360 & 0.070614 & 259.757425 & 15.153376 & $1.1822 E+07$ & 32558.23428 & 0.7028119415 & 0.7028119266 \\
\hline
\end{tabular}




\section{D4. Changes in Matrix Heat Capacity}

The final seven worksheets examine the effoct of matrix heat capacity. The matrix heat capacity is entered in cell B23. The section contains worksheets with the matrix heat capacity ranging from between 0-1 (kJ/kg-K). 
Spreadsheet for Calculation of Stream-Water Saturation Profiles

Injection Temperature $650 \cdot \mathrm{K}$
Inlital Temperature

$300 \cdot \mathrm{K}$
Motrlx Beat Capacily 0.0400 (tJ/kB)

MATIIXPROPERTIES

Porealsy
0.10
$N=$
3.00

Cp Matrix

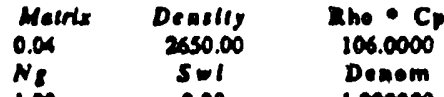

1.000000

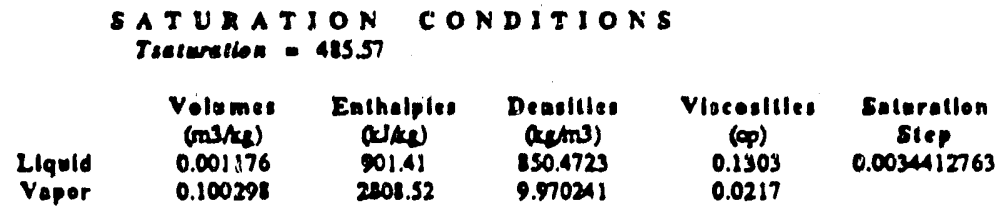

$\begin{array}{ccc} & \text { Temperelure } & \text { Veleclty } \\ \text { Injection } & 650 & 1.000000 \\ \text { Inlitiol } & 300 & 0.055466 \\ & & 6 \\ \text { Injection } & 650 & 6.88 \\ \text { Initial } & 300 & 1004.63\end{array}$

DOUMDARY CONDITIONE

Hear Wove

$\begin{array}{cccc}\text { Cepectiy Velectiy } & \text { Densliy } & \text { Entholpy } \\ 230 & 0.162970 & 6.222648 & 3199.91\end{array}$

$\begin{array}{llll}2.30 & 0.162970 & 6.282648 & 3199.91 \\ 4.16 & 0.451575 & 1004.6293 & 11270\end{array}$

$\begin{array}{cccc}G & F & \text { Gemme } & \text { Thele } \\ 6.88 & 6.88 & \text { (42123.87 } & 22023.87 \\ 1004.63 & 1004.63 & 399418.71 & 113218.71\end{array}$

\begin{tabular}{|c|c|c|c|c|c|c|c|c|}
\hline \multirow[b]{2}{*}{$\begin{array}{l}\text { Trniline } \\
\text { Lendine }\end{array}$} & \multirow[b]{2}{*}{$\begin{array}{c}\text { Inllial } \\
\text { So.HI } \\
1 \\
1\end{array}$} & \multirow[b]{2}{*}{$\begin{array}{c}\text { Gress } \\
5 v \cdot 20= \\
0.5 \\
0.5\end{array}$} & \multirow{2}{*}{$\begin{array}{c}\text { S a O C } \\
\text { Vopor } \\
\text { Soluralion } \\
0.1632 \\
0.6911\end{array}$} & \multicolumn{2}{|c|}{ CONDITI } & \multirow[b]{2}{*}{$\begin{array}{l}\text { Upatream } \\
\text { Flc } \\
\text { Velocliy } \\
0.761219 \\
0.055466\end{array}$} & \multirow[b]{2}{*}{$\begin{array}{c}\text { Shock } \\
\text { velecliy } \\
0.0866 \\
0.5936\end{array}$} & \multirow[b]{2}{*}{$\begin{array}{c}\text { Vopor } \\
\text { Frase Flow } \\
0.9995 \\
0.9930\end{array}$} \\
\hline & & & & $\begin{array}{l}\text { Objectlve } \\
\text { Functlen } \\
\text {-1.4ASIE-09 } \\
\text {-3.400SE-08 }\end{array}$ & $\begin{array}{c}\text { Treephene } \\
\text { Weve } \\
\text { Velocliy } \\
0.0866 \\
0.5936\end{array}$ & & & \\
\hline Inllins & $\begin{array}{l}\text { dKrv/dSr } \\
1.0000 \\
1.0000\end{array}$ & $\begin{array}{c}d \mathrm{Krl} / \mathrm{dSI} \\
0.0562 \\
0.2462\end{array}$ & $\begin{array}{c}\text { Total } \\
\text { Mobllity } \\
39.4665 \\
32.1462\end{array}$ & $\begin{array}{l}\text { dFv/dSv } \\
0.0114 \\
0.0780\end{array}$ & $\begin{array}{c}\mathbf{G} \\
124.0622 \\
2095822\end{array}$ & $\begin{array}{c}P^{\prime} \\
10.3845 \\
15.8449\end{array}$ & $\begin{array}{c}\text { Gammu } \\
\text { S.8229E+0S } \\
7.1938 E+05\end{array}$ & $\begin{array}{l}\text { Theta' } \\
25365.6429 \\
33199.3395\end{array}$ \\
\hline
\end{tabular}

MATERIA\& DALANCES

Inolde
Mous S1.620698
Eathalpy S5852441

Ialilol

20.023 .7721

Predueed

$55.7228 \mathrm{NO}$

6279.7488

Delenee
0.0003
-166.4695

Error

0.00034

0.26865 
Injectlon Temperature $650 \cdot \mathrm{K}$
Inllbl Temperature

$300 \cdot \mathrm{x}$
Matrix Beat Copacliy

0.0400 (kJ/LE)

\section{SATURATION PROFILE}

\begin{tabular}{|c|c|c|c|c|c|c|c|c|}
\hline $\begin{array}{c}\text { Vepor } \\
\text { Sateratlea }\end{array}$ & $\begin{array}{c}\text { Vapor } \\
\text { Frec Plow }\end{array}$ & $\mathrm{Krr} / \mathrm{dSr}$ & dKrVASI & $\begin{array}{l}\text { Total } \\
\text { Mobility }\end{array}$ & dF v/dSV & lambda & $\begin{array}{l}\text { Mase } \\
\text { Inolde }\end{array}$ & $\begin{array}{l}\text { Enthalpy } \\
\text { Inslde }\end{array}$ \\
\hline $\begin{array}{l}1.0000 \\
1.0000\end{array}$ & & & & & & $\begin{array}{l}0.000000 \\
0.086570\end{array}$ & & \\
\hline $\begin{array}{l}0.8632 \\
0.1597\end{array}$ & $\begin{array}{l}0.999507 \\
0.999467\end{array}$ & $\begin{array}{l}1.0000 \\
1.0000\end{array}$ & $\begin{array}{l}0.0562 \\
0.0590\end{array}$ & $\begin{array}{l}39.816544 \\
29.729128\end{array}$ & $\begin{array}{l}0.011373 \\
0.012016\end{array}$ & $\begin{array}{l}0.066570 \\
0.091471\end{array}$ & $\begin{array}{l}0.595832 \\
0.619488\end{array}$ & $\begin{array}{l}55588.783670 \\
2911.204472\end{array}$ \\
\hline $\begin{array}{l}0.8563 \\
0.8529\end{array}$ & $\begin{array}{l}0.99924 \\
0.999380\end{array}$ & $\begin{array}{l}1.0000 \\
1.0000\end{array}$ & $\begin{array}{l}0.0619 \\
0.0649\end{array}$ & $\begin{array}{l}39.571787 \\
39.414525\end{array}$ & $\begin{array}{l}0.012612 \\
0.013369\end{array}$ & $\begin{array}{l}0.096535 \\
0.101764\end{array}$ & $\begin{array}{l}0.644755 \\
0.691302\end{array}$ & $\begin{array}{l}3028.456739 \\
3141.074679\end{array}$ \\
\hline $\begin{array}{l}0.494 \\
0.4460\end{array}$ & $\begin{array}{l}0.999332 \\
0.999283\end{array}$ & $\begin{array}{l}1.0000 \\
1.0000\end{array}$ & $\begin{array}{l}0.0680 \\
0.0712\end{array}$ & $\begin{array}{l}39.257344 \\
39.100244\end{array}$ & $\begin{array}{l}0.014078 \\
0.014809\end{array}$ & $\begin{array}{l}0.107162 \\
0.112730\end{array}$ & $\begin{array}{l}0.729154 \\
0.768342\end{array}$ & $\begin{array}{l}3256.105120 \\
3373.595870\end{array}$ \\
\hline $\begin{array}{l}0.2425 \\
0.8391\end{array}$ & $\begin{array}{l}0.999230 \\
0.999170\end{array}$ & $\begin{array}{l}1.0000 \\
1.0000\end{array}$ & $\begin{array}{l}0.0744 \\
0.0777\end{array}$ & $\begin{array}{l}31.943229 \\
38.786299\end{array}$ & $\begin{array}{l}0.015564 \\
0.016341\end{array}$ & $\begin{array}{l}0.118473 \\
0.124391\end{array}$ & $\begin{array}{l}0.208895 \\
0.050842\end{array}$ & $\begin{array}{l}3493.595740 \\
3616.154566\end{array}$ \\
\hline $\begin{array}{l}0.1357 \\
0.1322\end{array}$ & $\begin{array}{l}0.999118 \\
0.999058\end{array}$ & $\begin{array}{l}1.0000 \\
1.0000\end{array}$ & $\begin{array}{l}0.0810 \\
0.0445\end{array}$ & $\begin{array}{l}38.629457 \\
38.472705\end{array}$ & $\begin{array}{l}0.017142 \\
0.017967\end{array}$ & $\begin{array}{l}0.130488 \\
0.136768\end{array}$ & $\begin{array}{l}0.891214 \\
0.939043\end{array}$ & $\begin{array}{l}3741.323230 \\
3869.153685\end{array}$ \\
\hline $\begin{array}{l}0.8288 \\
0.8253\end{array}$ & $\begin{array}{l}0.998994 \\
0.998928\end{array}$ & $\begin{array}{l}1.0000 \\
1.0000\end{array}$ & $\begin{array}{l}0.0280 \\
0.0915\end{array}$ & $\begin{array}{l}38.3160 \mathrm{~m} \\
38.15947\end{array}$ & $\begin{array}{l}0.014816 \\
0.019690\end{array}$ & $\begin{array}{l}0.143232 \\
0.149183\end{array}$ & $\begin{array}{l}0.985361 \\
1.033200\end{array}$ & $\begin{array}{l}3999.698978 \\
1133.013274\end{array}$ \\
\hline $\begin{array}{l}0.8219 \\
0.814\end{array}$ & $\begin{array}{l}0.998859 \\
0.998786\end{array}$ & $\begin{array}{l}1.0000 \\
1.0000\end{array}$ & $\begin{array}{l}0.0952 \\
0.0989\end{array}$ & $\begin{array}{l}38.003005 \\
37.446630\end{array}$ & $\begin{array}{l}0.020519 \\
0.021513\end{array}$ & $\begin{array}{l}0.156725 \\
0.163761\end{array}$ & $\begin{array}{l}1.082595 \\
1.133579\end{array}$ & $\begin{array}{r}4269.151883 \\
4408.171284\end{array}$ \\
\hline $\begin{array}{l}0.8150 \\
0.8116\end{array}$ & $\begin{array}{l}0.998711 \\
0.992632\end{array}$ & $\begin{array}{l}1.0000 \\
1.0000\end{array}$ & $\begin{array}{l}0.1027 \\
0.1065\end{array}$ & $\begin{array}{l}37.690355 \\
37.534180\end{array}$ & $\begin{array}{l}0.022463 \\
0.023439\end{array}$ & $\begin{array}{l}0.170993 \\
0.17426\end{array}$ & $\begin{array}{l}1.146188 \\
1240458\end{array}$ & $\begin{array}{l}1550.129152 \\
4695.04384\end{array}$ \\
\hline $\begin{array}{l}0.2081 \\
0.2047\end{array}$ & $\begin{array}{l}0.991549 \\
0.994463\end{array}$ & $\begin{array}{l}1.0000 \\
1.0000\end{array}$ & $\begin{array}{l}0.1104 \\
0.1144\end{array}$ & $\begin{array}{l}37.378107 \\
37.222140\end{array}$ & $\begin{array}{l}0.024443 \\
0.025473\end{array}$ & $\begin{array}{l}0.126061 \\
0.193902\end{array}$ & $\begin{array}{l}1.296425 \\
1354127\end{array}$ & $\begin{array}{l}4843.097125 \\
4984.228801\end{array}$ \\
\hline $\begin{array}{l}0.8012 \\
0.7978\end{array}$ & $\begin{array}{l}0.991374 \\
0.991281\end{array}$ & $\begin{array}{l}1.0000 \\
1.0000\end{array}$ & $\begin{array}{l}0.1185 \\
0.1227\end{array}$ & $\begin{array}{l}37.066279 \\
36.910526\end{array}$ & $\begin{array}{l}0.026530 \\
0.027616\end{array}$ & $\begin{array}{l}0.201953 \\
0.210217\end{array}$ & $\begin{array}{l}1.413603 \\
1.474892\end{array}$ & $\begin{array}{l}5148.542144 \\
5306.101222\end{array}$ \\
\hline 0.7944 & 0.998184 & 1.0000 & 0.1269 & 36.75484 & 0.028730 & 0.216698 & 1532035 & 5466.971469 \\
\hline 0.7909 & 0.991083 & 1.0000 & 0.1311 & 36.399354 & 0.029873 & 0.227398 & 1.603071 & 5631.219719 \\
\hline 0.7875 & 0.997978 & 1.0000 & 0.1355 & 36.443938 & 0.031045 & 0.236322 & 1.670045 & 5798.914232 \\
\hline 0.7840 & 0.997869 & 1.0000 & 0.1399 & 36.284638 & 0.032247 & 0.245472 & 1.732998 & 5970.124733 \\
\hline 0.7806 & 0.997756 & 1.0000 & 0.1444 & 36.133456 & 0.033420 & 0.254854 & 1.009975 & 6144.9221 .42 \\
\hline 0.7772 & 0.997639 & 1.0000 & 0.1490 & 35.978993 & 0.034743 & 0.264469 & 1.8183020 & 6323.380105 \\
\hline $\begin{array}{l}0.7737 \\
0.7703\end{array}$ & $\begin{array}{l}0.997517 \\
0.997391\end{array}$ & $\begin{array}{l}1.0000 \\
1.0000\end{array}$ & $\begin{array}{l}0.1536 \\
0.1583\end{array}$ & $\begin{array}{l}35.823452 \\
35.661634\end{array}$ & $\begin{array}{l}0.036037 \\
0.037364\end{array}$ & 0.274323 & 1.958181 & $\begin{array}{l}6505.572037 \\
6691574149\end{array}$ \\
\hline 0.7668 & 0.997260 & 1.0000 & 0.1631 & 35513942 & 0.036722 & 0.294762 & 2115039 & 6891574149 \\
\hline 0.7634 & 0.997124 & 1.0000 & 0.1620 & 35.359376 & 0.040114 & 0.305355 & $21 \% 833$ & 7075.320788 \\
\hline $\begin{array}{l}0.7599 \\
0.7565\end{array}$ & $\begin{array}{l}0.996944 \\
0.996838\end{array}$ & $\begin{array}{l}1.0000 \\
1.0000\end{array}$ & $\begin{array}{l}0.1729 \\
0.1779\end{array}$ & $\begin{array}{l}35.204940 \\
35.050635\end{array}$ & $\begin{array}{l}0.041539 \\
0.042998\end{array}$ & $\begin{array}{l}0.316201 \\
0.327306\end{array}$ & $\begin{array}{l}2.210937 \\
2367404\end{array}$ & $\begin{array}{l}7273.225475 \\
7475.260741\end{array}$ \\
\hline $\begin{array}{l}0.7531 \\
0.7496\end{array}$ & $\begin{array}{l}0.96888 \\
0.9 \% 5332\end{array}$ & $\begin{array}{l}1.0000 \\
1.0000\end{array}$ & $\begin{array}{l}0.1829 \\
0.1881\end{array}$ & $\begin{array}{l}34.596463 \\
34.742425\end{array}$ & $\begin{array}{l}0.04491 \\
0.046020\end{array}$ & $\begin{array}{l}0.336674 \\
0.350309\end{array}$ & $\begin{array}{l}2456387 \\
2447639\end{array}$ & $\begin{array}{l}7681.511067 \\
7892.062770\end{array}$ \\
\hline 0.7462 & 0.996371 & 1.0000 & 0.1933 & Mssess24 & 0.04754 & 0.362216 & 241516 & 107.004042 \\
\hline 0.7427 & 0.996204 & $\begin{array}{l}1.0000 \\
1.0000\end{array}$ & 0.1915 & 34.434761 & 0.049184 & 0.374398 & 2737974 & 8326.424993 \\
\hline $\begin{array}{l}0.7393 \\
0.7359\end{array}$ & $\begin{array}{l}0.996032 \\
0.995855\end{array}$ & $\begin{array}{l}1.0000 \\
1.0000\end{array}$ & $\begin{array}{l}0.2039 \\
0.2093\end{array}$ & $\begin{array}{l}34.211139 \\
34.127659\end{array}$ & $\begin{array}{l}0.050121 \\
0.052496\end{array}$ & $\begin{array}{l}0.346861 \\
0.399609\end{array}$ & $\begin{array}{l}2837073 \\
2938870\end{array}$ & $\begin{array}{l}2550.417702 \\
779.076255\end{array}$ \\
\hline $\begin{array}{l}0.7324 \\
0.7290\end{array}$ & $\begin{array}{l}0.995671 \\
0.995482\end{array}$ & $\begin{array}{l}1.0000 \\
1.0000\end{array}$ & $\begin{array}{l}0.2148 \\
0.2204\end{array}$ & $\begin{array}{l}33.974322 \\
33.821132\end{array}$ & $\begin{array}{l}0.054209 \\
0.055960\end{array}$ & $\begin{array}{l}0.412647 \\
0.425980\end{array}$ & $\begin{array}{l}3.043427 \\
3.150007\end{array}$ & $\begin{array}{l}9012496795 \\
250.77572\end{array}$ \\
\hline 0.7255 & 0.995286 & 1.0000 & 0.2060 & 33.668090 & 0.057751 & 0.439612 & 3.261072 & 494.018988 \\
\hline 0.7221 & 0.995084 & 1.0000 & 0.2317 & 33.515197 & 0.058512 & 0.453549 & 3.374257 & 912323651 \\
\hline 0.718 & 0.994576 & 1.0000 & 0.2075 & 33.362456 & 0.061454 & 0.46797 & 3.490521 & 995.79625 \\
\hline 0.7152 & 0.994661 & 1.0000 & 0.2433 & 13.209269 & 0.063367 & 0.482359 & 3.609839 & 10254.54482 \\
\hline 0.7118 & 0.99440 & 1.0000 & 0.2492 & 53.057436 & 0.065322 & 0.497242 & 3.732314 & 10518.677358 \\
\hline 0.7083 & 0.994211 & 1.0000 & 0.2552 & 32505161 & 0.057320 & 0.512450 & 3.858015 & 10783.307008 \\
\hline 0.7049 & 0.993976 & 1.0000 & 0.2613 & 32753046 & 0.069361 & 0.527990 & 3.907016 & 11063.547865 \\
\hline 0.7014 & 0.993734 & 1.0000 & 0.2674 & 32601091 & 0.071447 & 0.543867 & 4.119392 & 11344516892 \\
\hline 0.6980 & 0.993484 & 1.0000 & 0.2736 & 32449298 & 0.073578 & 0.560087 & 4.255220 & 11631.333650 \\
\hline 0.6946 & 0.993227 & 1.0000 & 0.2799 & 32297671 & 0.075754 & 0.576655 & 4.394577 & 11924.120355 \\
\hline $\begin{array}{l}0.6911 \\
0.0000\end{array}$ & 0.992963 & 1.0000 & 0.2262 & 32.146210 & 0.07797 & $\begin{array}{l}0.593577 \\
0.593577\end{array}$ & 4.537545 & 12223.001941 \\
\hline 0.0000 & & & & & & 1.000000 & & \\
\hline
\end{tabular}


Injection Temperature $650 \cdot \mathrm{K}$
Inlibl Temperalere

sos $x$
Mutrix Beat Cepacliy

0.0400 (WJ/kE)

TRAILING BOCK CALCULATIONG

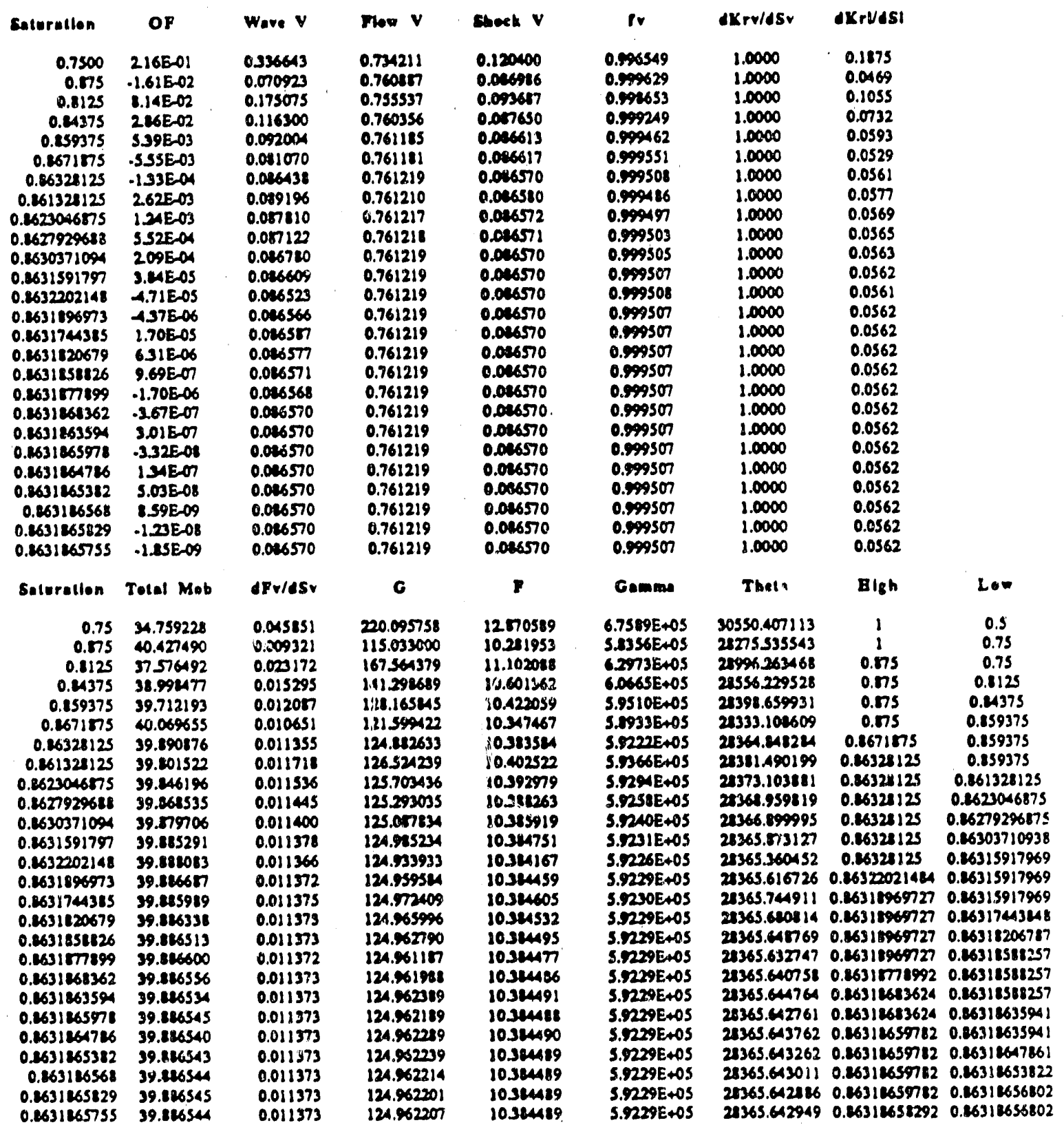


-230 .

Spreadsheet for Calculation of Stream-Water Saturation Profiles

Injecsion Temperalure sso $1 \mathrm{~K}$
Iallal Temperalure $300^{\circ} \mathrm{K}$
Melrix Beal Copoctiy 0.0400 (kJ/he)

LEADING SEOCK CALCULATIONS

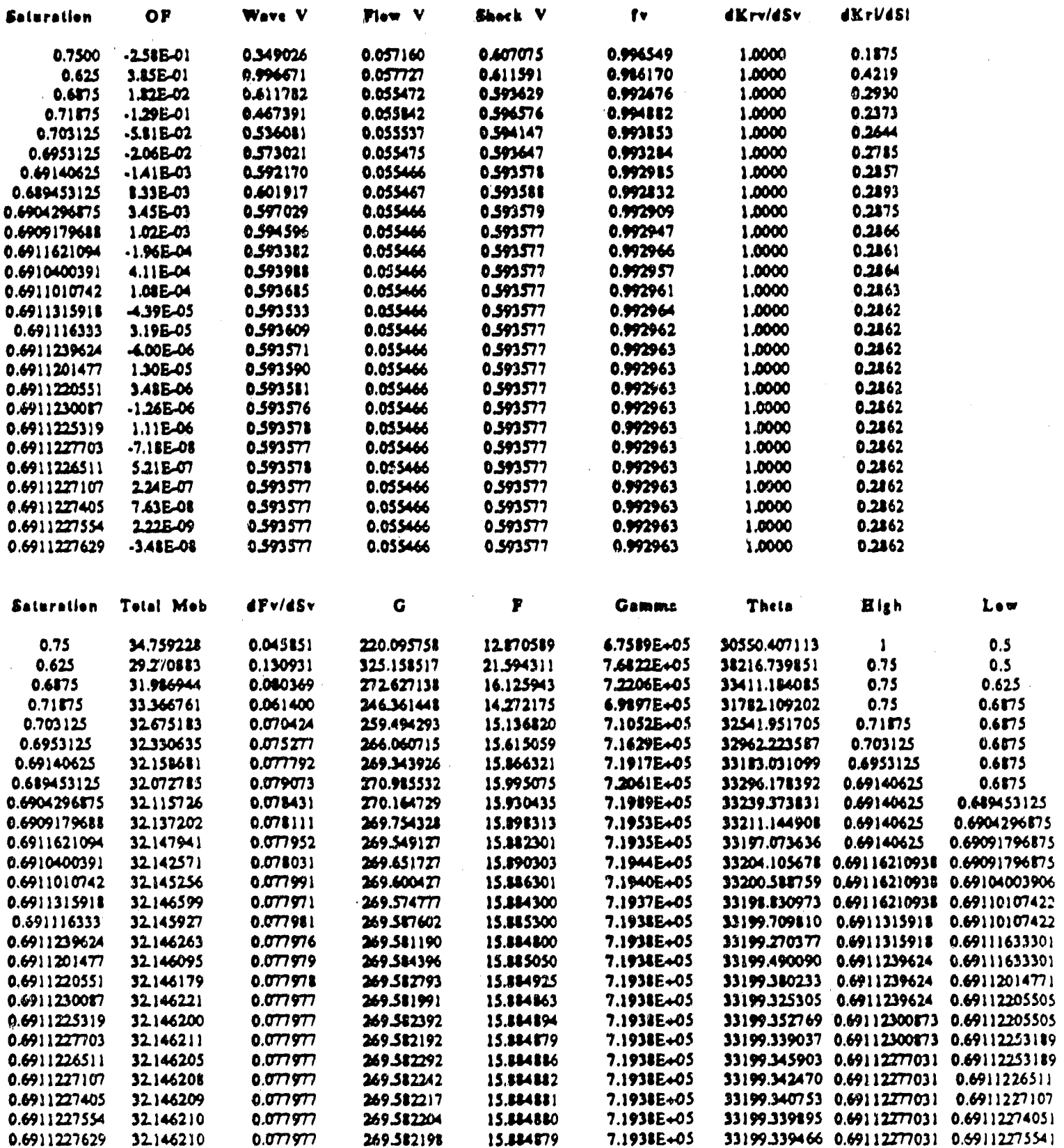


Spreadsheet for Calculation of Stream-Water Saturation Profiles

Injectlon Temperalure $650 \cdot \mathrm{K}$
Initial Temperalure

300 '
Molrix Beal Copoclly $0.2000\left(\mathrm{~kJ} / \mathrm{kg}_{\mathrm{B}}\right)$
MATRIXTROPERTIES

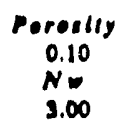

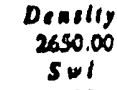

2650.00
$5 w 1$
0.00

Who $\cdot C_{p}$

330.0000

Denem

1.000000

SATURATION CONDITION

\begin{tabular}{|c|c|c|c|c|c|}
\hline 19 & $\begin{array}{c}\text { Volemes } \\
(103 / \mathrm{k}) \\
0.001176\end{array}$ & $\begin{array}{c}\text { Eatholpiss } \\
\text { oukes } \\
901.41\end{array}$ & $\begin{array}{c}\text { Demallies } \\
\text { acumis) } \\
\text { iso.4723 }\end{array}$ & $\begin{array}{c}\text { Visecelitios } \\
\text { (\%p) } \\
0.1303 \\
0.0217\end{array}$ & $\begin{array}{c}\text { saturetion } \\
\text { 8fep } \\
0.0022453538\end{array}$ \\
\hline
\end{tabular}

\begin{tabular}{|c|c|c|c|c|c|c|}
\hline \multicolumn{7}{|c|}{$1 \%$} \\
\hline $\begin{array}{l}\text { Injerilon } \\
\text { Inlilal }\end{array}$ & $\begin{array}{c}\text { Temperafure } \\
650 \\
300\end{array}$ & $\begin{array}{l}\text { Veloclly } \\
1.000000 \\
0.023600\end{array}$ & $\begin{array}{c}\text { copecliy } \\
2.30 \\
4.16\end{array}$ & $\begin{array}{l}\text { Velecliy } \\
0.033025 \\
0.110201\end{array}$ & $\begin{array}{l}\text { Denslty } \\
6.812648 \\
1004.6293\end{array}$ & $\begin{array}{c}\text { Encholpy } \\
3199.91 \\
112.70\end{array}$ \\
\hline $\begin{array}{l}\text { Insectlon } \\
\text { Intilat }\end{array}$ & $\begin{array}{l}650 \\
300\end{array}$ & $\begin{array}{c}\mathbf{c} \\
6.88 \\
1004.63\end{array}$ & $\begin{array}{c}P \\
6.88 \\
1004.63\end{array}$ & $\begin{array}{l}\text { Gamma } \\
3122523.87 \\
1544218.71\end{array}$ & $\begin{array}{l}\text { Thets } \\
22023.07 \\
113218.71\end{array}$ & \\
\hline
\end{tabular}

\begin{tabular}{|c|c|c|c|c|c|c|c|c|}
\hline $\begin{array}{l}\text { Trillins } \\
\text { Ladins }\end{array}$ & $\begin{array}{c}\text { Indinel } \\
\text { Sv.HI } \\
1 \\
1\end{array}$ & $\begin{array}{c}\text { Gueti } \\
\text { Sv.Low } \\
0.5 \\
0.5\end{array}$ & $\begin{array}{c}\text { Vaper } \\
\text { Soturatlen } \\
0.9125 \\
0.1002\end{array}$ & $\begin{array}{l}\text { Objerllve } \\
\text { Funetlon } \\
-1.2549 E-09 \\
6.9074 E-09\end{array}$ & $\begin{array}{c}\text { Twe.phase } \\
\text { Wure } \\
\text { Veloclly } \\
0.0305 \\
0.1897\end{array}$ & $\begin{array}{l}\text { Upstrenm } \\
\text { Flow } \\
\text { Veloclly } \\
0.706498 \\
0.023600\end{array}$ & $\begin{array}{l}\text { Shock } \\
\text { Veloclly } \\
0.0305 \\
0.1897\end{array}$ & $\begin{array}{c}\text { Vupor } \\
\text { Prac Flow } \\
0.9999 \\
0.9983\end{array}$ \\
\hline \multirow[t]{2}{*}{$\begin{array}{l}\text { Trilline } \\
\text { Leading }\end{array}$} & $\begin{array}{l}d X r v / d S v \\
1.0000 \\
1.0000\end{array}$ & $\begin{array}{l}d K r l / d S I \\
0.0230 \\
0.1197\end{array}$ & $\begin{array}{l}\text { Total } \\
\text { Mobllity } \\
421491 \\
37.0199\end{array}$ & $\begin{array}{l}\text { dFv/dSV } \\
0.0043 \\
0.0269\end{array}$ & $\begin{array}{c}\mathbf{G} \\
13.5243 \\
177.8856\end{array}$ & $\begin{array}{c}F^{\prime} \\
10.0728 \\
11.3599\end{array}$ & $\begin{array}{c}\text { Gamma } \\
\text { 24088E+06 } \\
\text { 24917E+06 }\end{array}$ & $\begin{array}{l}\text { Thelo' } \\
28091.7631 \\
29222.8569\end{array}$ \\
\hline & $\begin{array}{c}\text { Mass } \\
\text { Enthalpy }\end{array}$ & $\begin{array}{c}\text { Innide } \\
83.636756 \\
1738015362\end{array}$ & $\begin{array}{c}\text { Inltial } \\
100.462933 \\
15421.8712\end{array}$ & $\begin{array}{l}\text { Injecled } \\
6.882648 \\
202023.0721\end{array}$ & $\begin{array}{l}\text { Produced } \\
23.708774 \\
2671.9077\end{array}$ & $\begin{array}{c}\text { Eelence } \\
0.0001 \\
-33.7006\end{array}$ & $\begin{array}{l}\text { Error } \\
0.0000 \% \\
0.0191 \%\end{array}$ & \\
\hline
\end{tabular}


$-232$.

Spreadsheet for Calculation of Stream-Water Saturation Profiles

Injection Temperalure

$630 \cdot \mathbf{K}$
Inlilal Temperalure

$300 \cdot \boldsymbol{x}$
Matrix Beal Capacliy

0.2000 (kJ/kg)

SATURATION PROFILE

\begin{tabular}{|c|c|c|c|c|c|c|c|c|}
\hline $\begin{array}{c}\text { Vopor } \\
\text { Suturation }\end{array}$ & $\begin{array}{c}\text { Vapor } \\
\text { Prac Plew }\end{array}$ & $\mathrm{dKr} / \mathrm{dSV}$ & dKrVASI & $\begin{array}{c}\text { Tolal } \\
\text { Mobllity }\end{array}$ & $d F \nabla / d S v$ & lambda & $\begin{array}{l}\text { Mass } \\
\text { Inside }\end{array}$ & $\begin{array}{l}\text { Enihalpy } \\
\text { Inclde }\end{array}$ \\
\hline $\begin{array}{l}1.0000 \\
1.0000\end{array}$ & & & & & & $\begin{array}{l}0.000000 \\
0.030501\end{array}$ & & \\
\hline $\begin{array}{l}0.9125 \\
0.9102\end{array}$ & $\begin{array}{l}0.999878 \\
0.999668\end{array}$ & $\begin{array}{l}1.0000 \\
1.0000\end{array}$ & $\begin{array}{l}0.0230 \\
0.0212\end{array}$ & $\begin{array}{l}42149052 \\
42045755\end{array}$ & $\begin{array}{l}0.004318 \\
0.004557\end{array}$ & $\begin{array}{l}0.030504 \\
0.032196\end{array}$ & $\begin{array}{l}0.109447 \\
0.142920\end{array}$ & $\begin{array}{l}95248.933937 \\
4079.181063\end{array}$ \\
\hline 0.9080 & 0.999857 & 1.0000 & 0.0254 & 41.942478 & 0.00480 & 0.033939 & 0.150571 & 4208.588211 \\
\hline 0.9058 & 0.999846 & 1.0000 & 0.0266 & 41.139223 & 0.005098 & 0.035735 & 0.15462 & 4395.385193 \\
\hline $\begin{array}{l}0.9035 \\
0.9013\end{array}$ & $\begin{array}{l}0.999835 \\
0.999123\end{array}$ & $\begin{array}{l}1.0000 \\
1.0000\end{array}$ & $\begin{array}{l}0.0279 \\
0.0292\end{array}$ & $\begin{array}{l}41.735990 \\
41.632720\end{array}$ & $\begin{array}{l}0.005320 \\
0.005589\end{array}$ & $\begin{array}{l}0.037584 \\
0.039485\end{array}$ & $\begin{array}{l}0.166595 \\
0.174974\end{array}$ & $\begin{array}{l}4465581443 \\
4597214601\end{array}$ \\
\hline 0.5990 & 0.999110 & 1.0000 & 0.0306 & 41.529592 & 0.005166 & 0.041440 & 0.183603 & 4730.280314 \\
\hline 0.8968 & 0.99979 & 1.0000 & 0.0320 & 41.426428 & 0.008150 & 0.043450 & 0.192485 & 4R64.103237 \\
\hline 0.8945 & 0.999782 & 1.0000 & 0.0334 & 41.323288 & 0.006442 & 0.045514 & 0.201623 & 3000.200041 \\
\hline 0.0923 & 0.999767 & 1.0000 & 0.0348 & 41.220172 & 0.006742 & 0.047633 & 0.211022 & 3.38 .284410 \\
\hline 0.1900 & 0.999752 & 1.0000 & 0.0363 & 41.117081 & 0.001050 & 0.049809 & 0.220685 & 5277.246049 \\
\hline 0.8878 & 0.999736 & 1.0000 & 0.0378 & 41.014016 & 0.007366 & 0.052040 & 0.230615 & 5417.810882 \\
\hline 0.8855 & 0.999119 & 1.0000 & 0.0393 & 40.91097 & 0.007690 & 0.054329 & 0.240817 & 5559.881060 \\
\hline 0.8833 & 0.999701 & 1.0000 & 0.0409 & 40.207964 & 0.008022 & 0.056675 & 0.251294 & 5703.514961 \\
\hline $\begin{array}{l}0.8811 \\
0.7788\end{array}$ & $\begin{array}{l}0.999683 \\
0.999663\end{array}$ & $\begin{array}{l}1.0000 \\
1.0000\end{array}$ & 0.0424 & $\begin{array}{l}40.704979 \\
40.602021\end{array}$ & 0.008362 & 0.059079 & 0.262050 & S448.731192 \\
\hline 0.5766 & $0.99 \% 643$ & 1.0000 & 0.0457 & $\begin{array}{l}40.002021 \\
40.499091\end{array}$ & $\begin{array}{l}0.008711 \\
0.002068\end{array}$ & $\begin{array}{l}0.061542 \\
0.064064\end{array}$ & $\begin{array}{l}0.273089 \\
0.224116\end{array}$ & $\begin{array}{l}5995.548596 \\
6143.926254\end{array}$ \\
\hline 0.8743 & $0.99 \times 623$ & 1.0000 & 0.0474 & 40.396190 & 0.009433 & 0.066645 & 0.296034 & 6294.063485 \\
\hline 0.8721 & 0.999601 & 1.0000 & 0.0491 & 40.293318 & 0.009807 & 0.069257 & 0.307947 & 645.799852 \\
\hline 0.1698 & 0.999579 & 1.0000 & 0.0508 & 40.190476 & 0.010190 & 0.071991 & 0.320159 & 6599.215167 \\
\hline 0.6676 & 0.999555 & 1.0000 & 0.0526 & 40.087664 & 0.010581 & 0.074755 & 0.332676 & 6754.329490 \\
\hline 0.8653 & 0.999531 & 1.0000 & 0.0544 & 39.944883 & 0.010981 & 0.077582 & 0.345501 & 6911.163137 \\
\hline 0.6631 & 0.999506 & 1.0000 & 0.0562 & 39.882133 & 0.011390 & 0.020472 & 0.351638 & 7069.733679 \\
\hline 0.2608 & 0.999480 & 1.0000 & 0.0581 & 39.79415 & 0.011808 & 0.083426 & 0.372092 & 7230.070951 \\
\hline 0.1586 & 0.999453 & 1.0000 & 0.0600 & 39.676729 & 0.012235 & 0.026443 & 0.385868 & 7392.157049 \\
\hline 0.8564 & $0.99 \times 425$ & 1.0000 & 0.0619 & 39.574076 & 0.012672 & 0.019526 & 0.399969 & 7556.106339 \\
\hline 0.8341 & 0.999396 & 1.0000 & 0.0639 & 39.471456 & 0.013117 & 0.092674 & 0.414402 & 721,850459 \\
\hline 0.8519 & 0.999366 & 1.0000 & 0.0658 & 39.368871 & 0.013572 & 0.095887 & 0.429170 & 7889.411322 \\
\hline 0.1496 & 0.999335 & 1.0000 & 0.0678 & 39.266319 & 0.014037 & 0.099168 & $0.4 \times 1472$ & $\cos 8.901121$ \\
\hline 0.8474 & 0.999303 & 1.0000 & 0.0699 & 99.163803 & 0.014510 & 0.102516 & 0.459731 & 8230.252332 \\
\hline 0.2451 & 0.999270 & 1.0000 & 0.0720 & 39.001322 & 0.014994 & 0.105932 & 0.475534 & 403.517717 \\
\hline 0.8429 & 0.999236 & 1.0000 & 0.0741 & 38.95887 & 0.015487 & 0.109418 & 0.491692 & 2578.720331 \\
\hline 0.8406 & 0.999200 & 1.0000 & 0.0762 & 38.256468 & 0.015990 & 0.112972 & 0.508211 & 7755.0183523 \\
\hline 0.8324 & 0.999164 & 1.0000 & 0.014 & 31.754097 & 0.016504 & 0.116597 & 0.525004 & coss.030942 \\
\hline 0.0361 & 0.999126 & 1.0000 & 0.0205 & 38.651763 & 0.017027 & 0.120293 & 0.542341 & 9116.186540 \\
\hline 0.1339 & 0.999087 & 1.0000 & 0.0828 & 38.549467 & 0.017560 & 0.124060 & 0.559978 & 9299.374578 \\
\hline 0.8317 & $0.99 \% 047$ & 1.0000 & 0.0450 & 38.447210 & 0.011103 & 0.127900 & 0.577919 & AMA.619626 \\
\hline 0.8294 & 0.999006 & 1.0000 & 0.0773 & 38.344991 & 0.011657 & 0.131813 & 0.596387 & 9671.846575 \\
\hline 0.8272 & 0.998964 & 1.0000 & 0.0896 & 38.242813 & 0.019222 & 0.135800 & 0.615177 & 1661320632 \\
\hline 0.1249 & 0.998920 & 1.0000 & 0.0920 & 38.140674 & 0.019797 & 0.139862 & 0.634365 & 10052947332 \\
\hline 0.2227 & 0.998875 & 1.0000 & 0.0943 & 38.038576 & 0.020382 & 0.143999 & 0.653956 & 10246.672541 \\
\hline 0.2204 & 0.998828 & 1.0000 & 0.0967 & 37.936520 & 0.020978 & 0.148213 & 0.673956 & 10442582455 \\
\hline 0.8182 & 0.998781 & 1.0000 & 0.0992 & 37.034505 & 0.021566 & 0.152503 & 0.694372 & 10640.703615 \\
\hline 0.8159 & 0.988731 & 1.0000 & 0.1016 & 37.732532 & 0.022204 & 0.156871 & 0.715209 & 10441.062901 \\
\hline 0.8137 & 0.998681 & 1.0000 & 0.1041 & 37.630602 & 0.022833 & 0.161318 & 0.736473 & 11043.687544 \\
\hline 0.8114 & 0.902629 & 1.0000 & 0.1067 & 37.528715 & 0.023474 & 0.165244 & 0.758171 & 11248.605129 \\
\hline $0 . \cos 2$ & 0.998575 & 1.0000 & 0.1092 & 37.426872 & 0.024126 & 0.170451 & 0.720308 & 11455.043599 \\
\hline 0.0070 & 0.998520 & 1.0000 & 0.1118 & 37.325073 & 0.024790 & 0.175138 & 0.202891 & 11665.431261 \\
\hline 0.0047 & 0.992464 & 1.0000 & 0.1144 & 37.223319 & 0.025465 & 0.179908 & 0.825927 & 11077.396791 \\
\hline 0.2025 & 0.998406 & 1.0000 & 0.1171 & 37.121611 & 0.026152 & 0.14760 & 0.049421 & 12091.769239 \\
\hline $\begin{array}{l}0.1002 \\
0.0000\end{array}$ & 0.998447 & 1.0000 & 0.1197 & 37.019918 & 0.026850 & $\begin{array}{l}0.189696 \\
0.119696\end{array}$ & 0.773381 & 12308.578034 \\
\hline 0.0000 & & & & & & 1.000000 & & \\
\hline
\end{tabular}




$$
-233 .
$$

Spreadsheet for Calculation of Stream-Water Saturation Profiles

Injection Temperature $650 \cdot \mathrm{K}$
Inlilal Temperature

$300 \cdot x$
Motrix Beot Capoclly 0.2000 (kJ/ke)

TRAILING SBOCK CALCULATIONS

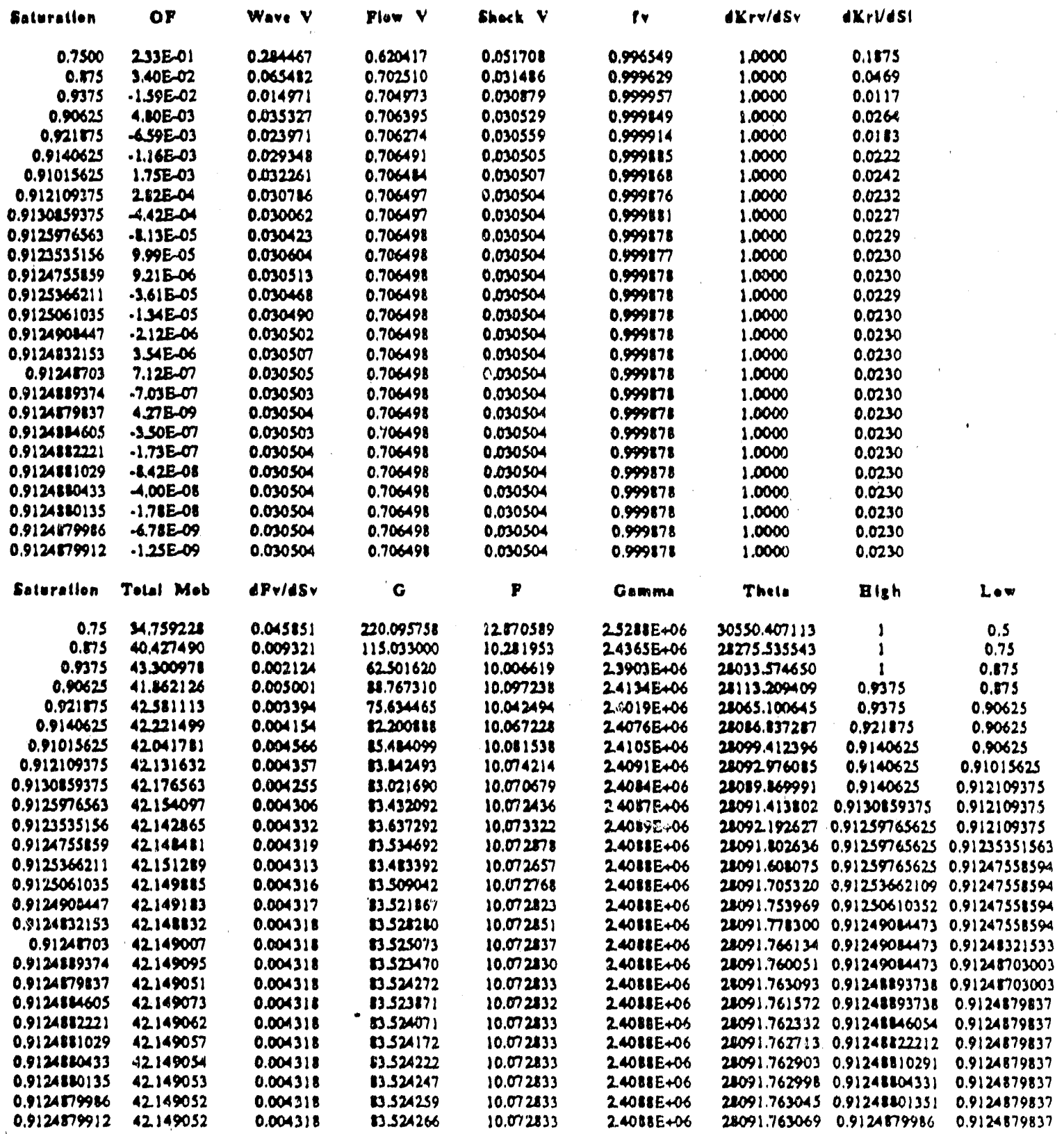


Injectlon Temperalure $650 \cdot \mathrm{K}$
Inlilal Temperature $300^{\circ} x$
Malrix Deal Copocliy 0.2000 (kJ/kg)

LEADING EROCK CALCULATIONS

\begin{tabular}{|c|c|c|c|c|c|c|c|c|}
\hline Saturallen & OP & Wave $V$ & Flow V & Cheek V & Ir & dKrv/dSv & $d K r L d s \mid$ & \\
\hline $\begin{array}{l}0.7500 \\
0.775\end{array}$ & $\begin{array}{r}\text { 1.J2E-01 } \\
-1.27 \mathrm{E}-0 \mid\end{array}$ & $\begin{array}{l}0.322936 \\
0.065154\end{array}$ & $\begin{array}{l}0.024013 \\
0.024323\end{array}$ & $\begin{array}{l}0.191599 \\
0.193020\end{array}$ & $\begin{array}{l}0.926549 \\
0.99 \% 629\end{array}$ & $\begin{array}{l}1.0000 \\
1.0000\end{array}$ & $\begin{array}{l}0.1875 \\
0.0469\end{array}$ & \\
\hline 0.8125 & $.261 \mathrm{E}-02$ & 0.163712 & 0.023622 & 0.189797 & 0.996553 & 1.0000 & 0.1055 & \\
\hline 0.78123 & 4.49E,-02 & 0.234662 & 0.023655 & 0.109952 & 0.997778 & 1.0000 & 0.1436 & \\
\hline 0.796875 & 7SOE-03 & 0.197207 & 0.023601 & 0.109704 & 0.991253 & 1.0000 & 0.1238 & \\
\hline 0.2046875 & $-9.75 E_{-}-03$ & 0.179958 & 0.023602 & 0.189710 & 0.99463 & 1.0000 & 0.1144 & \\
\hline 0.20001125 & $-1.24 \mathrm{E}-\mathrm{CB}$ & 0.126455 & 0.023600 & 0.129696 & 0.994362 & 1.0000 & 0.1191 & \\
\hline 0.798128125 & $3.20 \mathrm{E}-03$ & 0.192799 & 0.023600 & 0.189697 & 0.091309 & 1.0000 & 0.1214 & \\
\hline $\begin{array}{l}0.7992046575 \\
0.2002929618\end{array}$ & $9.23 \mathrm{E}-\mathrm{OA}$ & 0.190619 & 0.023600 & 0.189696 & 0.991335 & 1.0000 & 0.1202 & \\
\hline 0.2002929688 & $\begin{array}{r}-1.61 \mathrm{EOA} \\
3.10 \mathrm{E}-\mathrm{OA}\end{array}$ & $\begin{array}{l}0.119535 \\
0.190076\end{array}$ & $\begin{array}{l}0.023600 \\
0.023600\end{array}$ & $\begin{array}{l}0.189696 \\
0.189696\end{array}$ & $\begin{array}{l}0.998349 \\
0.998342\end{array}$ & $\begin{array}{l}1.0000 \\
1.0000\end{array}$ & $\begin{array}{l}0.1196 \\
0.1199\end{array}$ & \\
\hline 0.200170894 & $1.09 \mathrm{E}-04$ & 0.189105 & 0.023600 & 0.109696 & 0.991345 & 1.0000 & 0.1198 & \\
\hline 0.2002319336 & $.260 \mathrm{E}-0 \mathrm{~S}$ & 0.189670 & 0.023600 & 0.189696 & 0.998347 & 1.0000 & 0.1197 & \\
\hline 0.200201416 & $4.16 \mathrm{E}-\mathrm{OS}$ & 0.189738 & 0.023600 & 0.119696 & 0.998346 & 1.0000 & 0.1190 & \\
\hline 0.2002166748 & $7.81 E-06$ & 0.119704 & 0.023600 & 0.189696 & 0.991346 & 1.0000 & 0.1197 & \\
\hline $0.10022 \times 3012$ & $-9.11 E-06$ & 0.189687 & 0.023600 & 0.189696 & 0.998347 & 1.0000 & 0.1197 & \\
\hline 0.2002204895 & $-6.51 E-07$ & 0.189695 & 0.023600 & 0.109696 & 0.998347 & 1.0000 & 0.1197 & \\
\hline 0.2002125822 & 3.58E-06 & 0.189700 & 0.023600 & 0.109696 & 0.998347 & 1.0000 & 0.1197 & \\
\hline 0.2002195358 & 8.46E-06 & 0.189898 & 0.023600 & 0.189696 & 0.998347 & 1.0000 & 0.1197 & \\
\hline 0.2002200127 & 4.06E-OI & 0.119696 & 0.023600 & 0.109696 & 0.991347 & 1.0000 & 0.1197 & \\
\hline 0.0002202311 & $-1.23 E-09$ & $0.18 \% 6 \%$ & 0.0236000 & 0.189696 & 0.098347 & 1.0000 & 0.1197 & \\
\hline $\begin{array}{l}0.1002201319 \\
0.1002201915\end{array}$ & $\begin{array}{l}1.42 E-07 \\
9.61 E-099\end{array}$ & $\begin{array}{l}0.189696 \\
0.119696\end{array}$ & $\begin{array}{l}0.023600 \\
0.023600\end{array}$ & $\begin{array}{l}0.189696 \\
0.189696\end{array}$ & $\begin{array}{l}0.998347 \\
0.998347\end{array}$ & $\begin{array}{l}1.0000 \\
1.0000\end{array}$ & $\begin{array}{l}0.1197 \\
0.1197\end{array}$ & \\
\hline 0.1002202213 & $-5.65 \mathrm{E}-08$ & 0.119696 & 0.023600 & 0.109696 & 0.991347 & 1.0000 & 0.1197 & \\
\hline 0.0002202044 & $-234 E-08$ & 0.109696 & 0.023600 & 0.189696 & 0.991347 & 1.0000 & 0.1197 & \\
\hline 0.2002201919 & $-6.91 E-09$ & 0.109696 & 0.023600 & 0.189696 & 0.991347 & 1.0000 & 0.1197 & \\
\hline Soturallen & Total Mob & APv/ASv & C & $P$ & Cammon & Thate & Bleh & Low \\
\hline 0.73 & 34.739228 & 0.045851 & 220.095751 & 12510519 & $25218 E 406$ & 30550.407113 & 1 & s \\
\hline 0.875 & 40.427490 & 0.000321 & 115.033000 & 10.211953 & 2.436SE+06 & 22275.535543 & $i$ & 0.75 \\
\hline 0.8125 & 37.576492 & 0.023172 & 167.561379 & 01.102088 & 24827E+06 & 28996.263468 & 0.675 & $0.75^{\circ}$ \\
\hline 0.78125 & 36.162941 & 0.033213 & 193.830069 & 11.837827 & 25057E-06 & 296128221736 & 0.8125 & $\begin{array}{c}0.75 \\
0.7119\end{array}$ \\
\hline $\begin{array}{l}0.796879 \\
0.804675\end{array}$ & $\begin{array}{l}36.264574 \\
37.222259\end{array}$ & $\begin{array}{l}0.027913 \\
0.024972\end{array}$ & 100.697224 & 11.436916 & 249428406 & 29290.507675 & 0.8125 & $\begin{array}{l}0.78125 \\
0.796 \mathrm{~ns}\end{array}$ \\
\hline 0.100011125 & $\begin{array}{l}377222259 \\
37.049346\end{array}$ & $\begin{array}{l}0.025472 \\
0.026675\end{array}$ & $\begin{array}{l}174.130101 \\
177 A 14013\end{array}$ & $\begin{array}{l}11.28172\} \\
11347315\end{array}$ & $\begin{array}{l}248614 E+06 \\
2.4913 E+06\end{array}$ & $\begin{array}{l}29136.547240 \\
29211.764167\end{array}$ & $\begin{array}{c}0.8125 \\
0.8046775\end{array}$ & $\begin{array}{l}0.796775 \\
0.796775\end{array}$ \\
\hline 0.791828125 & 36.956943 & 0.027229 & 179.055618 & 11.391607 & 24924E+06 & 29250.690295 & 0.10071125 & 0.796775 \\
\hline 0.7991046775 & 37.001140 & 0.026981 & 178.234815 & 11.369339 & 24020E+06 & 29231.1117508 & 0.00071125 & $0.79612812 \mathrm{~s}$ \\
\hline $\begin{array}{l}0.1002929681 \\
0.8000418281\end{array}$ & 37.023242 & 0.026127 & 177.82414 & 11.351293 & 249178406 & 20221.414371 & 0.0007125 & 0.7990046875 \\
\hline 0.0000418281 & $\begin{array}{l}37.012191 \\
37.017717\end{array}$ & $\begin{array}{l}0.026904 \\
0.026266\end{array}$ & $\begin{array}{l}178.02 \% 15 \\
177.927014\end{array}$ & 11,363208 & $\begin{array}{l}249108406 \\
249118+06\end{array}$ & 20226.259023 & Q.c002029677s & \\
\hline 0.2002319336 & 37.020479 & O.R2547 & 179.575714 & $1195 \times 70$ & 249178406 & $\begin{array}{l}32223.1424969 \\
29222624239\end{array}$ & $\begin{array}{l}0.200292 \% 6175 \\
0.20029246175\end{array}$ & $\begin{array}{l}0.000046128113 \\
0.00017089844\end{array}$ \\
\hline 0.800201416 & 37.019098 & 0.026856 & 177.0134 & $113 \cos 59$ & 24917E+06 & 20223.229486 & 0.10023193359 & 0.0001706984 \\
\hline 0.2002166741 & 37.019789 & 0.026131 & 177.818539 & 11360014 & $249178+06$ & 20222926140 & 0.20023193359 & 0.20020141602 \\
\hline 0.0002243042 & 37.020134 & 0.02549 & 177.882127 & 1135942 & 24917E,06 & 29222775513 & 0.20023173359 & $\begin{array}{l}0.0002166741 \\
0.0002166748\end{array}$ \\
\hline 0.0002185822 & $\begin{array}{l}37.01961 \\
37.019875\end{array}$ & $\begin{array}{l}0.026150 \\
0.026151\end{array}$ & $\begin{array}{l}177.835333 \\
177.846936\end{array}$ & $\begin{array}{l}11.355021 \\
11.355071\end{array}$ & $\begin{array}{l}299178+06 \\
24917 E+06\end{array}$ & 292221051185 & 0.2002243042 & 0.2002166748 \\
\hline 0.10002195351 & 37.019918 & 0.026150 & i77.6e6135 & iIsscoso & 24917 E.06 & 292221770099 & $\begin{array}{l}0.1002204185 \\
0.0002204095\end{array}$ & $\begin{array}{l}0.2002167111 \\
0.80021858215\end{array}$ \\
\hline 0.0002200127 & 37.01940 & 0.028850 & 177.88573 & 11.359939 & 24917E+06 & 27222200642 & 0.0002204895 & 0.00021253583 \\
\hline 0.0002201319 & $\begin{array}{l}\$ 7.019931 \\
37.01945\end{array}$ & $\begin{array}{l}0.026150 \\
0.026150\end{array}$ & $\begin{array}{l}177.0185333 \\
177.085634\end{array}$ & 11.35993 & 249176.06 & 202221155913 & 0.8002204893 & 0.00022001266 \\
\hline 0.2002201915 & $\begin{array}{l}37.016045 \\
37.019948\end{array}$ & $\begin{array}{l}0.006850 \\
0.026150\end{array}$ & $\begin{array}{l}177.885634 \\
177.805583\end{array}$ & $\begin{array}{l}\text { II.j35936 } \\
\text { Ii.s5993s }\end{array}$ & $\begin{array}{l}249178+06 \\
249178+06\end{array}$ & $\begin{array}{l}29222858271 \\
29222857095\end{array}$ & 0.10022025104 & $\begin{array}{l}0.00022001266 \\
0.00022013117\end{array}$ \\
\hline 0.0002202213 & $37.01 \% 49$ & 0.026150 & i77.86ssss: & IIIs9934 & $24917 E+06$ & 29222156504 & 0.80023025101 & 0.20022010148 \\
\hline 0.0002202064 & 37.019949 & 0.026850 & 177.81557! & IIssposs & 24917E-06 & 29222856100 & 0.00022022128 & 0.00022019144 \\
\hline 0.0002201989 & 37.019948 & 0.026150 & 177.815577 & 11.359935 & $2.49176+08$ & 292221156248 & 0.80022020631 & 0.20022019141 \\
\hline
\end{tabular}


Spreadsheet for Calculation of Stream-hater Saturation profiles

Injectlon Temperature $650 \cdot x$
Inlilal Temperature $300^{\circ} \mathrm{K}$
Matrix Eeat Capacily $1.0000(\mathrm{~kJ} / \mathrm{kt})$

\begin{tabular}{|c|c|c|c|c|}
\hline & & $A T R \backslash X$ & PRO & $\mathrm{R} T \mathrm{ES}$ \\
\hline $\begin{array}{c}\text { Poroslily } \\
0.10 \\
N . \\
3.00\end{array}$ & $c_{p}$ & $\begin{array}{l}\text { Mairlx } \\
1.00 \\
N 8 \\
1.00\end{array}$ & $\begin{array}{c}\text { Dewally } \\
2650.00 \\
S w 1 \\
0.00\end{array}$ & $\begin{array}{l}\text { Rho CF } \\
2650,0000 \\
\text { Denom } \\
1.000000\end{array}$ \\
\hline
\end{tabular}

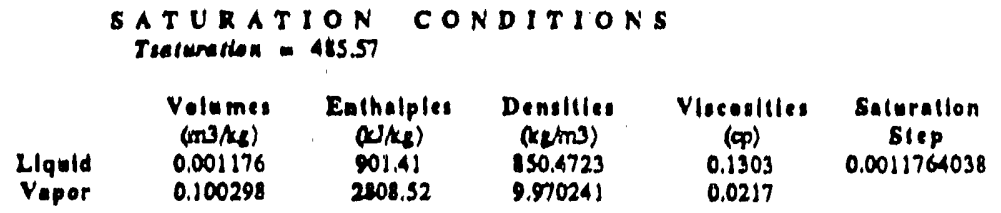

DOUNDARY CONDITIONS

Heal

Capacily

Velocliy

$2.30 \quad 0.006622$

0.006622
0.015992

Denally

6.882648

1004.6293

Enthalpy

3199.91

0.010726

C.88

1004.63
P Gomm.

$6.18 \quad 15524523.87$

1004.63

7268218.71
Thete.

2023.87

113218.71

\begin{tabular}{|c|c|c|c|c|c|}
\hline \multirow{2}{*}{$\begin{array}{l}\text { S B O C X } \\
\text { Vapor } \\
\text { solurallon } \\
0.9570 \\
0.0981\end{array}$} & \multicolumn{2}{|c|}{ CONDITIONS } & \multirow[b]{2}{*}{$\begin{array}{l}\text { Upolrenm } \\
\text { Flom } \\
\text { Velocliy } \\
0.692182 \\
0.010726\end{array}$} & \multirow[b]{2}{*}{$\begin{array}{l}\text { Shock } \\
\text { Velocliy } \\
0.0068 \\
0.0414\end{array}$} & \multirow[b]{2}{*}{$\begin{array}{c}\text { Vapor } \\
\text { Frac Flow } \\
1.0000 \\
0.9998\end{array}$} \\
\hline & $\begin{array}{l}\text { Objecilve } \\
\text { funcllon } \\
1.4356 \mathrm{E}-09 \\
4.0467 \mathrm{E}-09\end{array}$ & $\begin{array}{c}\text { Two.Phese } \\
\text { Wave } \\
\text { velocliy } \\
0.0068 \\
0.0414\end{array}$ & & & \\
\hline $\begin{array}{c}\text { Total } \\
\text { Mobllity } \\
4.1912 \\
41.4190\end{array}$ & $\begin{array}{c}d P v / d S v \\
0.0010 \\
0.0060\end{array}$ & $\begin{array}{c}\mathbf{G} \\
46.1514 \\
95.5899\end{array}$ & $\begin{array}{c}F^{\prime} \\
9.9819 \\
10.1346\end{array}$ & $\begin{array}{l}\text { Camma } \\
1.1611 E+07 \\
1.16 \mathrm{ME}+07\end{array}$ & $\begin{array}{c}\text { Thelo' } \\
22(1) 11.8389 \\
28146.0687\end{array}$ \\
\hline
\end{tabular}

MATERIAL IALANCES

Mass 065999 Encholyy 747635.0622

Inllial 100,462933 726821.8712

Invecied 6.812648 22023.7721
Prodused

10.773512

$\begin{array}{cc}\text { Dolance } & \text { Error } \\ 0.0000 & 0.0000 \% \\ .3 .6947 & 0.0005 \%\end{array}$


.236.

Spreadsheet for Calculation of Stream-Water Saturation Proflies

Injection Temperature $650 \cdot \mathrm{K}$
Inlibat Temperalure

$300^{\circ} \mathrm{x}$
Matrlx Beal Capacliy

1.0000 (kJ/kE)

SATURATION PROFILE

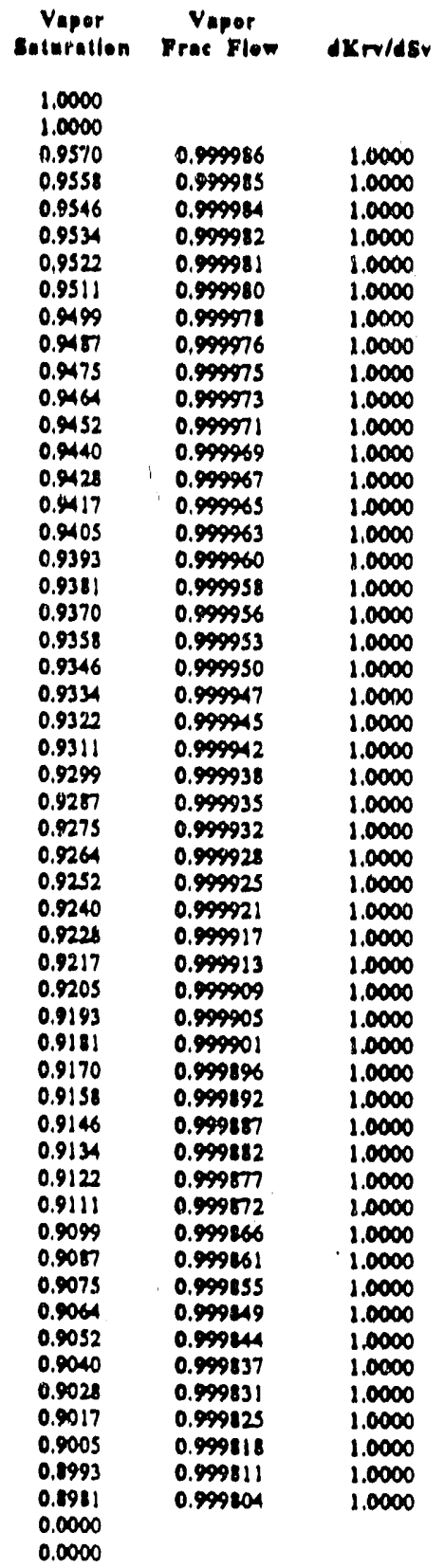

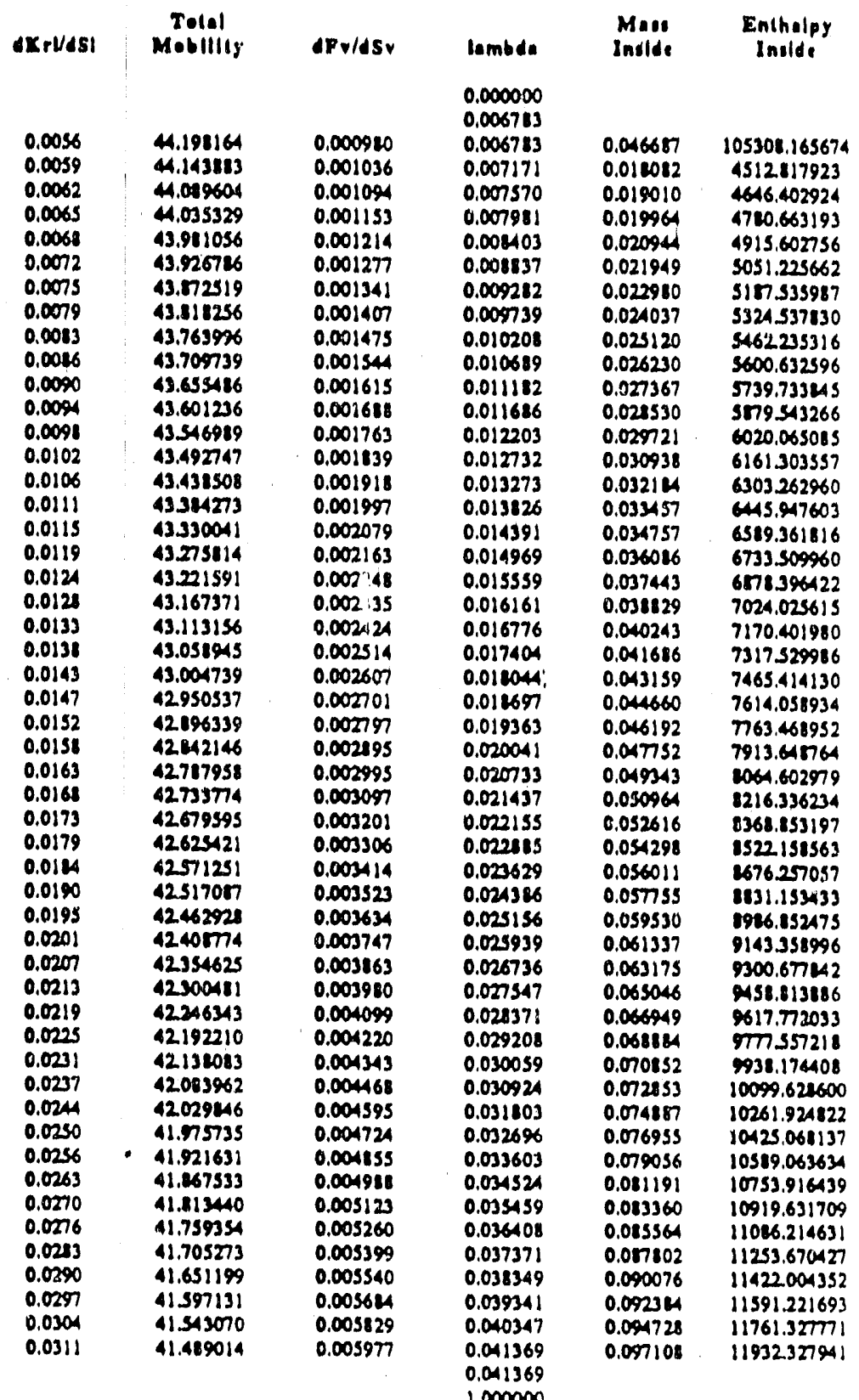


Spreadsheet for Calculation of Stream-Water Saturation Profiles

Injeclion Temperature $680 \cdot \mathrm{K}$
Intilal Temperature

soo ons
Matrix Bual Copacliy

$1.0000\left(x_{J} / k_{2}\right)$

TRAILING SBOCK CALCULATIONS

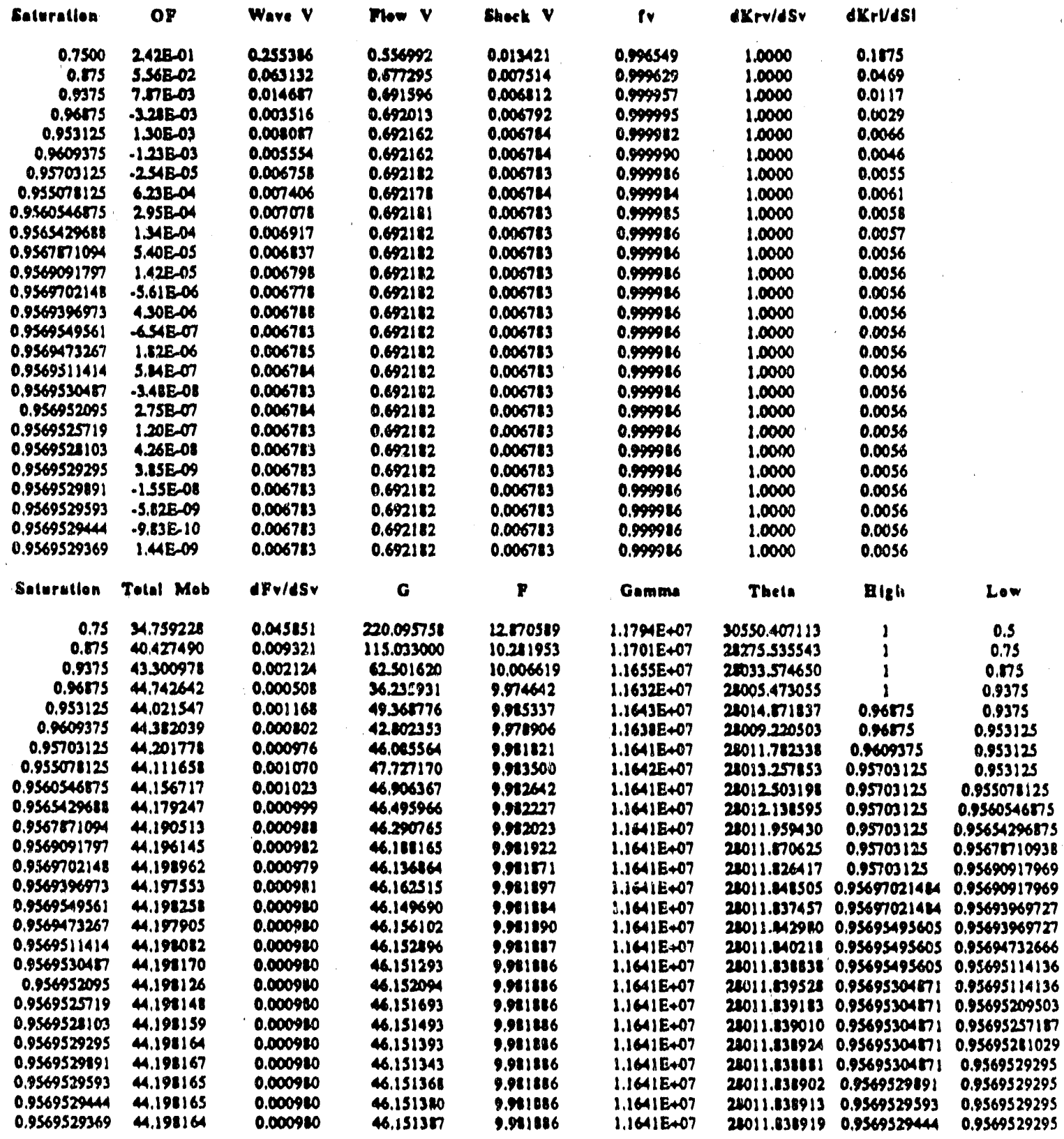


Spreadsheet for Calculation of Stream-Water Saturation Profiles

Injection Temporature sso $*$
Inllibl Temperuture

$300 \cdot x$
Matrlx Beal Cepacily $1.0000(\mathrm{~kJ} / \mathrm{kg})$

LEADING SBOCX CALCULATIONS

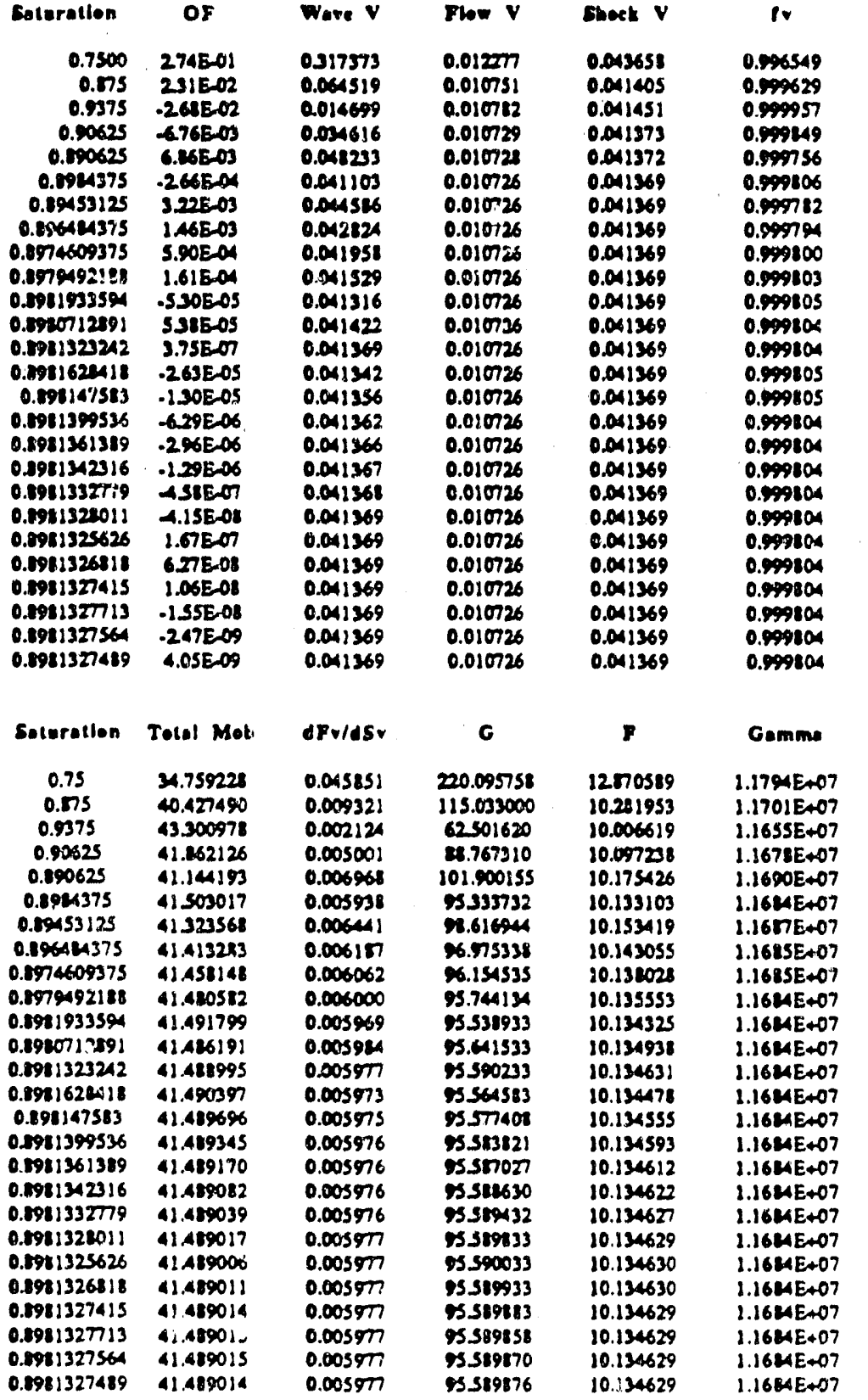

$\begin{array}{ll}\text { dKrv/dSr } & \text { KKrVdSI } \\ 1.0000 & 0.1175 \\ 1.0000 & 0.0169 \\ 1.0000 & 0.0117 \\ 1.0000 & 0.0261 \\ 1.0000 & 0.0359 \\ 1.0000 & 0.0309 \\ 1.0000 & 0.0331 \\ 1.0000 & 0.0321 \\ 1.0000 & 0.0315 \\ 1.0000 & 0.0312 \\ 1.0000 & 0.0311 \\ 1.0000 & 0.0312 \\ 1.0000 & 0.0311 \\ 1.0000 & 0.0311 \\ 1.0000 & 0.0311 \\ 1.0000 & 0.0311 \\ 1.0000 & 0.0311 \\ 1.0000 & 0.0311 \\ 1.0000 & 0.0311 \\ 1.0000 & 0.0311 \\ 1.0000 & 0.0311 \\ 1.0000 & 0.0311 \\ 1.0000 & 0.0311 \\ 1.0000 & 0.0311 \\ 1.0000 & 0.0311 \\ 1.0000 & 0.0311\end{array}$

\begin{tabular}{|c|c|c|}
\hline There & Bigh & Low \\
\hline 1550.407113 & 1 & 0.5 \\
\hline 275.535543 & 1 & 0.75 \\
\hline $\begin{array}{l}033.574650 \\
1113.209409 \\
1111.920650 \\
114.727756 \\
1162581158\end{array}$ & $\begin{array}{c}1 \\
0.9375 \\
0.90625 \\
0.00525 \\
0.0984375\end{array}$ & $\begin{array}{l}0.875 \\
0.875 \\
0.775 \\
0.090625 \\
0.090625\end{array}$ \\
\hline $\begin{array}{l}153.472957 \\
149.055510\end{array}$ & $\begin{array}{l}0.894375 \\
0.0944375\end{array}$ & $\begin{array}{c}0.09453125 \\
0.89644375\end{array}$ \\
\hline $\begin{array}{l}1146.210487 \\
1145.201343\end{array}$ & $\begin{array}{l}0.894375 \\
0.8944375\end{array}$ & $\begin{array}{c}0.8974609375 \\
0.89794921875\end{array}$ \\
\hline $\begin{array}{l}1146.340220 \\
1146.070608\end{array}$ & $\begin{array}{l}0.89819335938 \\
0.89819335938\end{array}$ & $\begin{array}{l}0.89794921875 \\
0.89207128906\end{array}$ \\
\hline $\begin{array}{l}145.935932 \\
146.003259\end{array}$ & $\begin{array}{c}0.89119335938 \\
0.8981628418\end{array}$ & $\begin{array}{l}0.19813232422 \\
0.19813232422\end{array}$ \\
\hline $\begin{array}{l}146.036931 \\
146.053768\end{array}$ & $\begin{array}{l}0.89814758301 \\
0.89113995361\end{array}$ & $\begin{array}{l}0.89813232422 \\
0.89813232422\end{array}$ \\
\hline $\begin{array}{l}1146.062188 \\
1146.065398\end{array}$ & $\begin{array}{l}0.09813613892 \\
0.8911323157\end{array}$ & $\begin{array}{l}0.69813232422 \\
0.19813232422\end{array}$ \\
\hline $\begin{array}{l}146.068503 \\
146.069555\end{array}$ & $\begin{array}{l}0.09113327789 \\
0.89813280106\end{array}$ & $\begin{array}{l}0.89813232422 \\
0.09813232422\end{array}$ \\
\hline $\begin{array}{l}146.069029 \\
146.068766\end{array}$ & $\begin{array}{l}0.89813280106 \\
0.59813280106\end{array}$ & $\begin{array}{l}0.89813256264 \\
0.09813268185\end{array}$ \\
\hline 46.068634 & 0.89813220106 & 0.89813274145 \\
\hline 46.067700 & 0.19113277125 & 0.09813274145 \\
\hline
\end{tabular}

28146.0687330 .891132756350 .89813274145 
Injection Temperatere $650 \cdot \mathrm{K}$
Iallat Temperacture $300 x$
Mairly Beat Copecliy

$\$ .0000$ (kJ/ke)

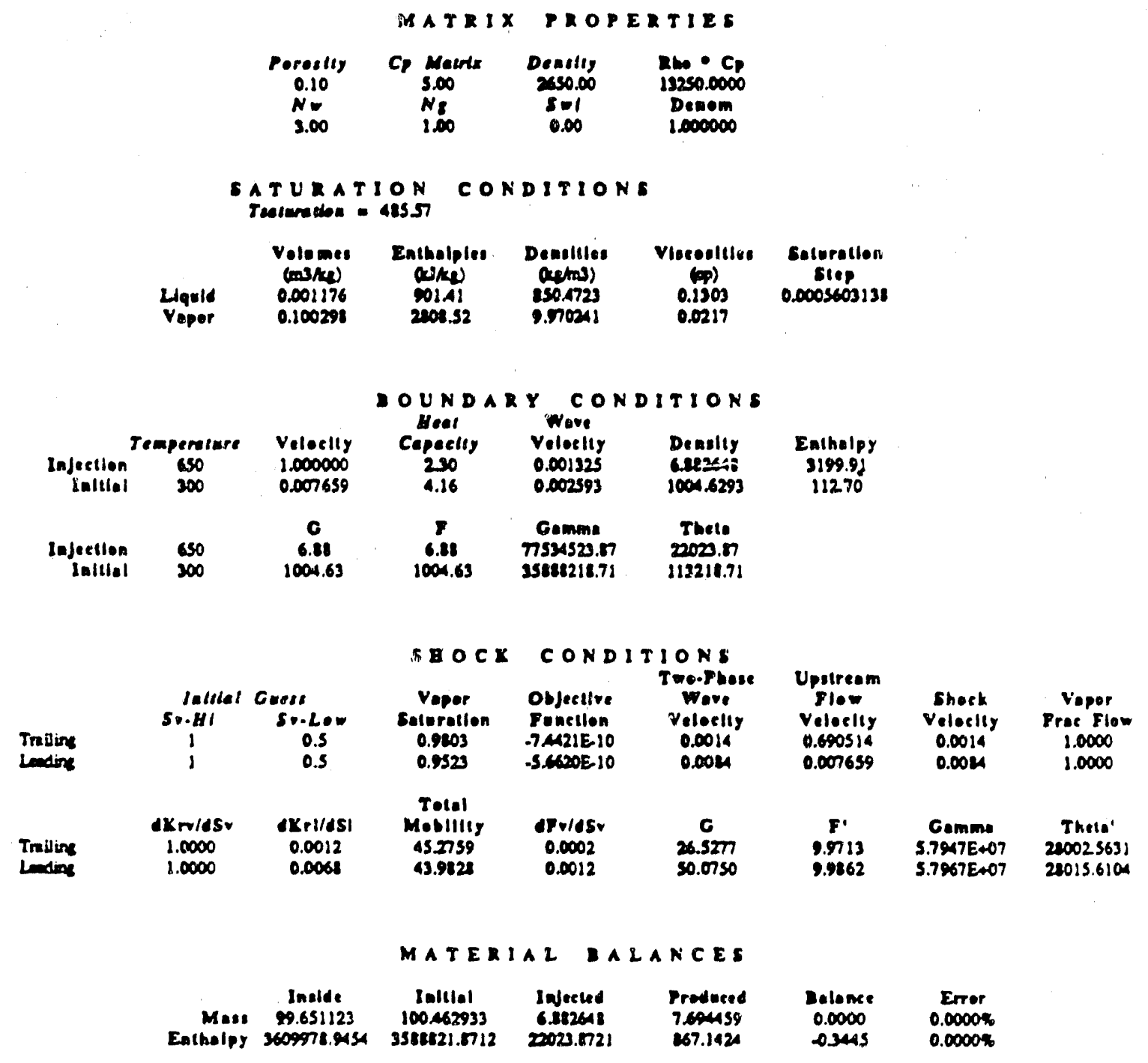


-240 .

Spreadsheet for Calculation of Stream-Water Saturation Profiles

Injectlon Temperature $650 \cdot \mathbf{X}$
Inltbl Temperalure $300 \bullet$
Matrix Beat Copacity

$\$ .0000\left(\mathrm{KJ} / \mathrm{k}_{\mathrm{g}}\right)$

SATURATION PROFILE

\begin{tabular}{|c|c|c|c|c|c|c|c|c|}
\hline $\begin{array}{c}\text { Vapor } \\
\text { Colteretlon }\end{array}$ & $\begin{array}{c}\text { Vepor } \\
\text { Frac Fiow }\end{array}$ & AKrr/dSv & UXPVASI & $\begin{array}{c}\text { Tolal } \\
\text { Moblility }\end{array}$ & APr/dSr & lambda & $\begin{array}{l}\text { Mens } \\
\text { Inolde }\end{array}$ & $\begin{array}{l}\text { Enthalpy } \\
\text { Inside }\end{array}$ \\
\hline $\begin{array}{l}1.0000 \\
1.0000\end{array}$ & & & & & & $\begin{array}{l}0.000000 \\
0.001372\end{array}$ & & \\
\hline $\begin{array}{l}0.9603 \\
0.9797\end{array}$ & $\begin{array}{l}0.999999 \\
0.999999\end{array}$ & $\begin{array}{l}1.0000 \\
1.0000\end{array}$ & $\begin{array}{l}0.0012 \\
0.0012\end{array}$ & $\begin{array}{l}45.275034 \\
45.250061\end{array}$ & $\begin{array}{l}0.000199 \\
0.000210\end{array}$ & $\begin{array}{l}0.001972 \\
0.001452\end{array}$ & $\begin{array}{l}0.009444 \\
0.002148\end{array}$ & $\begin{array}{c}106387.959165 \\
4651.756898\end{array}$ \\
\hline $\begin{array}{l}0.9792 \\
0.9766\end{array}$ & & & & & $\begin{array}{l}0.000222 \\
0.000235\end{array}$ & $\begin{array}{l}0.001535 \\
0.001620\end{array}$ & $\begin{array}{l}0.002250 \\
0.002354\end{array}$ & \\
\hline $\begin{array}{l}0.9716 \\
0.9781\end{array}$ & $\begin{array}{l}0.999998 \\
0.999998\end{array}$ & $\begin{array}{l}1.0000 \\
1.0000\end{array}$ & $\begin{array}{l}0.0014 \\
0.0014\end{array}$ & $\begin{array}{l}45.196315 \\
45.172413\end{array}$ & $\begin{array}{l}0.000235 \\
0.000247\end{array}$ & $\begin{array}{l}0.001620 \\
0.001707\end{array}$ & $\begin{array}{l}0.002354 \\
0.002461\end{array}$ & $\begin{array}{l}4924.063440 \\
5060.677899\end{array}$ \\
\hline $0 .: 775$ & 0.999998 & 1.0000 & 0.0015 & 45.146571 & 0.000260 & 0.001797 & 0.002569 & 5197.626860 \\
\hline 0.9769 & 0.99998 & 1.0000 & 0.0016 & 45.120699 & 0.000274 & 0.001889 & 0.002681 & 5334.881609 \\
\hline 0.9764 & 0.59499 & 1.0000 & 0.0017 & 45.094227 & 0.000257 & 0.001944 & 0.002794 & 5472453033 \\
\hline 0.9758 & 0.999998 & 1.0000 & 0.0018 & 45.068956 & 0.000301 & 0.002080 & 0.002910 & 5610.342022 \\
\hline 0.9753 & 0.999997 & 1.0000 & 0.0018 & 45.043015 & 0.000316 & 0.002180 & 0.003029 & 5748 44940 \\
\hline 0.9747 & o.segen & 1.0000 & 0.0019 & 45.017215 & 0.000330 & 0.002281 & 0.003149 & 5877.076272 \\
\hline 0.9741 & $0.99 i s \pi$ & 1.0000 & 0.0020 & 44991345 & 0.000345 & 0.002385 & 0.001273 & 6025.923327 \\
\hline 0.9736 & $0.99 \sin$ & 1.0000 & 0.0021 & 44.965475 & 0.000361 & 0.002491 & 0.003398 & 6165.091534 \\
\hline 0.7730 & 0.999997 & 1.0000 & 0.0022 & $44.93 \% 06$ & 0.000377 & 0.002600 & 0.003526 & 6304581797 \\
\hline 0.9725 & $0.999 \operatorname{sic}$ & 1.0000 & 0.0023 & 44.913737 & 0.000393 & 0.002711 & 0.003657 & C44.395022 \\
\hline 0.8719 & $0.9999 \%$ & 1.0000 & 0.0024 & 4.887269 & 0.000409 & 0.002825 & 0.003790 & 654532118 \\
\hline 0.9713 & $0.9 \operatorname{sens}$ & 1.0000 & 0.0025 & 4.262001 & 0.000428 & 0.002941 & 0.003925 & 6724.993904 \\
\hline 0.8708 & $0.9949 \%$ & 10000 & 0.0026 & 4.836133 & 0.000443 & 0.003060 & 0.004063 & 6205.781566 \\
\hline 0.9702 & o.spiges & 1.0000 & 0.0027 & 4.810266 & 0.000461 & 0.003181 & 0.004204 & 7006.895748 \\
\hline 0.9697 & 0.999095 & 1.0000 & 0.0028 & 4.784399 & 0.000478 & 0.003304 & 0.004347 & 7141.337461 \\
\hline 0.9691 & 0.999995 & 1.0000 & 0.0029 & 44.758532 & 0.000497 & 0.003430 & 0.004492 & 7290.107625 \\
\hline 0.9685 & 0.999995 & 1.0000 & 0.0030 & 44.732666 & 0.000515 & 0.003558 & 0.004640 & 7432207164 \\
\hline 0.4880 & 0.999994 & 1.0000 & 0.0031 & 4.706101 & 0.000534 & 0.003689 & 0.004790 & 7574.637005 \\
\hline 0.9674 & $0.99 \% 9$ & 1.0000 & 0.0032 & 4.680936 & 0.000553 & 0.003822 & 0.004943 & 717.398079 \\
\hline 0.9669 & 0.999994 & 1.0000 & 0.0033 & 44.655071 & 0.000573 & 0.003957 & 0.005099 & 7860.491315 \\
\hline 0.9663 & 0.999993 & 1.0000 & 0.0034 & 4.629207 & 0.000593 & 0.004095 & 0.005257 & 2003.917650 \\
\hline 0.9657 & 0.999993 & 1.0000 & 0.0035 & $4 . \cos 344$ & 0.000613 & 0.004236 & 0.005417 & $8147.67 \mathrm{EO}^{21}$ \\
\hline 0.9652 & 0.999993 & 1.0000 & 0.0036 & 44.577481 & 0.000634 & 0.004379 & 0.005581 & 291.773368 \\
\hline 0.9646 & 0.999992 & 1.0000 & 0.0038 & 44551618 & 0.000655 & 0.004525 & 0.005746 & 2436.204634 \\
\hline $0.96 \times 1$ & 0.999992 & 1.0000 & 0.0039 & 44.525756 & 0.000677 & 0.004673 & 0.005915 & 8520.972764 \\
\hline 0.9635 & 0.999992 & 1.0000 & 0.0040 & 4hagesen & 0.00059 & 0.004823 & 0.005026 & 1726.078707 \\
\hline 0.9629 & 0.999991 & 1.0000 & 0.0041 & 44,474033 & 0.000721 & 0.004976 & 0.006259 & 1871.523413 \\
\hline 0.9624 & 0.999991 & 1.0000 & 0.0012 & 44,448173 & 0.000743 & 0.005132 & 0.004435 & 9017.307836 \\
\hline 0.9618 & 0.999990 & 1.0000 & 0.0044 & 4.422313 & 0.000766 & 0.005290 & 0.006614 & 9163.432933 \\
\hline 0.9612 & 0.999950 & 1.0000 & 0.0045 & 44396454 & 0.000789 & 0.005451 & 0.006795 & 9309.899662 \\
\hline 0.9607 & 0.999989 & 1.0000 & 0.0046 & 44370595 & 0.000213 & 0.005614 & 0.006979 & 958.708987 \\
\hline 0.9601 & 0.999919 & 1.0000 & 0.0048 & 44344736 & 0.000137 & 0.005779 & 0.001166 & 903.26181 \\
\hline 0.9596 & 0.999989 & 1.0000 & 0.0049 & 44318579 & 0.000861 & 0.005848 & 0.007355 & 9751.359281 \\
\hline 0.9590 & 0.999988 & 1.0000 & 0.0050 & 44.293022 & 0.000886 & 0.005119 & 0.007547 & 9099.202190 \\
\hline 0.954 & 0.999988 & 1.0000 & 0.0052 & 44267165 & 0.000911 & 0.006292 & $0.0 \mathrm{mal}$ & 10047.391568 \\
\hline 0.9579 & 0.999987 & 1.0000 & 0.0053 & 4241309 & 0.000937 & 0.006468 & 0.007938 & 10195.925393 \\
\hline 0.9573 & 0.999017 & 1.0000 & $0.005 s$ & 4.215454 & 0.000963 & 0.006446 & 0.001138 & 10344.813642 \\
\hline 0.5568 & 0.599926 & 1.0000 & 0.0056 & 4.119599 & 0.000969 & 0.008127 & 0.008341 & 10494.048298 \\
\hline 0.9562 & 0. reveses & 1.0000 & 0.0058 & 44.163745 & 0.001015 & 0.007011 & 0.002546 & 10643.633345 \\
\hline 0.9556 & 0.999015 & 1.0000 & 0.0059 & 4.137592 & 0.001042 & 0.007197 & 0.00175 & 20793.569770 \\
\hline 0.9551 & $0.9 \sin$ & 1.0000 & 0.0061 & 44.112039 & 0.001070 & 0.007326 & 0.001965 & 10943.858563 \\
\hline 0.9545 & 0.99994 & 1.0000 & 0.0062 & 44.046187 & 0.001097 & 0.007577 & 0.009179 & $110 \times 500716$ \\
\hline 0.9540 & 0.999983 & 1.0000 & 0.0064 & 44.060335 & 0.001125 & 0.007771 & 0.009395 & 11245.497227 \\
\hline 0.5534 & 0.999912 & 1.0000 & 0.0065 & 4.034nes & 0.001154 & 0.007968 & $0.00 \% 614$ & $113 \% 6.449092$ \\
\hline 0.5528 & 0.999982 & 1.0000 & 0.0067 & 4.002634 & 0.001113 & 0.008167 & 0.009835 & i1548.557315 \\
\hline 0.9523 & 0.999981 & 1.0000 & 0.0068 & 43.912785 & 0.001212 & 0.008369 & 0.010060 & 11700.622898 \\
\hline 0.0000 & & & & & & 0.008369 & & \\
\hline 0.0000 & & & & & & 1.000000 & & \\
\hline
\end{tabular}


Spreadsheet for Calculation of Stream-Water Saturation Profiles

Injectlon Temperalure $650 \cdot \mathrm{K}$
Inlibal Temperalure $300 \cdot \boldsymbol{K}$
Matrix Beat Capaelty

$5.0000 \cdot(4 J / k g)$

TRAILING SBOCK CALCULATIONE

\begin{tabular}{|c|c|c|c|c|c|c|c|c|}
\hline Soturation & OP & Wev/d $V$ & Plow V & Check $V$ & iv & AKrv/dSV & exrvesi & \\
\hline $\begin{array}{r}0.7500 \\
0.175 \\
0.9375\end{array}$ & $\begin{array}{l}245 E-01 \\
6.10 E-02 \\
132 E-02\end{array}$ & $\begin{array}{l}0.247360 \\
0.062548 \\
0.014623\end{array}$ & $\begin{array}{l}0.539426 \\
0.671036 \\
0.624513\end{array}$ & $\begin{array}{l}0.002854 \\
0.001563 \\
0.001391\end{array}$ & $\begin{array}{l}0.996549 \\
0.999629 \\
0.999957\end{array}$ & $\begin{array}{l}1.0000 \\
1.0000 \\
1.0000\end{array}$ & $\begin{array}{l}0.1875 \\
0.0469 \\
0.0117\end{array}$ & \\
\hline 0.96175 & 2.13E-03 & 0.003500 & 0.690419 & 0.001373 & 0.999995 & 1.0000 & 0.0029 & \\
\hline 0.924375 & -5.14E-OA & 0.000258 & 0.690505 & 0.001372 & 0.999999 & 1.0000 & 0.0007 & \\
\hline $\begin{array}{r}0.8765625 \\
0.92046775\end{array}$ & $\begin{array}{l}5.20 E-04 \\
.236 E-0 S\end{array}$ & $\begin{array}{l}0.001952 \\
0.001348\end{array}$ & $\begin{array}{l}0.690505 \\
0.690514\end{array}$ & $\begin{array}{l}0.001372 \\
0.001372\end{array}$ & $\begin{array}{l}0.999998 \\
0.999999\end{array}$ & $\begin{array}{l}1.0000 \\
1.0000\end{array}$ & $\begin{array}{l}0.0016 \\
0.0011\end{array}$ & \\
\hline 0.972515625 & 264E- 04 & 0.001636 & 0.600512 & 0.001372 & 0.999998 & 1.0000 & 0.0014 & \\
\hline 0.9794921075 & 1.17E-OA & 0.001489 & 0.090514 & 0.001372 & 0.999999 & 1.0000 & 0.0013 & \\
\hline 0.9799104688 & ASSEDOS & 0.001418 & 0.690514 & 0.001372 & 0.999999 & 1.0000 & 0.0012 & \\
\hline 0.9202246094 & $1.07 E-05$ & 0.001383 & 0.690514 & 0.001372 & 0.999999 & 1.0000 & 0.0012 & \\
\hline 0.9103466797 & $651 E-06$ & 0.001366 & 0.600514 & 0.001372 & 0.999999 & 1.0000 & 0.0012 & \\
\hline 0.9002286445 & 210E-06 & 0.001374 & 0.690514 & 0.001372 & 0.999999 & 1.0000 & 0.0012 & \\
\hline $\begin{array}{l}0.9103161621 \\
0.9203009033\end{array}$ & $\begin{array}{l}.221 \mathrm{E}_{0} 06 \\
.5 .26 \mathrm{E}-08\end{array}$ & $\begin{array}{l}0.001370 \\
0.001372\end{array}$ & 0.690514 & 0.001372 & 0.999999 & 1.0000 & 0.0012 & \\
\hline 0.9102932739 & $1.02 E-06$ & $\begin{array}{l}0.001372 \\
0.001373\end{array}$ & $\begin{array}{l}0.690514 \\
0.690514\end{array}$ & $\begin{array}{l}0.001372 \\
0.001372\end{array}$ & $\begin{array}{l}0.999990 \\
0.999999\end{array}$ & $\begin{array}{l}1.0000 \\
1.0000\end{array}$ & $\begin{array}{l}0.0012 \\
0.0012\end{array}$ & \\
\hline 0.9202970886 & 4. $.0 \mathrm{E}-\mathrm{O}$ & 0.001373 & 0.690514 & 0.001372 & 0.999999 & 1.0000 & 0.0012 & \\
\hline 0.910298996 & 211E-OI & 0.001372 & 0.090514 & 0.001372 & 0.999999 & 1.0000 & 0.0012 & \\
\hline 0.9102999496 & 7.60E-O8 & 0.001372 & 0.690514 & 0.001372 & 0.999999 & 1.0000 & 0.0012 & \\
\hline 0.9203004265 & $8.72 E-09$ & 0.001372 & 0.690514 & 0.001372 & 0.999999 & 1.0000 & 0.0012 & \\
\hline 0.9203006649 & $.249 E-08$ & 0.001372 & 0.690514 & 0.001372 & 0.999999 & 1.0000 & 0.0012 & \\
\hline 0.9103005457 & $-8.11 \mathrm{E}-09$ & 0.001372 & 0.690514 & 0.001372 & 0.999999 & 1.0000 & 0.0012 & \\
\hline 0.9203004661 & 3.08E 10 & 0.001372 & 0.690514 & 0.001372 & 0.999999 & 2.0000 & 0.0012 & \\
\hline & $-3.90 E-09$ & 0.001372 & 0.690514 & 0.001372 & 0.999999 & 1.0000 & 0.0012 & \\
\hline & $\begin{array}{l}-1.00 E-09 \\
.7 .44 E_{10}\end{array}$ & 0.001372 & 0.690514 & 0.001372 & 0.999999 & 1.0000 & 0.0012 & \\
\hline & -7.MAE 10 & 0.001372 & 0.690514 & 0.001372 & 0.999499 & 1.0000 & 0.0012 & \\
\hline Saturation & Total Mob & $d F v / d S v$ & $\boldsymbol{c}$ & $\mathbf{P}$ & Comma & Theto & Bleh & $L \bullet=$ \\
\hline $\begin{array}{r}0.75 \\
0.875\end{array}$ & $\begin{array}{l}34.759228 \\
40.427490\end{array}$ & $\begin{array}{l}0.045851 \\
0.009321\end{array}$ & $\begin{array}{l}220.1095758 \\
115.033000\end{array}$ & $\begin{array}{l}12070589 \\
10.281953\end{array}$ & $\begin{array}{l}\text { S.8117E } 407 \\
\text { S.802SE }+07\end{array}$ & $\begin{array}{l}30550.407113 \\
22275.535543\end{array}$ & $\begin{array}{l}1 \\
1\end{array}$ & $\begin{array}{l}0.5 \\
0.75\end{array}$ \\
\hline 0.9375 & 43.300978 & 0.002124 & 62501620 & 10.006619 & S.7975E 407 & 24033574650 & 1 & 0.175 \\
\hline 0.96575 & 44.742642 & 0.000508 & 36.225931 & 9.974642 & S.79S5E 007 & 22005.473055 & $i$ & 0.9375 \\
\hline $\begin{array}{r}0.944375 \\
0.9765625\end{array}$ & $\begin{array}{l}45.464019 \\
45.103332\end{array}$ & $\begin{array}{l}0.000124 \\
0.000283\end{array}$ & $\begin{array}{l}23.103016 \\
29.669508\end{array}$ & $\begin{array}{l}9.970782 \\
9.972083\end{array}$ & $\begin{array}{l}5.794 \mathrm{E} \times 07 \\
5.7950 E+07\end{array}$ & $\begin{array}{l}22002081444 \\
21003.224190\end{array}$ & $\stackrel{1}{0.924375}^{1}$ & $\begin{array}{l}0.9675 \\
0.96175\end{array}$ \\
\hline 0.91046775 & 45.2133704 & 0.000195 & 26,34297 & 9.911303 & S.7947E+07 & 22002538594 & 0.944375 & 0.9765625 \\
\hline 0.978515625 & 45.193516 & 0.000237 & 28.027903 & 9.971657 & $5.7948 E N 07$ & 2200249861 & 0.92046875 & 0.9765625 \\
\hline $\begin{array}{l}0.9794921775 \\
0.9799804688\end{array}$ & $\begin{array}{l}45.232610 \\
45.261157\end{array}$ & $\begin{array}{l}0.000216 \\
0.000205\end{array}$ & $\begin{array}{l}27.207100 \\
26.796698\end{array}$ & $\begin{array}{l}9.971471 \\
9.971385\end{array}$ & $\begin{array}{l}\text { S.7947E } 407 \\
5.7947 E+07\end{array}$ & $\begin{array}{l}21002616718 \\
21002610825\end{array}$ & $\begin{array}{l}0.95046775 \\
0.92046775\end{array}$ & $\begin{array}{l}0.971515625 \\
0.9794921875\end{array}$ \\
\hline 0.9202245094 & 45.272430 & 0.000200 & 26591498 & 9.97133 & S.7947E-07 & 21002574258 & 0.86046075 & 0.97991046875 \\
\hline 0.9203466797 & 45.272067 & 0.000198 & 25.488197 & 9.971323 & S.7n+7E+07 & 22002556313 & 0.92046775 & 0.91022460938 \\
\hline 0.9202856445 & 45.275249 & 0.000199 & 26.540198 & 9.971333 & 5.79475407 & 210025852.57 & 0.91034667969 & 0.92022460938 \\
\hline 0.9203161621 & 45276658 & 0.000198 & 26314547 & 9.971328 & $5.7947 E+07$ & 22002560718 & 0.96034667969 & 0.91028564453 \\
\hline 0.9203009033 & 45275953 & 0.000199 & 26.527373 & 9.971330 & $5.7947 E+07$ & 22002563016 & 0.91031616211 & 0.92028564453 \\
\hline 0.9202937739 & 45.275601 & 0.000199 & 26.533785 & 9.971332 & $5.7947 E+07$ & 22002564136 & 0.90030090332 & 0.9202856493 \\
\hline 0.92029 ' 1886 & 4.527577 & 0.000199 & 26.530579 & 9.971331 & 5.7947E 407 & 22002563576 & 0.91030090332 & 0.91029327393 \\
\hline 0.910298996 & 45.275865 & 0.000199 & 26528976 & 9.971331 & $5.7947 E+07$ & 21002563296 & 0.92030090332 & 0.90029708862 \\
\hline $0.9102999 \pi 96$ & 45.279909 & 0.000199 & 26.528174 & 9.971331 & S.7947E+07 & 28002563156 & 0.92030090332 & 0.91029899597 \\
\hline 0.98030042 .65 & 45.275931 & 0.000199 & 26527773 & 9.971330 & S.7947E-07 & 22002363016 & 0.92030090332 & 0.98029994965 \\
\hline 0.9103006649 & 45.275942 & 0.000199 & 26.527573 & 9.971330 & $5.7947 E 407$ & 28002563051 & 0.01030090332 & 0.91030042648 \\
\hline 0.9103005457 & 45.275937 & 0.000199 & 26.527673 & 9.971330 & S.7947E+07 & 28002563069 & 0.9003006449 & 0.91030042648 \\
\hline $\begin{array}{l}0.9203004261 \\
0.9203005159\end{array}$ & $\begin{array}{l}45.275934 \\
45.275935\end{array}$ & $\begin{array}{l}0.000199 \\
0.000199\end{array}$ & $\begin{array}{l}26.527723 \\
26527698\end{array}$ & $\begin{array}{l}9.971330 \\
9.971330\end{array}$ & $\begin{array}{l}S .7947 E+07 \\
S .7947 E+07\end{array}$ & $\begin{array}{l}25002563077 \\
22002563073\end{array}$ & $\begin{array}{l}0.92030054569 \\
0.92030054569\end{array}$ & $\begin{array}{l}0.91030042648 \\
0.91030048609\end{array}$ \\
\hline 0.920300501 & 45.275935 & 0.000199 & 26.52711 & 9.971330 & S.7947ES07. & 29002563075 & 0.92030051589 & 0.92030048609 \\
\hline 0.9203004935 & 45.275934 & 0.000199 & 26.527717 & 9.971330 & S.7947E407 & 28002563076 & 0.95030050099 & 0.98030048609 \\
\hline
\end{tabular}


Spreadsheet for Calculation of Stream-Water Saturation Profiles

Injectlon Temperalure $650 \cdot \mathrm{K}$
Inillal Temperalure

$300 \mathrm{r}$
Matrix Beal Capacliy

5.0000 (WJike)

LEADING SBOCX CALCULATIONE

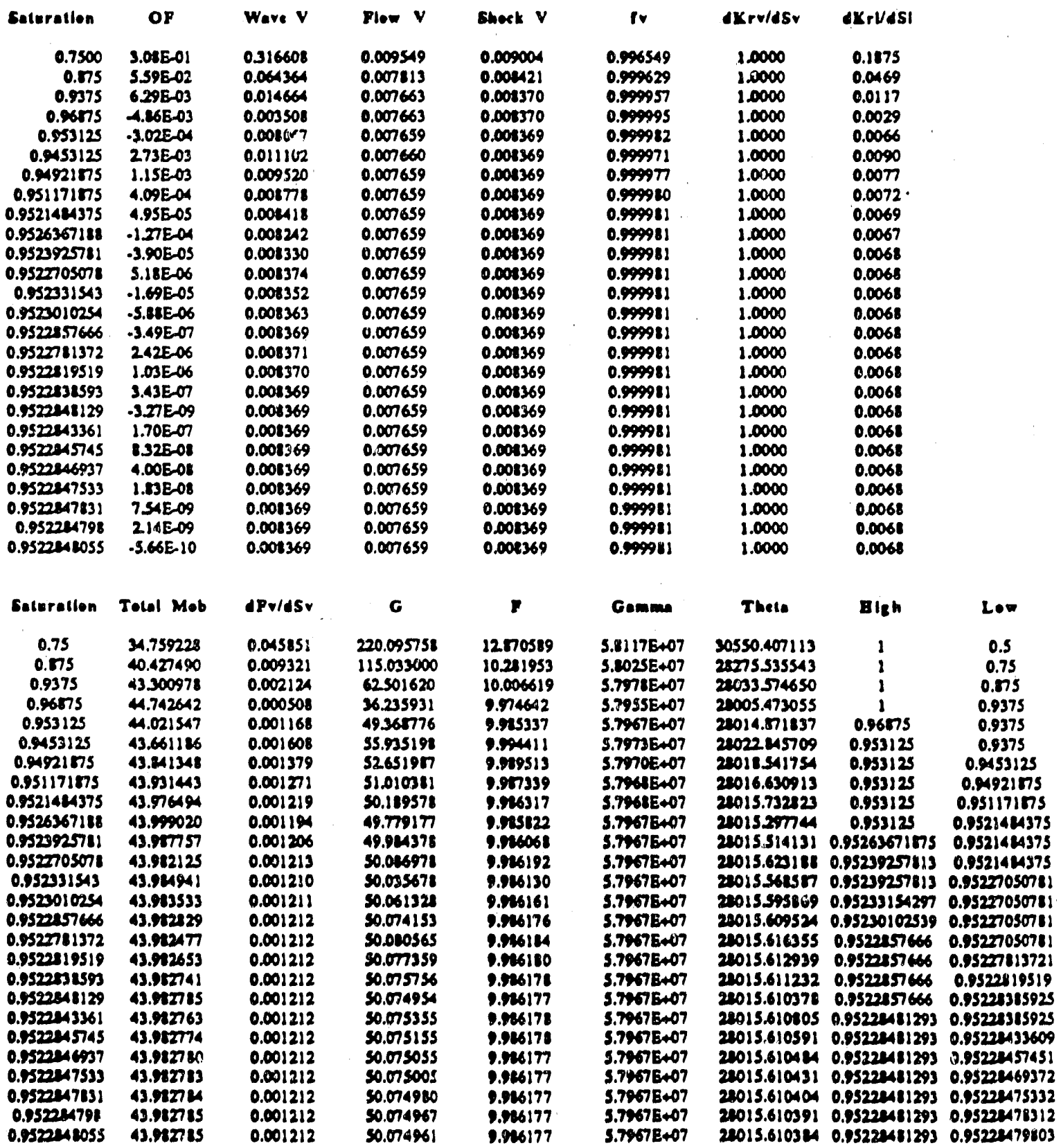


Spreadsheet for Calculation of Stream-Water Saturation Profiles

Infection Temperature $650 \mathrm{~K}$
Inlibl Temperalure

$300 \cdot \mathbf{K}$
Metrix Beal Capectily

$25.0000(k J / k E)$
MATRIX PROPEKTIES

\begin{tabular}{|c|c|c|c|}
\hline $\begin{array}{l}\text { eresiliy } \\
0.10 \\
N= \\
3.00\end{array}$ & C) $\begin{array}{c}\text { Mourdx } \\
25.00 \\
N_{8} \\
1.00\end{array}$ & $\begin{array}{c}\text { Densley } \\
2650.00 \\
5=1 \\
0.00\end{array}$ & $\begin{array}{l}x_{10} \cdot C_{p} \\
6250.0000 \\
D=00 m \\
1.000000\end{array}$ \\
\hline
\end{tabular}

SATURATION CONDITIONS Treicredon $=485.57$

Velumes 0.001176 0.001176

Enthalples

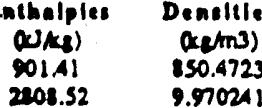

$$
\begin{aligned}
& \text { (qp) } \\
& 0.1303
\end{aligned}
$$

$0.021 \%$
Seturation

step 0.00025732

voper

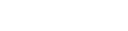

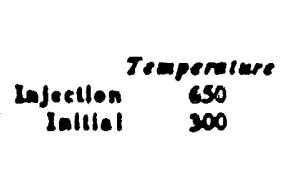

Injerllon 650

inllial 300

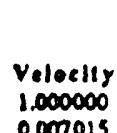

0.007015

\section{6}

1004.63
DOUNDAR$$
\text { Hea! }
$$

Cepecily

230

4.16

Velectiy

0.000265

0.000488

Density

G.432648

1004.6293
Comme

30754523.87

178928218.71

Theto

20025

100463
Emthelpy

3199.91

11270

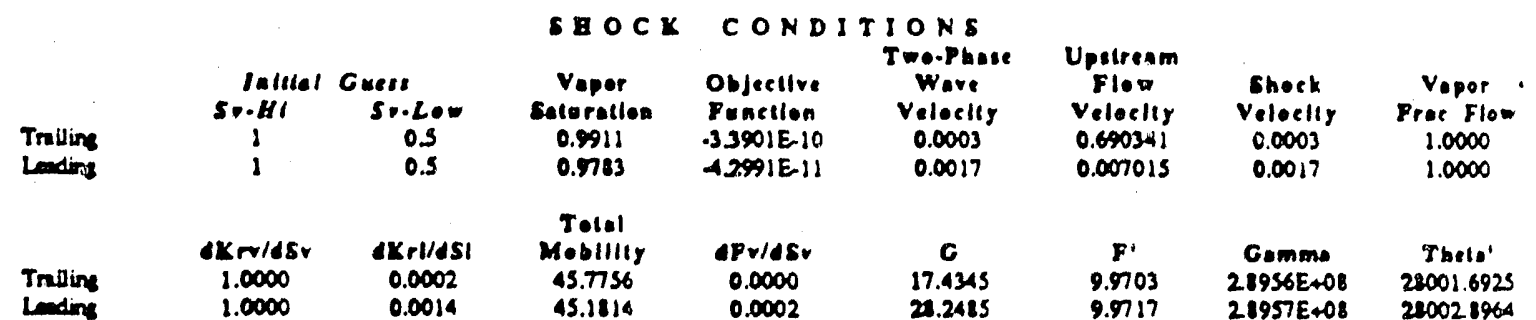

MATERIAL BALANCES

Inelde
Mass 100292015
Eathalpy j7920051531s
Inlilal

100.462933

17892821.712
Injecked

6.832648

$22023.872 \mathrm{~J}$
Produeed
Belane

0.0000

$-0.0278$
Errer

0.00004

$0.0000 \%$ 
Spreadsheet for Calculation ut Stream-Water Saturation Profiles

Injection Temperature $650 \bullet \mathrm{K}$
Inllial Temperature $300 \cdot \boldsymbol{K}$
Malrix Beat Copaclt:

25.0000 (kJ/ke)

SATURATION PROPILE

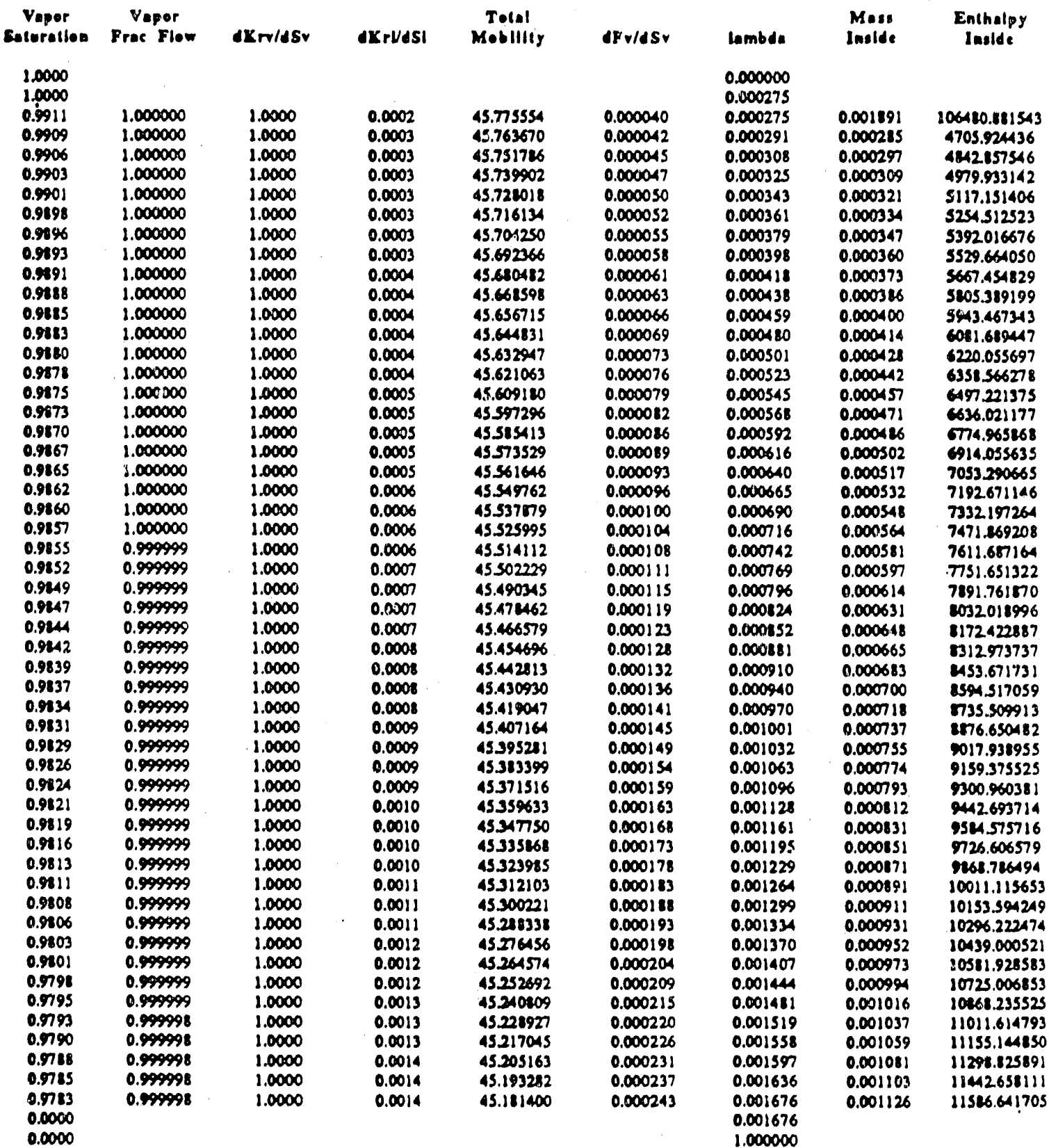


Spreadsheet for Calculation of Stream-Water Saturation Profiles

Inection Temperature $650 \bullet \mathbf{K}$
Inlibal Temperoture

300
Matrlx Deat Capacliy

35.0000 (kJ/kE)

TRAILING. SBOCE CALCULATIONS

\begin{tabular}{|c|c|c|c|c|c|c|c|c|}
\hline Sularatlon & OP & Wave V & Flow V & Sheck Y & Pv & dKrv/dSr & dXrt/dSI & \\
\hline $\begin{array}{r}0.7500 \\
0.775 \\
0.9375 \\
0.9675 \\
0.944375 \\
0.9921175 \\
0.91828125 \\
0.990234375 \\
0.9912109375 \\
0.9907226563 \\
0.9909667969 \\
0.991048672 \\
0.9911499023 \\
0.99111193448 \\
0.991104126 \\
0.9911111554 \\
0.99111155701 \\
0.9911174774 \\
0.9911144311 \\
0.99111189079 \\
0.99111191463 \\
0.9911192656 \\
0.99111193252 \\
0.9911192954 \\
0.99111192805 \\
0.9911119273\end{array}$ & $\begin{array}{l}245 \mathrm{E}-01 \\
621 \mathrm{E}-02 \\
1 . \mathrm{A3E}-02 \\
3.23 \mathrm{E}-03 \\
5.03 \mathrm{E}-04 \\
-6.24 \mathrm{E}-05 \\
205 \mathrm{E}-04 \\
5.79 \mathrm{E}-05 \\
-5.68 \mathrm{E}-06 \\
252 \mathrm{E}-05 \\
9.57 \mathrm{E}-06 \\
1.90 \mathrm{E}-06 \\
-1.90 \mathrm{E}-06 \\
-7.29 \mathrm{E}-09 \\
9.43 \mathrm{E}-07 \\
4.63 \mathrm{E}-09 \\
230 \mathrm{E}-07 \\
1.11 \mathrm{E}-07 \\
5.21 \mathrm{E}-08 \\
221 \mathrm{E}-08 \\
7.54 \mathrm{E}-09 \\
1.25 \mathrm{E}-10 \\
-3.59 \mathrm{E}-09 \\
-1.73 \mathrm{E}-09 \\
-2.03 \mathrm{E}-10 \\
-3.39 \mathrm{E}-10\end{array}$ & $\begin{array}{l}0.245631 \\
0.062426 \\
0.014610 \\
0.003506 \\
0.000258 \\
0.000212 \\
0.000420 \\
0.000333 \\
0.000269 \\
0.000300 \\
0.000244 \\
0.000277 \\
0.000273 \\
0.000275 \\
0.000276 \\
0.000275 \\
0.000275 \\
0.000275 \\
0.000275 \\
0.000275 \\
0.000275 \\
0.000275 \\
0.000275 \\
0.000275 \\
0.000275 \\
0.000275\end{array}$ & 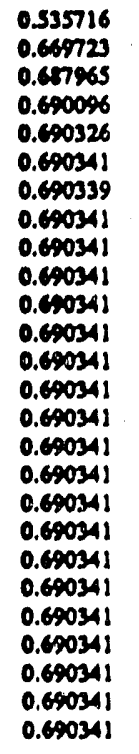 & $\begin{array}{l}0.000575 \\
0.000315 \\
0.000279 \\
0.000275 \\
0.000275 \\
0.000275 \\
0.000275 \\
0.000275 \\
0.000275 \\
0.000275 \\
0.000275 \\
0.000275 \\
0.000275 \\
0.000275 \\
0.000275 \\
0.000275 \\
0.000275 \\
0.000275 \\
0.000275 \\
0.000275 \\
0.000275 \\
0.000275 \\
0.000275 \\
0.000275 \\
0.000275 \\
0.000275\end{array}$ & $\begin{array}{l}0.09549 \\
0.90 \% 29 \\
0.999957 \\
0.999995 \\
0.199999 \\
1.000000 \\
1.000000 \\
1.000000 \\
1.000000 \\
1.000000 \\
1.000000 \\
1.000000 \\
1.000000 \\
1.000000 \\
1.000000 \\
1.000000 \\
1.000000 \\
1.000000 \\
1.000000 \\
1.000000 \\
1.000000 \\
1.000000 \\
1.000000 \\
1.000000 \\
1.000000 \\
1.000000\end{array}$ & $\begin{array}{l}1.0000 \\
1.0000 \\
1.0000 \\
1.0000 \\
1.0000 \\
1.0000 \\
1.0000 \\
1.0000 \\
1.0000 \\
1.0000 \\
1.0000 \\
1.0000 \\
1.0000 \\
1.0000 \\
1.0000 \\
1.0000 \\
1.0000 \\
1.0000 \\
1.0000 \\
1.0000 \\
1.0000 \\
1.0000 \\
1.0000 \\
1.0000 \\
1.0000 \\
1.0000\end{array}$ & $\begin{array}{l}0.1875 \\
0.0469 \\
0.0117 \\
0.0029 \\
0.0007 \\
0.0002 \\
0.0004 \\
0.0003 \\
0.0002 \\
0.0003 \\
0.0002 \\
0.0002 \\
0.0002 \\
0.0002 \\
0.0002 \\
0.0002 \\
0.0002 \\
0.0002 \\
0.0002 \\
0.0002 \\
0.0002 \\
0.0002 \\
0.0002 \\
0.0002 \\
0.0002 \\
0.0002\end{array}$ & \\
\hline Saturation & Tolaf Mob & dFv/ASV & $\mathbf{C}$ & $\boldsymbol{P}$ & Gemma & Thete & Aleh & $L \bullet W$ \\
\hline $\begin{array}{r}0.75 \\
0.875 \\
0.9375 \\
0.9675 \\
0.944375 \\
0.9921875 \\
0.98128125 \\
0.990234375 \\
0.9912109375 \\
0.9907226563 \\
0.9909667969 \\
0.9910186672 \\
0.9911499023 \\
0.9911193848 \\
0.991104126 \\
0.9911117554 \\
0.99111155701 \\
0.9911174774 \\
0.9911114311 \\
0.99111169019 \\
0.9911191463 \\
0.99111192656 \\
0.99111193252 \\
0.99111192954 \\
0.99111192105 \\
0.991119273\end{array}$ & $\begin{array}{l}34.759228 \\
40.427490 \\
43.300976 \\
44.742642 \\
45.464089 \\
45.824889 \\
45.644485 \\
45.734616 \\
45.779787 \\
45.757237 \\
45.768512 \\
45.774150 \\
45.776969 \\
45.775559 \\
45.774854 \\
45.775207 \\
45.775313 \\
45.775471 \\
45.775515 \\
45.775537 \\
45.775548 \\
45.775554 \\
45.775556 \\
45.775555 \\
45.775554 \\
45.775554\end{array}$ & $\begin{array}{l}0.045851 \\
0.009321 \\
0.002124 \\
0.0005018 \\
0.000124 \\
0.000031 \\
0.000070 \\
0.000048 \\
0.000039 \\
0.000043 \\
0.000041 \\
0.000040 \\
0.000040 \\
0.000040 \\
0.000040 \\
0.000040 \\
0.000040 \\
0.000040 \\
0.000040 \\
0.000040 \\
0.000040 \\
0.000040 \\
0.000040 \\
0.000040 \\
0.000040 \\
0.000040\end{array}$ & $\begin{array}{l}220.095758 \\
115.033000 \\
62.501620 \\
36.235931 \\
23.103086 \\
16.536663 \\
19.819875 \\
18.176269 \\
17.357466 \\
17.767868 \\
17.562667 \\
17.460067 \\
17.405766 \\
17 \text { A34416 } \\
17.47242 \\
17.40829 \\
17.437623 \\
17.436020 \\
17.435211 \\
17.43417 \\
17.434617 \\
17.434517 \\
17.434467 \\
17.43492 \\
17.434504 \\
17.434510\end{array}$ & $\begin{array}{l}12.770519 \\
10.211953 \\
10.006619 \\
9.974642 \\
9.770712 \\
9.970308 \\
9.970469 \\
9.770372 \\
9.970357 \\
9.970344 \\
9.970345 \\
9.970341 \\
9.970339 \\
9.770340 \\
9.970340 \\
9.710340 \\
9.970340 \\
9.970340 \\
9.970340 \\
9.970340 \\
9.970340 \\
9.970340 \\
9.970340 \\
9.970340 \\
9.970340 \\
9.910340\end{array}$ & 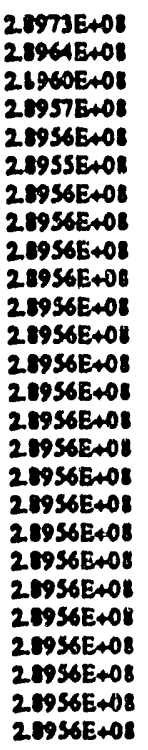 & $\begin{array}{l}30550.407113 \\
25275.535543 \\
22033.574650 \\
22005.473055 \\
22002.011441 \\
22001.664696 \\
22001.005609 \\
22001.721151 \\
22001.609725 \\
22001.704641 \\
22001.697011 \\
22001.693448 \\
22001.691554 \\
22001.692448 \\
22001.692391 \\
220011.692672 \\
22001.692560 \\
22001.692504 \\
22001.692476 \\
22001.692462 \\
22001.692455 \\
22001.692451 \\
22001.692450 \\
22001.692450 \\
22001.692451 \\
22001.692451\end{array}$ & $\begin{array}{c}1 \\
1 \\
1 \\
\vdots \\
1 \\
0.99211875 \\
0.9921875 \\
0.9921875 \\
0.9912109375 \\
0.9912109375 \\
0.9912109375 \\
0.9912109375 \\
0.991114990234 \\
0.99111193477 \\
0.99111934477 \\
0.99111193477 \\
0.99111934477 \\
0.9911193477 \\
0.9911193477 \\
0.9911193477 \\
0.9911193477 \\
0.9911199477 \\
0.99111932516 \\
0.99111929536 \\
0.99111921046\end{array}$ & $\begin{array}{c}0.5 \\
0.75 \\
0.775 \\
0.9375 \\
0.96175 \\
0.944375 \\
0.984375 \\
0.91128125 \\
0.990234375 \\
0.990234375 \\
0.99072265625 \\
0.99096679688 \\
0.99101866719 \\
0.99108886719 \\
0.99108826719 \\
0.99110412598 \\
0.99111175537 \\
0.99111557007 \\
0.99111747742 \\
0.9911143109 \\
0.991111390793 \\
0.99111914635 \\
0.99111926556 \\
0.991111926556 \\
0.99111926556 \\
0.991111926556\end{array}$ \\
\hline
\end{tabular}


Spreadsheet for Calculation of Stream-Water Saturation Profiles

Infection Tamperature $650 \mathrm{~K}$
Inlelal Temperature

$200 \cdot \mathbf{K}$
Matrix Beat Capaclly

25.0000 (kJ/kg)

\section{LEADING GBOCI CALCULATIONG}

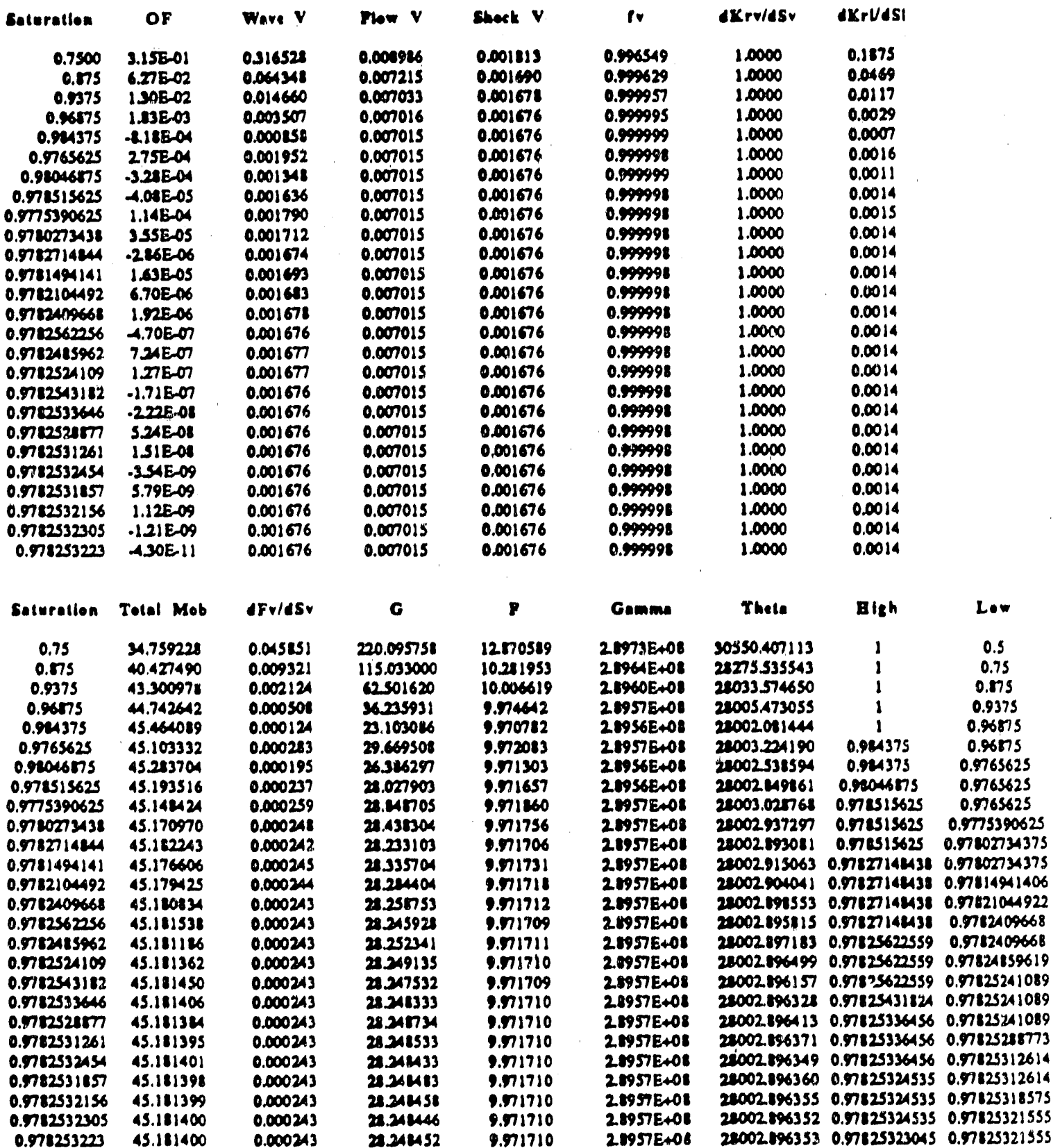




\section{APPENDIX E COMPUTER PROGRAMS}

This appendix contains the source code for the programs to do the path integration and to calculate the shock conditions. All the programs are written in the $\mathrm{C}$ programming language and were implemented using the BSD 4.3 Unix compiler. The eigenvalue routines were from the IMSL library and were linked to the $C$ code.

Each problem has its own set of source code. The techniques used to trace the composition paths and the shock conditions are similar for the steam-oil-water problem in 84.2 and the $\mathrm{CO}_{2}$-oil-water problem in $\$ 4.3$. This results in a large amount of overlap between the two sets of source code, but time constraints dictated the large amount of duplication.

\section{E.1 STEAM-OIL-WATER CODE}

The code for the steam-oil-water problem is divided into a main library of programs that calculate the properties of each composition point. These properties are the phase saturations, the phase densities, the phase viscosities and the relative permeabilities. Using these properties, the eigenvalues and eigenvectors are calculated and reported.

Each individual composition point is represented by a $\mathrm{C}$ structure that holds all the information about a composition point. These composition poirts are stored in a two-way linked list so the user can traverse the list in any direction. This makes backtracking along a path relatively simple.

There are two main programs used to calculate the steam-oil-water solutions. The first of these, tracepath integrates from a given initial composition along a user specified path. A simplified flow char for tracepath is shown in Figure E.1.

The program integrates along the path selected by the user. The integration continues along this path until a path switch is detected or the path reached the edge of the current hodograph space. Path switched are detected by taking the dot product of the all the current eigenvectors with the previous path eigenvector. If the current eigenvector nearest in direction to the previous eigenvector is along a different path, a path switch is indicated and the user is alerted.

The results of the integration are written to a file named "path.pts." This file contains all the information needed to construct the saturation, temperature and now velocity profiles. Each line of the filc represents one composition point with the following information:

1. The path eigenvalue. This is represents the velocity of the composition point and is used to determine the location of the composition point at a given time.

2. The composition point. This is given by the temperature, now velocity, stcam saturation, oil saturation, and water saturation in that order.

3. The two eigenvalues at the composition point. The small eigenvalue is listed followed by the large eigenvalue.

The second program is called jump. This program is used to find the discontinuities in the final solution. The program uses a Newton-Rhapson iteration to calculate the composition point that matches the downstream conditions, given an upstream composition point. The Rankine-Hugoniot conditions across the shock require that the shock velocity can be calculated using a material balance on the water, oil, or enthalpy components, and that these three velocities be cqual. This condition was given in Eq. 2.35 . 


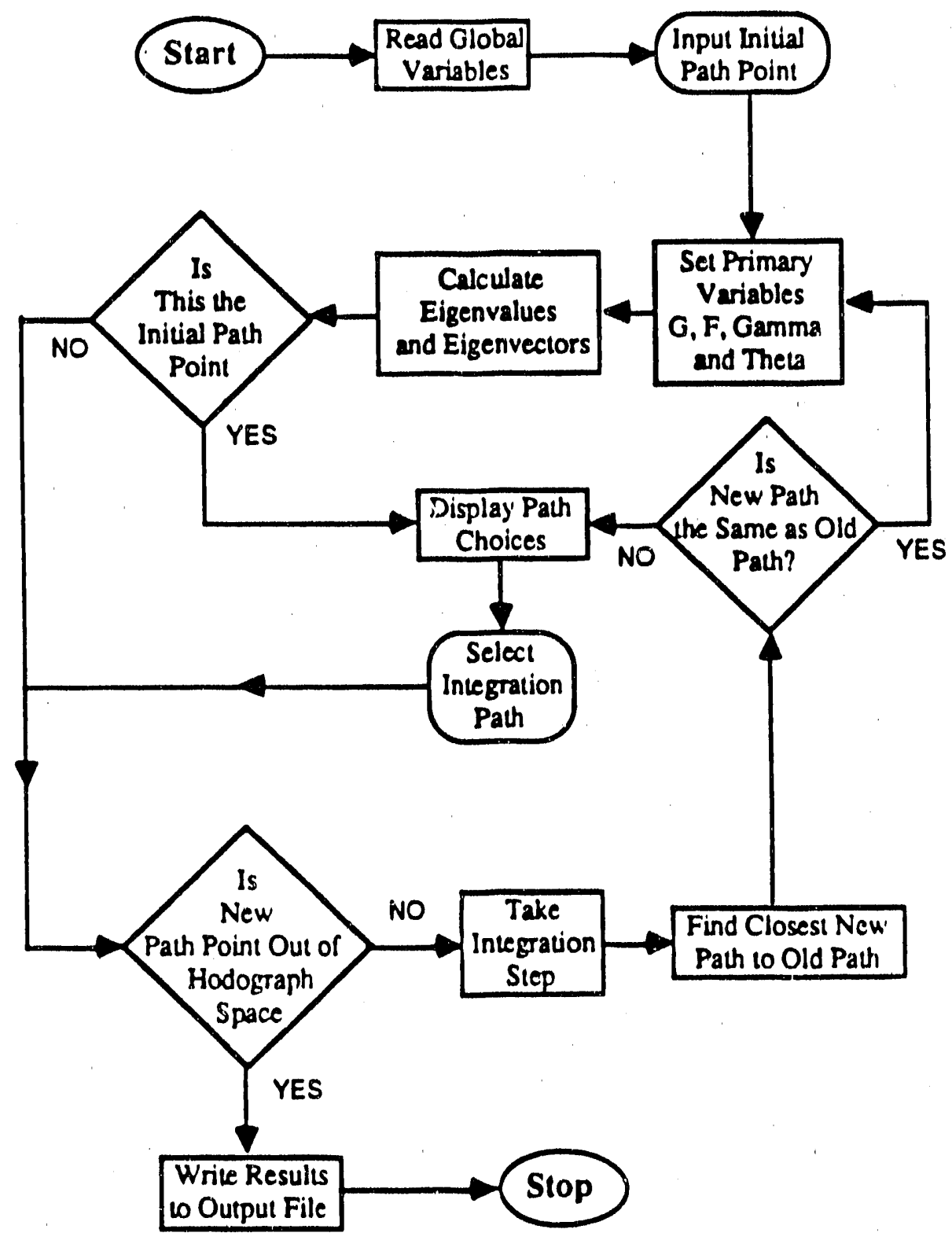

Figure E.1: Basic Flow Chart for the tracepath Program that performs the path integration for the steam-oil-water problem.

The program begins by requesting the upstream composition point. The primary variables and eigenvalues are calculated for this point. Then the user is asked for the kind of shock to be found, intermediate discontinuity or genuine shock. If an intermediate discontinuity is required, the direction of the shock is specified next by telling the program to match the eigenvalue of the upstream of downstream point. Once the direction of the intermediate discontinuity is selected, the actual eigenvalue to match (small or large) is requested. 
Selecting the genuine shock gives no specific shock velocity as with an internediate discontinuity. The shock velocity may be set, by giving a value to the next prompt, or left indeterminate by entering a question mark (?).

Finally, an initial guess of the downstream composition point is entered. The initial guess is s:quired regardless of the type of shock. The range of convergence for these problems is small and if the initial guess is not excellent, the system will fail to converge.

The program begins searching for the downstream composition point that connects to the upstream point via the specified shock. The first step is to calculate the flow velocity of the downstream composition point. This is done be equating the Rankine-Hugoniot conditions for the oil component and the enthalpy component. This results in the following equation for the downstream velocity,

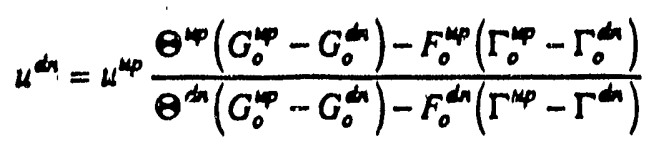

Solving for the downstream flow velocity by Eq. E.1 forces the shock velocities of the oil and enthalpy components to be equal.

Depending on the specification of the shock velocity, the program searches for the roots of one or two objective functions. $F_{1}$ matches the shock velocity of the water component to the oil component and $F_{2}$ matches the shock velocity of the water component to either the given velocity in the case of a genuine shock or the specified eigenvalue in the case of the intermediate discontinuity. The solution is found when both these functions are zero.

and

$$
I_{1}=\Lambda_{w}-\Lambda_{o}=0
$$

$$
F_{2}= \begin{cases}\Lambda_{w}-\lambda & \text { for intermediate discontinuity } \\ \Lambda_{\text {given }} & \text { for specified genuine shock } \\ 0 & \text { for unspecified genuine shock }\end{cases}
$$

Convergence tolerance is set to $10^{-8}$ for both functions. The derivatives are calculated by forward difference using a step of 0.001 for saturation and 1.0 for temperature. These derivatives overshoot the solution when the initial guess is far from a root. To remedy this, an under relaxation factor of 0.3 is applied when the absolute values of either $g_{1}$ or $F_{2}$ is greater than $10^{-4}$. The flow cart for jump is given in Figure E.2. 

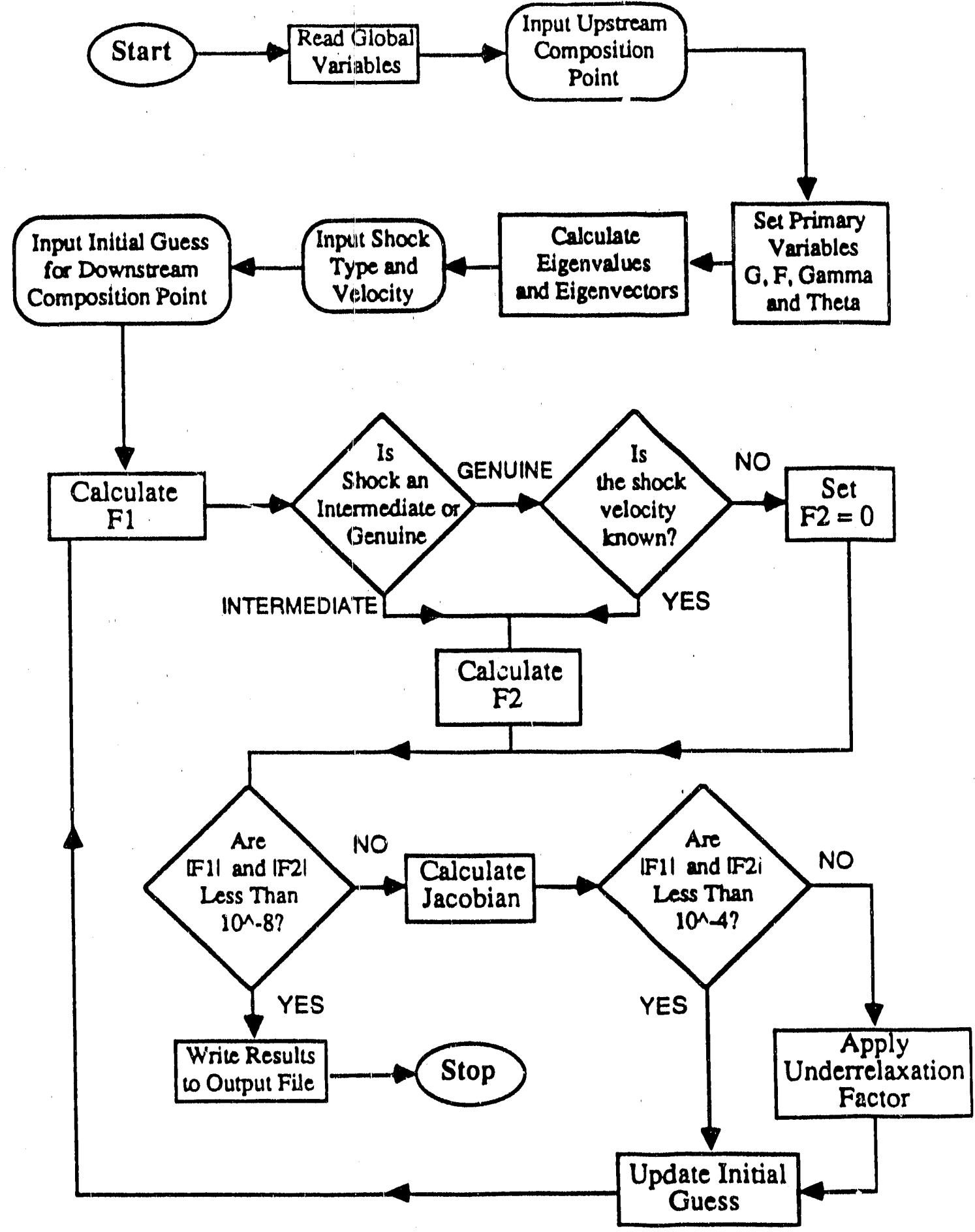

Figure E.2: Basic Flow Chart for the Jump Program that calculates the shock conditions for the steamoil-waler problem. 

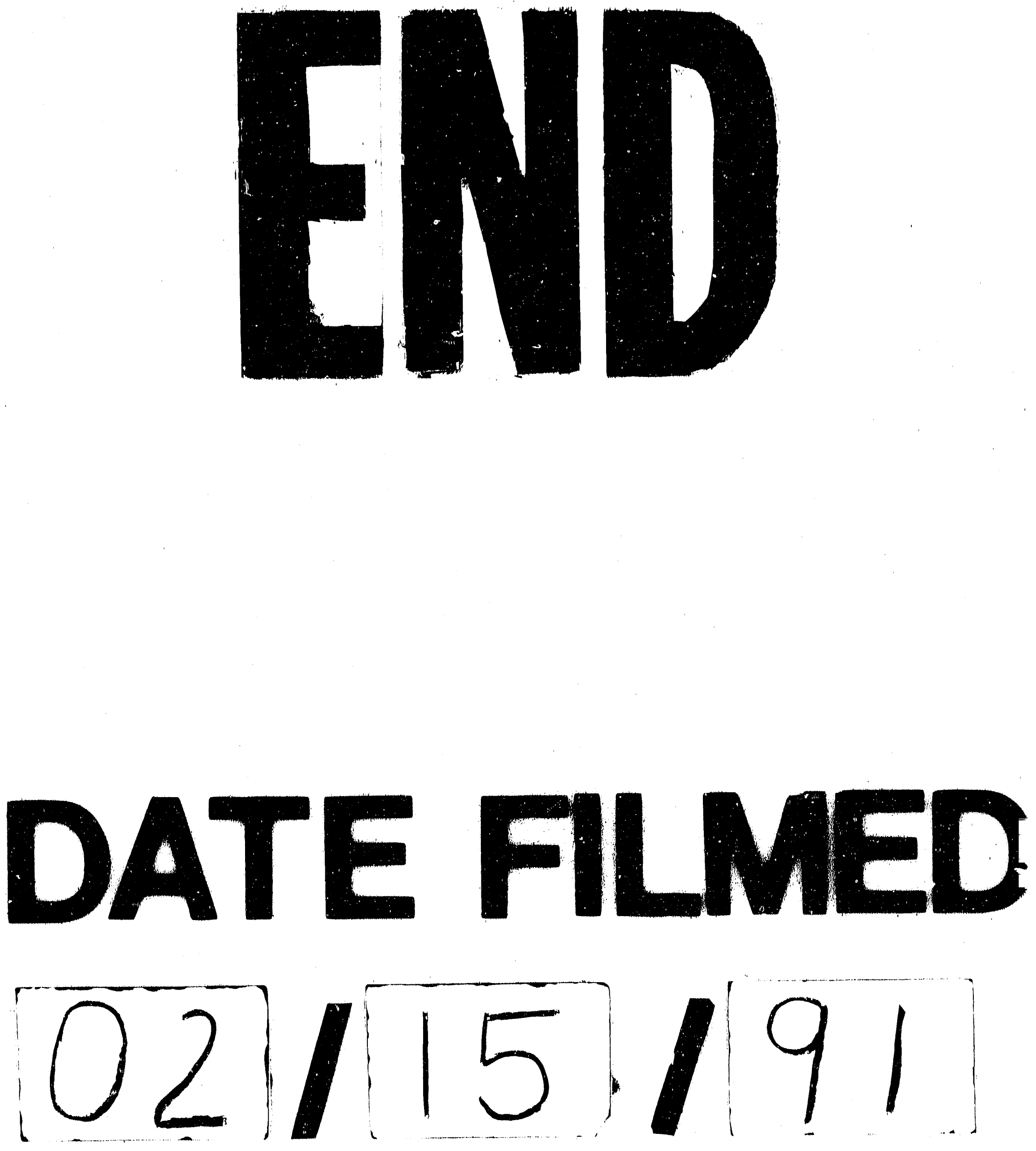